\title{
Heavy and Thermal Oil Recovery Production Mechanisms
}

\author{
By \\ Anthony R. Kovscek, Principal Investigator \\ Louis M. Castanier, Technical Manager
}

\section{Final Technical Progress Report}

\section{For the Period September 1, 2000 - December 31, 2003}

\author{
Work Performed Under Contract DE-FC26-00BC15311 \\ Prepared for \\ U.S. Department of Energy \\ Assistant Secretary for Fossil Energy
}

Sue Mehlhoff, Project Manager

National Petroleum Technology Office

P.O. Box 3628

Tulsa, OK 74101

Prepared by

Stanford University

Department of Petroleum Engineering

Green Earth Sciences, Bldg., Room 080B

367 Panama Street

Stanford, CA 94305-2220 


\section{DISCLAIMER}

This report was prepared as an account of work sponsored by an agency of the United States Government. Neither the United States Government nor any agency thereof, nor any of their employees, makes any warranty, express or implied, or assumes any legal liability or responsibility for the accuracy, completeness, or usefulness of any information, apparatus, product, or process disclosed, or represents that its use would not infringe privately owned rights. Reference herein to any specific commercial product, process, or service by trade name, trademark, manufacturer, or otherwise does not necessarily constitute or imply its endorsement, recommendation, or favoring by the United States Government or any agency thereof. The views and opinions of authors expressed herein do not necessarily state or reflect those of the United States Government or any agency thereof. 


\title{
ACKNOWLEDGEMENTS
}

Financial support for research in the area of heavy oil and thermal recovery mechanisms was provided the U.S. Department of Energy under Award No. DE-FC2600BC15311.

We acknowledge contributions from the Stanford University Petroleum Research Institute (SUPRI-A) Industrial Affiliates. All of these sources of support are acknowledged gratefully.

\author{
Aera Energy LLC \\ BP Exploration \\ ChevronTexaco \\ Conoco-Phillips \\ PDVSA Intevep \\ Shell Oil Company Foundation \\ Total \\ Tyco Thermal Controls
}




\section{SUMMARY}

The Stanford University Petroleum Research Institute (SUPRI-A) studies oil recovery mechanisms relevant to thermal and heavy-oil production. The scope of work is relevant across near-, mid-, and long-term time frames. In August of 2000 we received funding from the U. S. DOE under Award No. DE-FC26-00BC15311 that completed December 1, 2003. The project was cost shared with industry.

Heavy oil (10 to $\left.20^{\circ} \mathrm{API}\right)$ is an underutilized energy resource of tremendous potential. Heavy oils are much more viscous than conventional oils. As a result, they are difficult to produce with conventional recovery methods. Heating reduces oil viscosity dramatically. Hence, thermal recovery is especially important because adding heat, usually via steam injection generally improves displacement efficiency. The objectives of this work were to improve our understanding of the production mechanisms of heavy oil under both primary and enhanced modes of operation. The research described spanned a spectrum of topics related to heavy and thermal oil recovery and is categorized into: (i) multiphase flow and rock properties, (ii) hot fluid injection, (iii) improved primary heavy-oil recovery, (iv) in-situ combustion, and (v) reservoir definition. Technology transfer efforts and industrial outreach were also important to project effort.

The research tools and techniques used were quite varied. In the area of experiments, we developed a novel apparatus that improved imaging with X-ray computed tomography (CT) and high-pressure micromodels etched with realistic sandstone roughness and pore networks that improved visualization of oil-recovery mechanisms. The CT-compatible apparatus was invaluable for investigating primary heavy-oil production, multiphase flow in fractured and unfractured media, as well as imbibition. Imbibition and the flow of condensed steam are important parts of the thermal recovery process. The high-pressure micromodels were used to develop a conceptual and mechanistic picture of primary heavy-oil production by solution gas drive. They allowed for direct visualization of gas bubble formation, bubble growth, and oil displacement. Companion experiments in representative sands and sandstones were also conducted to understand the mechanisms of cold production. The evolution of in-situ gas and oil saturation was monitored with CT scanning and pressure drop data. These experiments highlighted the importance of depletion rate, overburden pressure, and oil-phase chemistry on the cold production process. From the information provided by the experiments, a conceptual and numerical model was formulated and validated for the heavy-oil solution gas drive recovery process.

Also in the area of mechanisms, steamdrive for fractured, low permeability porous media was studied. Field tests have shown that heat injected in the form of steam is effective at unlocking oil from such reservoir media. The research reported here elucidated how the basic mechanisms differ from conventional steamdrive and how these differences are used to an advantage. Using simulations of single and multiple matrix blocks that account for details of heat transfer, capillarity, and fluid exchange between matrix and fracture, the importance of factors such as permeability contrast between matrix and fracture and oil composition were quantified. Experimentally, we examined 
the speed and extent to which steam injection alters the permeabillity and wettability of low permeability, siliceous rocks during thermal recovery. Rock dissolution tends to increase permeability moderately aiding in heat delivery, whereas downstream the cooled fluid deposits silica reducing permeability. Permeability reduction is not catastrophic. With respect to wettability, heat shifts rock wettability toward more water wet conditions. This effect is beneficial for the production of heavy and medium gravity oils as it improves displacement efficiency.

A combination of analytical and numerical studies was used to examine the efficiency of reservoir heating using nonconventional wells such as horizontal and multilateral wells. These types of wells contact much more reservoir volume than conventional vertical wells and provide great opportunity for improved distribution of heat. Through simulation and analytical modeling of the early-time response of a reservoir to heating with a horizontal well showed that cyclic steam injection is an effective technique to heat a reservoir prior to continuous injection during a gravity drainage process.

New techniques for defining reservoir flow characteristics were developed to help us improve the recovery efficiency of heavy oil. A streamline approach was proposed and developed for inferring field-scale effective permeability distributions based on dynamic production data such as water-cut curve or tracer response. The basic idea is to relate production well data directly to the behavior of individual streamlines. Thus, flow patterns are studied in concert with geology to determine the success of recovery processes. This procedure, is robust, and reduces dramatically the computational size and time required for history matching and prediction of future recovery history. Extensions of the procedure show that the method is easily constrained to existing geological data.

\section{INTRODUCTION}

The United States continues to rely more extensively on imported oil year by year. Over the past 25 years, U.S. petroleum consumption has been growing at an average rate of $0.5 \%$ per year. The fraction of oil imported has grown from $28 \%$ of U. S. consumption in 1983 to about $55 \%$ today. Yet, the current situation has not emerged because the U.S. lacks substantial oil and gas resources. Rather, we have not been successful at conducting the research and development to develop cost-effective production techniques that allow us to convert resources into reserves. A case in point is heavy oil. Estimates place the total heavy resource (less than $20^{\circ} \mathrm{API}$ ) in the Western Hemisphere well in excess of 6 trillion bbl. The heavy oil resource in the U.S. alone is in the neighborhood of 200 billion bbl. The central problem with heavy-crude-oil production is that the oil is far more viscous than water or conventional crude oil. Because fluid flow resistance is proportional to viscosity, high viscosity frustrates production. 
This project improved our understanding of primary and thermal heavy-oil recovery mechanisms. As a result, recovery efficiency may increase and oil may be recovered where it is not producible by conventional means. In other words, this project began the development of the pathway to convert heavy-oil resources to reserves. Thermal methods, especially steam injection, where heat is used to lower oil viscosity, and carefully engineered primary (cold) production, are the best techniques for increasing production from heavy and fractured reservoirs. Hence, work was focused on thermal recovery and cold production.

The research tools and techniques employed were varied. Roughly equal weight was given to experimental investigation as it was to theory development and simulation. A guiding principle for the research effort was that experiments, physical data, and physical observations provide the ground truth against which models are judged for completeness and accuracy. Novel apparatus were developed that allow high-resolution imaging of multiphase flow through porous media. These apparatus proved invaluable for investigating primary heavy-oil production, multiphase flow in fractured and unfractured media, and for probing the effect of temperature on petrophysical properties.

\section{Organization of Report}

The research tasks are classified in five broad topical areas: (1) multiphase flow and rock properties, (2) hot-fluid injection, (3) improved primary (cold) recovery, (4) insitu combustion, and (5) reservoir definition. Technical transfer was integral to the research effort. Accordingly, this report is organized to summarize and document research performed in each area as well as to provide a discussion of technical transfer efforts and technical achievements. 


\section{EXPERIMENTAL}

Experimental studies were conducted to probe heavy and thermal oil recovery mechanisms. They are discussed in each area, as appropriate. Results obtained and related discussion are given in each topic area.

\section{RESULTS AND DISCUSSION}

A full spectrum of research in heavy and thermal oil recovery mechansms was undertaken. Results obtained and related discussion are given in the section, "Results and Discussion," in each topic area.

\section{CONCLUSIONS}

Each section lists its conclusions separately. 


\section{TABLE OF CONTENTS}

Acknowledgements $\quad$ iv

Summary $\quad$ V

Introduction vi

Organization of Report vii

Experimental viii

Results and Discussion viii

Conclusions viii

Area 1. Multiphase Flow and Rock Properties 1

References 2

One Dimensional Imbibition, Scaling, and Nonequilibrium Effects 3

(Task 1)

Introduction 3

Experimental One-dimensional Imbibition 3

Cocurrent Versus Counter Current Imbibition 5

Scaling Group $\quad 5$

$\begin{array}{lr}\text { Nonequilibrium Effects } & 9\end{array}$

Mathematical Analysis $\quad 10$

Simulation Results $\quad 11$

Experimental Apparatus $\quad 11$

$\begin{array}{ll}\text { Results and Discussion } & 13\end{array}$

$\begin{array}{lr}\text { Conclusions } & 13\end{array}$

$\begin{array}{ll}\text { Dynamic Relative Permeability from CT Data } & 19\end{array}$

(Task 1a)

Introduction $\quad 19$

$\begin{array}{ll}\text { Method } & 19\end{array}$

Results and Discussion $\quad 21$

Conclusions $\quad 26$

References $\quad 26$

Experimental and Analytical Study of Multidimensional Imbibition in $\begin{array}{lr}\text { Fractured Porous Media } & 29\end{array}$

(Task 1a)

$\begin{array}{ll}\text { Introduction } & 29\end{array}$

$\begin{array}{lr}\text { Experimental } & 30\end{array}$ 
Results and Discussion 31

Analytical Model $\quad 33$

Physically Correct Transfer Functions $\quad 35$

$\begin{array}{ll}\text { Conclusions } & 41\end{array}$

References $\quad 42$

Foam Generation at Low Surfactant Concentration 43

(Task 1b)

Introduction $\quad 43$

Model 43

$\begin{array}{ll}\text { Experimental } & 46\end{array}$

Results and Discussion $\quad 47$

Conclusions $\quad 52$

References

Scaling of Foamed Gas Mobility in Heterogeneous Porous Media 55

(Task 1b)

Introduction $\quad 55$

Foam Mobility $\quad 56$

Scaling of Foam Mobility $\quad 59$

$\begin{array}{ll}\text { Experimental } & 61\end{array}$

Results and Discussion $\quad 63$

$\begin{array}{ll}\text { Conclusions } & 70\end{array}$

$\begin{array}{ll}\text { References } & 71\end{array}$

Thermally Induced Fines Mobilization and Wettibility 75

(Task 1c)

$\begin{array}{ll}\text { Introduction } & 75\end{array}$

Wettability Alteration $\quad 75$

$\begin{array}{ll}\text { Experimental Investigation } & 77\end{array}$

Surface Forces $\quad 82$

Results and Discussion $\quad 85$

Quartz-Kaolinite System $\quad 86$

Quartz-Illite System $\quad 88$

$\begin{array}{lr}\text { Conclusions } & 89\end{array}$

$\begin{array}{lr}\text { References } & 90\end{array}$ 
Area 2. Hot-Fluid Injection 94

References $\quad 95$

A Numerical Analysis of the Single-Well Steam-Assisted Gravity

Drainage Process 96

(Task 2a)

Introduction $\quad 96$

Model Description 96

Results and Discussion $\quad 98$

Discussion of Early-Time Analysis $\quad 100$

Sensitivity Analysis 100

$\begin{array}{ll}\text { Sensitivity Cases } & 102\end{array}$

Discussion of Sensitivity Analysis 104

Conclusions 104

References 105

Analytical Model for Cyclic Steam Stimulation of a Horizontal Well 106

(Task 2a)

Introduction 106

Model Development 106

Injection and Soak Periods 107

Heat Remaining in the Reservoir 109

Production Period 111

Property Correlations $\quad 113$

Algorithm for Calculation Scheme 114

Results and Discussion $\quad 114$

Conclusions 119

$\begin{array}{ll}\text { References } & 120\end{array}$

Efficiency and Oil Recovery Mechanism of Steam Injection Into Low 121

Permeability, Hydraulically Fractured Reservoirs

(Task 2b)

Model Framework 121

Results and Discussion $\quad 122$

Efficiency 122

Stability in High Porosity Rock 124

Summary $\quad 125$

Conclusions 125

References 126 
Porosity and Permeability Evolution Accompanying Hot Fluid Injection 128 Into Diatomite

(Task 2b)

References

An Experimental Investigation of the Effect of Temperature on Recovery147 Of Heavy Oil From Diatomite

(Task 2b)

$\begin{array}{lr}\text { Introduction } & 147\end{array}$

$\begin{array}{lr}\text { Imbibition Potential } & 148\end{array}$

$\begin{array}{lr}\text { Experimental } & 149\end{array}$

$\begin{array}{ll}\text { Results and Discussion } & 150\end{array}$

Outcrop Core/Water/Mineral/Oil $\quad 150$

Outcrop Core/Water/Crude/Oil 151

Field Core/Water/Crude/Oil 152

Remaining Oil Saturation $\quad 153$

$\begin{array}{lr}\text { Interpretation } & 154\end{array}$

Scaling $\quad 154$

Wettability Alteration $\quad 155$

A Mechanism for Wettability Alteration 158

$\begin{array}{lr}\text { Conclusions } & 160\end{array}$

$\begin{array}{lr}\text { Nomenclature } & 161\end{array}$

$\begin{array}{ll}\text { References } & 162\end{array}$

Appendix A-Imbibition Potential 166

$\begin{array}{lr}\text { Appendix B-Experimental Details } & 169\end{array}$

$\begin{array}{lr}\text { Coreholder } & 169\end{array}$

$\begin{array}{ll}\text { CT Scanner } & 169\end{array}$

Rock 170

$\begin{array}{ll}\text { Fluids } & 170\end{array}$

$\begin{array}{ll}\text { Procedure } & 171\end{array}$

Area 3. Mechanisms of Primary Heavy Oil Recovery 173

A Microvisual Study of Solution Gas Drive Mechanisms in Viscous Oils 174 (Task 3a)

$\begin{array}{ll}\text { Introduction } & 174\end{array}$

Dynamics of Bubble Growth in Porous Media 175

$\begin{array}{ll}\text { Diffusion-Dominated Coalescence } & 176\end{array}$

$\begin{array}{ll}\text { Pressure-Driven coalescence } & 178\end{array}$

$\begin{array}{lr}\text { Experimental } & 183 \\ & 183\end{array}$

$\begin{array}{lr}\text { Pressure Vessel } & 183\end{array}$

$\begin{array}{ll}\text { Optics } & 186\end{array}$ 
Pressure and Fluid Delivery Systems 186

Capillary Tube Experiment 187

Results and Discussion 189

Viscous Oil 192

Comparison Among Observations and Calculations 196

Summary and Conclusions 198

Nomenclature 198

References 200

Heavy Oil Solution Gas Drive: Effect of Oil Composition and Rock 202

Consolidation

(Task 2a)

References 203

Model and Simulation of Cold Production Using a Population Balance 204

Approach

(Task 3b)

Introduction 204

Population Balance Model of Solution Gas Drive 105

Results and Discussion 205

Conclusion 209

References $\quad 210$

Area 4. In situ Combustion $\quad 211$

Solvent and Air Injection Processes 212

(Task 4a)

Introduction $\quad 212$

Experimental 212

Results and Discussion 213

Metallic Additives 216

$\begin{array}{ll}\text { (Task 4b) } & 216 \\ \text { Introduction } & 216\end{array}$

Results and Discussion 216 
Area 5. Reservoir Definition 221

A Streamline Based Technique for History Matching Production Data 222 (Task 5)

$\begin{array}{ll}\text { Introduction } & 222\end{array}$

$\begin{array}{ll}\text { Precious Work } & 223\end{array}$

$\begin{array}{ll}\text { Method } & 224\end{array}$

$\begin{array}{ll}\text { Permeability Updates } & 227\end{array}$

Steps of the Approach $\quad 227$

$\begin{array}{ll}\text { Results and Discussion } & 228\end{array}$

$\begin{array}{ll}\text { Conclusions } & 234\end{array}$

$\begin{array}{ll}\text { Nomenclature } & 234\end{array}$

$\begin{array}{ll}\text { References } & 235\end{array}$

History Matching Constrained to Geostatistical Data 238

(Task 5)

$\begin{array}{ll}\text { Introduction } & 238\end{array}$

Gauss-Markov Random Functions (GMrf) 238

Integration of GMrf into Streamline-Based History-Matching 239

$\begin{array}{ll}\text { Algorithm } & 240\end{array}$

Results and Discussion - GMrf)

Cases 1: Five-Spot, Isotropic Permeability Field, $M=1$

Cases 2: Five-Spot, Isotropic Permeability Field, M=2.5 242

Cases 3: Five-Spot, Anisotropic Permeability Fields 249

Cases 4: Prediction of Reservoir Performance Using the
History-Matched Model M=5

$\begin{array}{ll}\text { Computational Cost } & 256\end{array}$

$\begin{array}{ll}\text { Remarks } & 256\end{array}$

Streamline-Based Ranking $\quad 257$

$\begin{array}{ll}\text { Method } & 258\end{array}$

$\begin{array}{ll}\text { Conclusions } & 258\end{array}$

$\begin{array}{ll}\text { References } & 259\end{array}$

Area 6. Technology Transfer 261

Industrial Review Meeting $\quad 261$

$\begin{array}{ll}\text { Technical Presentations } & 261\end{array}$

Conference Presentations $\quad 262$

$\begin{array}{ll}\text { Technical Papers } & 263\end{array}$

Reviewed Manuscripts $\quad 264$

$\begin{array}{ll}\text { Website } & 265\end{array}$

$\begin{array}{ll}\text { Short Course } & 265\end{array}$

International Cooperation $\quad 266$

IEA Presentations 266 


\section{LIST OF TABLES}

Area 1. Multiphase Flow and Rock Properties

One Dimensional Imbibition, Scaling, and Nonequilibrium Effects

(Task 1a)

1. Viscosity of Wetting and Nonwetting Phases

2. Summary of Experimental Systems and the Ratio Viscosities of Wetting and Nonwetting Phases

Dynamic Relative Permeability from CT Data

(Task 1a)

1. Characteristics of the Berea Sandstone and Diatomite Cores

Foam Generation at Low Surfactant Concentration

(Task 1b)

1. Parameter Values

2. Limiting Capillary Pressure and Surface Tension V ( $\sigma$ from

Bertin et al., 1999)

Scaling of Foamed Gas Mobility in Heterogeneous Porous Media (Task 1b)

1. Properties of the Porous Media

2. Parameters for Gas-Mobility Calculations

3. Properties of the Porous Media

Thermally Induced Fines Mobilization and Wettability

(Task 1c)

1. Summary of Experimental Conditions

\section{Area 2. Hot-Fluid Injection}

\section{A Numerical Analysis of the Single-Well Steam-Assisted Gravity Drainage Process \\ (Task 2a)}

1. Grid, Rock, and Oil Property Description

2. Operating Conditions for Early-Time Performance Study 
Analytical Model for Cyclic Steam Stimulation of a Horizontal Well (Task 2a)

1. Physical Properties for Semi-Analytic Calculation and Simulation

Efficiency and Oil Recovery Mechanism of Steam Injection Into Low Permeability, Hydraulically Fractured Reservoirs

(Task 2b)

1. Breakthrough Comparisons in HCPVI for the Three Increasing Porosity Cases

Area 3. Mechanisms of Primary Heavy Oil Recovery Model and Simulation of Cold Production Using a Population Balance Approach

(Task 3b)

1. Porous Medium Characteristics

205

2. Fluid Properties

\section{Area 4. In-Situ Combustion}

Solvent and Air Injection Processes

(Task 4a)

1. Dilution of Cold Lake Crude Oil 212

2. Example Calculation for Solvent Injection 214

3. Example Production Data 214

\section{Area 5. Reservoir Definition}

History Matching Constrained to Geostatistical Data

(Task 5)

1. Summary of Flow Simulation Parameters for the Four Cases

2. Computational Work 


\section{LIST OF FIGURES}

\section{Area 1. Multiphase Flow and Rock Properties}

\section{One Dimensional Imbibition, Scaling, and Nonequilibrium Effects}

(Task 1a)

1. Schematic diagram of imbibition cell (a) exploded view and (b) front view illustrating injection/production configuration for countercurrent imbibition

2. CT-derived saturation maps for counter-current imbibition in water-wet diatomite. Fluid pairs are indicated on the top of each profile. Time is given in minutes beneath each saturation map

3. Nonwetting fluid recovery versus absolute time for countercurrent imbibition with varying nonwetting fluid viscosity

4. Correlation of recovery by countercurrent imbibition from diatomite

5. $S_{w}$ versus $x$ plots for different times. Simulations of co-current imbibition for a mobility ratio of 0.4

6. $S_{w}$ versus $x / \sqrt{t}$ plots. Simulations of co-current imbibition (final time: $9.65 \mathrm{~h}$ ) with four different mobility ratios

7. $S_{O}$ versus $x / \sqrt{t}$ plots for co-current imbibition in a diatomite core with air/decane system

8. $S_{w}$ versus $x / \sqrt{t}$ plots for counter-current imbibition in a diatomite core with decane/water system (total number of pixels: 200)

9. $S_{o}$ versus $x / \sqrt{t}$ plots for co-current imbibition in a diatomite core with air/decane system (total number of pixels: 300 )

10. $S_{w}$ versus $x / \sqrt{t}$ plots for counter-current imbibition in a diatomite core with decane/water system (total number of pixels: 300 )

11. $S_{O}$ versus $x / \sqrt{t}$ plots for co-current imbibition in a diatomite core with air/blandol system (total number of pixels: 300 )

12. $S_{w}$ versus $x / \sqrt{t}$ plots for counter-current imbibition in a diatomite core with blandol/water system (total number of pixels: 300 ) 
13. $S_{O}$ versus $x / \sqrt{t}$ plots for co-current imbibition in a diatomite core with air/decane system (total number of pixels: 200)

14. $S_{w}$ versus $x / \sqrt{t}$ plots for counter-current imbibition in a diatomite core with decane/water system (total number of pixels: 200)

\section{Dynamic Relative Permeability from CT Data}

(Task 1a)

1. Capillary pressure for Berea sandstone

2. Experimentally determined water relative permeability curve for Berea sandstone

3. Saturation profile obtained by simulation with measured $k_{r w}$ compared to the experimental profile for Berea sandstone

4. Capillary pressure curve used for interpretation of diatomite imbibition experiments. Data points are from Akin and Kovscek (1999)

5. Experimentally determined water relative permeability curve for outcrop diatomite

6. Saturation profile obtained by simulation with measured $k_{r w}$ compared to the experimental profile for outcrop diatomite

\section{Experimental and Analytical Study of Multidimensional Imbibition in Fractured Porous Media}

(Task 1a)

1. Possible imbibition patterns in (a) 1-D geometry (plane source),

(b) 2-D geometry (line source), and (c) 3-D geometry (point source).

Lines indicate front position as a function of time

2. The core holder: Frontal view

3. CT saturation image for $0.32 \mathrm{PV}$ imbibed, "filling-fracture." Aperture $=0.1 \mathrm{~mm}$. Injection rate $=1 \mathrm{cc} / \mathrm{min}$. Injection is from lower left corner and production from lower right corner

4. CT saturation image for 0.32 PV imbibed. "Instantly-filled fracture." Aperture $=0.025 \mathrm{~mm}$. Injection rate $=1 \mathrm{cc} / \mathrm{min}$. Injection is from lower left corner and production from lower right corner 
5. The average water saturation in the rock scales linearly with time ("fillingfracture" regime)

6. Water Iso-saturation curves for different times obtained with new approach (air-water imbibition, $\mathrm{q}=1 \mathrm{cc} / \mathrm{min}$, and $w_{f}=0.1 \mathrm{~mm}$ )

7. CT images for "filling-fracture" system for different times. Water injection at $1 \mathrm{cc} / \mathrm{min}$ in a fracture $0.1 \mathrm{~mm}$ thick. Injection is from lower left corner and production from lower right corner

8. Schematic representation of the characteristic length versus time

9. Shape factor versus time

10. Section of the grid (a) and (b) matrix relative permeability and capillary pressure functions used for the numerical simulations

11. Comparison of the average water saturation versus dimensionless time among the experimental results, analytical model, fine-grid numerical simulation, and the modified dual-porosity approach

12. Comparison of the extent of imbibition between experimental data, analytical model, modified dual-porosity and fine-grid simulation. Fracture aperture $=0.1 \mathrm{~mm}$. Injection rate $=1 \mathrm{cc} / \mathrm{min}$. Injection is from the lower left corner

\section{Foam Generation at Low Surfactant Concentration}

(Task 1b)

1. Model transient flowing bubble texture profiles (a) wt $\%$ case, (b) $0.1 \mathrm{wt} \%$ case; (c) $0.02 \mathrm{wt} \%$ case; (d) $0.01 \mathrm{wt} \%$ case; (e) $0.005 \mathrm{wt} \%$ case

2. $\quad$ Experimental (symbols connected by dashed lines) and model (solid lines) transient aqueous-phase saturation profiles. (a) $1 \mathrm{wt} \%$ case; (b) $0.1 \mathrm{wt} \%$ case; (c) $0.02 \mathrm{wt} \%$ case; (d) 0.01 case; (e) $0.005 \mathrm{wt} \%$ case

3. Experimental (symbols connected by dashed lines) and model (solid lines) transient pressure profiles. (a) $1 \mathrm{wt} \%$ case; (b) $0.1 \mathrm{wt} \%$ case; (c) $0.02 \mathrm{wt} \%$ case; (d) $0.01 \mathrm{wt} \%$ case; (e) $0.005 \mathrm{wt} \%$ case 


\section{Scaling of Foamed Gas Mobility in Heterogeneous Porous Media}

(Task 1b)

1. Prediction of the network model for flowing foam fraction compared to the experimental data of Friedmann et al. (1991)

2. Ratio of gas mobility in low and high permeability zones. Porosity of low permeability layer is varied and all other terms held constant

3. Apparatus for heterogeneous foam-displacement experiments. The mass flow meter is removed if foamer solution is injected

4. Pressure drop results for gas-only injection into heterogeneous system

5. Liquid production from each core for gas only injection into heterogeneous system

6. Gas Darcy velocity at the inlet to each core and the apparent ratio of sandstone to A10 gas mobility

7. Pressure drop results for simultaneous gas and liquid injection into heterogeneous system

8. Liquid production from each core for simultaneous injection of gas and liquid into heterogeneous system

9. Pressure drop response for gas-only and co-injection into A10 synthetic sandstone

10. Pressure response for gas-only and co-injection into sandstone

\section{Thermally Induced Fines Mobilization and Wettability}

(Task 1c)

1. Triangles in 1(a) through 3(b) illustrate pore spaces. Gray color symbolizes the water phase, black color designates oil phase, and round white circles represent clay particles

2. SEM images from Berea sandstone core used in experiments. (a) before and, (b) after waterflood

3. Experimental apparatus

4. Compositional chromatography of effluent fines collected (low salinity injection, test \#2 in core \#1, pH=7, 0.01 M NaCl) 
5. Permeability change during experiments. Open symbols indicate no fines in effluent, whereas closed symbols indicate fines production

6. View of the filtrate collected during test $3\left(120^{\circ} \mathrm{C}\right)$ from core 2 . Bright portions have large density

7. Sphere-plate system for modeling fines stability

8. Components of sphere-plate interaction and total potential at $\mathrm{pH}=7$ and $[\mathrm{Na}+]=0.1 \mathrm{M}$

9. Experimental and calculated zeta potential as a function of $\mathrm{pH}$ at constant salinity (a) Quartz, 1E-3M, NaNO3, (b) Kaolinite, E-4M NaCl

10. Detachment temperature obtained for quartz-kaolinite systems with $\xi$ potential measurements provided by Lorentz. Shading is in degree Celsius $\left({ }^{\circ} \mathrm{C}\right)$

11. Detachment temperature obtained for quartz-illite system. Shading is in degree Celsius $\left({ }^{\circ} \mathrm{C}\right)$

\section{Area 2. Hot-Fluid Injection}

\section{A Numerical Analysis of the Single-Well Steam-Assisted Gravity Drainage Process}

(Task 2a)

1. Schematic of grid system: (a) parallel to well bore and (b) perpendicular to wellbore

2. Recovery factor for the first year of production

3. Recovery factor for 10 years of production

4. Cumulative steam oil ratio for 10 years of production

5. Recovery factor versus time, sensitivity analysis

103

6. Cumulative steam oil ratio, sensitivity analysis 


\section{Analytical Model for Cyclic Steam Stimulation of a Horizontal Well}

(Task 2a)

1. (a) Schematic of heated area geometry; (b) differential element of the heated area

2. Comparison of semi-analytic model and reservoir simulation results 116

$\begin{array}{ll}\text { 3. Comparison of steam temperature } & 117\end{array}$

4. Sensitivity of cumulative oil production to the duration of injection 118

5. Sensitivity of cumulative oil production to heat losses 119

\section{Efficiency and Oil Recovery Mechanism of Steam Injection Into Low Permeability, Hydraulically Fractured Reservoirs}

(Task 2b)

1. Fraction of recovery for multi-layer case

2. Cumulative recovery for two different relative permeability curves for the "thief" model. The thermal basecase is compared to the waterflood case

\section{Porosity and Permeability Evolution Accompanying Hot Fluid Injection Into Diatomite}

(Task 2b)

1. Schematic of experimental setup

2. Porosity images for outcrop and field diatomite cores

3. Viscosity of oils and brine versus temperature

4. Measured interfacial tension between oils and water versus temperature

5. Spontaneous water imbibition in oil-field outcrop diatomite core at elevated temperatures: mineral oils

6. Spontaneous water imbibition in oil-filled diatomite cores at elevated temperatures: crude oil

7a Water saturation profile for water imbibition in oil-filled outcrop core (0-10) at $180^{\circ} \mathrm{C}$ : crude oil 
$7 \mathrm{~b}$ Water saturation distribution at $x_{D}=0.38$ versus time for crude oil/outcrop core $(0-10)$ at $\mathrm{T}=180^{\circ} \mathrm{C}$ and $S_{w i} 136$

8a Water saturation profiles for water imbibition in oil-filled field core (F-2) at $\mathrm{T}=120^{\circ} \mathrm{C}$ : crude oil

8b Water saturation profile for water imbibition in oil-filled field core (F-15) at $\mathrm{T}=180^{\circ} \mathrm{C}$ : crude oil

$8 \mathrm{c} \quad$ Water saturation distribution at $x_{D}=0.35$ versus time for crude oil/field core (F-15) at $\mathrm{T}=180^{\circ} \mathrm{C}$ and $S_{w i}=0$

9a Water saturation profile for water imbibition in oil-filled field core (F-25) at $\mathrm{T}=180^{\circ} \mathrm{C}$ : crude oil (dashed lines indicate forced imbibition)

$9 \mathrm{~b}$ Water saturation distribution at $x_{D}=0.32$ versus time for crude oil/field core (F-25) at $\mathrm{T}=180^{\circ} \mathrm{C}$ and $S_{w i}=30 \%$

10. Correlation between remaining oil saturation and temperature for diatomite core

11. Scaled spontaneous water imbibition in oil-filled outcrop diatomite core at elevated temperatures: mineral oils

12. Scaled spontaneous water imbibition in oil-filled diatomite cores at elevated temperatures: crude oil

13. Effect of temperature on imbibition potential for water imbibition in oil-filled diatomite core

14. Effect of fines production on water imbibition in oil saturated diatomite core: $\mathrm{n}$-decane

\section{Area 3. Mechanisms of Primary Heavy Oil Recovery}

\section{A Microvisual Study of Solution Gas Drive Mechanisms in Viscous Oils (Task 3a)}

1. Variation in diffusion coefficient of $\mathrm{CH}_{4}$ with oil viscosity

2. Variation in gas phase volume with time (constant pressure decline rate) 
3. (a) Side view of two gas bubbles moving towards each other in a narrow pore. (b) Cross-sectional view showing wetting-liquid distribution in a square pore and illustrating the geometry of the corner flow problem

4. Example calculation illustrating the effect of oil viscosity on pressuredriven coalescence of two gas bubbles in a cornered capillary

5 (a) Pressure-vessel developed for holding micromodels and

(b) schematic of apparatus

6 (a) Schematic of top view of micromodel illustrating injection and production ports and channels and (b) scanning electron microphotograph of micromodel etched pore pattern at 500X magnification

7. (a) Schematic of capillary tube apparatus for gravity-driven drainage of oil lens and (b) optical microphotograph of triangular capillary cross section

8. Pressure versus time for pressure depletion of $\mathrm{CO}_{2}$ saturated water in a micromodel

9. Bubble nucleation and growth in $\mathrm{CO}_{2}$ saturated water: (a) recently nucleated bubble at $0 \mathrm{~s}$, (b) bubble growth at $0.2 \mathrm{~s}$, (c) gas bubble at $0.9 \mathrm{~s}$ after growing to fill pore body, (d) bubbles expand and coalesce at $1.2 \mathrm{~s}$, (e) at $2.4 \mathrm{~s}$ bubble expands, (f) bubble is mobilized and leaves the pore space where it was nucleated at $2.8 \mathrm{~s}$

10. Repeated bubble nucleation and growth at the same site as shown in Fig. 9a recently nucleated buble at $0 \mathrm{~s}$, (b) bubble growth at $0.5 \mathrm{~s}$, (c) gas Bubble at $0.8 \mathrm{~s}$ after growing to fill pore body, (d) bubbles expands into Adjacent pore space at 1.0

11. Solubility of $\mathrm{CO}_{2}$ in the viscous mineral oil Kaydol as a function of pressure 193

12. Pressure versis time for pressure depletion of $\mathrm{CO}_{2}$ saturated viscous oil in a micromodel

13. Bubble nucleation and growth in $\mathrm{CO}_{2}$ saturated viscous oil: (a) recently Nucleated bubble at $0 \mathrm{~s}$, (b) bubble growth at $1 \mathrm{~min} 31 \mathrm{~s}$, (c) gas bubble Grows to fill pore body at $2 \mathrm{~min} 50 \mathrm{~s}$, (d) bubbles expands into adjacent Pore space at 3 min $6 \mathrm{~s}$, (f) bubble is mobilized and leaves the pore space Where it was nucleated at $3 \min 8 \mathrm{~s}$

14. Bubble coalescence in viscous oil: (a) three bubbles arrayed in pores at $0 \mathrm{~s}$,

(b) coalescence of upper two bubbles at $10 \mathrm{~s}$ as gas flows into pore space

(c) drainage of thick liquid lens and coalescence of gas bubble at $42 \mathrm{~s}$ 
15. Distance-time plot of coalescence in capillary tubes. Solid line shows calculations using physical model; dashed lines are experiments

\section{Model and Simulation of Cold Production Using a Population Balance Approach}

(Task 3b)

1. Match of the IN bubble population balance model to the experimental pressure data of Firoozabadi et al. (1992). Withdrawal rate is $1.44 \mathrm{~cm}^{3} /$ day $\left(5.10 \times 10-5 \mathrm{ft}^{3} /\right.$ day $)$ and the number of bubbles nucleated is 0.0124 bubbles $/ \mathrm{cm}^{3}$ (350 bubbles $/ \mathrm{ft}^{3}$ )

2. Match of the IN bubble population balance model to the experimental pressure data of Firoozabadi et al. (1992). Withdrawal rate is $7.20 \mathrm{~cm}^{3} /$ day $\left(2.50 \times 10^{-4} \mathrm{ft}^{3} /\right.$ day $)$ and the number of bubbles nucleated is 0.0353 bubbles $/ \mathrm{cm}^{3}$ (1000 bubbles $\left./ \mathrm{ft}^{3}\right)$

3. Match of the IN bubble population balance model to viscous-oil solution gas drive pressure data. Withdrawal rate is $6.00 \mathrm{~cm}^{3} /$ hour $\left(2.10 \times 10^{-4} \mathrm{ft}^{3} / \mathrm{hr}\right)$ and the number of bubbles nucleated is 0.490 bubbles $/ \mathrm{cm}^{-4} \mathrm{ft}^{3} / \mathrm{day}$ ) (14,000 bubbles/ $\mathrm{ft}^{-4} \mathrm{ft}^{3} /$ day)

\section{Area 4. In-Situ combustion}

\section{Solvent and Air Injection Processes}

(Task 4a)

1. Viscosity of mixtures of Cold Lake oil and n-decane. Concentration reported in volume per cent

\section{Metallic Additives}

(Task 4b)

1. Effluent gas composition, no additives

2. Effluent gas composition, tin with $5 \% \mathrm{Sn} \mathrm{cl}_{2}$ solution 


\section{Area 5. Reservoir Definition}

\section{A Streamline Based Technique for History Matching Production Data (Task 5)}

1. Comparison of permeability fields (a) reference, (b) intial, and (c) history matched. Shading indicates permeability in md

2. Fractional flow curves for test case

3. Pressure history for test case

\section{History Matching Constrained to Geostatistical Data}

(Task 5)

1. Reference permeability field, histogram and variogram $100 \times 100$

2. Permeability field at iterations $1,2,5$ and 9 . The left column is prior to the GMrf process, while the right is after the GMrf and therefore consistent with geology

3. History-matched permeability field $(100 \times 100)$, five-spot, $M=1$

4. Match in water-cut, five-spot, $100 \times 100$ grid-blocks, $M=1$

5. History-matched permeability field $(100 \times 100)$, five-spot, $M=2.5$

6. Match in water-cut five-spot $100 \times 100$ grid-blocks, $M=2.5$

7. Reference permeability field, histogram and variogram $200 \times 200$ grid blocks

8. History-matched permeability field (200×200 grid-blocks), five-spot, $M=1$

9. Match in water-cut, five-spot, $200 \times 200$ gridblocks, $M=1$

10. Reference and history-matched permeability fields

11. Match and prediction of water-cut. Matched to a production history of 150 days. The rest is prediction

12. Match and prediction of cumulative oil production. Matched to a production history of 0.75 PVI. The rest is predictioin 


\section{Area 1. Multiphase Flow and Rock Properties}

Application of enhanced oil recovery processes and simulation of these processes demands understanding of the physics of displacement and accurate representation of constitutive relations such as relative permeability and capillary pressure. We developed new experimental apparatus and interpretation procedures to collect this type of information. A common tool throughout much of the experimental portion of the research is the imaging of oil, water, and gas saturations in situ. This is accomplished primarily through the use of X-ray computed tomography $(\mathrm{CT})$. X-ray CT allows us to obtain the position and shapes of displacement fronts in porous media as a function of time.

Our work in the area of multiphase flow was divided into (a) Imbibition, (b) Mobility Control of Steam, and (c) Temperature Effects of Relative Permeability.

Task 1a considered imbibition in one-dimensional and multi-dimensional systems. Water imbibition is fundamental to steamdrive and waterflood performance of fractured and unfractured rocks. The rate and the extent of imbibition depend critically on the wettability of the rock and the viscosity of the wetting and nonwetting phases. Other factors include: fluid/fluid interfacial tension (IFT), pore structure, the initial water saturation of the rock, and relative permeability curves. Water injection, steam injection, and $\mathrm{CO}_{2}$ injection in a water-alternating gas (WAG) fashion all rely to some extent on capillary imbibition to aid oil production. Steam injection is, for all practical purposes, carried out under saturated conditions with some fraction of the injected steam in the liquid phase. Likewise, initial heating of a cold reservoir is accompanied by condensation and flow of the resulting hot water away from the injector (Hoffman and Kovscek, 2003). In $\mathrm{CO}_{2}$ injection under both miscible and immiscible conditions, water slugs are usually injected with the objective of controlling $\mathrm{CO}_{2}$ mobility. Thus, capillary phenomena are important to most recovery techniques of interest in low permeability media.

Work in Area 1a is summarized in a series of sections. The first section is entitled "One dimensional imbibition, scaling, and nonequilibrium effects," whereas the second is "Dynamic relative permeability from CT data." The third section that summarizes our efforts in Task 1a is "Experimental and analytical study of multidimensional imbibition in fractured porous media."

Task $1 \mathrm{~b}$ examined the gas mobility reduction properties of aqueous foams. Mobility control of enhanced oil recovery fluids such as steam or $\mathrm{CO}_{2}$ is an important problem. Aqueous surfactants have shown great promise for gas mobility control. Foam is a dispersion of gas within a surfactant-laden continuous liquid phase. The surfactant provides a measure of stability against coalescence of bubbles; foaming surfactants increase significantly the period of time that any individual bubble persists. Effective foams can be formed with the addition of surfactant chemical to brine at concentrations on the order of 0.05 to $1 \mathrm{wt} \%$. In the presence of foamed gas, gas and liquid flow behavior is determined by bubble size or foam texture.

Our work in this area was two fold. Effort was directed toward understanding and modeling foam generation and stability at low surfactant concentrations. Foam displacement experiments were conducted at a variety of surfactant concentrations in homogeneous one 
dimensional sandpacks. Modeling and interpretation of the experiments was also accomplished. This work is summarized in the section, "Foam generation and stability at low surfactant concentration" Second, we expanded our mechanistic model to foam processes in heterogeneous porous media (Kovscek et al., 1997). Specifically, we began with a mechanistic description of aqueous foam behavior in one-dimensional media and conducted a thorough analysis of how the physically-based foam-simulation parameters scale with permeability and rock type. The two major components of the population balance model considered were bubble size and gas mobility. Bubble size is determined by the interplay of foam generation and coalscence forces that are described by rate expressions. Thus, by considering individually the mathematical expressions and rate constants describing foam generation and coalescence, we ascertained the manner in which gas-phase mobility shifts as permeability is increased or decreased. This work is entitled "Scaling of foamed gas mobility in heterogeneous porous media."

Task 1c developed an understanding of the mechanisms by which rocks become more water wet during thermal operations. Reservoir rocks are thought to become more water wet during thermal recovery. To date, no plausible mechanism describes this shift toward water wetness. Fines mobilization presents one pathway to create fresh water wet surfaces. Work in Area 1c is summarized as "Thermally induced fines mobilization and wettability."

\section{References}

Hoffman, B. T. and A. R. Kovscek, 2003. "Light-Oil Steamdrive in Fractured Low Permeability Reservoirs," SPE 83491, Proceedings of the SPE Western Region/AAPG Pacific Section Joint Meeting, Long Beach, CA, May 19-24. 


\section{One Dimensional Imbibition, Scaling, and Nonequilibrium Effects}

\section{(Task 1a)}

\section{Introduction}

Imbibition studies are naturally divided into experiments that probe behavior in the matrix, flow in fractures, and matrix/fracture interactions. To advance the state of the art of analysis of imbibition experiments, we developed an unconventional mode of collecting CT data. In a conventional experiment, X-ray CT data is taken in slices normal to the central (i.e., long) axis of the core or sand pack. The porous medium is translated through the CT scanner in a series of steps with a CT exposure collected at every step. Thus, many CT exposures are needed to visualize the progress of fluid and data is missing from the volume of porous medium lying between scan positions. Figure 1 illustrates the "imbibition cell" that we developed to ameliorate the problems associated with conventional scanning. It is possible to examine the entire length of a core with a single CT scan. Thus, the cross-section of the coreholder and the water jacket shown schematically in Fig. 1b represents the scanned volume. In addition to providing temperature control, the shape of the water jacket and the large mass density of water minimizes imaging problems (cf. Akin and Kovscek, 2003) for a discussion of CT imaging applications and the associated difficulties). In the schematic, flow is one dimensional and imbibition may occur cocurrently (water and oil flow the same direction) or countercurrently (water and oil flow in opposite directions). The endcaps for the core are designed to accommodate a variety of flow conditions. CT images obtained are of unprecedented resolution and the CT image is easily converted to quantitative saturation information.

\section{Experimental One-dimensional Imbibition}

The imbibition cell and the unconventional scan geometry became a cornerstone of our approach to CT imaging of multiphase flow in porous media. With the apparatus illustrated in Fig. 1, we contrasted cocurrent and counter-current flow characteristics, validated a new scaling group for one-dimensional counter-current imbibition, demonstrated that it is possible to measure and quantify nonequilibrium multiphase flow effects during cocurrent and counter current imbibition, and, most importantly, we developed a technique to extract dynamic relative permeability from the shape and time evolution of saturation profiles measured within the imbibition cell. Each of these advances is discussed briefly. 


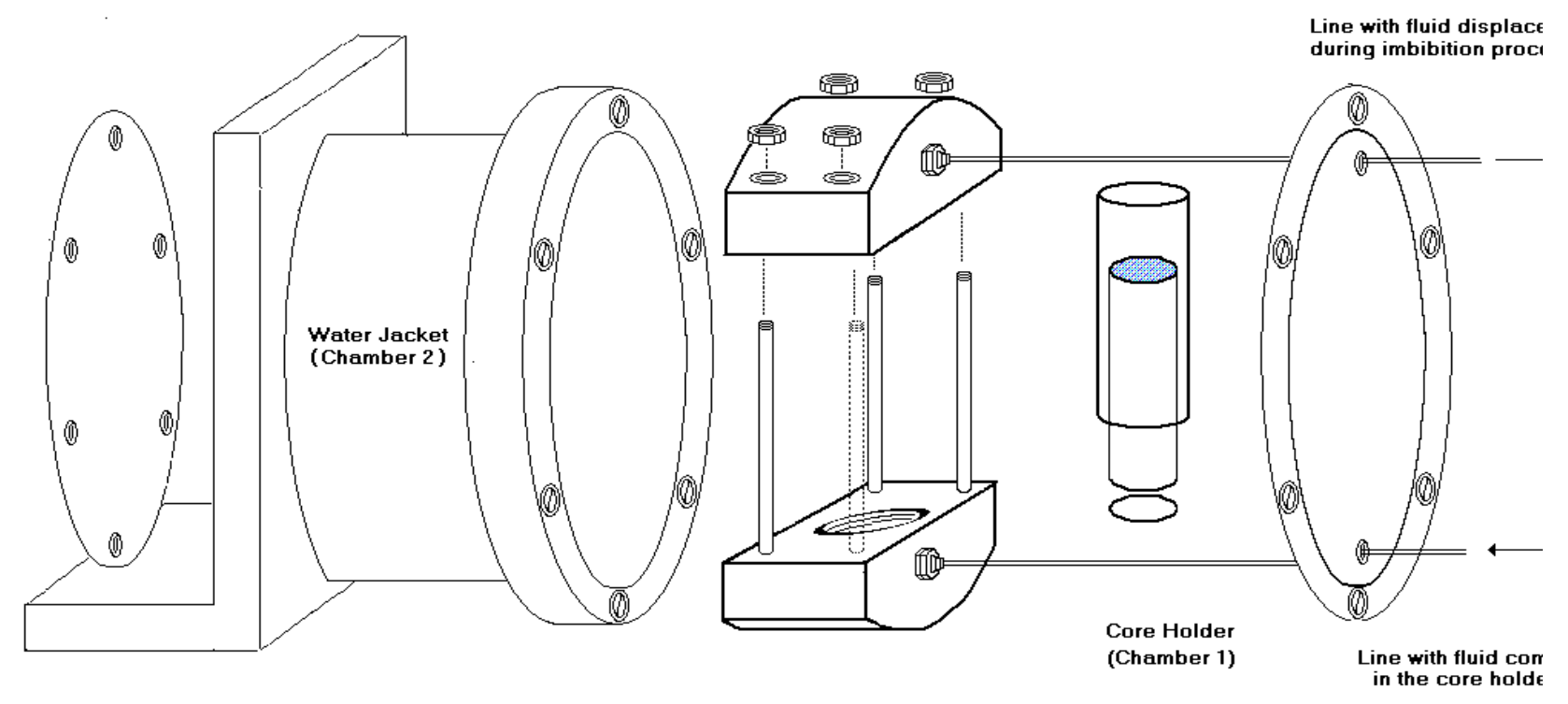

(a)

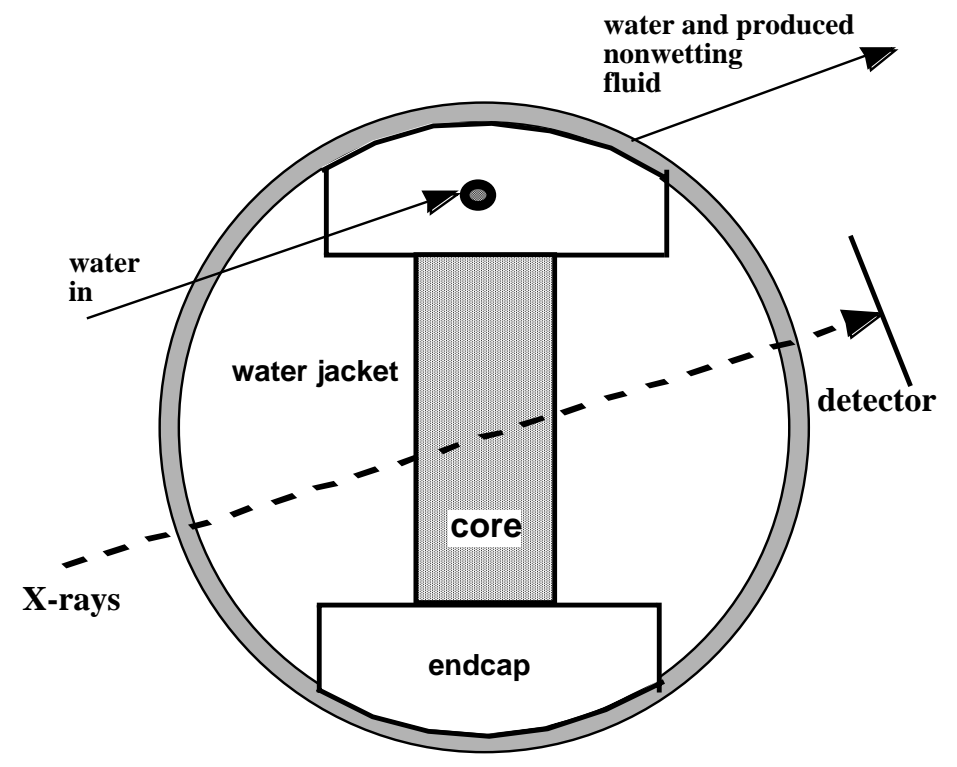

(b)

Figure 1. Schematic diagram of imbibition cell (a) exploded view and (b) front view illustrating injection/production configuration for countercurrent imbibition. 


\section{Cocurrent Versus Counter Current Imbibition}

There has been considerable debate recently as to whether cocurrent and countercurrent imbibition mechanisms differ fundamentally. The implications are profound for the modeling of oil recovery from fractured media. The imbibition cell allowed direct measurement of the shape and time progression of saturation fronts inside of a porous medium imbibing water spontaneously. Essentially, our experimental results showed that cocurrent imbibition exhibits displacement characteristics that are relatively pistonlike, whereas countercurrent imbibition profiles are much more diffuse (Zhou et al., 2002). We interpreted these measurements to indicate that during cocurrent imbibition water advances through pores and throats relatively uniformly displacing nonwetting fluid. During countercurrent flow, water movement is more dominated by flow along the nooks and crannies of the pore space. Water saturation builds in pore corners whereas the nonwetting fluid occupies the center of pore spaces as well as the largest pores. Interestingly, an independent study utilizing a new nuclear magnetic resonance technique with resolution on the $10 \mu \mathrm{m}$ scale confirmed and cited our interpretation as a cornerstone study (Chen et al., 2003).

\section{Scaling Group}

Our interpretation of imbibition displacement also shed new light on scale up of imbibition phenomena. Scaling of imbibition oil recovery has been pursued for roughly 40 years (Aronofsky et al., 1958; Mattax and Kite, 1962). The chief reason for our dogged pursuit of scaling is that one extrapolates accurately from the laboratory to the field when scaling is accurate. Most scaling approaches to date were empirical and did not address the possible significant difference in water and oil mobility that arises for moderately to more viscous oils. Experiments conducted in the imbibition cell illustrated in Fig. 1 employed nonwetting fluids whose viscosity varied by 4 orders of magnitude (Zhou et al., 2002). Results showed unequivocally that the advance of wetting liquid slows and saturation patterns become increasingly diffuse as the ratio of nonwetting to wetting liquid viscosity increases. Figure 2 presents a montage of saturation profiles as measured by X-ray CT for water displacing air, water displacing $n$-decane $\left(\mu_{\mathrm{o}}=0.84 \mathrm{cP}\right)$, and water displacing a viscous mineral oil $\left(\mu_{\mathrm{o}}=25 \mathrm{cP}\right)$. Tables 1 and 2 provide a summary of the experimental conditions. The elapsed time from the introduction of water is given in the figure caption for each exposure for each combination of wetting and nonwetting fluid. In short, the experimental results taught us that scaling of countercurrent imbibition needed to be revisited.

Table 1. Viscosity of Wetting and Nonwetting Phases

\begin{tabular}{|l|c|}
\hline Fluid & Viscosity (mPa.s) \\
\hline Air & 0.018 \\
Decane & 0.84 \\
Blandol & 25 \\
Water & 1.0 \\
\hline
\end{tabular}


Table 2. Summary of Experimental Systems and the Ratio

Viscosities of Wetting and Nonwetting Phases

\begin{tabular}{|llc|}
\hline $\begin{array}{l}\text { Imbibition } \\
\text { process }\end{array}$ & System & $\begin{array}{l}\text { Ratio of } \\
\text { viscosities }\end{array}$ \\
\hline Co-current & Air - Decane & 0.021 \\
& Air - Blandol & 7.2 \\
\hline \multirow{3}{*}{ Counter-current } & $\begin{array}{l}\text { Decane - } \\
\text { Water }\end{array}$ & 0.84 \\
& Blandol - Water & 25 \\
\hline
\end{tabular}

Rather than proceed phenomenologically, scaling of counter current recovery was examined theoretically from a fresh perspective. Full details are found in (Zhou et al., 2002). Essentially, flow is assumed to be one-dimensional, gravity is negligible, and the fluid are incompressible. Applying Darcy's law and invoking capillarity yields after considerable manipulations:

$$
\mathrm{u}_{\mathrm{w}}=\mathrm{k} \frac{1}{\sqrt{\mu_{\mathrm{w}} \mu_{\mathrm{nw}}}} \frac{\sqrt{\mathrm{k}_{\mathrm{rnw}} \mathrm{k}_{\mathrm{rw}}}}{\sqrt{\mathrm{M}}+\frac{1}{\sqrt{\mathrm{M}}}} \frac{\partial \mathrm{P}_{\mathrm{c}}}{\partial \mathrm{S}_{\mathrm{w}}} \frac{\partial \mathrm{S}_{\mathrm{w}}}{\partial \mathrm{x}}
$$

where $\mathrm{M}\left(=\left(\mu_{\mathrm{nw}} \mathrm{k}_{\mathrm{rw}} / \mu_{\mathrm{w}} \mathrm{k}_{\mathrm{rnw}}\right)\right)$ is the mobility ratio.

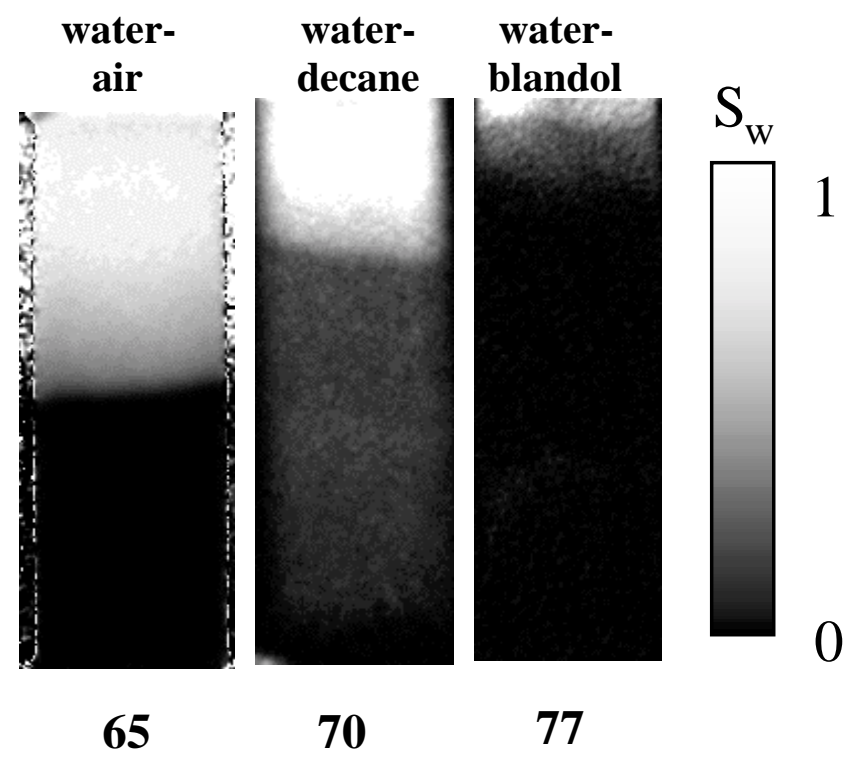

Figure 2. CT-derived saturation maps for counter-current imbibition in water-wet diatomite. Fluid pairs are indicated on the top of each profile. Time is given in minutes beneath each saturation map. 
The form of Eq. 1 results from the restriction to counter-current flow. It suggests that counter-current imbibition is diffusion-like and controlled by the product of the mobility in both phases. Note that the effects of viscosity are accounted for by $\sqrt{\mu_{\mathrm{nw}} \mu_{\mathrm{w}}}$ and $\sqrt{\mathrm{M}}+\frac{1}{\sqrt{\mathrm{M}}}$. The values of the second term are not very sensitive to $\mathrm{M}$, when $\mathrm{M}$ is of order unity. However, when the value of $M$ is substantially different from unity, the contribution of $\sqrt{M}+\frac{1}{\sqrt{M}}$ can be significant. The following dimensionless time follows from Eq. 1.

$$
\mathrm{t}_{\mathrm{D}}=\mathrm{t} \sqrt{\frac{\mathrm{k}}{\phi}} \frac{\sigma}{\mathrm{L}_{\mathrm{c}}^{2}} \sqrt{\lambda_{\mathrm{rw}}^{*} \lambda_{\mathrm{rnw}}^{*}} \frac{1}{\sqrt{\mathrm{M}^{*}}+\frac{1}{\sqrt{\mathrm{M}^{*}}}}
$$

where $\lambda_{\mathrm{r}}^{*}\left(=\mathrm{k}_{\mathrm{r}}^{*} / \mu\right)$ is a characteristic mobility for the wetting and nonwetting phases and $\mathrm{M}^{*}$ $\left(=\lambda_{\text {rw }}^{*} / \lambda_{\text {rnw }}^{*}\right)$ is a characteristic mobility ratio. Here, end-point relative permeabilities are used when calculating $\lambda_{\mathrm{r}}^{*}$ and $\mathrm{M}^{*}$. Equation 2 is similar to prior empirically determined (Zhang et al., 1996) scaling of $t_{D}$ with respect to phase viscosity. In the limit of $M^{*}$ approaching unity, $t_{D}$ varies inversely with the geometric mean of $\mu_{\mathrm{nw}}$ and $\mu_{\mathrm{w}}$. Note also that Eq. 2 explains water/gas imbibition scaling. For water/gas cases, $\sqrt{\mathrm{M}^{*}}<1 / \sqrt{\mathrm{M}^{*}}$ and the scaling of $t_{\mathrm{D}}$ with viscosity is approximately $1 / \mu_{\mathrm{w}}$ as indicated empirically (c.f., Handy, 1960). In short, our work resolves the discrepancies in scaling of counter current imbibition introduced by prior researchers.

The above discussion is based on the assumption that the relative permeability and the capillary pressure functions are similar for all of the measurements. In counter-current imbibition, the total water imbibed is controlled by matrix adjacent to the fracture. Thus, if $S_{w}$ at the inlet does not vary significantly, the oil recovery is proportional to $\sqrt{t_{D}}$ until the imbibition front reaches the outlet boundary. The capillary driving force, $\partial \mathrm{P}_{\mathrm{c}} / \partial \mathrm{S}_{\mathrm{w}}$, increases as water saturation decreases, and, consequently, the imbibition rate is larger at low water saturation than at high water saturation. Therefore, the oil recovery may depart from $\sqrt{t_{D}}$ (see discussion).

To recap, the oil recovery from counter-current imbibition is approximately proportional to the square-root of time during the initial infinite-acting period. The dimensionless time for counter-current imbibition can be scaled by Eq. 2, which is more general and explains the empirical findings of Zhang et al. (1996).

Figures 3 and 4 display the efficacy of the scaling group given in Eq. 2. For reference, Fig. 3 presents the raw data as interpreted by CT scanning. Note that recovery rate decreases substantially as the viscosity ratio between nonwetting and wetting fluids increases. To obtain oil recovery, the CT-measured saturation data are averaged over the entire core. Figure 4 summarizes the new scaling approach. Note that recovery has been scaled to the fraction of recoverable oil. For scaling, the endpoint relative permeabilities must be chosen. In concert with our interpretation of relative permeability from spontaneous imbibition dynamics (Schembre and Kovscek, 2001, 2003), the end points for water/air displacements are $\mathrm{k}_{\mathrm{rw}}^{*}$ equal to roughly 0.14 
and $\mathrm{k}_{\mathrm{rnw}}^{*}$ is chosen as 0.60 . In the water/oil cases, $\mathrm{k}_{\mathrm{rw}}^{*}$ and $\mathrm{k}_{\mathrm{rnw}}^{*}$ are set to 0.14 and 0.45 , respectively. These endpoint values are representative of fine-grained, strongly water-wet diatomite (Schembre and Kovscek, 2001). The recovery from the water/air system and all of the water/oil systems agree reasonably well. Within experimental scatter, the data are reduced to a single curve in spite of the fact that the nonwetting fluid viscosity varies by 4 orders of magnitude. The new dimensionless time improves significantly the scaling of spontaneous imbibition in low permeability porous media.

The good scaling of recovery from systems with different viscosity ratios suggests that the general shapes of the recovery curves as a function of dimensionless time are similar over the viscosity range studied. The scaling of the simulation results demonstrate that the end-point mobility ratio contributes to imbibition rate significantly. The end-point mobility ratio is also a measure of the wettability of the systems. For strongly water-wet rocks, such as clean sandstone, the relative permeability to water can be very small, resulting in small end-point mobility ratios. On the other hand, for weakly water-wet systems, the water-end point relative permeability can be large, resulting in large end-point mobility ratios. An interesting application of Eq. 2 as discussed later, is that the scaling group is useful for interpreting the wettability of porous media (Tang and Kovscek, 2002A, 2002B).

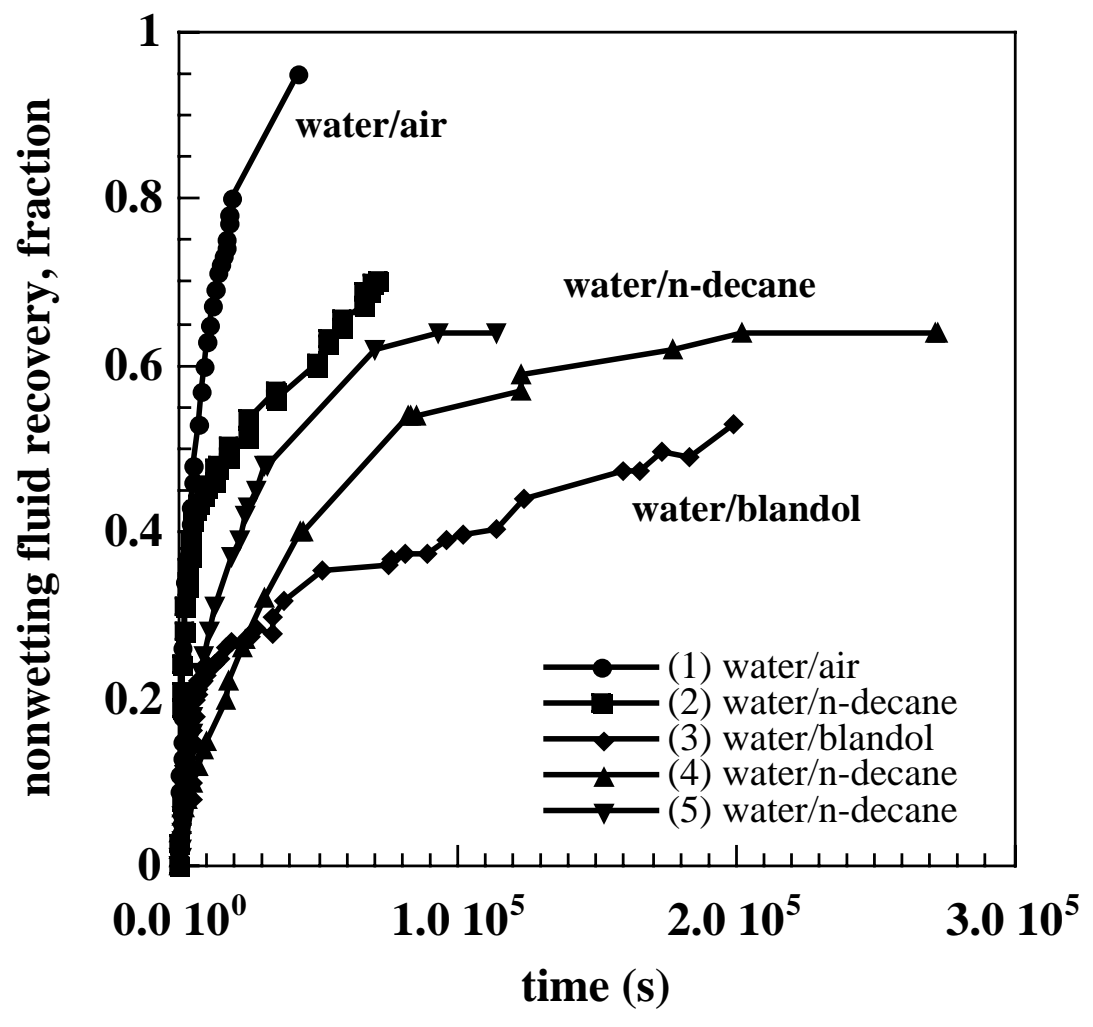

Figure 3. Nonwetting fluid recovery versus absolute time for countercurrent imbibition with varying nonwetting fluid viscosity. 


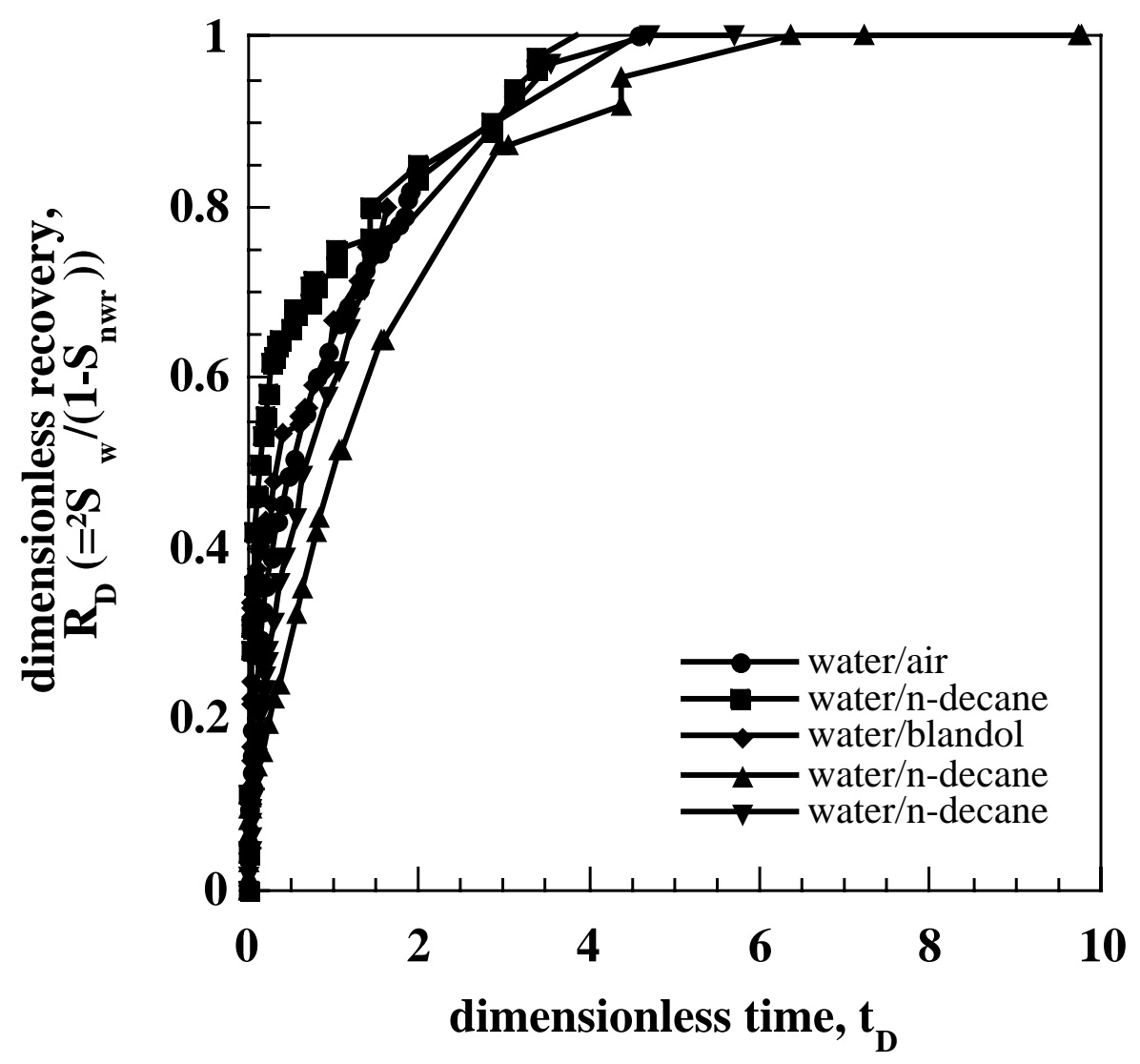

Figure 4. Correlation of recovery by countercurrent imbibition from diatomite.

\section{Nonequilibrium Effects}

Imbibition is, fundamentally, a nonequilibrium process (Silin and Patzek, 2003). By imbibing water, a porous medium is attempting to minimize the free energy of the wettingnonwetting phase interface and thereby come to a minimum equilibrium state. Nevertheless, it has been rather difficult to demonstrate conclusively that nonequilibrium effects change significantly the flow expected when equilibrium effects dominate flow. The imbibition cell yields experimental results of sufficient precision to allow unambiguous interpretation of nonequilibrium effects. Our results in this area are unpublished to date. Hence, a fair amount of detail is given here to substantiate the approach and results.

This study deals with the processes of counter-current imbibition and the role of nonwetting phase viscosity. We intend to examine imbibition performance over a 5-order of magnitude range of non-wetting phase viscosity. 


\section{Mathematical Analysis}

The flow of two immiscible fluids in a porous medium can be modeled using Darcy's and mass conservation laws. In the following mathematical model of counter-current imbibition, local equilibrium is assumed to be reached in both phases. Therefore relative permeabilities and capillary pressure are unique functions of the water saturation.

Neglecting the gravity forces, Darcy's law in one dimension gives an expression of the velocity of each phase (Marle, 1981),

and

$$
\begin{aligned}
& u_{w}=-\frac{k k_{r w}}{\mu_{w}} \frac{\partial p_{w}}{\partial x} \\
& u_{o}=-\frac{k k_{r o}}{\mu_{o}} \frac{\partial p_{o}}{\partial x}
\end{aligned}
$$

where $\mathrm{k}$ is the permeability, $\mathrm{k}_{\mathrm{r}}$ the relative permeability, and $\mu$ the phase viscosity. The pressure in the two fluids is linked by the capillary pressure:

$$
P_{c}=p_{o}-p_{w}=\sigma \sqrt{\frac{\phi}{k}} J
$$

where $\sigma$ is the interfacial tension, $\phi$ the porosity, and $\mathrm{J}$ is called the Leverett function. With the assumption of local equilibrium, we can find expressions for $\mathrm{J}\left(\mathrm{S}_{\mathrm{w}}\right), \mathrm{k}_{\mathrm{rw}}\left(\mathrm{S}_{\mathrm{w}}\right)$ and $\mathrm{k}_{\mathrm{ro}}\left(\mathrm{S}_{\mathrm{w}}\right)$. Mass conservation allows us to link the water saturation to its respective velocity:

$$
\phi \frac{\partial S_{w}}{\partial t}+\nabla \cdot u_{w}=0
$$

and

$$
\phi \frac{\partial\left(1-S_{w}\right)}{\partial t}+\nabla \cdot u_{o}=0
$$

Moreover, if we substitute the expression of $\mathrm{u}_{\mathrm{w}}$ (Eq. 3) into the Eq. 6, we can write,

with

$$
\frac{\partial}{\partial x}\left(D\left(S_{w}\right) \frac{\partial S_{w}}{\partial x}\right)=\frac{\partial S_{w}}{\partial t}
$$

$$
D\left(S_{w}\right)=-\frac{k k_{r o}}{\phi \mu_{o}} f\left(S_{w}\right) \frac{d P_{c}}{d S_{w}}
$$

The function $\mathrm{f}$ is called the fractional flow and is defined as

$$
f\left(S_{w}\right)=\frac{1}{1+\frac{k_{r o}}{k_{r w}} \frac{\mu_{w}}{\mu_{o}}}
$$


Equation 8 is described as a non-linear diffusion equation with a diffusion coefficient equal to D. The diffusion coefficient is a strongly non linear function of saturation and has a skewed bell shape (Chen et al., 1995). An important parameter in petroleum engineering is the mobility ratio which is defined as:

$$
M=\frac{k_{r w}}{k_{r o}} \frac{\mu_{o}}{\mu_{w}}
$$

Nevertheless, this mathematical model is based on the assumption of local equilibrium. According to Silin and Patzek (2003), the classical equations may not be valid for the first stage of capillary imbibition in a porous medium or at the oil-water interface. Viscous or other modes of dissipation may result in behavior that deviates from the classical formulation.

\section{Simulation Results}

The flow simulator Eclipse provides us with saturation values along the core at different times. Some numerical simulations have been performed to model the co-current imbibition mechanism in an horizontal core so the gravity forces are not taken into account. The code has a grid of 200 blocks and requires the input of rock and fluid properties. The porosity of the rock is 0.71 , and the permeability is $6.6 \mathrm{mD}$. The interfacial tension is set to $51.4 \mathrm{mN} / \mathrm{m}$ (Zhou et al., 2002). Also, the relative permeability end-points are 1 for oil and 0.5 for water.

Figure 5 shows the plot of the water saturation profiles obtained along the core at different times. Water viscosity is $1 \mathrm{cp}$ and oil viscosity is $2.5 \mathrm{cp}$. Figure 6 displays the plot of the water saturation versus the similarity variable $\eta=\frac{x}{\sqrt{t}}$ for a variety of mobility ratios.

We note that the saturation profiles collapse for the different times and a given mobility ratio. This is the behavior of a diffusion equation such as Eq. 8. The saturation front appears larger for a smaller value of the mobility ratio.

\section{Experimental Apparatus}

The apparatus shown in Fig. 1 is used to conduct experiments that verify the measurability of nonequilibrium effects during imbibiton. The setup allows for both co-current and counter-current imbibition in spontaneous and forced modes. Several experiments have been conducted in order to analyze the process of counter-current imbibition in diatomite cores. The value of the mobility ratio has been modified by changing the viscosity of the non-wetting phase from 0.018 to 0.84 to $25 \mathrm{cp}$. 


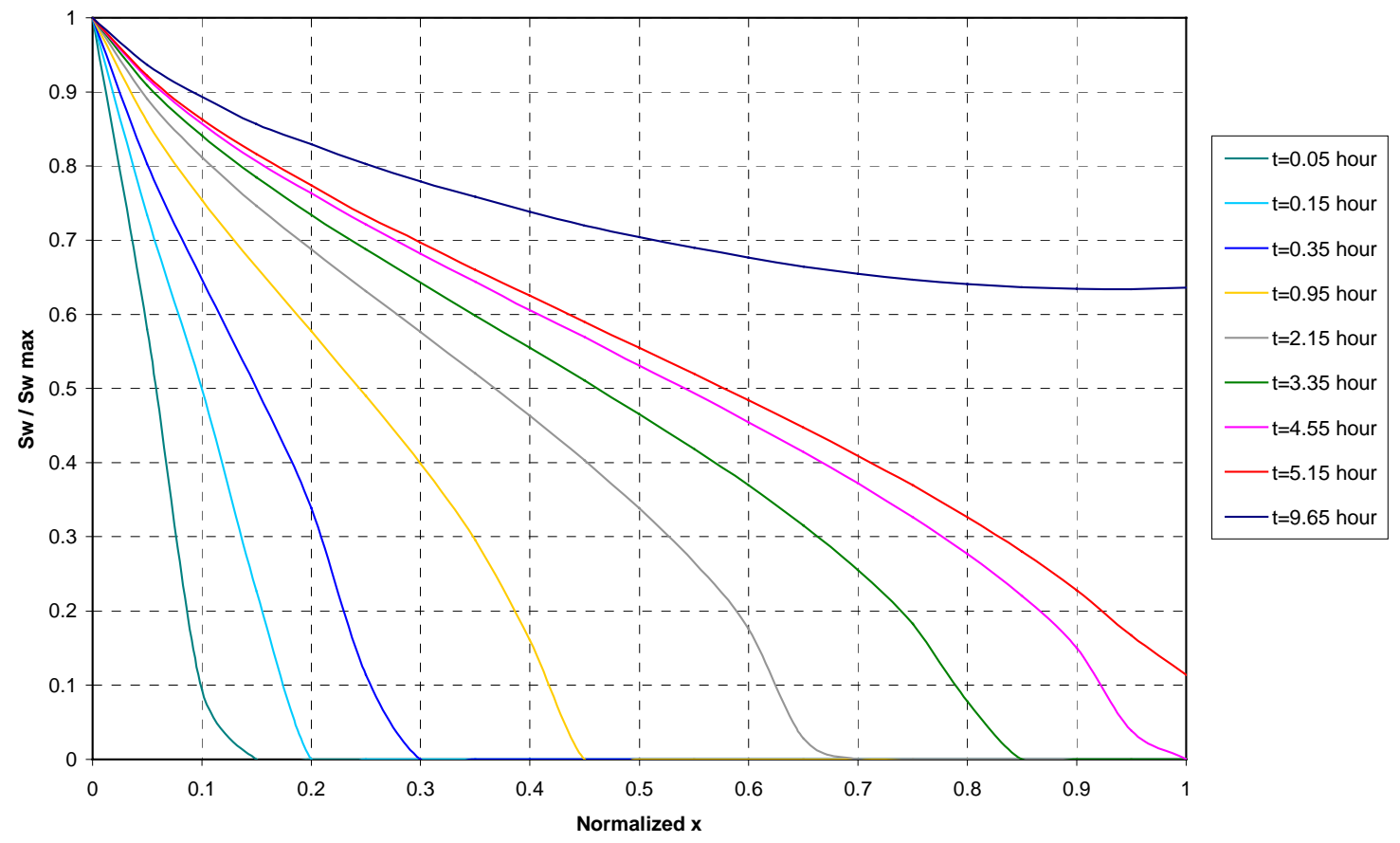

Figure 5. $\quad \mathrm{S}_{\mathrm{w}}$ versus x plots for different times. Simulations of co-current imbibition for a mobility ratio of 0.4 .

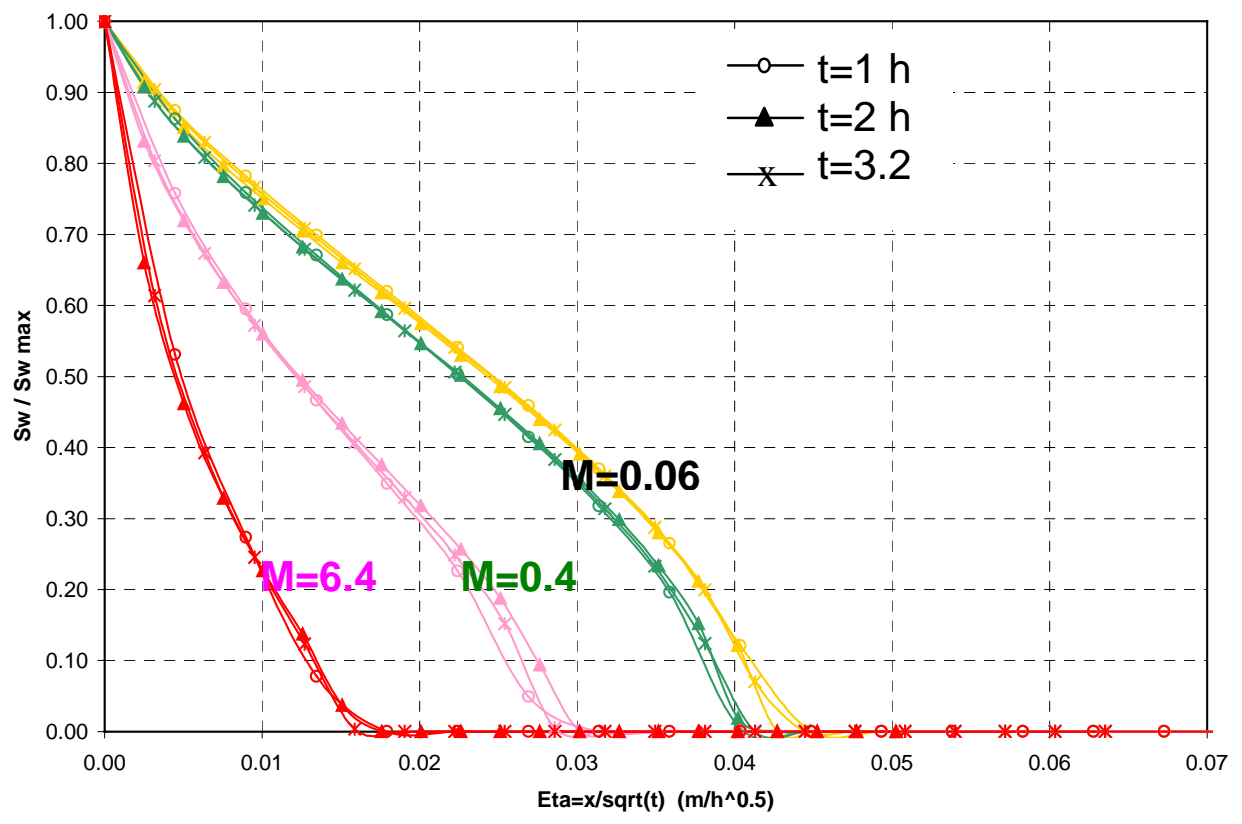

Figure 6. $\mathrm{S}_{\mathrm{w}}$ versus $\frac{x}{\sqrt{t}}$ plots. Simulations of co-current imbibition (final time: $9.65 \mathrm{~h}$ ) with four different mobility ratios. 
The core is placed into the special imbibition cell and the waterjacket is filled with water to limit the artifacts due to the scanning. Liquid can be injected at the top of the core while the bottom face is kept sealed, or vice-versa. For a counter-current imbibition experiment, we inject water at the top face of the core which has been previously saturated with oil. Due to the capillary forces, water flows through the pores of the rock and the oil is pushed out. The cylindrical core made of outcrop diatomite has a length of $9.5 \mathrm{~cm}$ and a diameter of $2.5 \mathrm{~cm}$. Samples are moderately to strongly water-wet. Diatomite is convenient and interesting for study, but results obtained are certainly not specific to diatomite.

\section{Results and Discussion}

The CT images were analyzed by subtracting images. The porosity and the saturation of the wetting phase as a function of time are computed. Figures 7-12 show the standardized saturation (ratio of wetting-phase saturation upon maximum wetting-phase saturation) versus the

variable $\eta=\frac{x}{\sqrt{t}}$ for counter-current imbibition processes. The ratio of viscosities $\frac{\mu_{\text {non wetting }}}{\mu_{\text {wetting }}}$ varies from 0.021 to 7.2 for co-current experiments and from 0.84 to 25 for counter-current experiments.

First, none of the data collapses as the classical equations suggest that they should do. This is not simply a case of experimental uncertainty. Data are spread widely, for example Fig. 11. Moreover, as time progresses curves progress from the right to the left because $\eta$ is inversely proportional to the square root of time.

Second, it seems that curves corresponding to air-oil systems are more spread than those representing oil-water systems (Figs. 9-14). Lines for different positions x seem to collapse when viscosities of each phase are closer. This result could be due to nonequilibrium effects which are more important when viscosities are very different.

Although we obtained the same results in two studies with decane as the non-wetting phase (Figs. 10 and 12), the curves did not collapse for a study with blandol as non-wetting phase (Figs. 14 and 16). It seems that non-equilibrium effects are sensitive to viscosity and the viscosity ratio. The pixel number refers to the position into the core where the saturation is computed (pixel 0: top of the core and pixel 200: bottom of the core ; total number of pixels: 200).

\section{Conclusions}

Based on the local equilibrium assumption, the mathematical study of counter-current imbibition shows that the equation governing the flow is a type of diffusion equation for both counter and cocurrent flow. Therefore, we expect that the plot of water saturation versus the similarity variable $\eta$ collapses all saturation profiles independent of time. Simulations for cocurrent imbibition exhibit this property of the equations. However, experimental data do not 
verify this property. Furthermore, it appears that cocurrent and counter-current processes do not have the same sensitivity to mobility ratio. Also, the shape of some experimental curves could be explained by nonequilibrium effects. The smaller the mobility ratio, the more important the nonequilibrium effects.

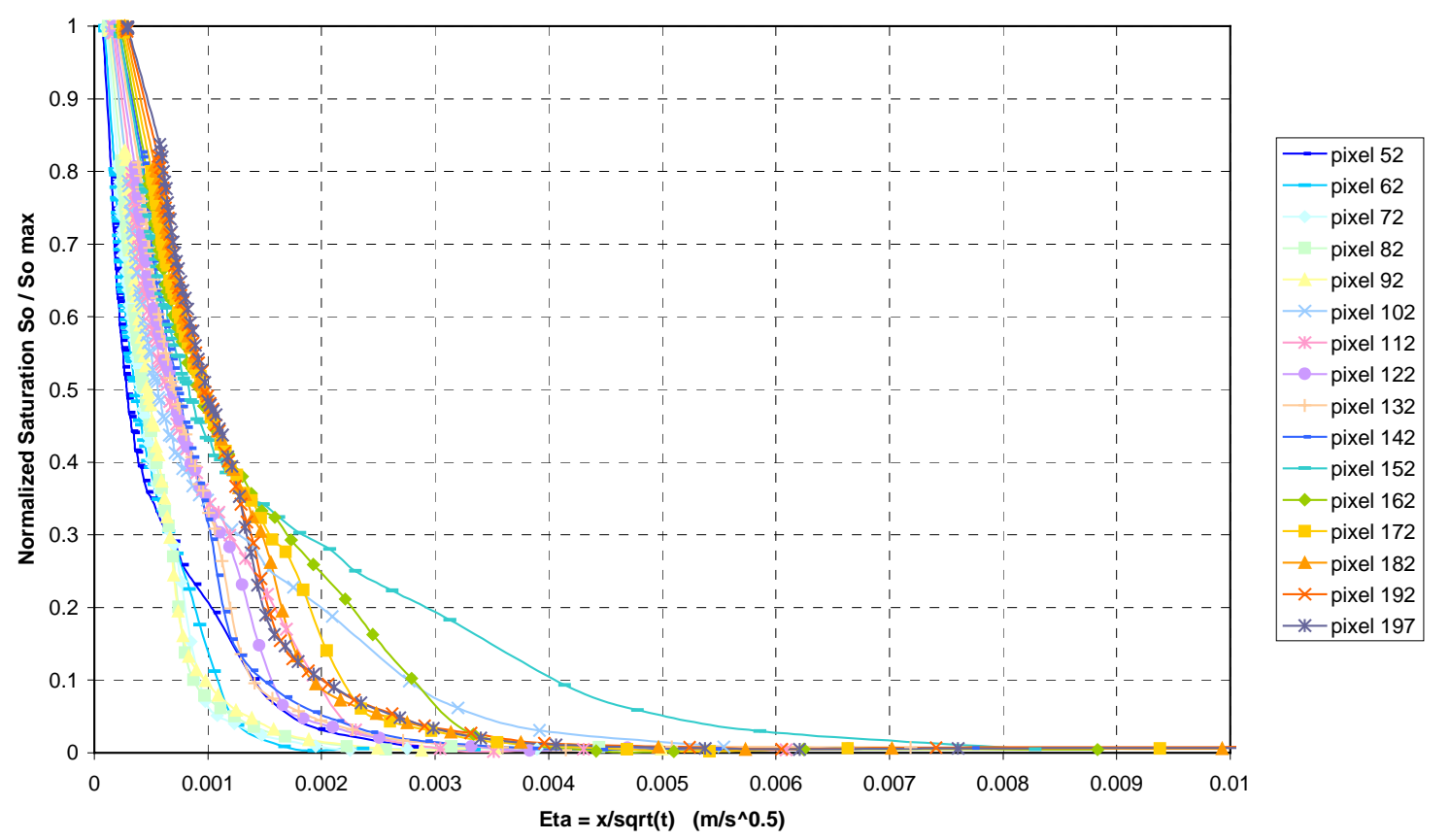

Figure 7. $\mathrm{S}_{\mathrm{o}}$ versus $\frac{x}{\sqrt{t}}$ plots for co-current imbibition in a diatomite core with air/decane system. 


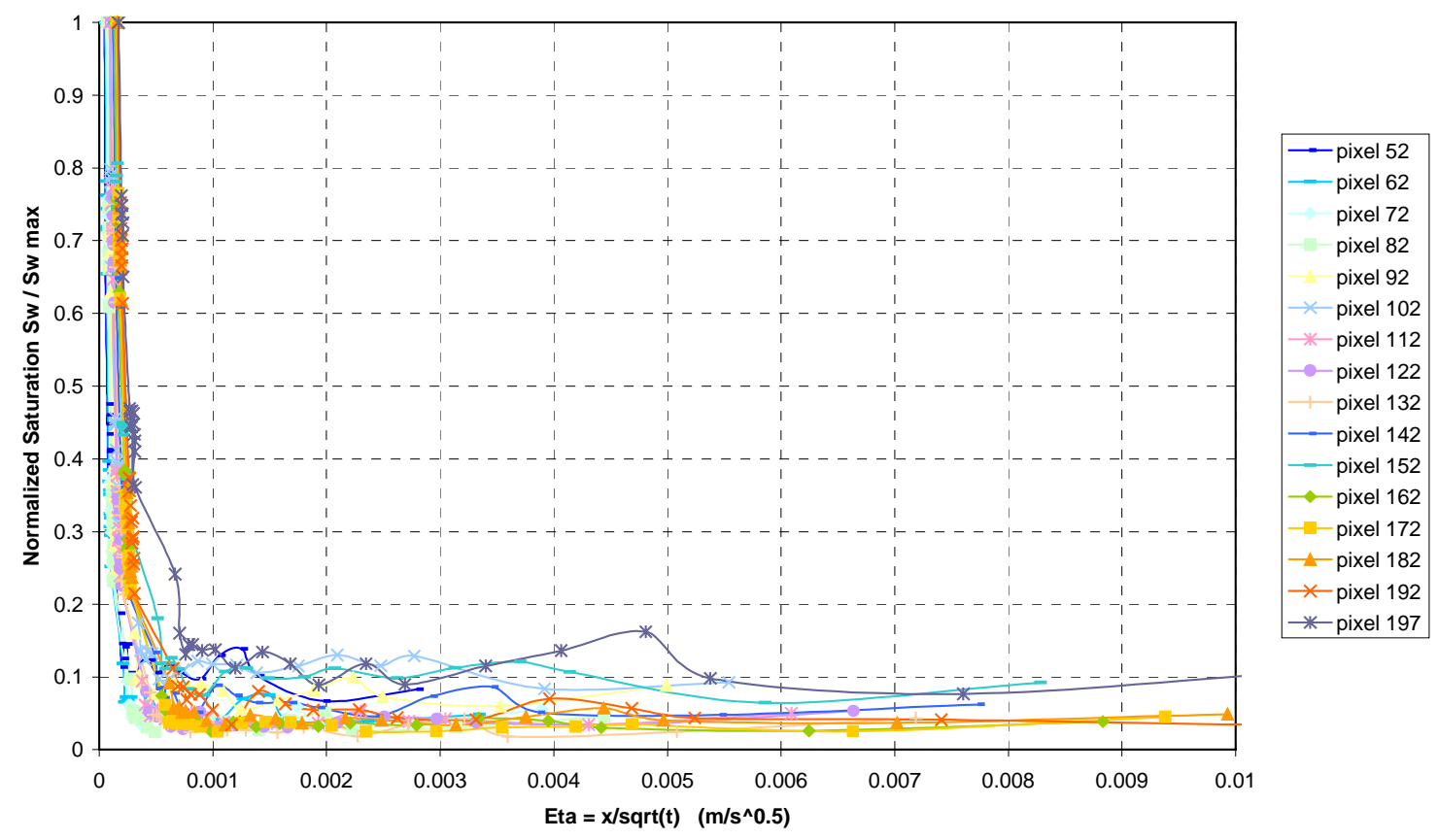

Figure $8 . \mathrm{S}_{\mathrm{w}}$ versus $\frac{x}{\sqrt{t}}$ plots for counter-current imbibition in a diatomite core with decane/water system (total number of pixels: 200).

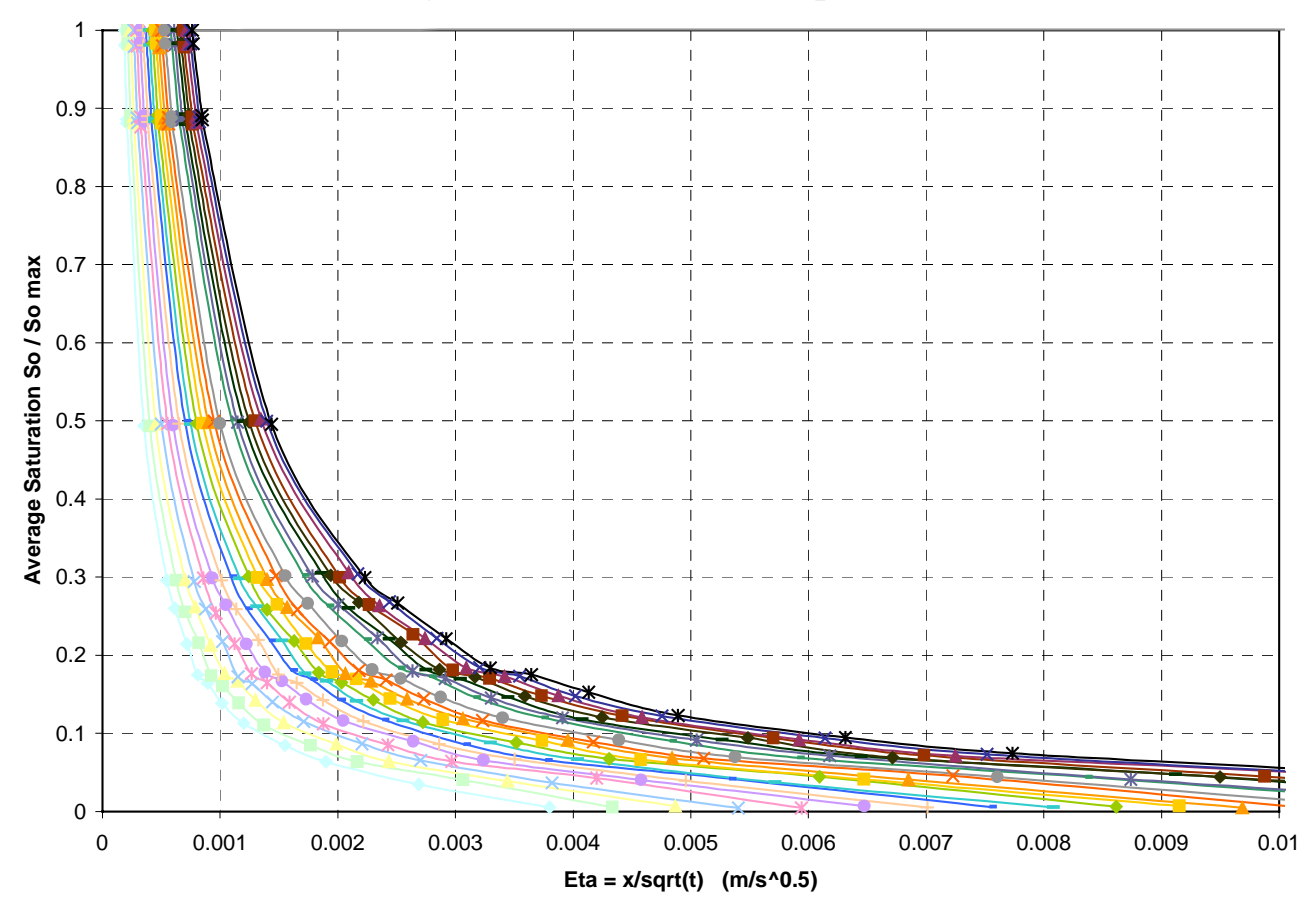

Figure 9. $\mathrm{S}_{\mathrm{o}}$ versus $\frac{x}{\sqrt{t}}$ plots for co-current imbibition in a diatomite core with air/decane system (total number of pixels: 300 ). 


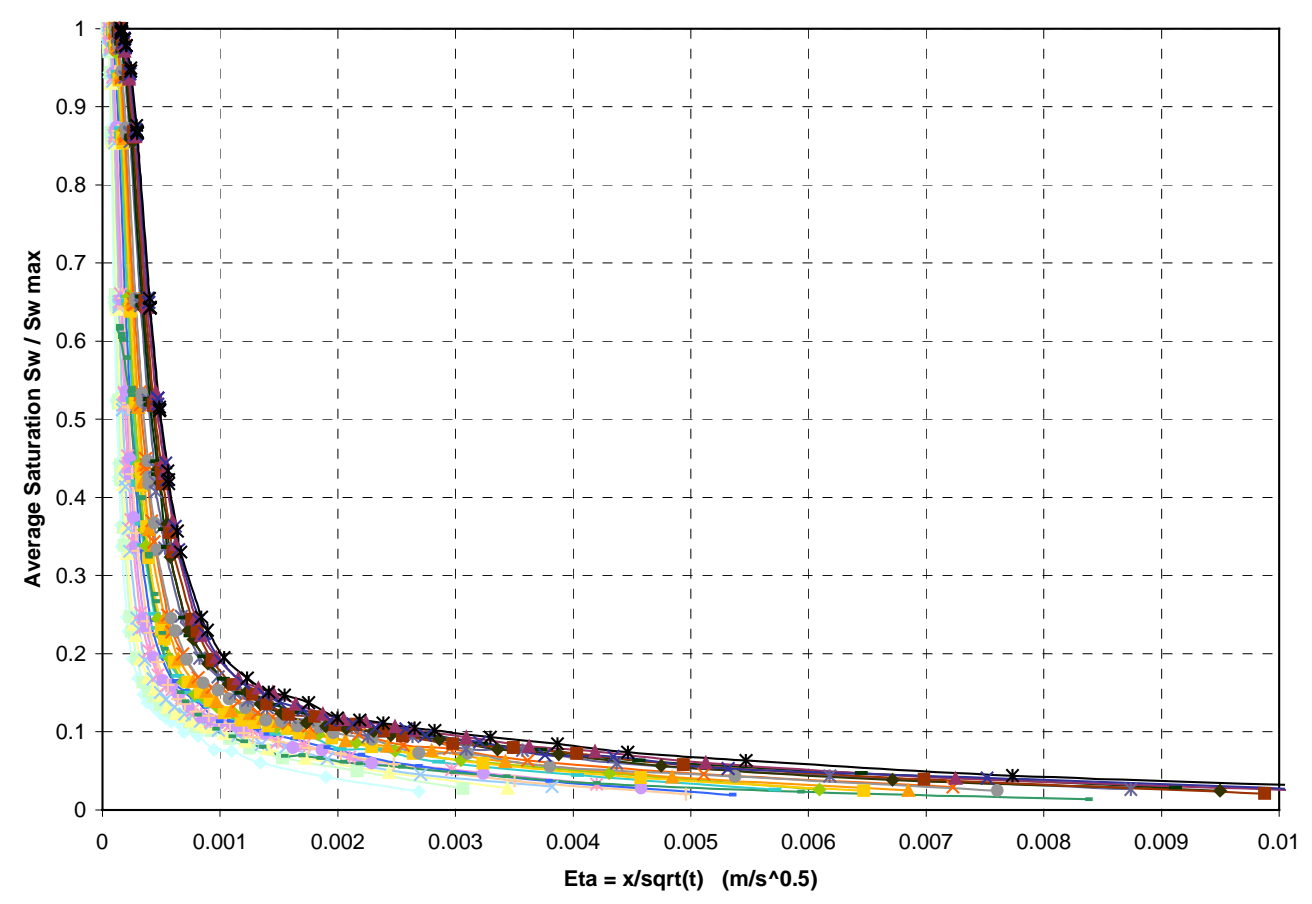
core with decane/water system (total number of pixels: 300).

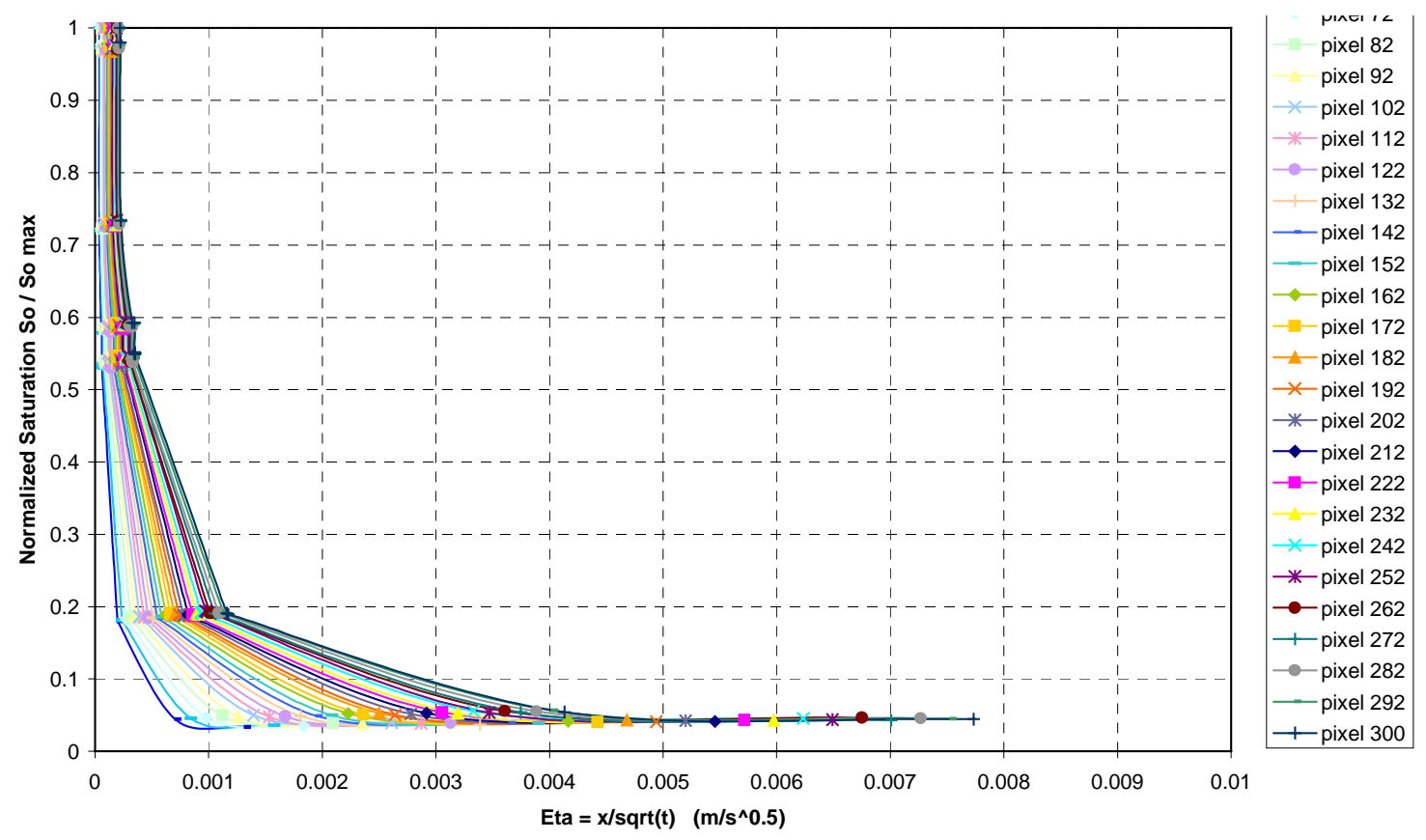

Figure 11. $\mathrm{S}_{\mathrm{o}}$ versus $\frac{x}{\sqrt{t}}$ plots for co-current imbibition in a diatomite core with air/blandol system (total number of pixels: 300 ). 


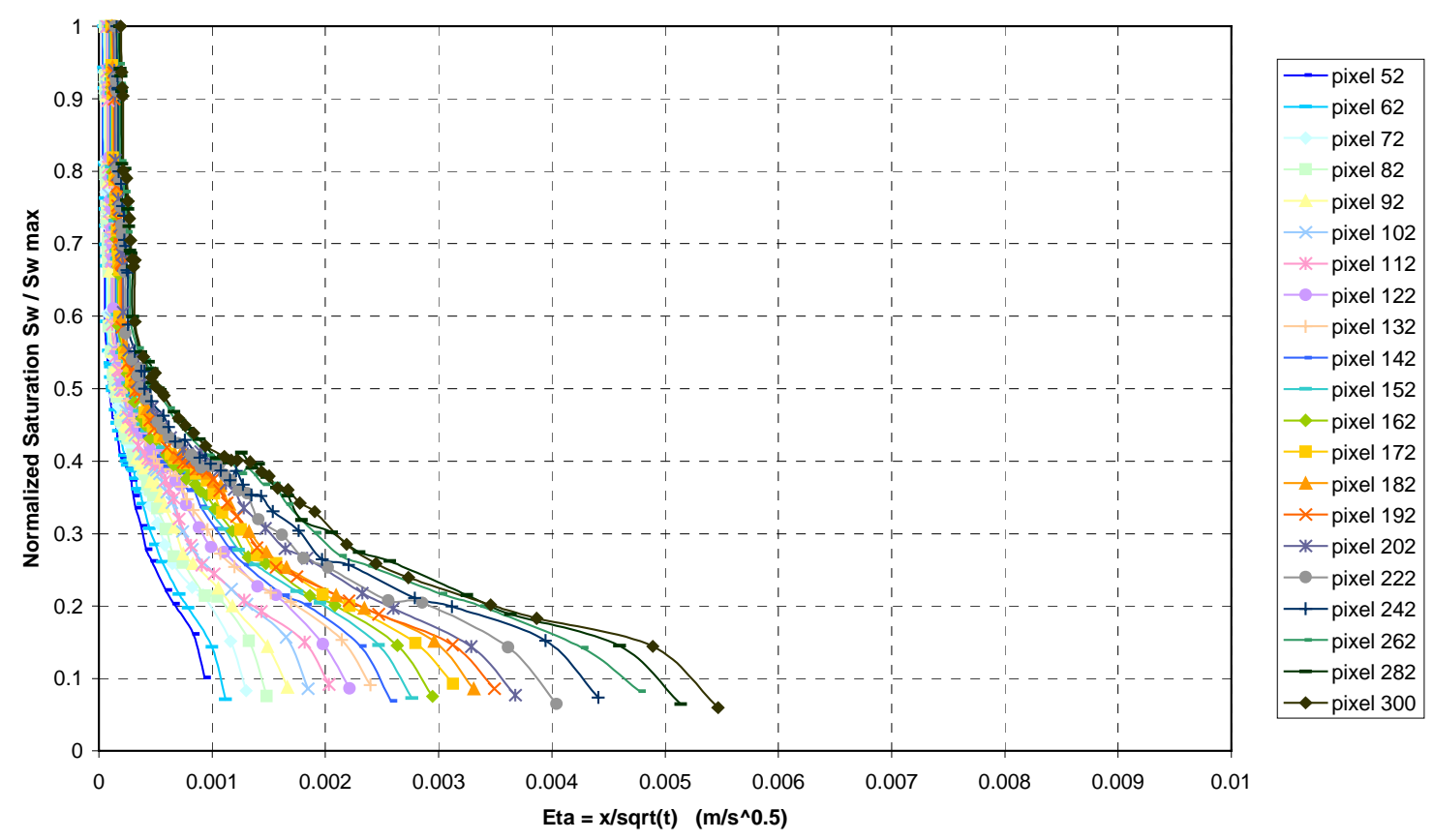

Figure 12. $\mathrm{S}_{\mathrm{w}}$ versus $\frac{x}{\sqrt{t}}$ plots for counter-current imbibition in a diatomite core with blandol/water system (total number of pixels: 300 ).

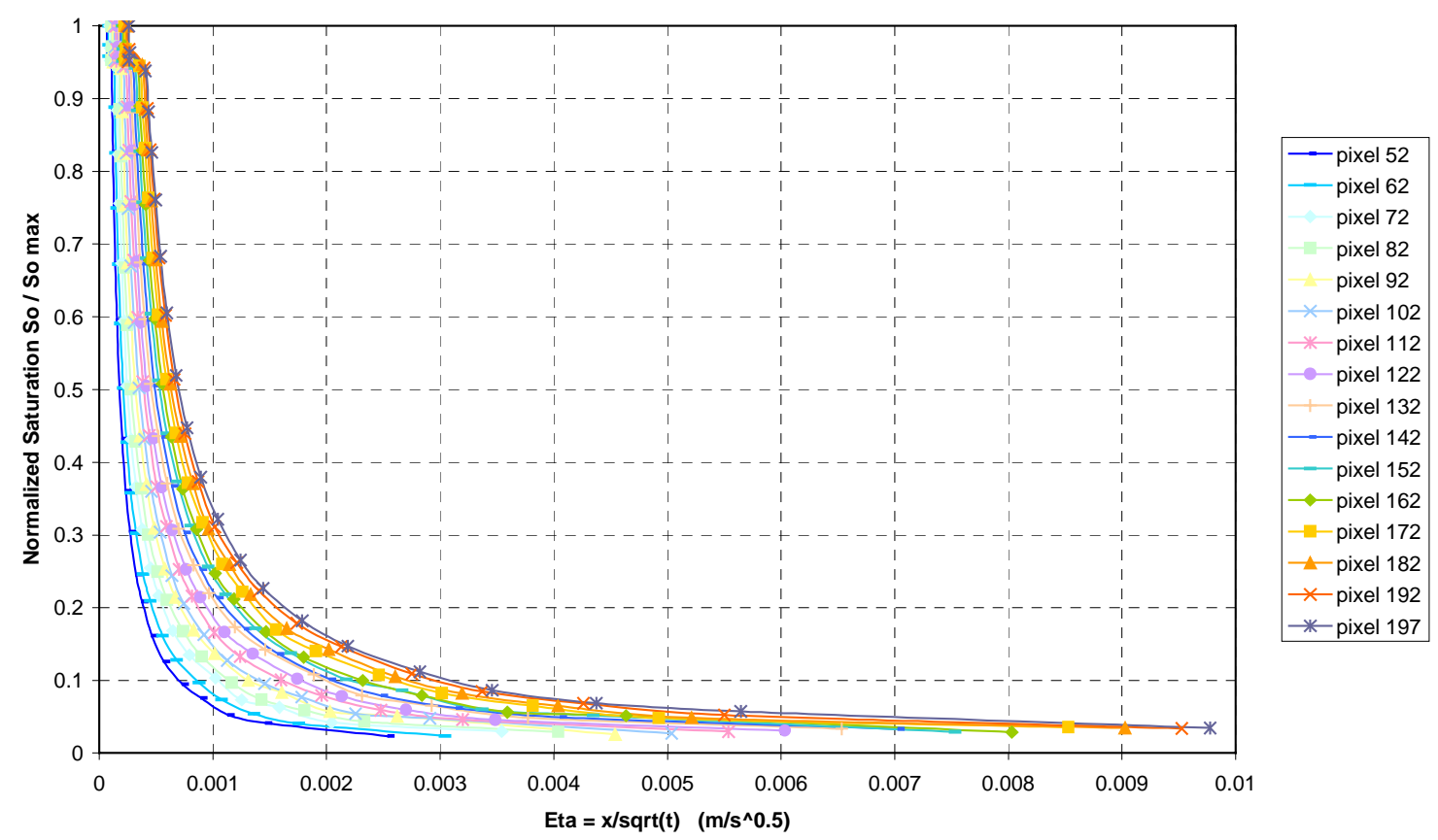

Figure $13 . \mathrm{S}_{\mathrm{o}}$ versus $\frac{x}{\sqrt{t}}$ plots for co-current imbibition in a diatomite core with air/decane system (total number of pixels: 200). 


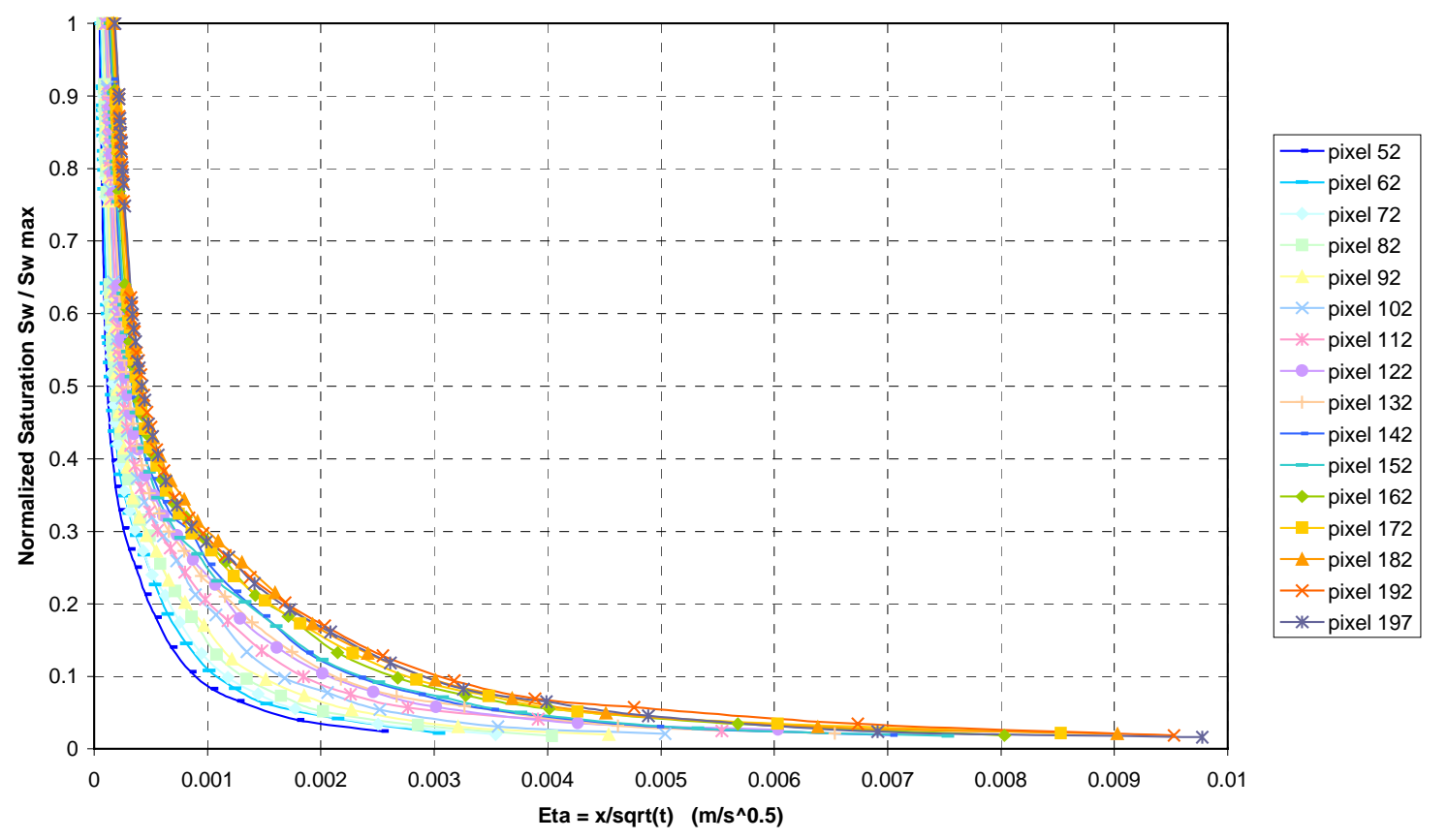

Figure 14: $\mathrm{S}_{\mathrm{w}}$ versus $\frac{x}{\sqrt{t}}$ plots for counter-current imbibition in a diatomite core with decane/water system (total number of pixels: 200). 


\section{Dynamic Relative Permeability from CT Data}

\section{(Task 1a)}

\section{Introduction}

The laboratory methods used to calculate relative permeability functions are grouped into centrifuge, steady- and unsteady-state techniques. The centrifuge method has been improved (Hirasaki et al., 1992, 1995), yet, it has potential pitfalls associated with experiments and interpretation. Additionally, concerns remain regarding the replacement of viscous forces with a range of centrifugal forces for unsteady state displacement processes that are rate dependent (Ali, 1997). Steady- state methods offer disadvantages, especially in the case of low permeability rocks where it is laborious to reach multiple steady states, and capillary forces and capillary end effects are significant (Firoozabadi and Aziz, 1988; Kamath et al., 1993). Capillary pressure has a significant effect on saturation distribution and recovery, and capillary forces dominate multiphase flow in low-permeability rocks and fractured reservoirs. In developing unsteady-state methods for low-permeability systems, it is necessary to account for capillary pressure when obtaining the relative permeability curve. Akin and Kovscek (1999) proved that the use of the (unsteady) JBN technique leads to inaccurate assessment of relative permeability in some cases. Thus, most conventional unsteady techniques do not apply.

\section{Method}

In the method proposed here, two-phase relative permeability curves are obtained from experimental in-situ, saturation profiles measured during spontaneous imbibition experiments. A previously measured capillary pressure versus saturation relationship is needed. Each relative permeability value at a particular saturation is treated independently when the experiment begins with low or zero initial water saturation. For experiments where the core is partially saturated with water initially, the water saturation increases almost uniformly in time throughout the core. Fitting relative permeability curves to a pre-determined functional shape is not necessary in either case. This section proceeds by developing the various equations needed for interpreting the data. In all cases, the relative permeability curve obtained is input for numerical simulation and the agreement between input and computed saturation profiles as a function of time checked.

The equations necessary for data processing of experiments are developed next. First cases with little or no initial water saturation are considered. Then, equations appropriate to cases with medium to high initial water are developed. The method differs slightly between these two cases.

The model begins from one-dimensional flow of two immiscible phases in porous media. The effect of gravity is neglected, but this is not a necessary assumption as shown elsewhere (Schembre and Kovscek, 2003). For the wetting-phase,

$$
u_{w}=-\frac{k k_{r w}}{\mu_{w}} \frac{\partial p_{w}}{\partial x}
$$


where $\mathrm{u}_{\mathrm{w}}$ is the Darcy velocity, $\mathrm{k}$ is the absolute permeability, $\mathrm{k}_{\mathrm{rw}}$ is the relative permeability, $\mu_{\mathrm{w}}$ viscosity, and $\frac{\partial p_{w}}{\partial x}$ is the pressure gradient of the wetting phase.

The Darcy velocity is a superficial velocity that is identically equal to $\left.\phi S_{w} \frac{d x}{d t}\right|_{S_{w}}$. Substituting this expression for $\mathrm{u}_{\mathrm{w}}$ in Eq. 1 results in

$$
\left.\phi S_{w} \frac{d x}{d t}\right|_{S_{w}}=-\frac{k k_{r w}}{\mu_{w}} \frac{\partial p_{w}}{\partial x}
$$

where $\left.\frac{\mathrm{dx}}{\mathrm{dt}}\right|_{\mathrm{S}_{\mathrm{w}}}$ is the interstitial velocity of a particular saturation $S_{\mathrm{w}}$ as a function of time and $\phi$ is the porosity. Integrating with respect to time, the relative permeability of a particular $S_{\mathrm{w}}$ is

$$
k_{r w}=\left(\frac{\left.x\right|_{S_{w}} S_{w}}{-\left.\int_{0}^{t}\left(\frac{\partial p_{w}}{\partial x}\right)\right|_{S_{w}} d t}\right) \frac{\mu_{w} \phi}{k}
$$

In this function, the porosity, viscosity and, permeability are independently measurable and constant. The position of a particular saturation as a function of time, $\left.\mathrm{x}\right|_{\mathrm{s}_{\mathrm{w}}}$ and the pressure gradient history are measured during the experiment. Thus, Eq. 3 allows direct computation of $\mathrm{k}_{\mathrm{rw}}$ solely from measured data. A similar development for the non-wetting phase yields

$$
k_{r n w}=\frac{\mu_{n w} \phi S_{n w}}{k}\left(\frac{\left.x\right|_{S_{n w}}}{\left.\int_{0}^{t} \frac{\partial p_{n w}}{\partial x}\right|_{S_{n w}} d t}\right)
$$

where $\frac{\partial p_{n w}}{\partial x}$ is the non-wetting phase pressure gradient. Equations 3 and 4 are related by the summation of phase saturations $\left(\mathrm{S}_{\mathrm{w}}+\mathrm{S}_{\mathrm{nw}}\right)$ to 1 .

In cases with initial water saturation and where the water saturation history is diffuse, it is difficult to implement the method above. Multiple locations for a given saturation value at a specific time lead to uncertainty in the assessment of the velocity of the saturation value. For cases with initial water, a variant of the method of Sahni et al. (1998) for gravity drainage is developed.

The Darcy velocity and conservation equation for each phase are again used. Rather than integrating Darcy's Law, the material balance for each phase is integrated. The in-situ saturation measurement and an independently measured capillary pressure relationship are still needed. The 
result for the wetting phase is,

$$
k_{r_{w}}=-\frac{\mu_{w} \phi}{k} \frac{\left.\int \frac{\partial S_{w}}{\partial t}\right|_{x, t} d x}{\left.\frac{\partial p_{w}}{\partial x}\right|_{x, t}}
$$

and similarly, for the non-wetting phase,

$$
k_{r n w}=-\frac{\mu_{n w} \phi}{k} \frac{\left.\int \frac{\partial S_{n w}}{\partial t}\right|_{x, t} d x}{\left.\frac{\partial p_{n w}}{\partial x}\right|_{x, t}}
$$

\section{Results and Discussion}

An extensive validation exercise for this approach is documented elsewhere (Schembre and Kovscek, 2003). Here, we focus on a demonstration using experimental data obtained from the imbibition cell. Water saturation profiles measured by X-ray CT during spontaneous imbibition water imbibition into air-filled Berea sandstone and diatomite are used. The properties of each system are summarized in Table 1. The column "voxel resolution" refers to the number of CT-measured saturation data along the $\mathrm{z}$-axis of the core.

Table 1: Characteristics of the Berea Sandstone and Diatomite Cores

\begin{tabular}{|lcc|}
\hline & Berea sandstone & Diatomite \\
\hline Porosity[fraction] & 0.22 & 0.68 \\
Permeability[md] & 780.0 & 6.6 \\
Diameter[cm] & 2.43 & 2.43 \\
Length [cm] & 8.8 & 9.02 \\
voxel resolution $^{*}$ & 210 & 225 \\
\hline
\end{tabular}

* total number of measured $\mathrm{S}_{\mathrm{w}}$ values along z-axis of core

The imbibition capillary pressure curve given by Sinnokrot et al. (1969) for Berea sandstone, was modified for different fluid system and petrophysical properties (ie, $\mathrm{k}, \phi)$ by using the Leverett J-function (Leverett, 1941; Ma et al., 1991) That is, the required curve was first nondimensionalized and then rescaled according to $\mathrm{k}, \phi$, and $\sigma(\theta=0)$. The capillary pressure relationship used in this case is shown in Fig. 1. The relative permeability curve obtained is shown in Fig. 2. The values of $\mathrm{k}_{\mathrm{rw}}$ obtained are reasonable. Moreover, the endpoint $\mathrm{k}_{\mathrm{rw}}$ obtained agrees with endpoints found in the literature for Berea sandstone (Lerdahl et al., 2000; Oak et al., 1990).

A comparison of the CT-determined experimental water saturation profiles versus time and the profiles obtained by simulating the core with the measured relative permeability is shown in Fig. 3. The results match especially well given the scatter in the experimental data. Notice that the shape and position of the imbibition front are well reproduced. Since the pressure gradient within the core was not measured in the experiments, it was not possible to find the non-wetting 
phase relative permeability. A linear $\mathrm{k}_{\mathrm{rg}}$ relationship is used for simulation. Because the gas is inviscid, the shape of $\mathrm{k}_{\mathrm{rg}}$ versus $\mathrm{S}_{\mathrm{w}}$ has little effect on the computed saturation profiles in Fig. 3.

We also used diatomite during experiments because there is very little known about petrophysical properties in diatomite. The use of the classical unsteady state method in the determination of relative permeability for diatomite surely leads to inaccurate results because of low permeability and corresponding capillary effects. The same experimental method as in the case of the Berea sandstone was used to obtain the water saturation profiles. The core is initially air-filled.

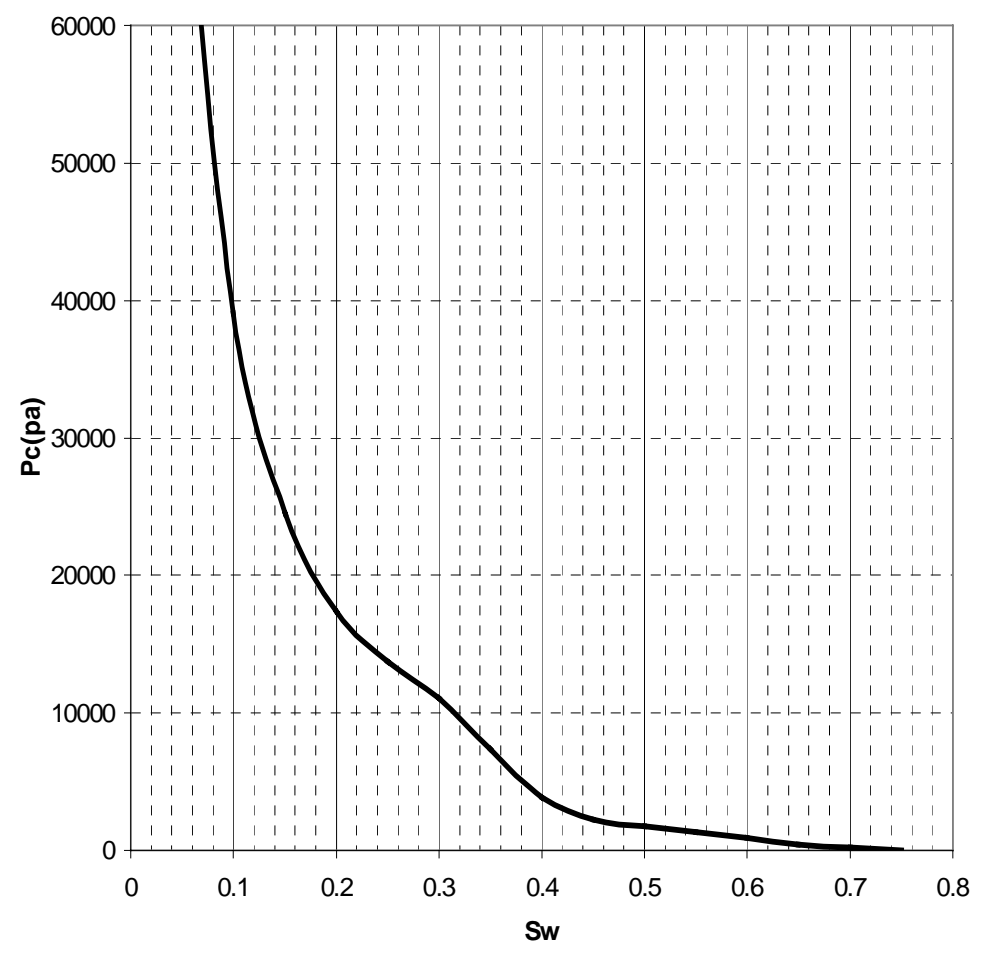

Figure 1. Capillary pressure for Berea Sandstone. 


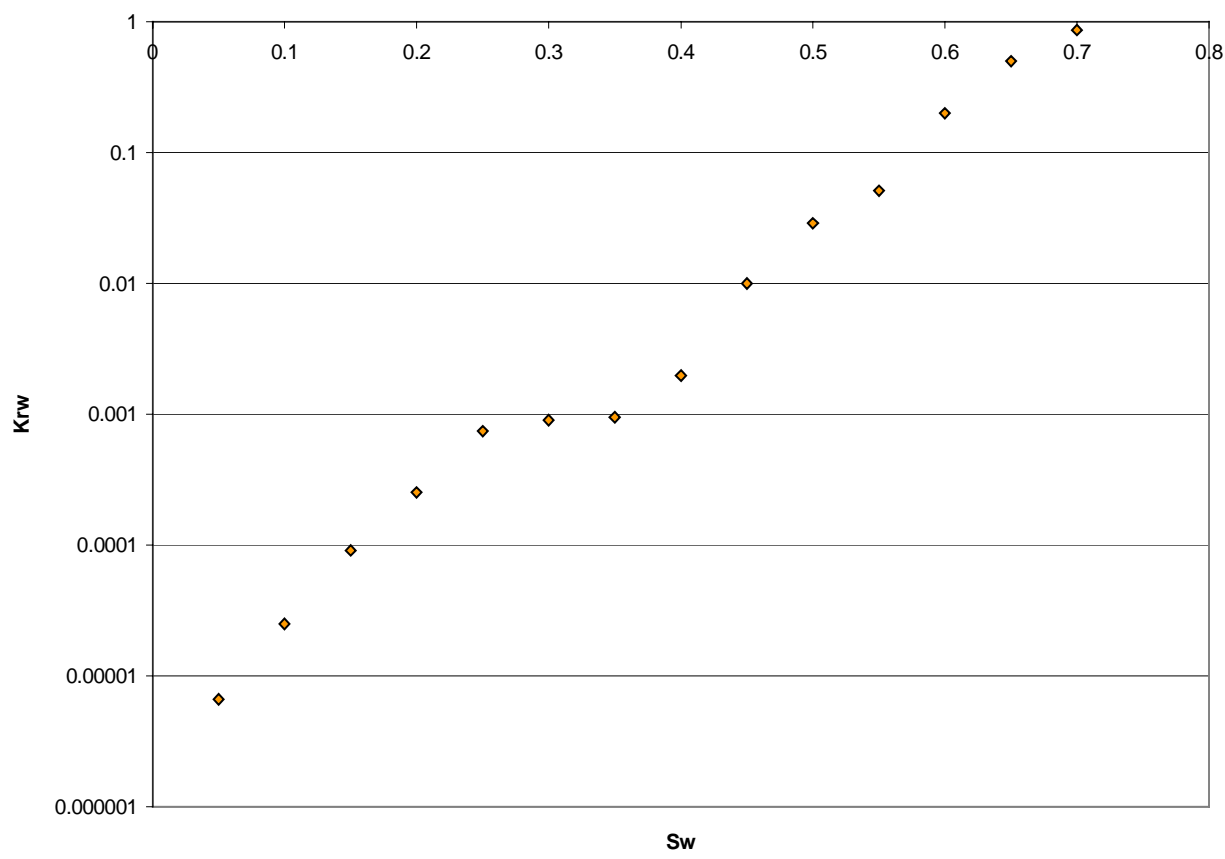

Figure 2. Experimentally determined water relative permeability curve for Berea sandstone.

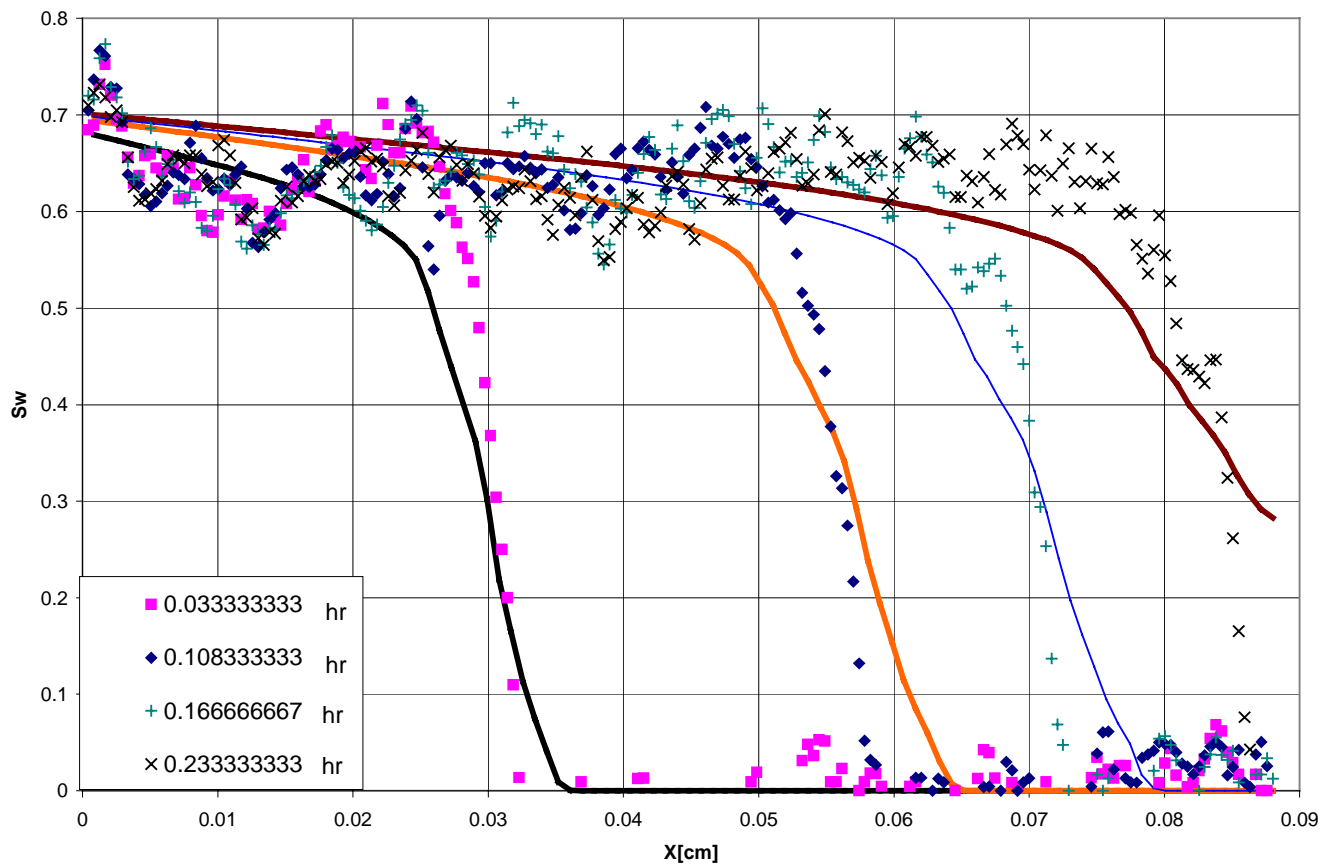

Figure 3. Saturation profile obtained by simulation with measured krw compared to the experimental profile for Berea sandstone. 
The input imbibition capillary pressure curve was measured with the porous-plate method as reported by Akin and Kovscek (1999). It is similar to another curve reported by Kumar and Beatty (1995), but not shown here. The capillary pressure used is given in Fig. 4. The relative permeability curve obtained from the measured $S_{w}$ profile is shown in Fig. 5. At high water saturation, the relative permeability is found to decrease as $S_{\mathrm{w}}$ increases. The reason for this artifact is shown in Fig. 6. Here, the experimental water saturation profile and the profile obtained by simulating the imbibition process are compared. High water saturations within the experiments advance slowly at earlier time. Note the first profile for a time of $0.0069 \mathrm{hr}$. This is indicative of flow that is not one dimensional at small times. Therefore, retardation exists for saturation values greater than 0.7 . The solid line gives the relative permeability curve used for simulation:

$$
k_{r w}\left(S_{w}\right)=5 E^{-5} \exp \left(8.3355 S_{w}\right)
$$

Despite the difficulties in measuring $\mathrm{k}_{\mathrm{rw}}$ at large $\mathrm{S}_{\mathrm{w}}$, the results obtained from simulation (solid line) with Eq. 7 match the saturation-history data well, as shown in Fig. 6. Additionally, the data points in Fig. 6 and the extrapolation at high water saturations indicate a low endpoint value of roughly 0.2 for $\mathrm{k}_{\mathrm{rw}}$. This is indicative of and consistent with the strong water-wetness of these core samples (Akin et al., 2000; Zhou et al., 2002).

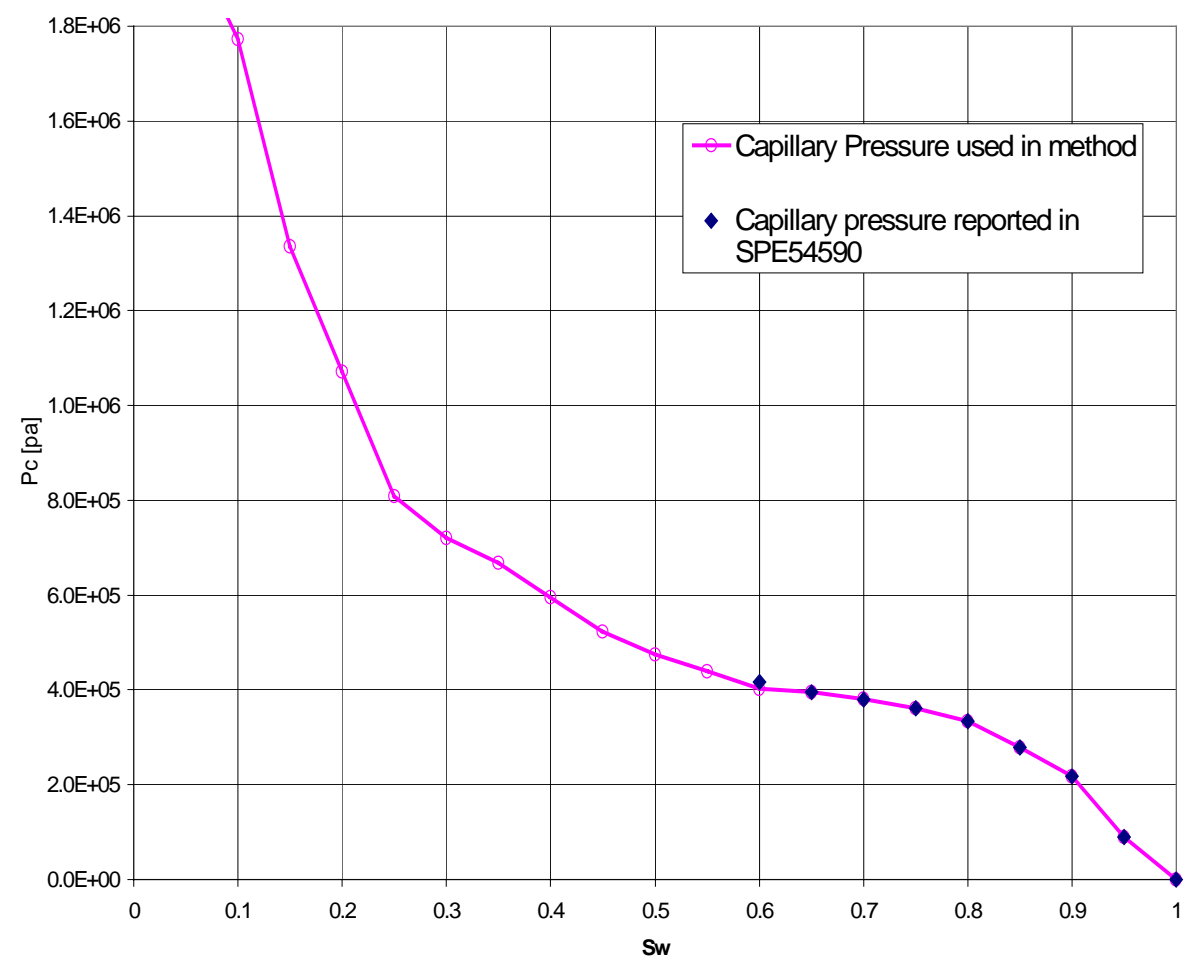

Figure 4. Capillary pressure curve used for interpretation of diatomite imbibition experiments. Data points are from Akin and Kovscek (1999). 


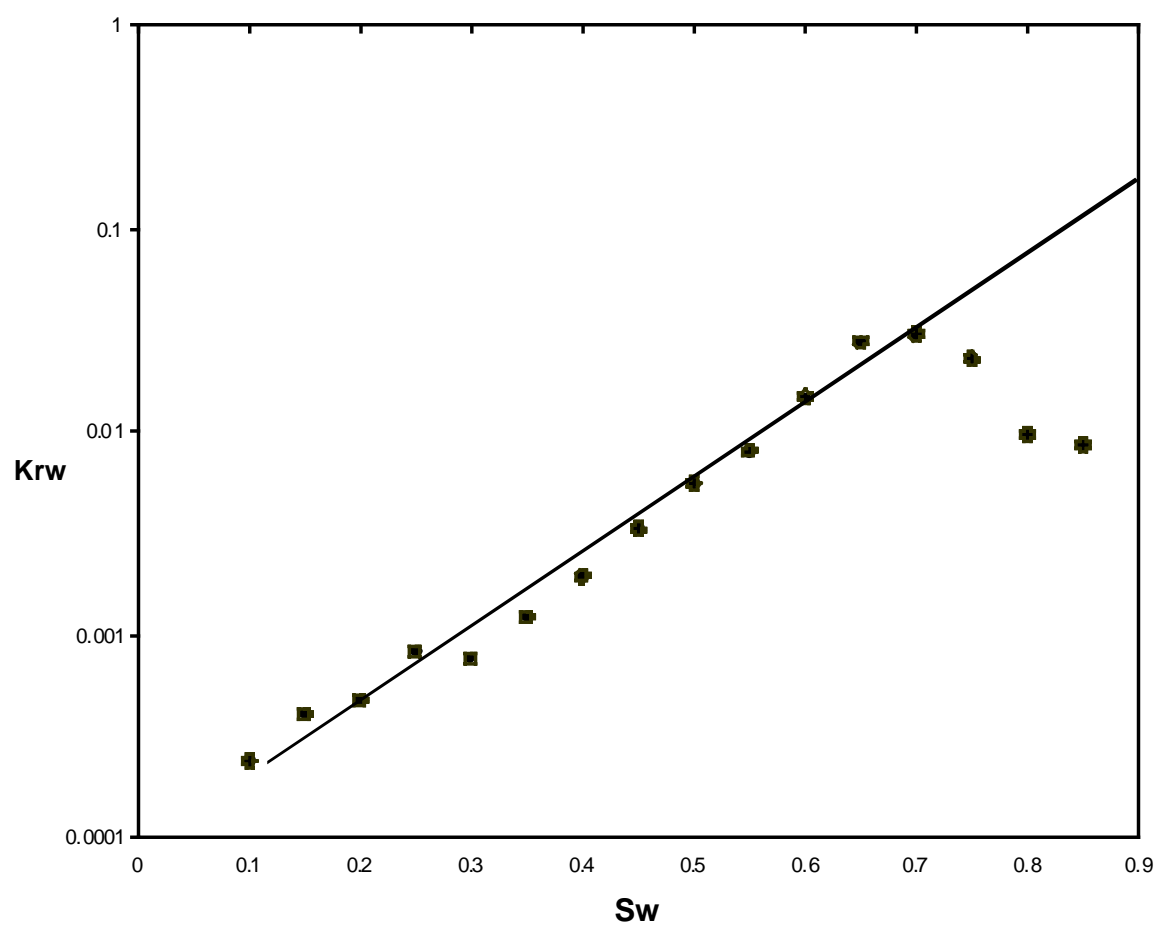

Figure 5. Experimentally determined water relative permeability curve for outcrop diatomite.

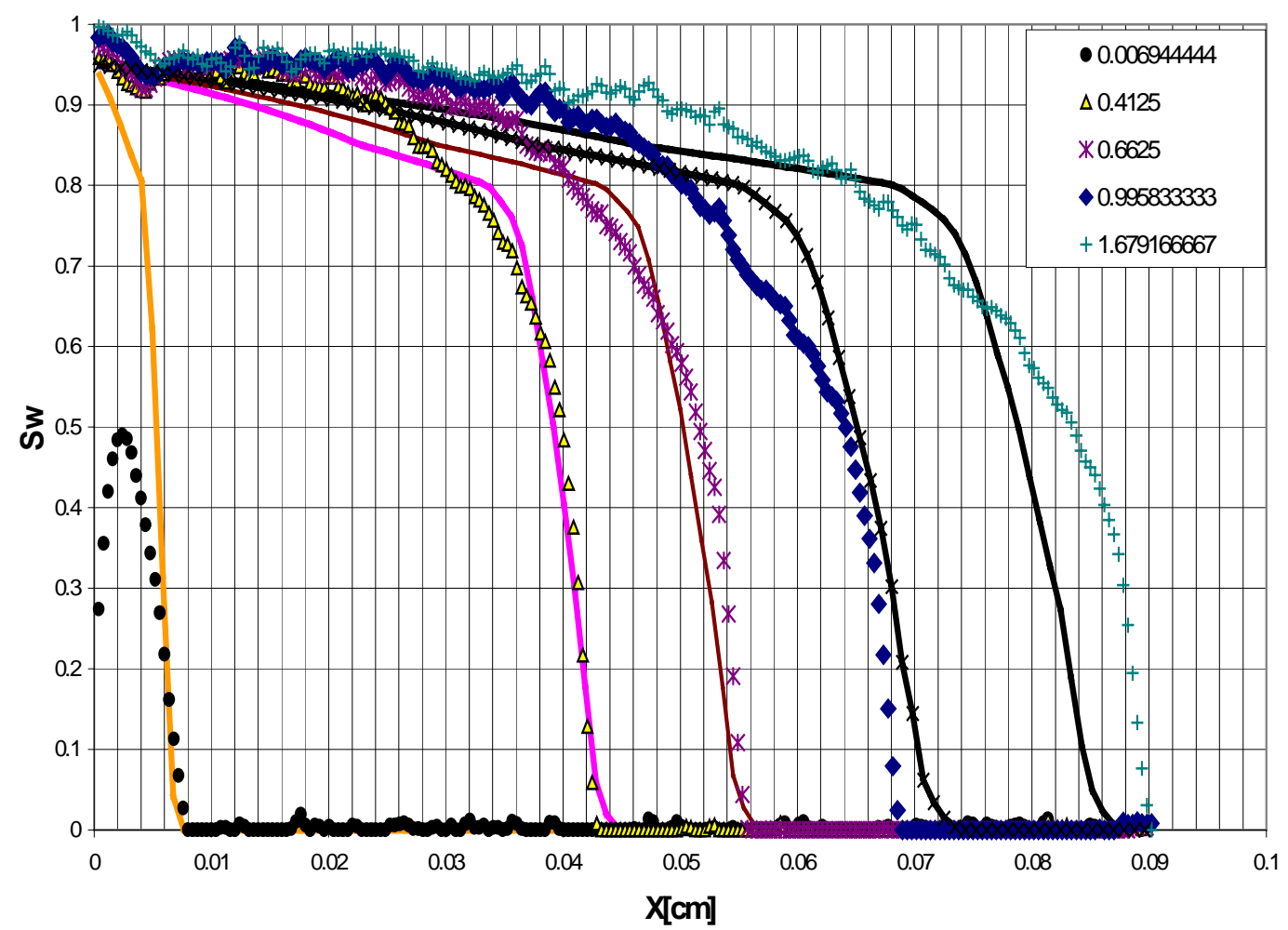

Figure 6. Saturation profile obtained by simulation with measured krw compared to the experimental profile for outcrop diatomite. 


\section{Conclusions}

In summary, a method has been developed to determine dynamic relative permeability from the water saturation profile history obtained during X-ray CT monitored spontaneous imbibition experiments. The method was tested using synthetic data for the water-air and wateroil cases. In addition, the method was adapted for cases with large initial water saturation. Computed relative permeability values matched well the input curves, suggesting reliability for these systems. In the water-oil case, discrepancies among the measured and input oil relative permeability values were attributed to numerical inaccuracies in the simulator. The air-phase pressure gradient can be neglected in the calculations of water relative permeability. A sensitivity study showed that neglecting the oil-phase pressure gradient might lead to error in high water saturation values for viscous oils.

The procedure was used to determine the water relative permeability for two experimental cases with Berea sandstone and quarried diatomite. In both cases, the relative permeability curves obtained were used to simulate the experimentally measured water saturation profile versus time. The computed saturation history reproduced the experimental behavior well. Importantly, this method allows measurement of relative permeability from a single experiment. Potentially this represents a great time savings.

\section{References}

Akin, S., Kovscek, A.R., 1999. Imbibition studies of low-permeability porous media, SPE 54590. Proceedings of the SPE Western Regional Meeting, Anchorage, May 26-29.

Akin, S. and Kovscek, A.R. 2003. "Computerised Tomography in Petroleum Engineering Research," in Applications of Computerized X-ray Tomography in Geology and Related Domains, Jacobs, P., Mees, F., Swennen, R, Van Geet, M. (eds), Special Publication, Geological Society, London.

Chen, Q, Gingras, M. K., and Balcom, B. J., 2003.“A Magnetic Resonance Study of Pore Filling Processes During Spontaneous Imbibition in Berea Sandstone," Journal of Chemical Physics, 119(18) 9609-9616.

Chen J., et al. 1995. "Theoretical investigation of counter-current imbibition in fractured reservoir matrix blocks", SPE 29141, Feb.

Firoozabadi, A., Aziz, K., 1988. Relative permeabilities from centrifuge data, SPE 15059.Proceedings of the $56^{\text {th }}$ California Regional Meeting of the SPE, Oakland, April 24.

Handy L., Determination of effective capillary pressures for porous media from imbibition data, Petroleum Transactions, AIME, 1960. 
Hirasaki, G.J., Rohan, J.H., Dudley, J.W., 1992. Modification of centrifuge and software for determination of relative permeability curves, SPE 25290. Proceedings of the Annual Technical Conference, Washington D.C., October 4-7.

Hirasaki, G.J., Rohan, J.H., Dudley J.W., 1995. Interpretation of oil-water relative permeabilities from centrifuge experiments, SPE 24879. SPE Adv. Tech. Series. 3 (1). 66-75.

Kamath, J., deZabala, E.F., Boyer, R.E., 1993. Water/oil relative permeability endpoints of intermediate-wet, low permeability rocks, SPE 26092. Proceedings of the Western Regional Meeting, Anchorage, May 26-28.

Kumar, M., Beatty, F.D., 1995. Cyclic steaming in heavy oil diatomites, SPE 29623. Proceedings of the Western Regional Meeting, Bakersfield, March 8-10.

Lerdahl, T.R., Øren, P., Bakke, S., 2000. A predictive network model for three-phase flow in porous media, SPE 59311. Proceedings of the SPE/DOE Improved Oil Recovery Symposium, Tulsa, April 3-5.

Leverett, M.C., 1941. Capillary behavior in porous solids. Petroleum Transactions, AIME 142, 152.

Ma S., Jiang, M. X., Morrow N.R., 1991. Correlation of capillary pressure relationships and calculations of permeability, SPE 22685. Proceedings of the $66^{\text {th }}$ Annual Technical Conference and Exhibition, Dallas, October 6-9.

Marle C.M., Multiphase flow in porous media, Editions Technip, 1981.

Mattax, C. C. and Kyte, J. R. (Jun 1962). "Imbibition Oil Recovery from Fractured Water-Drive Reservoirs.” Soc. Pet. Eng. J. 2: 177-184.

Oak, M.J., Baker, L.E., Thomas, D.C., 1990. Three-phase relative permeability of Berea sandstone. Journal of Petroleum Technology 42. 1054-61, August.

Sahni, A., Burger, J., Blunt, M., 1998. Measurements of three phase relative permeability during gravity drainage using CT scanning, SPE 39655. Proceedings of the SPE/DOE Improved Oil Recovery Symposium held at Tulsa, April 19-22.

Schembre, J. M. and A. R. Kovscek, 2001. " Direct Measurement of Dynamic Relative Permeability from CT-Monitored Spontaneous Imbibition Experiments," SPE 71484 Proceedings of the SPE Annual Technical Conference and Exhibition, New Orleans, Sept. 30 - Oct. 3.

Schembre, J. M. and A. R. Kovscek, 2003. "A Technique for Measuring Two-Phase Relative Permeability in Porous Media via X-ray CT Measurements, Journal of Petroleum Science and Engineering, 39(1\&2), 159-174. 
Silin, D. and T. W. Patzek, 2003. “On Barenblatt's Model of Spontaneous Countercurrent Imbibition," Transport in Porous Media, to appear.

Sinnokrot, A.A., Ramey, H.J., Marsden, S.S., 1969. Effect of temperature level upon capillary pressure curves, SPE 2517. Proceedings of the SPE $44^{\text {th }}$ Annual Fall meeting, Denver, Sept 28-Oct. 1.

Tang, G.Q. and A. R. Kovscek, 2002 "Experimental Study of Heavy Oil Production from Diatomite Core at Elevated Temperatures, " SPE 75132 Proceedings of the SPE/DOE Thirteenth Symposium on Improved Oil Recovery, Tulsa, April 13-17.

Tang, G-Q. and A. R. Kovscek, 2002B. "Wettability Alteration of Diatomite Induced by HotFluid Injection" SPE 77461, Proceedings of the SPE Annual Technical Conference and Exhibition, San Antonio, Sept. 29 - Oct. 2.

Zhang, X., Morrow, N. R. and Ma, S. 1996. "Experimental Verification of a Modified Scaling Group for Spontaneous Imbibition.” Soc. Pet. Eng. Res. Eng. 11: 280-28.

Zhou, D., L. Jia, J. Kamath, and A. R. Kovscek, 2002. "Scaling of Counter-Current Imbibition Processes in Low-Permeability Porous Media," Journal of Petroleum Science and Engineering, 33(1-3), 61-74. 


\section{Experimental and Analytical Study of Multidimensional Imbibition In Fractured Porous Media}

\section{(Task 1a)}

\section{Introduction}

Our understanding of imbibition behavior in fractured systems, and models for matrixfracture transfer, are largely based on such one-dimensional experiments as reported above. Nevertheless, even a rudimentary exploration of imbibition suggests than mechanisms in more than one dimensions are intricate. For example, the transfer of water and oil across the matrix fracture interface is governed by a balance of viscous forces driving flow through fracture and capillary suction that draws water out of the fracture. Similarly, imbibition time scales can be significantly large and comparable to the time required to fill fractures with wetting fluid. Thus, the overall time scaling for imbibition may differ from the results given in one-dimensional experiments.

Figure 1 illustrates schematically how time scaling differs with dimension. Where initial water saturation is low, results (e.g., Akin et al., 2000) have shown in one-dimensional media that there is a well-defined spreading front parallel to the surface exposed to the imbibing fluid. This fluid advances at a speed proportional to the square root of time, as shown in Fig. 1a. This is the basis for the so-called "square root of time models" of imbibition. Assume that the same physical process occurs in all directions in multidimensional porous media, even against the force of gravity. Next, consider a two-dimensional medium where water imbibes from a stationary line source, Fig. 1b; the progress of water imbibition in the $\mathrm{x}$-direction is proportional to the square root of time, and, likewise, in the z-direction. Thus, in two-dimensional media, the overall mass of water imbibed is proportional to the product of the extent of imbibition in each direction. Imbibition in this case should scale linearly with time. Later, this is shown to be true experimentally. Finally, this analysis suggests that three-dimensional water imbibition from a point source scales with time to the three-halves power, Fig. 1c.
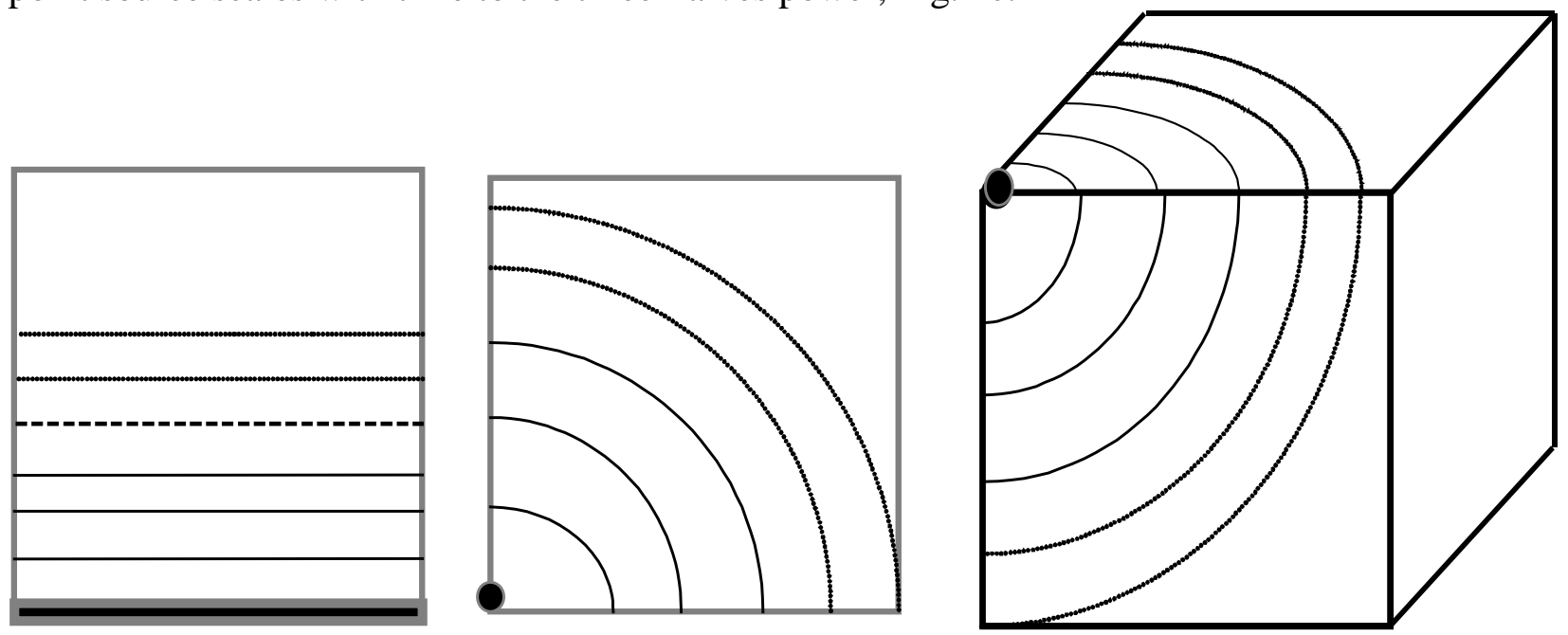

Figure 1. Possible imbibition patterns in (a) 1-D geometry (plane source),(b) 2-D geometry (line source), and (c) 3-D geometry (point source). Lines indicate front position as a function of time. 


\section{Experimental}

Experimental equipment was designed based upon our success with the unconventional scan geometry of the one-dimensional imbibition cell. The apparatus insures minimal artifacts while imaging and the collection of maximum saturation information. It is described next.

Due to the cubic geometry of the cores as illustrated in Fig. 2 and our desire to measure in-situ saturations with X-ray CT, conventional core holders could not be used. The basic idea of the experimental design given in Fig. 2 is to avoid drastic changes of the density of the object scanned by presenting a circular cross-section to the scanner. Initially the outside surface of the Berea sandstone (Cleveland Quarries) samples were coated and sealed with Marine Epoxy (Tap Marine Plastic \#314). The cores were then potted in vertical cylindrical PVC containers with the same epoxy. Once the core was covered by epoxy in the PVC container, the bottom face of the cylinders was cut open to expose the rock. Sufficient core was removed so that no trace of epoxy remained on this face (verified by subsequent CT scans).

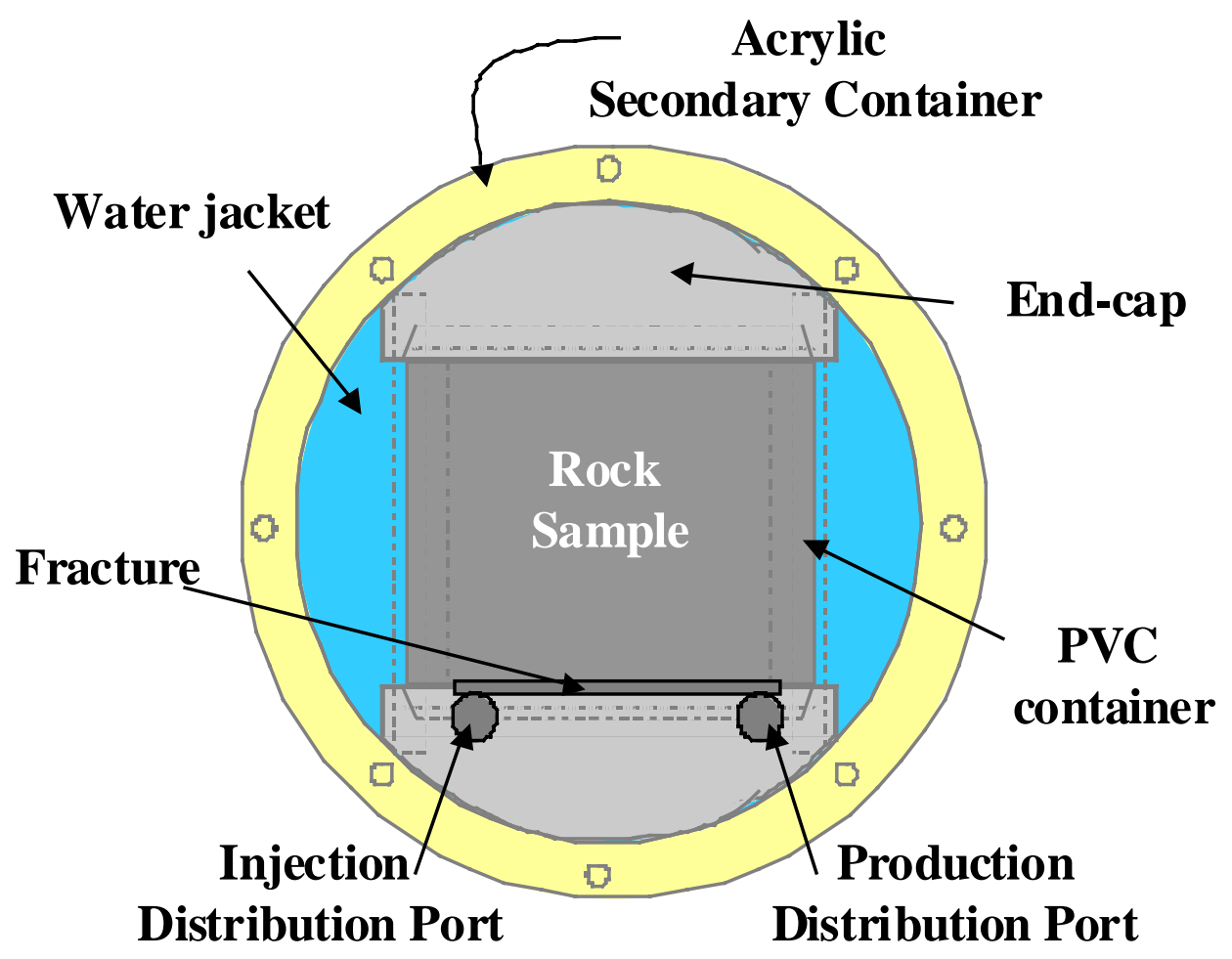

Figure 2. The core holder: Frontal view

This PVC core-holder was placed in a second horizontal cylindrical acrylic container, as shown in Fig. 2. A cylindrical shape was used to allow the core to be rotated in any position around the horizontal axis. Once filled with water, the second container serves as a water jacket to lessen the contrast in density between the acrylic and PVC/epoxy and to achieve some measure of temperature control. Thus, the cross-sectional shape for scanning is symmetric and circular, improving image quality. The scanning plane is the cross section shown in Fig. 2. 
Acrylic end-caps were machined to mate with the circular PVC core-holder. The end caps have 2 perforations (inlet and outlet) to give planar 2D flow across the face of the block. The endcaps (one at the top and one at the bottom) were attached to the PVC coreholder by means of through bolts and sealing O-rings. Fracture apertures were set by means of metallic shims (essentially feeler gauges of precise thickness) placed between the block and end-cap. Thus, a uniform fracture aperture is guaranteed along the entire core. Using this system, fluids are injected and produced in any combination desired. The end-caps are also shaped so that core and end-caps fit the inner diameter of the water jacket when assembled. This combination of endcaps, core, and water jacket allowed the coreholder to be set in a unique position. Identical cross sections are compared from experiment to experiment.

Prior to each experiment, cores were dried to zero water saturation in a vacuum oven. The core holder was assembled and the fracture aperture set. A number of experiments at constant water injection rates into the fracture were conducted. Injection rates varied from $0.1 \mathrm{cc} / \mathrm{min}$ to 4 $\mathrm{cc} / \mathrm{min}$. Different fracture apertures also were used, going from very narrow $(0.025 \mathrm{~mm})$ to wide $(0.1 \mathrm{~mm})$. Setting the flow rate with fixed fracture thickness, CT images were obtained to observe the progress of multidimensional imbibition. Water was injected on the left and produced from the right-hand side in order to have uniform advance of water along the bottom face of the core. Once the injection started, the core was scanned at the same location for successive times. Further experimental details and the equations for processing of the raw CT images are given by RangelGerman and Kovscek (2002).

\section{Results and Discussion}

Figures 3 and 4 show typical experimental results and illustrate the presence of two different fracture-flow regimes. Both images were taken after $0.32 \mathrm{PV}$ of water had imbibed into the core. White shading indicates water and black shading indicates air. Both systems have an injection rate into the fracture of $1 \mathrm{cc} / \mathrm{min}$. The chief difference between the two experiments is the fracture aperture. A wide fracture of $0.1 \mathrm{~mm}$ (Fig. 3) leads to relatively slow water advance through the fracture and a two-dimensional imbibition pattern. On the other hand, the fracture fills with water quickly when the aperture is narrow, $0.025 \mathrm{~mm}$ (shown in Fig. 4, the imbibition pattern is one-dimensional in this case). We have coined the behavior illustrated in Fig. 3 as the "filling-fracture" regime whereas that shown in Fig. 4 is termed an "instantly-filled" fracture.

The instantly-filled behavior is analogous to one-dimensional imbibition and requires little further comment here. The filling-fracture regime shows a variable length plane source due to relatively slow water flow through fractures. Water horizontal advance is controlled by the interaction between the matrix and the fracture as shown in Figs. 3 and 4. Because the advance of water in the horizontal and vertical directions each scale linearly with the square root of time, the mass of water imbibed scales linearly with time. That is, the mass imbibed in this twodimensional geometry is proportional to the product of these two length scales. 


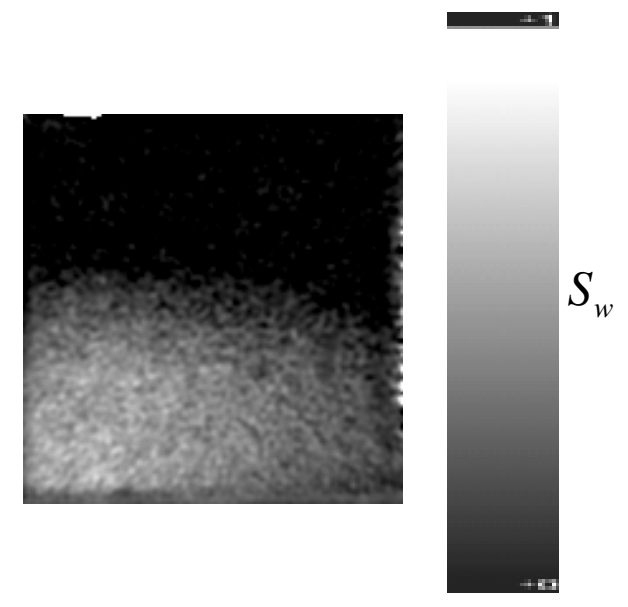

Figure 3. CT saturation image for $0.32 \mathrm{PV}$ imbibed, "filling-fracture." Aperture $=0.1 \mathrm{~mm}$. Injection rate $=1 \mathrm{cc} / \mathrm{min}$. Injection is from lower left corner and production from lower right corner.
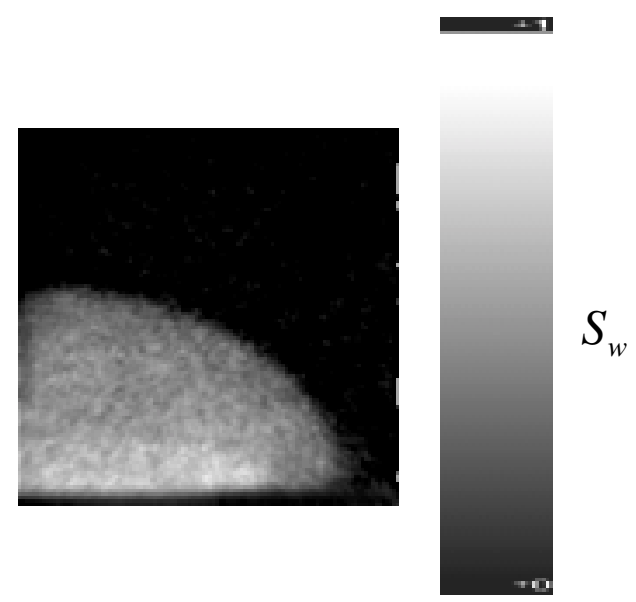

Figure 4. CT saturation image for $0.32 \mathrm{PV}$ imbibed. "Instantly-filled fracture". Aperture $=0.025 \mathrm{~mm}$. Injection rate $=1 \mathrm{cc} / \mathrm{min}$. Injection is from lower left corner and production from lower right corner.

Figure 5 summarizes the "filling-fracture" portion of all air-water experiments conducted. Within experimental uncertainty, the mass imbibed scales linearly with time before the advancing water front reaches the end of the fracture. Note that Fig. 5 shows an experiment where the initial water saturation was about 0.22 . The rate of imbibition is less because capillary forces are weaker than the cases with zero initial water saturation; however, a linear relationship between the mass of water imbibed and time is apparent. 


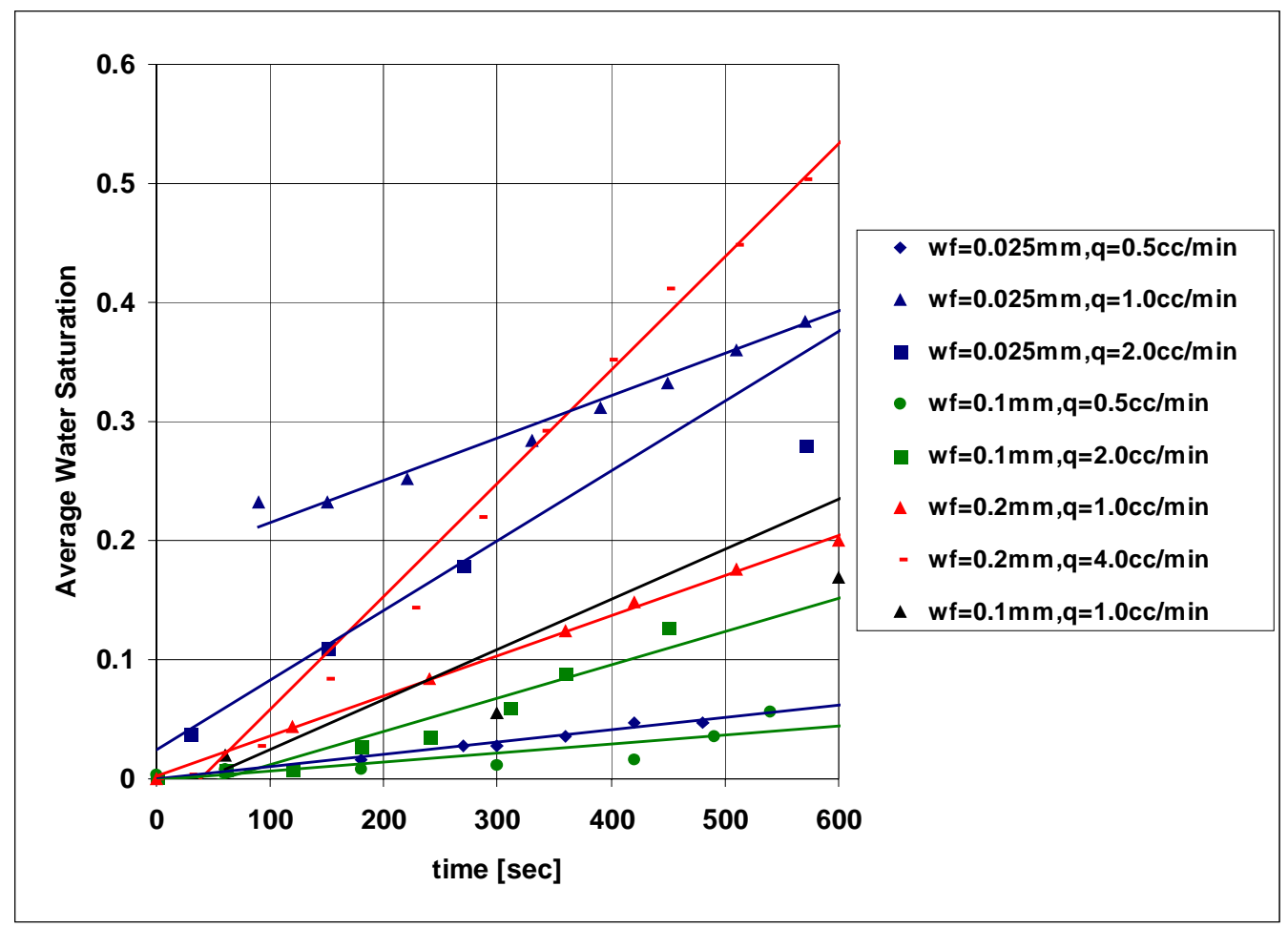

Figure 5. The average water saturation in the rock scales linearly with time ("filling-fracture" regime).

\section{Analytical Model}

The experimental results indicate clearly the existence of at least two regimes during capillary-dominated imbibition in fractured porous media. A precursor to developing more physical expressions for matrix-fracture transfer to be employed in numerical reservoir simulators is to develop an analytical model that describes the experiments. The model is useful for scaleup, for describing matrix-fracture transfer in a multiblock system, and if integrated provides the functional dependence with time of shape factors that describe matrix-fracture transfer in simulation.

Full details of the model are given by Rangel-German and Kovscek (2002, 2003). In summary, the imbibition process is approximated by a diffusion-like equation with a capillarypressure-based hydraulic diffusivity. Three assumptions were used: 1) the water saturation in the matrix is constant initially, 2) the water saturation in the matrix at an infinite distance from the fracture remains constant, and 3) the convection/diffusion equation with constant diffusivity, $\alpha_{h}$, applies for this problem. Linear superpostion is used to develop the saturation distribution of water in the matrix. Mass balance is applied to track the progress of water in the fracture.

Figure 6 displays typical results from the analytical model. Compare to Fig. 7 and it is found that the model well represents the experiment. The characteristic time, $t_{c}$, is the boundary between imbibition regimes. It can be found implicitly from Eq. 20 when $\mathrm{X}$ is set to the fracture 
characteristic length. For example, with the parameters used for the calculations in Fig. 6 , $t_{\mathrm{Dc}}$ equals 1800 and in dimensional quantities $t_{c}$ equals $12.5 \mathrm{~min}$. This compares favorably with the air-water experimental results in Fig. 7 where the critical time is between 10 and 15 min. Also note that the calculations well represent the shape and velocity of the water front.

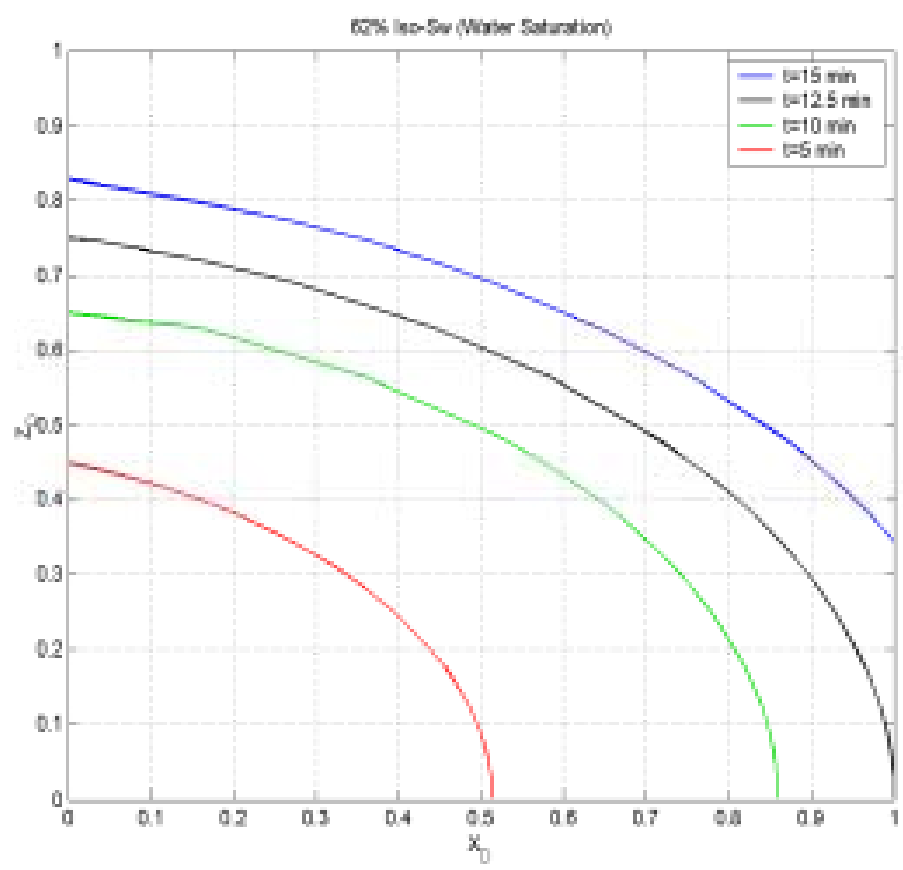

Figure 6. Water Iso-saturation curves for different times obtained with new approach. (air-water imbibition, $\mathrm{q}=1 \mathrm{cc} / \mathrm{min}$, and $\mathrm{w}_{\mathrm{f}}=0.1 \mathrm{~mm}$ ).

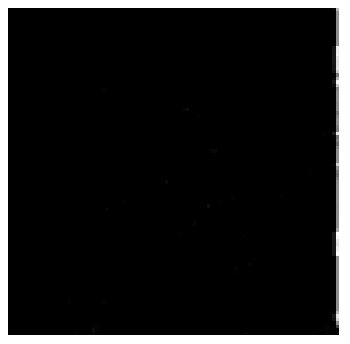

$1 \min (0.032 \mathrm{PV})$

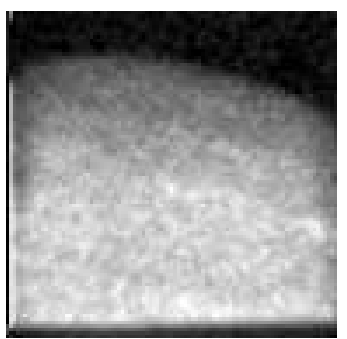

$20 \min (0.64 \mathrm{PV})$

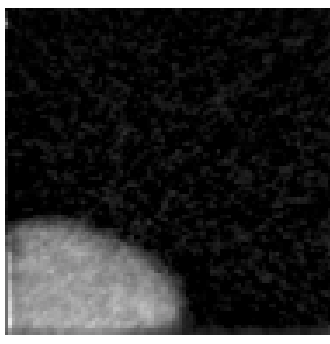

$5 \min (0.016 \mathrm{PV})$

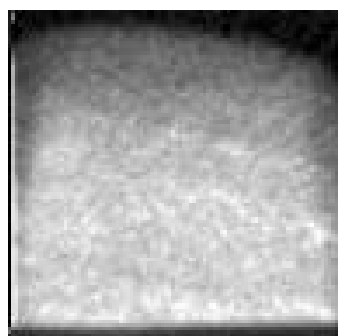

$25 \min (0.8 \mathrm{PV})$

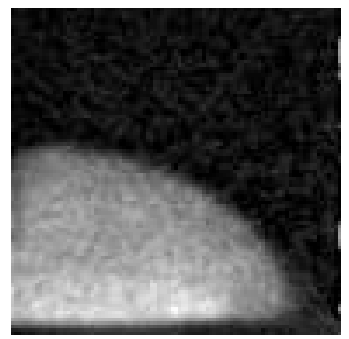

$10 \min (0.32 \mathrm{PV})$

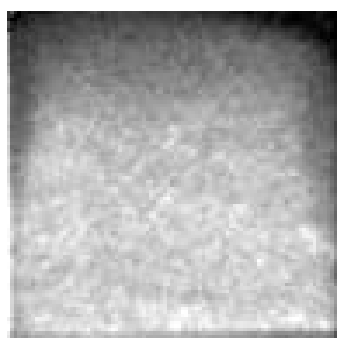

$30 \min (0.96 \mathrm{PV})$

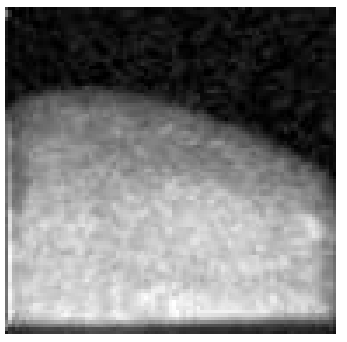

$15 \min (0.48 \mathrm{PV})$

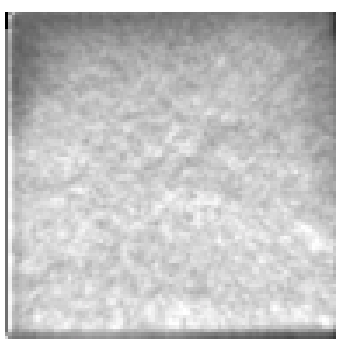

$1 \mathrm{hr}(1.92 \mathrm{PV})$

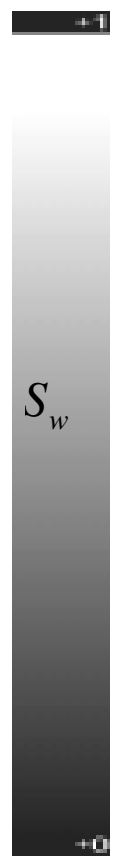

Figure 7. CT images for "filling-fracture" system for different times. Water injection at $1 \mathrm{cc} / \mathrm{min}$ in a fracture $0.1 \mathrm{~mm}$ thick. Injection is from lower left corner and production from lower right corner. 
The model allows us to develop explicit expressions for the rate of water transfer to the matrix, $R_{\mathrm{ff}}$. In the filling fracture regime the rate is obtained as

$$
R_{f f}=\frac{d Q_{m}(t)}{d t}=q_{w, i n j}\left(1-e^{t D} \operatorname{erfc}\left[\sqrt{t_{D}}\right]\right)
$$

where the dimensionless time is

$$
t_{D}=4\left(\frac{\phi}{\phi_{f}}\right)^{2} \frac{\alpha_{h}}{w_{f}^{2}} t
$$

In these equations, $Q_{m}$ is the volume of water imbibed into the matrix, $\mathrm{q}_{\mathrm{w} \text {,inj }}$ is the volumetric injection rate of water into the fracture, $\phi$ is the matrix porosity, $\phi_{\mathrm{f}}$ is the fracture porosity, $\alpha_{\mathrm{h}}=\mathrm{kk}_{\mathrm{rw}} / \mu_{\mathrm{w}}\left(\mathrm{dP}_{\mathrm{c}} / \mathrm{dS}_{\mathrm{w}}\right)$ is the hydraulic diffusivity of the matrix, and $\mathrm{w}_{\mathrm{f}}$ is the fracture aperture. The rate of transfer in the instantly-filled regime, $R_{i f}$, is a constant with respect to the

square-root of time. Thus for both regimes and accounting for initial water saturation, $S_{w i}$, the equation for average water saturation in the matrix block is:

$$
\overline{S_{w}}=t R_{f f}\left[1-H\left(t-t_{c}\right)\right]+\left[{ }_{c} R_{f f}+\left(t-t_{c}\right)^{0.5} R_{i f}\right] H\left(t-t_{c}\right)+\overline{S_{w i}}
$$

where $\mathrm{H}$ is the Heaviside function; and $\mathrm{t}_{\mathrm{c}}$ is the characteristic time for the fracture. The characteristic time, $t_{c}$, is the boundary between both regimes. The fracture fills instantly with respect to the matrix if $t_{D}\left(=t / t_{c}\right)$ is large.

\section{Physically Correct Transfer Functions}

Our next goal was to reduce the experimental data and the analytical model to a form appropriate for inclusion in a dual-porosity reservoir simulator. Due to the variable-extent of the imbibing area in the filling-fracture regime, it cannot be approached in as straight-forward a fashion as the instantly-filled fracture regime. To obtain a characteristic, dimensionless solution, a dimensional analysis is performed. It has two objectives: (i) to obtain a better understanding of the definition of shape factor within the dual-porosity/dual-permeability formulation, and (ii) provide a general shape factor for any kind of geometry, shape and regime that can be applied to current commercial numerical simulators without major coding complications.

One of the general dual-porosity (and dual-permeability) or MINC models is that these formulations were developed for pseudo-steady state flow between the fracture and the matrix, and/or uniformly distributed fractures that are totally immersed instantaneously. As shown both experimentally and analytically in the previous sections (and detailed in Rangel-German, 2002), there is a sizeable and varied suite of physical phenomena such as fractures being partially covered by water or parallel fractures that are not necessarily connected, and therefore the pressure distribution between them is non-uniform. These cases lead to more efficient ultimate recovery because they occur as co-current flow modes. Current numerical formulations lack the 
ability of matching the transient behavior in the matrix or considering blocks that are partially covered by water and, therefore, lead to overly pessimistic predictions of performance.

Discrepancies exist for values of shape factors proposed by different investigators. Exponential approximations or using only the first term of the summations in the analytical solutions helps keep the calculations efficient by giving a constant shape factor; however, these approaches do not necessarily capture the physics of the process. Apparently, this is a common case of trade-off between computational efficiency and accuracy. Nevertheless, the definition of the transfer function

$$
\tau=\frac{\sigma k V}{\mu}\left(\widetilde{p_{m}}-p_{f}\right)
$$

suggests a physically accurate formulation that is also computationally efficient. Equation 4 is a Darcy's law type equation applied to a characteristic distance from the fracture to some position within the matrix block. Dimensional analysis teaches that $\sigma$ is expressed as:

$$
\sigma(t)=\frac{A(t)}{V l^{*}(t)}
$$

where $A(t)$ is the area of the matrix block contacted by water, $V$ is the bulk volume of the rock matrix block and $l^{*}$ is the distance between the saturation at the fracture and the matrix average saturation, $\overline{S_{w}}$. Thus, the transfer function due to imbibition is

$$
\tau_{w}=\sigma_{S} V \alpha_{h}\left(S_{w \max }-\overline{S_{w m}}\right)
$$

with $\sigma$ calculated using Eq. 5.

The analytical model summarized in the previous section also includes an equation for the water advance in a single fracture in the presence of capillary imbibition into adjacent matrix blocks. This equation was obtained by coupling the matrix and fracture implicitly, and it is used here to derive or estimate the transfer functions needed in numerical reservoir simulation. The area of the matrix block contacted by water, $A(\mathrm{t})$, is obtained easily as shown by Rangel-German and Kovscek (2002).

The only term missing to compute the shape factor in Eq. 5 is the distance between the water saturation at the fracture and the matrix average water saturation, $l^{*}$. For a steady-state problem, it is clear that $l^{*}$ is the half distance between the fractures (i.e. half-length of the matrix block). However, for unsteady state behavior such as early-time filled-fracture or during the filling-fracture regime, the behavior of $l^{*}$ is not obvious. In this work, a simple analytical method to obtain $l^{*}$ is proposed. The integral of the saturation distribution over the entire domain (n-D, if needed) is equal to the average saturation in the matrix times a characteristic distance to the centroid of the domain. The characteristic distance, $l^{*}$, for one set of fractures is 


$$
l^{*}(t)=\frac{\int_{0}^{L_{x}} S_{w}(x, t) d x}{\overline{S_{w m}}(t)}
$$

thus, $l^{*}$ depends on the shape of the matrix block. Figure 8 shows a schematic of the distance, $l^{*}$ for water saturation, as a function of time.

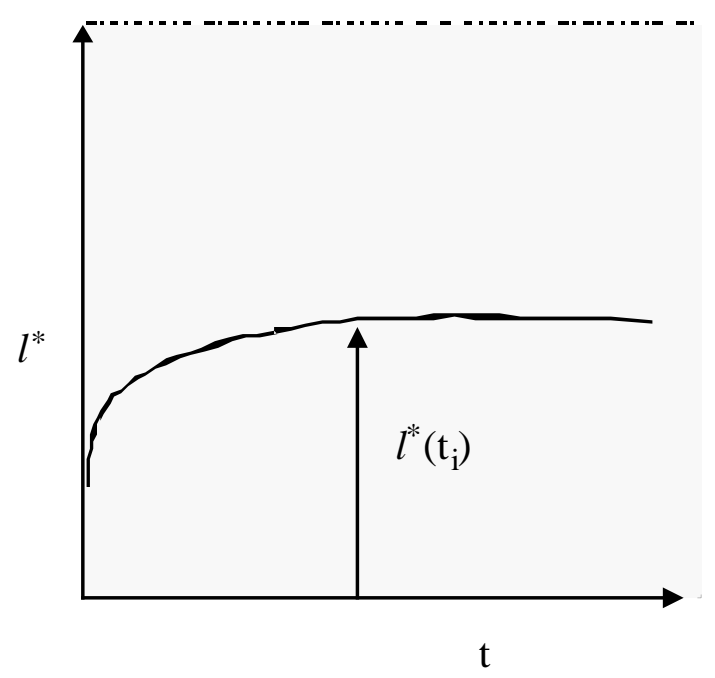

Figure 8. Schematic representation of the characteristic length versus time.

An analogous $\sigma(t)$ is needed when dealing with pressure; thus, $l_{\mathrm{p}}^{*}$ is obtained in a manner similar to Eq. 7, but using the pressure solution and the average pressure in the matrix:

$$
l_{p}^{*}(t)=\frac{\int_{0}^{L_{x}} p_{w}(x, t) d x}{\overline{p_{w m}}(t)}
$$

The characteristic length, $l^{*}$, is computable from experimental results obtained with CT scanning. Every CT image (corresponding to a single time) of every experiment is translated into a bitmap file containing the water saturation value at each pixel. The integral of Eq. 7 is calculated numerically. Then, one computes the average water saturation in the matrix. Figure 9 illustrates a plot of the shape factor, $\sigma(t)$, calculated by means of Eqs. 6 and 8 against dimensionless time, $t_{D}=\frac{\alpha_{h} t}{L_{x}^{2}}$, on a log-log plot. Four different experiments from Rangel-German and Kovscek (2002) that span a variety of behavior are analyzed. These integrals (Eqs. 7 and 8) can also be obtained from the analytical or numerical solution of the distribution of saturations and pressures, respectively. 


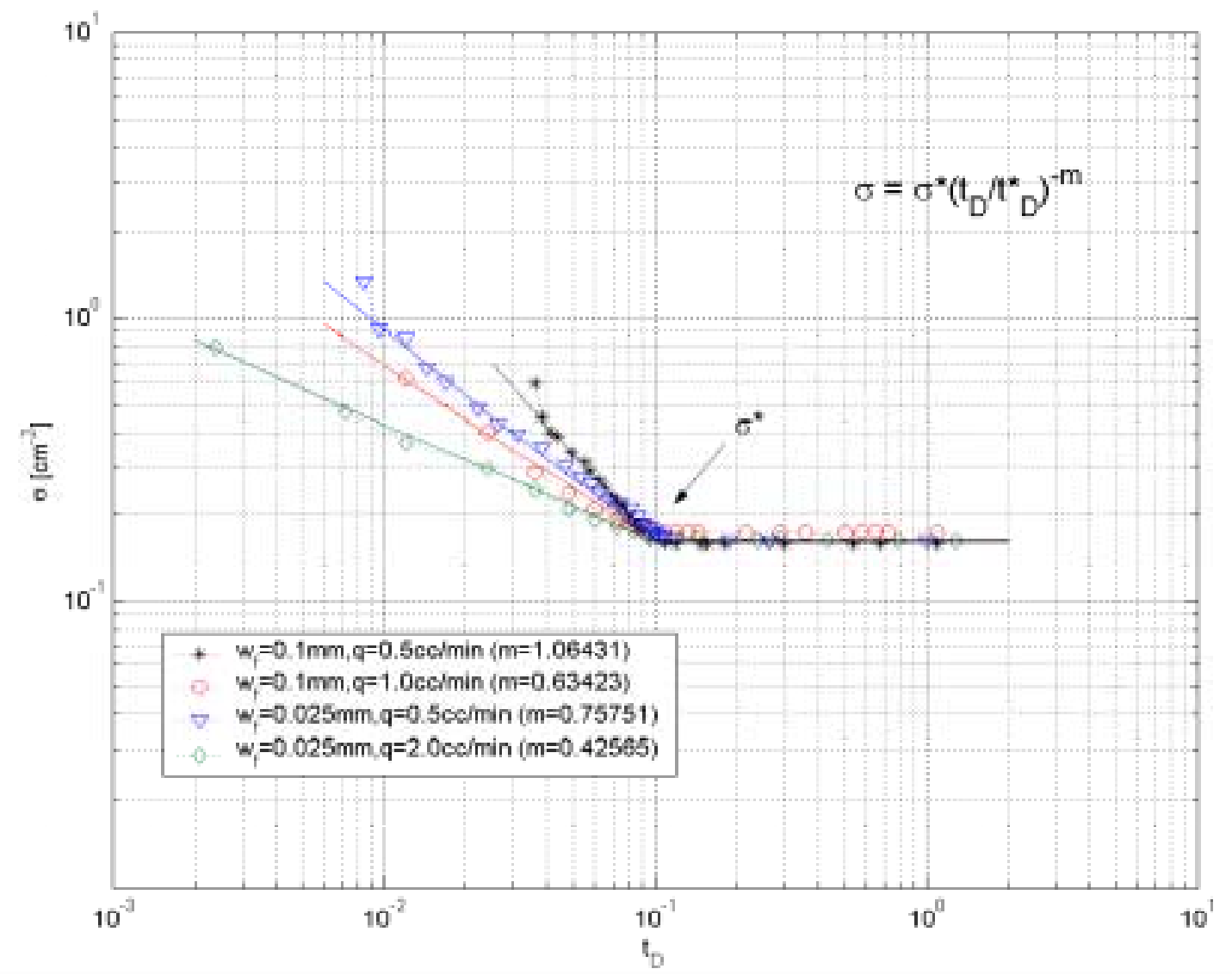

Figure 9. Shape factor versus time.

All of the experiments converge to the constant shape factor, $\sigma^{*}$, at the same characteristic dimensionless time of approximately, $t^{*}{ }_{D}=0.1$. In general, the new shape factor is written as:

$$
\sigma=\sigma *\left(\frac{t_{D}}{t_{D}^{*}}\right)^{-m} \text { for } t_{D}<t_{D}{ }^{*}
$$

and

$$
\sigma=\sigma * \text { for } t_{D} \geq t_{D}^{*}
$$

where $t^{*}{ }_{D}$ is 0.1 and $m$ is a function of the flow rate and the fracture aperture. Note that this formulation converges to the classical constant $\sigma$, and also has a time varying portion for $t_{D}$ less than $t_{D}{ }^{*}$. This makes its implementation in any dual-porosity simulator relatively straightforward. Alternately, the time dependence could be converted to an aqueous phase saturation dependence that is a function of matrix-block geometry, capillary pressure, and relative permeability functions.

Figure 9 also provides information on the physics of the fracture filling and imbibition processes. For instance, the experiment performed at a flow rate and fracture aperture of 2.0 $\mathrm{cc} / \mathrm{min}$ and $0.025 \mathrm{~mm}$, respectively, corresponds to a narrow fracture filling rapidly at a high flow rate; therefore, the one-dimensional advance occurs early in the experiment and the centroid of the average water saturation, $l^{*}$, is closer to 0.5 as the front becomes more uniform. This case 
exhibits the smallest and most constant $\sigma$ during the transient period. On the other hand, the experiment performed at a flow rate and fracture aperture of $0.5 \mathrm{cc} / \mathrm{min}$ and $0.1 \mathrm{~mm}$, respectively, corresponds to a relatively wide fracture filling slowly at a low flow rate (see Fig. 3). The centroid of the average water saturation has a small value initially; hence a large value of shape factor is obtained. It is important to mention that both the instantly-filled and filling-fracture regimes display similar behavior when using the definition of shape factor given by Eq. 5. Thus, the expression for shape factor is general.

A numerical validation of the proposed shape factor is presented next with comparisons made to the experimental data, analytical model (above), the modified dual-porosity formulation with the new time-dependent shape factor and transfer function, and full-physics numerical simulation. The new dual-porosity formulation was coded into a simple simulator. Additionally, a fine-grid numerical simulation was also performed to match the results using Eclipse 100 (Geoquest 2000). Figure 10(a) illustrates a section of the grid used in the numerical simulation. The matrix block is described by a 10x10x10 grid, and the fracture is comprised in a 10x10x1 grid with dimensions corresponding to the experimental apparatus shown in Fig. 2. Fig. 10(b) illustrates the matrix capillary pressure and relative permeability functions. The fine-grid simulation aims to mimic both the CT-experimental and analytical results; therefore, properties of both matrix and fracture are the same as those from the experiments described in Rangel-German (2003). Full details of the set up of the validation are given in Rangel-German and Kovscek (2003).

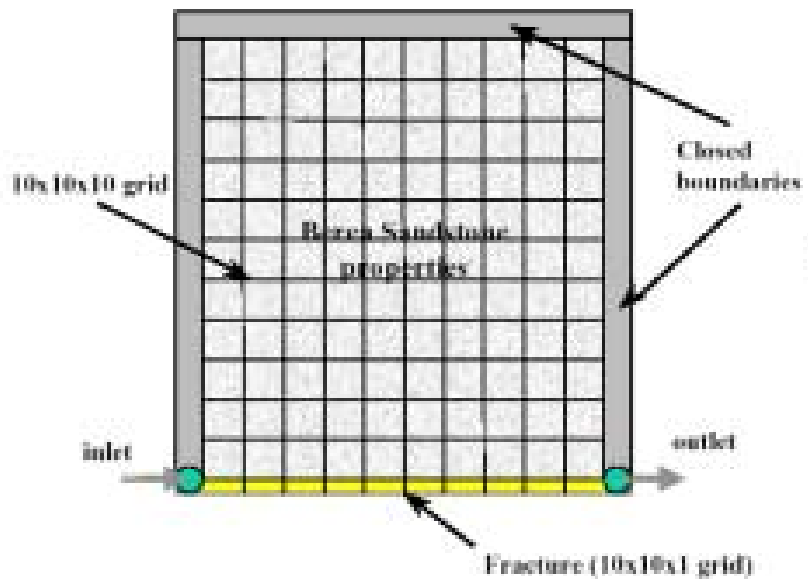

(a)

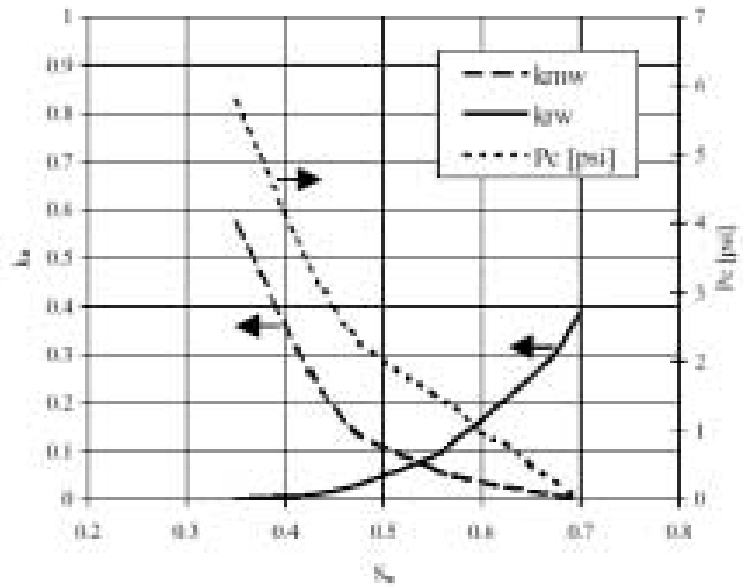

(b)

Figure 10. Section of the grid (a) and (b) matrix relative permeability and capillary pressure functions used for the numerical simulations.

Figures 11 and 12 contrast the results with all of the approaches. Figure 11 shows the average water imbibed versus time. Agreement among all approaches is excellent; but of course the analytical and new dual-porosity approaches execute much more quickly than the fine-grid simulation. Figure 12 compares the extent of imbibition (iso-saturation curves/maps) for different 
times obtained with the experimental set-up, the analytical model described in the previous sections, the modified 'dual-porosity' approach, and the fine-grid simulation. The squares within the modified dual porosity portion of Fig. 12 illustrate the fraction of the matrix filled with water. Figure 12 indicates that not only does the analytical model and now dual-porosity approach get the imbibed volumetrics correct, but the water is also distributed correctly.

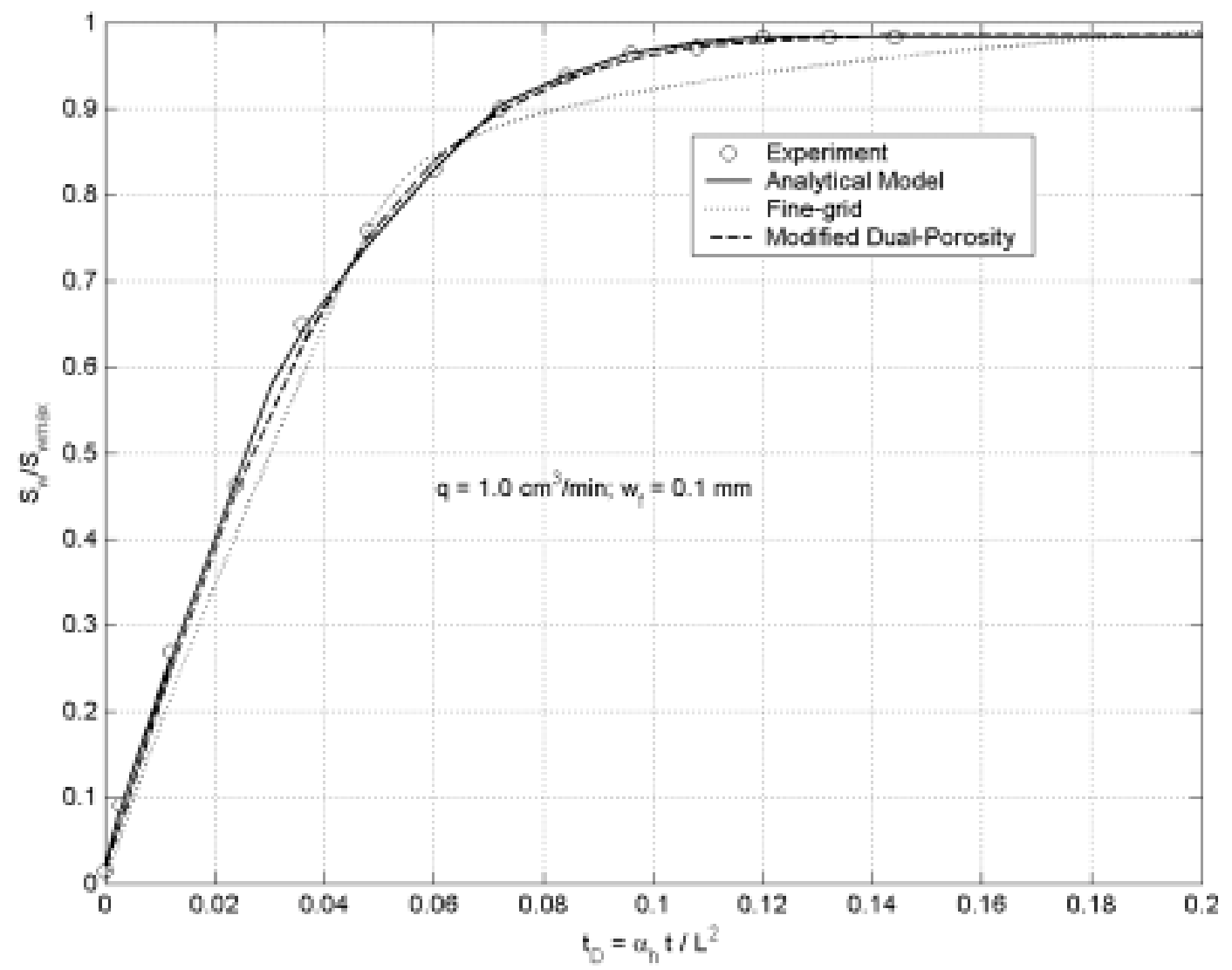

Figure 11. Comparison of the average water saturation versus dimensionless time among the experimental results, analytical model, fine-grid numerical simulation, and the modified dual-porosity approach. 
X-ray CT Scanning Core Experiment:
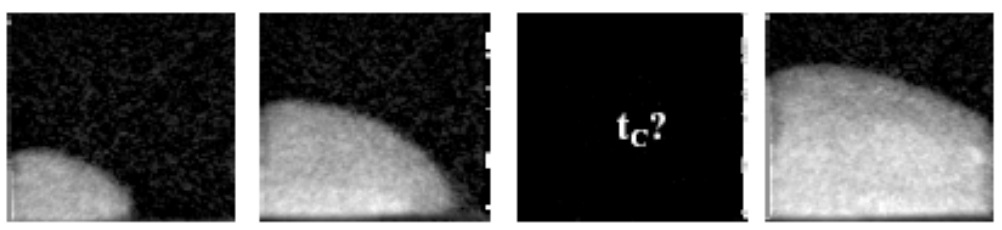

Analytical Model, $\overline{S_{w}}=t R_{f f}\left[1-H\left(t-t_{c}\right)\right]+\left[t_{c} R_{f f}+\left(t-t_{c}\right)^{0.5} R_{f f}\right] H\left(t-t_{c}\right)+\overline{S_{w t}}$ :

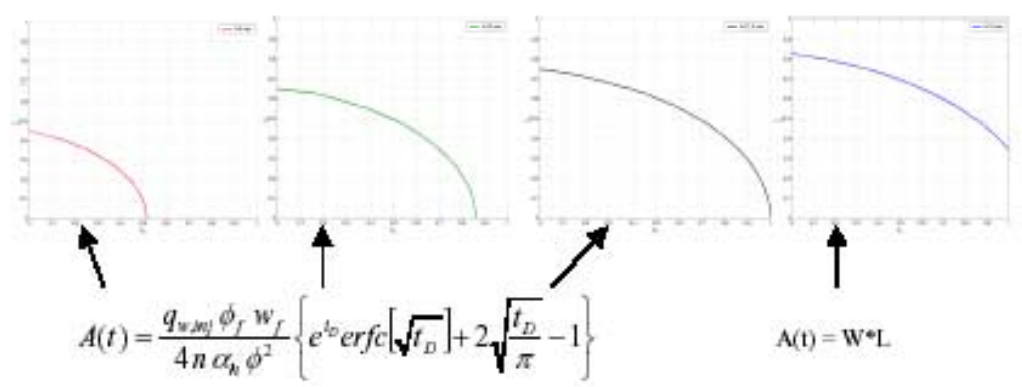

Modified Dual-Porosity, $1 \times 1 \times 1$ matrix and $1 \times 1 \times 1$ fracture:
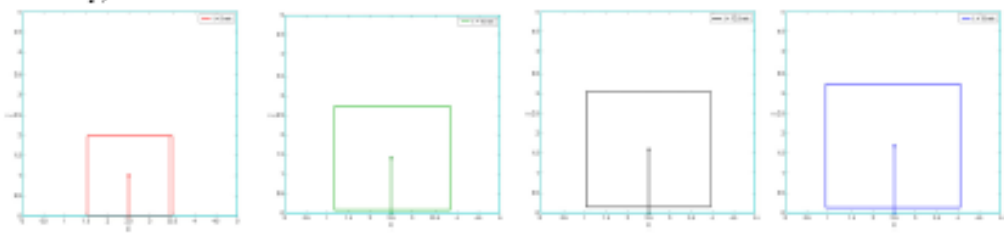

$$
\text { With } \tau=V \alpha_{n} \sigma\left(S_{\text {mix }}-\overline{S_{\text {wav }}}\right) \text { where } \sigma(t)=\frac{A(t)}{V l^{*}(t)} \text { and } l^{*}(t)=\frac{\int_{0}^{t_{x}} S_{w}(x, t) d x}{\overline{S_{\text {wam }}}(t)}
$$

Fine-grid Single-Porosity, 10x10x10 matrix and $10 \times 10 \times 1$ fracture:
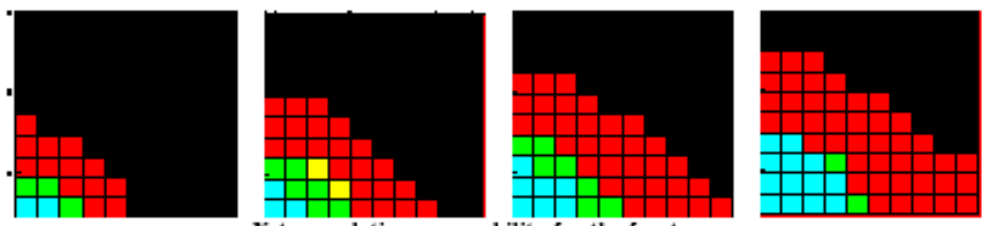

Figure 12. Comparison of the extent of imbibition between experimental data, analytical model, modified dual-porosity and fine-grid simulation. Fracture aperture $=$ $0.1 \mathrm{~mm}$. Injection rate $=1 \mathrm{cc} / \mathrm{min}$. Injection is from the lower left corner.

\section{Conclusions}

In summary, the pseudo-steady state approach for describing matrix-fracture transfer leads to constant shape factors. However, multi-phase flow in fractured porous media is not wellapproximated by this assumption as illustrated by our experiments. Clearly, there are multiple modes of fracture and matrix fill up. The behavior of the shape factor is clearly time dependent, and therefore the use of a constant shape factor is incorrect. Formulations for modeling flow in fractured porous media were, thus, reconsidered. One of the advantages of the new shape factor is that it is based on dimensional analysis. It embodies the full physics of matrix-fracture transfer. 


\section{References}

Akin, S., Schembre, J.M., Bhat, S.K., and Kovscek, A.R., 2000. Spontaneous imbibition characteristics of diatomite. J. Petrol. Sci. \& Eng., 25: 149-165.

GEOQUEST, 2000. Eclipse 100 Technical Description 2000A, GeoQuest, Schlumberger.

Rangel-German, E. R. and A. R. Kovscek, 2002. "Experimental and Analytical Study of Multidimensional Imbibition in Fractured Porous Media," Journal of Petroleum Science and Engineering, 36(1-2), 45-60.

Rangel-German. E. R. and A. R. Kovscek, 2003. "Time-Dependent Matrix-Fracture Shape Factors for Partially and Completely Immersed Fractures," SPE 84411, Proceedings of the SPE Annual Technical Conference and Exhibition, Denver, Oct. 5-8. 


\section{Foam Generation at Low Surfactant Concentration}

\section{(Task 1b)}

\section{Introduction}

This project was designed to obtain an understanding of how overall recovery, pressure drop profiles, and water saturation profiles shift with changing surfactant concentration. There are both theoretical and experimental components. A quantitative model is necessary to interpret the experimental results to follow, because the mechanisms of gas mobility reduction by foam derive from the flow resistance of discrete bubbles. Thus, it seems natural to compute directly gas mobility from the texture or number concentration of bubbles inside porous media. A balance that tracks the evolution of the number concentration of bubbles is one means to link bubbles and gas mobility. Foam is treated mathematically as a nonreactive, time-dependent component of the gas phase. Previous work has formulated a population balance approach that is mechanistic in that well documented foam mechanisms are incorporated into a continuum balance equation for foam (Kovscek et al., 1995; Kovscek et al., 1997). The theory reproduces well experimental foam behavior at surfactant concentrations around $1 \mathrm{wt} \%$. However, this approach has not been tested over a variety of surfactant concentrations and especially at low concentration where foam is weak.

\section{Model}

In addition to the requisite material balances for water, surfactant, and nitrogen chemical species, a transient balance on the mean bubble size is written. In one dimension (Kovscek et al. 1995):

$$
\frac{\partial}{\partial t}\left[\phi\left(S_{f} n_{f}+S_{t} n_{t}\right)\right]+\frac{\partial}{\partial x}\left(u_{f} n_{f}\right)=\phi S_{g}\left[k_{1} v_{w} v_{f}^{1 / 3}-k_{-1} v_{f} n_{f}\right]+Q_{b}
$$

where $t$ denotes time, $\phi$ is porosity, $S$ is saturation, $n$ is bubble concentration (bubbles per unit volume of gas), $u$ is Darcy velocity, $\mathrm{v}$ is the interstitial velocity $(v=u / S \phi), k_{1}$ is the generation rate constant, $k_{-1}$ is the coalescence rate constant, and $Q_{\mathrm{b}}$ is a source/sink term for bubbles. The subscript $\mathrm{f}$ refers to flowing foam, $\mathrm{t}$ to trapped foam, $\mathrm{g}$ to the gas phase, and $\mathrm{w}$ to the aqueous phase. Units of the various terms are listed in Table 1. We refer to number concentration, foam texture, and bubble texture synonymously throughout this work.

Note the similarity of Eq. 1 with the usual mass and energy balances for transport processes in porous media. The first term on the left of Eq. 1 is the accumulation of foam bubbles and the second term is the flux of foam bubbles. The first term on the right of Eq. 1 represents the net generation of foam bubbles, i.e., generation minus coalescence. Foam generation is taken as a power-law expression that is proportional to the interstitial liquid velocity multiplied by the $1 / 3$ power of the gas velocity. The origin of these velocity dependencies for generation may be found elsewhere (Kovscek and Radke, 1996). 
Table 1. Parameter Values

\begin{tabular}{|c|c|c|c|}
\hline $\begin{array}{r}\text { Two-phase } \\
\text { param }\end{array}$ & & $\begin{array}{r}\text { Population ba } \\
\text { param }\end{array}$ & \\
\hline parameter & value & parameter & value \\
\hline $\mathrm{k}$ & $7 \mu \mathrm{m}^{2}$ & $\mathrm{k}_{1}$ & $7.5 \times 10^{12} \mathrm{~s}^{1 / 2} \mathrm{~m}^{-13 / 3}$ \\
\hline$\phi$ & 0.35 & $\mathrm{k}_{-1}^{\mathrm{o}}$ & $100 \mathrm{~m}^{-1}$ \\
\hline $\mathrm{a}$ & 3 & $\alpha$ & $\begin{array}{l}2.5 \times 10^{-14} \\
\operatorname{Pas}^{2 / 3} \mathrm{~m}^{10 / 3}\end{array}$ \\
\hline $\mathrm{b}$ & 1.8 & $X_{f}$ & 0.10 \\
\hline $\mathrm{k}_{\mathrm{rw}}^{\mathrm{o}}$ & 0.7 & & \\
\hline $\mathrm{k}_{\mathrm{rg}}^{\mathrm{o}}$ & 1 & \multicolumn{2}{|c|}{$\begin{array}{l}\text { Capillary pressure function } \\
\text { after Pruess (1987) }\end{array}$} \\
\hline$S_{\text {wir }}$ & 0.06 & \multirow{3}{*}{\multicolumn{2}{|c|}{$\begin{aligned} \mathrm{P}_{\mathrm{c}}=\sigma & \sqrt{\frac{\phi}{\mathrm{k}}}\left(1.417\left(1-\mathrm{S}_{\mathrm{wd}}\right)\right. \\
& \left.-2.120\left(1-\mathrm{S}_{\mathrm{wd}}\right)^{2}+1.263\left(1-\mathrm{S}_{\mathrm{wd}}\right)^{3}\right)\end{aligned}$}} \\
\hline$\mu_{w}$ & $1.0 \mathrm{mPa}-\mathrm{s}$ & & \\
\hline$\mu_{\mathrm{g}}$ & $0.018 \mathrm{mPa}-\mathrm{s}$ & & \\
\hline
\end{tabular}

Foam coalesces when bubbles flow into so-called termination sites (Jiménez and Radke, 1989; Kovscek and Radke, 1994). Hence, foam coalescence is taken as proportional to the interstitial flux of foam, $\mathrm{v}_{\mathrm{f}} \mathrm{n}_{\mathrm{f}}$. The coalescence rate constant varies strongly with the local capillary pressure, surfactant formulation, and aqueous-phase concentration. A high rate of coalescence is expected at low surfactant concentration because there is little surfactant to exert a stabilizing effect. A dramatically lower rate is expected when the concentration is high and the surfactant is known to produce stable foam, provided that the porous medium does not exceed limiting capillary pressure.

The effects of surfactant concentration and capillary pressure are embodied in the coalescence rate constant. The following functional relationship is employed:

$$
k_{-1}=k_{-1}^{o}\left(\frac{P_{c}}{P_{c}^{*}-P_{c}}\right)^{2}
$$

where $\mathrm{k}_{-1}{ }^{0}$ is a scaling factor that is set constant and $\mathrm{P}_{\mathrm{c}}{ }^{*}$ is the limiting capillary pressure, $\mathrm{k}_{-1}$ becomes very large as $\mathrm{P}_{\mathrm{c}}$ and $\mathrm{P}_{\mathrm{c}}{ }^{*}$ approach equality. Khatib et al. (1988) first suggested that foam-film strength was directly related to gas mobility reduction in the presence of foam. For a given surfactant and concentration, they observed dramatic foam coarsening at a particular capillary pressure that they termed the "limiting capillary pressure," $\mathrm{P}_{\mathrm{c}}{ }^{*}$. Above $\mathrm{P}_{\mathrm{c}}{ }^{*}$, foam films are unstable, coalescence of foam lamellae is significant, and high gas mobility emerges. Below $\mathrm{P}_{\mathrm{c}}{ }^{*}$, coalescence is significantly less and gas mobility is low. The major task of this work is to infer the functional form of $\mathrm{P}_{\mathrm{c}}{ }^{*}$ with respect to surfactant concentration from laboratory experiments and to simulate these experiments quantitatively. 
To solve Eq. 1, additional information on the convection of foam and wetting aqueous phase is required. Darcy's law is retained including the formalism of multiphase relative permeability curves. For the gas phase, we replace viscosity with an effective viscosity relation for foam:

$$
\mu_{f}=\mu_{g}+\frac{\alpha n_{f}}{v_{f}^{1 / 3}}
$$

where $\alpha$ is a constant of proportionality. This expression follows directly from theoretical studies of bubble flow in capillary tubes and porous media (Bretherton, 1961; Hirasaki and Lawson, 1985; Wong et al. 1995a, 1995b).

Foam also reduces gas mobility by reducing the gas-phase relative permeability. Because the portion of gas that actually flows partitions into the largest pores with the least flow resistance, we employ a so-called "Stone-type" relative permeability model (Stone, 1970), although the ideas employed here predate Stone (Saraf and Fatt, 1967). Hence, the relative permeability of the flowing foam is only a function of $S_{f}$. Because the wetting phase partitions selectively to the smallest pore space, the aqueous-phase relative permeability is unaffected by flowing and stationary foam. This statement is well established by experiment (Bernard et al., 1965; Holm, 1968; Sanchez et al., 1986; Huh and Handy, 1989; DeVries and Wit, 1990). Aqueous phase relative permeability remains solely a function of $S_{w}$. Clearly, the relative permeability of the stationary foam is zero. A standard exponent relative permeability model is

used

$$
\begin{gathered}
k_{r w}=k_{r w}^{o} S_{w d}^{a} \\
k_{r f}=k_{r g}^{o} S_{f d}^{b}
\end{gathered}
$$

with $\mathrm{a}$ and $\mathrm{b}$ representing the exponents for water and gas flow, respectively. The superscript $\mathrm{o}$ indicates the endpoint relative permeability. The reduced saturations, $S_{\mathrm{id}}$, are written

$$
\begin{aligned}
& S_{f d}=X_{f}\left(1-S_{w d}\right) \\
& S_{w d}=\frac{\left(S_{w}-S_{w i r}\right)}{\left(1-S_{w i r}\right)}
\end{aligned}
$$

where $X_{f}=S_{f} / S_{g}$ is the fraction of the gas phase that is flowing and $S_{\text {wir }}$ is the irreducible aqueous-phase saturation. The relative permeability exponents and endpoint relative permeabilities represent values for non-dispersed, continuum gas-liquid flow in the porous medium. 


\section{Experimental}

The apparatus consists of a $0.51 \mathrm{~m}$ (20 in) long aluminum sandpack with a $0.051 \mathrm{~m}$ ( 2 in) inner diameter. It was packed with dry 100-120 mesh Ottawa sand in an upright position and pneumatic vibrators were used to ensure good settling. Screens at the inlet and outlet faces of the sandpack prevented washout of sand grains. The average porosity and permeability of pack 1 are $35 \%$ and $6.7 \mu \mathrm{m}^{2}$, whereas for pack 2 the values are $36 \%$ and $7.0 \mu \mathrm{m}^{2}$. CT images, as described later, confirmed that the packs were homogeneous. Permeability values reported are permeability to brine.

The sandpack attaches to an L-shaped mounting bracket for bolting of the apparatus to a precision positioning system (Compumotor, RP240, Parker Hannifin Corp.). Displacements were conducted with the core oriented horizontally. Positioning errors are not detectable with this system. Note that the geometry for CT scanning is conventional so that multiple cross sections are examined as the core translates through the CT scanner. The core length is too great to be suitable for the unconventional geometry employed in our imbibition studies. Fairly long porous media need to be used during foam experiments because the inlet sections of cores are subjected to strong foam generation and coalescence forces embodied in Eq. 1.

Aluminum pressure taps were welded to the surface of the coreholder. Each tap was machined to accept a filter and thereby sand migration through the tap was avoided. Pressure response was measured by a single Paroscientific pressure transducer (model 43K-101) connected to a 7-way valve. Measurement of six independent pressures is possible. The valve was switched manually and a PC was used to collect absolute pressure data from the transducer. The total acquisition time for a set of pressure data is roughly 30 to 40 s.

Nitrogen and surfactant solution are co-injected at constant rates using a Matheson 0-10 SCCM (standard cubic centimeters per minute) mass flow meter (model 8240) and an ISCO (model 500D) syringe pump, respectively. Experiments are conducted at backpressures of roughly $690 \mathrm{kPa}$ (100 psi) using a Grove Mitey-Mite dome loaded backpressure regulator (Model S-91LW).

In-situ saturation information was collected with the CT scanner. Scans were made on 13 cylindrical volume sections perpendicular to the central axis of the core. Scan locations were not evenly spaced so that scanning through pressure ports was avoided. The acquisition time of one image is $7 \mathrm{~s}$ and the processing time is about $40 \mathrm{~s}$. The total time of measurement is short enough to capture accurately the position of the front. Raw CT number data are converted to porosity and saturation distribution information according to the algorithms summarized by Akin and Kovscek (2003).

The surfactant used to make the foamer solution is an $\alpha$-alpha olefin sulfonate (AOS 1416) supplied by Shell Chemical Co. Sodium chloride $(\mathrm{NaCl})$ at a concentration of $0.5 \mathrm{wt} \%$ in distilled water was the brine. Surfactant solution concentrations employed in the experiments were $0.005,0.01,0.02,0.1$, and $1.0 \mathrm{wt} \%$ active surfactant. The less concentrated solutions were prepared by diluting the $1 \mathrm{wt} \%$ foamer solution with a $0.5 \mathrm{wt} \%$ brine. The critical micelle concentration (CMC) of the solution is roughly $0.005 \mathrm{wt} \%$ (Bertin et al. 1999) and the nominal solution viscosity is $1 \mathrm{mPa}$. 
The experimental procedure is relatively simple. The pack was prepared and mounted on the positioning system and then $\mathrm{N}_{2}$ was injected to assure a dry pack. The system back pressure was increased to $690 \mathrm{kPa}(100 \mathrm{psi})$ and dry CT scans were made at the prescribed positions. Carbon dioxide $\left(\mathrm{CO}_{2}\right)$ was injected to displace the $\mathrm{N}_{2}$. At least 10 pore volumes of $0.005 \mathrm{wt} \%$ foamer solution were injected to saturate the pore space with liquid and satisfy adsorption of surfactant to the sand. The advantage of $\mathrm{CO}_{2}$ is that it dissolves readily in water ensuring that all gas is removed. Periodically, the pressure lines were opened to purge any gas that had accumulated. Then wet CT images were collected.

The gas-liquid mixture was not foamed before injection and the injection rates are constant throughout the experiments. The foamer solution injection rate was fixed at $0.07 \mathrm{~mL} / \mathrm{min}$ and the gas flow was set to $3.8 \mathrm{SCCM}$ (standard cubic centimeters per minute). This translates to a superficial gas velocity of about $0.39 \mathrm{~m} / \mathrm{d}$ and a gas fractional flow of $88 \%$ at the nominal system back pressure of $690 \mathrm{kPa}$. The total superficial velocity, including liquid injection, is 0.44 $\mathrm{m} / \mathrm{day}$.

Following the completion of an experiment, the core was flushed with $\mathrm{CO}_{2}$ and then copious (10-20 PV) amounts of foamer solution of the next larger concentration were injected to resaturate the sandpack. The CT scanner was used to monitor the progress of resaturation thereby verifying that all gas had been ejected from the core. The experiments were performed in order of increasing surfactant concentration to assure that surfactant desorption from solid did not affect results. The first pack $\left(6.7 \mu \mathrm{m}^{2}\right)$ was used for solutions of $0.005,0.01,0.02$, and $1 \mathrm{wt} \%$ surfactant, and the second pack $\left(7 \mu \mathrm{m}^{2}\right)$ was employed for the $0.1 \mathrm{wt} \%$ solution.

\section{Results and Discussion}

To model the transient foam displacements, Eqs. 1-4 are solved with a standard fullyimplicit, simultaneous-solution finite difference method with upstream weighting of the phase mobilities. Additionally, water and nitrogen mass balances are computed. Because the sand packs are presaturated with surfactant solution, there is no need to compute a surfactant mass balance. The primary variables are water-phase pressure, gas-phase saturation, and bubble density. Boundary and initial conditions mirror the experiments: gas and liquid are injected at constant mass rates and the outlet pressure is fixed at $690 \mathrm{kPa}(100 \mathrm{psi})$. The surfactant and gas are not foamed before injection. Therefore, no bubbles are injected and $Q_{b}$ is set to zero. The initial conditions include $S_{\mathrm{w}}$ equal to $1, \mathrm{n}_{\mathrm{f}}$ equal to 0 , and the appropriate uniform surfactant concentration depending upon the experiment under consideration.

Tables 1 and 2 list the model parameters including standard multiphase flow information as well as population balance parameters. Also given in Table 1 is the capillary pressure versus water saturation function (Pruess 1987). The parameter $\alpha$ in the expression for effective foam viscosity varies with surface tension (Hirasaki and Lawson 1985). As Table 2 shows, the surface tension changes by only $2.9 \mathrm{mN} / \mathrm{m}$ over the entire range of concentrations employed. Accordingly, $\alpha$ is held fixed in the simulations to follow. The ratio of foam generation to coalescence rate constants, $\mathrm{k}_{1} / \mathrm{k}_{-1}^{\mathrm{O}}$, was chosen to yield bubbles with undistorted spherical radii of about $0.4 \mathrm{~mm}$ for the $1 \mathrm{wt} \%$ case. Further information on parameter estimation for population 
balance models is discussed in some detail elsewhere (Kovscek and Radke 1994; Kovscek et al. 1995).

Table 2. Limiting Capillary Pressure and Surface TensionV

( $\sigma$ from Bertin et al., 1999)

\begin{tabular}{|l|l|l|}
\hline Concentration $(\mathrm{wt} \%)$ & $\sigma(\mathrm{mN} / \mathrm{m})$ & $\mathrm{P}_{\mathrm{c}}{ }^{*}(\mathrm{kPa})$ \\
\hline \hline 1.0 & 31.1 & 30.4 \\
\hline 0.10 & 31.9 & 17.6 \\
\hline 0.020 & 32.8 & 7.19 \\
\hline 0.010 & 33.1 & 3.67 \\
\hline 0.005 & 34.0 & 2.34 \\
\hline
\end{tabular}

The critical capillary pressure, $\mathrm{P}_{\mathrm{c}}{ }^{*}$, as a function of surfactant concentration is required to simulate the experiments. Because the surfactant concentration in each experiment is constant, we set $\mathrm{P}_{\mathrm{c}}{ }^{*}$ at each concentration rather than assuming a functional form for $\mathrm{P}_{\mathrm{c}}{ }^{*}$ versus surfactant concentration. Table 2 lists the value of $\mathrm{P}_{\mathrm{c}}$ * found for each concentration and the experimentally determined surface tension as a function of concentration for this particular surfactant at a brine concentration of $0.5 \mathrm{wt} \%$. As expected, $\mathrm{P}_{\mathrm{c}}{ }^{*}$ is large when the surfactant concentration is large and decreases as the concentration approaches the CMC (Aronson et al., 1994).

The modeling results for foam texture evolution at each surfactant concentration are presented first. Unfortunately, no experimental method exists to measure directly foam texture in situ. High surfactant concentration results are shown first and then subsequent simulations at lower concentration follow. Next, the effect of foam texture on gas mobility is judged from measured and computed aqueous-phase saturation profiles as a function of time. Pressure drop profiles and the match between experiment and model complete this section.

The predicted bubble concentration along the length of the core as a function of time is given for each of the different surfactant concentrations in Fig. 1. Figure 1a represents the largest concentration while Fig. 1e shows the lowest. Time is given nondimensionally in pore volumes of total injection with the exit pressure used to calculate the volumetric gas rate. In Fig 1a, representing the $1 \mathrm{wt} \%$ concentration experiment, a region of net generation is found near the inlet. Bubble concentration is nearly constant at all time levels beyond roughly the first quarter of the sand pack until the concentration peaks immediately upstream of the front. Physically, the net rate of foam generation is large at the front. Thus, the bubble concentration goes through a maximum before decreasing to zero downstream of the front where there is no gas, and hence, no bubbles (Kovscek et al., 1997). The relatively constant foam concentration between the inlet region and the foam front is about 22 bubbles $/ \mathrm{mm}^{3}$. A foam bubble of this size has a diameter of roughly $0.44 \mathrm{~mm}$ when taken out of the porous medium and allowed to assume a spherical shape. For the simulation of the $1 \mathrm{wt} \%$ concentration, the breakthrough time of bubbles is about 1.2 PVI.

The bubble concentration profiles shown in Fig. $1 \mathrm{~b}$ for the $0.1 \mathrm{wt} \%$ concentration are lower than the values for the $1 \mathrm{wt} \%$ case. The lower surfactant concentration results in a lesser critical capillary pressure for foam coalescence, as given in Table 2. As a result, the foam coalescence rate increases and the average bubble concentration decreases. Disregarding the inlet 
region and the elevation of bubble concentration at the front, the flowing bubble concentration in this case is about 3.5 bubbles/ $\mathrm{mm}^{3}$ corresponding to an undistorted spherical bubble diameter of $0.82 \mathrm{~mm}$.

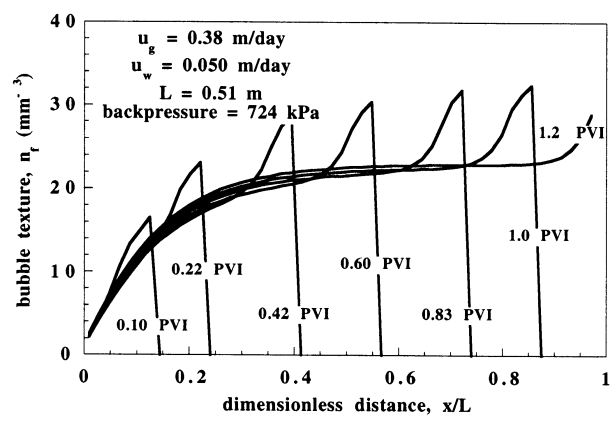

(a)

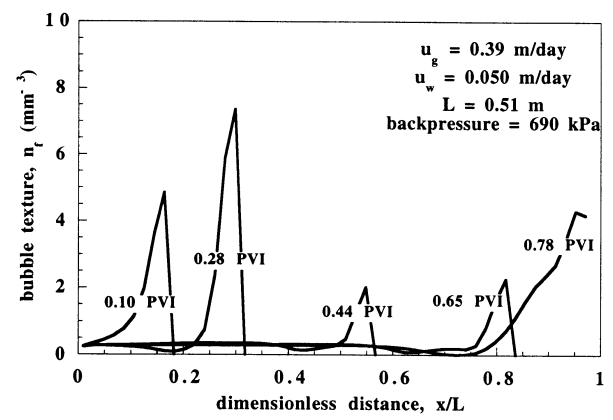

(c)

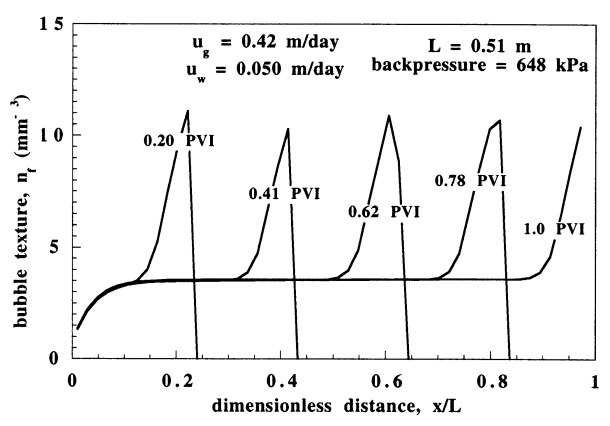

(b)

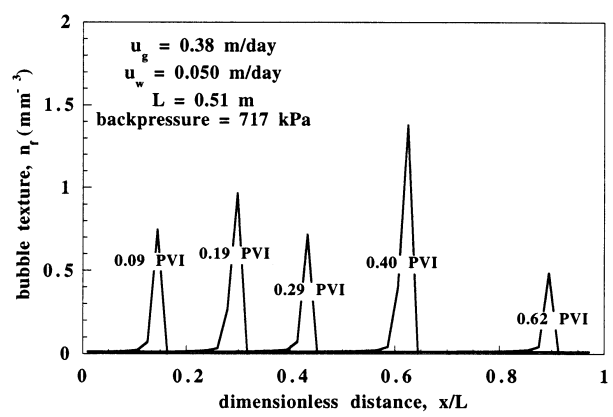

(d)

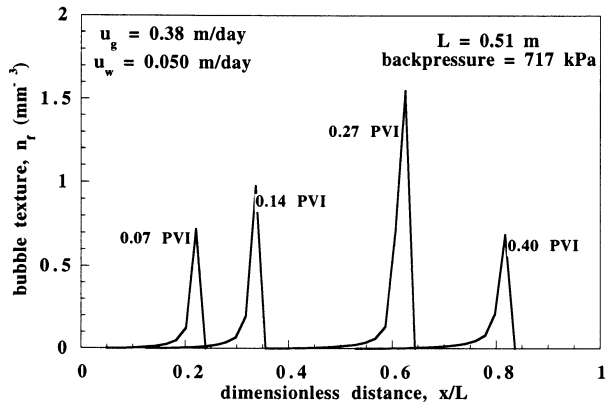

(e)

Figure 1. Model transient flowing bubble texture profiles (a) $1 \mathrm{wt} \%$ case; (b) $0.1 \mathrm{wt} \%$ case; (c) $0.02 \mathrm{wt} \%$ case; (d) $0.01 \mathrm{wt} \%$ case; (e) $0.005 \mathrm{wt} \%$ case.

An elevation in foam texture in the region of the front is also found in the lower surfactant concentration cases. The process of snap-off of gas bubbles is purely a mechanical process and continues regardless of the concentration of surfactant. This is reflected in the net foam generation rate of Eq. 1. As a consequence, bubbles are always generated when the water saturation is high and the capillary pressure is less than critical such as when gas first enters a water saturated region of the porous medium. Foam coalescence forces quickly destroy most of this initial foam because the surfactant concentration is low and thus the limiting capillary pressure for foam coalescence is reached quickly. Correspondingly, the magnitude of the 
elevation in foam texture at the front also decreases with decreasing surfactant concentration. In Fig. 1c the maximum texture is 8 bubbles $/ \mathrm{mm}^{3}$ while in Fig. $1 \mathrm{~d}$ it is 4 bubbles/ $\mathrm{mm}^{3}$. There is little difference in the bubble texture profiles of Figs. 1d and 1e because the limiting capillary pressure, as given in Table 2, has changed little between these two cases.

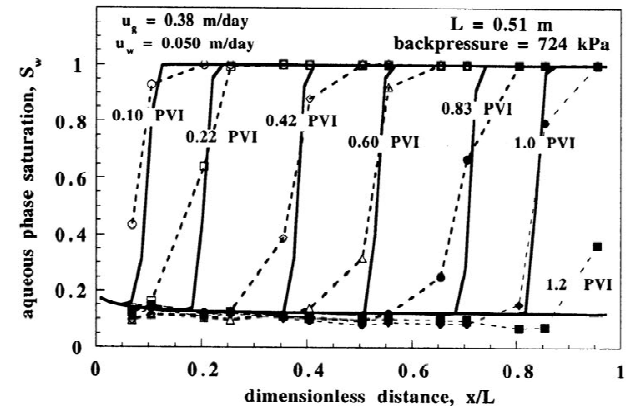

(a)

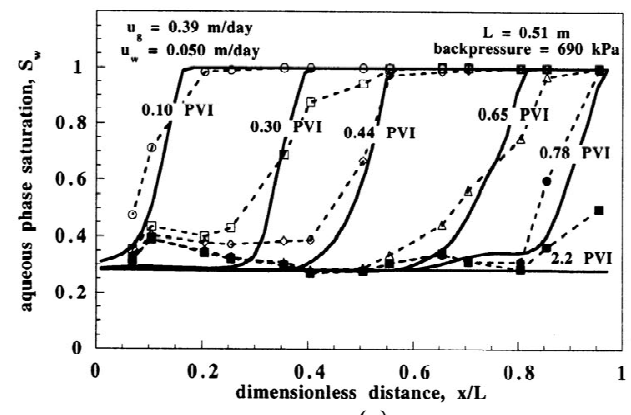

(c)

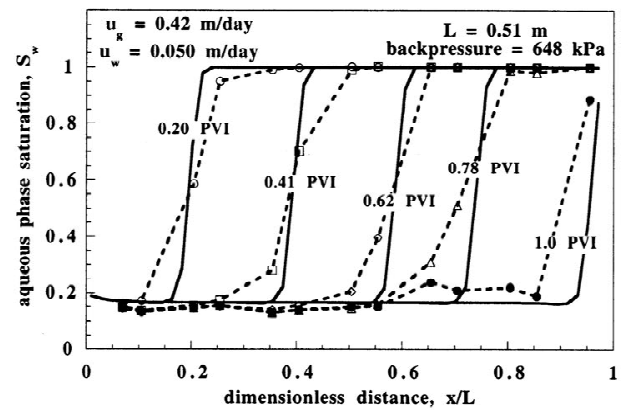

(b)

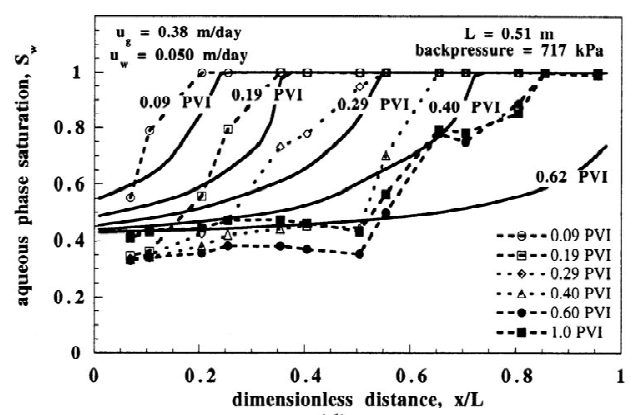

(d)

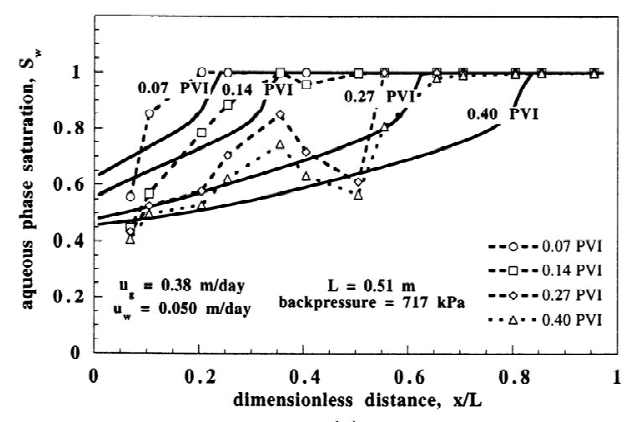

(e)

Figure 2. Experimental (symbols connected by dashed lines) and model (solid lines) transient aqueous-phase saturation profiles. (a) $1 \mathrm{wt} \%$ case; (b) $0.1 \mathrm{wt} \%$ case; (c) $0.02 \mathrm{wt} \%$ case; (d) $0.01 \mathrm{wt} \%$ case; (e) $0.005 \mathrm{wt} \%$ case.

The one-dimensional aqueous-phase saturation profiles as a function of time for each case are presented in Fig. 2. Time is again given nondimensionally in pore volumes. One dimensional saturation data is obtained by averaging the voxel by voxel CT-measured saturations for each cross section. In Fig. 2, symbols give the experimentally measured points and they are connected by dashed lines to guide the eye. The solid lines represent model predictions based upon the foam textures in Fig. 1 and Eqs. 3 to 5 for gas mobility. In general, transient displacement is efficient where bubble concentrations are large such as in the $1 \mathrm{wt} \%$ surfactant concentration case in Fig. 
28a, and displacement efficiency decreases as surfactant concentration and bubble texture decrease. Also note that the saturation change across the front is steep and sharp when the foam is strong as shown in Figs 2a and 2b.

The match between experiment and model is quite good in all cases prior to breakthrough and the onset of end effects, except perhaps the $0.005 \mathrm{wt} \%$ case. Displacement efficiency declines with decreasing surfactant solution concentration in both the simulations and experiments. Also, notice that the predicted saturation profiles across the displacement front in Figs. 2c through $2 \mathrm{e}$ become more diffuse as the surfactant concentration decreases. Predictions echo experiments in this regard. It is important to recall that these results are not achieved by history matching each experiment. The only parameter that changes in each simulation is the value of $\mathrm{P}_{\mathrm{c}}^{*}$. This parameter is physical and directly related to surfactant concentration (Aronson et al., 1994).

The experimental displacement results summarized in Fig. 2e show a region at $\mathrm{x} / \mathrm{L}$ roughly equal to 0.35 where desaturation of the aqueous phase is inefficient. Displacement is not uniform across the cross section, and a region of $100 \%$ aqueous phase saturation remains in the upper portion of the cross section. The simulations are one dimensional and cannot account for such selective displacement at low surfactant concentration. Nevertheless, the simulations reproduce, qualitatively, the low displacement efficiency at this surfactant concentration.

The second type of in-situ data collected was pressure drop. The lower limit of accuracy for the pressure measurement system used is about 0.14 to $0.34 \mathrm{kPa}(0.02$ to $0.05 \mathrm{psi})$. Experimental and simulated pressure drop profiles as a function of time are presented in Fig. 3 for each of the surfactant concentrations. The match between model and experiment is acceptable. Large bubble concentrations lead to significant gas-phase flow resistance and low aqueous-phase saturation. Therefore, the $1 \mathrm{wt} \%$ case with the finest bubble texture, as shown in Fig. 3a, demonstrates the largest overall pressure drop. As the bubble textures decline with surfactant concentration, the predicted pressure drops decline also.

For the $1 \mathrm{wt} \%$ and $0.1 \mathrm{wt} \%$ cases in Figs. $3 \mathrm{a}$ and $3 \mathrm{~b}$ where significant foam and pressure drops are generated, it is easy to distinguish the foam-filled region of the sand pack in the pressure drop profiles. For example in Fig. $3 \mathrm{~b}$ at $0.41 \mathrm{PVI}$, a relatively steep pressure gradient due to the presence of foam extends from the inlet to $\mathrm{x} / \mathrm{L}$ equal to 0.43 . The pressure drop in the remainder of the sand pack is small corresponding to the single-phase flow of water.

To improve the clarity of Figs. $3 \mathrm{c}$ to $3 \mathrm{e}$, a legend displays the symbol and time corresponding to each experimental data point. Difficulties were encountered in measuring the small pressure drops characteristic of the lower surfactant concentrations, especially at the inlet of the sandpack. Gas migrated into some of the pressure lines during the experiment. In these instances, large, aphysical, positive pressure gradients were measured. These erroneous pressure data likely originated from gas entering the sintered filters inside the pressure taps. Accordingly, erroneous pressure information has been deleted from Figs. 3d and 3e. The portion of the data presented in Fig. 3c that is in error is not clear, so all information is retained. Despite these problems, experiment and simulation agree on the general magnitude of pressure drop.

A feature of the model that bears further discussion is the predicted pressure drop profiles for the low surfactant concentration cases in the region immediately upstream of the foam front. 
Examine the pressure profile at 0.44 PVI in Fig. $3 \mathrm{c}$ and the corresponding bubble concentration profile in Fig. 3c. The pressure gradient is relatively shallow in the foam-filled region except immediately before the front. Here, the gradient is much steeper than in the front region because bubble concentration is elevated at the front. This elevation in texture reduces gas mobility causing the pressure gradient to steepen.

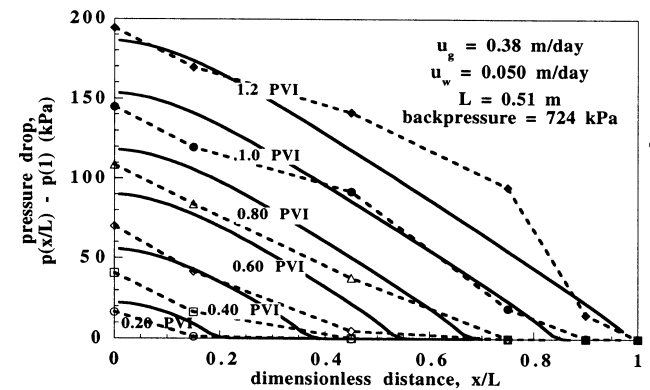

(a)

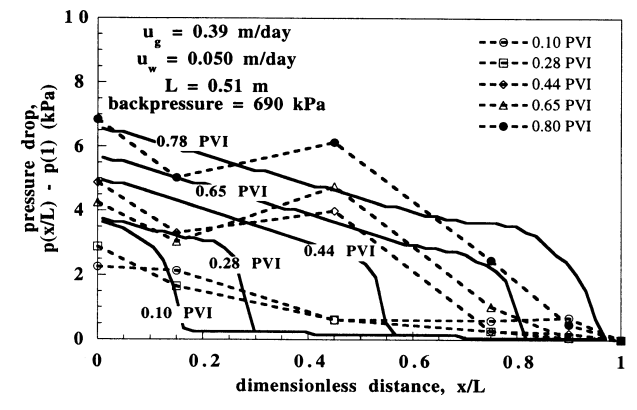

(c)

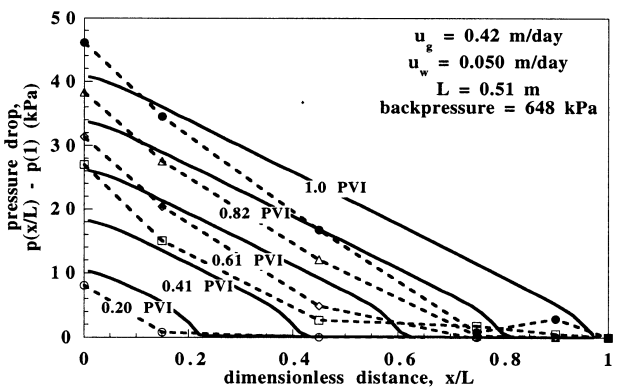

(b)

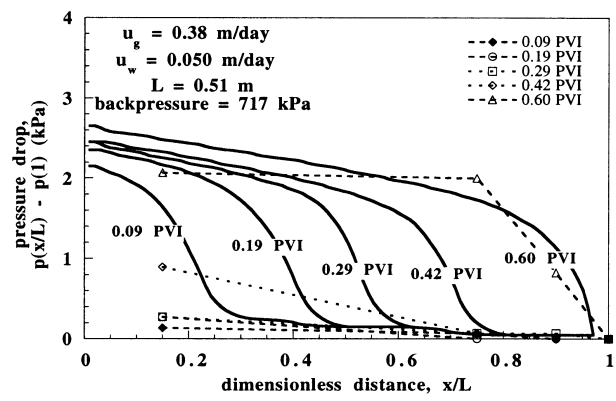

(d)

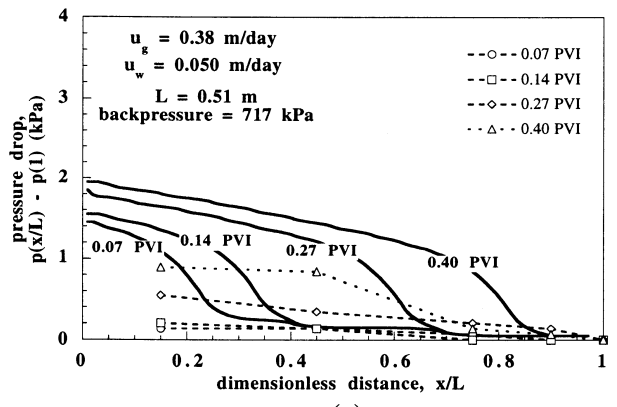

(e)

Figure 3. Experimental (symbols connected by dashed lines) and model (solid lines) transient pressure profiles. (a) $1 \mathrm{wt} \%$ case; (b) $0.1 \mathrm{wt} \%$ case; (c) $0.02 \mathrm{wt} \%$ case; (d) $0.01 \mathrm{wt} \%$ case; (e) $0.005 \mathrm{wt} \%$ case. 


\section{Conclusions}

An experimental study of foam generation and propagation as a function of aqueous surfactant concentration was conducted in $7 \mu \mathrm{m}^{2}$, homogeneous sand packs. The in-situ phase saturation and pressure distribution were measured during the experiments. As expected, the displacement efficiency decreases and gas mobility increases with decreasing surfactant concentration. This experimental trend indicates that foam weakens as the surfactant concentration decreases. However, even weak foam improves displacement somewhat.

Such results are best understood considering the role of foam bubble size, or synonymously foam texture, in setting the mobility of foamed gas in porous media. Many small bubbles result in a low mobility foam and few large bubbles result in a high mobility foam. Foam bubbles, at fixed injection rates of liquid and gas, grow in volume as surfactant concentration decreases because foam coalescence forces are inversely proportional to surfactant concentration.

These ideas are expressed quantitatively by the incorporation of the role of foam texture on displacement in porous media using a mechanistic, mean, bubble-size conservation equation. In essence, foam is treated as a nonchemical, reactive, component of the gas phase and the evolution of foam texture is modeled explicitly using rate equations for foam generation and coalescence. Coalescence as a function of surfactant concentration is modeled employing the notion of limiting capillary pressure for foam stability. That is, the maximum suction capillary pressure that foam films can withstand decreases with surfactant concentration. The foam bubble population balance model predicts accurately the location of saturation and pressure fronts across the range of concentrations studied. This is achieved by properly accounting for limiting capillary pressure as a function of aqueous surfactant concentration and without parameter adjustment.

\section{References}

Aronson, A. S., Bergeron, V., Fagan, M. E. and Radke, C. J. (1994). "The Influence of Disjoining Pressure on Foam Stability and Flow in Porous Media." Colloids and Surfaces A: Physicochemical Eng. Aspects 83: 109-120

Bernard, G. G., Holm, L. W. and Jacobs, L. W. (1965). "Effect of Foam on Trapped Gas Saturation and on Permeability of Porous Media to Gas." Soc. Pet. Eng. J. 5(4): 295-300.

Bretherton, F. P. (1961). “The Motion of Long Bubbles in Tubes.” J. Fluid Mech. 10: 166-188.

De Vries, A. S. and Wit, K. (1990). "Rheology of Gas/Water Foam in the Quality Range Relevant to Steam Foam.” Soc. Pet. Eng. Res. Eng. 5(2): 185-192.

Hirasaki, G. J. and Lawson, J. B. (1985). "Mechanisms of Foam Flow in Porous Media: Apparent Viscosity in Smooth Capillaries.” Soc Pet. Eng. J. 25(2): 176-190.

Holm, L. W. (1968). "The Mechanism of Gas and Liquid Flow Through Porous Media in the Presence of Foam.” Soc. Pet. Eng. J. 8(4): 359-369. 
Huh, D. G. and Handy, L. L. (1989). "Comparison of Steady- and Unsteady-State Flow of Gas and Foaming Solution in Porous Media." Soc. Pet. Eng. Res. Eng. 4(1): 77-84.

Jiménez, A. I. and Radke, C. J. (1989). Dynamic Stability of Foam Lamellae Flowing Through a Periodically Constricted Pore. in Oil-Field Chemistry: Enhanced Recovery and Production Stimulation. J. K. Borchardt and T. F. Yen, eds., Washington, D.C., American Chemical Society. 396: 460-479.

Khatib, Z. I., Hirasaki, G. J. and Falls, A. H. (1988). "Effects of Capillary Pressure on Coalescence and Phase Mobilities in Foams Flowing Through Porous Media." Soc. Pet. Eng. Res. Eng. 3(3): 919-926.

Kovscek, A. R. and C. J. Radke, "Fundamentals of Foam Transport in Porous Media," in Foams: Fundamentals and Applications in the Petroleum Industry, Schramm, L. L. Ed., ACS Advances in Chemistry Series No. 242, American Chemical Society, Washington D.C., 115-163 (1994).

Kovscek, A. R., T. W. Patzek, and C.J. Radke "A Mechanistic Population Balance Model for One Dimensional Foam Flow in Boise Sandstone," Chemical Engineering Science, 50(23), 3783-3799 (1995).

Kovscek, A. R., and C. J. Radke, "Gas-Bubble Snap-Off Under Pressure Driven Flow in Constricted Noncircular Capillaries," Colloids and Surfaces A: Physicochemical and Engineering Aspects, 117, 55-76 (1996).

Kovscek, A. R., T. W. Patzek, and C. J. Radke. "Mechanistic Foam Flow Simulation in Heterogeneous and Multidimensional Porous Media," Society of Petroleum Engineers Journal, 2(4), 511-526 (1997).

Pruess, K. (1987) "TOUGH User's Guide," Rep NUREG/CR-4645, Nucl. Reg. Commission, Washington D.C.

Stone, H. L. (1970). "Probability Model for Estimating Three-Phase Relative Permeability." J. Pet. Tech. 22(2): 214-218.

Wong, H., Radke, C. J. and Morris, S. (1995). "The Motion of Long Bubbles in Polygonal Capillaries: I. Thin Films.” J. Fluid Mech. 292: 71-95.

Wong, H., Radke, C. J. and Morris, S. (1995). "The Motion of Long Bubbles in Polygonal Capillaries: II. Drag, Fluid Pressure, and Fluid Flow.” J. Fluid Mech. 292: 95-110 


\title{
Scaling of Foamed Gas Mobility in Heterogeneous Porous Media
}

\author{
(Task 1b)
}

\section{Introduction}

The presence of heterogeneity frustrates, to some extent, our ability to displace or remove crude oil during the injection of gases such as steam and carbon dioxide. High permeability stratified zones preferentially carry most of the injected fluid. The physical explanation is simple. The mobility, $\lambda$, of a Newtonian injection fluid is generally largest in the most permeable zones and, hence, injected fluids prefer to channel and follow the path of highest mobility. This fact makes it difficult to establish uniform injection profiles around wells in heterogeneous formations and to sweep effectively regions of oil fields or aquifers that lie far from wells.

One possibility to overcome the problem of channeling is to design a displacement so that injected fluid mobility adjusts as a function of the permeability of the medium and/or the velocity of the injected fluid. Thus, injected fluid mobility increases as porous medium permeability decreases. Some authors refer to this concept as selective mobility reduction (Heller, 1994). Foam flow in heterogeneous porous media appears to exhibit these attributes (c.f., Bertin et al., 1999).

Experimental evidence indicates that foam can indeed reduce gas mobility more effectively in high permeability strata contrary to the results of displacement with a Newtonian fluid. For instance, Casteel and Djabbarah (1988) examined $\mathrm{CO}_{2}$ displacement efficiency with foaming agents in parallel one-dimensional porous media with different permeabilities. They found that foam increased resistance to flow in the high permeability core and diverted injected $\mathrm{CO}_{2}$ to the low permeability porous medium. Bertin et al. (1999) constructed a heterogeneous porous medium with a permeable sand surrounding a sandstone core. The permeability contrast was about 70:1. Experiments with and without capillary communication between the two media were conducted. Without surfactant, and thus foam, desaturation was inefficient and little gas was injected into the low permeability sandstone. In communicating and noncommunicating systems with foam, a significant fraction of the injected gas was diverted to the low permeability medium and overall displacement from both low and high permeability zones improved significantly. Other studies regarding heterogeneous porous media also found that foam had the capability to reapportion a fraction of injected fluids from high to low permeability strata (Robin, 1987; Llave et al., 1990; Yaghoobi and Heller 1996; Hirasaki et al., 1997A, 1997B).

Our work establishes from theoretical considerations the conditions for effective fluid diversion by foam. We also provide experimental support for the predictions developed. Specifically, we address the adjustment of foam mobility as permeability varies within a porous medium. Local equilibrium and scaling concepts are developed to estimate how foam bubble size adjusts with respect to porous medium properties. These estimates of bubble size are then used to obtain gas mobility in the presence of foam. To the extent possible, the mathematical framework employed is a continuum approach consistent with Darcy law description of multiphase flow in porous media. The description to follow is somewhat abbreviated as this research has appeared in print (Kovscek and Bertin 2003A, 2003B). 


\section{Foam Mobility}

Mobility of foamed gas in porous media can be expressed in a manner analogous to the flow of continuous Newtonian or nonNewtonian fluids

$$
\lambda_{f}=\frac{K k_{r f}}{\mu_{f}}
$$

where $\lambda$ is the phase mobility, $\mathrm{K}$ is the absolute permeability, $k_{r}$ is relative permeability and $\mu$ is the viscosity. The subscript $\mathrm{f}$ refers to flowing foam. The mobility of trapped or stationary foam is identically 0 . Equation 1 indicates that foam mobility is naturally divided into relative permeability and effective viscosity portions. Our task was to ascertain how foam mobility adjusts with respect to medium permeability, porosity, capillary pressure, surfactant properties, and velocities of the gas and wetting liquid phases. All of these factors are significant.

Because foam partitions into stationary and flowing fractions, it is necessary to determine the flowing foam fraction $X_{f}=S_{f} / S_{g}$ to compute the gas-phase relative permeability in the presence of foam. Our starting point to derive the macroscopic scaling dependencies was a network percolation analysis that yields the following expression:

$$
X_{f}=\left[\psi_{1} \frac{\nabla p}{n_{f} K^{1 / 2}} \frac{f_{c}}{f-f_{c}}\right]^{\eta}
$$

where $\Psi_{1}$ is a constant of proportionality, $\mathrm{f}$ is the percolation fraction, $f_{c}$ is the percolation threshold, and $\eta$ is a percolation exponent that is equal to 0.4 for 3D lattices (Stauffer 1985).

The sublinear scaling of $X_{\mathrm{f}}$ indicated by an $\eta$ is 0.4 demonstrates a very weak dependence of all variables and parameters on $\mathrm{X}_{\mathrm{f}}$. This agrees with the limited number of experiments conducted. Gillis and Radke (1990) found no dependence of $\mathrm{X}_{\mathrm{f}}$ in regard to gas velocity whereas Friedmann et al. (1991) reported a slight increase in $\mathrm{X}_{\mathrm{f}}$ with gas velocity. Unfortunately, Gillis and Radke (1990) do not report the pressure gradients at which their measurements were made and so a direct comparison cannot be made to their work.

We proceed with the data of Friedmann et al. (1991) and treat it as a self-consistent and closed set. Values of $\mathrm{X}_{\mathrm{f}}$ are obtained as a function of gas velocity from their Fig. 5b. The pressure gradient corresponding to each $\mathrm{X}_{\mathrm{f}}$ is read from their Fig. 6 . They also present a model for bubble size as a function of system parameters. We calculate the equilibrium bubble size by equating their Eqs. 13 and 14 and solving for $n_{f}$. These equations give rate expressions for bubble generation and coalescence, respectively. The kinetic constants for calculating nf are drawn from their Table 1. This results in $n_{f}$ values on the order of $5 \times 10^{4}$ bubbles $/ \mathrm{mm}^{3}$. The numerical value of $K$ is set to $0.5 \mu \mathrm{m}^{2}$. The percolation network parameters are $\eta=0.4, f_{c}=0.25$, and $f=1$ to represent the large fraction of pores filled by gas during strong foam flow. The proportionality constant $\psi_{1}$ is obtained as a best fit to the experimental data. 
The result of this exercise is given in Fig. 1. The general trend of increasing $X_{f}$ with velocity is reproduced very well. For zero gas frontal advance rate, the new theory predicts no flowing foam consonant with physical reasoning. Thus, the functionality expressed in Eq. 2 appears reasonable. Foam is mobilized as the pressure gradient is increased. The permeability dependence of Eq. 2 indicates that it is easier to mobilize a long bubble than it is a short bubble. On the other hand, increased foam texture and surface tension decrease the fraction of mobile gas.

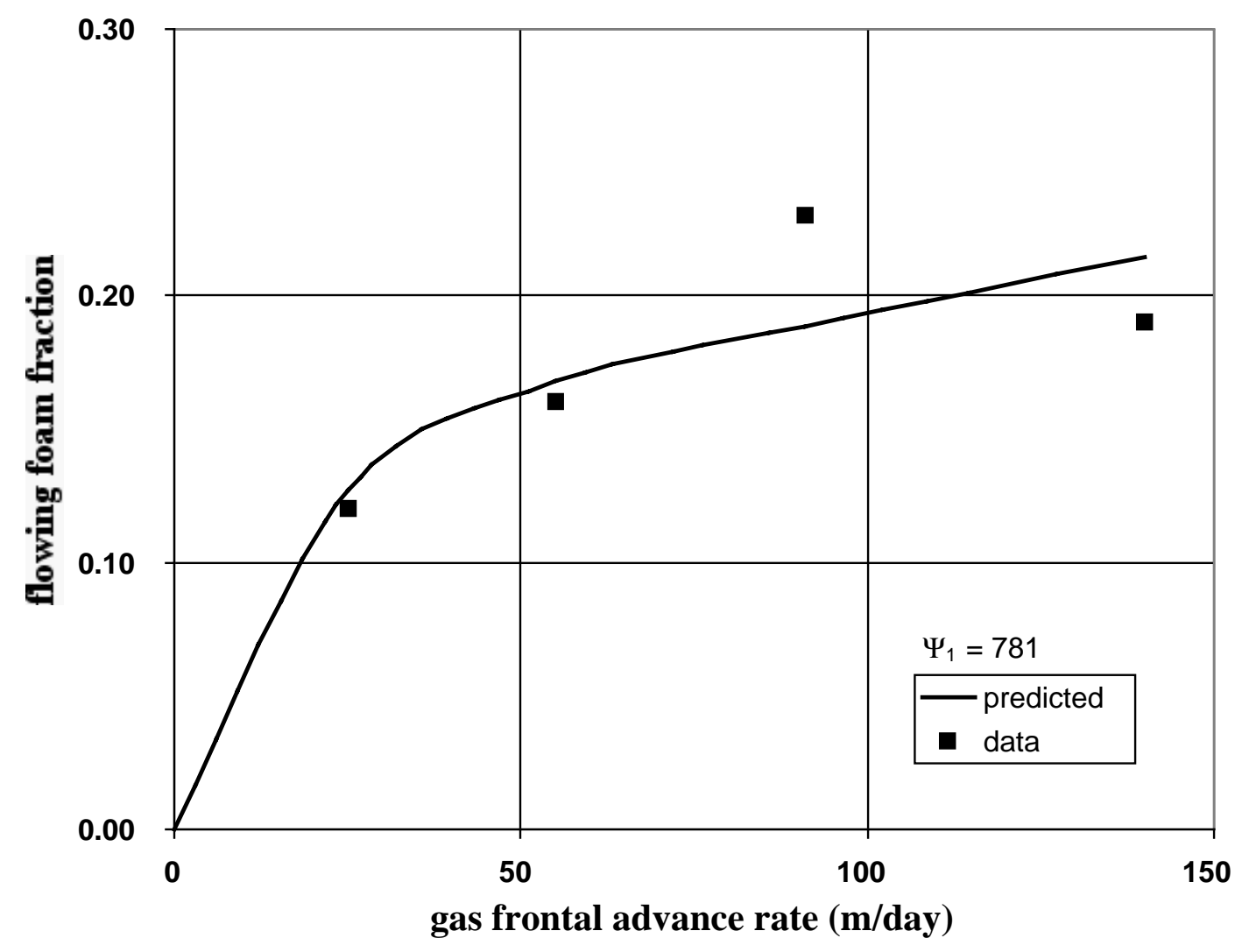

Figure 1. Prediction of the network model for flowing foam fraction compared to the experimental data of Friedmann et al (1991).

A note is required on the magnitude of $\psi_{1}$. The foam textures computed by Friedmann $e t$ al. (1991) are exceptionally fine. Ettinger and Radke (1992) measured effluent bubble sizes from similar Berea sandstone of 100 to 500 bubbles $/ \mathrm{mm}^{3}$; these values are about two orders of magnitude smaller than the computed $\mathrm{n}_{\mathrm{f}}$ of Friedmann et al. (1991). A reduction in $n_{f}$ by about 100 reduces $\psi_{1}$ by two order of magnitude for the exercise shown in Fig. 1.

Setting the right hand side of Eq. 1 of the previous section (Foam Generation at Low Surfactant Concentration) and $\mathrm{Q}_{\mathrm{b}}$ equal to zero equates the rate of foam generation and coalescence yielding local equilibrium. Solving the resulting expression for the local equilibrium 
foam texture $\mathrm{n}_{\mathrm{f}}^{\text {eq }}$ and making appropriate substitutions for $\mathrm{k}_{-1}$ yields (Kovscek and Bertin, 2003A):

$$
n_{f}^{e q}=\left(\frac{k_{1}}{k_{-1}^{o}}\right)\left(\frac{P_{c}^{*}-P_{c}}{P_{c}}\right)^{2}\left(\frac{v_{w}}{v_{f}^{2 / 3}}\right)
$$

The velocity dependencies reflect the alteration in bubble size that gives foam its unique rheological properties. Foam texture becomes finer and thus mobility decreases when liquid injection is increased at a constant gas injection rate; on the other hand, foam becomes coarser if the gas velocity is increased while the liquid velocity is held constant. Equation 3 indicates an effect of permeability on $n_{f}^{e q}$ indirectly through $P_{c}$, the ratio $k_{l} / k_{-1}$, and the velocity ratio $\mathrm{v}_{\mathrm{w}} / \mathrm{v}_{\mathrm{f}}^{2 / 3}$. The ratio $k_{1} / k_{-1}^{o}$ sets the general magnitude of foam texture and it varies with porous medium characteristics such as pore size and the topology of the pore space.

It has been argued elsewhere that on the order of one bubble resides in every pore for strong foam (Bertin et al., 1998). Following this line of reasoning, the ratio $k_{1} / k_{-1}^{o}$ for granular media should scale as the number of pores, $\mathrm{n}_{\mathrm{p}}$, according to

$$
\frac{k_{1}}{k_{-1}^{o}} \sim n_{p} \sim\left(\frac{\phi^{3}}{(1-\phi)^{2}} \frac{1}{K}\right)^{3 / 2}
$$

Equation 4 results from the Kozeny-Carman relationship. Thus, the scaling for $n_{f}^{e q}$ becomes,

$$
\begin{array}{ll}
n_{f}^{e q} \sim\left(\frac{\phi^{3}}{(1-\phi)^{2}} \frac{1}{K}\right)^{3 / 2}\left(\frac{P_{c}^{*}-P_{c}}{P_{c}}\right)^{2} \frac{v_{w}}{v_{f}^{2 / 3}} ; & P_{c}<P_{c}^{*} \\
n_{f}^{e q}=0 ; & P_{c} \geq P_{c}^{*}
\end{array}
$$

Effective viscosity of the flowing foam is the other factor describing foamed gas mobility. It is expressed as (Falls et al., 1988; Ettinger and Radke, 1992; Friedmann et al. 1992; Kovscek and Radke, 1994, Kovscek et al., 1995; Fergui et al., 1998),

$$
\mu_{f}=\mu_{g}+\frac{\alpha n_{f}}{v_{f}^{c}}
$$

where $\alpha$ is a constant of proportionality that varies with $K$ and the surfactant type through the interfacial tension. Additionally, $v(=u / \phi S)$ is the interstitial velocity and $\mathrm{c}$ is an exponent that expresses the shear thinning behavior of foam. The subscript $g$ indicates an unfoamed continuous gas phase. Equation (6) results from extensions of the flow of bubbles in capillary tubes (Bretherton, 1961; Wong et al., 1995A, 1995B) to foam flow (Hirasaki and Lawson, 1985). These studies indicate that the theoretical value of $c$ is close to $1 / 3$. In the limit of no flowing foam, that is $\mathrm{n}_{\mathrm{f}}=0$, we obtain the bulk gas viscosity. 
The work of Hirasaki and Lawson (1985) teaches about the permeability dependence of $\alpha$. After appropriate substitution into Eq. 6 and simplification, we obtain the following scaling for the effective viscosity of a foamed gas phase:

$$
\mu_{f} \cong n_{f} \frac{R^{3}}{R_{c}} \frac{1}{v_{f}^{1 / 3}} \cong \frac{\beta^{3 / 2} K^{3 / 2} P_{c}}{2 \sigma} \frac{n_{f}}{v^{1 / 3}}
$$

where the capillary pressure, $P_{c}$, is recognized as equal to $2 \sigma / R_{c}$. Comparison of Eq. 2 with Eq. 6 teaches that $\alpha$ scales as $\mathrm{K}^{3 / 2} \mathrm{P}_{\mathrm{c}}$ indicating that the effective viscosity of a foamed gas at fixed texture and velocity will increase as permeability and capillary suction increase. Falls et al. (1988) also describe a $\mathrm{K}^{3 / 2}$ dependence for effective viscosity.

\section{Scaling of Foam Mobility}

Substitution of Eqs. 6 and 2 into Eq. 1 completes the exercise to obtain the scaling trends for foam mobility. The expression reads

$$
\begin{array}{ll}
\lambda_{f}=\psi_{3} K^{1+a \eta} k_{r g}^{o}\left(\frac{(1-\phi)^{2}}{\phi^{3}}\right)^{3(1+a \eta) / 2}\left(\frac{P_{c}}{P_{c}^{*}-P_{c}}\right)^{2(1+a \eta)}\left(\frac{1}{P_{c}}\right)\left(\frac{v_{f}^{1+2 a \eta / 3}}{v_{w}^{1+a \eta}}\right) \nabla p^{a \eta} ; P_{c}<P_{c}^{*} \\
\lambda_{f}=\lambda_{g} ; & P_{c} \geq P_{c}^{*}
\end{array}
$$

where the terms a and $\eta$ arise from the exponent model for gas relative permeability and the percolation network scaling of $S_{f}$ near the percolation threshold. The proportionality constant $\psi_{3}$ is composed primarily of surface tension and the proportionality constant $\beta$.

While quite involved, Eq. 8 incorporates all of the features that foam flow in porous media has exhibited. For instance, foam mobility is large if the surfactant is weak or if concentration is low through the term including $P_{c}{ }^{*}$. Conversely, foam mobility is low if $P_{c}{ }^{*}$ is large. The experimentally observed trend that $\lambda_{f}$ increases with $v_{f}$ while liquid injection rate is held constant is found (c.f., Ettinger and Radke, 1991; Persoff et al., 1991; Kovscek et al., 1995). Similarly, $\lambda_{f}$ varies inversely with increasing $v_{w}$ when gas injection rate is fixed. To expect functional dependencies simpler than given in Eq. 8 is naïve given the complex rheology displayed by foam.

Table 1. Properties of the Porous Media

\begin{tabular}{|c|c|c|}
\hline & high permeability & low permeability \\
\hline \hline$K\left(\mu \mathrm{m}^{2}\right)$ & 5.35 & 0.686 \\
\hline$\phi$ & 0.395 & 0.244 \\
\hline$S_{w c}$ & 0.10 & 0.25 \\
\hline
\end{tabular}


Table 2: Parameters for Gas-mobility Calculations

\begin{tabular}{|c|c|c|}
\hline parameter & value & capillary pressure \\
\hline$\mu_{w}$ & $1 \mathrm{mPa} \mathrm{s}$ & $P_{c}=\sigma \sqrt{\frac{\phi}{K}}\left(1.417\left(1-S_{w D}\right)\right.$ \\
& & $\left.-2.120\left(1-S_{w D}\right)^{2}+1.263\left(1-S_{w D}\right)^{3}\right)$ \\
\hline$\sigma$ & $32 \mathrm{mN} / \mathrm{m}$ & $S_{w D}=\frac{S_{w}-S_{w c}}{1-S_{w c}}$ \\
\hline$a$ & 2.5 & \\
\hline$\eta^{*}$ & 0.4 & \multicolumn{2}{|c}{} \\
\hline$P_{c}$ & $20 \mathrm{kPa}$ &
\end{tabular}

It must be noted, however, that this particular scaling of foam mobility is unlikely to apply to carbonates in a quantitative sense. Equation 4 originates from sands and $n_{p}$ cannot be scaled in the same way for carbonates, or high-porosity porous media. Nevertheless, the idea that the ratio of foam germination to termination sites scales as the number of pores should remain true. For rocks that do not follow Eq. 4, an appropriate relationship can be substituted and the remainder of the exercise repeated.

Consider, briefly, trends for heterogeneous porous media as predicted by Eq. 8. It suggests that foam mobility increases with permeability to a power greater than 1 if all other factors are fixed. This is counter to the goals of fluid diversion. Fortunately, the remaining terms teach us the conditions where foam is an effective mobility control and diversion agent.

Next consider the role of porosity on foam mobility through a simple calculation. We choose the conditions in Table 1 for representative high and low permeability strata along with a $P_{c}{ }^{*}$ equal to $20 \mathrm{kPa}$, an $S_{w, s s}$ in the sandstone of 0.5 , and an $S_{w, A 10}$ of 0.16 . Parameter values are physical and result from experiments discussed in relation to the experiment to follow. Capillary pressure is obtained from the Leverett J-function and dimensionalizing by the ratio $\sigma(\phi / K)^{1 / 2}$. Table 2 summarizes other relevant parameter values. Also for the sake of highlighting the role of porosity, we set the liquid and gas velocity, as well as the pressure drop, equal in each layer. The porosity of the high permeability medium is held fixed while the porosity of the lower permeability rock is varied from 0.15 to 0.40 . The ratio of the gas mobility in the low upon high permeability layer is then computed according to Eq. 8. The result is given in Fig. 2. When the porosity is about the same as in both media, $\lambda_{\mathrm{f}, \text { low }} / \lambda_{\mathrm{f} \text {,high }}$ is less than 1 indicating that diversion is not expected. Around $\phi$ equal to 0.35 the ratio is unity. As the porosity of the lower permeability medium decreases, the ratio $\lambda_{\mathrm{f}, \mathrm{ss}} / \lambda_{\mathrm{f}, \mathrm{A} 10}$ increases. This result occurs because the first term in parenthesis on the right of Eq. 8 increases as porosity decreases. Recall that this term originates from the scaling of foam germination and termination sites. For most sands and sandstones, permeability decrease correlates with porosity decrease. Thus, $(1-\phi)^{2} / \phi^{3}$ counteracts the reduction in mobility indicated by the superlinear scaling of $\lambda_{\mathrm{f}}$ with permeability. In cases where the porosity contrast is large, the term $(1-\phi)^{2} / \phi^{3}$ indicates that it should be relatively easy to obtain foamed gas injection into low permeability zones. 


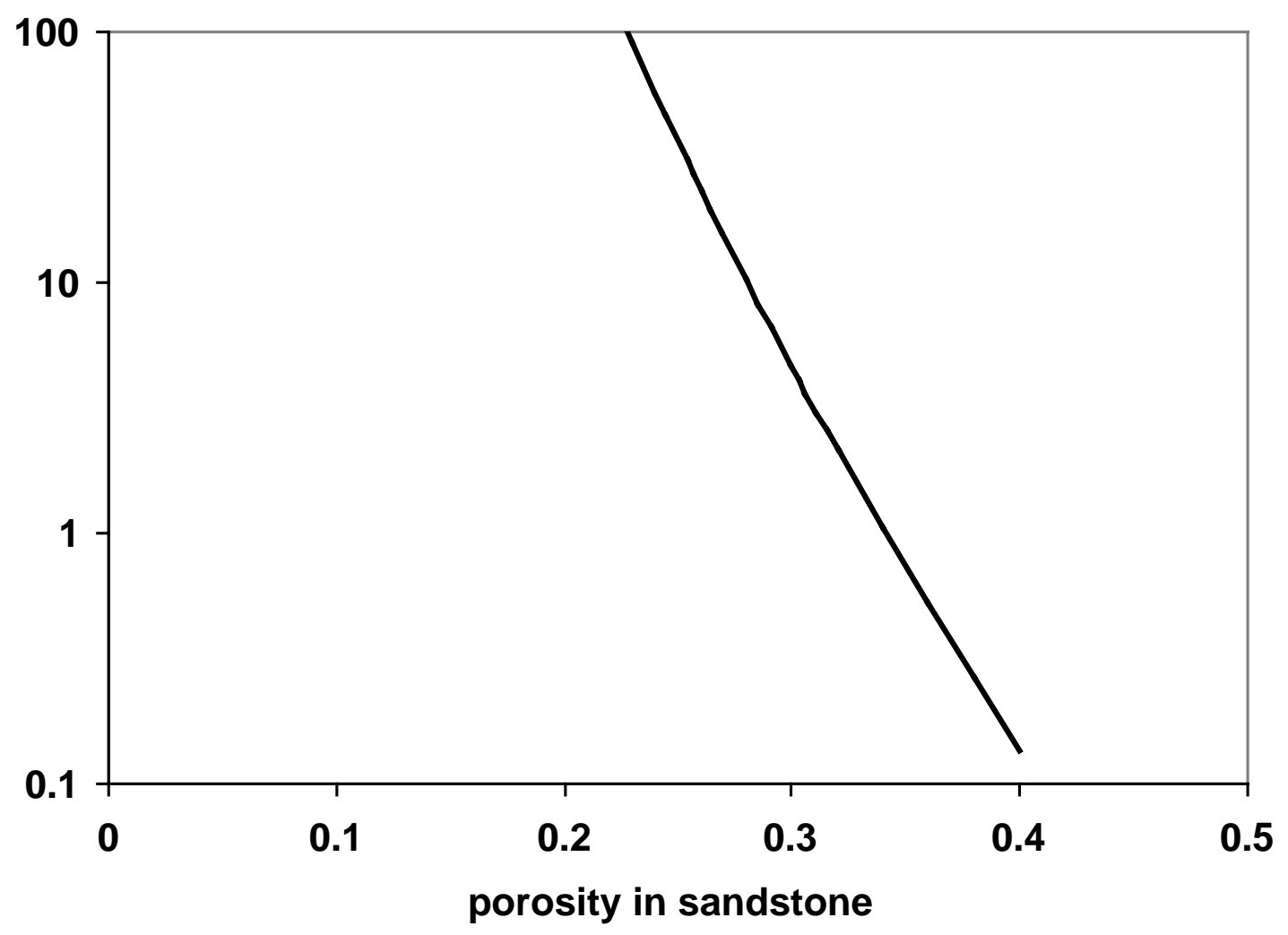

Figure 2. Ratio of gas mobility in low and high permeability zones. Porosity of low permeability layer is varied and all other terms held constant.

\section{Experimental}

An experimental setup was prepared to characterize foam flow in individual homogeneous cores and then to consider gas mobility in the composite system as shown in Fig. 3. The porous media were A-10 (US Filtration Co.) a high permeability, consolidated, synthetic rock and Vosges sandstone. The A-10 is pure silica while the Vosges sandstone contains some clay material. Table 3 lists the properties and dimensions of each core. The permeability contrast was about 8 to 1 , whereas the porosity contrast was roughly 2 to 1 . The different cross-sectional areas gave roughly similar pore volume within each core.

The cores were prepared by affixing an aluminium plate to each end via a clamp. The plates contain ports for fluid injection/production and pressure measurement. The bare rock surfaces were coated with Araldite 2013 (Ciba Specialty Chemicals) a metal bonding adhesive that is viscous, nonwetting to silica, and so does not penetrate the rock matrix. Likewise, this adhesive bonds the metal plate to each face of the core. After drying for 24 hours, the length of each core was wrapped with epoxy-coated fiberglas twine to add mechanical strength. This system can withstand absolute pressures of about $500 \mathrm{KPa}$. We chose to work with cores that communicate only through inlet and exit conditions to more easily characterize the mobility 
within each porous medium. The effect of capillary communication and cross flow among porous media is considered experimentally in another paper (Bertin et al. 1999).

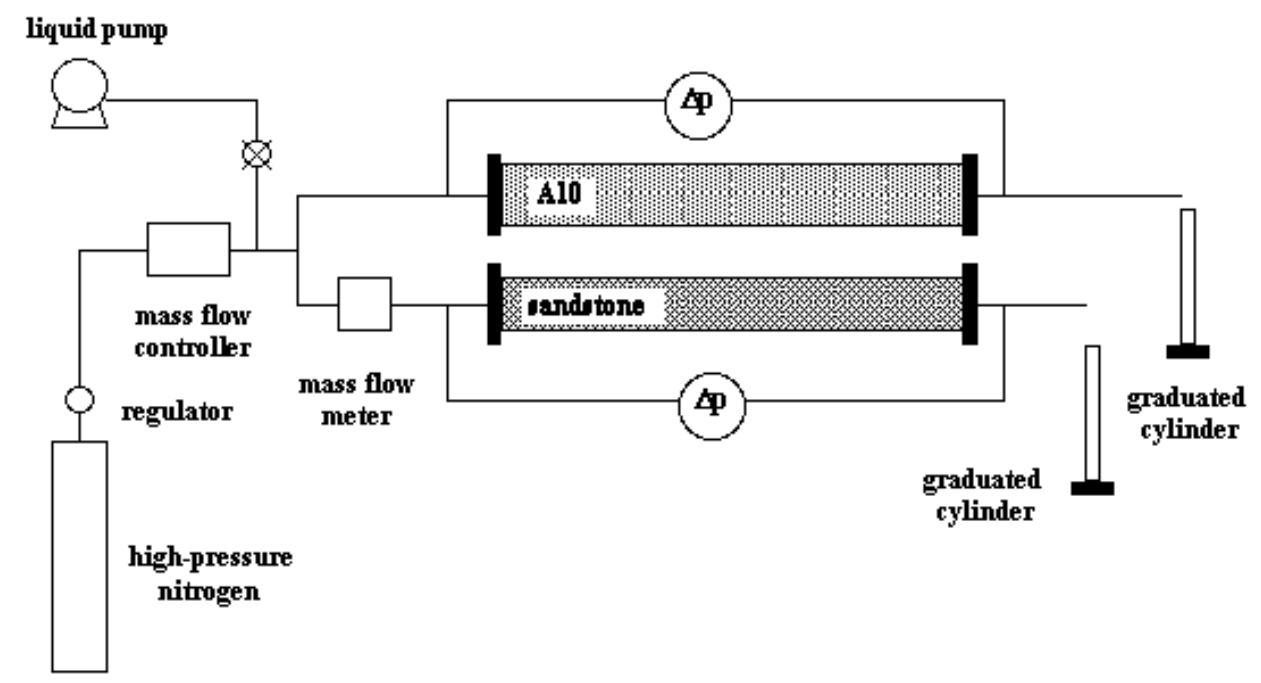

Figure 3. Apparatus for heterogeneous foam-displacement experiments. The mass flow meter is removed if foamer solution is injected.

The aqueous foamer solution was composed of potassium iodide (KI) and Witconate AOS 38 (Witco), an $\alpha$-olefin sulfonate with carbon chain length of 14 to 16 . Surfactant concentration was $0.2 \mathrm{wt} \%$ active chemical in a brine of $1 \mathrm{wt} \% \mathrm{KI}$. The CMC is approximately $0.01 \mathrm{wt} \%$ and surface tension is $32 \mathrm{mN} / \mathrm{m}$. Solution viscosity is $1 \mathrm{mPa}$. Cores were saturated with foamer solution by first injecting bone-dry $\mathrm{CO}_{2}$ to displace and/or evaporate any fluid. Dryness was assured by measuring core weight. From 5 to $10 \mathrm{PV}$ of foamer solution were then injected to satisfy rock adsorption and dissolve any residual $\mathrm{CO}_{2}$. Measurement of rock permeability to the foamer solution provided a check that there was no residual gas in the system.

Bottled nitrogen was the gas source for foam displacement. Total gas injection was controlled using a 0-5 SCCM (standard cubic centimeters per minute) mass flow controller (Bronkhorst). The gas stream was split using a tee and the flow resistance in each porous medium set the fraction of gas injected into each core. In experiments with injection of only gas, the portion of the gas injected into the sandstone is measured by a mass flow meter (Bronkhorst). Atmospheric back pressure was applied and the production streams were not mingled. The effluent from each individual core was collected and measured as a function of time. Pressure transducers (0 to $100 \mathrm{KPa}$, Rosemount) measured the pressure drop across each core and a PC recorded individual measurements. 
Table 3. Properties of the Porous Media

\begin{tabular}{|c|c|c|}
\hline & $\mathrm{A}-10$ & Vosges sandstone \\
\hline $\mathrm{K}\left(\mu \mathrm{m}^{2}\right)$ & 5.35 & 0.686 \\
\hline$\phi$ & 0.395 & 0.244 \\
\hline $\mathrm{L}(\mathrm{m})$ & 0.48 & 0.497 \\
\hline cross-sectional area $\left(\mathrm{m}^{2}\right)$ & $1.96 \times 10^{-3}$ & $2.62 \times 10^{-3}$ \\
\hline pore volume $\left(\mathrm{m}^{3}\right)$ & $3.72 \times 10^{-4}$ & $3.18 \times 10^{-4}$ \\
\hline
\end{tabular}

\section{Results and Discussion}

Several injection conditions were imposed. First, gas alone was injected into the composite system. No restrictions were placed on the gas injection; each porous medium accepted a portion of the injected gas according to the foamed-gas mobility within the core. This served as a confirmation of the diversion properties of foam measured in a separate study (Bertin et al., 1999) with a substantially different apparatus and porous medium geometry. Second, foamer solution and gas were coinjected simultaneously. This was done so as to verify the liquid velocity dependence in Eq. 24 of Kovscek and Bertin (2001). Finally, the pressure response of each individual core to simultaneous injection of gas and liquid was measured so as to establish a baseline.

The porous media are fully saturated with surfactant solution and nitrogen is injected at $1.5 \mathrm{SCCM}\left(25 \mathrm{~mm}^{3} / \mathrm{s}\right)$. The production from each core is measured as is the pressure drop. In this experiment, a mass flow meter is used downstream of the mass flow controller at the inlet of the sandstone to measure the fraction of gas accepted by the sandstone. Comparison of injected gas rate and the measured collection of effluent serve as a mutual check for accuracy. Figure 4 presents the pressure response accompanying gas injection and Figure 5 the liquid production. The maximum pressure drop measured over the system is a little more than $0.3 \mathrm{bar}(30 \mathrm{kPa})$ and is relatively low because liquid is not injected into both of the porous media. The steady increase in pressure drop at all times prior to breakthrough is indicative of foam generation and control of gas mobility. Breakthrough occurs first in the sandstone at $0.7 \mathrm{PV}$ (318 min). At roughly $0.71 \mathrm{PV}$ (328 $\mathrm{min}$ ), foam begins to exit the A10. After gas production begins, the experimental system approaches a pseudosteady state pressure drop as the cores gradually dry out. Gas was injected for several hours after breakthrough, but collapse of the foam never occurred. The average water saturation of each core was calculated from the liquid effluent collected. At breakthrough, $S_{\mathrm{w}}$ averages 0.16 in the $\mathrm{A} 10$ and 0.50 in the sandstone. 


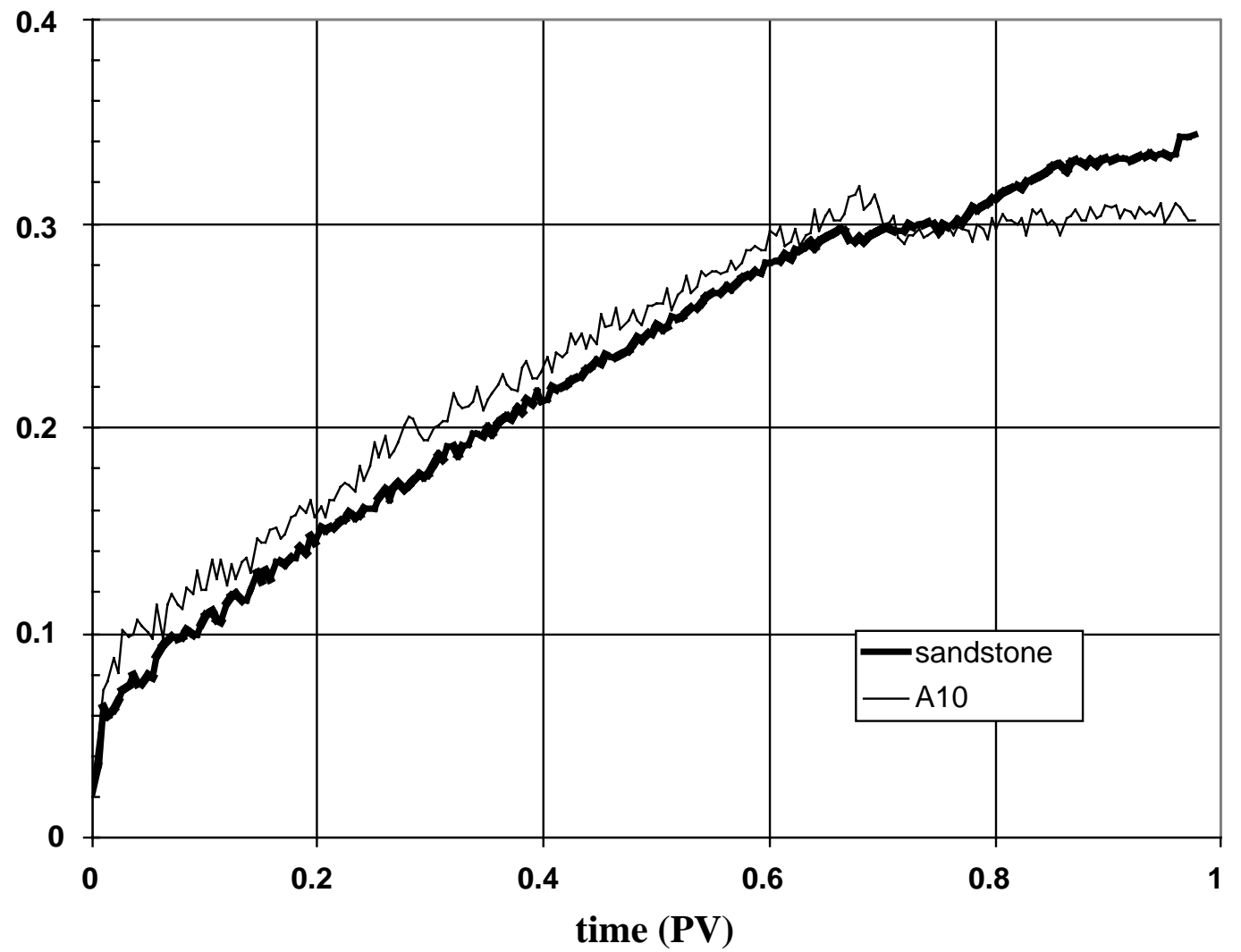

Figure 4. Pressure drop results for gas-only injection into heterogeneous system. 


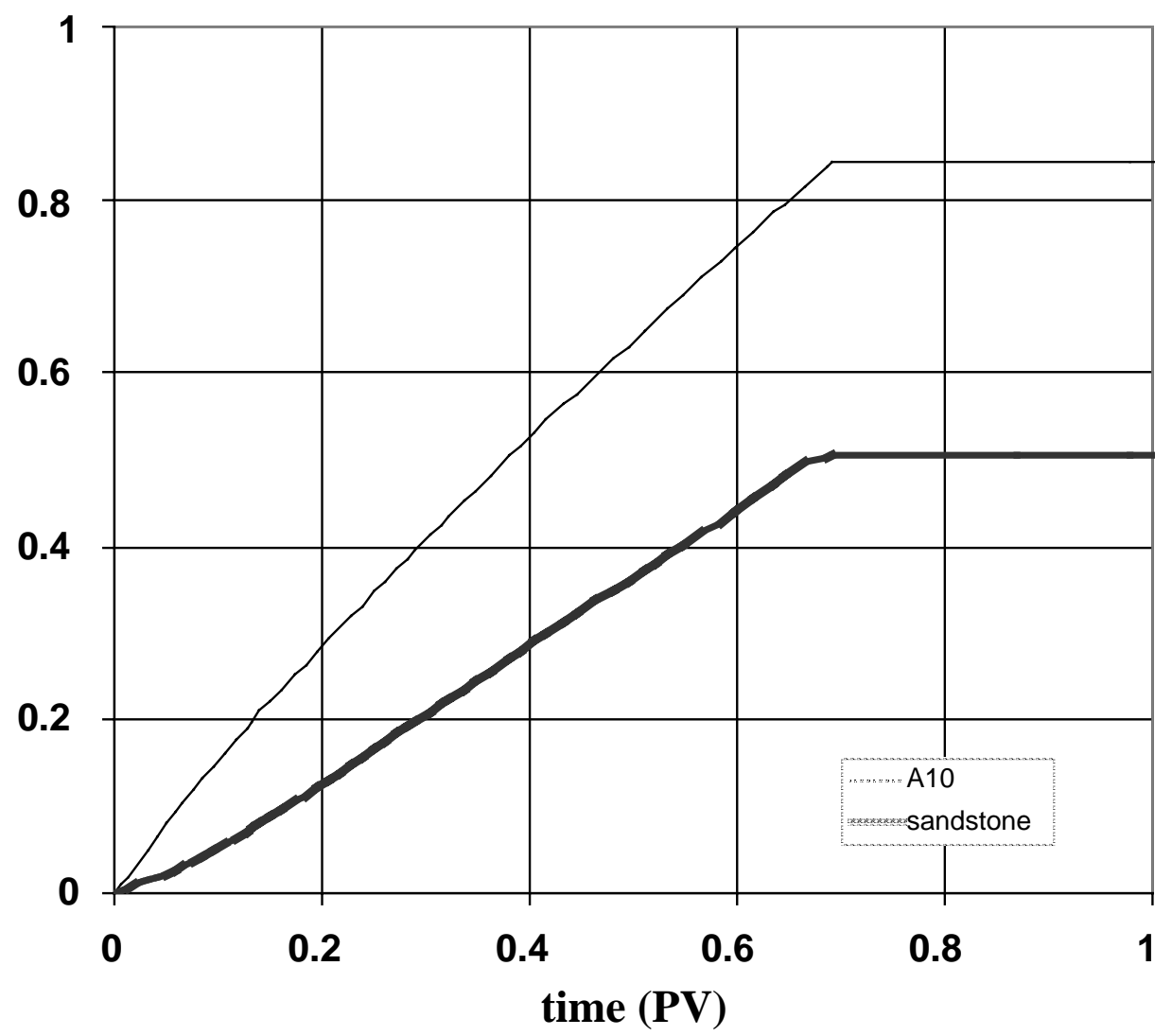

Figure 5. Liquid production from each core for gas only injection into heterogeneous system.

Because no liquid is injected, we measure the gas injection rate into each core as a function of time. Figure 6 presents the gas Darcy velocity at the inlet to each core. Initially, gas is more mobile in the higher permeability A10 and it accepts a greater fraction of the injection. Gradually, gas mobility in the A10 drops so that the sandstone accepts a greater fraction of the injected gas. The excellent diversion of gas to the sandstone despite a contrast in permeability of almost 8 to 1, can be explained through the ratio of gas mobility in the sandstone upon that in the A10, $\lambda_{\mathrm{f}, \mathrm{ss}} / \lambda_{\mathrm{f}, \mathrm{A} 10}$, computed with Eq. 8 . The measured absolute permeability and porosity of each core are taken from Table 3. Capillary pressure is computed from the Leverett J-function and dimensionalized according to $\sigma(\phi / K)^{1 / 2}$ (see Table 2 of Kovscek and Bertin, 2001). The limiting capillary pressure is set to $20 \mathrm{kPa}$ according to the work of Apaydin and Kovscek (2001) who employed a similar surfactant solution. Next, the gas velocity in each porous medium at gas breakthrough is taken from Fig. 6 and converted to interstitial velocity using the average gas saturation at breakthrough. That is, $S_{\mathrm{g}}$ is set to 0.5 for the sandstone and 0.85 for the A10. With the assembled information and Eq. $8, \lambda_{\mathrm{f}, \mathrm{ss}} / \lambda_{\mathrm{f}, \mathrm{A} 10}$ is equal to $0.868\left(v_{w, A 10} / v_{w, s s}\right)^{1+a \eta}$ at breakthrough. Thus, before consideration of liquid velocity effects, we expect gas mobility to have the possibility of being roughly equal in each porous medium. This agrees well with the experimentally observed trend that $\lambda_{\mathrm{f}, \mathrm{ss}} \lambda_{\mathrm{f}, \mathrm{A} 10}$ is of order 1 as shown in Fig. 6. 


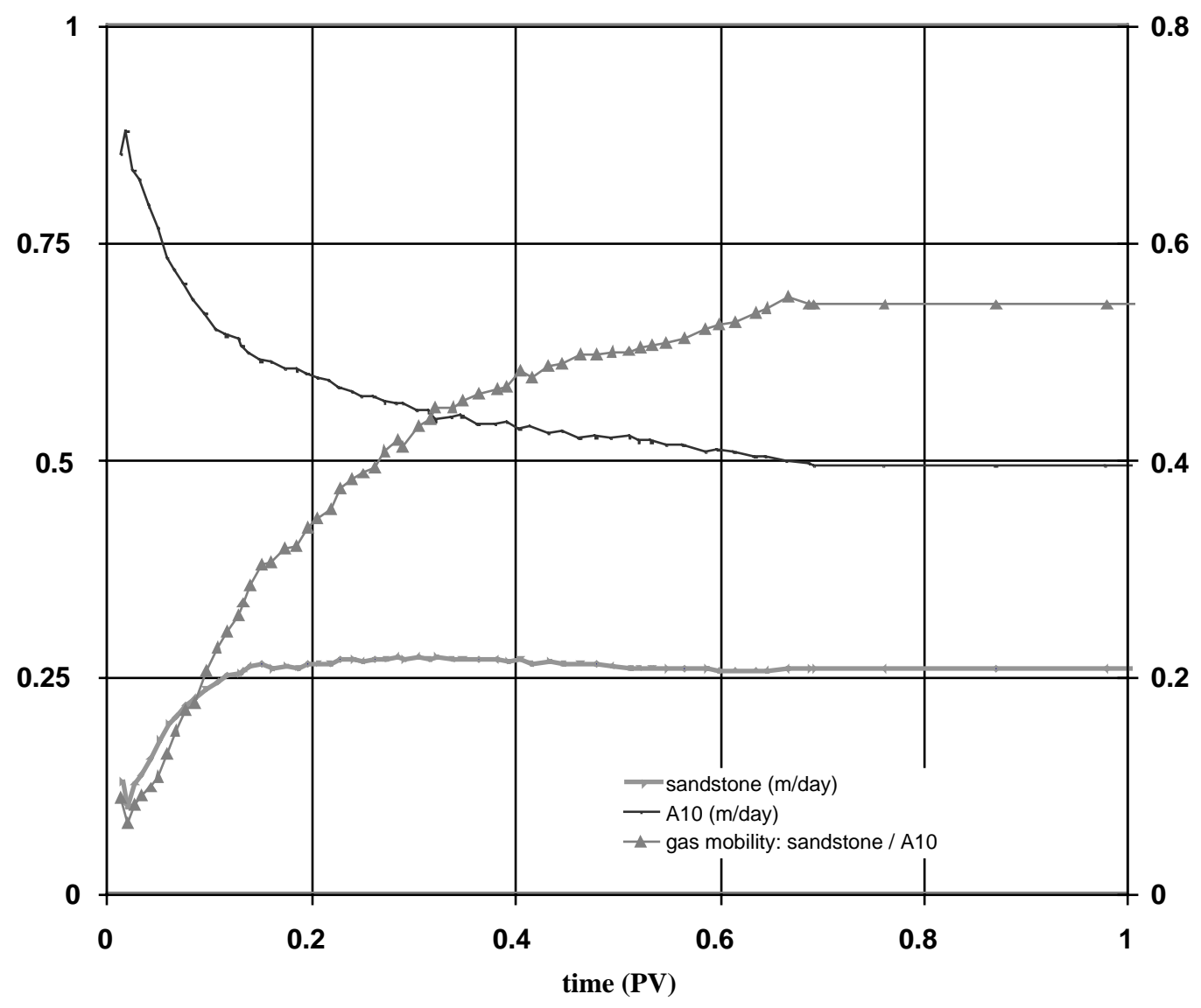

Figure 6. Gas Darcy velocity at the inlet to each core and the apparent ratio of sandstone to A10 gas mobility.

In the next set of experiments, gas and foamer solution are injected simultaneously. As in the previous experiments, the pore space is fully saturated with surfactant solution initially. The total liquid flow rate is $9 \mathrm{~mL} / \mathrm{hr}\left(2.5 \mathrm{~mm}^{3} / \mathrm{s}\right)$ and the gas rate is again $1.5 \mathrm{SCCM}\left(25 \mathrm{~mm}^{3} / \mathrm{s}\right)$ as in the prior gas-only injection. The overall gas fractional flow is 0.90 . Each core accepts whatever portion of the injected fluid it desires and, thus, the fraction of gas and liquid entering each core is variable over the duration of the experiment. Unfortunately, we do not possess a means to quantify the gas fractional flow entering each core. However, transparent tubing connected the various flow devices at the inlet of the apparatus and qualitative observations were possible.

Figure 7 displays the pressure response of the system. It exceeds that of the previous experiment because liquid is coinjected. Gas breaks through in the sandstone at $0.43 \mathrm{PV}$ (180 min). At this point, the pressure drop across the system is 0.5 bar $(50 \mathrm{kPa})$. The initial pressure response and steady increase of pressure accompanying gas injection again indicate foam generation and mobility control of the gas. Gas production from the A10 never occurs, even after greater than $10 \mathrm{PV}$ (4200 min) of injection. After breakthrough in the sandstone, pressure increases steadily, but much more slowly. It reaches a plateau of approximately 1.4 bar (140 kPa) at roughly $9.5 \mathrm{PV}$ of total injection. After breakthrough, the pressure drops measured across each 
porous medium are not equal. This is probably a capillary effect. Because gas never breaks through in the high-permeability A10, a gas-phase pressure is measured on the upstream side while on the downstream side a liquid-phase pressure is measured. After breakthrough in the sandstone, gas is present on both the upstream and downstream sides. Nevertheless, the rate of pressure increase is the same for each medium and the difference in pressure between the two media after breakthrough is virtually constant.

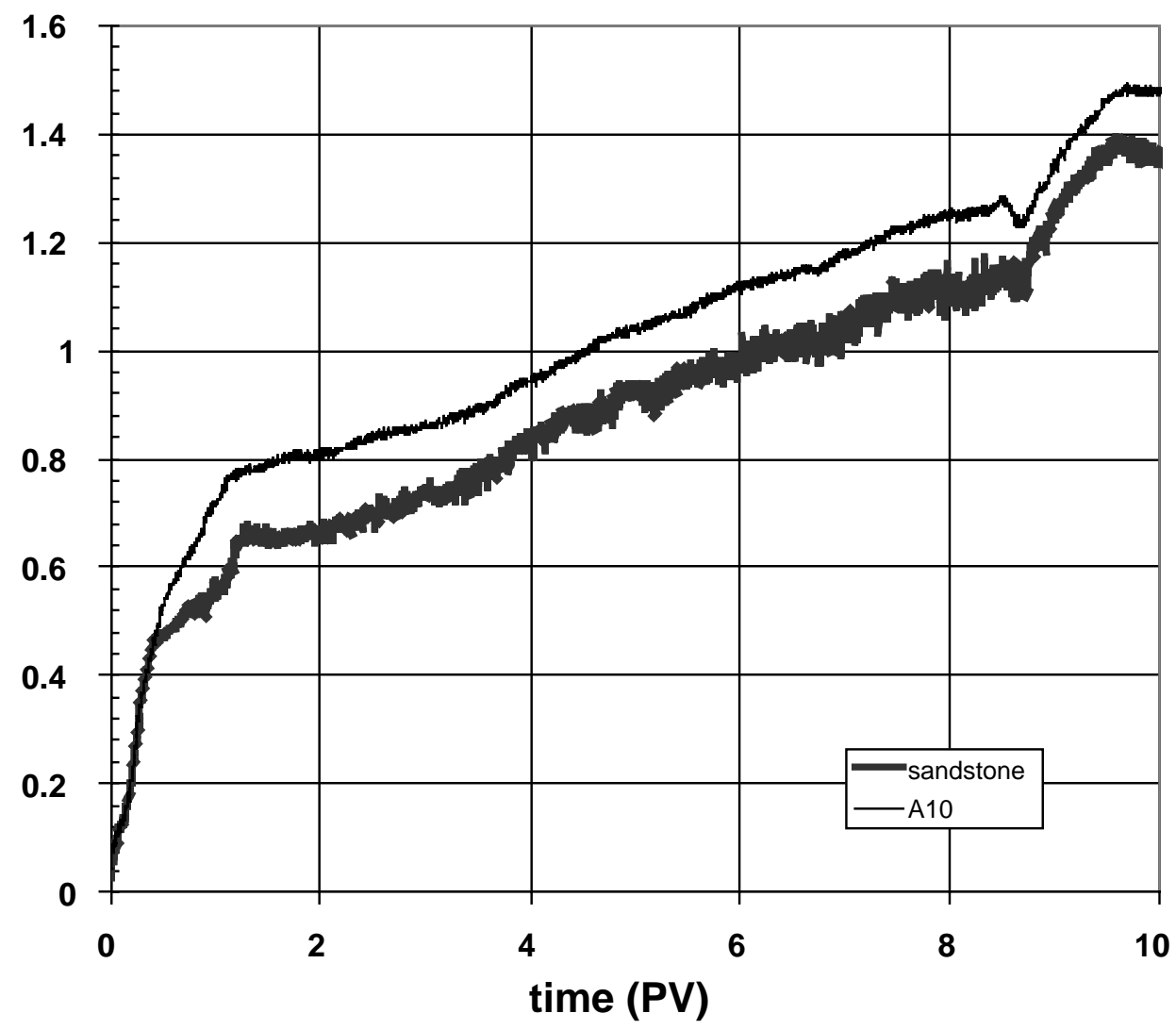

Figure 7. Pressure drop results for simultaneous gas and liquid injection into heterogeneous system.

The produced liquid from each porous medium as a function of time is presented in Figure 8. Initially, the sandstone accepts very little gas. As verified visually, the major portion of the gas enters the more permeable A10 at very small times. Once foam is generated in the A10, gas is diverted to the sandstone and production of liquid from the sandstone increases. At this stage, the sandstone accepts an increasing fraction of the gas, very little of the injected liquid, and production of liquid is fairly efficient. Visual estimates place the gas fractional flow at the inlet of the sandstone in excess of $99.9 \%$ (one part in a thousand is liquid), at this stage. In contrast the A10, accepts a greater fraction of liquid and a lesser fraction of gas. The production rate from the A10 drops. The system reaches a state where the higher permeability layer is virtually blocked to gas flow. The majority of the injected gas enters the sandstone. Liquid production from the A10 is rather steady and exceeds 1 because of the continuous liquid injection into the system. 


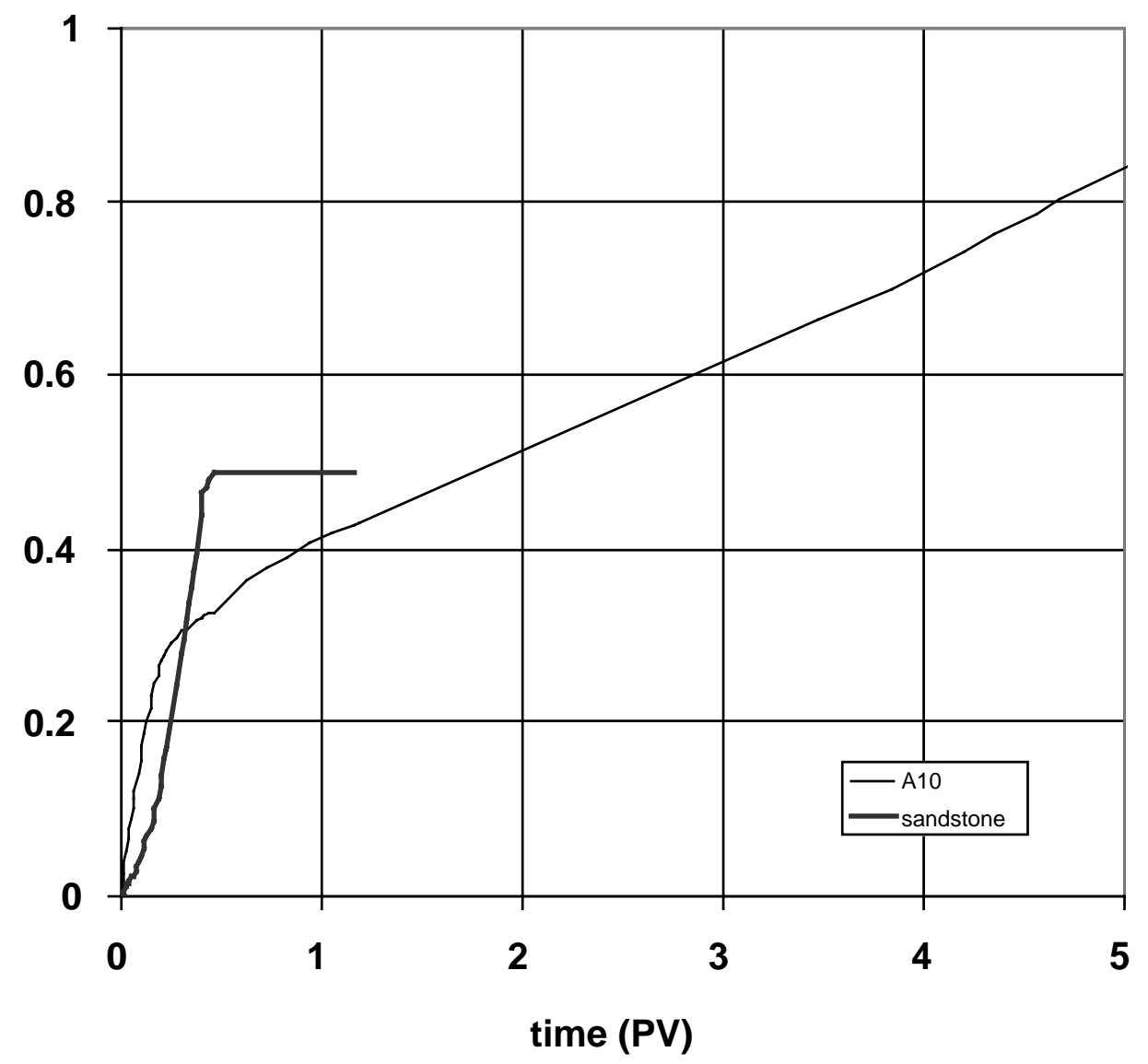

Figure 8. Liquid production from each core for simultaneous injection of gas and liquid into heterogeneous system.

Mass balance gives the average water saturation of the sandstone at breakthrough equal to 0.5 , assuming negligible liquid injection. This is identical to the experiment with injection of only gas. After breakthrough, the fraction of liquid entering the sandstone increases somewhat and visual estimates place the gas fractional flow between 95 and $99 \%$. This is consistent with the increase in pressure drop after gas breakthrough. The increased liquid flow leads to increased foam generation and reduced gas mobility as indicated by Eq. 8 .

The blockage of the higher permeability A10 can be better understood by considering the liquid velocity dependence in Eq. 8 of Kovscek and Bertin (2001). The liquid velocity exponent is larger than that for the gas velocity. Because the A10 accepts most of the injected liquid, the liquid velocity is relatively high within the A10 and the gas mobility is expected to be low. In this case, mobility is low enough to lead to blockage of the high permeability core.

Gas-only injection as well as simultaneous surfactant solution and nitrogen injection experiments were conducted to contrast with the above heterogeneous experiments. In these cases, the cores are fully saturated with surfactant solution initially. The rate for gas-only injection is $1.5 \mathrm{SCCM}$. For coinjection, the gas rate is $1.5 \mathrm{SCCM}$ and the liquid injection rate is $0.15 \mathrm{~cm}^{3} / \mathrm{min}$. 
Figure 9 reports the pressure response for the A10 synthetic sandstone. With coinjection of gas and liquid, the measured pressure drop is about $0.1 \mathrm{bar}(10 \mathrm{kPa})$ greater than without liquid injection. The dip in both pressure responses at roughly $0.8 \mathrm{PV}$ of injection corresponds to gas breakthrough. On the other hand, Figure 10 gives data for similar experiments conducted in the Vosges sandstone. Here, the pressure drop for coinjection far exceeds that of gas-only injection. Gas breakthrough in the sandstone is at slightly more than $0.5 \mathrm{PV}$ for the gas-only case, while breakthrough occurs at roughly $0.7 \mathrm{PV}$ when gas and surfactant solution are coinjected. At 0.7 $\mathrm{PV}$, the pressure drop during coinjection is already 0.7 bar, whereas the pressure drop in the gasonly case is about 0.4 bar. Hence, the difference between gas-only injection and coinjection appears to be more significant in the case of lower permeability porous media. We also note that the relatively slow increase in pressure drop after breakthrough during coinjection might be diagnostic of an end effect (Apaydin and Kovscek, 2001).

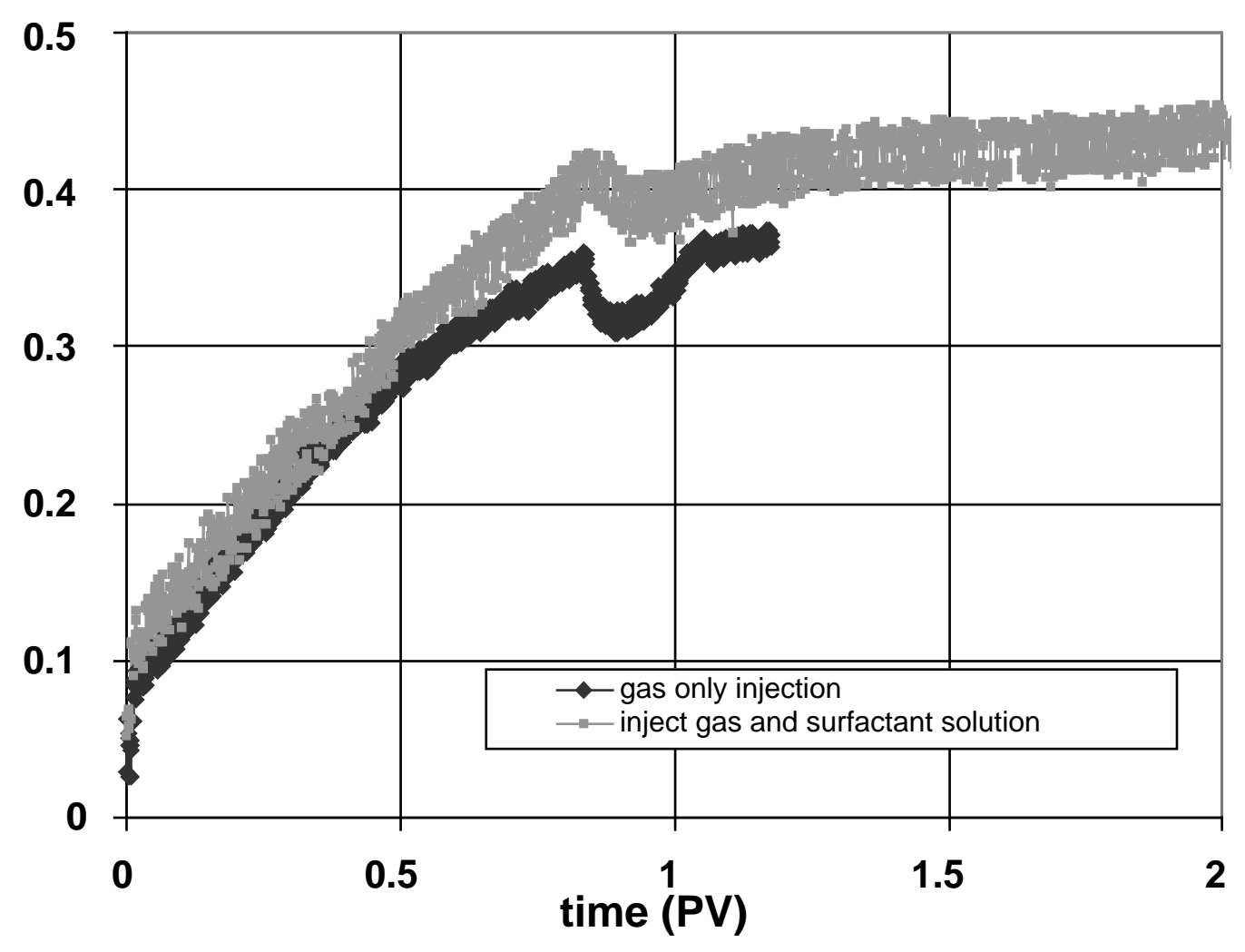

Figure 9. Pressure drop response for gas-only and co-injection into A10 synthetic sandstone. 


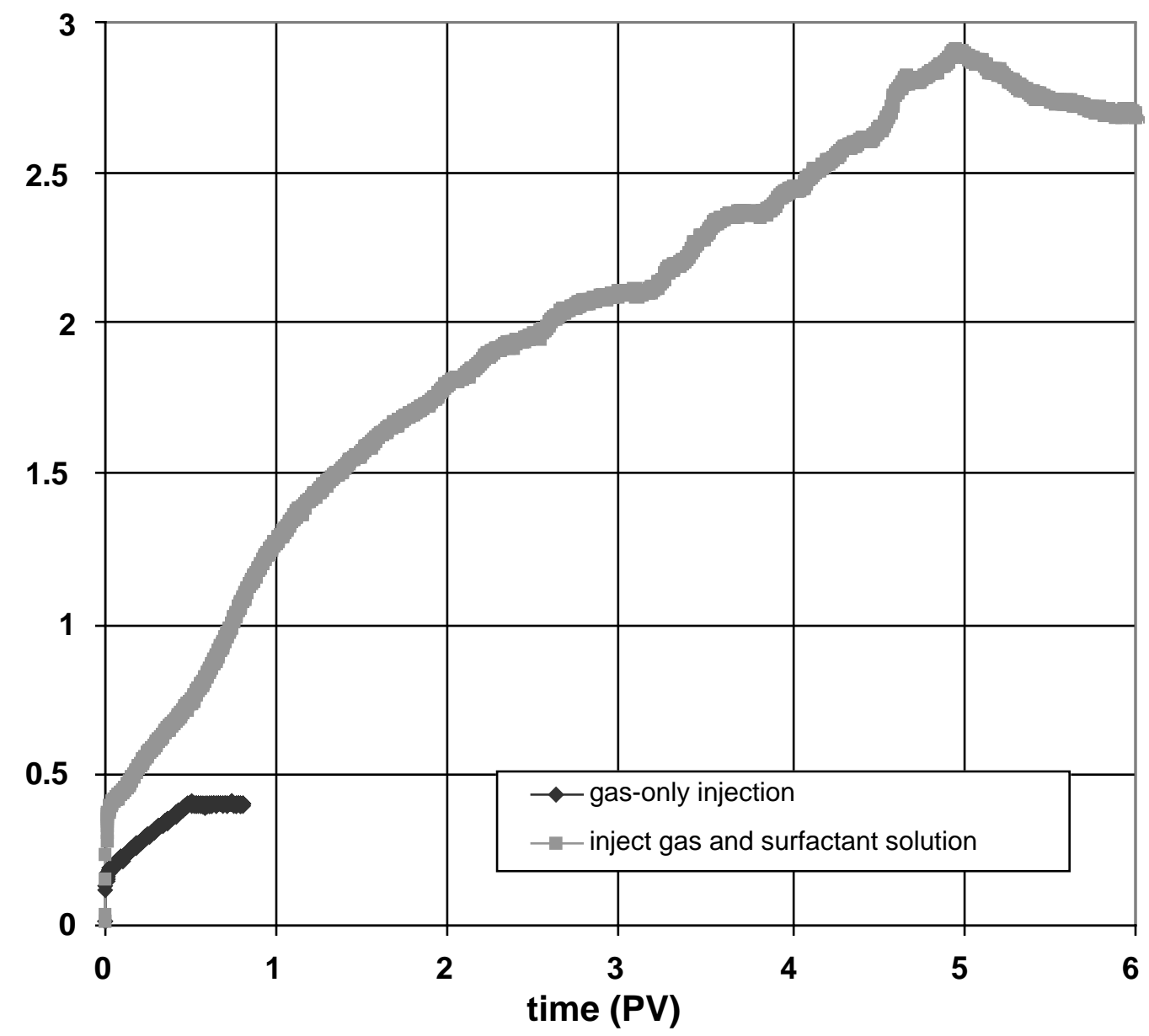

Figure 10. Pressure response for gas-only and co-injection into sandstone.

It is interesting to contrast the measured pressure drop among the various cases. In the case of coinjection into the heterogeneous system, the maximum pressure drop is about 1.2 bar $(120 \mathrm{KPa})$. Recall that the sandstone carries all of the injected gas in this case. When this amount of gas is injected along with surfactant solution into the sandstone alone, the overall pressure drop is more than twice as much, 2.5 bar $(250 \mathrm{kPa})$. The chief difference is the amount of liquid flowing in the sandstone in each of these experiments. As Eq. 8 indicates, the gas mobility is lower, and the pressure drop correspondingly greater, when the liquid interstitial velocity is greater.

\section{Conclusions}

An experimental and scaling study of foamed gas mobility in heterogeneous porous media was performed. The scaling study demonstrated that important factors such as permeability, porosity, capillary pressure, limiting capillary pressure for foam collapse, and liquid and gas 
velocity can be combined to describe gas mobility upon assumption of local equilibrium of foam generation and coalescence rates. Experiments supported predictions of the scaling exercise. Effective diversion of gas to low permeability zones is possible with foam. The experiments also highlight the important role of liquid velocity on gas diversion. Foamed gas mobility is inversely proportional to liquid velocity. A zone of a porous medium that receives the majority of liquid during coinjection of surfactant solution and gas exhibits low gas mobility. It is within these zones that the possibility for complete blockage to gas exists.

\section{References}

Apaydin, O. and A. R. Kovscek, "Surfactant Concentration and End Effects on Foam Flow in Homogeneous Porous Media," Transport in Porous Media, 43(3), 511-536 (2001).

Bertin, H.J., Quintard, M.Y., and Castanier, L.M.: 1998, Modeling Transient Foam Flow in Porous Media Using a Bubble Population Correlation, Society of Petroleum Engineers Journal 3(4), 356-362.

Bertin, H.J., Apaydin O.G., Castanier, L.M., and Kovscek, A.R.: 1999, Foam Flow in Heterogeneous Porous Media, Society of Petroleum Engineers Journal 4(2), 75-82.

Bertin, H.J.: 2000, Foam Diversion Modeling Using a Bubble-Population Correlation, , Proceeding of the SPE/DOE Symposium on Enhanced Oil Recovery, Tulsa, OK, U.S.A., SPE 59366.

Casteel, J.F. and Djabbarah, N.F.: 1988, Society of Petroleum Engineers Reservoir Engineering 3, 1186-1192.

Chambers, K.T. and Radke, C.J.: 1991, Capillary Phenomena in Foam Flow Through Porous Media, in Interfacial Phenomena in Petroleum Recovery, Morrow, N.R. ed., 191-255, Marcel Dekker, NY.

Cheng, L., Kam, S. I., Delshad, M., and Rossen, W.R.: 2001, Simulation of Dynamic Foam-Acid Diversion Process, Proceedings of the SPE European Formation Damage Conference, The Hauge, Netherlands, SPE 68916.

Cohen, D., Patzek, T.W., and Radke, C.J.: 1997, Onset of Mobilization and the Fraction of Trapped Foam in Porous Media, Transport in Porous Media 28, 253-284.

Corey, A.T.: 1954, The Interrelation Between Gas and Oil Relative Permeabilities, Producer's Monthly 19, 38-41.

de Gennes, P. G.: 1992, Conjectures on Foam Mobilization, Revue De L'Institut Francais Du Pétrole 47(2), 249-254. 
Ettinger, R.E. and Radke, C.J.: 1992, Influence of Foam Texture on Steady Foam Flow in Berea Sandstone, Society of Petroleum Engineers Reservoir Engineering 7, 83-90.

Falls, A.H., Hirasaki, G.J., Patzek, T.W., Gauglitz, P.A., Miller, D.D., and Ratulowski, T: 1988, Development of a Mechanistic Foam Simulator: The Population Balance and Generation by Snap-Off, Society of Petroleum Engineers Reservoir Engineering 3, 884-892.

Fergui, O., Bertin, H. and Quintard, M.Y: 1998, Transient Aqueous Foam Flow in Porous Media: Experiments and Modeling, Journal of Petroleum Science and Engineering 20, 9-29.

Friedmann, F., Chen, W.H., and Gauglitz, P.A.: 1991, Experimental and Simulation Study of High-Temperature Foam in Porous Media, Society of Petroleum Engineers Reservoir Engineering 6, 37-45.

Gillis, J.V. and Radke, C.J.: 1990, A Dual Gas Tracer Technique of Determining Trapped Gas Saturation During Steady Foam Flow in Porous Media, Proceedings of the $65^{\text {th }}$ SPE Annual Technical Conference, New Orleans, LA, U.S.A. SPE 20519.

Hanssen, J. E.: 1993A, Foam as a Gas Blocking Agent in Petroleum Reservoirs I: Empirical Observations and Parametric Study, Journal of Petroleum Science and Engineering 10, 135-156.

Hanssen, J. E.: 1993B, Foam as a Gas Blocking Agent in Petroleum Reservoirs II: Mechanisms of Gas Blockage By Foam, Journal of Petroleum Science and Engineering 10, 117-133.

Heller, J.P.: 1994, $\mathrm{CO}_{2}$ Foams in Enhanced Oil Recovery, in Foams in the Petroleum Industry, Schramm, L.L. ed., 201-234, Amercan Chemical Society, Washington D.C.

Hirasaki, G.J. and Lawson, J.B.: 1985, Mechanisms of Foam Flow in Porous Media: Apparent Viscosity in Smooth Capillaries, Society of Petroleum Engineers Journal 25(2), 176-190.

Hirasaki, G.J.: 1989A, The Steam Foam Process, Journal of Petroleum Technology 41(5), 449456.

Hirasaki, G.J.: 1989B, The Steam Foam Process, Supplement to SPE 19505 Society of Petroleum Engineers, Richardson, TX.

Hirasaki, G.J., Miller, C.A., Szafranski, R., Lawson, J.B., and Akiya, N.: 1997A, Surfactant/Foam Process for Aquifer Remediation, Proceedings of the SPE Annual Technical Conference, San Antonio, TX, U.S.A. SPE 39292.

Hirasaki, G.J., Miller, C.A., Szafranski, R., Lawson, J.B., Tanzil, D., Jackson; R.E., Londergan, J. and Mainardus, H.: 1997B, Field Demonstration of the Surfactant/Foam Process for Aquifer Remediation, Proceedings the SPE International Symposium on Oilfield Chemistry, Houston, TX, U.S.A. SPE 39292.

Jiménez, A. I. and Radke, C.J.: 1989, Dynamic Stability of Foam Lamellae Flowing Through a Periodically Constricted Pore, in Oil Field Chemistry: Enhanced Recovery and Production Stimulation, Borchardt, J.K. and Yen, T.F. eds., 460-469, Amercan Chemical Society, Washington D.C. 
Kharabaf, H. and Yortsos, Y.C.: 1998, Pore Network Model for Foam Formation and Propagation in Porous Media, Society of Petroleum Engineers Journal 13(1), 42-53.

Khatib, Z.I., Hirasaki, G.J. and Falls, A.H.: 1988, Effects of Capillary Pressure on Coalescence and Phase Mobility in Foams Flowing in Porous Media, Society of Petroleum Engineers Reservoir Engineering 3, 919-926.

Llave, F.M., Chung, F.T-H., Louvier, R.W. and Hudgins, D.A., 1990, Foams as Mobility Control Agents for Oil Recovery by Gas Displacement, Proceeding of the $7^{\text {th }}$ SPE/DOE Symposium on Enhanced Oil Recovery, Tulsa, OK, U.S.A., SPE 20245.

Kibodeaux, K., Zeilinger, S. C., and Rossen, W.R.: 1994, "Improved Prediction of Foam Diversion in Matrix Acidization," Proceedings of the $69^{\text {th }}$ SPE Annual Technical Conference, New Orleans, LA U.S.A. SPE 28550.

Kovscek, A.R. and Radke, C.J.: 1994, Fundamentals of Foam Transport in Porous Media, in Foams in the Petroleum Industry, Schramm, L.L. ed., 115-163, Amercan Chemical Society, Washington D.C.

Kovscek, A.R., Patzek, T.W., and Radke, C.J.: 1995, A Mechanistic Population Balance Model for Transient and Steady State Foam Flow in Boise Sandstone, Chemical Engineering Science 50(23), 3783-3799.

Kovscek, A.R. and Radke, C.J.: 1996, Gas Bubble Snap-Off Under Pressure Driven Flow in Unconstricted Noncircular Capillaries, Colloids and Surfaces A 117, 55-76.

Laidlaw, W.G., Wilson, W.G., and Coombe, D.A.: 1993, A Lattice Model of Foam Flow in Porous Media: A Percolation Approach, Transport in Porous Media 11, 139-159.

Myers, T.J. and Radke, C.J.: 1999, Transient Foam Displacement in the Presence of Residual Oil: Experiment and Simulation Using a Population Balance Model, Proceedings of the SPE Annual Technical Conference, Houston, TX, U.S.A. SPE 56412.

Persoff, P., Radke, C.J., Pruess, K., Benson, S.M., and Witherspoon, P.A.: 1991, A Laboratory Investigation of Foam Flow in Porous Media at Elevation Pressure, Society of Petroleum Engineers Reservoir Engineering 6, 365-371.

Robin, M.: 1987, Laboratory Evaluation of Foaming Additives Used to Improve Steam Efficiency, Proceedings of the $62^{\text {nd }}$ SPE Annual Technical Conference, Dallas, TX, U.S.A. SPE 16729.

Rossen, W.R.: 1988, Theories of Foam Mobilization Pressure Gradient, Proceeding of the $6^{\text {th }}$ SPE/DOE Symposium on Enhanced Oil Recovery, Tulsa, OK, U.S.A., SPE 17358.

Rossen, W.R. and Gauglitz, P.A.: 1990, Percolation Theory of Creation and Mobilization of Foams in Porous Media, American Institute of Chemical Engineers Journal 36(8), 11761188 . 
Rossen, W.R. and Zhou, Z. H..: 1996, Modeling Foam Mobility at the Limiting Capillary Pressure, Society of Petroleum Engineeers Advanced Technoloyg Series 3(1), 146-153.

Rossen, W. R. and Lu Q.: 1997, Effect of Capillary Crossflow on Foam Improved Oil Recovery, Proceedings of the SPE Western Regional Meeting, Long Beach, CA U.S.A., SPE 38319.

Stauffer, D.: 1985, Introduction to Percolation Theory, Taylor and Francis, London

Tanzil, D., Hirasaki, G.J., and Miller, C.A.: 2000, Mobility of Foam in Heterogeneous Porous Media: Flow Parallel and Perpendicular to Stratification, , Proceedings of the SPE Annual Technical Conference, Dallas, TX U.S.A. SPE 63228.

Yaghoobi, H. and Heller, J.P.: 1996, "Effect of Capillary Contact on $\mathrm{CO}_{2}$-Foam Mobility in Heterogeneous Core Samples," Proceedings of Permian Basin Oil and Gas Recovery Conference, Midland, TX, U.S.A. SPE 35169.

Zehrboub, M., Ben-Naceur, K., Toubol, E., and Thomas, R.: 1994, Matrix Acidizing: A Novel Approach to Foam Diversion, Society of Petroleum Engineers Production \& Facilities, May, 121-126.

Zeilinger, S. C., Wang, M., Kibodeaux, and Rossen, W.R.: 1995, Improved Prediction of Foam Diversion in Matrix Acidization, Proceedings of the SPE Production Operations Symposium, Oklahoma City, OK U.S.A. SPE 29529.

Zhou, Z. H. and Rossen, W. R.: 1992, Applying Fractional-Flow Theory to Foams for Diversion in Matrix Acidization, Proceedings of the $67^{\text {th }}$ Annual SPE Technical Conference and Exhibition, Washington D.C. U.S.A. SPE 24660.

Zhou, Z. H. and Rossen, W. R.: 1992, Applying Fractional-Flow Theory to Foam Processes at the "Limiting Capillary Pressrue", Proceedings of the SPE/DOE ${ }^{\text {th }}$ Symposium on Enhanced Oil Recovery, Tulsa, OK. U.S.A. SPE 24180. 


\section{Thermally Induced Fines Mobilization and Wettability}

\section{(Task 1c)}

\section{Introduction}

Thermal recovery research and field operations indicate that reservoir rock exposed to high temperature undergoes a systematic shift in wettability toward water wetness (Bennion and Thomas, 1992; Al-Hadhrami and Blunt, 2001). The consequence of a shift toward water wetness is that steam and steam condensate become more effective displacement agents and the rock matrix imbibes water more effectively. It is well understood that wettability influences the flow and relative permeability characteristics of porous media (Bennion and Thomas, 1992; DiCarlo et al., 1998). Examination of temperature as a variable on relative permeability has been conducted since the 1960's (Edmonson, 1965; Poston et al., 1967; Sinnokrot et al., 1969). The effects of fluid properties, such as $\mathrm{pH}$ and salinity, on fines migration and permeability are discussed extensively in the formation damage literature (Young, 1979; Kia, 1987; Zhou et al., 1997; Vaidya and Fogler, 1990; Gunter et al., 1992). Yet, controversy exists regarding the origin of temperature effects and their extent. To date, no plausible mechanism describes this shift toward water wetness. Fines mobilization presents one pathway to create fresh water wet surfaces.

In order to understand the mechanisms of fines mobilization, we review and use DLVO (Derjaguin, Landau, Verway and Overbeek) theory. It explains the stability of colloidal systems as a result of the balance of attractive and repulsive forces. As will be shown, DLVO calculations predict a profound effect of temperature on fines stability. Release of fine clay material is predicted at conditions $(\mathrm{pH}$, salinity, temperature) obtained during steamflood. Predictions are presented in the form of isotherm maps. They are confirmed experimentally by core waterfloods performed in Berea sandstone at temperatures ranging from 20 to $200^{\circ} \mathrm{C}$. Permeability reduction is observed with temperature increase and fines mobilization occurs repeatably at a particular temperature that varies with solution $\mathrm{pH}$ and ionic strength. A scanning electron microscope (SEM) was used to analyze composition of the effluent samples collected during experiments. This study shows that temperature is a decisive factor in fines release and the temperature at which the detachment of fines occurs is well predicted by DLVO theory.

Discussion of this topic is organized in the following fashion. First a scenario for wettability alteration at elevated temperature is given. Second, corefloods with hot water are used to show that temperature mobilizes fines in Berea sandstone. Third, surface forces, so called DLVO theory, are used to explain experimental observations.

\section{Wettability Alteration}

The mechanism for formation of mixed wettability in initially water-wet rocks that has come to be accepted in recent years is that asphaltenes are deposited on pore walls when aqueous wetting films coating solid grains are unstable (Kovscek et al., 1993). This mechanism explains the formation of continuous oil-wet pathways on pore walls and the origin of oil-wet and water- 
wet surfaces within the same core (Kovscek et al., 1993). For all practical purposes, asphaltenes adhere irreversibly to siliceous or carbonaceous surfaces. Solvents such as hot pyridine are required to remove asphaltene from solid material (Windholz et al., 1976).

A possible scenario for wettability shift toward water wetness as a function of fines detachment is shown in Fig 1. Figures 1.1.a, 1.2.a and 1.3.a represent mixed-wet systems with oil-wet fines attached to the matrix wall at initial reservoir temperature for three different initial scenarios; before (Fig. 1.1.a), during (Fig. 1.2.a) and after (Fig. 1.3.a), waterflood is performed. Dark shading denotes oil; whereas water is lightly shaded. Brackets located outside of the triangular pore indicate the section of the pore wall that is oil-wet.
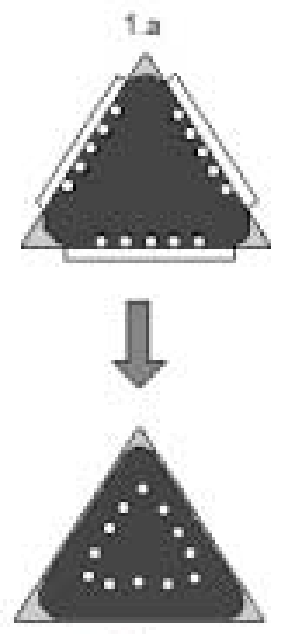

1.6
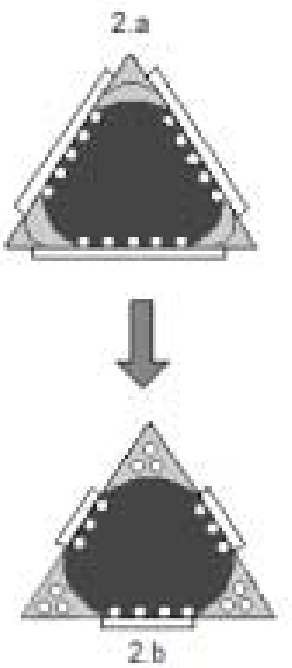
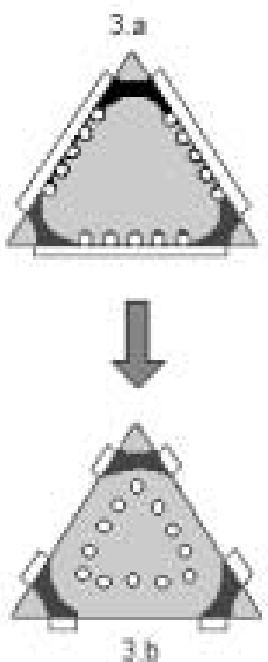

Figure 1. Triangles in 1.a through 3.b illustrate pore spaces. Gray color symbolizes the water phase, black color designates oil phase and, round white circles represent clay particles.

The same pore systems in Figs. 1.1.a, 1.2.a, and 1.3.a are represented at greater temperatures, in Figs. 1.1.b, 1.2.b, and 1.2.c, respectively. In all three figures, an increase of temperature is hypothesized to cause the detachment of fines from the walls. The grains underlying the fines are water-wet because oil never contacted them directly. The fraction of water-wet surface thereby increases. Thus, Fig 1.1.b represents a system with greater connate water saturation that has shifted to water wetness as a result of temperature increase. In Fig. 1.2.b we represent another possible scenario where fines detach from a portion of the wall. In this case, the shift to water wetness occurs in the area where the fines are exposed to water that has imbibed spontaneously. Fines detached from the wall could be present in both water and oleic phases. This possibility explains the existence of fines in water effluent reported by some authors (Tang and Kovscek 2002). In Fig. 1.3.b, there is also detachment of the fines from the pore walls during forced imbibition. Thus, Fig 1.3.b. represents a system with lower residual oil saturation and increased water wetness. 
Fines release and change in wettability, illustrated in Fig. 1, may occur under particular fluid conditions ( $\mathrm{pH}$, salinity, composition) and characteristics of the mineral surface. This mechanism is invalidated immediately if temperature cannot be shown to cause the release of fines from solid surfaces. Our next step is to conduct experiments that illustrate fines mobilization at elevated temperature.

\section{Experimental Investigation}

We designed experiments to (a) validate the hypothesis that temperature is crucial in fines release and, (b) map the sensitivity of the conditions to the temperature. The rock samples are Berea sandstone (Cleveland Quarries). The advantages of using Berea sandstone include the considerable amount of clays and, its large permeability that allows experiments to be performed in a relatively short time.

Figure 2(a) shows an image of the Berea Sandstone used, magnified at 250X with a scanning electron microscope (JEOL JSM-5600 LV). Note the fine material adhering to the sand grains. The area enclosed by the square was magnified 5000 times and it shows the classic platelike or, accordion-like mineral form of kaolinite

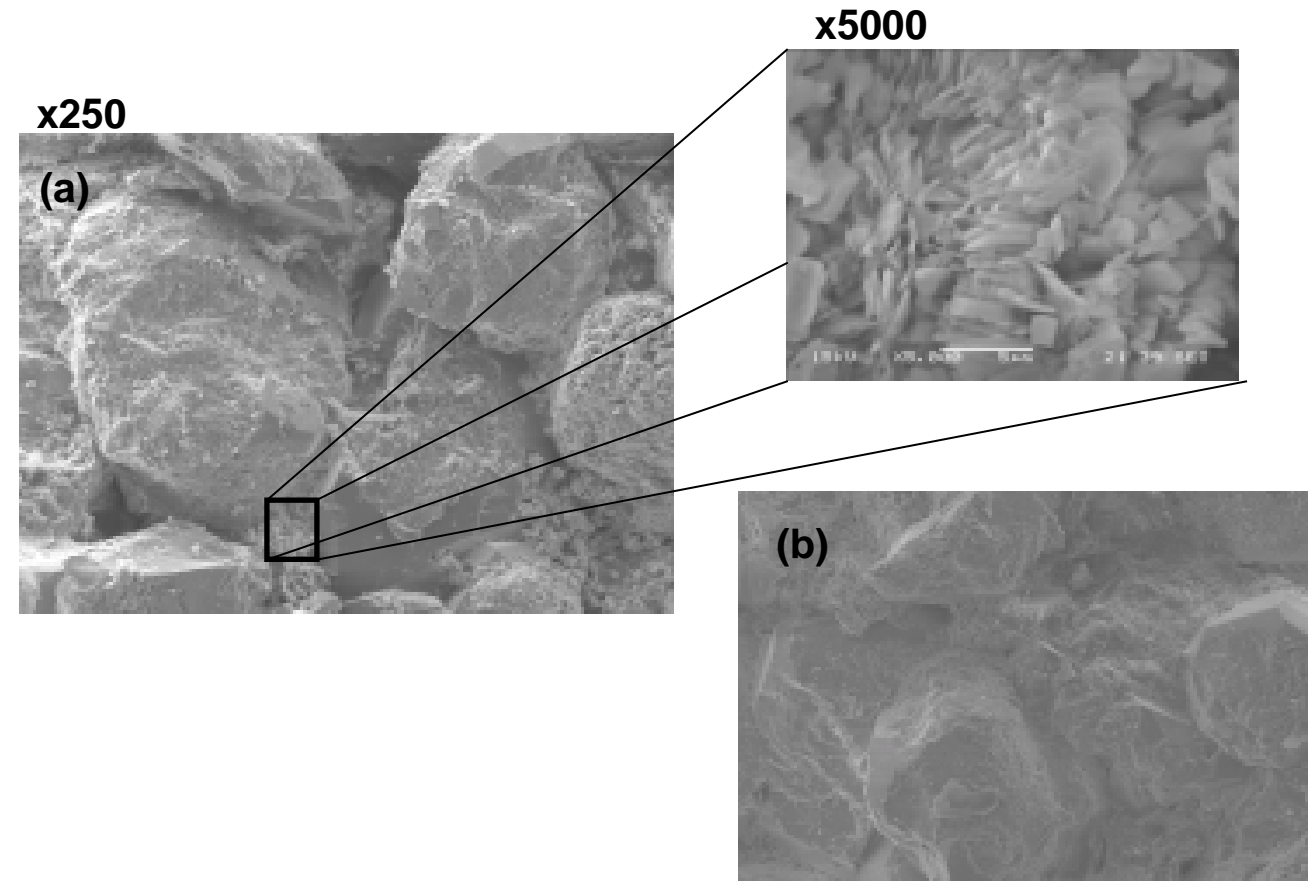

Figure 2. SEM images from Berea sandstone core used in experiments.

(a) before and, (b) after waterflood.

We performed 20 tests with unfired cores and 3 tests with a fired core. The cores were subject to single-phase water injection at different $\mathrm{pH}$, salinity, and temperatures. The salinity and $\mathrm{pH}$ of each of the injection fluid is listed in Table 1. 
Figure 3 shows the layout of experimental apparatus. The cores were cylindrical with length between 4 and $6 \mathrm{~cm}$ and diameter of $2.5 \mathrm{~cm}$. Core porosity ranged between 20 and $23 \%$. Cores were placed in vacuum and temperature of $50{ }^{\circ} \mathrm{C}$ for a minimum of ten hours. The apparatus is assembled by coating the core with a thin film of solvent resistant sealant (Fluoro Silicone Rubber) and placing the core inside FTP heat-shrink tubing (Plastic Professional). This covering serves as a high temperature sleeve. A net confining pressure of approximately 250-400 psia is applied. The cores were subject to waterflood at different conditions. Corefloods were performed at constant Darcy velocity of approximately $1.0 \mathrm{~m} /$ day. This injection flow rate is typical for fluid injection and, small enough to prevent "wormholes" at high temperatures.

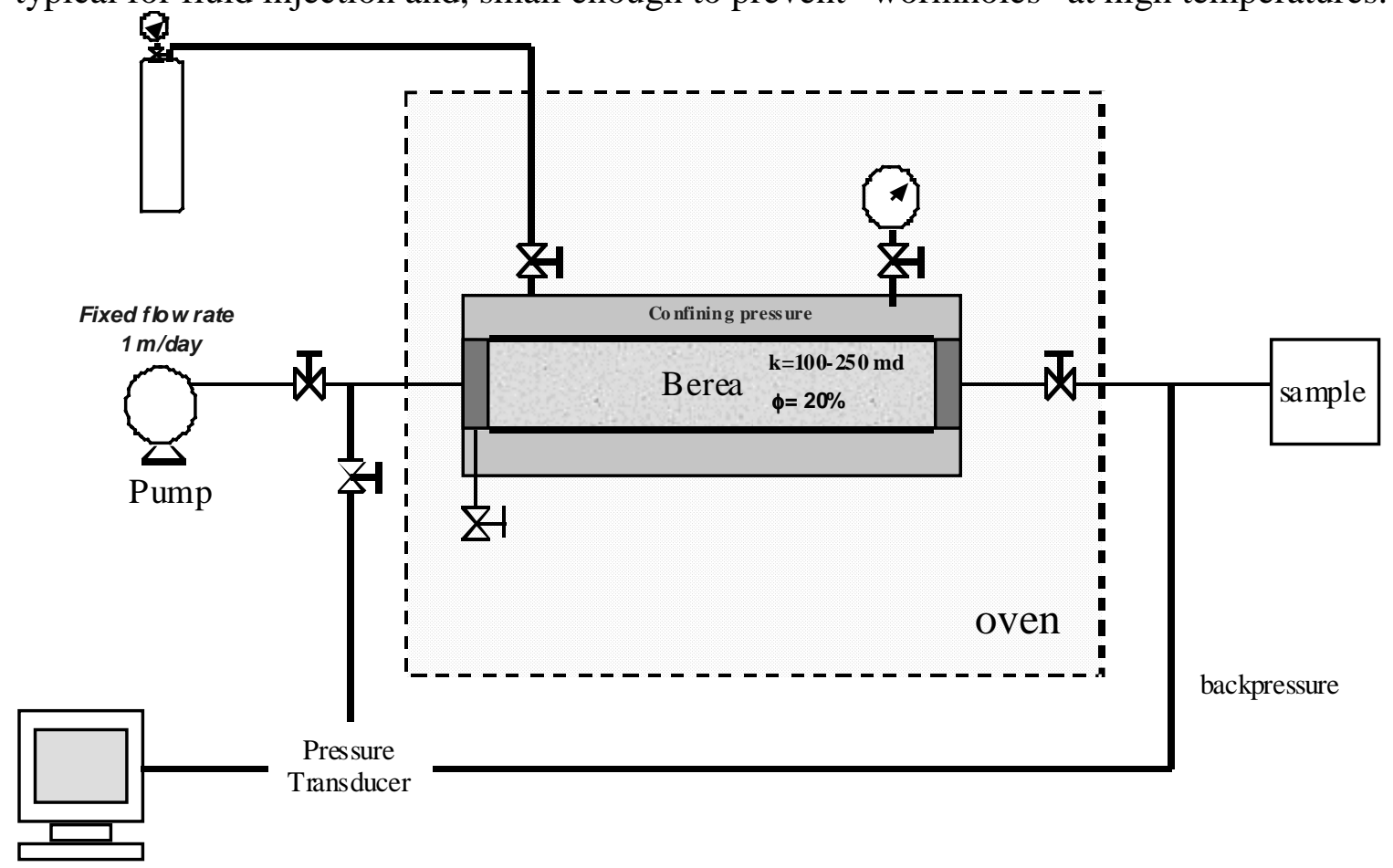

Figure 3. Experimental apparatus.

Experiments were performed at a variety of temperatures, salinities, and $\mathrm{pH}$, under isothermal conditions. The core holder is placed in the oven ( Blue-M thermostated to $\pm 1^{\circ} \mathrm{F}$ ) for waterflood at high temperature. Back- pressure is used in order to ensure single phase flow inside the core at high temperature. The $\mathrm{pH}$ of the solutions in all experiments was adjusted and controlled by using buffers. We used a buffer consisting of sodium hydroxide and potassium phosphate for $\mathrm{pH}$ 7. A solution of sodium bicarbonate and sodium carbonate was used for an alkaline $\mathrm{pH}$ of 10 . Cores were flushed with a copious amount of buffer solution prior to temperature to satisfy any losses of buffer to the rock.

Flow rate was held constant and, changes in permeability were observed by measuring the pressure drop across the core. Fines migration was verified by collecting effluent samples from experiments. Some effluent solutions were filtered and analyzed to verify the presence of clays.

The first experiment validated both the experimental setup and procedure. Also, this experiment verified that fines production was possible from this set of cores. In the first 
experiment, a core was subjected to $10^{-4} \mathrm{M} \mathrm{NaCl}$ solutions at $25^{\circ} \mathrm{C}$. Initially, the core was exposed to a high salinity solution $(1 \mathrm{M} \mathrm{NaCl})$ and permeability was allowed to stabilize. The core was then exposed to low salinity solution $\left(10^{-4} \mathrm{M} \mathrm{NaCl}\right)$. As the low salinity solution was injected, the core suffered an abrupt reduction of permeability to about $1 \%$ of the initial permeability and fines were observed in the effluent. The effluent collected during the injection of low salinity solution was analyzed using backscattered electron microscopy (BSE-SEM). Backscattered electron images give contrast based on atomic number and provide compositional information. Material with a greater atomic number appears brighter than lower atomic number material. X-rays are produced as a by-product of the interaction of the SEM electron beam with the atoms in the sample surface. These X-rays have energies that are characteristic of the atoms that produce them. Furthermore, the number of X-rays produced by the atoms of a particular element is related to the concentration of that element in the sample being scanned.

Table 1: Summary of Experimental Conditions.

\begin{tabular}{|c|c|c|c|c|c|}
\hline $\begin{array}{c}\text { Cores } \\
\text { t }\end{array}$ & Fired & $\begin{array}{l}\text { Sodium Chioride Consentration } \\
\text { (M) }\end{array}$ & $\mathrm{pH}$ & $\begin{array}{c}\text { Test } \\
\text { e }\end{array}$ & $\begin{array}{c}\text { Temperature } \\
\text { (e C) }\end{array}$ \\
\hline \multirow[t]{2}{*}{1} & No & 1 & 7 & 1 & 23 \\
\hline & & 0.000001 & & 2 & \\
\hline \multirow[t]{4}{*}{2} & No & 0.01 & 7 & 1 & 23 \\
\hline & & & & 2 & $\infty$ \\
\hline & & & & 3 & 120 \\
\hline & & & & 4 & 160 \\
\hline \multirow[t]{2}{*}{3} & No & 0.01 & 10 & 1 & 23 \\
\hline & & & & 2 & 80 \\
\hline \multirow[t]{3}{*}{4} & No & 005 & 10 & 1 & 23 \\
\hline & & & & 2 & 120 \\
\hline & & & & 3 & 160 \\
\hline \multirow[t]{3}{*}{5} & No & 02 & 10 & 1 & 23 \\
\hline & & & & 2 & 120 \\
\hline & & & & 3 & 160 \\
\hline \multirow[t]{3}{*}{6} & $\gamma \in \theta$ & 005 & 10 & 1 & 23 \\
\hline & & & & 2 & 120 \\
\hline & & & & 3 & 180 \\
\hline \multirow[t]{3}{*}{7} & No & 02 & 10 & 1 & 23 \\
\hline & & & & 2 & 120 \\
\hline & & & & 3 & 180 \\
\hline \multirow[t]{2}{*}{8} & No & 0.05 & 10 & 1 & 23 \\
\hline & & & & 2 & 160 \\
\hline
\end{tabular}

Figure 4 shows the composition obtained from the filtered material. Aluminum and silicon are present in large proportion indicating clays in the sample collected. The presence of potassium (K) might indicate the presence of illite (Hower, 1974). 


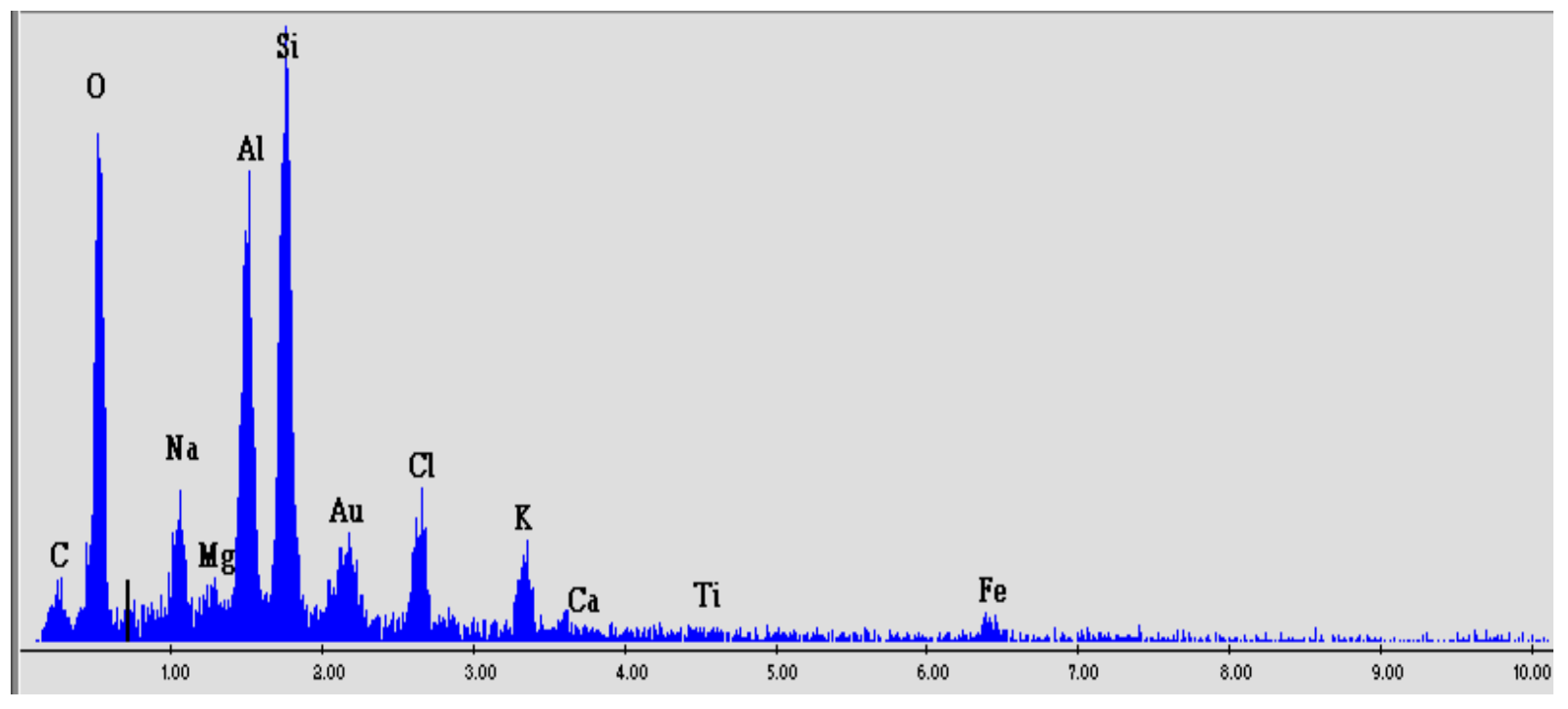

Figure 4. Compositional chromatography of effluent fines collected (low salinity injection, test \# 2 in core \#1, $\mathrm{pH}=7,0.01 \mathrm{M} \mathrm{NaCl}$ ).

The next experiments were performed at constant salinity and $\mathrm{pH}$ conditions, with changes in the temperature. Tests run in cores 2 and 3 compared two systems with identical brine concentrations but different pHs (7 and 10). Experiments in cores 3, 4, and 5 compare three systems with the same alkaline $\mathrm{pH}$, but different brine concentrations. Experiments in cores 4 and 6 compare the effects of fired core versus unfired core. Finally, further tests, performed in cores 7 and 8 , were conducted to establish repeatability. The selection of an alkaline $\mathrm{pH}$ comes from the observation that steam condensates have large $\mathrm{pH}$ typically ranging from 10 to 12 (Gunter et al., 1992).

The initial permeability for all experiments was calculated at room temperature. After stabilizing flow, temperature was increased to $80^{\circ} \mathrm{C}(353 \mathrm{~K}), 120^{\circ} \mathrm{C}(353 \mathrm{~K})$, and $180^{\circ} \mathrm{C}(453 \mathrm{~K})$, sequentially. Permeability and fines release were monitored continuously.

Figure 5 summarizes the permeability variation observed in each of the experiments performed. Open symbols indicate that the fluid collected was fine-free while filled symbols denote the presence of fines in the effluent. Identical symbols for different tests indicate tests performed at identical conditions. In all experiments, the cores that were not fired suffered reduction in the permeability as we increased the temperature. The permeability reduction might indicate the presence of swelling clays or that fine release occurs, but they do not mobilize. Nevertheless, the greatest reduction in permeability is associated with conditions that produce fines in the effluent.

Effluent collected from test \# 3 in core 2 was filtered and analyzed with the SEM technique. Figure 6 shows the texture image of a portion of the filter. In texture images, brighter objects are more distanced from the mean surface of the image. Compositional analysis of bright particles in Fig. 6 shows much silicon and aluminum among other elements. The fines produced are most likely the pore lining clays illustrated in Fig. 2(a). Figure 2(b) shows a portion of core 2 after test \# 3 was completed, the pore surface appear to be free of fines relative to Fig. 2(a). 


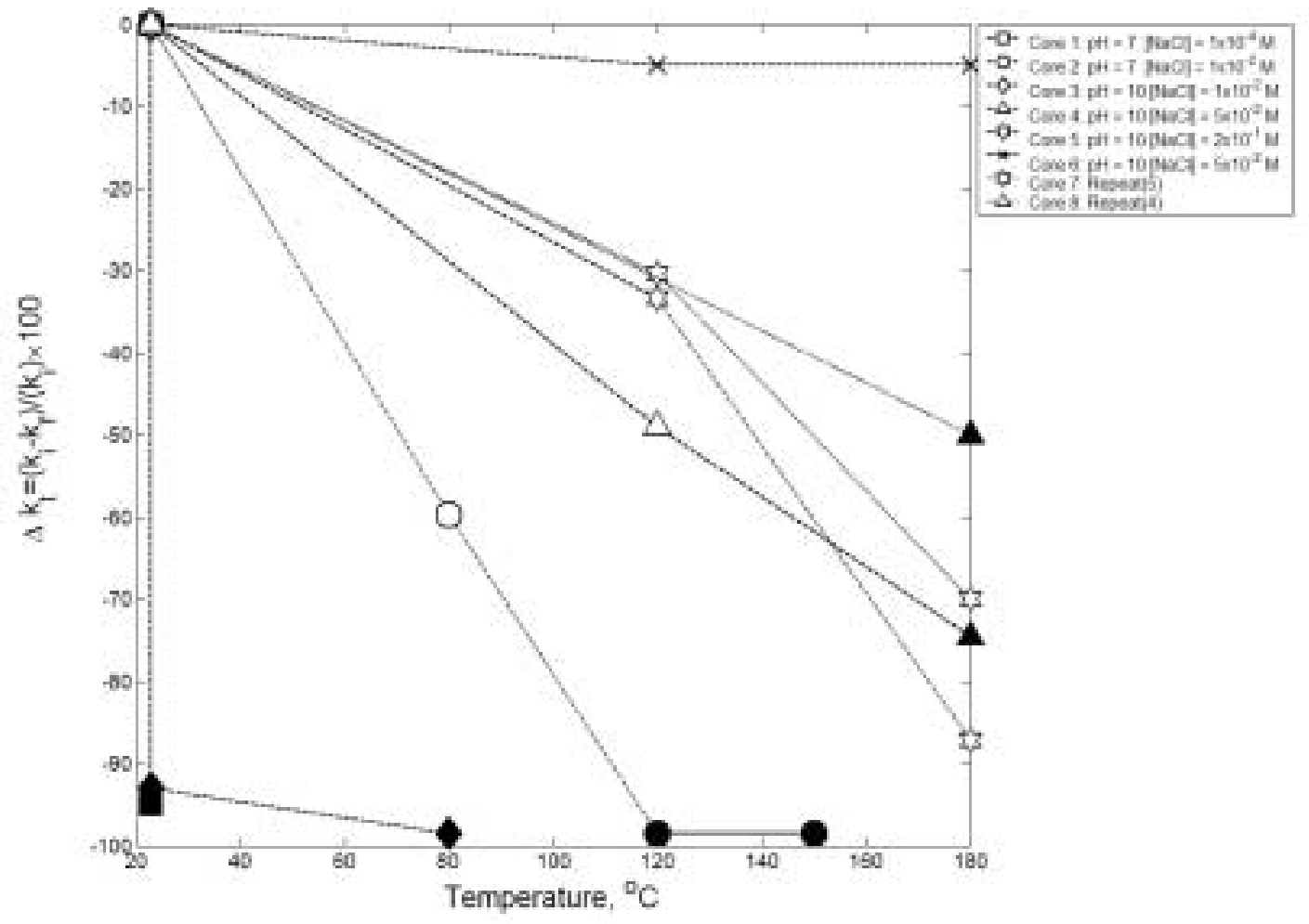

Figure 5. Permeability change during experiments. Open symbols indicate no fines in effluent, whereas closed symbols indicate fines production.

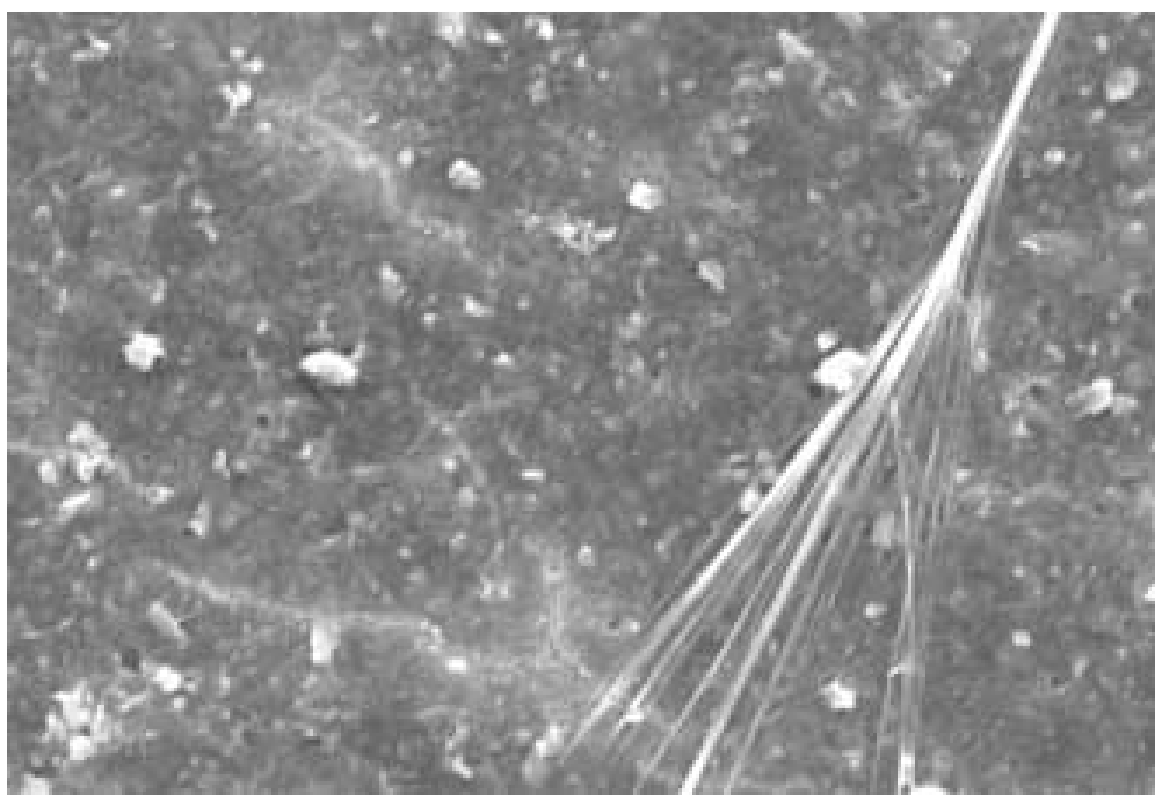

Figure 6. View of the filtrate collected during test $3\left(120^{\circ} \mathrm{C}\right)$ from core 2. Bright portions have large density. 
Experiments performed in cores 3, 4, and 5 compare the behavior of systems with alkaline $\mathrm{pH}$ for different salinity. As we increased the sodium chloride concentration, the reduction in permeability is less severe. None of the tests performed on core 5 produced fines. Hot waterflood performed on the fired core 6 shows a slight increase of permeability as we increased the temperature opposed to the behavior of the rest of experiments. No fines production was observed from the fired core. The contrast of results between tests in cores 4 and 6 confirmed that once the stabilization of clays in the rock is achieved by means of high salinity, the increase in temperature did not induce the detachment of fines. Finally, experiments performed in cores 7 and 8 repeated results and the observations made in similar tests performed on cores 5 and 4, respectively.

\section{Surface Forces}

In order to understand the relationship of the experimental variables with temperature and, predict fines detachment with increase in temperature, we invoke surface forces theory. Surface forces are generally mentioned in research concerning formation damage associated with permeability impairment due to fines migration (Bennion and Thoma, 1992; Young, 1979; Kia, 1987; Zhou et al., 1997; Vaidya and Fogler, 1990; Gunter, 1992). Surface forces are also included in studies that link wettability with fluids composition, salinity and $\mathrm{pH}$ (Kovscek et al., 1993; Melrose, 1982; Takamura and Chow, 1983; Anderson, 1986; Churaev, 1988;Buckley et al., 1997; Hirasaki, 1991; Buckley et al., 1996). The focus of these investigations is primarily between surfaces forces and properties such as composition, salinity, and $\mathrm{pH}$. Temperature is seldom mentioned.

Forces acting between two charged colloidal particles in an electrolyte solution are either attractive $\left(\mathrm{F}_{\mathrm{A}}\right)$ or repulsive $\left(\mathrm{F}_{\mathrm{R}}\right)$. In principle, the stability of colloidal systems is explained by the balance of these two interactions. The net interaction is either repulsive or attractive. This idea is referred to as DLVO (Derjaguin, Landau, Verway, and Overbeek) theory. It is assumed that colloidal particles are very large compared with electrolyte ions. This makes it possible to express the interaction forces between particles, $\mathrm{F}_{\mathrm{T}}$, as a function of the interparticle distance, $\mathrm{h}$.

$$
F_{T}(h)=F_{A}(h)+F_{R}(h)
$$

The balance between the forces affecting a clay particle in a reservoir determines the stability or detachment of the clay particles.

The analysis assumes that a single phase wetting fluid is in contact with the pore surface and fines, and it models the system of a fine attached to a pore wall as a simple geometrical object (sphere, rod, plate), attached to a flat surface, as shown in Figure 7. The interaction between a clay particle and a pore surface is described by the following equation,

$$
V_{t}(h)=V_{L V A}(h)+V_{D L R}(h)+V_{B R}(h)+V_{H R}(h)+V_{I H L}(h)
$$


where $\mathrm{V}$ is the potential and subscripts represent: $\mathrm{t}$, total; LVA, the London Van Der Waals attraction; DLR, the double layer repulsion; BR, the Born repulsion, HR, the hydrodynamic repulsion; and IHL, interfacial hydrate layer. The resulting potential of a clay particle is repulsive (unstable) if $\mathrm{V}_{\mathrm{t}}$ is positive and, attractive (stable) if it is negative. The different composites of this equation and their computation are discussed next.

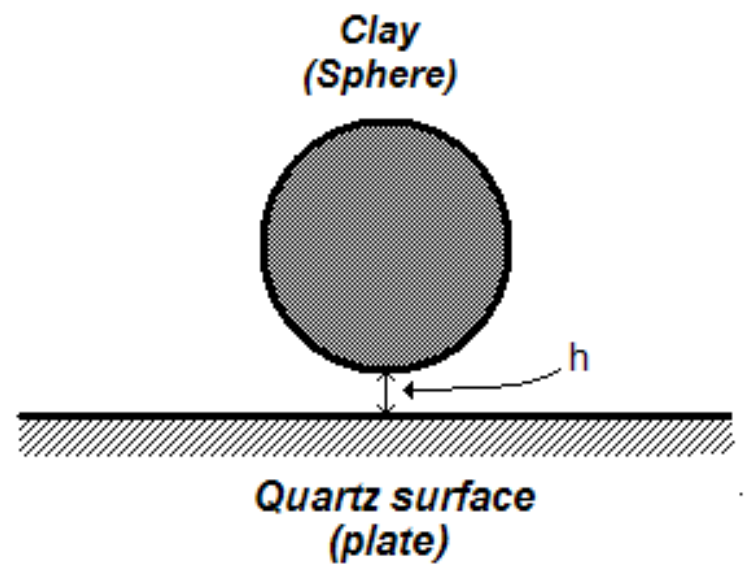

Figure 7. Sphere-plate system for modeling fines stability.

The London-Van der Waals attractive energy, $\mathrm{V}_{\mathrm{LVA}}(\mathrm{h})$, acts between interacting bodies over distances as large as $100 \AA$. Their magnitudes are comparable to that of the electrical double layer. In our system, we neglect the effects due to retardation of electromagnetic waves because the ratio of the distance of separation, $h$, to the particle fines radius, $r_{p}$, is much less than unity. Thus, the London Van-der Waals potential is estimated numerically by the expression for unretarded interaction (Hamaker, 1937),

$$
V_{L V A}(h)=-\frac{A}{6}\left\lfloor\frac{2(H+1)}{H(H+2)}+\ln \left(\frac{H}{H+2}\right)\right\rfloor
$$

where, $\mathrm{A}$ is the Hamaker constant, and $\mathrm{H}$ is the ratio of the distance of separation to particle radius, $\mathrm{H}=\mathrm{h} / \mathrm{r}_{\mathrm{p}}$. In order to proceed, a particle radius of $1 \mu \mathrm{m}$ is chosen consistent with the SEM image in Fig. 2. The attractive van der Waals forces are independent of changes in solution chemistry. The Hamaker constant characterizes the dispersion interaction between the fines and the pore walls separated by a film of wetting fluid. We estimated this constant using the known binary values for kaolinite-water-kaolinite, $\mathrm{A}_{\mathrm{kwk}}$, and silica-water-silica, $\mathrm{A}_{\mathrm{sws}}$ :

$$
\mathrm{A}_{\mathrm{swk}}=\sqrt{\mathrm{A}_{\mathrm{sws}} \mathrm{A}_{\mathrm{kwk}}}
$$

The value of the Hamaker constant estimated for the system silica-water-kaolinite is $2.26 \mathrm{E}-20 \mathrm{~J}$. This is close to the value used by Kia et al. (1987), 2.6E-20 J. We assume the Hamaker constant to be independent of the temperature. Studies of the effect of temperature on the Hamaker 
constant for ceramic systems report a change of $3 \%$ over the range $27-200{ }^{\circ} \mathrm{C}$ (French 2000), this change is very small considering the changes in the other terms in Eq. 1.

The double layer repulsion term, $\mathrm{V}_{\mathrm{DLR}}(\mathrm{h})$, is calculated assuming a constant charge condition as

$$
V_{D L R}(h)=\frac{\varepsilon r_{p}}{4}\left(\Phi_{01}^{2}+\Phi_{02}^{2}\right)\left[\left(\frac{2 \Phi_{01} \Phi_{02}}{\Phi_{01}^{2}+\Phi_{02}^{2}}\right) \ln \left(\frac{1+\exp (-\kappa h)}{1-\exp (-\kappa h)}\right)-\ln (1-\exp (-2 \kappa h)\rfloor\right.
$$

where $\kappa$ is the Debye-Huckel reciprocal length, $\varepsilon$ is the dielectric constant of the medium, and $\Phi_{\mathrm{oi}}$ is the surface potential of the wall $(\mathrm{i}=1)$ and particle $(\mathrm{i}=2)$. Surface potential cannot be measured directly but they are approximated by zeta potentials derived from measurements (e.g, electrophoretic mobility).

The Debye-Huckel reciprocal length term is given by

$$
\kappa=\sqrt{\frac{2 \mathrm{e}^{2} \mathrm{n}_{\mathrm{b}}}{\varepsilon k \mathrm{~T}}}
$$

Where $\mathrm{n}_{\mathrm{b}}$ is the total ion density in bulk. The surface potential of each surface, $\Phi_{\mathrm{o}}$, is approximated by the zeta-potential $\zeta_{\mathrm{i}}$, that is the electrical potential at the boundary between the

Stern layer and the diffuse layer. The zeta potential is approximated by the Nernst equation,

$$
\zeta_{i}=\Phi_{o_{i}}=-2.3\left(\frac{k T}{e}\right)\left(p H-p H_{o_{i}}\right)
$$

Where $\mathrm{pH}_{\mathrm{oi}}$ is the isoelectric point of surface $\mathrm{i}$.

The Born repulsion term, $\mathrm{V}_{\mathrm{BR}}(\mathrm{h})$, measures the short-range molecular interaction resulting from the overlap of electron clouds. It is suggested to follow the relationship ${ }^{39}$

$$
V_{B R}=\frac{A \delta^{6}}{7560}\left\lfloor\frac{8 r_{p}+h}{\left(2 r_{p}+h\right)^{7}}+\frac{6 r_{p}-h}{h^{7}}\right\rfloor
$$

Where $\delta$ is the collision diameter. It is assigned a value of approximately $5 \AA^{40}$. Finally, $\mathrm{V}_{\mathrm{HR}}(\mathrm{h})$ is the hydrodynamic potential and, $\mathrm{V}_{\mathrm{IHL}}(\mathrm{h})$ is the interfacial hydrate layer. The effects of these two terms are considered relatively small and can be neglected. Hydrodynamic forces were estimated for a similar system with flow rates an order of magnitude greater than those used in our experiments. Hydrodynamic forces remain negligible compared to the London-Van der Waals attractive forces (Khilar and Fogler, 1984). 
Figure 8 illustrates the three main components of a clay-quartz system at room temperature, $\mathrm{pH}$ of 7 , and brine concentration of $0.1 \mathrm{M}$. In this particular case, the resulting total potential is negative and translates into a stable (attractive) system. In order to study the joint effect of temperature, fluid salinity and $\mathrm{pH}$, we computed a series of such force versus distance curves. As temperature increases, the minimum in $\mathrm{V}_{\mathrm{t}}$ increases approaching the crossover from attractive to repulsive.

\section{Results and Discussion}

We define the detachment temperature of a fine as the temperature where the resulting potential, $\mathrm{V}_{\mathrm{t}}$, switches from attractive to repulsive for a given salinity and $\mathrm{pH}$. In the next section, we present the resulting potential for the sphere-plate system for various conditions in quartz-clay systems. Quartz is the most abundant fraction by weight in sandstone and is present largely in the form of sand grains with a lesser fraction present as fines. Clay minerals such as kaolinite and illite are non-swelling clays commonly found in Berea sandstone (Conway et al., 2000) and hydrocarbon producing formations (Hower, 1974; Scheuerman and Bergersen, 1990). As a final point, we assume that the clay surface charge is homogeneously distributed over the sphere.

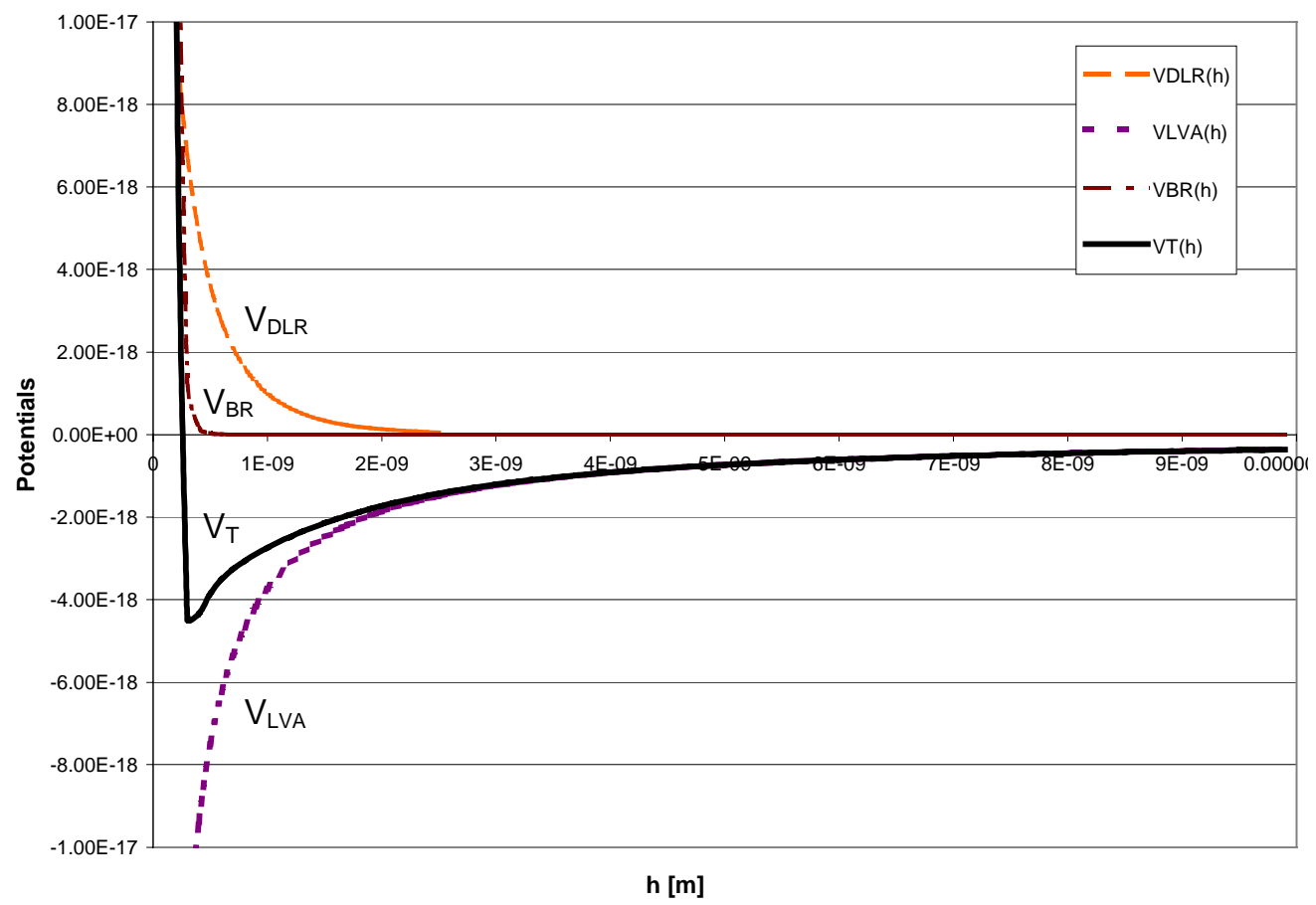

Figure 8. Components of sphere-plate interaction and total potential at $\mathrm{pH}=7$ and $[\mathrm{Na}+]=0.1 \mathrm{M}$. 


\section{Quartz-Kaolinite System}

Kaolinite is a common hydrous alumina silicate. Various zeta potential measurements are found in the literature (Lorenz, 1969; Williams and Williams, 1978,; Ramachandran and Somasundaran, 1986). We adjust the zeta potentials predicted by Eq. 8 to published values for kaolinite using regression. Figure 9 shows zeta potential as a function of $\mathrm{pH}$ at different temperatures, for (a) quartz and, (b) kaolinite correspondingly. The points indicate zeta potential reported (Ramachandran and Somasundaran, 1986) while lines represent the fit curve used in our model. Figure 10 shows zeta potential as a function of $\mathrm{pH}$ for quartz and kaolinite, this time at a constant temperature and various salinity values. We define the change of zeta potential as a function of temperature, by fitting values of zeta potential for different temperatures (Ramachandran and Somasundaran, 1986). Figure 11 compares the change of zeta potential with respect to temperature for quartz and kaolinite, by fixing a reference value at room temperature. The values for alumina (Pagneoux et al., 1998), silica (Dai and Chung, 1995), and bitumen (Dai and Chung, 1995) are shown for comparison. According to this plot, all materials follow a similar behavior with increasing temperature: the zeta potential becomes more negative as the temperature increases. This observation agrees with other studies (Dai and Chung, 1995).
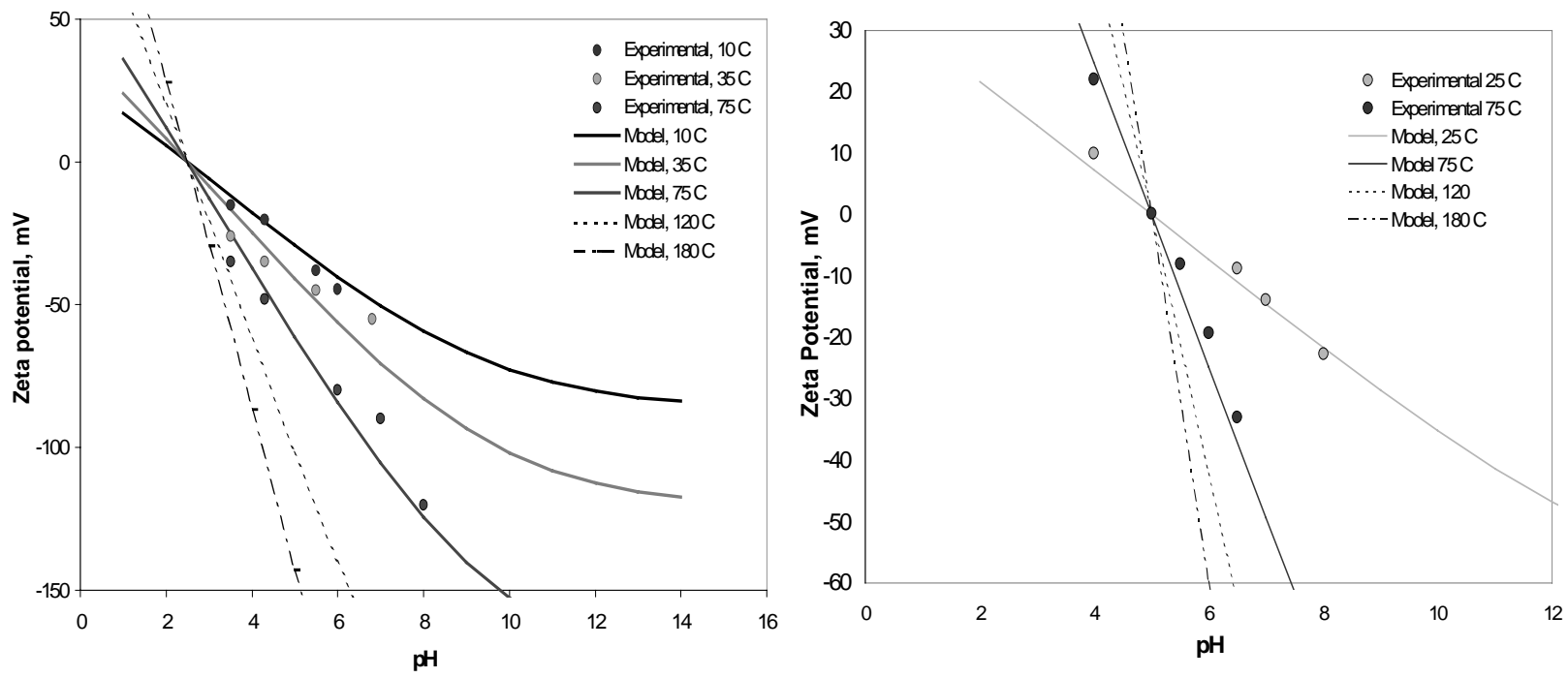

Figure 9. Experimental and calculated zeta potential of as a function of $\mathrm{pH}$ at constant salinity: (a) Quartz, 1E-3M NaNO3, (b) Kaolinite, E-4M NaCl.

The zeta potentials are used in the calculation of the total potential according to Eq. 2 . The total potential tends toward repulsive as the temperature increases. Repeated calculations of the total potential for different fluid $\mathrm{pH}$ and sodium chloride concentration allows prediction of the temperature at which the force between sphere (clay) and plate (grain surface) is no longer attractive. Figure 10 shows contour maps of detachment temperatures for a kaolinite-quartz system according to the experimental $\zeta$-potential curve reported by Lorenz (1969) from Fig. 9. 


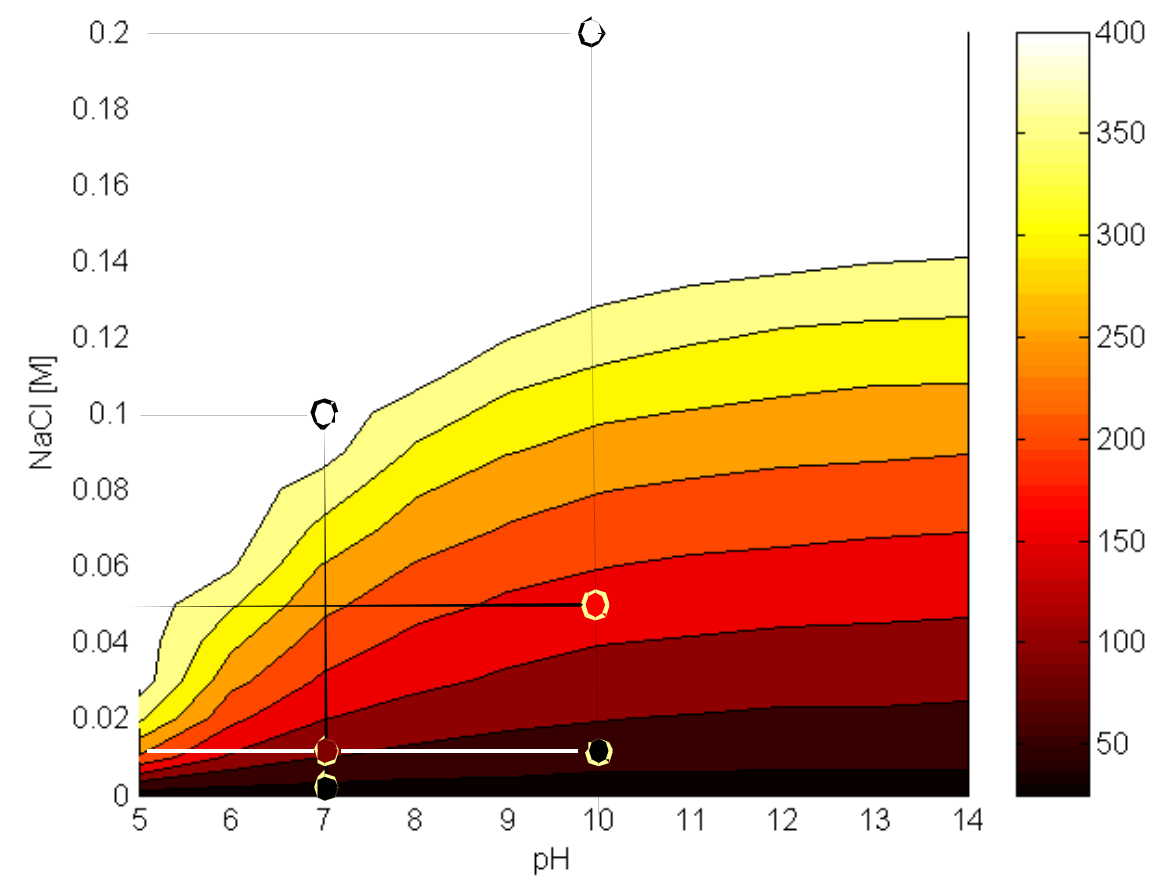

Figure 10. Detachment temperature obtained for quartz-kaolinite systems with $\zeta$-potential measurements provided by Lorenz. Shading is in degree Celsius $\left({ }^{\circ} \mathrm{C}\right)$.

The sodium chloride concentrations (salinity) ranges between 0.001 and $0.2 \mathrm{M}$ (100 and $12000 \mathrm{ppm}$ ) and $\mathrm{pH}$ values from 5 to 14 . For low salinity and high $\mathrm{pH}$, the resulting potential at room temperature is mostly repulsive indicating fines release. As we increase $\mathrm{pH}$ and decrease salinity, the detachment temperature decreases. For instance, the system with $\mathrm{pH} 7$ and salinity of $0.01 \mathrm{M}$ shows a detachment temperature of approximately $150{ }^{\circ} \mathrm{C}$ while the system with $\mathrm{pH} 10$ shows a detachment of temperature of only $50^{\circ} \mathrm{C}$. This is expected because the zeta potential becomes more negative with increasing $\mathrm{pH}$. As a consequence, the force between the clay and surface becomes repulsive at temperatures only slightly greater than room temperature.

We constructed and compared contour maps for the other two data sets of zeta values (Williams and Williams, 1978; Ramachandran and Somasundaran, 1986). The zeta potentials differ not only with respect to the range of values covered, but also in the isoelectric points. As expected, the sensitivity of the system to the bulk conditions depends on the zeta values used in the calculation. Nevertheless, all calculations predict considerable sensitivity to temperature. Zeta potentials reported by Lorenz (1969) lead to a greater temperature sensitivity as compared to the zeta potentials reported by Williams and Williams (1978) and Ramachandran and Somasundaran (1986). The position of the isoelectric point contributes to the sensitivity to temperature, as well. As the isoelectric point shifts toward the origin, the clay surface becomes negatively charged at a lower $\mathrm{pH}$. We considered the isoelectric point to be fixed in all calculations; however, the literature suggests that the isoelectric point shifts to lower $\mathrm{pH}$ as the temperature increases (Dai and Chung, 1995). Such behavior increases the sensitivity to temperature, in some cases. 
Figure 10 shows contour maps for kaolinite-quartz sytems assuming that fine clays have cylindrical shapes. We use the $\zeta$-potential provided by Lorenz (1969) similar to the calculations shown in Fig 12. The calculations of the double layer repulsive forces for the cylinder-plate system used the linear superposition approximation (LSA)(Mahmood et al., 2001, Gregory, 1975). The approximation yields results intermediate between constant charge and constant potential assumptions (Gregory, 1975). Cylinder-plate calculations predict a system more sensitive to temperature than the sphere-plate configuration. For comparison, we also performed calculations for plate-plate systems. The cylinder-plate and plate-plate systems showed roughly the same sensitivity to an increase in temperature.

Figure 10 also compares results obtained experimentally to the theoretical calculations described in this section. Circles indicate that $\mathrm{pH}$ and salinity of an experiment whereas the shading of the circle reports the temperatue at which fines were produced with the effluent. Results are mostly in agreement with calculations assuming sphere-plate systems and the $\zeta$ potential provided by Lorenz (1969). Overall, the temperature of fines production is well approximated by DLVO theory.

\section{Quartz-Illite System}

Illite, a potassium aluminum silicate hydroxide hydrate mineral of the clay group, is another common clay present in hydrocarbon reservoirs. It is formed during the alteration of silicate minerals such as mica and feldspar. Illite is also known as clay mica.

We repeated the calculations applied to the quartz-kaolinite system. The zeta potentials for illite (Horikawa, 1988) are more negative than those for kaolinite. Assuming that illite follows the same reduction in $\zeta$-potential versus temperature function as kaolinite, we calculate the detachment temperatures. Figure 11 shows the contour map of the detachment temperature for an illite-quartz system. The sodium chloride concentrations (salinity) range between 0.0 and $5 \mathrm{M}$ (100 and 250,000 ppm) and $\mathrm{pH}$ values between 5 and 14 . The quartz-illite system is more sensitive to changes in temperature. Thus, it is more prone to detachment than the quartzkaolinite system. The fact that the zeta potentials are more negative results in greater repulsive forces. The isotherms are almost horizontal indicating that the resulting potential shows the same behavior for the range of $\mathrm{pH}$ at a given salinity. 


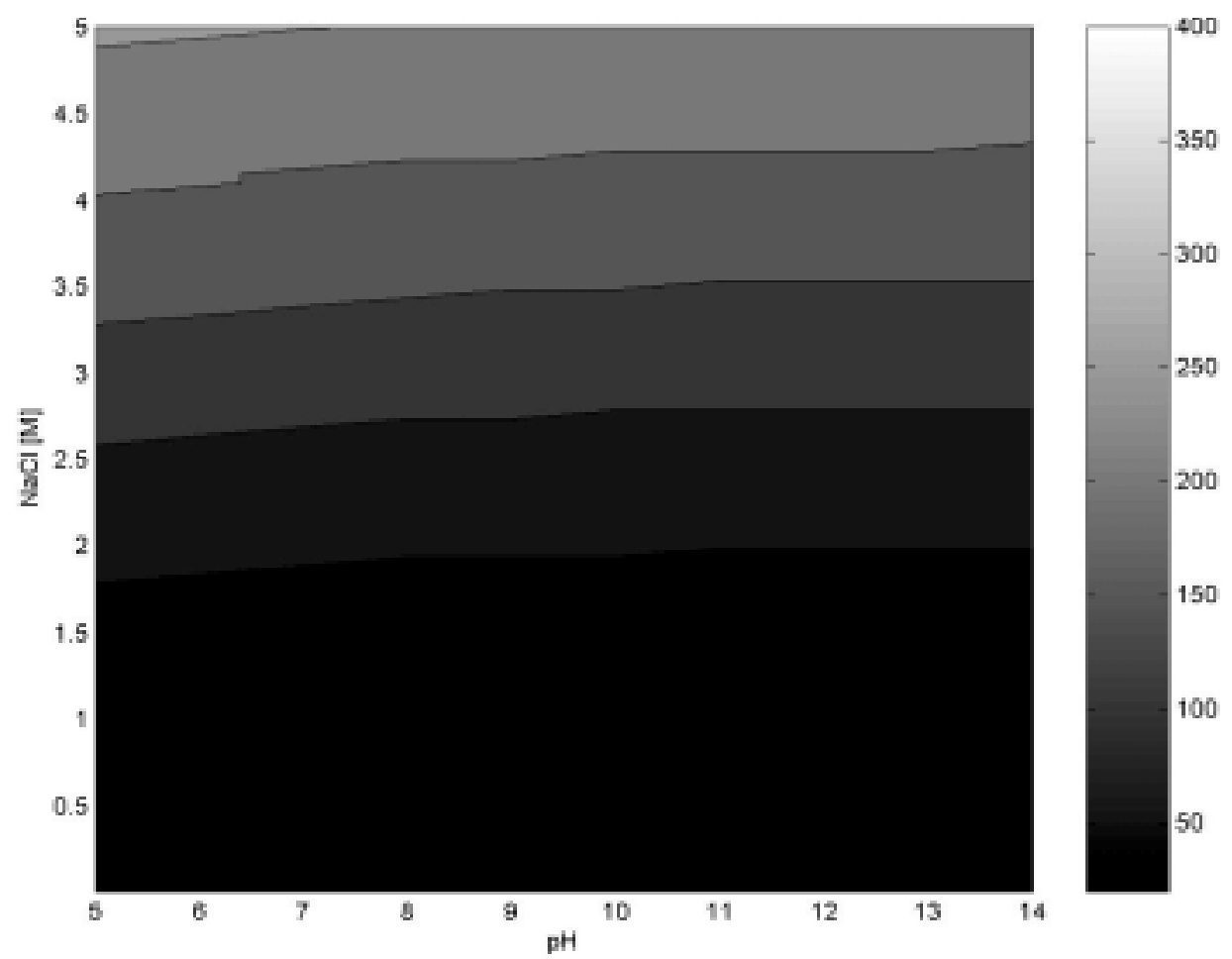

Figure 11. Detachment temperature obtained for quartz-illite system. Shading is in degree Celsius $\left({ }^{\circ} \mathrm{C}\right)$.

\section{Conclusions}

The interaction between fluid and surface forces is a dominant factor in wettability and formation damage. Interactions depend on factors including mineralogy of the porous medium, concentration and chemical composition of the fluids, $\mathrm{pH}$, and temperature of the system. Temperature is very little studied in the surface forces literature in relation to fines mobilization. In this work, we develop an understanding of fines mobilization through experiments and the role played by surface forces. Results confirm the decisive role of the interplay of temperature, $\mathrm{pH}$, salinity, and mineralogy on these phenomena. The value of the isoelectric point varies significantly with the surface mineralogy. This variation is responsible for the sensitivity that fines show to the change of fluid and temperature conditions. The results presented here taught that temperature increase allows fine release under conditions of high $\mathrm{pH}$ and moderate salinity. Fines detachment changes the nature of fluid-solid interactions in porous media supporting our hypothesis that fines mobilization is a mechanism for porous media to shift towards water wetness during thermal recovery operations. 


\section{References}

Anderson, W. G: "Wettability, Survey-Part 1: Rock/Oil/Brine Interacitons and the Effect of Core Handling on Wettability," SPE 13392. Journal of Petroleum Technology, October 1986. Pp 1125-1144.

Al-Hadhrami, H. S. and M. J. Blunt, "Thermally Induced Wettability Alteration to Improve Oil Recovery in Fractured Reservoirs," SPE Reservoir Evaluation and Engineering, June 2001, pp 179-186.

Bennion, D. B. and F. B. Thomas: "Formation damage Due to Mineral Alteration and Wettability Changes During Hot Water and Steam Injection in Clay-Bearing Sandstone Reservoirs," SPE paper 23783, proceedings of the SPE International Symposium on Formation Damage Control, held in Lafayette, Louisiana, February 26-2. 1992.

Buckley, J.S., K. Takamura and N.R. Morrow: "Influence of Electrical Surface Charges on the Wetting Properties of Crude Oil," SPE 16964. Proceedings of the 62nd SPE Annual Technical Conference and Exhibition, Dallas, TX. September 27-30, 1997.

Buckley, J. S.: "Asphaltene precipitation and Crude Oil Wetting," SPE Advanced Technology Series, Vol. 3. No. 1. pp 53-59.

Buckley, J. S., C Bosseau, and Yu Liu: "Wetting alteration by Brine and Crude Oil: From Contact angles to Cores," SPE 30765. SPE Journal, September 1996. pp 341-350.

Churaev, N. V. "Wetting Films and Wetting," Revue de Physique Appliquée. 23 (1988) No 6. pp 975-987.

Conway, M.W., R. E. Himes and R. Gray: "Minimizing Clay Sensitivity to Fresh Water Following Brine Influx," SPE 58748. Proceedings of the 2000 SPE International Symposium on Formation Damage Control held in Lafayette, LA, 23-24 February 2000.

Dai, Q and K. H. Chung: "Bitumen-sand Interaction in Oil in Sand Processing," Fuel. Vol 74. No. 12, pp. 1858-1864, 1995.

Diabira, I., L. M. Castanier, and A. R. Kovscek, "Porosity and Permeability Evolution Accompanying Hot Fluid Injection Into Diatomite," Petroleum Science and Technology, 19 (9\&10), 1167-1185 (2001).

DiCarlo, D. A., A. Sahni, and M. J. Blunt, "The effect of Wettabilility on Three-Phase Relative Permeability," SPE paper 49317, proceedings of the SPE Annual Technical Conference and Exhibition, held in New Orleans, Louisiana, 27-30 September 1998.

Edmonson, T. A.: "Effect of Temperature on Waterflooding," J. Cdn. Pet. Tech (Oct-Dec 1965) 236-42. 
French, R. H.: "Origins and Applications of London Dispersion Forces and Hamaker Constants in Ceramics,” J. Am. Ceram. Soc., 83 [9] 2117-46 (2000).

Gunter, W. D., Z. Zhou, and E. H. Perkins: "Modeling Formation Damage Caused by Kaolinite from 25 to 300 Centigrade In the Oil Sand Reservoirs of Alberta," SPE 23786. Proceedings of the SPE Intl. Symposium on Formation Damage Control, Louisiana, February 26-27, 1992.

Gregory, J.: "Interaction of Unequal Double Layers at Constant Charge," Journal of Colloid and Interface Science, Vol. 51, No. 1, April 1975.

Hamaker, H.C.: “The London-Van der Waals Attraction between Spherical Particles," Physics, 4, 1058 (1937).

Hirazaki, G. J.: “Wettability: Fundamentals and Surface Forces,” SPE 17367. SPE Formation Evaluation, June 1991, pp 217-226.

Horikawa, Y., R. S. Murray and J. P Quirk: "The Effect of Electrolyte Concentration on the Zeta Potentials of Homoionic Montmorillonite and Illite," Colloids and Surfaces, 32 (1988) pp. 181-195

Hower, W.F.: "Influence of Clays on the Production of Hydrocarbons," SPE paper 4785. Proceedings of the AIME Symposium on Formation Damage Control, held in New Orleans, LA, Feb. 7-8, 1974.

Khilar K.C. and H. S. Fogler: "Colloidal Induced Fine migration in porous media, Reviews in Chemical Engineering, n. 4, 1987, pp 41-108.

Khilar, K. C. and H. S. Fogler: "The existence of a Critical Salt Concentration for Particle Release," Journal of Colloid and Interface Science, Vol. 101, No. 1, September 1984.

Kia, S. F.: "Effect of Salt Composition on Clay Release in Berea Sandstone," SPE paper 16524. Proceedings of the SPE International Symposium on Oilfield Chemistry. San Antonio, Texas, February 4-6, 1987.

Kovscek, A. R., H. Wong and C. J. Radke: "A pore-Level Scenario for the Development of Mixed Wettability in Oil Reservoirs," Environmental and Energy Engineering, AIChE Journal, June 1993, Vol. 39, No. 6. pp 1072-1085.

Lorenz, P B. , Clays Clay Miner., 17 (1969) 223-251.

Mahmood, T., A. Amirtharajah, T. W. Sturm and K. E. Dennett: "A Micromechanics Approach for Attachment and Detachment of asymmetric Colloidal Particles," Colloids and Surfaces A: Physicochemical and Engineering Aspects 177 (2001) pp. 99-110. 
Melrose, J.C.: "Interpretation of Mixed Wettability States in Reservoir Rocks," SPE paper 10971, proceedings of the 57th SPE Annual Fall Technical Conference and Exhibition, New Orleans, LA, September 26-29, 1982.

Pagneoux, C., M. Serantoni, R. Laucournet, T. Chartier, and J. F. Baumard: "Influence of the Temperature on the Stability on Aqueous Alumina Suspension." J. Am. Ceram. Soc., 1998. pp 1935 - 1948.

Poston, S. W, S. Ysrael, A. K. M. S. Hossain, E. F. Montgomery and H. J. Ramey, "The effect of Temperature on Irreducible Water Saturation and relative Permeability of Unconsolidated Sands," SPE paper 1897, proceedings of the SPE 42nd Annual Fall Meeting, held in Houston, Oct 1-4, 1967.

Ramachandran, R. and P. Somasundaran: "Effect of Temperature on the Interfacial Properties of Silicates," Colloids and Surfaces, 21 (1986) 355-369.

Scheuerman, R. F. and B.M. Bergersen: "Injection-Water Salinity, Formation Pretreatment and Well-Operations Fluid-Selection Guidelines," SPE paper 18461. Journal of Petroleum Technology, July 1990. pp 836-845.

Sinnokrot, A. A., H.J. Ramey and S.S. Marsden: "Effect of Temperature Level upon Capillary Pressure Curves," SPE 2517. Proceedings of the SPE 44th Annual Fall meeting, Denver, Colorado (Sept 28-Oct. 1, 1969).

Takamura, K. and R. S. Chow: "A mechanism for Initiation of Bitumen displacement from Oil Sand," Journal of Canadian Petroleum Technology, Nov-Dec 1983, pp 22-30.

Tang, G. and A. R. Kovscek, "Wettability Alteration of Diatomite Induced by Hot-Fluid Injection," SPE paper 77461, the proceedings of 2002 Annual Technical Conference and Exhibition, held on 29 September to 2 October, 2002 in San Antonio, TX

Tang, G. and N. R. Morrow, "Influence of brine composition and fines migration on crude oil/brine/rock interactions and oil recovery," Journal of Petroleum Science and Engineering 24 (1999) 99-111.

Tang, G. and A. R. Kovscek, "Experimental Study of Heavy Oil Production from Diatomite by Water Imbibition at Elevated Temperatures," Proceedings of the SPE/DOE Thirteen Symposium on Improved Oil Recovery held in Tulsa, Oklahoma, 13-17 April 2002.

Tchistiakov, A.A., "Colloid Chemistry of In-Situ Clay-Induced Formation Damage," SPE paper 58747, proceedings of the 2000 SPE International Symposium on Formation Damage Control held in Lafayette, Louisiana, 23-24 February 2000.

Vaidya, R.N. and H.S. Fogler: "Fine Migration and Formation Damage:Influence of pH and ion Exhange," SPE paper 19413, proceedings of the SPE Formation Damage Control Symposium, Lafayette, Louisiana, February 22-23, 1990. 
Williams, D. J. A. and K. P. Williams: “Electrophoresis and Zeta Potential of Kaolinite,", Journal of Colloids and Interface Sciences, Vol 65 (1978) 79-87

Windholz, ed., The Merck Index: An Encyclopedia of Chemicals, Drugs, and Biologicals, Thirteenth Edition, Merck \& Co., Inc., Whitehouse Station, NJ, USA, 2001.

Young, B. M.: "Clay Stabilization Agents - Their Effectiveness in High Temperature Steam," SPE paper 7895, proceedings of the 1979 SPE of AIME Intl. Symposium on Oilfield and Geothermal Chemistry held in Houston, Texas, January 22-24, 1979.

Zhou, Z., B. Wiwchar, W. D. Gunter and, J.S. Dudley: "The Potential of Permeability Damage during the Thermal Recovery of Cold Lake Bitumen," Paper 97-102. Presented at the 48th Annual Technical Meeting of the Petroleum Society in Calgary, Alberta, Canada, June 8-22, 1997. 


\section{Area 2. Hot-Fluid Injection}

Steam injection is the most common form of enhanced oil recovery (Moridis, 2002). Heat in the form of steam is commonly injected into reservoirs containing heavy (cf. Prats, 1982) and medium (cf. Kovscek et al., 1997) gravity oils to reduce oil viscosity, cause volumetric expansion of oil, and thereby improve production. There were two primary thrusts to our efforts in the area of hot-fluid injection: (a) nonconventional wells for thermal oil recovery, (b) streamdrive in low permeability, fractured porous media. Task $2 \mathrm{a}$ consists entirely of modeling whereas Task $2 \mathrm{~b}$ has experimental and simulation components.

A combination of analytical and numerical studies was used to examine the efficiency of reservoir heating using nonconventional wells such as horizontal and multilateral wells. These types of wells contact much more reservoir volume than conventional vertical wells and provide great opportunity for improved distribution of heat. Through simulation and analytical modeling of the early-time response of a reservoir to heating with a horizontal, it is shown how gravity forces can be used to their maximum extent while minimizing the role of viscosity.

The motivation for examining steamdrive as a recovery option for fractured porous media is that field tests have demonstrated heat injected in the form of steam is effective at unlocking oil from such media. It was not clear, however, the manner in which the basic mechanisms differ from conventional steamdrive and how such differences could be used to advantage. Using simulations that account for details of heat transfer, capillarity, and fluid exchange in one, two, and three dimensions, the importance of factors such as the wettability of the matrix and oil composition are quantified. Experimentally, we have examined the speed and extent to which steam injection alters the permeabillity of low permeability rocks during thermal recovery. Increases in permeability due to rock dissolution aid injection and heat delivery to the rock, but downstream permeability reduction might limit applicability. We have also measured the improvement in recovery associated with the imbibition of hot water into porous media that are not initially strong water wet.

Discussion of Area 2a includes a summary of our work on single-well steamassisted gravity drainage entitled "A Numerical Analysis of the Single-Well SteamAssisted Gravity Drainage Process," and a more lengthy section entitled, "Analytical Model for Cyclic Steam Stimulation of a Horizontal Well."

Area $2 b$ first shows our results on heat transfer and oil recovery mechanisms in fractured, low permeability media. We take a pattern simulation approach and deconstruct the various mechanisms of oil production. A summary entitled "Efficiency and Oil Recovery Mechanism of Steam Injection into Low Permeability, Hydraulically Fractured Reservoirs" is presented. Then a longer report, "Steamdrive Stability in High Porosity Rock" is given. Then Task $2 b$ turns to experimental components. A brief discussion of permeability alteration during hot fluid injection is presented before turning to "An Experimental Investigation of the Effect of Temperature on Recovery of Heavy Oil From Diatomite." 


\section{References}

Kovscek, A. R., R. M. Johnston, and T. W. Patzek, "Evaluation of Rock/Fracture Interactions During Steam Injection Through Vertical Hydraulic Fractures," Society of Petroleum Engineers Production and Facilities, 12(2),. 100-105 (1997).

Prats, M.: "Thermal Recovery", Monograph - Volume 7, SPE of AIME, Henry L. Doherty Memorial Fund of AIME, 1982. 


\title{
A Numerical Analysis of the Single-Well Steam-Assisted Gravity Drainage Process
}

\author{
(Task 2a)
}

\section{Introduction}

Steam assisted gravity drainage (SAGD) is an effective method to produce heavy oil and bitumen. In a typical SAGD approach, steam is injected into a horizontal well located directly above a horizontal producer helping to displace heated oil. Single-well (SW) SAGD attempts to create a similar process using only one horizontal well. SAGD maximizes the role of gravity forces during steam flooding of heavy oils. As steam enters the reservoir, it heats the reservoir fluids and surrounding rock. Hot oil and condensed water drain through the force of gravity to a production well at the bottom of the formation. In conventional SAGD, steam is injected through a horizontal well placed directly above a horizontal producer. Thus, a steam chamber forms around the injection well. In single-well (SW) SAGD, a horizontal well is completed such that it assumes the role of both injector and producer (Falk et al., 1996). In a typical case, steam is injected at the toe of the well, while hot reservoir fluids are produced at the heel of the well. Recovery mechanisms are envisioned to be similar to conventional SAGD. Advantages of SWSAGD might include cost savings in drilling and completion and utility in relatively thin reservoirs where it is not possible to drill two vertically spaced horizontal wells. However, the process is technically challenging.

In a reservoir where cold oil is very viscous and will not flow easily, initial production rates via any gravity drainage process are very small. In a strict definition of SAGD, steam only enters the reservoir to fill void space left by produced oil. If the oil is cold, we must heat it to reduce viscosity. Therefore, initial heating of the area around the wellbore is required so that SAGD can take place. Efficient initial heating was the subject of this piece of research.

This work was grouped into two general topics: (i) a screening study of early time operating performance of SW-SAGD and (ii) a sensitivity analysis of the effect of reservoir and well completion parameters. Understanding the operating conditions to improve initial performance relates directly to understanding methods of heating the near-wellbore area at earlytime. A central idea realized here is that the near-well region must be heated rapidly and efficiently for significant early-time response. The sensitivity analysis helps us understand reservoir properties, fluid conditions, and well completion strategies where the process might be an appropriate production technique. This research has appeared as a report (TR 124) and as a journal publication (Elliot and Kovscek, 2001). Accordingly, the discussion is somewhat abbreviated.

\section{Model Description}

The thermal reservoir simulator STARS (CMG, 1998) is used to simulate various scenarios. The grid system and dimensions are illustrated in Fig. 1. Figure 1a displays the cross section along the length of the well and Fig. $1 \mathrm{~b}$ a cross section perpendicular to the well. The grid system is Cartesian with local grid refinement, as detailed in Fig. 1, immediately around the 
$800 \mathrm{~m}$ long well. An element of symmetry, with one boundary lying along the wellbore, is used to represent the reservoir volume. We assume that wells are developed in multiple patterns and thus all boundaries are no flux. The well spacing is $160 \mathrm{~m}$. The single horizontal well is represented using a discretized wellbore model that accurately represents fluid and heat transfer in the well (CMG, 1998). Additionally, the well is broken into two sections to simulate the placement of a packer. Each section is equal in length and they lie directly end to end. This gives us freedom to explore various completion strategies and operating conditions. Our initial simulation work suggested that this inject at the toe and produce at the heel strategy had greater probability of success than attempting to develop countercurrent steam and oil flow along the entire length of the well.

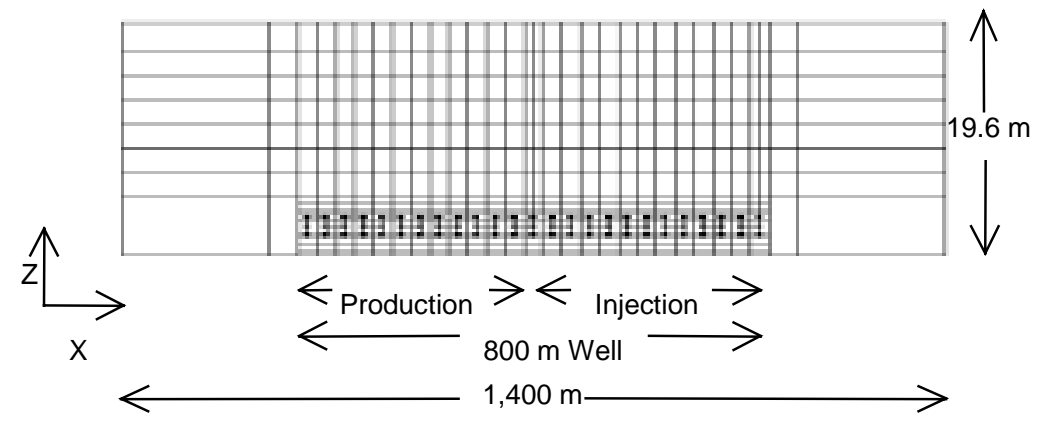

(a)

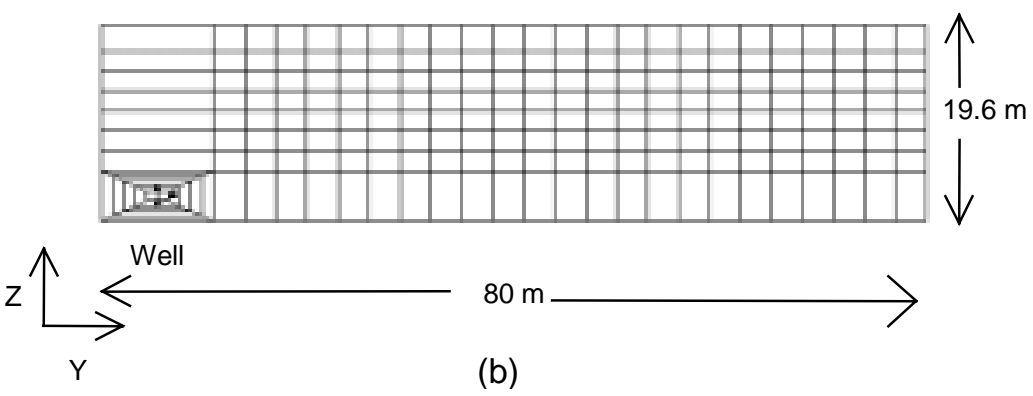

Figure 1. Schematic of grid system: (a) parallel to well bore and (b) perpendicular to wellbore.

Initially, the average reservoir pressure is $2,654 \mathrm{kPa}(380 \mathrm{psi})$, the pressure distribution is hydrostatic, and the initial reservoir temperature is $16{ }^{\circ} \mathrm{C}$. The horizontal permeability, $\mathrm{k}_{\mathrm{h}}$, is 3400 $\mathrm{md}$, whereas the vertical permeability, $\mathrm{k}_{\mathrm{v}}$, is $680 \mathrm{md}$. Hence, the ratio $\mathrm{k}_{\mathrm{h}}: \mathrm{k}_{\mathrm{v}}$ is about 5 to 1 . The oil viscosity at the initial reservoir temperature is $4.043 \mathrm{~Pa}-\mathrm{s}$ (4043 cP). An increase of oil temperature to $100{ }^{\circ} \mathrm{C}$ decreases the oil viscosity to $0.030 \mathrm{~Pa}-\mathrm{s}(30 \mathrm{cP})$. Additional model details are documented in Elliot and Kovscek (2001).

Four base cases were created to explore a range of early-time procedures. Briefly the cases represent SAGD operating conditions, extreme pressure differential conditions where steam is injected near the fracturing or parting pressure of the formation, cyclic steam injection, and steam circulation through the well. For a maximum injection pressure of $10,000 \mathrm{kPa}$, as reported in Table 2, the reservoir depth would have to be greater than roughly $450 \mathrm{~m}$ to avoid fracturing, 
assuming that overburden pressure increases at $22.6 \mathrm{kPa} / \mathrm{m}$. Similar maximum pressures have been reported in the field (Liderth, 1995). Arbitrarily, $100 \mathrm{~d}$ was chosen as the duration of attempts to heat the near-well region. In all cases, SAGD conditions follow this initial period.

\section{Results and Discussion}

To heat the near-wellbore area and improve the initial production response of SAGD, we combined the operating conditions displayed in Table 1 into seven cases: (i) SAGD operation conditions from the start, (ii) extreme pressure differential between injector and producer sections for 100 days followed by SAGD, (iii) steam circulation for 100 days followed by SAGD, (iv) circulate for 100 days followed by 100 days of an extreme pressure differential followed by SAGD, (v) cycle once followed by SAGD, (vi) cycle twice followed by SAGD, and (vii) cycle three times followed by SAGD. In each case except the first, an initial preheating phase precedes SAGD.

Results of this screening study are displayed in Figures 2 to 4 . Figures 2 and 3 display recovery factor histories for the first year of production and for 10 years of production, respectively. Recovery factor refers to the fraction of oil produced from the entire simulation volume including the $600 \mathrm{~m}$ of reservoir beyond the toe of the well. The cumulative steam oil ratio (CSOR) versus time curves for each case are displayed in Fig. 4. It is obvious from the curves that it is possible to improve initial production response. In general, cyclic steaming as applied in Cases 5 to 7 leads to the most rapid oil recovery. However, the late time recovery performances shown in Fig. 3 display similar behavior for all cases. Recovery factor ranges from $19-22 \%$ after 3650 days of injection and all curves increase at similar rates.

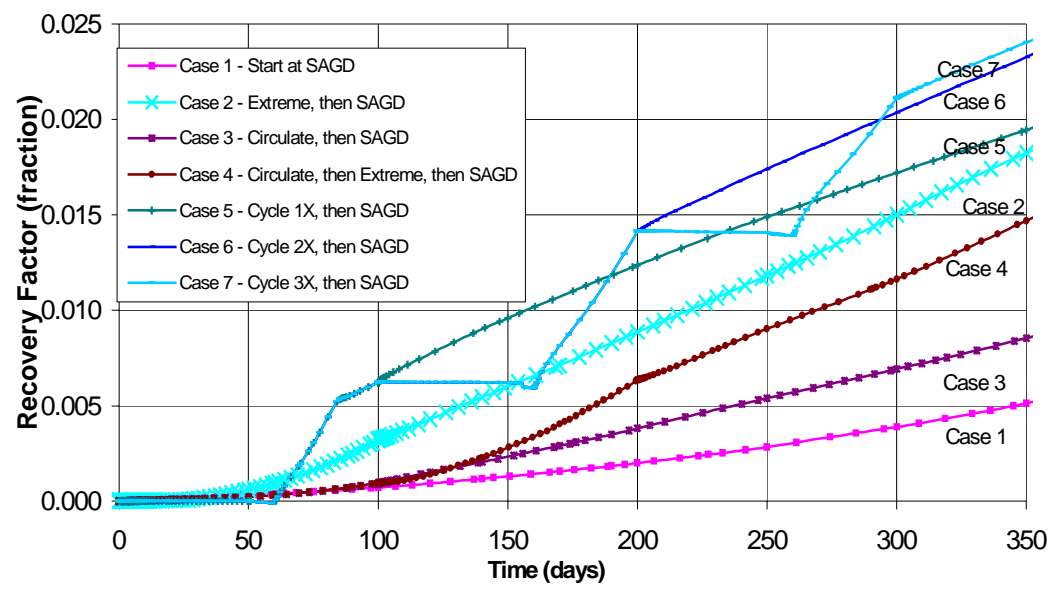

Figure 2. Recovery factor for the first year of production. 


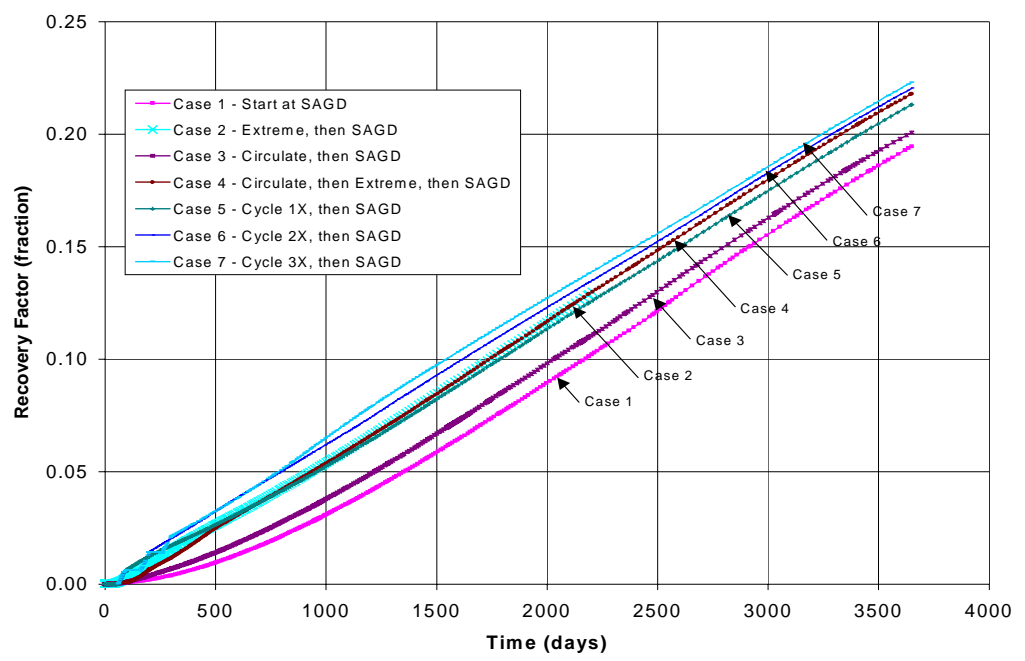

Figure 3. Recovery factor for 10 years of production.

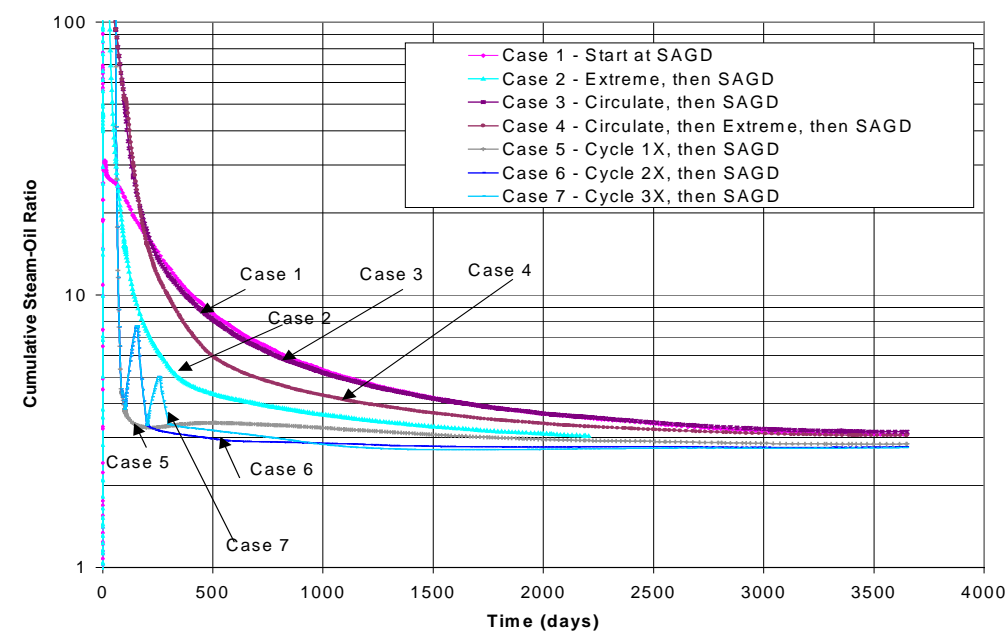

Figure 4. Cumulative steam oil ratio for 10 years of production.

Case 1 represents a base case in which SAGD was initiated from the beginning without preheating. This case produced the lowest percent recovery curve. In Case 2, we increase the injection rate constraint which thereby increases the pressure differential between the injection and production wells; hence, the conditions are referred to as extreme. The pressurization of the system improves production somewhat relative to the base case.

The "circulate" phase in Cases 3 and 4 is a modified form of steam circulation in the well. Steam is injected so as to maintain the initial reservoir pressure. We did not simulate true steam circulation where steam that exits the tubing may only flow in the well before it is produced. A true circulating case in which the near-wellbore area is heated only by conduction would be inefficient, and the other techniques that we explore present better options. Circulation here is similar to the SAGD case: steam may replace oil volume in the reservoir when oil is produced. 
The cumulative steam oil ratio displayed in Fig. 4 is standard: the cumulative steam volume injected as cold water equivalent volume divided by the cumulative hydrocarbon production. The CSOR varies substantially among the studies during the initial period because significantly different production and injection schemes were used. At late time, however, the CSOR for all cases averages roughly 3.0. Note, however, that the cyclic cases perform somewhat better, with regard to CSOR, in initial and late-time response.

\section{Discussion of Early-Time Analysis}

The problem of improving early-time performance of SW-SAGD transforms, essentially, into a problem of heating rapidly the near-wellbore area to create conditions that allow gravity drainage of oil. More specifically, for a steam chamber to grow, oil viscosity must be low enough so that fluid drains creating voidage for steam to fill. After the conditions necessary for gravity drainage of oil have been initiated by preheating, the SW-SAGD process allows for continuous steam chamber growth and oil production. Comparing the various simulation results, cyclic steam injection appears to be the most efficient method of heating the near-wellbore area. Heating is much more rapid than relying on conduction alone.

An important general observation is that regardless of the process, early-time procedures should be carried out to maximize steam injection and heat delivery to the reservoir. The goal of any early-time procedure should be to heat the near-wellbore area as uniformly as possible. This goal is easier to achieve when operating at a maximum steam temperature. Later in the SAGD process, pressure can and should be reduced to a target operating pressure that optimizes efficiency and production rate.

The late time performance for all of the cases is favorable regardless of the early-time process. This confirms Joshi's finding that a steam chamber will grow in the reservoir. Favorable recovery factors are obtainable regardless of the injection/ production configuration (Joshi, 1986).

There are obvious factors that will improve or inhibit SW-SAGD performance. For example, lower viscosity will certainly improve response, as will larger rock permeability and system compressibility. The actual variance in performance due to differing reservoir parameters is an interesting problem that we begin to address using a sensitivity analysis in the next section.

\section{Sensitivity Analysis}

We performed a sensitivity analysis of various reservoir parameters to gain a better understanding of their effect on production performance. Oil viscosity and gas content, reservoir thickness, and horizontal to vertical permeability anisotropy were studied.

The sensitivity analysis base case varies slightly from the base case used in the early-time analysis. Table 2 displays the operating conditions for the sensitivity analysis base case. The base case consists of two steam injection cycles followed by SAGD operating conditions. We adjusted the operating conditions in an attempt to reduce steam short-circuiting, reduce steam-oil ratio, and 
to improve efficiency. During the initial cyclic period, we reduced the maximum reservoir pressure to $8,000 \mathrm{kPa}$ from $10,000 \mathrm{kPa}$ while keeping the rest of the operating conditions the same. The steam injection temperature was $296{ }^{\circ} \mathrm{C}$, that corresponds to a steam pressure of 8004 $\mathrm{kPa}$. During SAGD, operating conditions were chosen so that the process operated near the original reservoir pressure of $2654 \mathrm{kPa}$. Maximum injection pressure was set slightly above initial reservoir pressure at $3230 \mathrm{kPa}$; minimum production pressure was set slightly below initial reservoir pressure at $2230 \mathrm{kPa}$. The steam injection temperature was reduced to $238{ }^{\circ} \mathrm{C}$ from 295 ${ }^{\circ} \mathrm{C}$. Initial reservoir conditions were chosen to approximate field conditions (Oballa and Buchanan 1996). The rate constraints remained the same at $300 \mathrm{~m}^{3} / \mathrm{d}$ maximum liquid production and $200 \mathrm{~m}^{3} / \mathrm{d}$ maximum steam injection rate.

Table 1. Grid, Rock, and Oil Property Description.

\begin{tabular}{|c|c|c|c|}
\hline \multicolumn{2}{|c|}{ Grid System } & \multicolumn{2}{|c|}{ Rock Properties } \\
\hline $\begin{array}{l}\text { total no. } \\
\text { blocks }\end{array}$ & 5,568 & porosity & $33 \%$ \\
\hline $\mathrm{x}$-dimension & $1,400 \mathrm{~m}$ & $\mathrm{k}_{\mathrm{h}}$ & $3,400 \mathrm{mD}$ \\
\hline y-dimension & $80 \mathrm{~m}$ & $\mathrm{k}_{\mathrm{V}}$ & $800 \mathrm{mD}$ \\
\hline z-dimension & $19.6 \mathrm{~m}$ & & \\
\hline well length & $800 \mathrm{~m}$ & & \\
\hline \multicolumn{2}{|c|}{ Reservoir Properties } & \multicolumn{2}{|c|}{ Oil Properties } \\
\hline initial pressure & $2,654 \mathrm{kPa}$ & components & water, oil, and gas \\
\hline $\begin{array}{l}\text { initial } \\
\text { temperature }\end{array}$ & $16^{\circ} \mathrm{C}$ & $\begin{array}{l}\text { initial } \\
\text { composition }\end{array}$ & $90 \%$ (mole) oil \\
\hline initial $\mathrm{S}_{\mathrm{o}}$ & $85 \%$ & & $10 \%$ (mole) gas \\
\hline initial $S_{w}$ & $15 \%$ & $\begin{array}{l}\text { viscosity } \\
\text { (mPa-s) versus } \\
\text { temperature }(\mathrm{K}) \\
\text { function }\end{array}$ & $\mu=1.74 \times 10^{-6} \exp \frac{6232.74}{T}$ \\
\hline
\end{tabular}

Between the injection and production sections of the horizontal well, a $30 \mathrm{~m}$ long unperforated section was added. This is an attempt to force steam to penetrate substantially far into the reservoir and reduce the amount of steam short-circuiting. 
Table 2: Operating Conditions for Early-Time Performance Study

\begin{tabular}{|l|c|c|c|c|}
\hline Property & \multicolumn{4}{|c|}{ Operating Condition } \\
\hline $\begin{array}{l}\text { steam } \\
\text { temperature } \\
\left({ }^{\circ} \mathrm{C}\right)\end{array}$ & SAGD & Extreme & Cyclic & Circulating \\
\hline $\begin{array}{l}\text { max. injection } \\
\text { rate }(\mathrm{CWE} \\
\left.\mathrm{m}^{3} / \text { day }\right)\end{array}$ & 295 & 295 & 295 & 295 \\
\hline $\begin{array}{l}\text { max. injection } \\
\text { pressure }(\mathrm{kPa})\end{array}$ & 10,000 & 600 & 300 & 300 \\
\hline $\begin{array}{l}\text { max. } \\
\text { production } \\
\text { rate }\left(\mathrm{m}^{3} / \mathrm{day}\right)\end{array}$ & 300 & 600 & 10,000 & 10,000 \\
\hline $\begin{array}{l}\text { min. } \text { production } \\
\text { pressure }(\mathrm{kPa})\end{array}$ & 500 & 500 & 300 & 300 \\
\hline
\end{tabular}

\section{Sensitivity Cases}

The various cases created in the sensitivity analysis include: (i) a base case with the properties in Table 1 and operating conditions shown in Table 3, (ii) dead oil to examine the effect of solution gas, (iii) an increase in pay thickness to $28 \mathrm{~m}$, (iv) a reduction in pay thickess to $4 \mathrm{~m}$, (v) permeability anisotropy $\mathrm{k}_{\mathrm{h}}: \mathrm{k}_{\mathrm{v}}$ of 1 , (vi) $\mathrm{k}_{\mathrm{h}}: \mathrm{k}_{\mathrm{v}}$ equal to 2 , (vii) $\mathrm{k}_{\mathrm{h}}: \mathrm{k}_{\mathrm{v}}$ equal to 10 , (viii) oil viscosity of $20 \mathrm{~Pa}-\mathrm{s}$ at initial reservoir temperature $(20,000 \mathrm{cP})$, and (iv) oil viscosity of $40 \mathrm{~Pa}-\mathrm{S}$ $(40,000 \mathrm{cP})$ at initial reservoir temperature.

All calculations in the sensitivity cases include a separation of $30 \mathrm{~m}$ between injector and producer sections of the well to achieve better steam distribution in the reservoir. Two periods of cyclic steam injection precede continuous injection and production. In both periods, steam is injected for 50 days at a rate of $300 \mathrm{~m}^{3} /$ day with a maximum allowed pressure of $8000 \mathrm{kPa}$. The soak period is 10 days and the length of production is a relatively short 40 days to avoid large heat withdrawal from the reservoir. Continuous SAGD operating conditions follow. All of the various sensitivity runs follow this procedure.

The recovery factor versus time and cumulative steam-oil ratio (CSOR) versus time curves are presented in Figs. 5 and 6, respectively. Again, recovery refers to the entire simulation volume. Significant variation is found in production response between most of the cases. Briefly, production is greatest when the reservoir permeability is isotropic, and is worst when the initial oil viscosity is high or the oil-saturated thickness is small. The steam-oil ratio is largest for the short formation thickness of $4 \mathrm{~m}$. The next largest CSOR is for the viscous $40 \mathrm{~Pa}$-s initial oil case. The smallest CSOR is found for the case with a pay zone thickness of $28 \mathrm{~m}$ and for cases where $\mathrm{k}_{\mathrm{h}}: \mathrm{k}_{\mathrm{v}}$ approaches 1 . Note that recovery factors are less here, Fig. 5, compared to the early-time study, Fig. 3, because the steam injection pressure has been reduced substantially as seen by comparing Tables 1 and 2. 


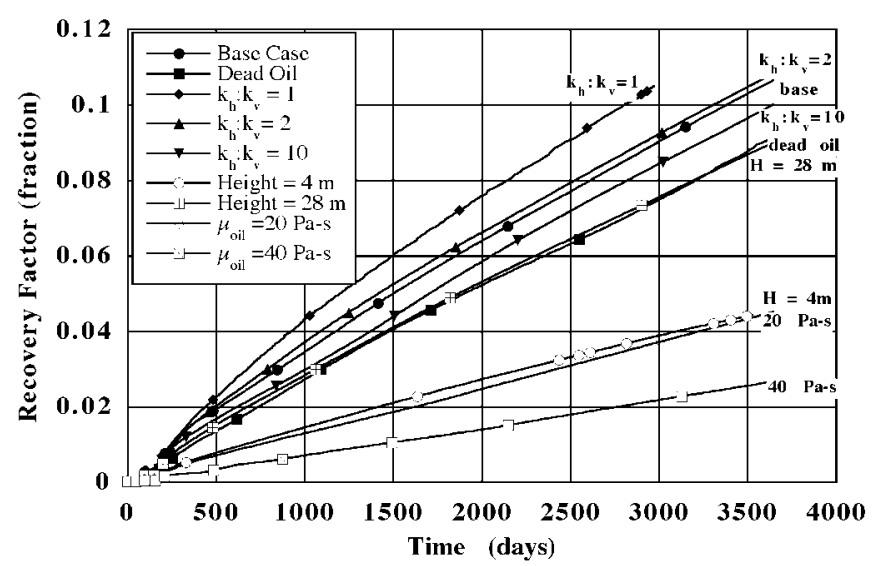

Figure 5. Recovery factor versus time, sensitivity analysis.

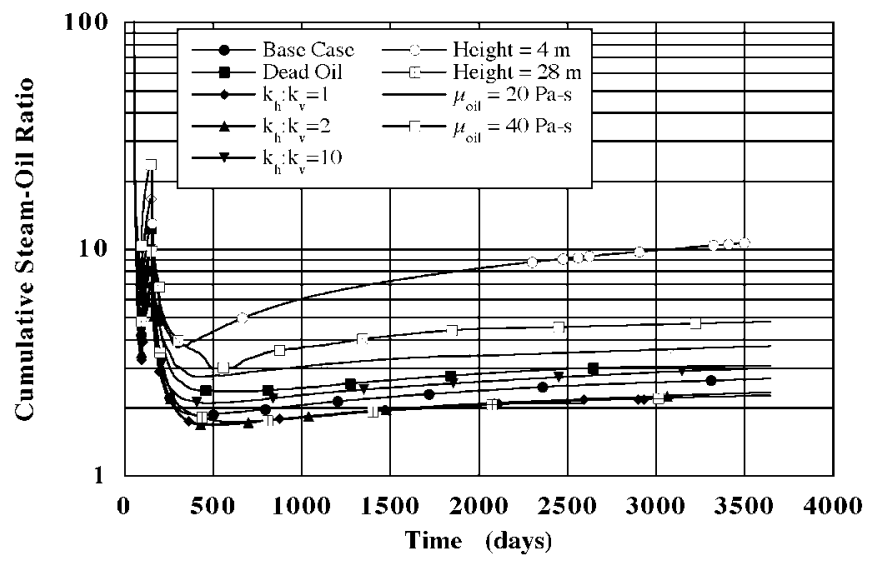

Figure 6. Cumulative steam oil ratio, sensitivity analysis.

Due to space restrictions, it is impossible to display the instantaneous production rates, bottomhole pressure, and temperature profiles for all of the sensitivity cases. However, a brief discussion of the results of each of the sensitivity cases will be given.

The base case oil viscosity is $4.043 \mathrm{~Pa}$-s at initial reservoir conditions. Two additional cases were created by increasing the initial viscosity to $20 \mathrm{~Pa}$-s and then $40 \mathrm{~Pa}$-s. The recovery factors at 3650 days (10 years) for these two less favorable cases were $4.5 \%$ and $2.7 \%$, respectively, as compared to $10.6 \%$ for the base case. The cumulative steam-oil ratios were also unfavorable. The CSOR were 3.7 and 4.8 after 10 years for the 20 and $40 \mathrm{~Pa}$-s cases, respectively. The problem is quite simple: it is hard for the viscous oil to drain and therefore it cannot be replaced by steam. In the base case, the average oil production rate is $75 \mathrm{~m}^{3} /$ day over the 3650 days of production and excluding the startup period. For the $20 \mathrm{~Pa}$-s case the average oil production rate is $30 \mathrm{~m}^{3} /$ day while for the $40 \mathrm{~Pa}-\mathrm{s}$ case it is $20 \mathrm{~m}^{3} /$ day. As the viscosity increases 
the average oil production rate decreases, it becomes difficult to meet the steam injection target, and the size of the steam chamber is smaller compared to the less viscous cases.

The base horizontal to vertical permeability ratio, $\mathrm{k}_{\mathrm{h}}: \mathrm{k}_{\mathrm{v}}$, is 4.25 . The vertical permeability was adjusted to create three more cases with ratios of 1,2 , and 10 . In all cases, the horizontal permeability remained fixed at $3400 \mathrm{mD}$. Larger ratios present less favorable conditions for upward steam migration and oil drainage. As $\mathrm{k}_{\mathrm{h}}: \mathrm{k}_{\mathrm{v}}$ increases, cumulative recovery at a given time decreases and the CSOR increases, as shown in Figs. 5 and 6.

The base case oil composition contained $10 \%$ gas by mole which translates to a solution gas oil ratio of about $28 \mathrm{SCF} / \mathrm{STB}$. An additional case that models a dead oil was created by reducing the solution gas to $1 \%$ by mole. The two effects of solution gas in regard to the oil phase are to increase oil-phase compressibility and reduce the oil-phase viscosity. Oil phase viscosity is computed as a mole fraction weighted sum of the oil and gas component viscosities (CMG 1998). Recovery factor and CSOR were less favorable with the dead oil at $9.2 \%$ and 3.0 after 10 years as compared to $10.4 \%$ and 2.8 for the base case. Likewise, the average oil production rate is 64 $\mathrm{m}^{3} /$ day over the ten year period as compared to $80 \mathrm{~m}^{3} /$ day for the base case.

\section{Discussion of Sensitivity Analysis}

Taken together, the results from the sensitivity analysis suggest that application of SWSAGD to exceptionally viscous oils will be difficult. When the initial viscosity is greater than 10 $\mathrm{Pa}-\mathrm{s}$, oil drainage becomes very slow and it is difficult to form a large steam chamber. Likewise, the application of SW-SAGD to thin oil zones with thickness of roughly $4 \mathrm{~m}$ does not appear to be feasible. A steam chamber of significant height must develop for efficient oil drainage. On the other hand, the 19.6 and $28 \mathrm{~m}$ thick cases showed significant recovery and little difference was found between theses two cases.

The presence of solution gas also aids recovery somewhat. Even though the oil is viscous and the amount of gas low in both cases, volumetric expansion of the oil is aided by solution gas and cumulative recovery increases by $10 \%$ relative to the dead oil case at 3650 days.

As expected, oil recovery improves as the permeability anisotropy decreases. Both steam injection and oil drainage are aided as the vertical permeability increases relative to the horizontal. Resistance to flow in the vertical direction decreases relative to horizontal. Additionally, less short circuiting occurs as $\mathrm{k}_{\mathrm{v}}$ increases relative to $\mathrm{k}_{\mathrm{h}}$.

\section{Conclusions}

Here it is shown that to improve early-time performance of SW-SAGD, it is necessary to heat the near-wellbore region rapidly and uniformly to reduce oil viscosity and promote gravity drainage. Cyclic steaming, as a predecessor to SW-SAGD, represents the most thermally efficient 
early-time heating method. Uniform heating along the length of the wellbore appears achievable with cyclic steam injection. Immediately placing a cold well on SAGD hinders the early-time heating process and initial production response in this case will be low. Regardless of the earlytime process, it should be performed to provide maximum heat delivery to the reservoir. Additionally, despite different initial procedures, the oil production rates after several years of steam injection are all very similar.

The sensitivity analysis performed here indicates that SW-SAGD is most applicable to heavy oils with initial viscosity below $10 \mathrm{~Pa}-\mathrm{s}$ (10,000 cP) Additionally, the reservoir must be sufficiently thick to allow significant vertical steam chamber growth. Recovery from thin oil zones is not significant or efficient. The sensitivity analysis also indicates that the presence of relatively small amounts of solution gas aids the recovery process by enhancing volumetric expansion of the oil on heating.

\section{References}

Computer Modelling Group LTD. 1998. STARS Version 98 User's Guide. CMG Calgary, Alberta, Canada.

Elliot, K. T. and A. R, Kovscek, "A Numerical Analysis of the Single-Well Steam Assisted Gravity Drainage Process," Petroleum Science and Technology, 19 (7 and 8), 733-760, 2001; also TR 124, DOE DE-FC26-00BC1531, 2001.

Falk, K., Nzekwu, B., Karpuk, B., and Pelensky, P. 1996 World Oil. July: 85-95.

Liderth, D. 1995. Daily Oil Bulletin, May 2, 1995. 


\section{Analytical Model for Cyclic Steam Stimulation of a Horizontal Well}

\section{(Task 2a)}

\section{Introduction}

The previous section illustrated that cyclic steaming as a precursor to steam-assisted gravity drainage may be an effective means of stimulating a heavy-oil formation thereby producing oil at appreciable rates relatively quickly. This work concerns the application of horizontal wells to thermal oil recovery. It consists of an analytical model developed to calculate oil recovery and reservoir heating during cyclic steam injection. It holds for heavy-oil, pressuredepleted reservoirs where the main driving force for production is gravity. Our objective is to present a relatively simple model taking into account gravity-drainage along the sides of the steamoil interface, the pressure draw down driving force and the drainage of oil through the steam zone.

The performance of horizontal wells may be predicted from empirical correlations, simple analytical models, or thermal reservoir simulation. Empirical correlations can be extremely useful for correlating data within a field and for predicting performance of new wells in that and similar fields. However, use of such correlations for situations much different from those that led to their development might be subject to large errors. On the other hand, one can use a compositional or black-oil thermal model to predict the performance of cyclic steam operations. Thermal models are based on the fundamental laws of conservation of mass and heat. Fluid flow is related to pressure gradient through the concept of relative permeability. In addition, a thermal model is sensitive to rock properties, fluid properties and geological features. Much of this information is often unknown and must be estimated from limited data and experience in similar situations. Furthermore, because of the complexity of the SAGD recovery process, the equations of a thermal simulator could be difficult and expensive to solve depending upon the exact scenario. Simply, an analytical model of cyclic steam injection is useful to expose the first-order mechanisms of reservoir heating and oil production.

\section{Model Development}

Cyclic steam injection, commonly referred to as "Huff-n-Puff" involves steam injection into a reservoir, followed by soaking, and finally the oil production period. As stated earlier, oil production is governed by the gravity driving force. The model developed in the following section incorporates gravity as a prime driving force for oil flow towards the well and thereby predicts the oil production flow rate per unit length of the horizontal well in the production period. Heat losses from the heated zone to the overburden and the adjacent unheated oil-bearing formation are also included.

By way of background, steam introduced at the bottom of the formation through a horizontal well, displaces the oil and rises to the top of the formation where it is trapped if an impermeable cap rock exists. We assume that the steam zone adopts a triangular shape in cross 
section as shown in Fig.1. The steam injection rate around the well remains constant over the entire injection interval. Therefore, the steam injection pressure generally remains constant over the injection process if the reservoir is pressure-depleted. Steam heats up the colder oil sand near the condensation surface and oil drains along the condensation surface by a combination of gravity and pressure difference into the production well. Steam condensate also drains toward the well. In addition, oil drains through the steam chamber into the production well. The mechanisms involved in oil production during cyclic steam injection are diverse and intricate. Reduction of oil viscosity as a result of an increase in the temperature greatly improves the production response. Gravitydrainage and pressure drawdown are the major mechanisms of oil production in the case of cyclic steaming.

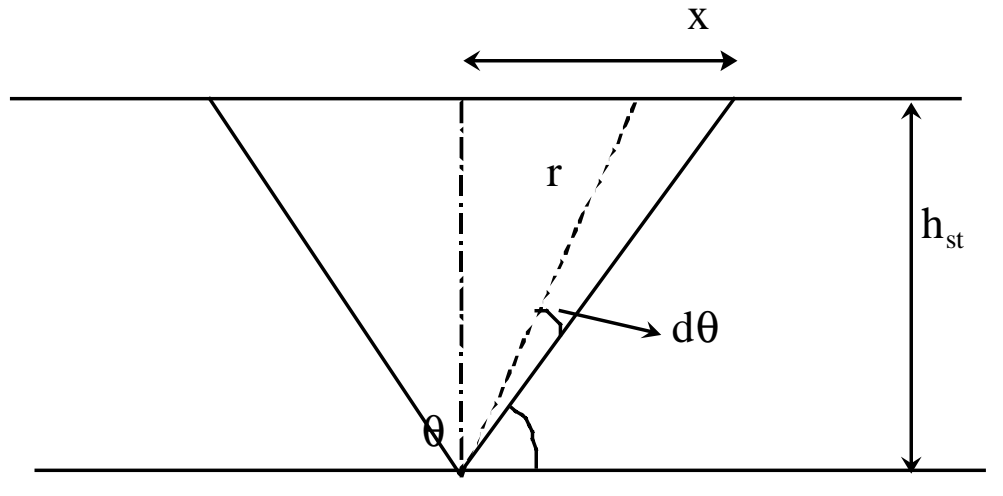

(a)

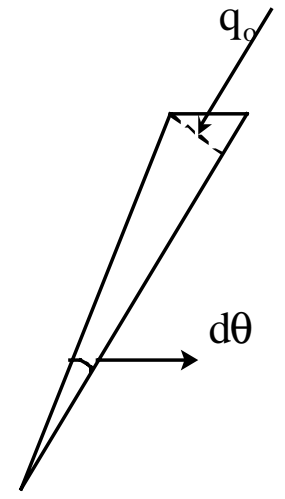

(b)

Figure 1. (a) Schematic of heated area geometry; (b) differential element of the heated area.

The model is divided into three periods: the injection period, the soaking period and the production period. Each is described in detail. In what follows, correlations and equations are given in field units $\left({ }^{\circ} \mathrm{F}\right.$, psi, ft, BTU, BPD, etc), unless otherwise noted.

\section{Injection and Soak Periods}

During the injection interval, heat losses from the steam zone to the reservoir are considered negligible although heat losses to the overburden must be considered. The oil sand near the wellbore is assumed to be at steam temperature $T_{s}$, the saturated steam temperature at the sand face injection pressure. Pressure fall-off away from the well during injection is neglected during this analysis. In the soaking period, heat is lost to the overburden and the reservoir. The temperature of the steam zone thus decreases while soaking.

There are certain important variables such as the steam zone volume and the zone horizontal range that need to be addressed. The steam zone volume, $\mathrm{V}_{\mathrm{s}}$, during injection is calculated using the approach of Myhill and Stegemeier (1978) as

$$
V_{s}=\frac{Q_{i} E_{h, s}}{M_{T} \Delta T}
$$


where, $\mathrm{E}_{\mathrm{h}, \mathrm{s}}$ is the thermal efficiency, $\Delta \mathrm{T}$ is the temperature rise of the steam zone above the initial reservoir temperature (assumed to be same as the temperature rise at down-hole condition, $\Delta \mathrm{T}_{\mathrm{i}}$ ), and $\mathrm{M}_{\mathrm{T}}$ is the total volumetric heat capacity of the reservoir. Heat capacity is the sum of the rock and fluid contributions and is written

$$
M_{T}=(1-\phi) M_{r r}+\sum_{\beta=w, o, g} \phi S_{\beta} M_{\beta}
$$

where, $\phi$ is the porosity, $S$ is the phase saturation, and the subscript $\beta$ refers to the individual phases. The quantity $Q_{i}$ is the cumulative heat injected including any remaining heat from the previous cycles. The heat injection rate is given by

$$
Q_{i}=w_{i}\left(C_{w} \Delta T+f_{s d h} \Delta H_{v d h}\right)
$$

where, $\mathrm{w}_{\mathrm{i}}$ is the mass rate of steam injection in the reservoir, $\mathrm{C}_{\mathrm{w}}$ is the average specific heat of water over the temperature range corresponding to $\Delta \mathrm{T}, \mathrm{f}_{\mathrm{sdh}}$ and $\Delta \mathrm{H}_{\mathrm{vdh}}$ are the steam quality and the latent heat of vaporization both at down-hole conditions, respectively.

The thermal efficiency is calculated from the function given by Myhill and Stegemeier

$$
E_{h, s}=\frac{1}{t_{D}}\left\{G\left(t_{D}\right)+\left(1-f_{h v}\right) \frac{U\left(t_{D}-t_{c D}\right)}{\sqrt{\pi}}\left[2 \sqrt{t_{D}}-2\left(1-f_{h v}\right) \sqrt{t_{D}-t_{c D}}-\int_{0}^{t_{c D}} \frac{e^{x} \operatorname{erfc}(\sqrt{x})}{\sqrt{t_{D}-x}} d x-\sqrt{\pi} G\left(t_{D}\right)\right]\right\}
$$

In the expression above, the heat losses to both, the overburden as well as the underburden are included. In the model proposed, the triangular cross sectional shape of the steam chamber is such that heat loss occurs only to the overburden. Thus, $\mathrm{E}_{\mathrm{h}, \mathrm{s}}$ needs to be modified to be consistent with our model. Now, $\mathrm{E}_{\mathrm{h}, \mathrm{s}}$ is defined as the ratio of the heat remaining in the zone to the total heat injected. Thus, the quantity $\left(1-\mathrm{E}_{\mathrm{h}, \mathrm{s}}\right)$ is the ratio of the heat lost to the total heat injected

$$
\mathrm{F}_{1}=1-\mathrm{E}_{\mathrm{h}, \mathrm{s}}
$$

Neglecting loss to the underburden, heat losses are approximately one-half of the value predicted by Eq. 4b. Therefore, the modified thermal efficiency is

In Eq. $4 \mathrm{a}, \mathrm{G}\left(\mathrm{t}_{\mathrm{D}}\right)$ is

$$
\left(\mathrm{E}_{\mathrm{h}, \mathrm{s}}\right)_{\bmod }=1-0.5 \mathrm{~F}_{1}=0.5\left(1.0+\mathrm{E}_{\mathrm{h}, \mathrm{s}}\right)
$$

$$
G\left(t_{D}\right)=2 \sqrt{\frac{t_{D}}{\pi}}-1+e^{t_{D}} \operatorname{erfc}\left(\sqrt{t_{D}}\right)
$$

The symbols $t_{D}$ and $t_{C D}$ represent dimensionless times given by

$$
t_{D}=4\left(\frac{M_{S H}}{M_{T}}\right)^{2} \frac{\alpha_{S H}}{h_{t}^{2}} t
$$


where, $\alpha_{\mathrm{SH}}$ is the shale thermal diffusivity, $\mathrm{t}$ is the time, $\mathrm{h}_{\mathrm{t}}$ is the total reservoir thickness and $\mathrm{M}_{\mathrm{SH}}$ is the shale volumetric heat capacity. The dimensionless critical time (Myhill and Stegemeier, 1978) is defined by

$$
e^{t_{c} D} \operatorname{erfc}\left(t_{c D}\right) \equiv 1-f_{h v}
$$

The quantity $f_{h v}$, the fraction of heat injected in latent form, is given by

$$
f_{h v}=\left(1+\frac{C_{w} \Delta T}{f_{s d h} \Delta H_{v d h}}\right)^{-1}
$$

The step-function $\mathrm{U}$ in Eq.4 is defined as

$$
\begin{array}{ll}
U(x)=0 & \text { for } x<0 \\
U(x)=1 & \text { for } x>0
\end{array}
$$

We assume that the steam zone shape has a triangular cross section with a y-directional length equal to the length of the horizontal well, L (Fig.1). The volume is written as

$$
V_{s}=R_{h} L h_{s t}
$$

where $R_{h}$ is one-half of the base of the triangular heated zone and $h_{s t}$ is the steam zone thickness also referred to as "Zone Horizontal Range". Rearranging Eq.10a, it follows that,

$$
R_{h}=\frac{V_{s}}{L h_{s t}}
$$

\section{Heat Remaining in the Reservoir}

Boberg and Lantz (1966) give the average temperature of the steam zone, $\mathrm{T}_{\mathrm{avg}}$, as

$$
T_{a v g}=T_{R}+\left(T_{s}-T_{R}\right)\left[f_{H D} f_{V D}\left(1-f_{P D}\right)-f_{P D}\right]
$$

The dimensionless parameters $\mathrm{f}_{\mathrm{VD}}, \mathrm{f}_{\mathrm{HD}}$ and $\mathrm{f}_{\mathrm{PD}}$ are functions of time and represent horizontal loss, vertical loss and energy removed with the produced fluids, respectively. Aziz and Gontijo (1984) define them according to the following expressions.

The horizontal heat losses are expressed as (Aziz and Gontijo, 1984):

$$
f_{H D}=\frac{1}{1+5 t_{D H}}
$$


where,

$$
t_{D H}=\frac{\alpha\left(t-t_{i n j}\right)}{R_{h}^{2}}
$$

The quantity $\mathrm{f}_{\mathrm{HD}}$ is the average unit solution for the one-dimensional heat conduction problem in the horizontal direction,

$$
\alpha \frac{\partial^{2} f}{\partial x^{2}}=\frac{\partial f}{\partial t}
$$

where $f=\left(\frac{T-T_{o}}{T_{S}-T_{o}}\right)$ is the dimensionless temperature and $\alpha$ is the reservoir thermal diffusivity.

The averaged solution for this equation after making use of the appropriate boundary conditions consistent with the geometry chosen for our model is:

$$
f_{H D}=\frac{1}{2}+\sqrt{\frac{t_{D H}}{\pi}}\left\{-2.0+\exp \left(-\frac{1}{t_{D H}}\right)\right\}+\operatorname{erf}\left(\frac{1}{\sqrt{t_{D H}}}\right)\left\{\left(\frac{2}{\sqrt{\pi}}-1\right) t_{D H}+\frac{1}{2}\right\}
$$

Similarly, the vertical heat losses are expressed as (Aziz and Gontijo, 1984):

$$
f_{V D}=\frac{1}{\sqrt{1+5 t_{D V}}}
$$

where,

$$
t_{D V}=\frac{4 \alpha\left(t-t_{i n j}\right)}{h_{t}^{2}}
$$

Note that for the first cycle, the initial amount of heat in the reservoir is set to zero. For all following cycles, the initial amount of energy is calculated based on steam zone volume and the average temperature at the end of the previous cycle. The average temperature at any time during the cycle is calculated using Eq.11 (Boberg and Lantz, 1966). Since Boberg and Lantz's equation assumes a cylindrical shape for the heated zone, this equation is just an approximation for the triangular shape being considered. However, we use the approximations for $f_{H D}$ and $f_{V D}$ as employed by Aziz and Gontijo (1984) who assume a conical shape with triangular cross section. This is identical to the cross sectional shape that we have considered. The only difference is that they rotate the triangular shape through $\pi$ radians whereas our coordinate system is Cartesian.

The term that accounts for the energy removed with produced fluids is given by

$$
f_{P D}=\frac{1}{2 Q_{M A X}} \int_{0}^{t} Q_{p} d t
$$

where,

$$
Q_{p}=5.615\left(q_{o} M_{o}+q_{w} M_{w}\right)\left(T_{a v g}-T_{R}\right)
$$


where $\mathrm{Q}_{\mathrm{MAX}}$ is the maximum heat supplied to the reservoir. It is calculated at the end of the soak period as the amount of heat injected plus the heat remaining in the reservoir from the previous cycle minus the losses to the over burden (Vogel, 1982),

$$
\begin{gathered}
Q_{M A X}=Q_{i n j}+Q_{\text {last }}-2 R_{h} L K_{R}\left(T_{s}-T_{R}\right) \sqrt{\frac{t_{\text {soak }}}{\Pi \alpha}} \\
Q_{i n j}=350.376 \bar{Q}_{i} w_{s} t_{i n j} \\
Q_{\text {last }}=V_{s} M_{T}\left(T_{\text {avg }}-T_{R}\right)
\end{gathered}
$$

where, $L$ is the length of the horizontal well, $K_{R}$ is the thermal conductivity of the reservoir, $t_{\text {soak }}$ is the soak time, $t_{\text {inj }}$ is the length of the injection cycle, $Q_{\text {last }}$ is the heat remaining from the previous cycle, $\bar{Q}_{i}$ is the amount of heat injected per unit mass of steam, and $\mathrm{w}_{\mathrm{s}}$ is the steam injection rate (cold water equivalent).

The steam pressure is calculated from the following approximate relationship (Prats, 1982):

$$
p_{s}=\left[\frac{T_{s}}{115.95}\right]^{4.4545}
$$

The volumetric heat capacities of oil and water are given as (Prats, 1982):

$$
\begin{aligned}
& M_{o}=(3.065+0.00355 T) \sqrt{\rho_{o}} \\
& M_{w}=\rho_{w} C_{w}
\end{aligned}
$$

As the integral for $\mathrm{f}_{\mathrm{PD}}$ is not easy to calculate, it is approximated by the following expression (Aziz and Gontijo, 1984),

$$
\Delta f_{P D}=\frac{5.615\left(q_{o} M_{o}+q_{w} M_{w}\right)\left(T_{a v g}^{n-1}-T_{R}\right) \Delta t}{2 Q_{M A X}}
$$

where, $\Delta \mathrm{t}$ is the time step, $\mathrm{n}$ is the time step number, $\mathrm{q}_{\mathrm{o}}$ and $\mathrm{q}_{\mathrm{w}}$ are the oil and water production rates, respectively. The average temperature of the steam zone in the ' $n-1$ 'th cycle is $T_{\text {avg }}^{n-1}$.

\section{Production Period}

In this period, the well is opened for oil flow from the reservoir. A new model is presented to predict oil production rates during the production period. Butler et al. (1980) presented a series of publications related to the gravity-drainage of heavy-oil reservoirs subjected to steam injection in which the theory was directed to linear flow from horizontal wells. In this development, a similar approach is used. However, the steam zone shape has been assumed to be a prism with 
triangular cross section and the horizontal well lies at the bottom edge. Figure 1 shows the cross sectional shape of the zone.

It is assumed in the derivation of the flow equation that the reservoir is initially saturated with oil and water, and it is pressure-depleted. After steam flooding, the steam chamber occupies a prismatic shape. Steam distribution along the well is assumed to be uniform. Heat transfer from the steam chamber to the neighboring oil zone is via conduction. During the injection period, the average temperature of the steam chamber is assumed to be the saturation temperature of the steam. The oil is heavy enough to permit the injected steam to go to the top of the reservoir as suggested by Van Lookeren (1977). The reservoir is assumed to be initially pressure-depleted and is only negligibly re-pressurized when steam is injected. Finally, the potential that causes flow of oil into the same well that acts as a production well is a combination of gravity forces and pressure drive.

In this approach, we assume that gravity-drainage takes place through the entire steam chamber. Thus, integration is over the entire steam chamber and proceeds angularly with the appropriate approximation for the area. Gravity and pressure drive are taken as the forces causing production. The steam zone growth is estimated in terms of the decrease in the angle of inclination of the zone with the horizontal, q. The thickness of the steam zone is assumed to be equal to the reservoir thickness. The heated-zone geometry used to derive the flow equation is given in Fig.1. The maximum heated dimension in the horizontal direction is $\mathrm{R}_{\mathrm{h}}$, while the maximum heated dimension in the vertical direction is the reservoir thickness $h$.

According to Darcy's law, the flow rate through an incremental area, dA, normal to the direction of flow [Fig.1(b)], is written

$$
d q_{o}=-\frac{k_{o}}{\mu_{o}}\left(\rho_{o}-\rho_{s}\right) \nabla \phi d A
$$

where, $\nabla \phi$ is the gradient of potential in the direction of flow, $\mu_{\mathrm{o}}$ is the dynamic viscosity of oil, $\mathrm{k}_{\mathrm{o}}$ is the effective permeability to oil, and $\rho_{\mathrm{o}}-\rho_{\mathrm{s}}$ is the density difference between the oil and steam. According to Hubbert (1956), the potential is expressed as

$$
\Delta \phi=\frac{p_{s}-p_{w f}}{\rho_{o}}+g \sin \theta \Delta h
$$

Here, $\mathrm{g}$ is the acceleration due to gravity. The differential area depends upon distance, $\mathrm{r}$, above the well:

$$
d A=2 r \sin \left(\frac{1}{2} d \theta\right) \approx r d \theta
$$

The oil-phase effective permeability, $\mathrm{k}_{\mathrm{o}}$ is the product of the absolute permeability, $\mathrm{k}$, and relative permeability, $\mathrm{k}_{\mathrm{ro}}$. With $\mathrm{r}$ equal to $\mathrm{h}_{\mathrm{st}} \operatorname{cosec}(\theta)$ and applying the approximation suggested in Eq.21, the differential area reduces to

$$
d A=L h_{s t} \operatorname{cosec} \theta d \theta
$$


It follows upon substitution of Eq.22 into Eq.20 that the flow rate becomes

$$
d q_{o}=-\frac{k_{o}}{\mu_{o}}\left(\rho_{o}-\rho_{s}\right)\left(\frac{p_{s}-p_{w f}}{\rho_{o}}+g h_{s t} \sin (\theta)\right) h_{s t} L \operatorname{cosec} \theta d \theta
$$

Integration over one-half of the heated area and multiplication by 2 yields

$$
\frac{q_{o}}{L}=-\frac{2 k_{o}}{\mu_{o}}\left(\rho_{o}-\rho_{s}\right)\left\lfloor 144.0 \frac{p_{s}-p_{w f}}{\rho_{o}} \log \left\{\frac{1}{\tan \left(\frac{\theta}{2}\right)}\right\}+g h_{s t}(\pi-2 \theta)\right\rfloor
$$

\section{Property Correlations}

A variety of property correlations are needed as constitutive relations. The steam viscosity and density are calculated by correlations given by Farouq Ali (1982). The average specific heat of water is obtained as

$$
C_{w}=\frac{h_{w}\left(T_{s}\right)-h_{w}\left(T_{R}\right)}{T_{s}-T_{R}}
$$

The water enthalpy correlation of Jones (1977) and the steam latent heat correlation of Farouq Ali (1982) are used to obtain water properties. The oil and water densities are approximated by according to the information given by Aziz (1984). These oil density correlations hold for oils with gravity less than $20^{\circ} \mathrm{API}$ and temperature less than $500^{\circ} \mathrm{F}$.

Fluid viscosities as a function of temperature are obtained from relations given by Jones (1977). This correlation for oil viscosity is applicable to oils with specific gravity less than $30^{\circ}$ API.

After the soak period and before the production begins, it is assumed that the only mobile phase around the well is water

$$
\bar{S}_{w}=1-S_{\text {orw }}
$$

Once the well is opened for production, it is assumed that the oil saturation around the well increases. Also, the water saturation is given by

$$
S_{w}=\bar{S}_{w}-\left(\bar{S}_{w}-S_{w i}\right) \frac{W_{p}}{W I P}
$$

where $\mathrm{W}_{\mathrm{p}}$ is the water production during the cycle and WIP is the amount of mobile water in place at the beginning of the cycle (Aziz and Gontijo, 1984). The normalized water saturation expression is

$$
S_{w}^{o}=\frac{S_{w}-S_{w i}}{1-S_{w i}-S_{o r w}}
$$


The analytical expressions presented by Farouq Ali (1982) for the relative permeabilities suggested by Gomaa (1980) are as follows:

$$
\begin{gathered}
k_{r w}=-0.002167 S_{w}^{o}+0.024167\left(S_{w}^{o}\right)^{2} \\
k_{r o}=-0.9416+\frac{1.0808}{S_{w}^{o}}-\frac{0.13856}{\left(S_{w}^{o}\right)^{2}}
\end{gathered}
$$

with,

$$
k_{r o}=1.0 \text { if } S_{w}^{o} \leq 0.2
$$

\section{Algorithm for Calculation Scheme}

These equations and ideas are translated into a seven-step algorithm:

- Initialize the model by inputting reservoir, fluid, and operational properties.

- Calculate steam zone geometry (volume, height, and thickness), temperature, and saturations during injection and at the start of the production cycle.

- Calculate oil and water production flow rates at small time steps within the production interval. Also, calculate the cumulative volume of fluids produced and check against original fluids in place.

- Calculate the average temperature of the heated zone during production and at the end of the production cycle.

- If additional steps are needed for cycle completion, go to stage 3, else proceed.

- Calculate the amount of fluids and heat remaining in the reservoir at the termination of the cycle, and thereby calculate recovery and oil-steam ratio (OSR).

- If a new cycle is required, repeat steps, else terminate calculations.

\section{Results and Discussion}

Similar reservoir and fluid models were assembled for comparison of the semi-analytical calculation versus thermal reservoir simulation using CMG STARS. Input parameters are summarized in Table 1. A two-dimensional homogeneous permeability field was constructed for simulation. There are 55 by 13 gridblocks in the horizontal and vertical directions, respectively. The grid is refined locally near the well. The horizontal direction is sufficiently large $(7,495 \mathrm{ft}$.) so as to represent a semi-infinite system in lateral extent. Injection and soak periods are set to 45 and 20 days, respectively. Three huff-n-puff periods are calculated and the elapsed production period increased for each subsequent cycle. The production periods are chosen as 50, 100, and 140 for the first, second, and third cycles.

Figure 2 illustrates the match between cumulative oil production from semi-analytical theory and reservoir simulation. Results for the first cycle match well. For the second and third cycle, the semi-analytical theory over predicts the cumulative oil production significantly. That is, the oil production rate from the semi-analytical theory is somewhat larger than that predicted by the reservoir simulator. 
Table 1. Physical Properties for Semi-Analytic Calculation and Simulation

\begin{tabular}{|l|l|}
\hline Variable & Value \\
\hline permeability (Darcy) & 1.5 \\
\hline porosity & 0.2 \\
\hline initial water saturation & 0.25 \\
\hline connate water saturation & 0.10 \\
\hline well radius (ft) & 0.31 \\
\hline residual oil saturation to steam & 0.05 \\
\hline residual oil saturation to water & 0.25 \\
\hline initial reservoir temperature $\left({ }^{\circ} \mathrm{F}\right)$ & 110 \\
\hline saturated steam temperature $\left({ }^{\circ} \mathrm{F}\right)$ & 365 \\
\hline thermal conductivity $\left(\mathrm{BTU} / \mathrm{ft}-\mathrm{D}-{ }^{\circ} \mathrm{F}\right)$ & 24.0 \\
\hline thermal diffusivity $\left(\mathrm{ft}^{2} / \mathrm{day}\right)$ & 0.48 \\
\hline time step $($ day) & 1.0 \\
\hline injected steam quality & 0.67 \\
\hline steam injection rate $(\mathrm{bbl} / \mathrm{d} / \mathrm{ft}$ of well) & 1.0 \\
\hline API gravity of oil & 14.0 \\
\hline injection pressure $(\mathrm{psi})$ & 150.0 \\
\hline pay thickness $(\mathrm{ft})$ & 80.0 \\
\hline
\end{tabular}

After substantial investigation, it has been found that the temperature of the steam zone as computed by both methods differed somewhat. Figure 3 presents the temperature of the steam zone versus time that complements the cumulative oil production in Fig. 2. The upper curve represents the temperature history as predicted by the semianalytical model and it well illustrate model assumptions. That is, temperature is constant during the injection period, relatively slow decline in temperature during soak, and then a somewhat greater decline in temperature during the production period. The greater decline in temperature during production signifies the production of hot reservoir fluids. The temperature history as predicted by the reservoir simulator has many of the same features as that from the semi-analytical theory. The primary difference between the two curves is the temperature reached during injection. The semi-analytical theory predicts a steam temperature of roughly $365^{\circ} \mathrm{F}$ commensurate with the injection bottom hole pressure of $150 \mathrm{psi}$ whereas the reservoir simulator has a temperature during injection of about $240{ }^{\circ} \mathrm{F}$. The temperature is lower in the simulation and thus the oil viscosity is greater as compared to the semianalytical theory. Because the oil viscosity is greater in the simulation, oil drains more slowly reducing the cumulative oil production as compared to the semi-analytical theory. No reason for the discrepancy in temperature is apparent. 


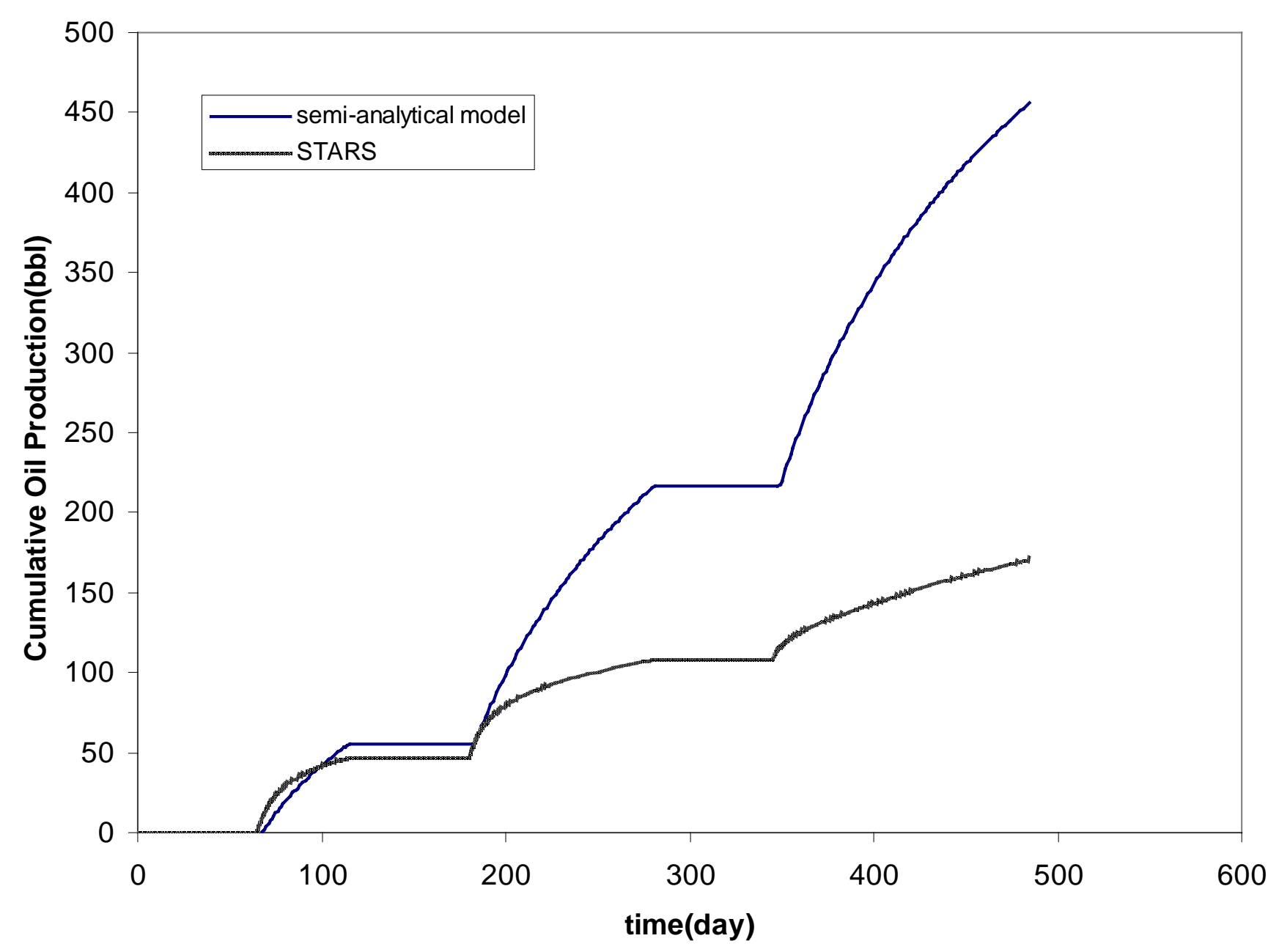

Figure 2. Comparison of semi-analytic model and reservoir simulation results.

Despite the lack of qualitative agreement, the semi-analytical theory is useful to display the relative sensitivity of various reservoir parameters. Figure 4 illustrates the effect of increasing the injection period to 100 days to increase the amount of energy input to the system. Again, three cycles are simulated. Figure 4 also shows the result obtained with 45 days of injection, as above. The additional heat benefits production in all cycles and, for instance, increases the cumulative oil recovered in the second cycle by almost $50 \%$.

On the other hand, the semi-analytical model is also helpful to illustrate parameters that have little influence on the results expected. Figure 5 summarizes the results from examining the sensitivity of production to heat losses to the overburden by adjusting the thermal conductivity of the overburden. Both semi-analytical and reservoir simulation results are given. Both display the same qualitative behavior and illustrate that decreasing the heat losses by one half changes the cumulative production very little. Thus, heat losses to the overburden are relatively unimportant during the initial stages of cyclic steaming. Most of the heat injected remains in the productive interval enhancing production. 


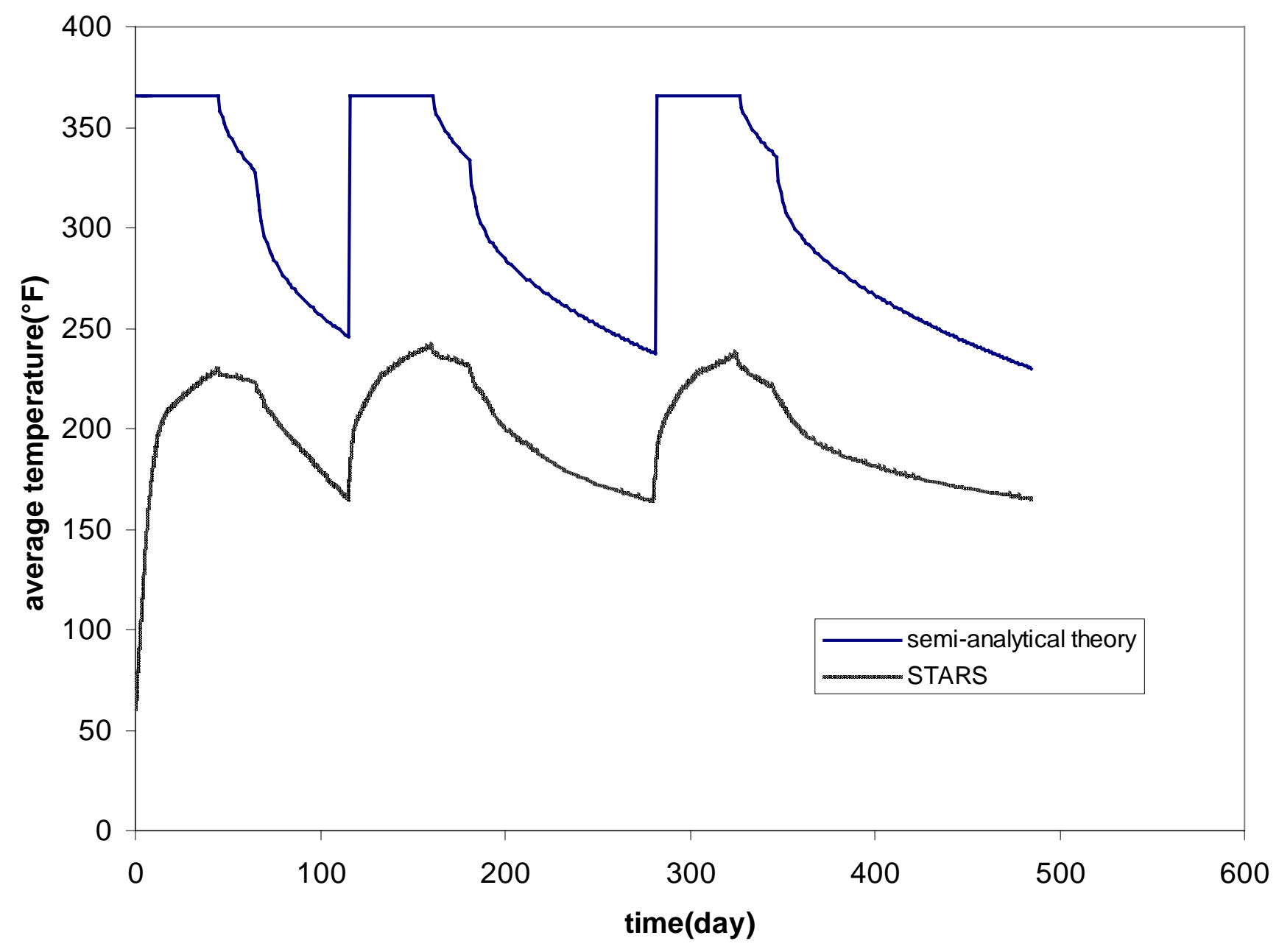

Figure 3. Comparison of steam zone temperature 


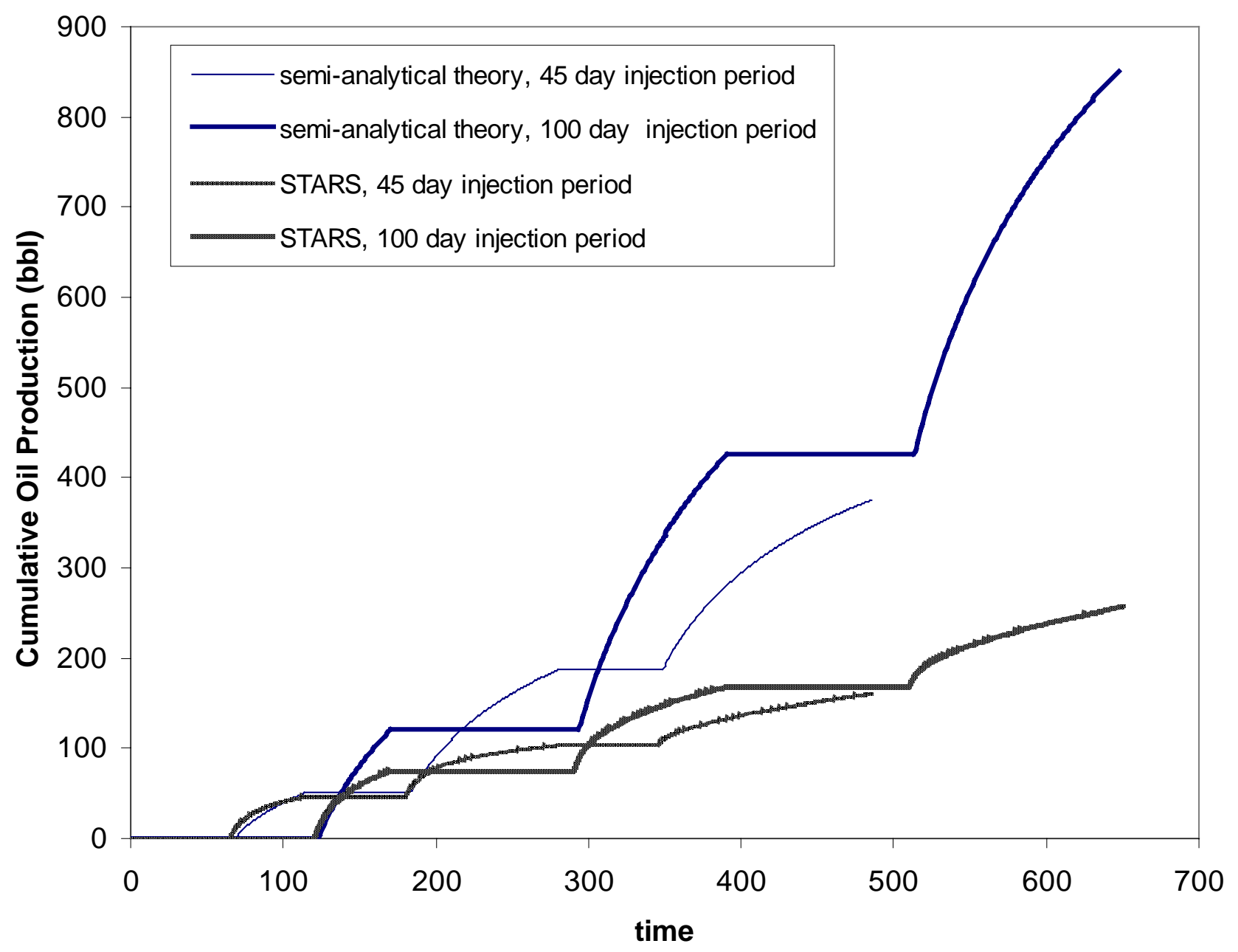

Figure 4. Sensitivity of cumulative oil production to the duration of injection. 


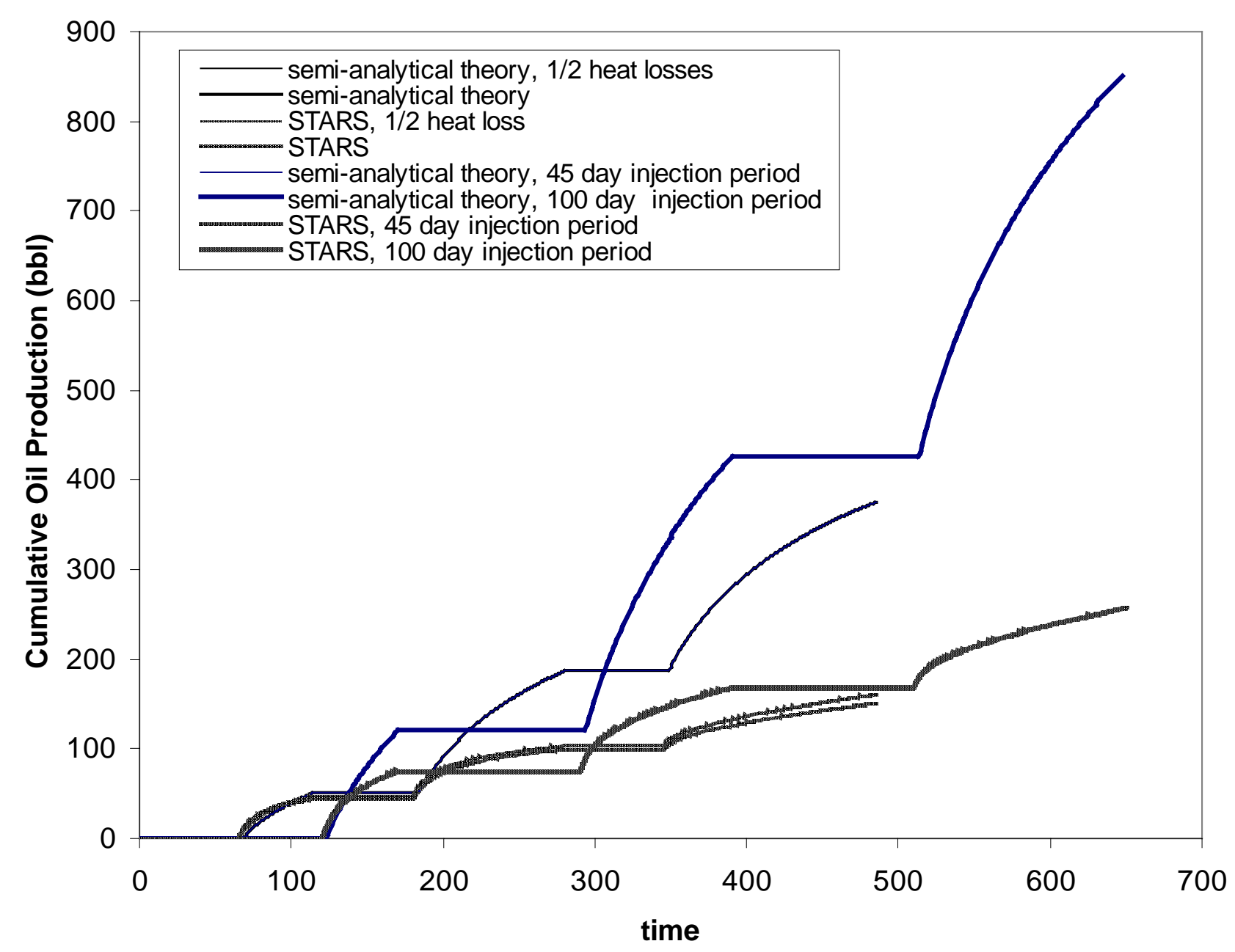

Figure 5. Sensitivity of cumulative oil production to heat losses.

\section{Conclusions}

A simple model has been developed for the cyclic steam process with a horizontal well. It incorporates a new flow equation for gravity drainage of heavy oil. Important factors such as pressure drive, gravity drive, and shape of the steam zone are incorporated into the model. Limited comparisons of the model to numerical simulation results indicate that the model captures qualitatively the essential physics of the recovery process. Nevertheless, the lack of quantitative agreement suggests that further research is needed to refine the model and complete a full verification exercise.

Generally, cyclic steaming appears to be effective in heating the near well area and the reservoir volume surrounding a horizontal well. A sensitivity analysis shows that the process is relatively robust over the range of expected physical parameters. As such, cyclic steaming is an 
effective process to warm cold reservoirs containing viscous oil prior to continuous steam injection processes such as steam-assisted gravity drainage.

\section{References}

Aziz, K. and Gontijo J.E.: "A Simple Analytical Model for Simulating Heavy-oil Recovery by Cyclic Steam in Pressure-Depleted Reservoirs", paper SPE 13037 presented at the 59th Annual Technical Conference and Exhibition, Houston, September 16-19, 1984.

Boberg, T.C. and Lantz, R.B.: "Calculation of the Production Rate of a Thermally Stimulated Well”, J.Pet.Tech. (Dec. 1966).

Farouq Ali, S.M.: "Steam Injection Theories - A Unified Approach", paper SPE 10746 presented at the California Regional Meeting of the SPE, San Francisco, March 24-26, 1982.

Jones, J.: "Cyclic steam Reservoir Model for Viscous Oil, Pressure-depleted, Gravity-drainage Reservoirs", SPE 6544, 47th annual California Regional Meeting of the SPE of AIME, Bakersfield, April 13-15, 1977.

Myhill, N.A. and Stegemeier, G.L.: "Steam Drive Correlation and Prediction”, J. Pet. Tech. (Feb. 1978) 173-182.

Prats, M.: "Thermal Recovery", Monograph - Volume 7, SPE of AIME, Henry L. Doherty Memorial Fund of AIME, 1982.

Van Lookeren: "Calculation Methods for Linear and Radial Steam Flow in Oil Reservoirs", J.Pet.Tech. (June, 1983) 427-439.

Vogel, V.: "Simplified Heat Calculation of Steamfloods", paper SPE 11219 presented at the 57th SPE Annual Fall Technical Conference and Exhibition, New Orleans, LA, September 2629, 1982. 


\title{
Efficiency and Oil Recovery Mechanism of Steam Injection into Low Permeability, Hydraulically Fractured Reservoirs
}

\author{
(Task 2b)
}

\section{Introduction}

Low-permeability fractured reservoirs contain a large volume of the worldwide oil resource (Saidi, 1983), yet production from these reservoirs has been modest. Primary production is low, usually $5 \%-10 \%$ of the original oil-in-place. Furthermore, waterfloods commonly suffer from low injectivity, poor sweep, and injector-to-producer linkage. These factors tend to degrade waterflood's effectiveness and have facilitated an attempt to use enhanced recovery techniques. At Teapot Dome Field in central Wyoming steam was injected into in a low-permeability fractured reservoir containing a light oil (32 ${ }^{\circ}$ API) (Olsen et al., 1992; Olsen et al., 1993; Doll et al., 1995). While this reservoir did not perform up to initial expectations, it showed that steam injection was feasible. Also, the importance of steam quality and the need for a sizeable $S_{\circ} \phi$ (the volume of oil per unit rock volume) was more clearly understood. Additional examples include fractured carbonates such as Ghaba North in Oman (27 API). A study is under way to determine if steam can increase production economically in such fields (cf. Al-Hadhrami and Blunt, 2001). Also, a pilot in the Yates Field of west Texas (30 API) has initiated and is showing promising results (Moritis, 2002).

The South Belridge field (California) has undergone a number of steam injection pilot tests into low permeability $(0.1 \mathrm{md})$ fractured reservoir containing light oil. Initial results from two of these pilots have demonstrated that these reservoirs respond very well to steam injection (Johnston and Shahin, 1995; Kovscek et al., 1996 I, II). A larger field project is underway at South Belridge but, as of yet, no data are reported. For these reasons, and because a significant amount of information about diatomite was made available to us, this study uses diatomite as its basis. Nevertheless, the work is generally applicable to low permeability fractured reservoirs that contain light oil.

The response to the steam injection at South Belridge was prompt and significant (Johnston and Shahin, 1995; Kovscek et al., 1996A, 1996B); however, the mechanisms for increased recovery are not fully understood. Subsequent pilots also showed good response, and field operators are considering expanding the pilot program to a much larger region. This work determined why the steamflood results were so much better than the water flood results, the mechanisms that are most important for recovery in this type of system, and how fluid displacement banks form. Additionally, this topic developed a better understanding of why steamdrive displacement in high porosity rock may be considered stable. Results have already been presented in the literature (Hoffman and Kovscek 2003, 2004). 


\section{Model Framework}

The simulator STARS (CMG, 2002) was used to perform various simulations of steam injection into low permeability, high porosity rock. One-dimensional and two dimensional (vertical and areal) calculations were made. Rock properties were representative of South Belridge and the fluid description was compositional incorporating 3 distinct components for the oil. Full details of the model setup are documented in the literature (Hoffman and Kovscek 2003, 2004); hence, only abbreviated details are provided here.

\section{Results and Discussion}

Three thermal recovery mechanisms (vaporization, thermal expansion, and viscosity reduction) were compared using the areal model descriptions to gauge the relative importance of each mechanism in light oil steamflood. For all reservoir descriptions, the primary recovery mechanism during the initial stages of steam injection is thermal expansion (Hoffman and Kovscek 2003). After water breakthrough, the importance of vaporization and viscosity reduction increase relative to the other mechanisms. The contribution of viscosity reduction peaks around a pore volume of steam injection while vaporization grows continuously as the distillate bank moves through to the producer.

\section{Efficiency}

The incremental recovery accompanying steam injection for low permeability systems has been shown to be quite high (Johnston and Shahin, 1995; Kovscek et al., 1996 I, II). One of the main reasons is that as steam is injected heat penetrates the lower permeability regions via conduction even where injected fluid is not able to do so. Although this phenomenon is explored from both a vertical efficiency and areal efficiency point of view, permeability distribution is shown to be the major cause for efficiency differences.

Figure 1 shows the cumulative recovery for both the waterflood and steamflood cases for the 2D vertical model (Hoffman and Kovscek 2003). The black lines represent the original relative permeability curves, and the gray set of lines corresponds to the more oil-wet relative permeability functions. For both cases, the incremental recovery is more than $35 \%$ of initial oil in place when compared to waterflood. Oil saturation is reduced in all layers as a result of steam injection. The remaining oil saturation in the low permeability layers is around 0.10. Once the heat has entered the low permeability layers, it enhances recovery by thermal expansion, vaporization and viscosity reduction. On the other hand, the final oil saturation in the low permeability layers for the waterflood is close to the original oil saturation (0.50). Further, even the most permeable layers are swept better by steam. Vaporization reduces the residual oil saturation in these regions to about 1\%; whereas, in the waterfloods the same areas have about $10 \%$ oil remaining. 


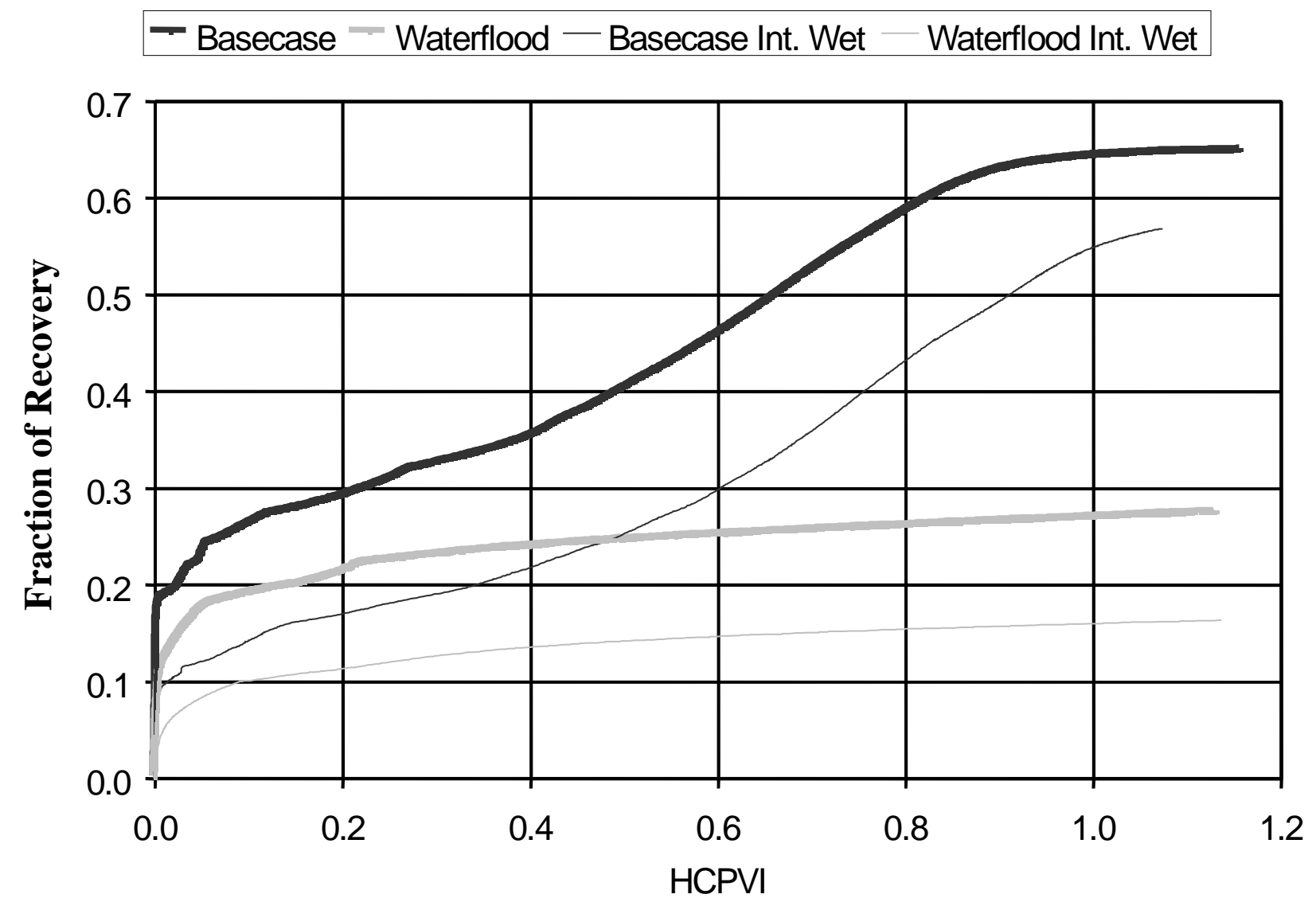

Figure 1. Fraction of recovery for multi-layer case.

The heterogeneous "thief" model is utilized to study the effects of areal heterogeneity on efficiency. Recall, both the producer and injector have hydraulic fractures that extend approximately three-quarters of the model width. In contrast to the vertical model that contains many thin heterogeneities, the areal heterogeneity model contains only three major high permeabilty zones, but they are wider. Figure 2 displays the cumulative recovery for the two different relative permeability curves. The difference between the thermal basecase and the waterflood case is much smaller for the areal case compared to the vertical case. For both relative permeability curves, the incremental recovery is around $15 \%$ of original oil in place. This recovery is about half that observed for the vertical case. 


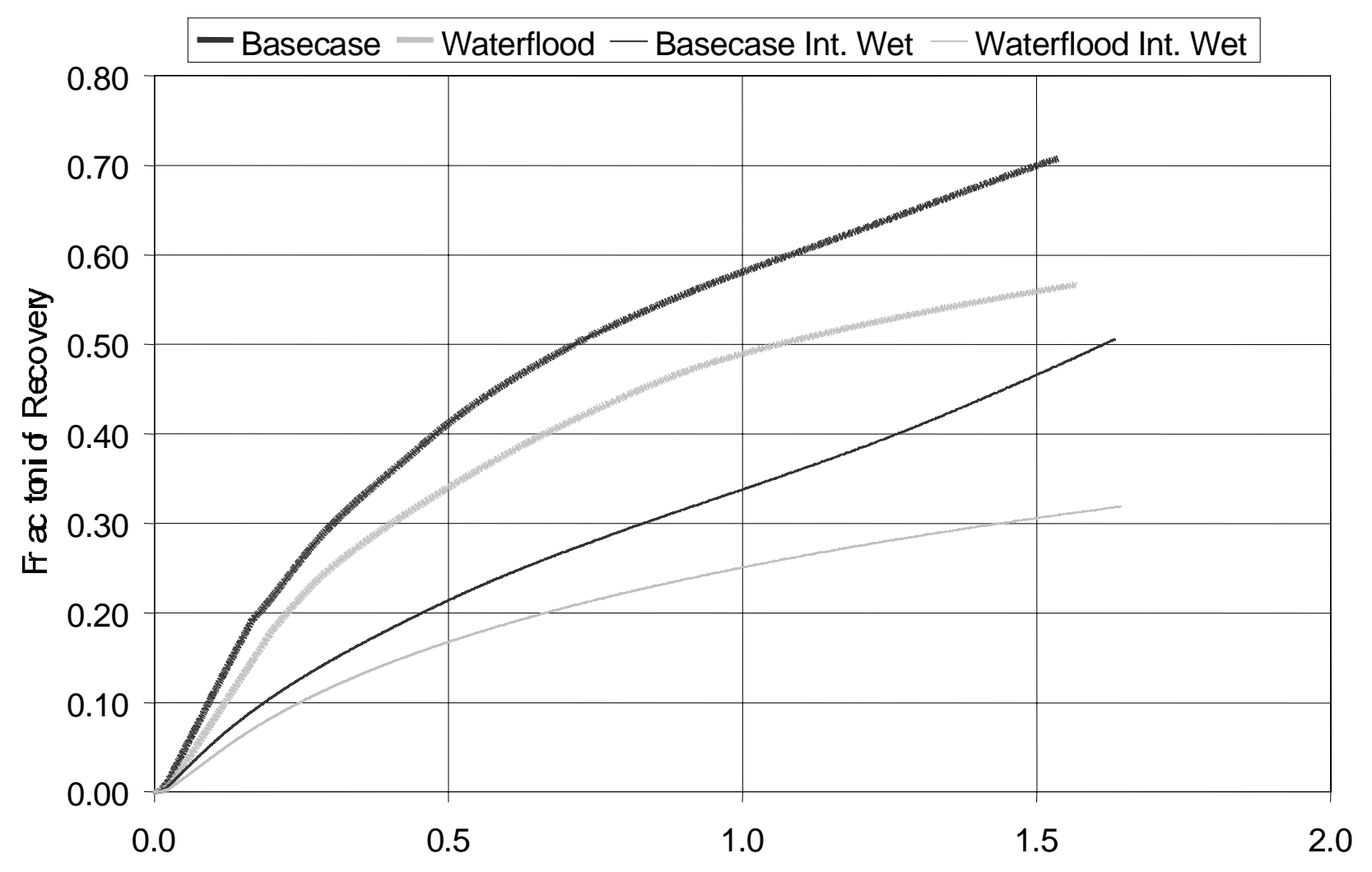

Figure 2. Cumulative recovery for two different relative permeability curves for the "thief" model. The thermal basecase is compared to the waterflood case.

\section{Stability in High Porosity Rock}

Instability occurs when an injected fluid bypasses a portion of the in-situ fluid that it is trying to displace. This often occurs when a fluid of high mobility displaces a fluid of lower mobility. Therefore, injection of a highly mobile gas such as steam would not appear to be effective in recovering viscous crude oils. However, the condensable nature of steam provides a self-stabilizing effect. As an unstable extension of steam begins to form into the cooler reservoir, heat dissipates from the extension to the reservoir. With loss of heat, the steam condenses to water and the instability is suppressed (Ali, 1982). The rate at which the heat will dissipate from the finger is a function of the thermal conductivity and heat capacity of the system along with the temperature difference between the steam and the reservoir. Analytical techniques as well as reservoir simulation (Hoffman and Kovscek, 2004) were used to examine the stability of steam displacement of oil in low permeability rock.

For a pressure of $500 \mathrm{psi}$ and less, the steam front is stable for all porosity values in our range. Above $2500 \mathrm{psi}$, the front will be unstable for all porosity values. Steam injection pressures ranging from 500 to 1200 psi have been reported for diatomite projects (Kovscek et al., 1996 A, 1996B). It is shown that the more porous diatomite layers could be close to the stability limit and unstable at the largest injection pressures. The effect of heterogeneity, however, is much 
more important with respect to steam flow than is the stability of the steam-water-oil displacement.

\section{Summary}

Table 1 compares breakthrough time for a homogeneous reservoir, a heterogeneous model that is relatively uncorrelation (Geomodel), and a heterogeneous model with high permeability "thief" zone connecting injection and producer. The last two columns of Table 1 compare how much faster a high porosity model (average porosity of 50\%) break through compared to a sandstone-type (25\% porosity) models.

Overall, there are noticeable differences between the stability of the displacing front as a function of the porosity. However, the differences varied considerably depending on geologic description and relative permeability conditions. The most difference observed is for the thief models, where the high porosity runs break through $23 \%$ and $28 \%$ faster than the sandstone porosity runs. The homogeneous models have the smallest difference $(4 \%-5 \%)$. Relative permeability has a small effect; the difference between the original and more oil-wet relative permeability curves change the answer only a few percent. Although porosity does have a role in stability, heterogeneity has a much greater affect. For all cases, the thief models break through in about half the time of the geomodel or homogeneous models.

\section{Table 1. Breakthrough Comparisons in HCPVI for the Three Increasing Porosity Cases}

\begin{tabular}{|c|l|c|c|c|c|c|}
\hline & \multirow{2}{*}{} & Mean Porosity $(\phi)=$ & \multicolumn{3}{|c|}{ Breakthrough (HCPVI) } & \multicolumn{2}{c|}{$\%$ faster than SS } \\
\cline { 3 - 7 } & $25 \%$ & $50 \%$ & $70 \%$ & $50 \%$ & $70 \%$ \\
\hline Original & Homogeneous & 0.38 & 0.36 & 0.36 & $4 \%$ & $5 \%$ \\
Relative & Geomodel & 0.35 & 0.31 & 0.29 & $10 \%$ & $16 \%$ \\
Permeability & Thief & 0.20 & 0.15 & 0.14 & $23 \%$ & $28 \%$ \\
\hline Oil-Wet & Homogeneous & 0.29 & 0.28 & 0.27 & $5 \%$ & $7 \%$ \\
Relative & Geomodel & 0.27 & 0.24 & 0.23 & $11 \%$ & $17 \%$ \\
Permeability & Thief & 0.18 & 0.14 & 0.12 & $23 \%$ & $29 \%$ \\
\hline
\end{tabular}

\section{Conclusions}

A pattern reservoir simulation study of steam injection into a hydraulically-fractured diatomite formation has been completed (Hoffman and Kovscek 2003, 2004). The information gained includes a better understanding of the recovery efficiencies, primary recovery mechanisms, and in-situ saturation and hydrocarbon composition profiles in steam drives. Multiple reservoir descriptions varied from homogeneous to quite heterogeneous. Viscosity, relative permeability, oil-phase composition, and other rock and fluid properties are realistic. 
Comparison among steamflood and waterflood results shows that the incremental recovery ranges from about $15 \%$ up to around $35 \%$ of initial oil in place. The distribution of permeability is a key factor in determining incremental recovery. As permeability becomes more correlated in a direction roughly parallel to pattern boundaries, recovery decreases. On the other hand, a highly variable, but uncorrelated, permeability distribution displays good recovery. Regardless of the distribution of permeability, it is apparent that steamflooding heterogeneous, low permeability, hydraulically-fractured systems significantly increases the recovery as compared to waterflood. It is likely that the recovery from low permeability naturally fractured reservoirs can be improved similarly using steam injection. These candidates suffer from problems similar to diatomite, such as below average primary recovery and poor waterflood performance.

For the reservoir descriptions and fluid properties employed here, thermal expansion of the hydrocarbon fluids accounts for about half of the incremental recovery early in the injection life, that is, until the cold water bank breaks through. At this point vaporization, viscosity reduction, and thermal expansion all contribute approximately equal amounts to the recovery. At late times and as the distillate bank approaches the producer, vaporization becomes the most important recovery mechanism.

A clearly differentiable distillate bank does not form. Instead there is a bank of distilled, condensed hydrocarbons and hot water. It is characterized by the lightest hydrocarbon component farthest downstream and grades to heavier components at its rear. The mixed distillate-hot-water bank is preceded by a cold water bank and followed by the steam front.

\section{References}

Al-Hadhrami, H. S. and Blunt, M. J.: Thermally Induced Wettability Alteration To Improve Oil Recovery in Fractured Reservoirs, SPE Reservoir Evaluation and Engineering 2001, June, 179-186.

CMG-Computer Modeling Group: STARS User Manual, Calgary, Alberta Canada, 2002.

Doll, T. E., Luers, D. K., Strong G. R., Schulte, R. K., Sarathi, P.S., Olsen, D. K. and Hendricks, M. L.: An Update of Steam Injection Operations at Naval Petroleum Reserve No. 3, Teapot Dome Field Wyoming: A Shallow Heterogeneous Light-Oil Reservoir, SPE 30286, presented at SPE International Heavy Oil Symposium in Calgary Alberta, June 1921,1995

Hoffman, B. T. and A. R. Kovscek, "Light-Oil Steamdrive in Fractured Low Permeability Reservoirs," SPE 83491, Proceedings of the SPE Western Region/AAPG Pacific Section Joint Meeting, Long Beach, CA, May 19-24, 2003.

Hoffman, B.T. and A. R. Kovscek, "Steamdrive Stability in High Porosity Rock," SPE 86985, Proceedings of the SPE International Thermal Operations and Heavy Oil Symposium and Western Regional Meeting, Bakersfield, CA, Mar 16-18, 2004. 
Johnston, R. M., and Shahin, G. T.: Interpretation of Steam Drive Pilots in the Belridge Diatomite, SPE 29621, presented at SPE Western Regional Meeting, Bakersfield, CA, March 8-10, 1995.

Kovscek, A. R., Johnson, R. M., and Patzek, T. W.: Interpretation of Hydrofracture Geometry During Steam Injection Using Temperature Transients I. Model Formulation and Verification, In Situ 1996, 20 (3) 251-285.

Kovscek, A. R., Johnson, R. M., and Patzek, T. W.: Interpretation of Hydrofracture Geometry During Steam Injection Using Temperature Transients II. Asymmetric Hydrofractures, In Situ 1996, 20 (3) 289-309.

Kovscek, A. R., R. M. Johnston, and T. W. Patzek: Evaulation of Rock/Fracture Interactions During Steam Injection Through Vertical Hydraulic Fractures, Society of Petroleum Engineers Production and Facilities 1997, 12(2), p. 100-105.

Moritis, G.: Biennial EOR Production Report - California steam EOR produces less; other EOR continues, Oil and Gas J., 2002, April 15, Tulsa, 72.

Olsen, D.K., Sarathi, P. S., Mahmood, S. M., Ramzel, E. B., and Roark, S. D.: Light Oil Steamflooding: A Laboratory Study of Oil Recovery from Oil-Wet and Water-Wet Porous Media from a 2-D Physical Model, SPE 23692, presented at SPE Latin American Petroleum Engineering Conference, Caracas, March 8-11, 1992.

Olsen, D. K., Sarathi, P. S., Hendricks, M. L., Schulte, R. K., and Giangiacomo, L. A.: Case History of Steam Injection Operations at Navel Petroleum Reserve No. 3, Teapot Dome Field, Wyoming: A Shallow Heterogeneous Light-Oil Reservoir, SPE 25786, presented at SPE International Thermal Operations Symposium in Bakersfield, February 8-10, 1993.

Saidi, A. M.: "Naturally Fractured Reservoirs," paper SPE 12270 presented at SPE Reservoir Simulation Symposium, San Francisco, November 15-18, 1983. 


\section{Porosity and Permeability Evolution Accompanying Hot Fluid Injection into Diatomite}

\section{(Task 2b)}

An experimental study of silica dissolution was performed to probe the evolution of permeability and porosity in siliceous diatomite during hot fluid injection such as water or steam flooding. This work is documented in a technical report (TR 123) and in the literature (Diabira et al., 2002); hence, only a summary is given here.

The results of this experimental investigation show that for some conditions of hot-water injection diatomite undergoes a reduction in porosity and permeability due to flow-induced compression and rearrangement of rock-mineral grains. The effects of this compression here were more severe than indicated by previous examinations of diatomite compressibility. In some cases, a roughly three-fold reduction in permeability was witnessed. Dissolution of the rock matrix is a slow process relative to compression and cannot compensate for reduction in permeability until very many pore volumes of fluid are injected. Based upon the results reported here, it would appear to be beneficial to consider the role of rock compression whenever hot fluid is injected into diatomite.

The pattern of dissolution observed by CT scans, SEM images, and visual inspection of cores is uniform dissolution of the rock matrix. No evidence of wormholes or other selective dissolution phenomena was witnessed. These observations concur with previous theoretical analyses of the dissolution of porous media.

\section{References}

Diabira, I., L. M. Castanier, and A. R. Kovscek, "Porosity and Permeability Evolution Accompanying Hot Fluid Injection into Diatomite," Petroleum Science and Technology, 19(9 and 10), 1167-1185 (2001). 


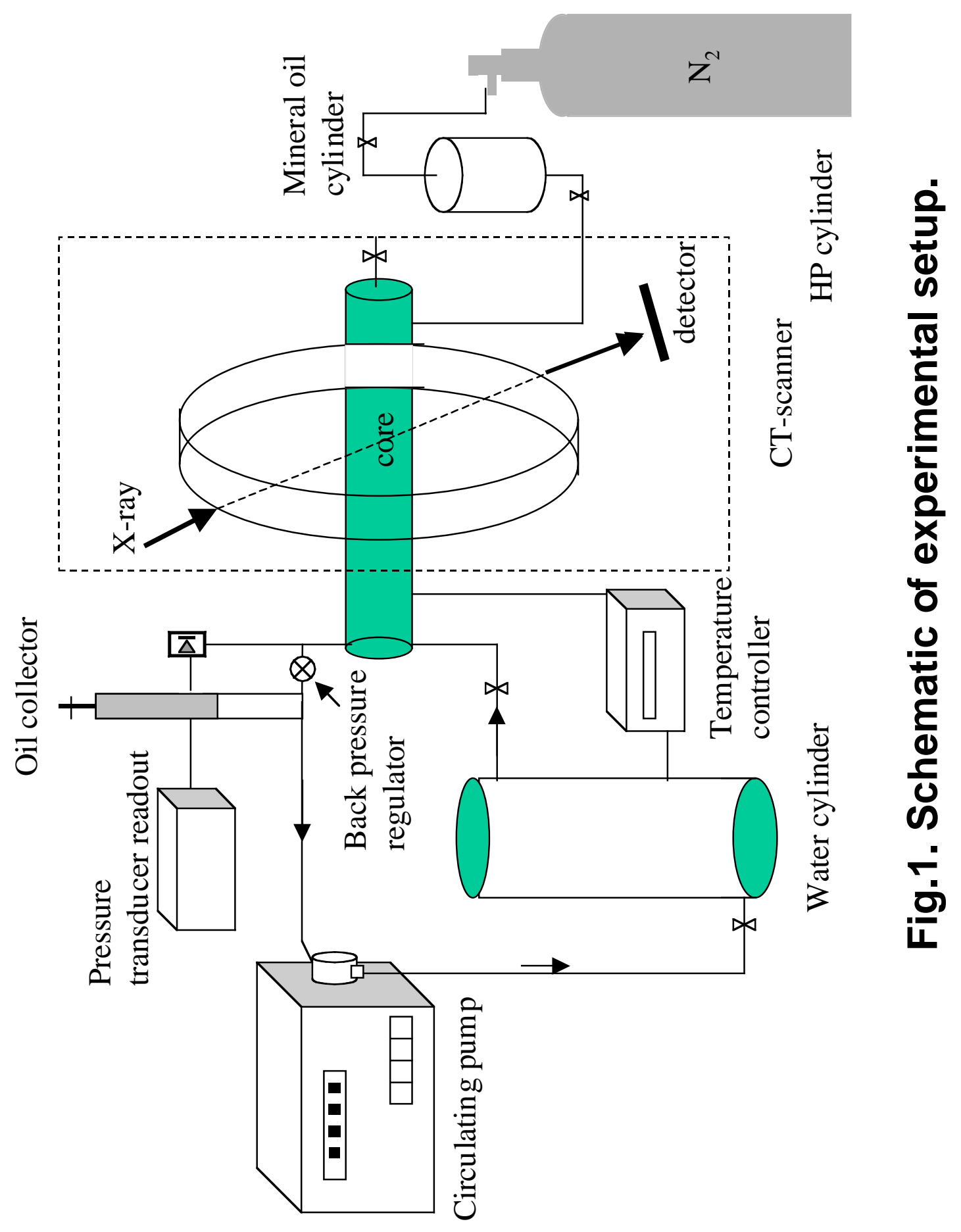




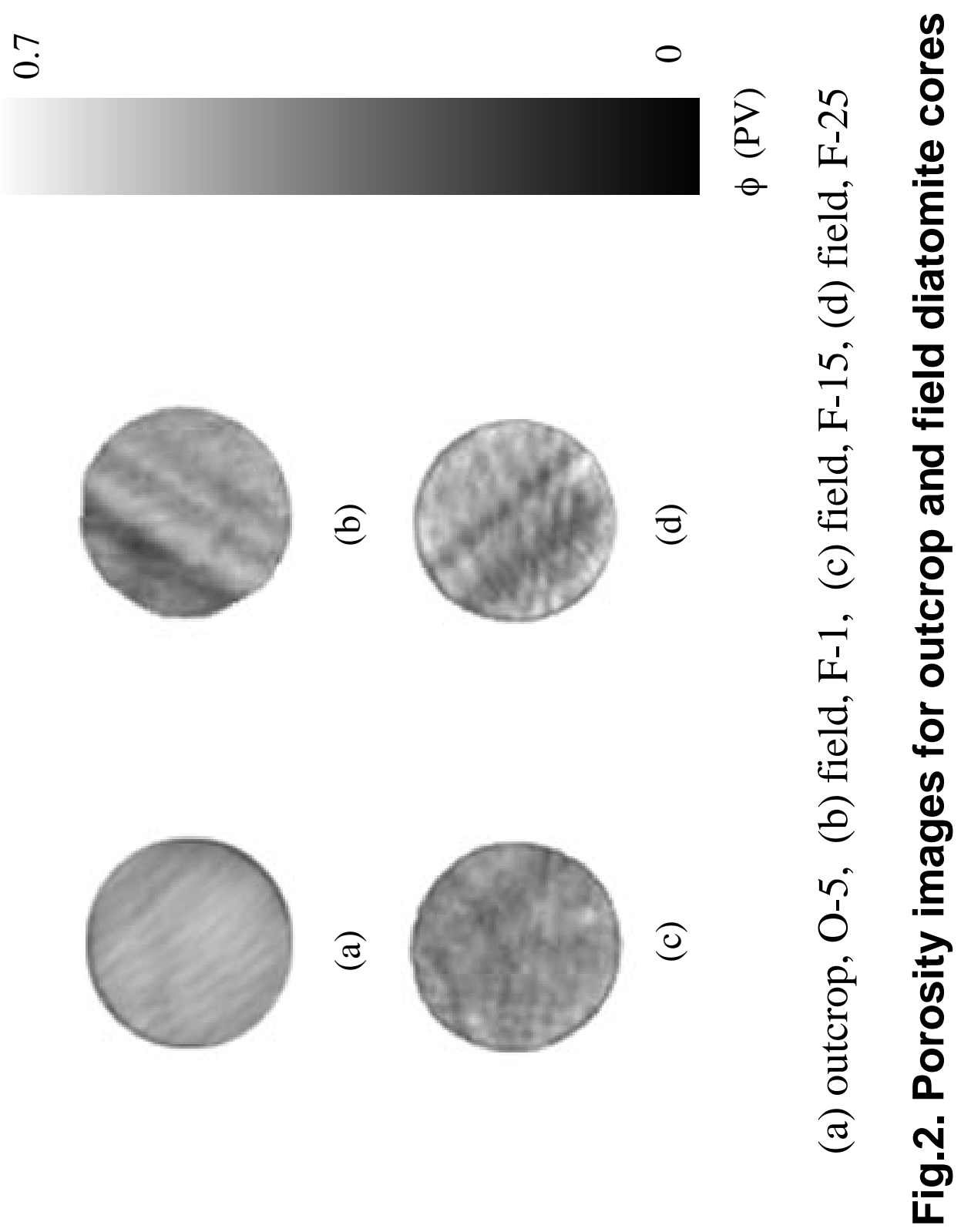




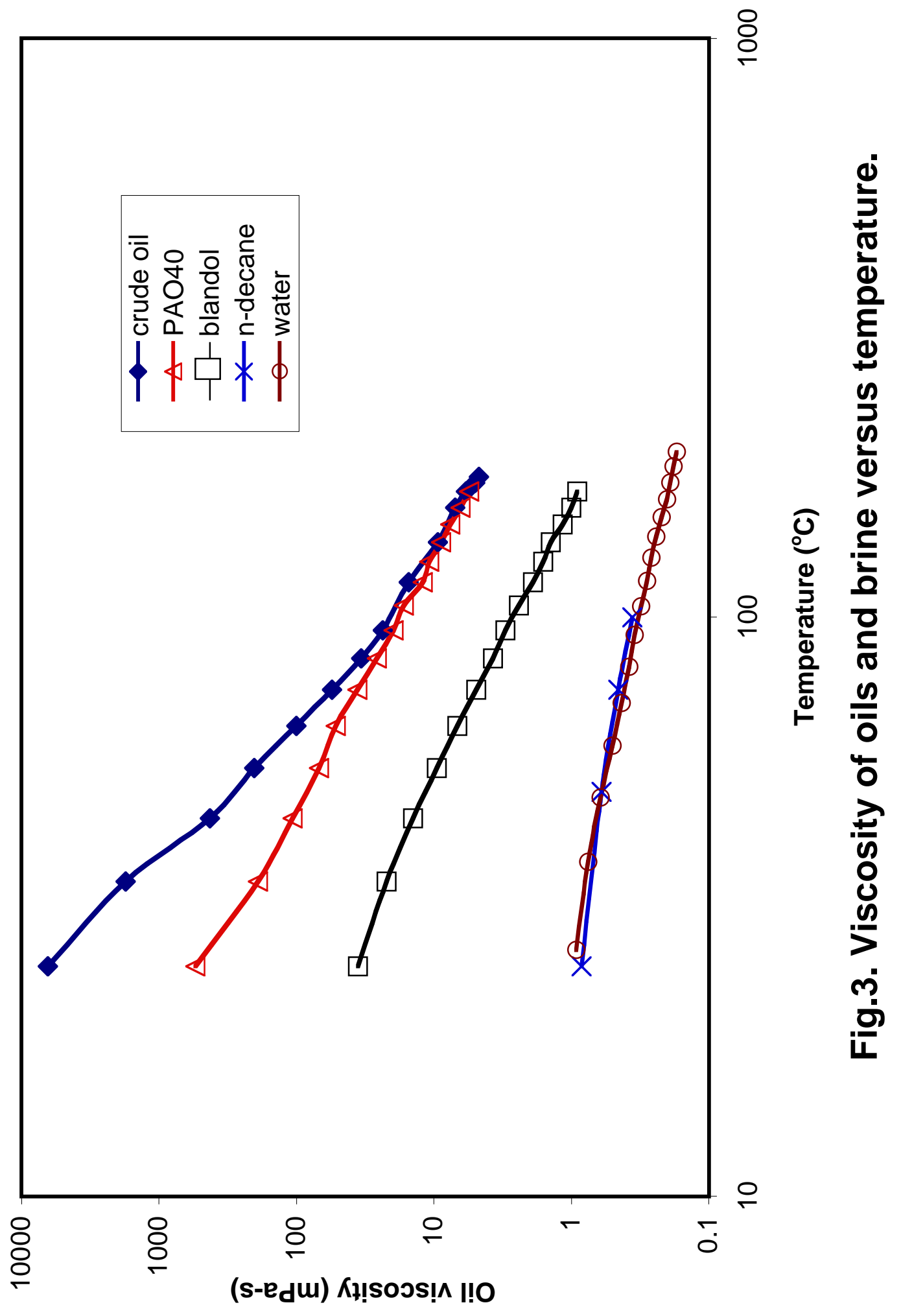




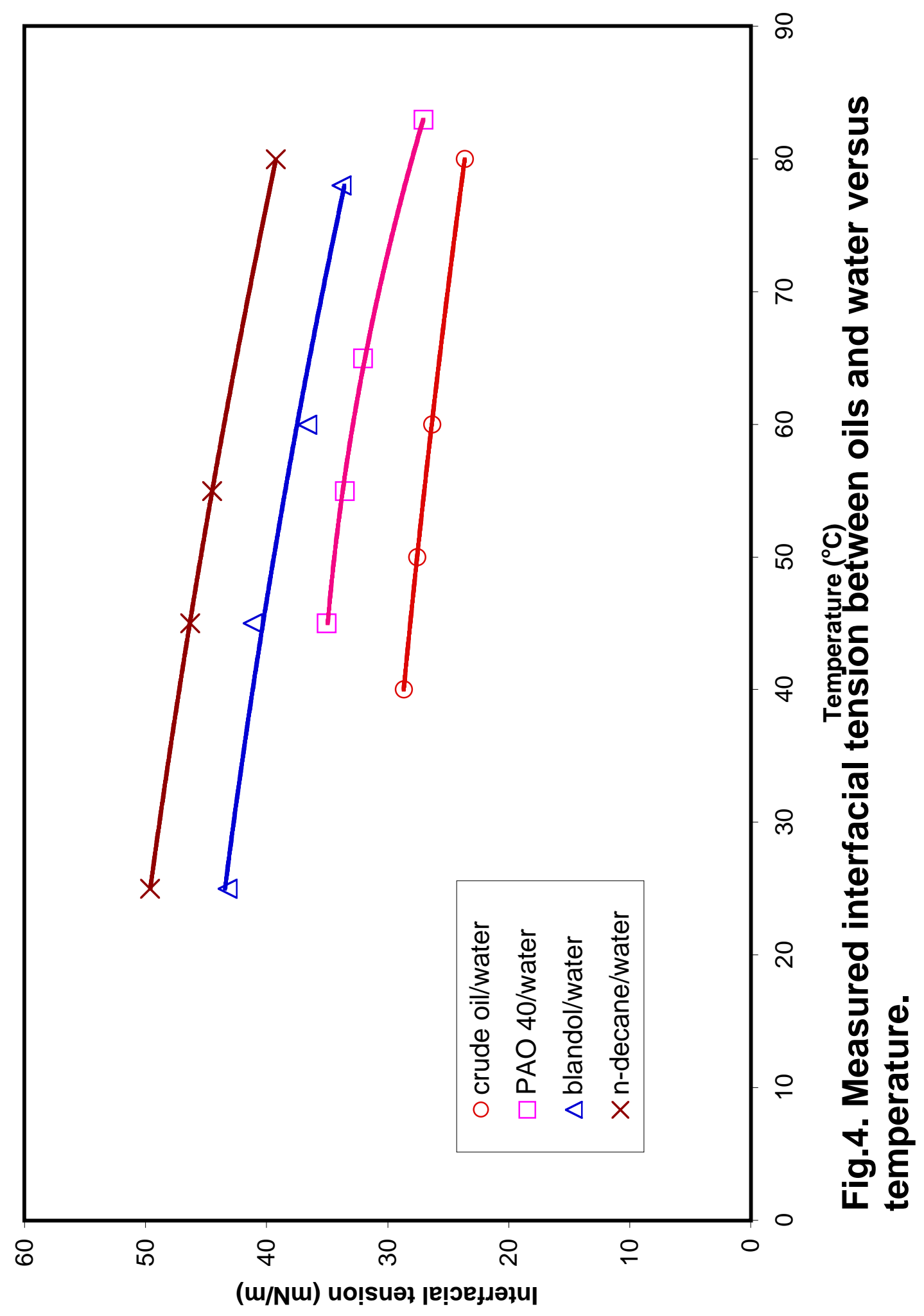




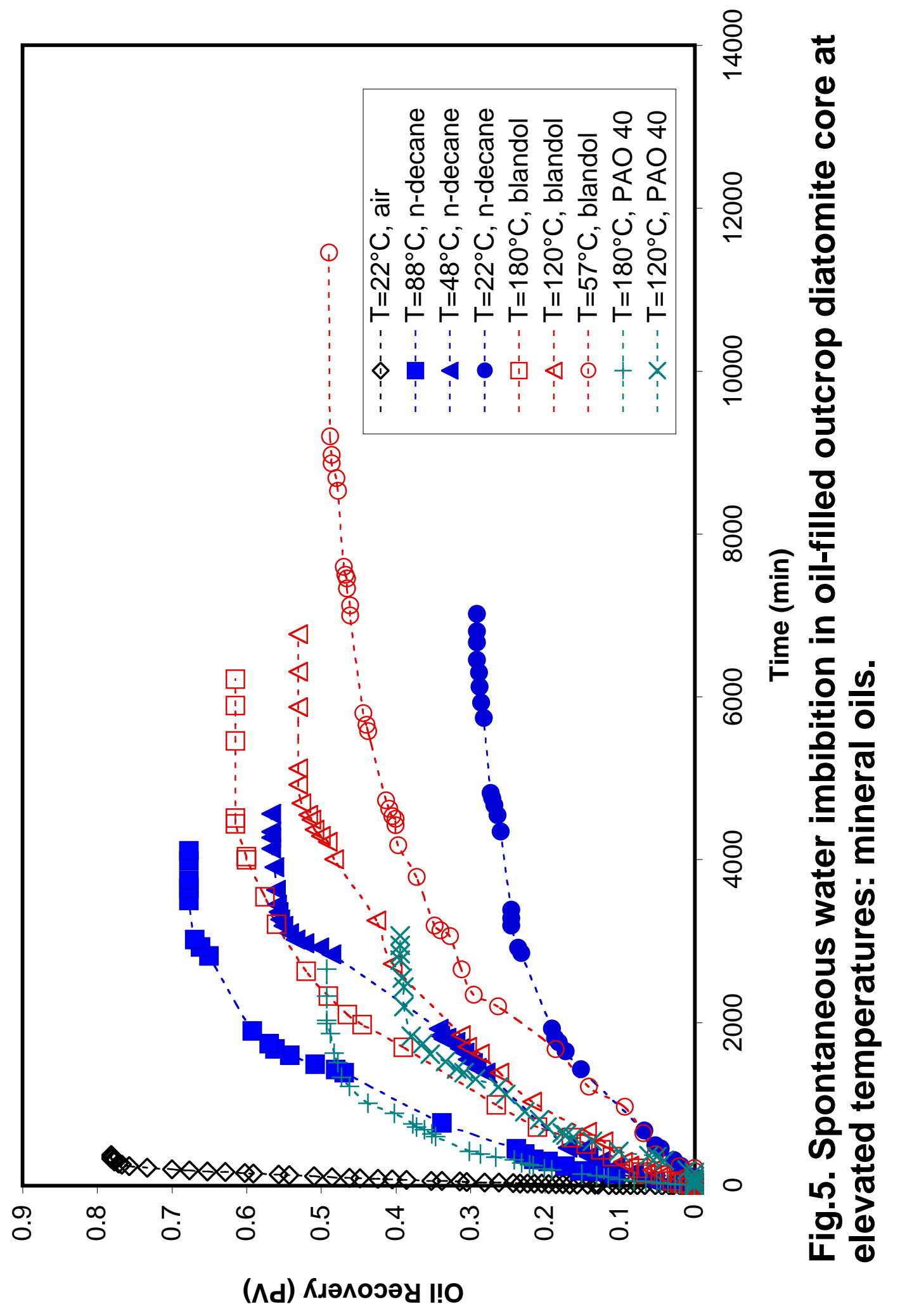




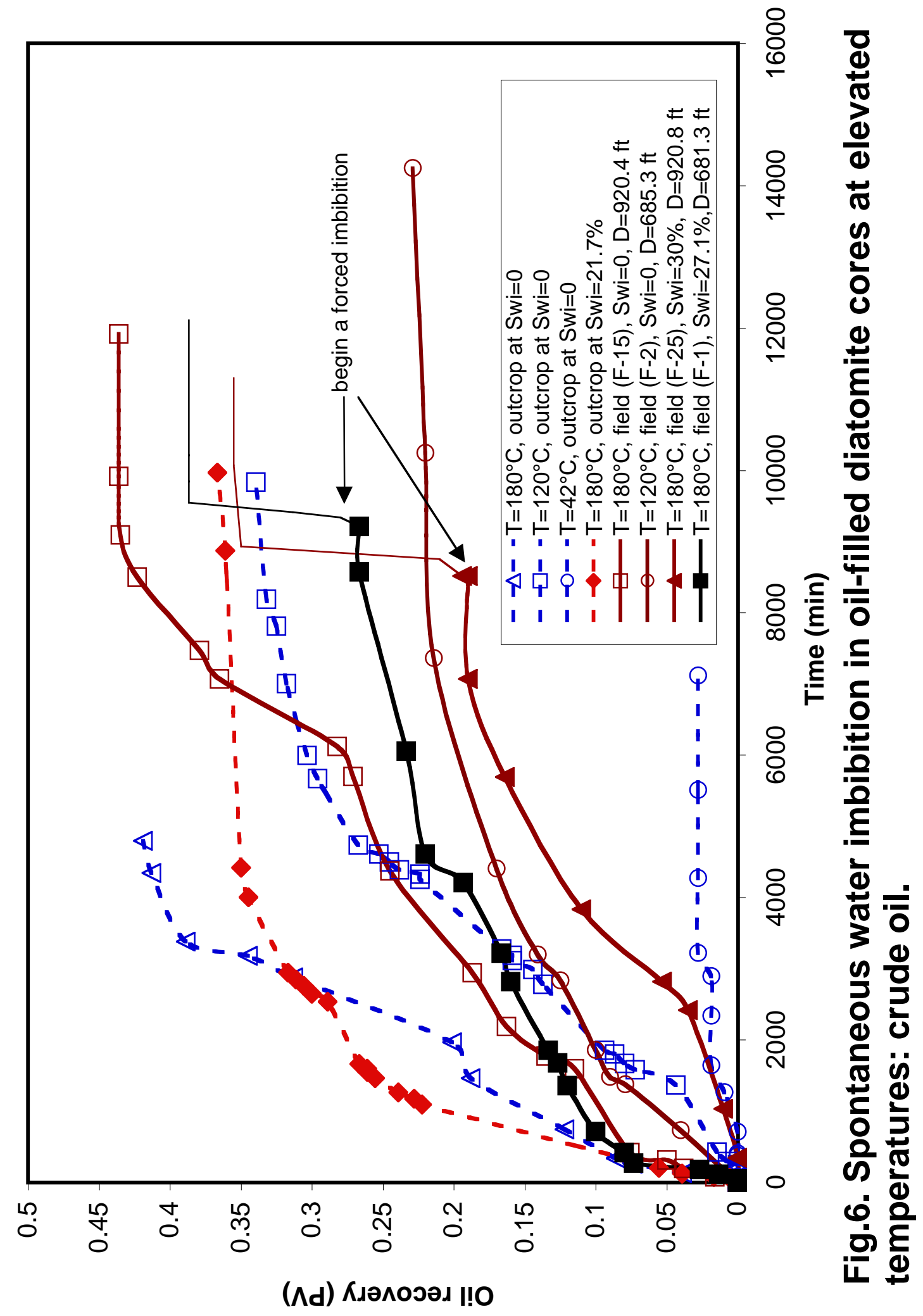




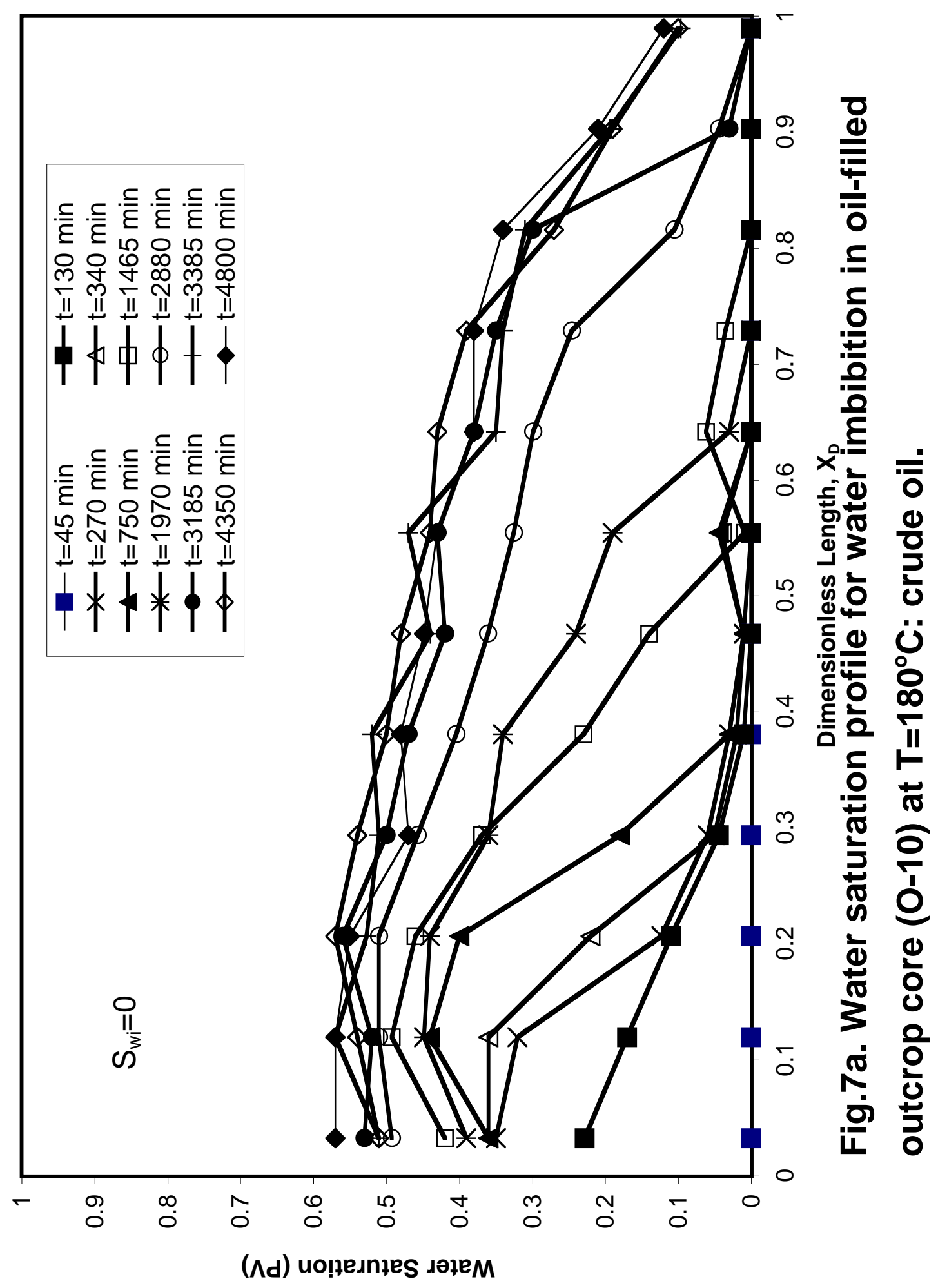




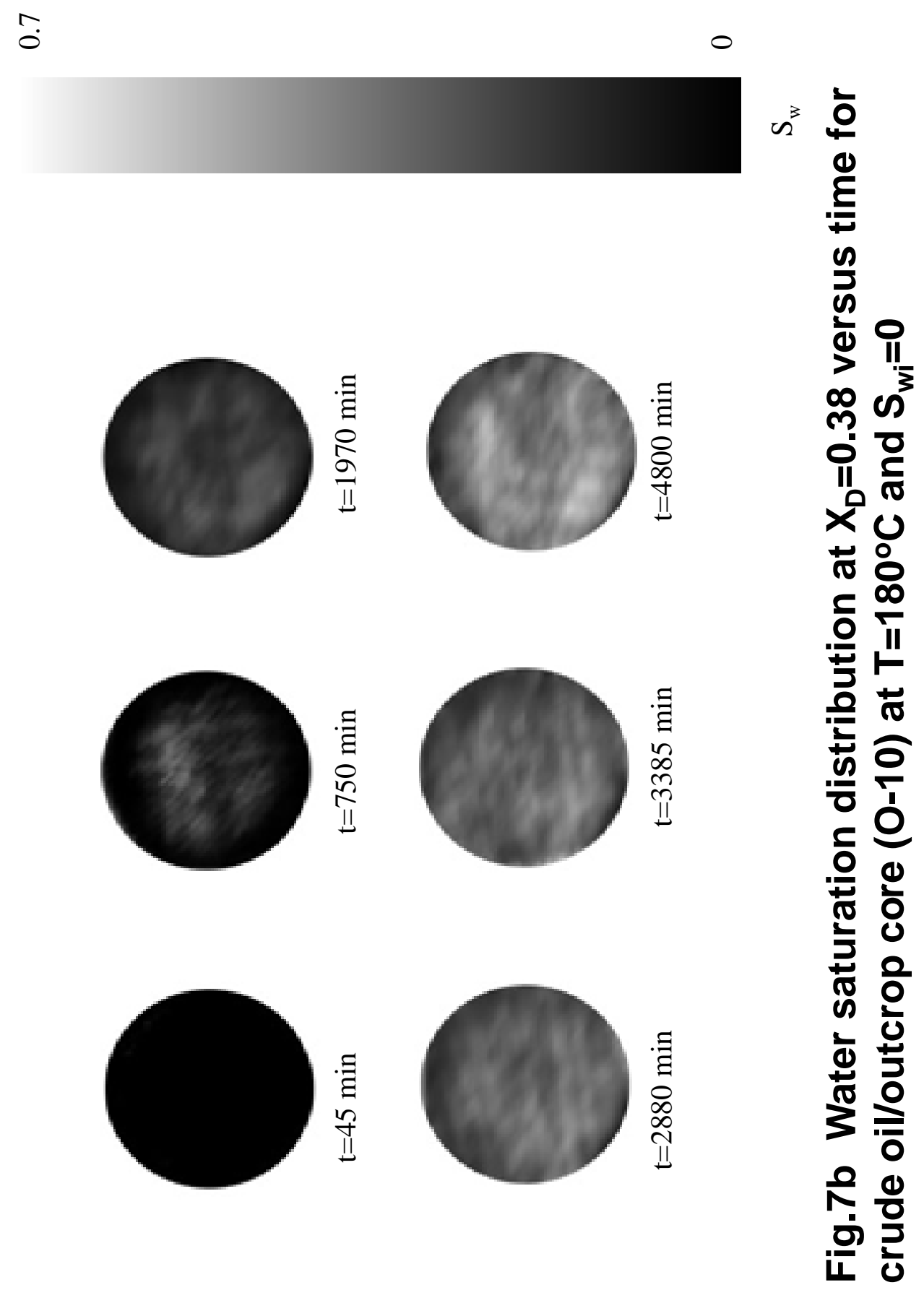




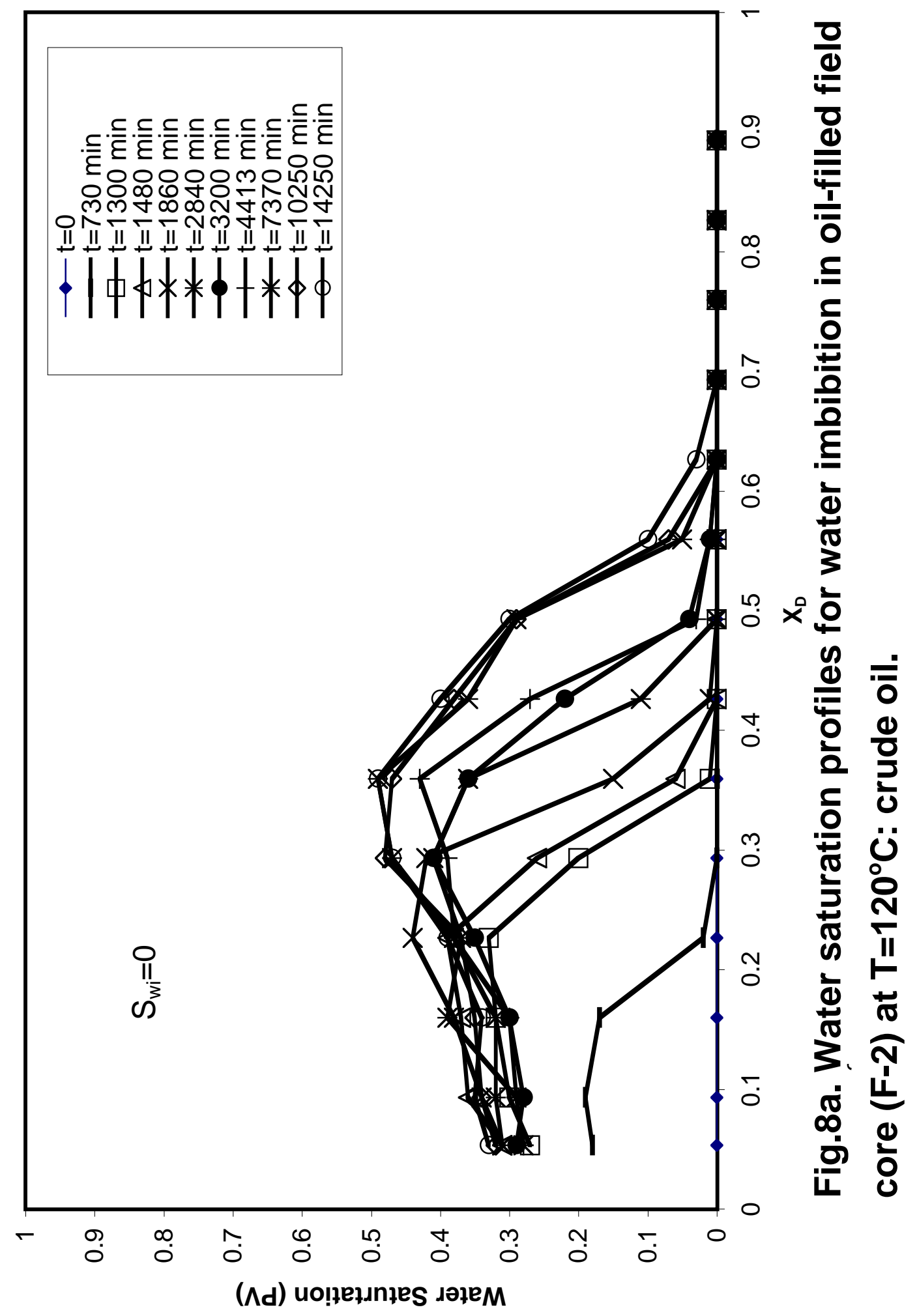




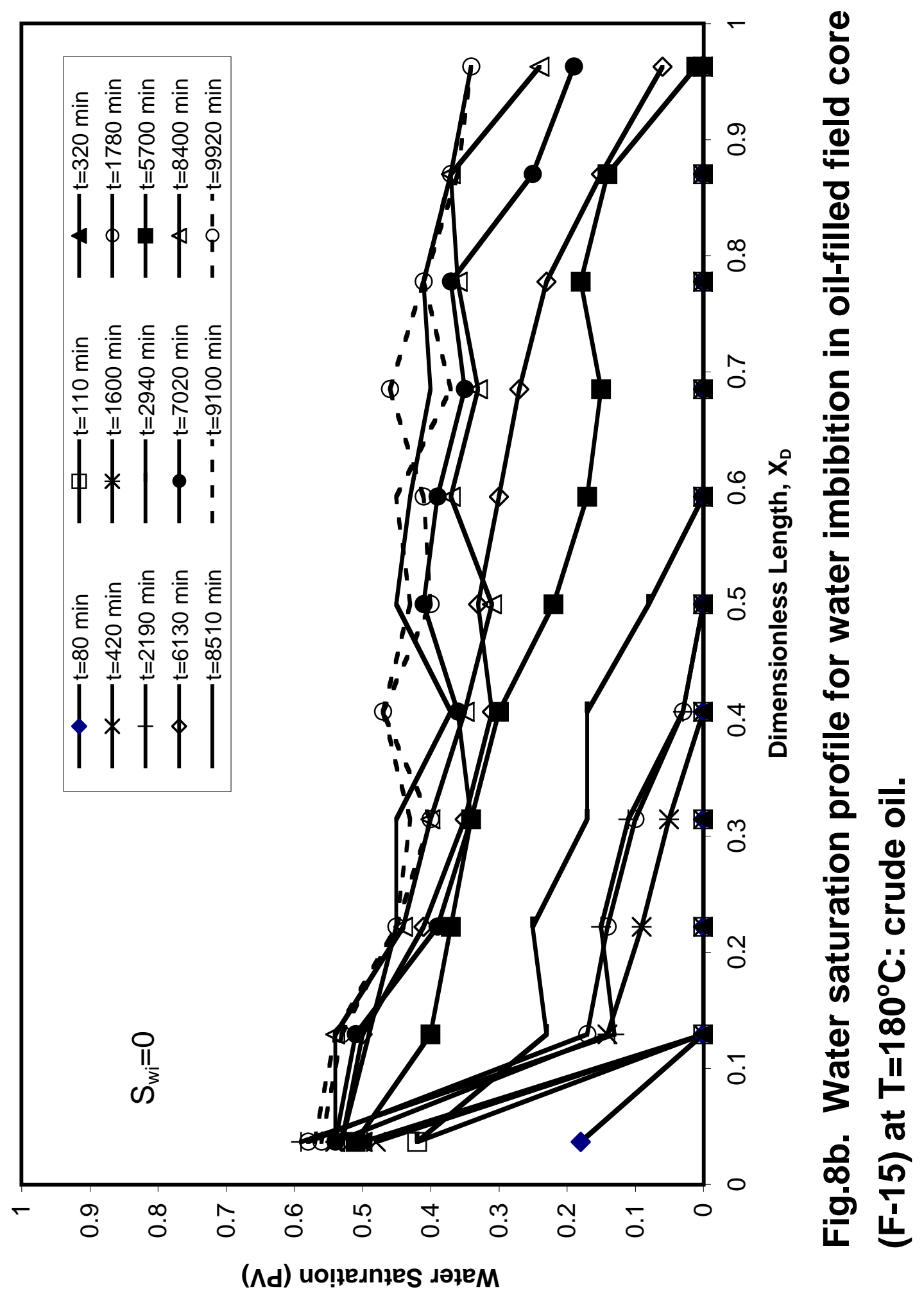



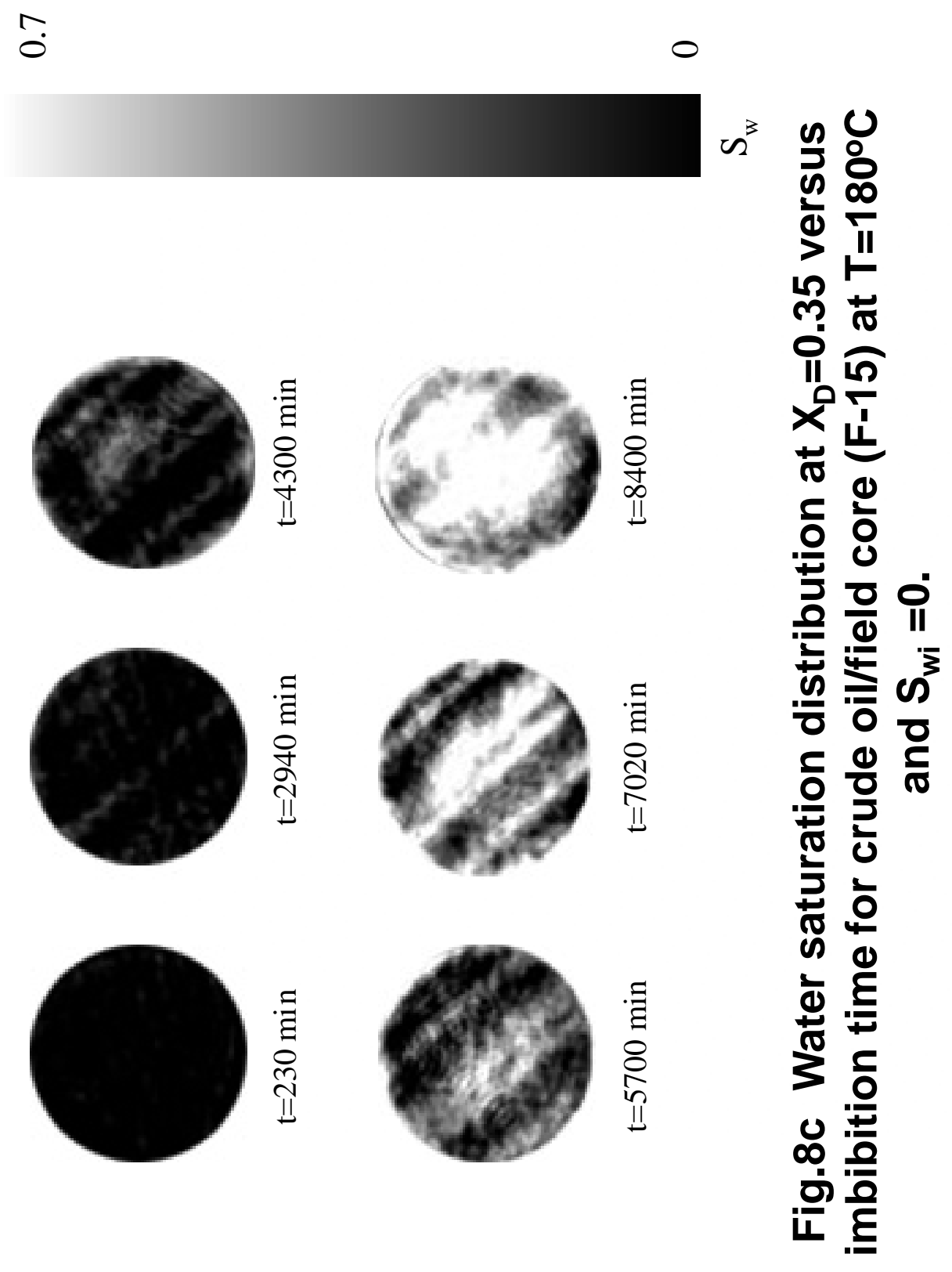


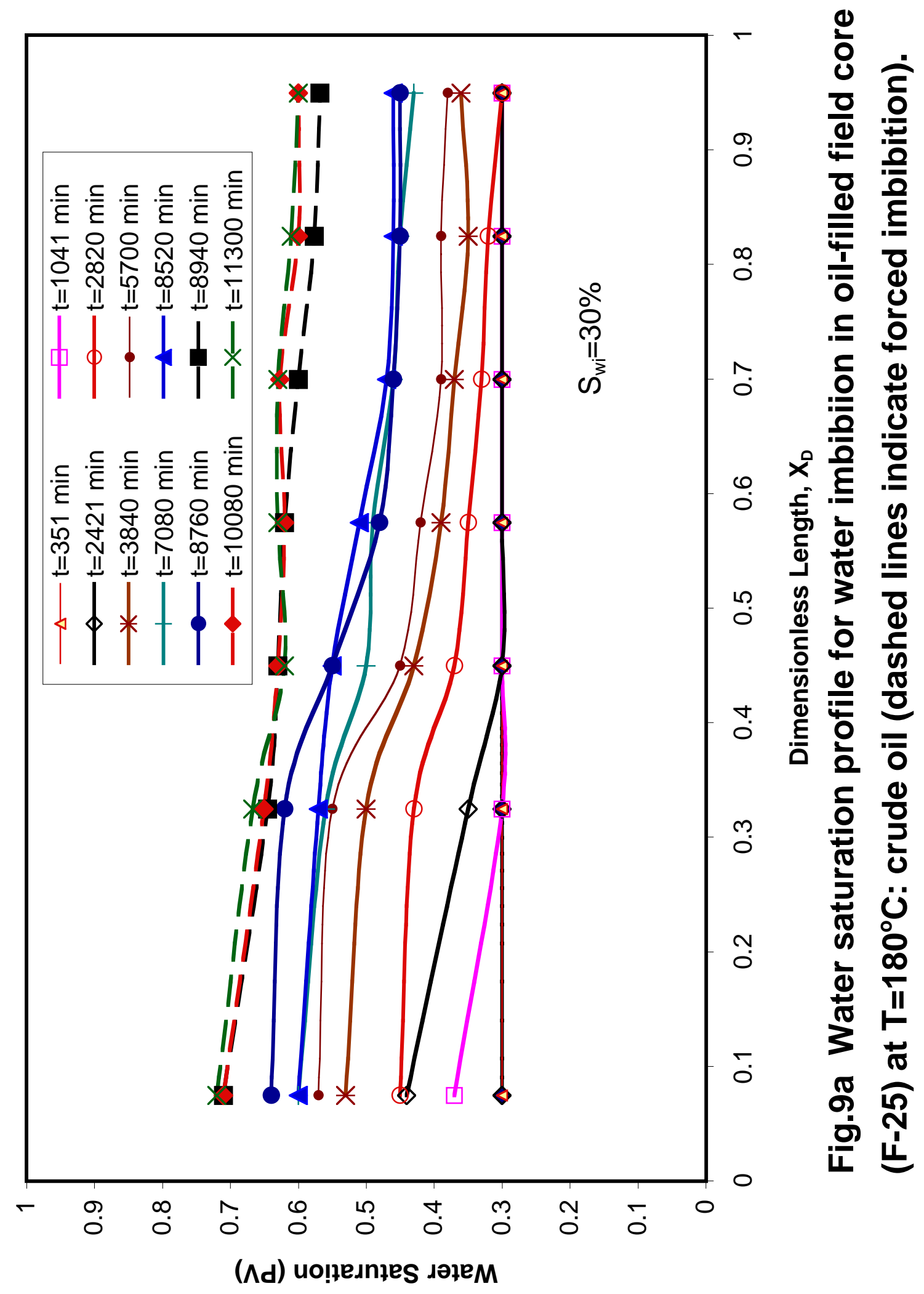



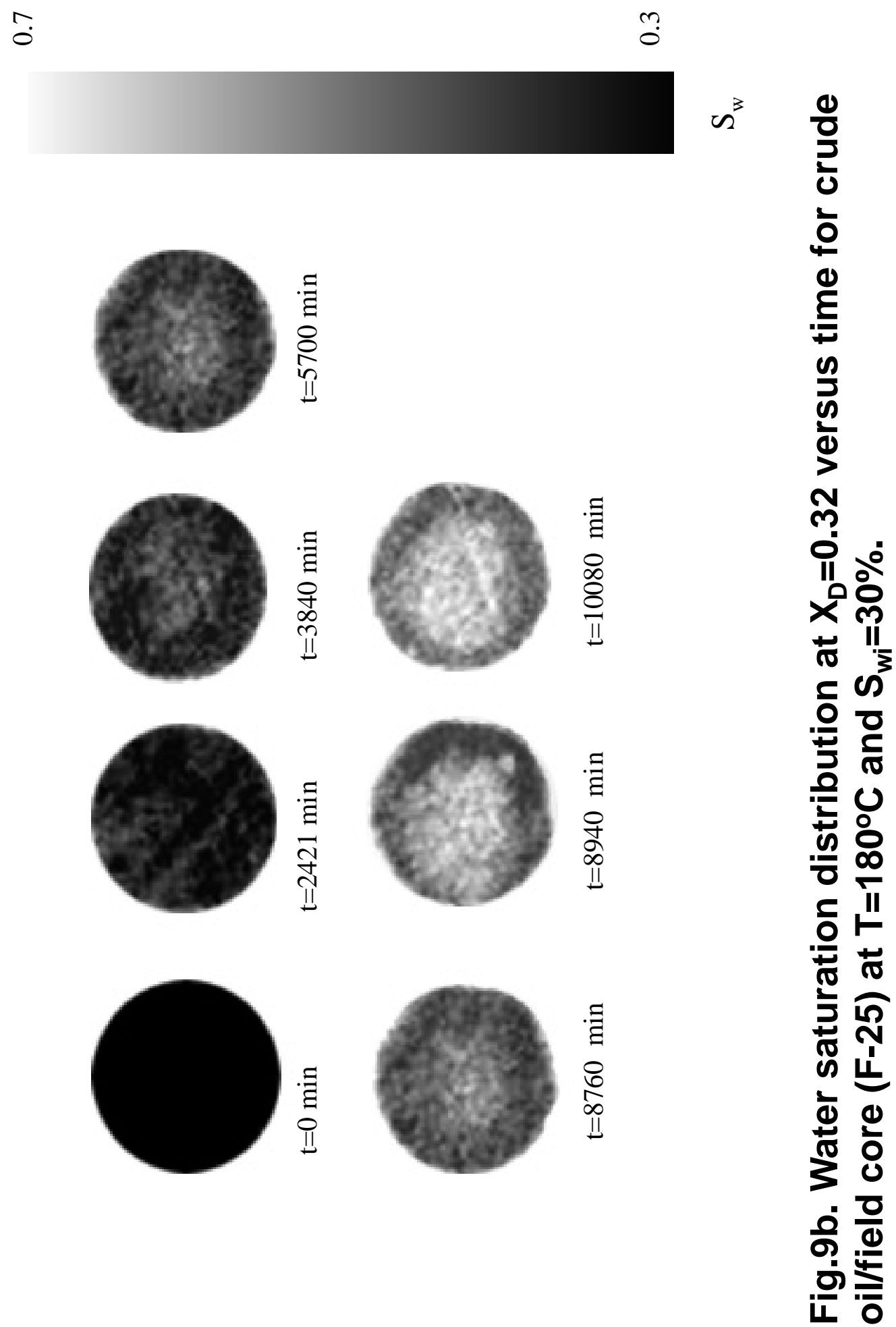


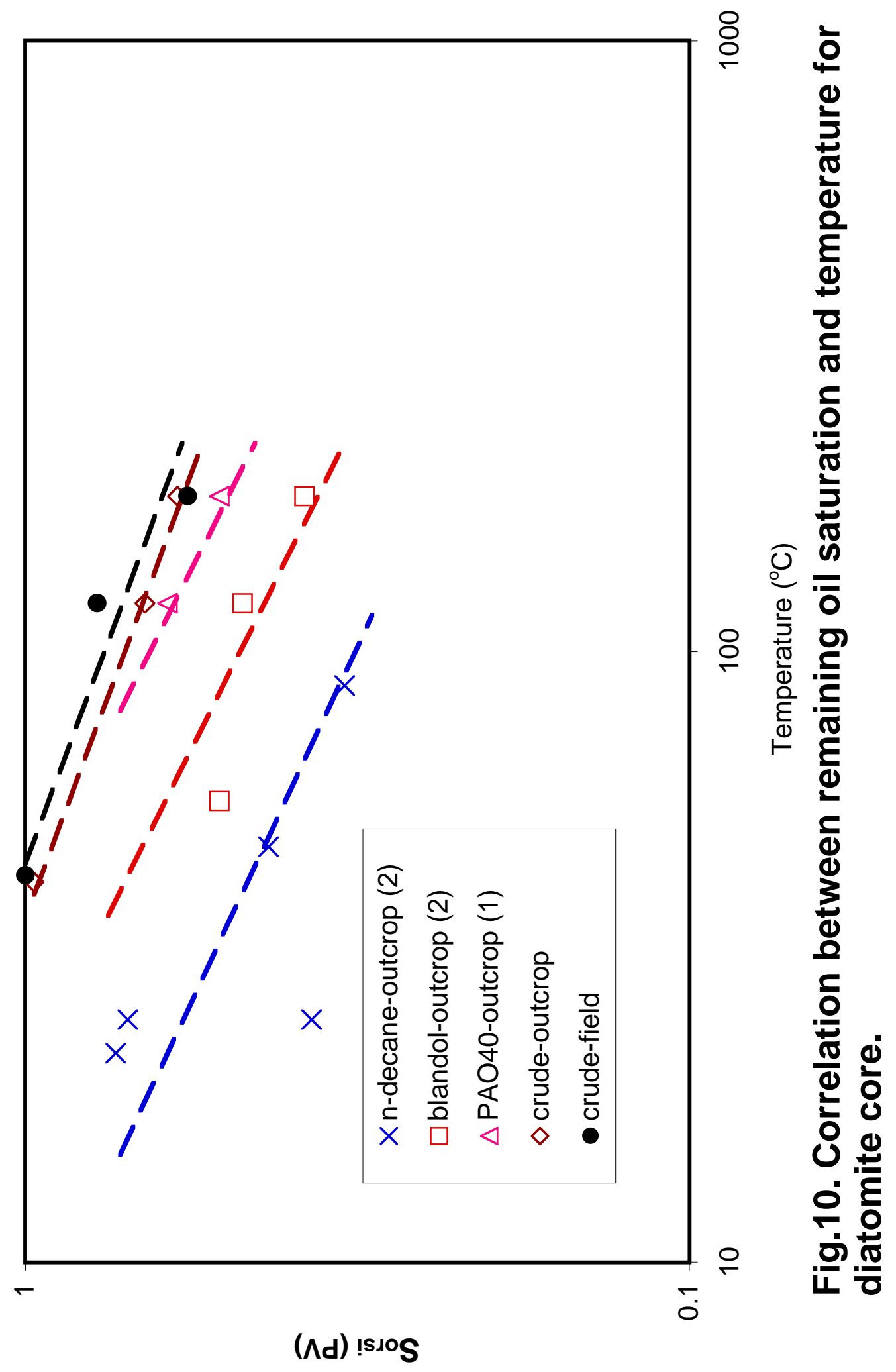




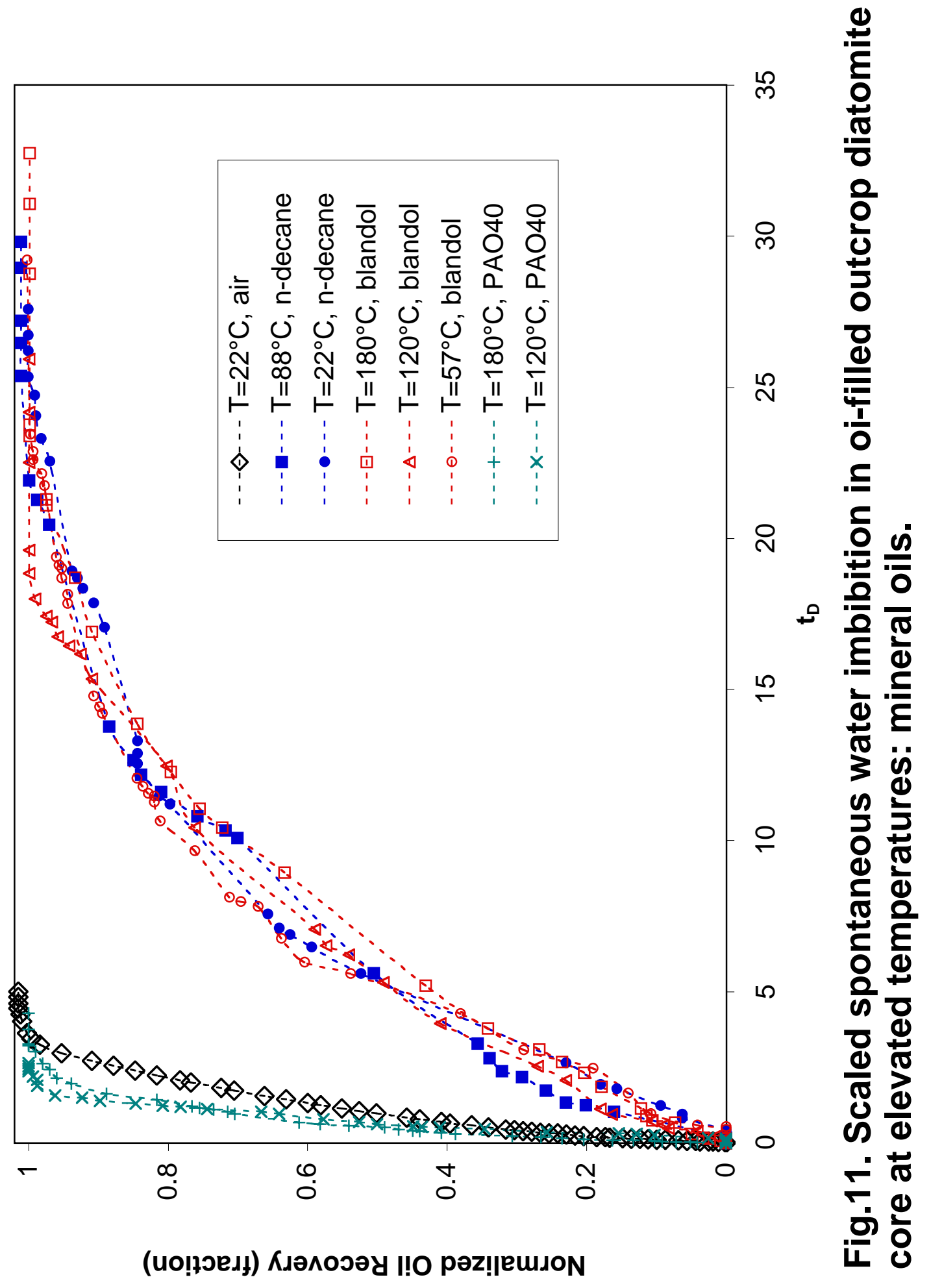




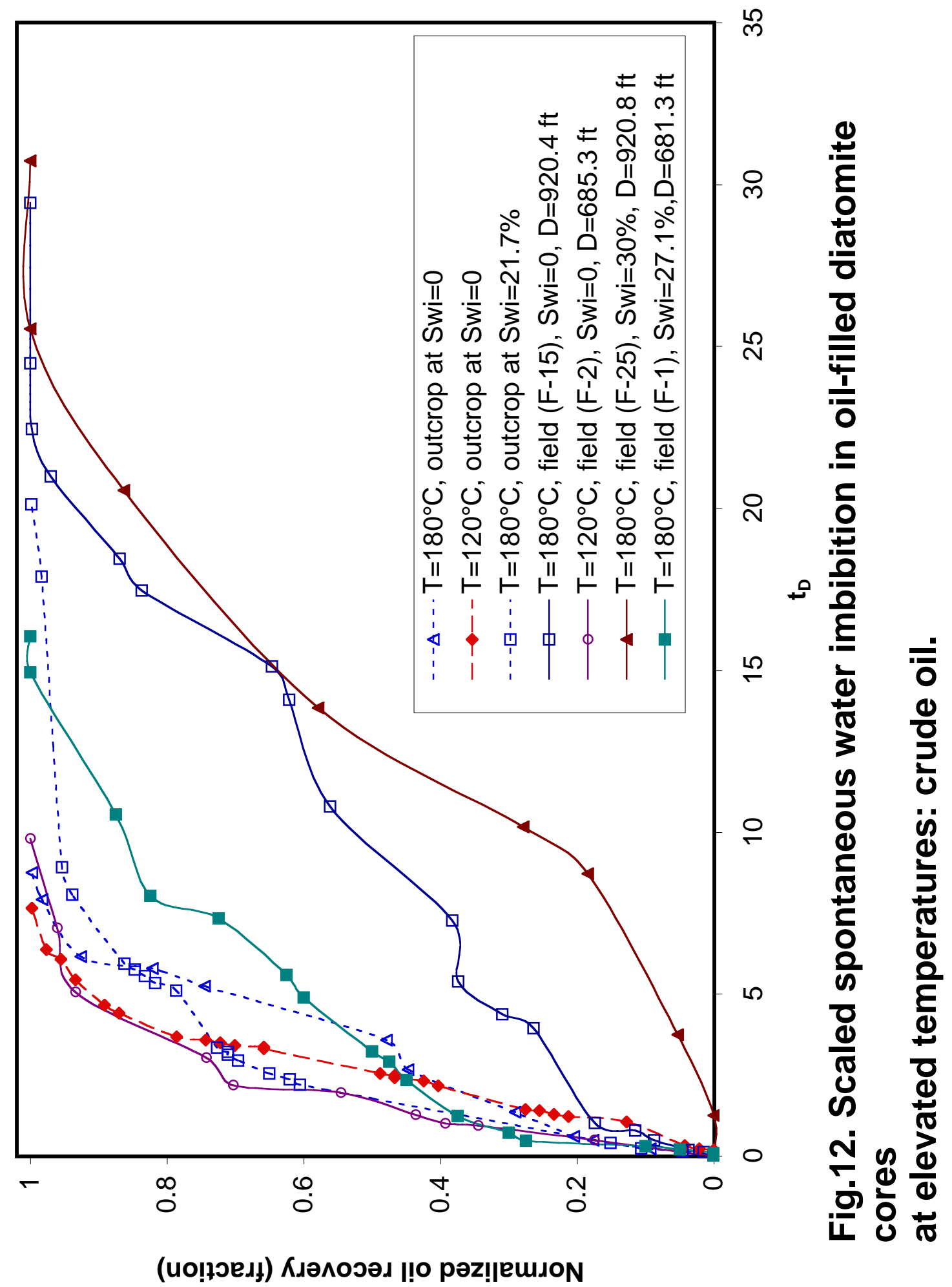



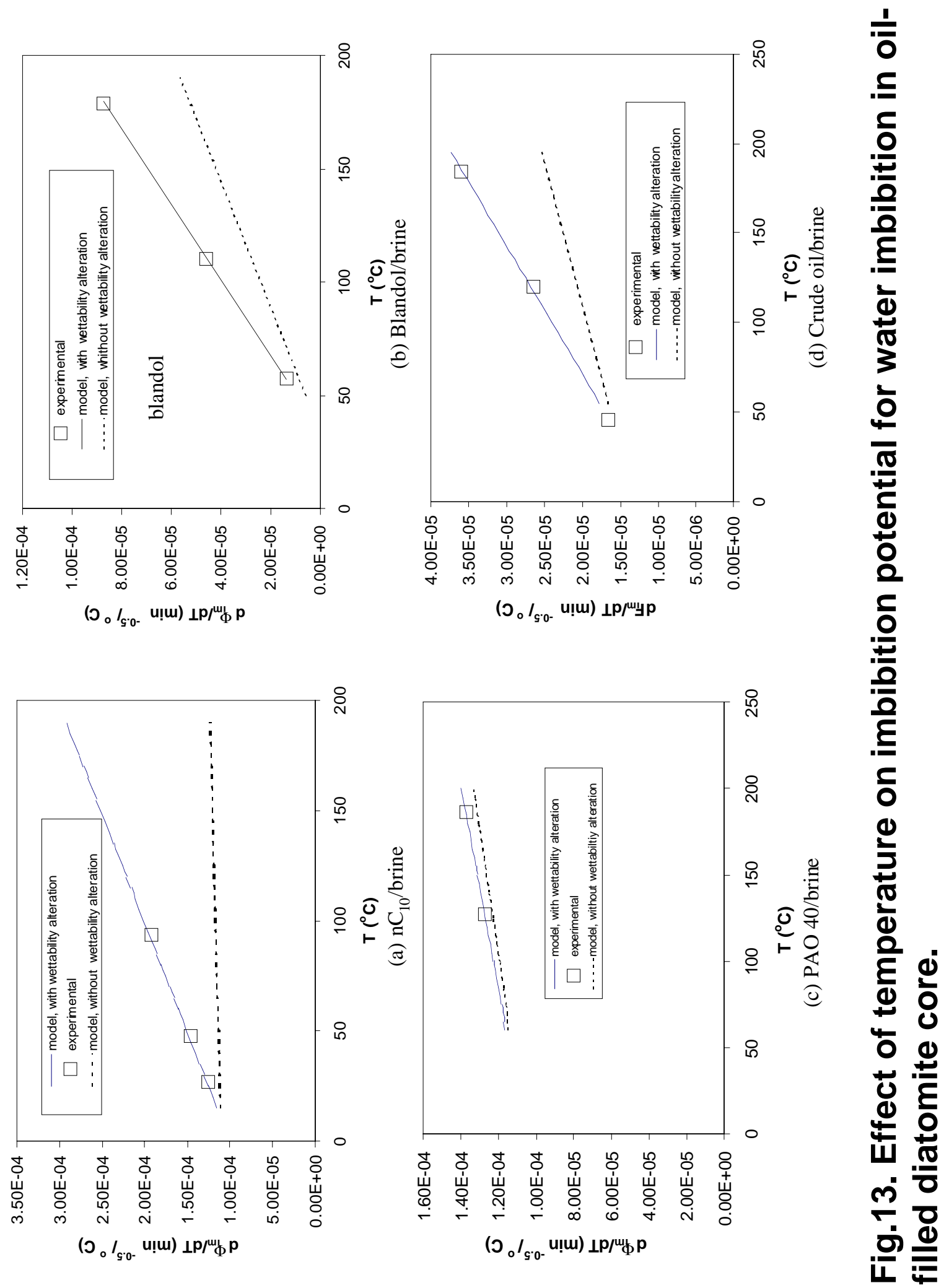


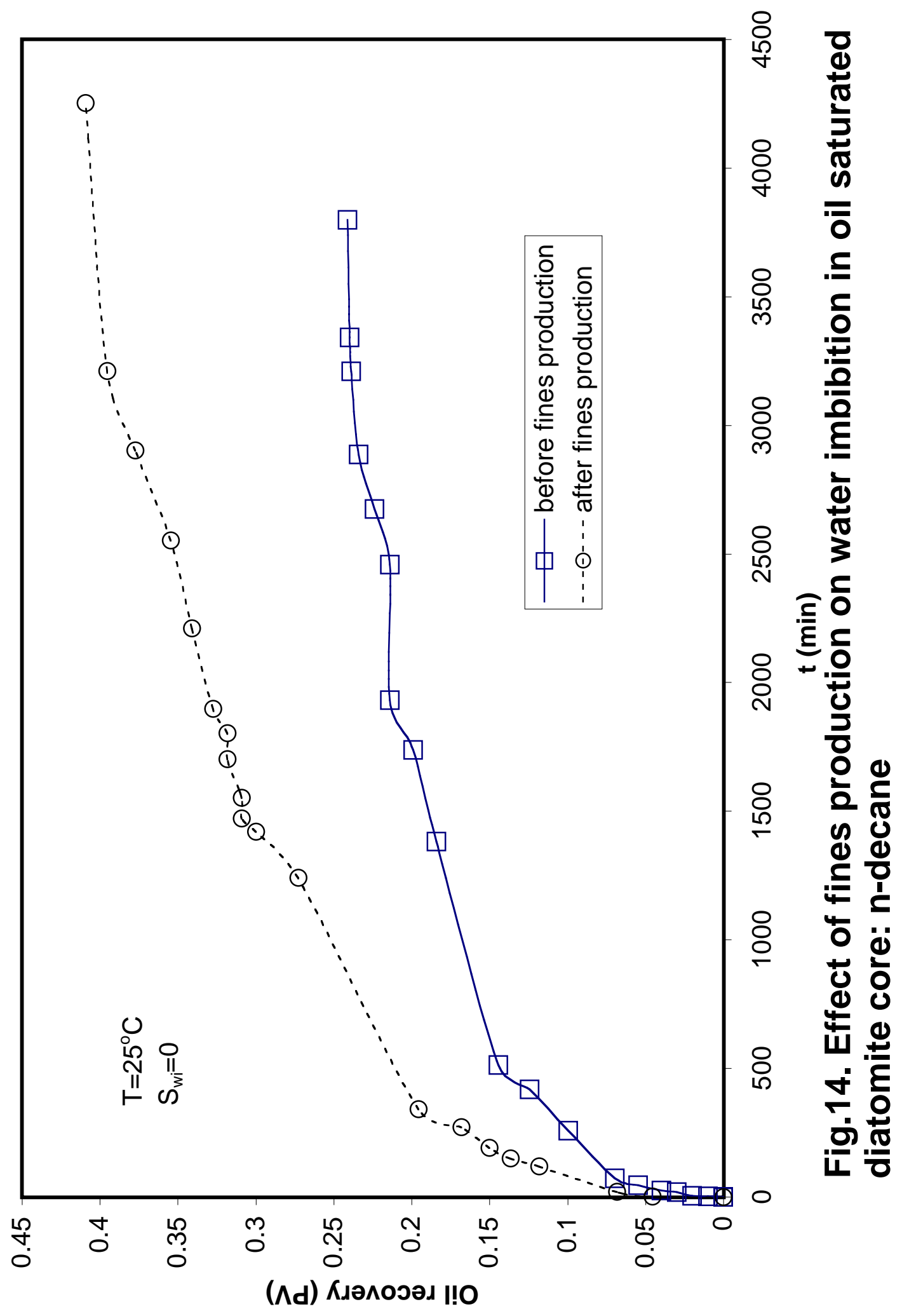




\section{An Experimental Investigation of the Effect of Temperature on Recovery of Heavy Oil From Diatomite}

\section{(Task 2b)}

\section{Introduction}

The ultimate goal of this work is to improve our understanding of multiphase fluid and heat flow characteristics of low-permeability, fractured rock. Oil production from many lowpermeability, fractured reservoirs is frustrated by not only low matrix permeability but also large oil viscosity and a matrix wettability state that is not sufficiently water wet to favor water imbibition during conventional water flooding (e.g., Al-Hadhrami and Blunt, 2001). Some diatomaceous reservoirs appear to exhibit these attributes. Thermal recovery using hydraulically fractured wells is one process to improve oil recovery and unlock these resources. Steam injection in either drive (Kovscet et al., 1996a, 1996b), or cyclic modes (Murer et al., 2000; Kumar and Beatty, 1995) is being tested for light and heavy-oil diatomite reservoirs.

Virtually all recovery processes for diatomite, and other tight fractured rocks, rely to an extent on free and/or forced imbibition (Akin et al., 2000). Capillary phenomena are equally important during steam injection into diatomite. Injected steam, especially at short times, is accompanied by condensation and flow of the resulting hot water away from the injector. In steam drive, a condensed hot-water bank precedes the steam zone within the reservoir; whereas, in cyclic steam operations the steam zone generally collapses during soak and production leaving a region filled with heated oil and condensed steam. A necessary precursor to elucidating thermal oil recovery processes in diatomite is to understand the imbibition characteristics of hot water.

Diatomite is relatively unstudied in the laboratory. Most previous studies were performed on sandstone (e.g., Bourbiaux and Kalaydijian, 2000; Jadhunandan and Morrow, 1991; Reis and Cil, 1993; Tang and Morrow, 1997) or chalk (e.g., Cuiec et al., 1994; Milter and Øxnevard, 1996) at low temperature. Diatomite is fine grained, exhibits a variety of pore length scales, and flow pathways are complex (Akin et al., 2000; Schwartz, 1988). Generalization of results across rock type from chalk or sandstone to diatomite is not currently possible. Further experiments probing the flow properties of this important class of rock are needed.

The first X-ray CT images of diatomite undergoing water injection were given by Wendel et al. (1998). Subsequently, spontaneous co-current (Akin et al., 2000) and counter-current (Zhou et al., 2002) imbibition characteristics were imaged with X-ray CT for a variety of mobility ratios, at room temperature. Regardless of imbibition mode, oil was recovered at the laboratory scale at appreciable rates with sensibly zero pressure drop. On the other hand, Kamath et al. (1995) showed that water-oil relative permeability end points could be increased by as much as $31 \%$ during forced displacement with increased flow rate and/or pressure drop.

This work studies experimentally the effect of temperature on water imbibition rate and ultimate recovery from oil-saturated core using diatomite from an outcrop and a heavy-oil field. The paper proceeds by discussing imbibition potential. It is a necessary tool for consistent 
interpretation of experimental results. Then, the high-pressure, high-temperature apparatus constructed to conduct spontaneous and forced imbibition experiments is described. Recovery data are interpreted using a dimensionless scaling group and an analytical model that exposes the role of wettability alteration, viscosity reduction, and oil/water interfacial tension.

\section{Imbibition Potential}

When gravity is negligible, spontaneous water imbibition in oil-saturated porous media is driven solely by capillary pressure, $P_{c}$

$$
P_{c}=\frac{2 \sigma}{r} \cos \theta
$$

where $\sigma$ is the interfacial tension between oil and water, $r$ is the radius of curvature of the interface, and $\theta$ is the water/oil/solid contact angle. When the rock is strongly water-wetting, the contact angle is close to zero and interfacial tension dominates the capillary pressure for a given porous medium. Because the interfacial tension decreases with increasing temperature, temperature usually decreases the capillary pressure. This has a negative effect on water imbibition rate for a rock/water/oil system.

On the other hand, the viscosity of oil and water phases is a function of temperature. Recently, it was demonstrated that the counter-current water imbibition rate in oil saturated porous media is expressed by Zhou et al. (2003) as,

$$
u_{w}=k \frac{1}{\sqrt{\mu_{w} \mu_{O}}} \frac{\sqrt{k_{r o} k_{r w}}}{\sqrt{M}+\frac{1}{\sqrt{M}}} \frac{d P_{c}}{d x}
$$

where $u_{w}$ is the time and space dependent Darcy velocity, $k$ is the permeability of the core, $k_{r w}\left(S_{w}\right)$ and $k_{r o}\left(1-S_{w}\right)$ are the relative permeability to water and oil, respectively, $M\left(=\mu_{o} k_{r w} / \mu_{w} k_{r o}\right)$ is the mobility ratio, and $\mathrm{x}$ is the axial distance. When temperature is increased, the oil-phase viscosity decreases and oil mobility improves. Thus, water imbibition as a function of temperature is governed by the competition between reduction in the viscosity of oil and water phases and interfacial tension in the absence of any alteration in wettability. When oil-phase viscosity decreases more rapidly with temperature as compared to interfacial tension, the water imbibition rate increases.

Because of the variation in physical properties with temperature (Al-Hadhrami and Blunt, 2001; Tang and Morrow, 1997; Anderson, 1986; McCaffery, 1972; Rao, 1999; Tang and Kovscek, 2002), interpretation of experimental results to follow is difficult without a quantitative tool accounting for the variation of interfacial tension, phase viscosities, and wettability with temperature. The tool employed here is the imbibition potential (Akin et al., 2000; Tang and Kovscek, 2002).

The imbibition potential derives from the work of Handy (1960) who demonstrated that imbibed water volume is proportional to the square root of the time in air saturated sandstone 
cores. Appendix A details a development similar to that of Handy, but accounting for the mobility of the wetting and nonwetting phases. The origin for these relations is Eq. 2. In short, the analysis indicates that the slope of the line for imbibed water volume versus the square root of imbibition time is written

$$
\Delta \bar{S}_{W}=\Phi_{i m} t^{0.5}
$$

where $\mathrm{t}$ is time, $\bar{S}_{w}$ is the spatial average water saturation, and $\Phi_{\text {im }}$ is the imbibition potential. Equation 3 implies that the imbibition potential is analogous to rate when time is expressed as a square root. Appendix A demonstrates that $\Phi_{\text {im }}$ is parameterized by capillary pressure, water and oil viscosity, relative permeability, and the absolute permeability.

Before proceeding, several comments are in order. Oil recovery curves in counter-current imbibition do not necessarily follow square-root-of-time behavior (e.g., Miller and Øxnevard, 1996; Parsons and Chaney, 1964; Lefebvre duPrey, 1978). If flow is counter-current at the pore level, square-root-of-time behavior is generally not observed unless the nonwetting phase is inviscid. Additionally, the redistribution of fluids in the pore space is not instantaneous during counter-current imbibition. This is another possible cause for counter-current imbibition results to deviate from square-root-of-time behavior (cf. Barenblatt et al., 2002). Hence, before applying Eq. 3, we check carefully that the saturation trends versus the square root of time are linear.

\section{Experimental}

Experimentally, we wish to obtain the change of average water saturation, measure the rate of imbibition, and determine fluid displacement patterns within the rock. X-ray CT meets these goals, is noninvasive, and measurements can be made without stopping an experiment. Figure 1 presents the experimental setup. The heart of the apparatus is a high-temperature, highpressure coreholder. Experimental procedures minimize the effects of rock compaction and volumetric expansion of fluids on oil recovery. Extensive details of the apparatus, cores, wetting and nonwetting fluids, and image processing of the raw CT data are presented in Appendix B.

Briefly, outcrop and field cores are both employed. Porosity of the samples ranges from 0.55 to 0.67 while permeability varies from 0.8 to 2.9 md, as documented in Fig. 2 and Table 1 . Field samples are cleaned before use, whereas outcrop core is not.

Three white oils (n-decane, blandol, and PAO40) and crude oil were used as the oil phases. The viscosity of these fluids at $22{ }^{\circ} \mathrm{C}$ varied from 0.93 to $6400 \mathrm{mPa}$-s. A relatively fresh synthetic brine with the composition reported in Table 2 was the aqueous phase. Figure 3 shows the viscosity versus temperature relationships; note the dramatic effect of temperature on the viscous mineral and crude oils. Figure 4 reports interfacial tension versus temperature for the various fluids. Tension is extrapolated to high temperature as explained in Appendix B and Table 3.

The apparatus, Fig. 1, is designed for both spontaneous countercurrent imbibition and cocurrent forced displacement. An initial condition is established and the coreholder brought to a constant temperature. All tests are conducted isothermally. Tests are initiated with zero initial 
water saturation, $S_{w i}$, and with $S_{w i}$ of about 0.30 . These different initial conditions bracket the range of observed saturation patterns within the core. At low $\mathrm{S}_{\mathrm{wi}}$, a water front develops characterized by a saturation pattern that becomes disperse over time, whereas at greater $S_{\text {wi }}$ more uniform fill up of the porous medium with no discernable front is noticed (Akin et al., 2000).

Brine is supplied at one face of the core at $0.5 \mathrm{~cm}^{3} / \mathrm{min}$. The outlet is kept closed and brine imbibes countercurrently. Water is supplied until no further change in water saturation is measured by CT.

\section{Results and Discussion}

Results are described in order of increasing complexity, that is, from outcrop cores and simple fluids to field cores and crude oil. The experimental program was designed to separate the effects of viscosity reduction of oil and water phases from beneficial alteration of wettability toward water wetness. In this manner, the magnitude of the principle mechanisms of thermal recovery from diatomite is probed.

In total, 18 experiments are reported, Table 1. The average increase in aqueous phase saturation versus the square root of time followed a linear trend as verified by the nearly unit values of correlation coefficient, $\mathrm{R}^{2}$. All lines were forced through the initial saturation. Hence, the imbibition potential is useful for sorting the macroscopic results. Table 1 summarizes all experiments by listing the best-fit imbibition potential, the correlation coefficient of the fit, and various quantities of interest. Fortunately, the outcrop sample permeabilities are all nearly equal allowing generalization without considering the role of permeability. Also tabulated is remaining saturation of the nonwetting phase after spontaneous imbibition, $S_{\text {orsi }}$.

The air-water/outcrop system exhibits the largest $\Phi_{i m}$ consistent with low gas viscosity and the extreme preference of the solid surface for water as opposed to air. The field core/water/heavy-oil at $43{ }^{\circ} \mathrm{C}$ exhibits the smallest. For each fluid system, the values of $\Phi_{\text {im }}$ presented in Table 1 indicate that an increase in temperature results in more rapid recovery consistent with reduced oil and water viscosity as temperature increases. Similarly, at a fixed temperature, the recovery rate decreases as the ratio of wetting to nonwetting phase viscosity decreases. Systems with initial water saturation also show lesser imbibition potential.

\section{Outcrop Core/Water/Mineral Oil}

Figure 5 shows recovery versus time for all outcrop core/water/mineral oil tests. We plot all data together for compactness and to emphasize the effectiveness of our scaling procedure to follow. A general trend for outcrop cores is a decrease in imbibition rate, as the nonwetting-phase viscosity increases. This observation is indicated by the time period for recovery in Fig. 5 and by imbibition potential in Table 1. There is some discrepancy in the ordering of imbibition potential with viscosity. For instance, compare water/blandol results with water/PAO40 results at $180{ }^{\circ} \mathrm{C}$. The more viscous PAO40 has larger imbibition potential. As discussed later, this effect is traced to different wettability among two different samples of quarry stone. 
A second trend is that remaining oil saturation following spontaneous imbibition decreases as temperature increases, as shown by Table 1 and Fig. 5. Consider the runs with blandol, the modestly viscous mineral oil. The test temperatures were 57, 120, and $180{ }^{\circ} \mathrm{C}$, respectively. The viscosity ratio of displacing phase (water) to displaced phase (oil) was about 0.062 at $57^{\circ} \mathrm{C}, 0.15$ at $120{ }^{\circ} \mathrm{C}$, and 0.217 at $180{ }^{\circ} \mathrm{C}$. Spontaneous imbibition recovery of blandol increases with temperature. The final oil recovery is about $0.49 \mathrm{PV}$ at $57{ }^{\circ} \mathrm{C}, 0.53 \mathrm{PV}$ at $120{ }^{\circ} \mathrm{C}$, and $0.62 \mathrm{PV}$ at $180^{\circ} \mathrm{C}$.

\section{Outcrop Core/Water/Crude Oil}

To increase system complexity in a logical fashion, the heavy diatomite crude oil was used next as the nonwetting phase in outcrop cores. A separate core was used for each test. Because the crude oil is very viscous at room temperature, the oil and coreholder were heated to $80^{\circ} \mathrm{C}$ to accelerate the oil injection process. After the core was saturated with the crude oil, the core was heated or cooled to test temperature and allowed to reach thermal equilibrium. Each core was aged with oil at the test temperature for 1 to 2 days before it was subjected to water imbibition. The oil is heavy and asphaltenic. No effect of aging time is apparent in our work.

Figure 6 shows the oil recovery curves for the crude oil as a function of temperature. All crude oil tests are plotted on a single figure to emphasize the success of our scaling efforts to follow. The average rate of imbibition for the outcrop/water/crude-oil system (dashed lines) increases by more than an order of magnitude as temperature increases from 42 to $180^{\circ} \mathrm{C}$. The oil recovery when $S_{w i}$ equals 0 and $T$ equals $42{ }^{\circ} \mathrm{C}$ is only $0.03 \mathrm{PV}$; however, the final oil recovery is about $0.42 \mathrm{PV}$ at $180{ }^{\circ} \mathrm{C}$ when $S_{w i}$ equals 0 . For the experiment conducted at $180{ }^{\circ} \mathrm{C}$ and $S_{w i}$ equal to $21.7 \%$, the water imbibition rate for the core is initially $(t<1500 \mathrm{~min})$ similar to the core at $T=180{ }^{\circ} \mathrm{C}$ and $S_{w i}=0$. After $1500 \mathrm{~min}$, water imbibition rate declines quickly indicating lesser capillary driving forces and greater flow resistance to oil production.

In-situ aqueous phase saturation profiles as a function of time are presented in Fig. 7 for an experiment at $180{ }^{\circ} \mathrm{C}$ with no initial water. Water propagates throughout the core. The imbibition front is relatively diffuse. Water advance is slower compared to previous mineral-oil cases. At $180{ }^{\circ} \mathrm{C}$, crude oil viscosity and the viscosity of the PAO40 are about the same. Yet, the outcrop/water/crude oil system without intial water saturation takes about 4,000 min to reach a stable water saturation profile. Experiments were halted when the water saturation profile displayed, sensibly, no change between subsequent CT exposures. Additionally, Fig. 7 indicates that the water saturation along the core is not uniform at the end of the experiment. Near the inlet the final water saturation is $0.55 \mathrm{PV}$, while near the outlet it is roughly $0.10 \mathrm{PV}$. 


\section{Field Core Water/Crude Oil}

Four field cores and crude oil were used for the remaining tests. The cores F-2 $(685.3 \mathrm{ft})$, and F-15 (920.8 ft) were used at 43,120 , and $180{ }^{\circ} \mathrm{C}$, and zero initial water saturation. The other two cores, F-25 (928.1 ft) and F-1 (681.3 ft), were used for experiments at $180{ }^{\circ} \mathrm{C}$ with initial water saturations of 30 and $27 \%$, respectively. Recovery versus time for these experiments (solid lines) is presented in Fig. 6.

The first test with F-2 at $43^{\circ} \mathrm{C}$ did not show any water imbibition. Hence, its imbibition potential is zero, Table 1 . This core was then heated to $120^{\circ} \mathrm{C}$ and allowed to reach thermal equilibrium. Then it was subjected to water imbibition. Oil viscosity at $120{ }^{\circ} \mathrm{C}$ is reduced to 12 $\mathrm{mPa}-\mathrm{s}$ and the mobility ratio is more favorable (i.e., smaller). About $0.22 \mathrm{PV}$ oil was produced by spontaneous water imbibition at $120^{\circ} \mathrm{C}$.

The second field core $(\mathrm{F}-15)$ was used at $180^{\circ} \mathrm{C}$ and zero initial water saturation. Here, the oil viscosity is $4.5 \mathrm{mPa}$-s at test conditions. The water imbibition rate at times less than $500 \mathrm{~min}$ increased significantly, as compared to results at $120{ }^{\circ} \mathrm{C}$. The rate declined somewhat in the later period, but it is still greater than rates for the $120{ }^{\circ} \mathrm{C}$ case. The final oil recovery is about $0.43 \mathrm{PV}$.

Figure 8 shows data obtained by X-ray CT for the experiments at 120 and $180^{\circ} \mathrm{C}$. The aqueous-phase saturation profiles in Fig. 8a teach that water imbibes instantly at $120{ }^{\circ} \mathrm{C}$, for all practical purposes. However, the imbibing front only progresses through a little more than one half of the core. After $7370 \mathrm{~min}$, the shape and position of the displacing front no longer evolves. The region of relatively high water saturation near the center of the core indicates low oil relative permeability. Combined with the somewhat elevated oil viscosity of $12 \mathrm{mPa}$-s at $120{ }^{\circ} \mathrm{C}$, flow resistance is high. The nonwetting phase has difficulty exiting the interior of the core.

Figure $8 \mathrm{~b}$ shows aqueous phase saturation profiles versus time for the $180{ }^{\circ} \mathrm{C}$ experiment. The displacement front is diffuse, but propagates throughout the entire core. At the end of the experiment, the water saturation is somewhat larger near the inlet as compared to the outlet. Water saturation images versus time are displayed in Fig. 8c for the cross section $X_{D}=0.35$ at 180 ${ }^{\circ} \mathrm{C}$. Dark shading indicates low $S_{w}$ while light indicates high $S_{w}$. Due to core heterogeneity, the water saturation is not uniform within the cross section at all times and water has not yet swept all of the cross-sectional area. It is apparent that water advance through some of the bedding layers is responsible for a major portion of the imbibition response. Note the almost pure white shading in these swept areas compared to the darker shading in the remainder of the cross section. As time progresses, the amount of white shading increases as water imbibes slowly into the unswept areas.

Subsequent experiments were conducted with initial water saturation. To establish initial water saturation, the coreholder was heated to $80{ }^{\circ} \mathrm{C}$. This reduced oil viscosity so that oil was pumped easiliy into the brine-saturated core. After oil breakthrough, the flow direction was reversed and oil was injected until no further water was produced. The produced water was collected and used to estimate the initial water saturation. The cores were aged at room temperature for slightly different amounts of time: F-25 was aged for 10 days while F-1 was aged for only 2 days, as a result of CT-scanner availability. Again, we note no effect of aging time for this heavy, asphaltenic crude oil. 
Recovery versus time is shown in Fig. 6. The total oil recovery by spontaneous water imbibition is about $27 \%$ of OOIP for core F-25 and about $40 \%$ of OOIP for core F-1. At the end of spontaneous imbibition, the cores were subjected to forced imbibition. The inlet pressure was about 4.03 $\mathrm{MPa}$ (585 psia) and outlet pressure was about $3.89 \mathrm{MPa}$ (565 psia). Hence, the pressure drop across the core was about $138 \mathrm{kPa}(20 \mathrm{psi})$. Forced water imbibition continued for about 2 days until no further oil was produced. The total oil recovery increased to about $52 \%$ of OOIP for core F-25 and 54\% of OOIP for core F-1. Desaturation by forced imbibition occurred quickly. Most of the additional oil was produced in less than $400 \mathrm{~min}$. These results indicate that the oil saturation remaining after spontaneous imbibition is well connected.

Forced displacement is effective for the diatomite system; however, the pressure gradient is roughly $1.8 \mathrm{MPa} / \mathrm{m}(80 \mathrm{psi} / \mathrm{ft})$. This is consistent with the results of Kamath et al. (1995). For a reservoir system, the imposed pressure gradient will obviously be less and oil production will occur by both spontaneous and forced water imbibition.

The water saturation profile along the core for the experiment with F-25 $\left(S_{w i}=30 \%\right)$ is presented in Fig. 9. The dashed lines are for forced water imbibition. This figure shows that the water-front is much more diffuse than that observed for the experiments without initial water saturation. It also teaches that forced water imbibition (following free imbibition) does not result in clearly defined water fronts or oil banks moving through the core. That is, the displacement remains diffuse throughout spontaneous and forced imbibition without the formation of a true oil bank. Figure $9 \mathrm{~b}$ shows that water distribution within the cross section is more uniform, as compared to Fig 8c. Nevertheless, bedding structures fill with water preferentially.

\section{Remaining Oil Saturation}

Figure 10 summarizes remaining oil saturation versus temperature for all systems. Clearly, $S_{\text {orsi }}$ declines as $T$ increases for each fluid-rock system. As oil becomes more viscous, the remaining oil saturation shifts toward large values.

In general, results for experiments with crude oil lag behind those for the white mineral oils. Remaining oil saturation is greater, for a given temperature, for experiments with crude oil as compared to those with mineral oil. This statement remains true even when crude oil and PAO40 viscosity are roughly equal at $180{ }^{\circ} \mathrm{C}$. Additionally, the apparent slope of the line describing the decline in $S_{\text {orsi }}$ with increasing $T$ is less for crude oil. As discussed subsequently, this indicates possible interaction of crude-oil constituents with rock that alters wettability toward mixed wetness.

From the imbibition curves at $180{ }^{\circ} \mathrm{C}$ for the field core/water/crude oil system, we do not find a systematic effect of initial water saturation on water imbibition rate. This may be caused by the heterogeneity of the field cores and the small sample set. Nevertheless, the remaining oil saturation is somewhat insensitive to the presence of initial water, Table 1. For the three cases at $180{ }^{\circ} \mathrm{C}, S_{\text {orsi }}$ averages 0.51 , and the largest $S_{\text {orsi }}(=0.57)$ is found for the case where $S_{\text {orsi }}$ is 0 . With forced water imbibition, the residual oil saturation could be as low as $0.35 \mathrm{PV}$. 


\section{Interpretation}

Counter to expectation, results for the outcrop/water/mineral oil system appear to exhibit some effects of changing wettability state versus temperature. Further, recovery results are qualitatively similar to our previous work at low temperature with the same rock ${ }^{15}$; however, imbibition rate is somewhat lower than expected for the experiments with blandol and n-decane. Compare $\Phi_{i m}$ for blandol, n-decane, and PAO40 in Table 1 . The more viscous PAO40 has larger $\Phi_{i m}$ across a range of $T$.

Outcrop cores were cut from two different blocks of quarried stone. Most of the experiments here were conducted with core from the second block and its wettability state differs from the first. To verify this statement, recovery versus time is scaled with appropriate dimensionless groups accounting for the effect of mobility ratio and interfacial tension, but not initial wettability state. Separate characteristic curves are obtained because the initial rock wettability state differs among samples.

The focus of our interpretation efforts are on determining the magnitude of the shift in wettability toward water wetness. We deconvolve reduction in phase viscosity and interfacial tension from any increase in water wetness. A mechanism for wettability alteration is also given.

\section{Scaling}

Although several scaling groups for counter-current imbibition have been proposed (e.g., Zhou et al., 2002; Zhang et al., 1996; Wang, 1999), we use exclusively our previously developed group (Zhou et al., 2002) resulting from Eq. 2,

$$
t_{D}=\sqrt{\frac{k}{\phi}} \frac{\sigma}{L_{C}^{2}} \sqrt{\lambda_{r w}^{*} \lambda_{r n w}^{*}} \frac{t}{\sqrt{M^{*}}+\frac{1}{\sqrt{M^{*}}}}
$$

The mobility ratio is incorporated explicitly and the group originates from restriction of the equations of motion to counter-current flow. It is most appropriate given the extreme variations in viscosity and mobility with temperature. The term $\lambda_{r}^{*}=k_{r}^{*} / \mu$ is a characteristic mobility for the wetting $(w)$ and non-wetting $(n w)$ phases, and $M^{*}=\lambda_{r w}^{*} / \lambda_{r n w}^{*}$ is a characteristic mobility ratio. End-point relative permeabilities are used for calculating $\lambda_{r}^{*}$ and $M^{*}$. The scaling is explicitly for spontaneous imbibition and $\mathrm{k}_{\mathrm{rw}}{ }^{*}$ is properly evaluated at $1-S_{\text {orsi }}$. Numerical values of $k_{r o}{ }^{*}$ and $k_{r w}{ }^{*}$ are set equal to 0.60 and 0.16 , respectively, consistent with previous work (Zhou et al., 2002; Schembre and Kovscek, 2002).

The characteristic length scale, $L_{c}$, is expressed as,

$$
L_{c}=\sqrt{\frac{V_{b}}{\sum \frac{A_{i}}{l_{i}}}}
$$


where $V_{b}$ is the bulk volume of the rock sample, $A_{i}$ is the area of a face open for imbibition in the $\mathrm{i}$ direction and $l_{\mathrm{i}}$ is the distance from the open face to the no-flow boundary in the $\mathrm{i}$ direction.

Tension measurements in Fig. 2 were extrapolated to the temperature of interest as described in Appendix B and Table 3.

Figure 11 presents the results from renormalizing the spontaneous imbibition behavior of the mineral oil/water/outcrop core systems. Contrast with Fig. 5 and note that scaled results separate clearly into two groups. The water/air/outcrop and water/PAO40/outcrop systems fall into one group. In a dimensionless time of roughly 2 these systems achieve residual non-wetting phase saturation. This is consistent with our prior results of imbibition in very strongly water wetting diatomite outcrop (Akin et al., 2000; Zhou et al., 2002).

The second group of results in Fig. 11 requires a dimensionless time of order 10 to come to final oil saturation. The existence of two distinct groups within the dimensionless data indicates that core samples are not uniform with respect to initial wettability state. Evidently, the air/water/outcrop and PAO40/water/outcrop systems are more strongly water wet than the remaining systems. This is consistent with the fact that cores were drawn from two different blocks of the same outcrop. These blocks were quarried at different times and locations.

We also scaled the water imbibition data for crude-oil tests. The results are presented in Fig.12. Obviously, the scaled experimental data for crude-oil systems are more scattered than those shown in Fig.11. The scaled data falls into at least two groups. All of the results for the outcrop core/water/crude oil system collapse onto a single curve, within experimental error. Cores for these experiments all came from the second block and so collapse to a single curve, as expected. The experimental results with field cores separate according to depth. Results from cores taken at roughly 680 feet nearly fall onto the outcrop results. Results from the field cores taken at about $920 \mathrm{ft}$ follow a different trend. These experiments require more than twice as long in a dimensionless sense to reach residual oil saturation.

\section{Wettability Alteration}

The scaling exercise demonstrated that there are at least two wettability states in the outcrop samples and two lithologies in the reservoir core. However, the degree and importance of change in the wettability state with temperature is not readily demonstrated. For this task, we employ the concept of imbibition potential.

Appendix A makes evident that imbibition potential is a function of viscosity, interfacial tension, wettability, mobility ratio, and rock properties when gravity is negligible. That is,

$$
\Phi_{\mathrm{i} m}=f\left(f_{\mu r}, f_{\sigma}, f_{w}, f_{r}\right)
$$


where $f_{\mu r}$ is the viscosity ratio function, $f_{\sigma}$ is an interfacial tension function, $f_{w}$ is the wettability function, and $f_{r}$ is the rock-property function. A differentiated form of imbibition potential with regard to temperature is

$$
\begin{aligned}
\frac{d \Phi_{i m}}{d T}= & \frac{\partial \Phi_{i m}}{\partial f_{\mu r}} \frac{d f_{\mu r}}{d T}+\frac{\partial \Phi_{i m}}{\partial f_{\sigma}} \frac{d f_{\sigma}}{d T} \\
& +\frac{\partial \Phi_{i m}}{\partial f_{W}} \frac{d f_{W}}{d T}+\frac{\partial \Phi_{i m}}{\partial f_{r}} \frac{d f_{r}}{d T}
\end{aligned}
$$

where $d \Phi_{i m} / d T, d f_{\mu R} / d T, d f_{\sigma} / d T, d f_{w} / d T$, and $d f_{r} / d T$ are the imbibition potential, viscosity ratio (oil to water), interfacial tension, wettability, and rock-property variation with temperature. These terms are measured independently through experiments. The terms $\partial \Phi_{i m} / \partial f_{\mu R}, \partial \Phi_{i m} / \partial f_{\sigma}$, $\partial \Phi_{i m} / \partial f_{W}$, and $\partial \Phi_{i m} / \partial f_{r}$ represent the variation of the functionality of imbibition potential with change in viscosity ratio, interfacial tension, wettability, and rock properties. We assume that the imbibition-potential functionality is constant, or is a very weak function of temperature and the fluids employed. The various partial derivatives in Eq. 7 are then constants. The following definitions result: $a_{1}=\partial \Phi_{i m} / \partial f_{\mu R}, a_{2}=\partial \Phi_{i m} / \partial f_{\sigma}, a_{3}=\partial \Phi_{i m} / \partial f_{w}$, and $a_{4}=\partial \Phi_{i m} / \partial f_{r}$, respectively. Upon substitution, Eq. 7 reduces to

$$
\frac{d \Phi_{i m}}{d T}=a_{1} \frac{d f_{\mu r}}{d T}+a_{2} \frac{d f_{\sigma}}{d T}+a_{3} \frac{d f_{W}}{d T}+a_{4} \frac{d f_{r}}{d T}
$$

When the factors, $a_{1}, a_{2}, a_{3}$ and $a_{4}$ are determined experimentally, or theoretically, the imbibition potential is predicted at different temperatures through integration of Eq. 8 .

With the measured imbibition potentials in Table 1, viscosity in Fig. 3, and interfacial tension in Fig. 4 and Table 3, we obtained $d \Phi_{i m} / d T, d f_{\mu R} / d T$, and $d f_{\sigma} / d T$ functions. However, the variation of wettability with temperature for water/oil/diatomite is currently unknown. Diatomite is a siliceous rock; therefore, we employed the contact angle versus temperature relationship measured for water/mineral oils/quartz systems by McCaffery $^{18}$ to determine the $d f_{w} / d T$ function for outcrop/water/n-decane. The term $d f_{r} / d T$ is set to 0 , because the experimental apparatus is designed to maintain a constant net confining stress regardless of temperature, and, accordingly, the effect of temperature on porosity and permeability is negligible.

Equation 8 is reduced to three terms with weight factors. By regressing the data for $d \Phi_{i m} / d T, d f_{\mu R} / d T, d f_{\sigma} / d T$, and $d f_{w} / d T$ at three different temperatures for the diatomite/water/ndecane system, the weight factors $a_{1}, a_{2}$, and $a_{3}$ are determined as $8.73 \times 10^{-6} \mathrm{mPa}-\mathrm{s}^{-1}-\mathrm{min}^{-0.5}$, $5.74 \times 10^{-4} \mathrm{~cm} /$ dyne-min ${ }^{0.5}, 1.78 \times 10^{-2} \mathrm{~min}^{-0.5}$, respectively. It was assumed that the measured weight factors do not change with temperature and are valid for all water/oil/diatomite systems in this study. This assumption is verified a posteriori.

One of the important implications of Eq. 8 is to identify wettability alteration with temperature. The terms $d \Phi_{i m} / d T, d f_{\mu R} / d T$, and $d f_{\sigma} / d T$ are obtained through water imbibition 
tests for various measured viscosity ratios and interfacial tension. The sole unknown effect is that of wettability. If $d f_{w} / d T$ is greater than 0 at a given temperature, wettability alteration occurs with an increase in temperature. The $\Phi_{i m}$ versus $T$ data shown in Table 1 are matched versus temperature for the water/Blandol, water/ PAO40, and water/crude oil systems by adjusting the $d f_{w} / d T$ function. For simplicity, a linear relationship is assumed,

$$
\frac{d f_{w}}{d T}=m T+b
$$

Figure 13 displays the results of this exercise for the various experiments in outcrop core. All views in Fig. 13 present the variation in imbibition potential with wettability alteration included as a solid line. Results when wettability alteration is not accounted for (i.e., $d f_{w} / d T=0$ ) are given as dashed lines. Experimental data are given as symbols. Values of the best-fit coefficients $\mathrm{m}$ and $\mathrm{b}$ are summarized in Table 4. Also tabulated is the percentage change in imbibition performance with increased temperature attributed to wettability. For water/n-decane the temperature range of interest is 22 to $88^{\circ} \mathrm{C}$. For the remaining systems The temperature range of interest is 40 to $180{ }^{\circ} \mathrm{C}$. All experiments in outcrop core exhibit some shift toward increased water wetness as temperature increases.

Figure 13a shows the imbibition potential variation with temperature obtained for the water/n-decane/diatomite system. Model data (solid line) well fits the experimentally measured data (symbols). Wettability alteration with temperature is significant for this system. Table 4 reports that the wettability effect increased imbibition potential by roughly $30 \%$. If wettability alteration is neglected altogether (dashed line), imbibition potential remains nearly unchanged with increase in temperature. This suggests that reduction in viscosity ratio, which increases imbibition potential is nearly offset by reduction in interfacial tension, that decreases imbibition potential. Recall that the core used for water/n-decane tests was not strongly water-wet initially; the remaining oil saturation decreased from 0.72 to $0.36 \mathrm{PV}$ as temperature increased from 25 to $88^{\circ} \mathrm{C}$.

Figure $13 \mathrm{~b}$ shows the measured and modeled imbibition potential variation versus temperature for outcrop/water/blandol. Compared to the water/n-decane/diatomite system, the wettability alteration with temperature is slightly less for the water/blandol/diatomite system, Table 4 . When wettability alteration with temperature is omitted from the analysis, the imbibition potential increases with temperature as indicated in Fig. 13b. This result teaches that the decrease in viscosity ratio with temperature overcomes the negative effect of a decrease in interfacial tension at elevated temperature.

The modeled and measured imbibition potential data for the outcrop/water/PAO-40 system are presented in Fig.13c. Table 4 shows only a $4 \%$ change in imbibition potential with temperature that is attributed to wettability alteration for this system. The cores used for these experiments were strongly water-wet (the first sample of outcrop) as indicated by the scaled results in Fig. 11. When the wettability alteration function is excluded from our analysis, the calculated imbibition potential still demonstrates a significant increase with temperature. The lines in Fig. 13c are nearly identical with and without wettability alteration. This indicates that the 
majority of the increase in imbibition performance for the outcrop/water/PAO40 system is from viscosity ratio reduction and improved oil mobility with temperature.

Figure $13 \mathrm{~d}$ presents the imbibition potential data for the outcrop/water/crude-oil system. Table 4 teaches that the shift in wettability increased performance by more than $12 \%$. Additionally, reduction in viscosity ratio plays a major role in improving imbibition potential, dashed line Fig. 13d. When wettability alteration is excluded from the interpretation, it is found that an increase in temperature still increased imbibition potential significantly. The wettability shift toward water wetness and the improvement in oil mobility both contributed significantly to the oil recovery with an increase in temperature from 43 to $180{ }^{\circ} \mathrm{C}$.

Unfortunately, a quantitative interpretation of the effects of wettability on the reservoir core/water/crude oil system is not possible. The data are too sparse and the 4 cores did not all behave similarly in a nondimensional sense, Fig. 12. Nevertheless, the outcrop core/water/crude oil results imply that both oil mobility improvement and wettability shift contribute to enhanced oil recovery in reservoir core.

The analysis summarized in Fig. 13 assumed that the coefficients $a_{1}, a_{2}$, and $a_{3}$ were constant and independent of the fluids within the core. With the values of $d f_{w} / d T$ for each fluid system reported in Table 4, constancy of these coefficients across all fluid systems is checked. Numerical averages and the variation of each coefficient are $a_{1}=8.7 \times 10^{-6} \pm 0.03 \times 10^{-6} \mathrm{mPa}^{-\mathrm{s}^{-1}}$ $\min ^{-0.5}, a_{2}=-5.69 \times 10^{-4} \mathrm{~cm} /$ dyne- $\min ^{0.5} \pm 0.16 \times 10^{-4}$, and $a_{3}=0.0168 \pm 0.0008 \mathrm{~min}^{-0.5}$. Within experimental error, these values are identical to the prior values of $\mathrm{a}_{1}, \mathrm{a}_{2}$, and $\mathrm{a}_{3}$. The assumption of invariant coefficients is self consistent.

\section{A Mechanism for Wettability Alteration}

Recently, Al-Hadhrami and Blunt (2001) proposed a model to explain the wettability alteration encountered during thermal operations in fractured carbonate systems. They assumed desorption of oil-wet asphaltene macromolecules from rock surfaces when the temperature was over a critical value. Thus, water-wet surface was produced. Similar opinions were also expressed by Hjelmeland and Larrondo (1986). This mechanism is not likely for siliceous surfaces. Laboratory experience suggests asphaltenes adhere irreversibly and stably (Clementz, 1982; Yan et al., 1997). Strong organic-phase solvents, such as hot pyridine, are generally needed to dissolve asphaltenes adsorbed on siliceous solids (Windholz et al., 1976).

There are at least two mechanisms for increase in water wetness with temperature in these experiments. The white oils, especially n-decane, exhibit solvent action on cores. Recall that the outcrop cores were dried but not cleaned. A fraction of the increase in water wetness with the outcrop core/water/n-decane system is likely due to dissolution of dirt or organic contamination on the rock surface. Simple cleaning action increases water wetness. Of course, experiments with crude oil do not exhibit this effect.

A second, and field relevant, mechanism for increase in water wetness with temperature is

fines detachment from rock surfaces. It is here that our efforts are concentrated. Oil-wet rock 
surfaces and mixed wettability result when polar, asphaltenic components of crude oil adhere to reservoir solids in a fashion perhaps similar to that suggested by Kovscek et al. (1993). An increase in temperature destabilizes fines attached to rock surfaces through a reduction or change of sign in colloidal attractive forces. Oil-coated fines cover the pore walls, but they are released from pore surfaces because colloidal adhesion forces decrease with increasing temperature. Fresh, water-wet rock surfaces underlying the fines are thereby exposed upon fines release. The increase in water-wet surface area results in an increase in water wetness for the entire core. In some regards, this mechanism is analogous to current thinking about wettability alteration accompanying dilute brine injection at constant temperature (Tang and Morrow, 1999). The following experimental phenomena support this proposed mechanism.

It is observed experimentally that an increase in water imbibition efficiency at high temperature is accompanied by fines production. Table 5 lists the feature of fines collected at various temperatures for counter-current water imbibition in oil (or air) saturated outcrop diatomite cores. Concurrent with an increase in oil production rate, appearance of fines in the core effluent is observed. The higher the temperature, the greater the fines production. Also, it is found that fines production correlates with nonwetting phase viscosity: the greater the oil viscosity the greater the fines production.

The size of produced fines tends to increase as the viscosity of the non-wetting phase increases. The total fines production during counter-current imbibition is about $98 \mathrm{mg}$ for crude oil and about $31 \mathrm{mg}$ for blandol after 50 and $22 \mathrm{PV}$ of hot water, respectively, were circulated across the core face. The cumulative imbibed water was about $0.43 \mathrm{PV}$ for the crude oil test and $0.61 \mathrm{PV}$ for blandol test. For water imbibition in air-saturated cores, we did not observe visible fines production.

The color of the fines transitions from light-yellow to brown as the non-wetting phase is switched from mineral oil to crude oil. In the case of crude oil, this implies that the fines adsorbed some polar oil components, such as asphaltenes and/or resins. When a drop of crude oil was placed into the produced solution containing fines and the solution was agitated, the oil drop disappeared quickly due to adsorption onto fines. Hence, these fines had affinity for oil and were possibly oil-wet.

To study further the above hypothesis, additional water imbibition experiments in $n$ decane-saturated diatomite were conducted using a single core. The core was saturated with ndecane and subjected to water imbibition at room temperature until no obvious oil production occurred. The oil recovery was about $0.28 \mathrm{PV}$ and no visible fines were produced.

Thereafter, the temperature was raised to $90^{\circ} \mathrm{C}$ and forced displacement was performed $(\Delta p=5 \mathrm{psi} / \mathrm{cm}$ ). More oil was produced accompanied with some fines production (about $27 \mathrm{mg}$ with size less than $3 \mu$ ). The final oil recovery was about $0.67 \mathrm{PV}$ after forced water imbibition. Next, the fluids in the core were removed by applying house vacuum at $T=60^{\circ} \mathrm{C}$.

After the core was dry and cold, it was re-saturated with n-decane and again subjected to spontaneous water imbibition at room temperature. The results are presented in Fig. 14. One finds that both water imbibition rate and the extent of oil recovery are improved after some fines production from this core. The permeability prior to fines production is $3.32 \mathrm{md}$, whereas it is 
$2.71 \mathrm{md}$ following fines production. As the permeability decreased by about $18 \%$, the increased water imbibition rate cannot be ascribed to an increase in permeability. The final oil recovery to spontaneous imbibition increases from $0.25 \mathrm{PV}$ to about $0.4 \mathrm{PV}$ after fines production; the modified Amott index is 0.37 before fines production and is 0.60 after fines production indicating a more water-wet state.

Because the temperature is the same for these two imbibition tests, we infer that the increase in imbibition efficiency is due to increase in water-wetness induced by hot-water injection and some fines production. The imbibition potential is $0.0047 \mathrm{~min}^{-0.5}$ for the first experiment and increases to $0.0077 \mathrm{~min}^{-0.5}$ following fines production.

\section{Conclusions}

Temperature affects profoundly water imbibition and oil recovery from oil-filled diatomite, a tight fractured siliceous rock. Imbibition rate increases and the remaining oil saturation to spontaneous imbibition of water decreases systematically with increasing temperature. Forced displacement, following spontaneous imbibition recovered significant oil. In field core, recovery increased from roughly $30 \%$ of OOIP following spontaneous imbibition of water to $50 \%$ with forced displacement.

Experiments are interpreted using imbibition potential. It is defined as the best-fit slope obtained from plotting average water saturation versus the square root of time. Imbibition potential is divided into contributions from viscosity ratio, interfacial tension, wettability, and rock properties. Imbibition results depend strongly on the initial wettability state of the rock. When the rock/water/oil system is initially strongly water wet, recovery performance increases with temperature mainly because oil viscosity decreases. On the other hand, when the rock is not strongly water wet, an increase in temperature also leads to an increase in water wetness and this aids imbibition performance too. There is competition between the beneficial effects of an increase in water-wetness and oil mobility as compared to the detrimental effect of decreasing interfacial tension with temperature. Over the range of temperatures examined here, wettability and mobility ratio improvements are dominant.

Production of oil-wet fines was observed at high temperature along with a corresponding increase in water imbibition rate and extent of oil recovery. It is proposed that wettability alteration at high temperature is related to fines detachment from pore surfaces. Detachment creates water-wet surfaces when the rock is siliceous.

Significantly, a scaling analysis correlated experimental results. The scaling group is physically-based; it incorporates mobility ratio, end-point wetting and nonwetting phase mobilities, and remaining oil saturation to spontaneous imbibition as a function of temperature. Thus, scale up of imbibition behavior from the laboratory conditions to thermal field operations appears feasible. 


\section{Nomenclature}

a constants in Eq. 12

A area, $\mathrm{cm}^{2}$

$b \quad$ constant in Eq. $13,{ }^{\circ} \mathrm{C}^{-1}$

$C_{0}, C_{1} \quad$ constants in corresponding states

relationship for interfacial tension

CT CT number, Hounsfields

$f \quad$ function

$k \quad$ permeability, md

$k_{r} \quad$ permeability

$L \quad$ length, m

$m \quad$ slope in Eq. $13,{ }^{\circ} \mathrm{C}^{-2}$

$M \quad$ mobility ratio

$P_{c} \quad$ capillary pressure, $\mathrm{Pa}$

$P V \quad$ pore volume

$r \quad$ characteristic radius, $m$

$S \quad$ saturation, fraction

$S_{\text {orsi }} \quad$ remaining oil saturation after spontaneous

imbibition

$T \quad$ temperature, ${ }^{\circ} \mathrm{C}$

$T_{c} \quad$ critical temperature, $\mathrm{K}$

$t \quad$ time, s or min

$x \quad$ distance, $\mathrm{m}$

$X_{D} \quad$ dimensionless length, fraction

$V \quad$ volume, $\mathrm{cm}^{3}$

Greek Symbols

$\phi \quad$ porosity, fraction

$\Phi_{i m} \quad$ imbibition potential, $\min ^{-0.5}$

$\sigma \quad$ interfacial tension, $\mathrm{N} / \mathrm{m}$

$\lambda$ mobility

$\theta \quad$ contact angle

Subscripts and Superscripts

a air

c characteristic

$o$ oil

$r \quad$ rock

w water, wettability

$\mu r \quad$ viscosity ratio 


\section{References}

Akin, S., Schembre, S.K., Bhat, S.K., and Kovscek, A.R., "Spontaneous Imbibition Characteristics of Diatomite," J. Pet. Sci. and Eng. 25 (2000) 149-165.

Al-Hadhrami, H.S., and Blunt, M.J., "Thermally Induced Wettability Alteration to Improve Oil Recovery in Fractured Reservoirs," Soc. Pet. Eng. Res. Eval. \& Eng (June 2001) 179-186.

Anderson, W.G., "Wettability Literature Survey-Part 2: Wettability Measurement," JPT (November 1986) 1246-1262.

Barenblatt, G.I., Patzek, T.W., and Silin, D.B., "The Mathematical Model of Non-Equilibrium Effects in Water-Oil Displacement," paper SPE 75169 presented at the 2002 SPE/DOE Symposium on Improved Oil Recovery, Tulsa, OK, April 13-17.

Birdi, K. S, ed. "Handbook of Surface and Colloid Chemistry," CRC Press, New York (1999).

Bourbiaux, B.J. and Kalaydjian, F.J., "Experimental Study of Cocurrent and Countercurrent Flows in Natural Porous Media," Soc. Pet. Eng. Res. Eval. \& Eng. (August 2000) 361368.

Carslaw, H. S. and Jaegar, J. C., Conduction of Heat in Solids, $2^{\text {nd }}$ Edition, Oxford University Press, Oxford: (1959) 297-326.

Clementz, D. M., "Alteration of Rock Properties by Adsorption of Petroleum Heavy Ends: Implication for Enhanced Oil Recovery," paper SPE 10683 presented at 1982 SPE/DOE Symposium on Enhanced Oil Recovery, Tulsa, OK, April 4-7.

Cuiec, L., Bourbiaux, J.B. and Kalaydjian, F.J., "Oil Recovery by Imbibition in Low Permeability Chalk,” SPEFE (September 1994) 200-208.

Handy, L.L., "Determination of Effective Capillary Pressure for Porous Media from Imbibition Data," Trans, AIME 219 (1960) 75-87.

Hjelmeland, O.S. and Larrondo, L.E., "Experimental Investigation of the Effects of Temperature, Pressure, and Crude Oil Composition on Interfacial Tension Properties," SPEFE (July 1986) 321-328.

Jadhunandan P.P and Morrow, N.R., "Spontaneous Imbibition of Water by Crude Oil/Brine/Rock Systems," In Situ 15(4) (1991) 319-345.

Kamath, J., deZabala, E.F., and Boyer, R.E., "Water/Oil Relative Permeability Endpoints of Intermediate-Wet, Low-Permeability Rocks,” SPEFE (March 1995) 4-10. 
Kovscek, A.R., Johnston, R.M., and Patzek, T.W., "Interpretation of Hydrofracture Geometry During Steam Injection Using Temperature Transients I. Model Formulation and Verification," In Situ 20(3) (1996A) 251-288.

Kovscek, A.R., Johnston, R.M., and Patzek, T.W., "Interpretation of Hydrofracture Geometry During Steam Injection Using Temperature Transients I. Model Formulation and Verification,” In Situ 20(3) (1996B) 289-309.

Kovscek, A.R., Wong, H., and Radke, C.J., "A Pore-Level Scenario for the Development of Mixed Wettability in Oil Reservoirs," Am. Inst. Chem. Eng. J. (June, 1993) 1072-1085.

Kumar, M., and Beatty, F.D., "Cyclic Steaming in Heavy Oil Diatomite," paper SPE 29623 presented at the 1995 SPE Western Regional Meeting, Bakersfield, CA, March 8-10.

Lefebvre Du Prey, E., "Gravity and Capillarity Effects on Imbibition in Porous Media," SPEJ (June 1978) 195-206.

Lide, D. R., ed., "Handbook of Chemistry and Physics,", $81^{\text {st }}$ edition, CRC Press, New York, (2000).

McCaffery, F.G., "Measurement of Interfacial Tension and Contact Angles at High Temperature and Pressure," J. Can. Pet Tech. (July-September 1972) 26-32.

Milter, J. and Øxnevard, R.I., , "Spontaneous Imbibition in Two Different Chalks Facies," Petroleum Geoscience 2, (1996) 231-240.

Murer, A.S., McClennen, K.L., Ellison, T.K., Larson, D.C., Timmer, R.S., Thomsen, M.A., and Wolcott, K.D., "Steam Injection Project in Heavy-Oil Diatomite," Soc. Pet. Eng. Res. Eval. \& Eng (February 2000) 2-12.

Parsons, R. W. and Chaney P.R., "Imbibition Model Studies on Water-Wet Carbonate Rocks," SPEJ (March 1964) 26-34.

Prats, M, Thermal Recovery, $2^{\text {nd }}$ Printing, Society of Petroleum Engineers, Dallas, TX: (1986) 220.

Rao, D. N., "Wettability Effects in Thermal Recovery Operations," Soc. Pet. Eng. Res. Eval. \& Eng (October 1999) 420-430.

Reid, R.C., Prausnitz, J.M., and Poling, B.E., "The Properties of Gases and Liquids," McGrawHill, New York (1987).

Reis, J.C. and Cil, M., "A Model for Oil Expulsion by Counter-Current Water Imbibition in Rocks: One Dimensional Geometry," J. Pet. Sci. and Eng, 10 (1993) 97-107. 
Ross, C.M. and Kovscek, A.R., "Pore Microstructure and Field Distribution in a Diatomaceous Reservoir," paper SPE 75190 presented at the 2002 SPE/DOE Symposium on Improved Oil Recovery, Tulsa, OK, April 13-17.

Schembre, J. M. and Kovscek, A.R. "A Technique for Measuring Two-Phase Relative Permeability in Porous Media via X-ray CT-Measurments," J. Pet. Sci and Eng 39(1-2) (2002) 159-174.

Schwartz, D.E.: "Characterizing the Lithology, Petrophysical Properties, and Depositional Setting of the Belridge Diatomite, South Belridge Field, Kern, County, California," in Studies of the Geology of the San Joaquin Basin, S.A. Graham and H.C. Olson (eds.), The Pacific Section of Society of Economic Paleontologists and Mineralogists, Los Angles, CA (1988) 281-301.

Tang, G. and Kovscek, A.R., "Experimental Study of Heavy Oil Production from Diatomite by Water Imbibition at Elevated Temperatures," paper SPE 75132 presented at the 2002 SPE/DOE Thirteenth Symposium on Improved Oil Recovery held in Tulsa, OK., April 13-17.

Tang, G., and Morrow, N.R., "Effect of Temperature, Salinity and Oil Composition on Wetting Behavior and Oil Recovery by Waterflooding;" Soc. Pet. Eng. Res. Eval. \& Eng, (1997) .

Tang, G.-Q. and Morrow, N. R, "Influence of Brine Composition and Fines Migration on Crude/Oil/Brine/Rock Interactions and Oil Recovery," J. Pet. Sci. and Eng. 24 (1999) 99111.

Wang, R. "Gas Recovery from Porous Media by Spontaneous Imbibition of Liquid,", MS thesis, University of Wyoming, Laramie, WY 82070 (1999).

Wendel, D.J., Kunkel, L.A., and Swanson, G.S., "Waterflood Potential of Diatomite: New Laboratory Methods," paper SPE 17439 presented at the 1998 SPE California Regional Meeting held in Long Beach, CA, March 23-25.

Windholz, M., Budavari, S., Stroumtsos, L. Y., and Fertig, M. N. eds, The Merck Index, Ninth Edition, MerckandCo. Inc., Rahway, NJ: (1976) 1033.

Withjack, E.M., "Computed Tomography for Rock Property Determination and Fluid Flow Visualization,” SPEFE (December 1988) 696-704.

Yan, J., Plancher, H., and Morrow, N. R., "Wettability Changes Induced by Adsorption of Asphaltenes," paper SPE 37232 presented at the 1997 SPE International Symposium on Oilfield Chemistry, Houston, TX., February. 18-21.

Zhang, X., Morrow, N.R., and Ma, S., "Experimental Verification of a Modified Scaling Group for Spontaneous Imbibition," SPERE (November 1996) 280-285. 
Zhou, D, Jia, L. Kamath, J., and Kovscek, A.R., "Scaling of Counter-Current Imbibition Processes in Low-Permeability Porous Media," J. Pet. Sci. and Eng, 33 (2002) 61-74. 


\section{Appendix A-Imbibition Potential}

The object of this appendix is to substantiate the functionality given in Eq. 3 where the volume of water imbibed is proportional to the square root of time. We develop approximate relations for both a frontal advance like pattern of water movement and a diffusive pattern of core fill up. The second object is to draw out the parameters that influence the magnitude of the proportionality constant.

The Bond number $\left(=\left(\rho_{\mathrm{w}}-\rho_{\mathrm{o}}\right) \mathrm{gk} / \sigma\right)$ for water-oil displacement here is of the order $10^{-10}$ assuming a $\mathrm{k}$ of $3 \mathrm{md}$, interfacial tension of $15 \mathrm{mN} / \mathrm{m}$, and a density difference of $200 \mathrm{~kg} / \mathrm{m}^{3}$. Buoyancy is negligible in comparison to capillarity.

A frontal advance analysis similar to Handy's (1960) begins with the assumption of onedimensional fluid flow, the capillary pressure varies linearly with $\mathrm{x}$ (i.e., $\mathrm{d} P_{c} / \mathrm{d} x=\Delta P_{\mathrm{c}} / x$ ), and strictly counter-current flow as described by Eq. 2 . Next, substitute $\mathrm{u}_{\mathrm{w}}\left(=(1 / \phi A) d V_{w} / d t\right)$ to obtain

$$
u_{w}=\frac{1}{A \phi} \frac{d V_{w}}{d t}=k \frac{\mathbf{1}}{\sqrt{\mu_{w} \mu_{o}}} \frac{\sqrt{k_{r w} k_{r o}}}{\sqrt{M}+\frac{1}{\sqrt{M}}} \frac{\Delta P_{C}}{x}
$$

where $\mathrm{V}_{\mathrm{w}}$ is the volume of water imbibed. In a frontal-advance analysis, the volume of water imbibed is related to the position of a front by mass balance according to

$$
x=\frac{V_{w}}{\phi A\left(\mathbf{1}-S_{\text {orsi }}\right)}
$$

Upon substituting Eq. A2 into Eq. A1, simplifying, separation of variables, and integration,

$$
V_{w}^{2}=2 k \frac{(\phi A)^{2}\left(\mathbf{1}-S_{o r s i}\right)}{\sqrt{\mu_{w} \mu_{o}}} \frac{\sqrt{k_{r w} k_{r o}}}{\sqrt{M}+\frac{1}{\sqrt{M}}} \Delta P_{c} t
$$

The volume of water imbibed is converted to the increase in the core-averaged aqueous-phase saturation after division by pore volume $\left(\Delta \bar{S}_{w}=V_{w} / \phi A L\right)$. After conversion of $V_{w}$ to $\Delta \bar{S}_{w}$ and taking the square root of the resulting expression, we obtain

$$
\Delta \bar{S}_{w}=\left(\frac{2 k\left(\mathbf{1}-S_{o r s i}\right)}{\sqrt{\mu_{w} \mu_{O}}} \frac{\sqrt{k_{r w} k_{r o}}}{\sqrt{M}+\frac{1}{\sqrt{M}}} \frac{\Delta P_{c}}{L^{2}}\right)^{0.5} t^{0.5}
$$

Equation A3 teaches that the volume of water imbibed versus time is a function of water and oil viscosity, interfacial tension, wettability, mobility ratio, and rock properties. 
It is arguable that a frontal advance analysis for imbibition may over simplify process physics. Some experimental data display a slow diffuse-like fill up for cores subjected to water. Alternately, we begin by stating continuity for the aqueous phase,

$$
\frac{\partial S_{w}}{\partial t}+\frac{\mathbf{1}}{\phi} \frac{\partial u_{w}}{\partial x}=\mathbf{0}
$$

where constant porosity and incompressibility is assumed. Substituting Eq. 2 of the main text for $u_{\mathrm{w}}$ and employing the equality $\partial P_{c} / \partial x=\left(\partial P_{c} / \partial S_{w}\right)\left(\partial S_{w} / d x\right)$ yields,

$$
\frac{\partial S_{w}}{\partial t}+\frac{\partial}{\partial x}\left(D\left(S_{w}\right) \frac{\partial S_{w}}{\partial x}\right)=\mathbf{0}
$$

where $\mathrm{D}(\mathrm{Sw})$ is the hydraulic diffusivity expressed as

$$
D\left(S_{w}\right)=\frac{k}{\phi} \frac{\mathbf{1}}{\sqrt{\mu_{w} \mu_{o}}} \frac{\sqrt{k_{r w} k_{r o}}}{\sqrt{M}+\frac{1}{\sqrt{M}}} \frac{\partial P_{C}}{\partial S_{w}}
$$

Equation A4 is a second order, nonlinear, partial differential equation. Note the similarity to the diffusion equation. Solution of Eq. A4 is nontrivial. A simplifying assumption is that a constant effective hydraulic diffusivity, $D_{\text {eff, }}$ is obtained by evaluating $D\left(\mathrm{~S}_{\mathrm{w}}\right)$ at some constant representative value. The assumption of constant hydraulic diffusivity allows straightforward analytical solution. The resulting expression (Carslaw and Jaegar, 1959) is

$$
S_{w}(x, t)=\left(\mathbf{1}-S_{\text {orsi }}\right) \operatorname{erfc}\left(\frac{x}{\mathbf{2} \sqrt{D_{\text {eff }} t}}\right)
$$

where $\operatorname{erfc}$ denotes the complementary error function.

The volume of water imbibed as a function of time is obtained upon integrating the water flux at the core inlet.

$$
V_{w}=\left.A \phi D_{\text {eff }} \int_{\mathbf{0}}^{t} \frac{\partial S_{w}(x, t)}{\partial x}\right|_{x=\mathbf{0}} d \tau
$$

Application of a Laplace Transform to the intergral yields

$$
\left.\int_{\mathbf{0}}^{t} \frac{\partial S_{w}(x, t)}{\partial x}\right|_{x=\mathbf{0}} d \tau=\frac{\mathbf{1}}{s} \frac{\left(\mathbf{1}-S_{\text {orsi }}\right)}{\sqrt{D_{e f f}}}
$$

where $\mathrm{s}$ is the Laplace transform of t. Substitution of Eq. A8 into Eq. A7 and application of the Laplace back-transform results in

$$
V_{w}=2 A \phi D_{\text {eff }}^{\mathbf{0 . 5}}\left(\mathbf{1}-S_{\text {orsi }}\right) \sqrt{\frac{t}{\pi}}
$$


Again, we convert the volume of water imbibed to the increase in the average water saturation of the core to obtain

$$
\Delta \bar{S}_{w}=\frac{2\left(1-S_{\text {orsi }}\right) D_{e f f}^{0.5}}{L \sqrt{\pi}} \sqrt{t}
$$

Equations A10 and A3 derive from substantially different analyses. Nevertheless, they display similarly that the change in the average water saturation is proportional to the square root of time. Further, the proportionality constant is parameterized by capillary pressure, phase mobilities, phase viscosity, and relative permeability. 


\section{Appendix B-Experimental Details}

This appendix details the experimental apparatus, fluids and fluid properties, and the procedures for the experiments and processing of data. Significant care was exercised in the choice of materials and in apparatus design, Figure 1. The major components of the apparatus are described next.

\section{Coreholder}

We fabricated a coreholder that resembles a Hassler-sleeve from aluminum (6061-T6). It is relatively transparent to X-rays and therefore CT-scanable. Cores with lengths from 2.5 to 7.5 $\mathrm{cm}$ ( 1 to $3 \mathrm{in})$ and $2.5 \mathrm{~cm}$ ( 1 in) diameter can be held. The apparatus is assembled by coating the core with a thin film of silicone gel (Ultra Copper High Temp RTV Silicone Gasket Maker) and placing the core inside PTFE heat-shrink tubing (Plastic Professional). The silicone-gel is nonwetting and does not penetrate the core. It serves to prevent any fluid bypass during injection once a confining pressure is applied; thus, the silicone gel-tubing combination serves as a hightemperature sleeve.

Mineral oil is used as a confining fluid in the annular space between the sleeve and the aluminum coreholder. A net confining pressure of roughly $1.4 \mathrm{MPa}$ (200 psi) is applied. Heating tape is wrapped around the outside of the coreholder and then covered with high-temperature insulation. A temperature controller maintains constant coreholder temperature. The thermocouple for feedback to the controller is located in the center coreholder between the coreholder and the heating tape. This system was designed and tested for service up to $260^{\circ} \mathrm{C}$ $\left(500{ }^{\circ} \mathrm{F}\right)$ and a maximum total confining pressure of $7 \mathrm{MPa}(1,000 \mathrm{psia})$. In addition to being compatible with a CT scanner, the main advantage of this design is that the effect of heating on rock compaction and fluid expansion is minimized. The system is brought to the temperature of interest at a constant difference between confining and pore pressure while fluids flow through the core.

The endcap contacting the core face contains fluid distribution channels of large dimension (order $\mathrm{mm}$ ) that allow water to be circulated across that face. The pressure drop for fluid flow through the endcap is sensibly zero.

The coreholder is attached to an L-shaped mounting bracket. This assembly is bolted to a stepper-motor driven precision positioning system (Compumotor RP240, Parker-Hannifin) that is used to position the core inside the CT gantry. Any position along the length of the core can be revisited with an accuracy of $\pm 0.01 \mathrm{~mm}$.

\section{CT Scanner}

The scanner is a fourth generation Picker 1200SX with a scan angle of $398^{\circ}$. The voxel dimension is $0.35 \mathrm{~mm}$ by $0.35 \mathrm{~mm}$ by $3 \mathrm{~mm}$. The tube current is $95 \mathrm{~mA}$ and the energy level of 
the radiation is $130 \mathrm{keV}$. The acquisition time of one image is roughly $6 \mathrm{~s}$, while the processing time is about $40 \mathrm{~s}$. Images are acquired in series and processing delayed until all the desired images are acquired. Thus, the total acquisition time to scan the length of the core is short enough to characterize the saturation profile along the core at a particular time.

\section{Rock}

Both field and outcrop cores are studied. All rock samples are in the opal-A phase. Outcrop diatomite cores (Grefco Quarry, Lompoc, CA) have a porosity, $\phi$, of $62-67 \%$ and an air permeability, $k$, of 2-4 md, as shown in Table 1 . They are almost pure white and the porosity is relatively uniform, as shown by the representative CT image in Fig.2a. This quarried diatomite represents an end member of so-called "clean" reservoir rock resulting from Late Miocene diatomaceous sediments (Ross and Kovscek, 2002). It provides a convenient and appropriate contrast to the reservoir samples used here. A previous study (Akin et al., 2000) characterized fully water-wet samples. We used a similar process to cut and shape the outcrop diatomite core samples as described in Akin et al. (2000) and Zhou et al. (2002). Our previous study did not suggest a need to clean outcrop samples. The cores were not cleaned but dried and preserved in an oven under house vacuum at a temperature of $50-60{ }^{\circ} \mathrm{C}$.

Field cores are from a heavy-oil field (Kern Co., CA). Only 4 samples were available. Core recovery from diatomite fields is frustratingly poor. Their porosity ranges from 55 to $59 \%$ and permeability from 0.8 to $1.3 \mathrm{md}$, as indicated by Table 1 . The field cores were sampled from depths between 650 to $980 \mathrm{ft}$ : F-1 depth=681.3 ft, F-2 depth $=685.3 \mathrm{ft}, \mathrm{F}-15 \mathrm{depth}=920.8 \mathrm{ft}$, and F-25 depth=928.1 ft. Cores were cleaned by injecting a mixture of methanol and toluene. After cleaning, they were preserved in synthetic reservoir brine and sealed in an airtight aluminum bag until used. The composition of the synthetic brine is listed in Table 2. Prior to an experiment, the brine was removed by drying carefully at $35^{\circ} \mathrm{C}$ under house vacuum to avoid inducing fractures. Measurement of permeability after drying verified that cores had not fractured, see Table 1. Figures $2 b$ to $2 d$ show representative porosity images obtained for the field cores. Bedding and banding structures are obvious. The length of the field cores was about 4.0 to $6.5 \mathrm{~cm}$ (1.6 to 2.6 in) and the diameter was $2.5 \mathrm{~cm}$ (1 in).

\section{Fluids}

Three white oils, n-decane, blandol (Witco Chemical), and PAO40 (Chevron Lubricants), and filtered/dewatered heavy crude oil were used as the oil phases. The viscosity at room temperature $\left(22{ }^{\circ} \mathrm{C}\right.$ ) for these oils is $0.93 \mathrm{mPa}-\mathrm{s}, 35 \mathrm{mPa}-\mathrm{s}, 407 \mathrm{mPa}-\mathrm{s}$, and 6,400 $\mathrm{mPa}-\mathrm{s}$, respectively. The $n$-decane was chosen because it has a viscosity similar to water for a range of temperature. In the next stages, a modestly viscous mineral oil, blandol, and more viscous PAO40 were used. Hence, the viscosity ratio is made progressively less favorable $\left(\mu_{\mathrm{w}} / \mu_{\mathrm{nw}}<1\right)$. The chief reason for using the mineral oils was to identify the effect of temperature on recovery due to oil viscosity reduction without contribution to recovery from wettability alteration via crude oilbrine-rock interaction. It is shown in the main text that this goal was not entirely realized. Lastly, 
we used crude oil to study further water imbibition behavior that could be attributed to both reduction of oil viscosity and wettability alteration accompanying an increase in temperature.

Figure 3 shows the measured viscosity versus temperature relationship for the oils and for water. It shows that the crude oil is the most sensitive to temperature. The viscosities of $n$-decane and water versus temperature were obtained from Lide (2000). Oil viscosity was measured with a Brookfield (LVT) viscometer. The synthetic reservoir brine in Table 2 was used as the water phase for all tests. It was prepared by dissolving salts in house distilled water. This dilute brine has the same viscosity versus temperature relation as pure water (Prats, 1986).

The interfacial tension between oil and water at various temperatures was measured using a Fisher Surface Tensiometer (Model 20). The liquids were preheated in an oven and then placed in the chamber for measurement. Due to the limitation of this instrument, we were only able to measure the IFT at temperatures below $80^{\circ} \mathrm{C}$, as shown in Fig.4. The data were used to estimate the tension as a function of temperature with the following corresponding states relationship (Birdi, 1986; Reid et al., 1987),

$$
\sigma=C_{O}\left(1-\frac{T}{T_{C}}\right)^{C_{1}}
$$

where $\sigma$ is the interfacial tension $(\mathrm{N} / \mathrm{m}), T$ is the temperature $(\mathrm{K}), C_{0}$ and $C_{1}$ are constants, and $T_{c}$ is the pseudocritical temperature $(\mathrm{K})$ of the oil and water mixture. The numerical value of $C_{l}$ is set to 1.22 (Reid et al., 1987). When extrapolating $\sigma$ data at $T_{1}$ to $\sigma$ at $T_{2}$, a ratio $\left(\sigma\left(T_{1}\right): \sigma\left(T_{2}\right)\right.$ ) is constructed so that the coefficient $C_{0}$ is eliminated. The mixture pseudocritical temperatures are then obtained by regression of the data in Fig. 3. The $\mathrm{T}_{\mathrm{c}}$ for $\mathrm{n}$-decane-water, blandol-water, PAO40-water, and crude oil-water systems are taken as 568, 580, 584, and $598 \mathrm{~K}$, respectively. Thereafter, we estimated the interfacial tension at temperatures outside the range of measurement.

\section{Procedure}

Figure 1 shows the complete experimental apparatus. Brine flows past one face of the core at $0.5 \mathrm{~cm}^{3} / \mathrm{min}$ via pump (reciprocating or syringe pump at high temperature). We study countercurrent behavior so the outlet of the coreholder was closed for all tests during the spontaneous imbibition phase. A backpressure regulator (model R3A-C, N-norgren Inc) elevated the system pressure to range between 2.7 and $4.1 \mathrm{MPa}$ (400 to $600 \mathrm{psia}$ ) such that water remained in the liquid phase throughout the duration of an experiment. The confining pressure was set from 4.1 $\mathrm{MPa}$ to $5.5 \mathrm{MPa}$ (600 to $800 \mathrm{psia}$ ) so as to maintain roughly $1.4 \mathrm{MPa}$ (200 psia) of net effective stress. All experiments were conducted under isothermal conditions.

Produced oil was collected in a graduated cylinder to provide a check on the mass balance and average water saturation as measured by X-ray CT scanning. After the produced water was separated from the oil, it was returned to a $300 \mathrm{~cm}^{3}$ water cylinder maintained at the experimental temperature. 
The core was placed horizontally within the CT scanner gantry, as indicated by Fig.1. Cylindrical volume sections were monitored at $5 \mathrm{~mm}$ intervals along the central axis of the core. About 8 to 10 images were collected sequentially to span the core from inlet to outlet at any particular time. For the tests with an initial water saturation of zero, images of the dry airsaturated cores were taken first. Then oil was injected into the core until $100 \%$ saturated with oil and a second set of images acquired. The voxel-by-voxel values of porosity were calculated (Withjack, 1988) as,

$$
\phi=\frac{C T_{o r}-C T_{a r}}{C T_{o}-C T_{a}}
$$

where CT represents CT number (Hounsfields). The subscripts or and ar signify oil-saturated rock and air saturated rock, respectively; whereas $o$ and $a$ represent the oil and air bulk fluid phases. Average core porosity is obtained by averaging porosity across each cross section and then averaging cross-sectional porosities.

Once the initial condition was established, the core was subjected to counter-current water imbibition. Water was supplied at the core face until no further change in water saturation was measured by CT. The voxel-by-voxel water saturation, $S_{w}$, of each cross-sectional area was computed using 6

$$
S_{w}=\frac{C T_{o w r}-C T_{o r}}{\phi\left(C T_{w}-C T_{o}\right)}
$$

where $\phi$ is the independently measured porosity from Eq. B2, the subscript $o w r$ refers to rock containing oil and water, the subscript $w$ denotes the bulk water phase. One-dimensional saturation profiles were obtained by averaging the voxel-by-voxel $S_{w}$ images from a cross section. Average water saturation within the core, $\bar{S}_{w}$, was obtained by averaging all of the cross-sectional $S_{w}$.

For the tests with nonzero $S_{w i}$, images of the dry air-saturated cores were taken first. Then the core was $100 \%$ saturated with water and a second set of images was acquired. Thereafter, oil was injected into the core to establish initial water saturation. The flow direction was reversed several times to obtain a uniform saturation distribution. When the initial water saturation was established, a third set of images were taken. 


\section{Area 3. Mechanisms of Primary Heavy Oil Recovery}

So-called cold production of heavy oil is attractive because steam injection is costly due to the operating and capital expenses for steam generation facilities. Sometimes primary recovery can be on the order of $10-15 \%$ from heavy-oil reservoirs. Most cases of significant cold production are associated with foamy crude oil and horizontal wells. Here, gas released from solution during pressure depletion remains dispersed as small bubbles rather than uniting to form a single phase. With foamy crude oil, the gas-oil ratio (GOR) is lower and the oil productivity of a well is increased over the unfoamed case.

A key to developing a mechanistic understanding of foamy-oil behavior is to delineate bubble growth, interaction, and flow mechanisms at the pore level. These were relatively unknown for foamy heavy oil at the start of this project. We used twodimensional micromodels with one to one representation of sandstone pore features to observe bubble size, nucleation, growth, and flow. The micromodels were subjected to a declining pressure and the dynamics of heavy-oil solution gas drive observed. By using various types of oil, we hope to delineate crude-oil conditions that are conducive to the formation of foamy oil. As pressure and temperature are important to crude-oil behavior, a major portion of the micromodel effort will be in the design of temperature controlled pressure vessel to conduct experiments at relevant temperatures and pressures. The major difficulty is the construction of an apparatus that provides a confining pressure to the micromodel and incorporates a transparent window for viewing the micromodel. The thickness of window, transparent confining fluid, and micromodel must remain thin for imaging with a high-power microscope objective. This work is summarized in the section entitled "A Microvisual Study of Solution Gas Drive Mechanisms in Viscous Oils."

Companion experiments were also conducted in sands and sandstone. The purpose of the experiments was to measure the evolution of oil and gas saturation in situ as well as pressure while undergoing primary production of foaming and non-foaming heavy crude oils. Production rate was varied to delineate critical rates of production necessary for foamy oil generation. Details in this subarea are entitled "Heavy Oil Solution Gas Drive: Effect of Oil Composition and Rock Consolidation."

Additionally, the experimental observations and results from the above were translated into a mechanistic model for cold production of heavy oil. The model is mechanistic and consistent with physical observations. Because unfoamed oil is a subset of foamy oil, the model addresses primary production of all heavy crudes. Information on foam-bubble size and the rheology of bubble-laden crude oil has been translated into a model and subsequently tested in a reservoir simulator. Our approach is based on a bubble population balance. In a population-balance method, the number density of bubbles (i.e., the number of bubbles per unit volume of gas) is tracked as a function of location and time. Number density is also referred to as bubble texture. This section is entitled, "Model and Simulation of Cold Production Using a Population Balance Approach." 


\section{A Microvisual Study of Solution Gas Drive Mechanisms in Viscous Oils}

\section{(Task 3a)}

\section{Introduction}

Primary production from some heavy-oil reservoirs operating under a solution-gas-drive mechanism exhibits unexpectedly high primary recovery with relatively low pressure decline rate, lower than expected producing gas oil ratios, and higher oil production rates. (Bora et al., 1997). The process of solution gas drive involves nucleation of gas bubbles as the pressure in the reservoir falls below the bubble point. It is postulated that these small bubbles are trapped and tend to remain dispersed in the oil for a relatively long period of time. Initial development of heavy-oil reservoirs under solution-gas drive has economic advantage because implementation of traditional thermal methods can be avoided or delayed, resulting in cost savings. For example, at Frog Lake, Alberta Canada, a study (Huang et al., 1997) concluded that cold production, the simultaneous production of oil and sand with the aid of progressive cavity pumps, can produce heavy oil $\left(12^{\circ}\right.$ API, 20,000 to $\left.50,000 \mathrm{cP}\right)$ without the introduction of any external thermal energy. Another study in the Hamaca area of the Orinoco Belt in Eastern Venezuela (De Mirabal et al., 1996,1997 ) attributed the $10 \%$ OOIP (original oil in place) recovery to foamy-oil behavior of the crude. It was postulated that the primary production mechanism of the Hamaca reservoirs is controlled by the in-situ formation of a non-aqueous, oil-continuous foam under solution gas drive conditions. Notably, recovery in this case was obtained without sand production.

To explain the above phenomena a number of mechanisms have been suggested that can be divided into two main categories. Geomechanical effects such as sand dilation and development of wormholes comprise the first category (Dusseault, 1993; Tremblay et al., 1999). Higher oil production rate with sand mobilization may lead to enhanced well drainage radius, grain movement, and gas bubble expansion. The second category suggests that the special properties of the flowing fluids, the gas and heavy oil, are the main reasons for high production (Pooladi-Darvish and Firoozabadi, 1999; Firoozabadi, 2001). These categories are not mutually exclusive.

In either event, retention of the evolved gas phase in the oil leads to maintenance of the reservoir energy. Although Dake (1978) does not explicitly discuss heavy oils, the general material balance equation is applicable to primary recovery by solution gas drive:

$$
R_{F}=\left(\frac{\left(B_{o}-B_{o i}\right)+\left(R_{s i}-R_{s}\right) B_{g}}{B_{o}+\left(R_{p}-R_{s}\right) B_{g}}\right)
$$

where $\mathrm{R}_{\mathrm{F}}$ is the fraction of the OOIP recovered, $\mathrm{B}$ is the formation volume factor, the subscripts $\mathrm{o}$ and $\mathrm{g}$ refer to oil and gas phases, respectively, and $\mathrm{R}$ is the gas-oil ratio. The subscripts $\mathrm{s}$ and si refer to the solution gas-oil ratio and its initial value whereas the subscript $\mathrm{p}$ refers to the cumulative gas-oil ratio of the oil and gas produced. For heavy oils, $\mathrm{B}_{\mathrm{o}}$ is close to 1 and varies little with pressure and/or the amount of gas in solution. Hence, the terms related to gas-oil ratio 
determine $R_{F}$. Equation 1 is inversely proportional to $R_{p}-R_{S}$. Thus, systems where the cumulative gas-oil ratio differs little from the solution gas-oil ratio, all other factors being equal, obtain maximum oil production. Gas that remains in the reservoir enhances the total reservoir system compressibility and results in greater cumulative production for a given pressure drop between the well and the reservoir. It is our postulate that gas is retained because it coalesces slowly and remains dispersed as bubbles that are on the order of pore-size dimensions.

The work presented here is directed towards developing a better understanding of the coalescence of bubbles during solution gas drive behavior in heavy oils. Specifically, we wish to understand the effect of oil-phase viscosity on the coalescence of gas bubbles trapped by narrow pore throats. Broadly speaking, bubble coalescence during solution gas drive is divided into two time regimes. The first is governed by the diffusion of gas through oil to bubbles, whereas the later is governed by pressure depletion and volumetric expansion that drains oil from between bubbles (Arora and Kovscek, 2000, 2003). The paper proceeds by discussing further the effect of oil-phase viscosity on these two regimes. This motivates additional experimental study of, especially, the late-time regime. A microvisual apparatus employing etched-silicon micromodels is then described along with results for bubble nucleation, growth, and coalescence. Discussion and conclusions round out the manuscript.

\section{Dynamics of Bubble Growth in Porous Media}

In bulk solution, gas nuclei appear due to thermal fluctuations when the pressure is below the bubble point (Scriven, 1959; Wilt, 1986). For gas/oil systems, homogeneous nucleation requires a supersaturation of several thousand $\mathrm{kPa}$ provided that the system is not close to critical (Kamath and Boyer, 1995). Supersaturation is the difference between the liquid-phase pressure at which liquid and gas are in equilibrium and the actual liquid-phase pressure. In porous media, values of supersaturation are not large, compared to bulk solution, indicating heterogeneous nucleation (Kamath and Boyer, 1995). Nucleation sites, pore-wall topology and capillary forces in combination with diffusion characteristics, such as the competition for solute between bubbles, determine the gas-phase occupancy of a porous medium.

There are two principal conceptual models for pressure and volume evolution during solution gas drive: (i) instantaneous nucleation (IN-Firoozabadi and Kashchiev, 1996) and (ii) heterogeneous nucleation in a progressive fashion (PN- $\mathrm{Li}$ and Yortsos, 1995). Instantaneous nucleation assumes that all bubbles nucleate from randomly located active sites at essentially the same time. Bubbles are not modeled as originating from preexisting bubbles trapped in crevices or the roughness of pore walls (Firoozabadi, 1997). Diffusional bubble growth is modeled as three-dimensional with shape factors to account for nonspherical bubble shapes. It follows that bubbles grow in a compact fashion such that bubbles are of roughly equal size throughout the porous medium.

On the other hand, PN assumes that bubbles are released from sites such as crevices or roughness on pore walls with poor liquid wetting characteristics. These sites become activated when the local supersaturation exceeds the capillary pressure of the site. Because the size of such crevices holding trapped gas varies, bubbles are released from pore walls at different degrees of supersaturation, that is progressively as the supersaturation increases. Bubbles grow as a result of 
diffusional transport of gas; however, the scaling of bubble growth in porous media is different as compared to bulk due to the underlying pore microstructure (Li and Yortsos, 1995; Satik et al., 1995). Bubble growth is complicated by the geometry of pore space, the availability of solute, and the pressure gradient.

El Yousfi et al. (1997) undertook a series of experiments in transparent two-dimensional representations of pore space (i.e., micromodels) to differentiate among the IN or PN model. They saturated the micromodels with a solution of carbon dioxide in water. Bubbles were nucleated at a fixed supersaturation by connecting the micromodel to a gas reservoir maintained at a pressure significantly less than the initial pressure of the micromodel $(300 \mathrm{kPa})$. Bubbles appeared progressively for a period of time when the pressure drop became sufficient to overcome capillary forces. Later, bubbles grew by diffusion and expansion of total volume. The series of events described is more similar to PN than to IN especially in regard to the release of preexisting small bubbles from pore walls. However, the duration of bubble production (1 hour) was about $15 \%$ of the length of time required to deplete the micromodel of pressure. Hence, they stated that the process might be approximated by IN if the longer time behavior is of interest. Subsequently, Arora and Kovscek $(2001,2003)$ implemented a bubble population balance model of solution gas drive incorporating PN physics. They found that the period of active bubble nucleation during pressure depletion was relatively short for the experiments modeled. In their calculations, the supersaturation was always nonzero, but increased only during the initial stages of pressure depletion. Their results supported the truncated progressive nucleation observations made by El Yousfi et al. (1997).

\section{Diffusion-Dominated Coalescence}

To study the role of oil-phase viscosity during the early diffusion controlled period of bubble growth and coalsecence, we employ the IN model (Firoozabadi and Kashchiev, 1996). This choice is made simply because it is relatively easy to expose the role of viscosity quantitatively within IN. Alternately, the PN model should lead to similar conclusions with respect to the role of viscosity on bubble growth and coalescence, but the time scaling may be different.

Equations 4-12 of Firoozabadi and Kashchiev (1996) are used to develop a first order differential equation (Eq. 13 of Firoozabadi and Kashchiev, 1996) for the volume of gas evolved. The equation is solved here with the Runge-Kutta method (Press et al., 1992). The diffusion coefficient plays a role in setting the kinetic constant (Eq. A4 of Firoozabadi and Kashchiev. (1996) for bubble growth. The infinitely-dilute, mutual binary diffusion coefficient, $\mathrm{D}_{\mathrm{AB}}$, is calculated for our work using the empirical correlation of Hayduk and Minhas (1982),

$$
\mathrm{D}_{\mathrm{AB}}=13.3 \times 10^{-8} \frac{\mathrm{T}^{1.47} \mu^{\varepsilon}}{\mathrm{V}_{\mathrm{A}}^{0.71}}
$$

where $\mathrm{T}$ is the absolute temperature, $\mu$ is the viscosity, $\mathrm{V}_{\mathrm{A}}$ is the molar volume, and the subscripts $\mathrm{A}$ and $\mathrm{B}$ represent the solute and solvent, respectively. According to Reid et al. (1987), $\mathrm{D}_{\mathrm{AB}}$ can 
be assumed to be a representative diffusion coefficient for concentrations of $\mathrm{A}$ as large as 10 mole percent. We assume for calculations that $\mathrm{CH}_{4}$ is the solute dissolved in oil. The exponent $\varepsilon$ is given by

$$
\varepsilon=\frac{10.2}{\mathrm{~V}_{\mathrm{A}}}-0.791
$$

From Tyn and Calus (1975), molar volume is estimated according to

$$
\mathrm{V}_{\mathrm{A}}=0.285 \mathrm{~V}_{\mathrm{C}}^{1.048}
$$

The critical volume, $\mathrm{V}_{\mathrm{C}}$, of methane is $270 \mathrm{~cm}^{3}$. A temperature of $37.8^{\circ} \mathrm{C}$ and bubblepoint pressure of 28.44 bars are taken for calculations. Figure 1 illustrates that the diffusion coefficient decreases by an order of magnitude as oil-phase viscosity increases from 100 to $1000 \mathrm{cP}$, all other factors being equal.

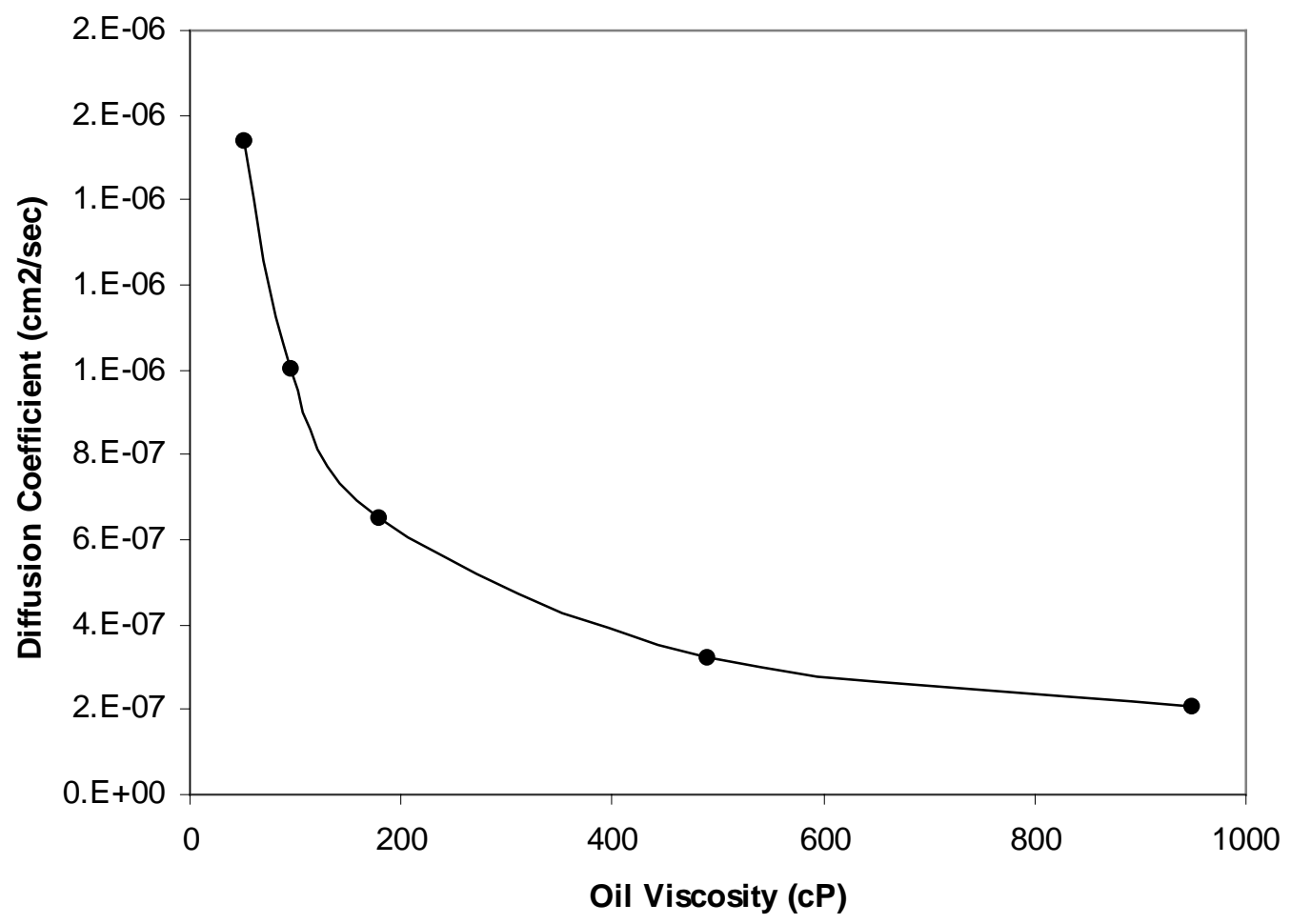

Figure 1. Variation in diffusion coefficient of $\mathrm{CH}_{4}$ with oil viscosity.

Additional parameter choices for bubble growth calculations include: a pressure depletion rate of $5 \mathrm{bar} /$ day, 5 bubbles nucleated per $\mathrm{cm}^{3}$ of liquid, and initial bubble radius of $1 \mu \mathrm{m}$. Other parameters are used as specified by Firoozabadi and Kashchiev (1996). Note again that the diffusion coefficient enters into the kinetic constant determining the rate of bubble growth. The 
growth of gas bubble volume as a function of time is calculated for oils with viscosities of 51, 95, 178, 450, and $950 \mathrm{cP}$. Results are presented in Fig. 2. Viscosity plays an important role in defining the coalescence dynamics of gas bubbles during the early diffusion dominated stage. Bubble volume increases much more slowly in viscous oils compared to light oils chiefly because gas diffuses much more slowly through viscous oil. In Fig. 2, it is clear that roughly doubling the oil-phase viscosity from 51 to $95 \mathrm{cP}$ more than halves the volume of a bubble at a given time.

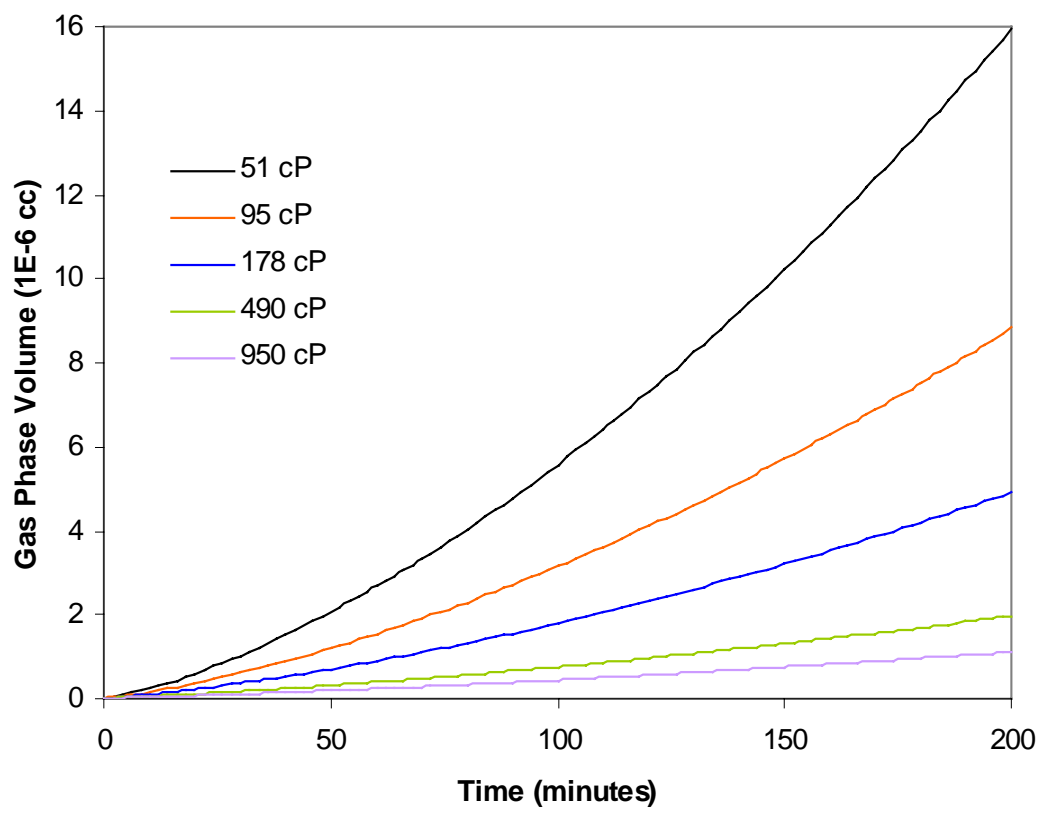

Figure 2. Variation in gas phase volume with time (constant pressure decline rate).

\section{Pressure-Driven Coalescence}

At times significantly later than bubble nucleation, coalescence is controlled mainly by the pressure drop across bubbles that results from flow of oil and volumetric expansion of bubbles. The liquid drains from thick-liquid bridges between bubbles, until they come close enough to coalesce. A physical model is developed next to illustrate the role of solution viscosity on the coalescence of gas bubbles.

Consider Fig. 3 to understand a possible geometry of bubbles trapped inside a pore. Such a configuration might arise in porous media if (i) the right bubble is held in place by a pore throat and the left bubble is driven forward, (ii) the bubbles are arrayed in a channel that is perpendicular to the flow direction such that the resulting pressure drop drives both bubbles toward one another, or (iii) the bubbles are oriented vertically and the lower bubble rises under the forces of buoyancy. In Fig. 3, oil is the wetting phase, gas is the non-wetting phase, and there is no aqueous phase present. The pore has a noncircular cross-sectional area, such as a triangle or square, Fig. 3b. Noaxisymmetric, cornered cross-sectional areas reflect the corners, nooks, and crannies of real pores in porous media. Diffusional bubble growth at this stage is slow and 
neglected. The flow of oil out of the lens occurs through the bulk oil held by capillarity in the pore corners. Transport through the oil films that line the straight section of the bubble-pore wall interface is ignored. Flow resistance in thin films is inversely proportional to film thickness to the third power; film flow is highly resistive compared to the bulk flow of liquids in corners. Any effect of gravity is ignored at the pore level. Finally, we assume that no thin-film forces are present to stabilize gas-oil interfaces.

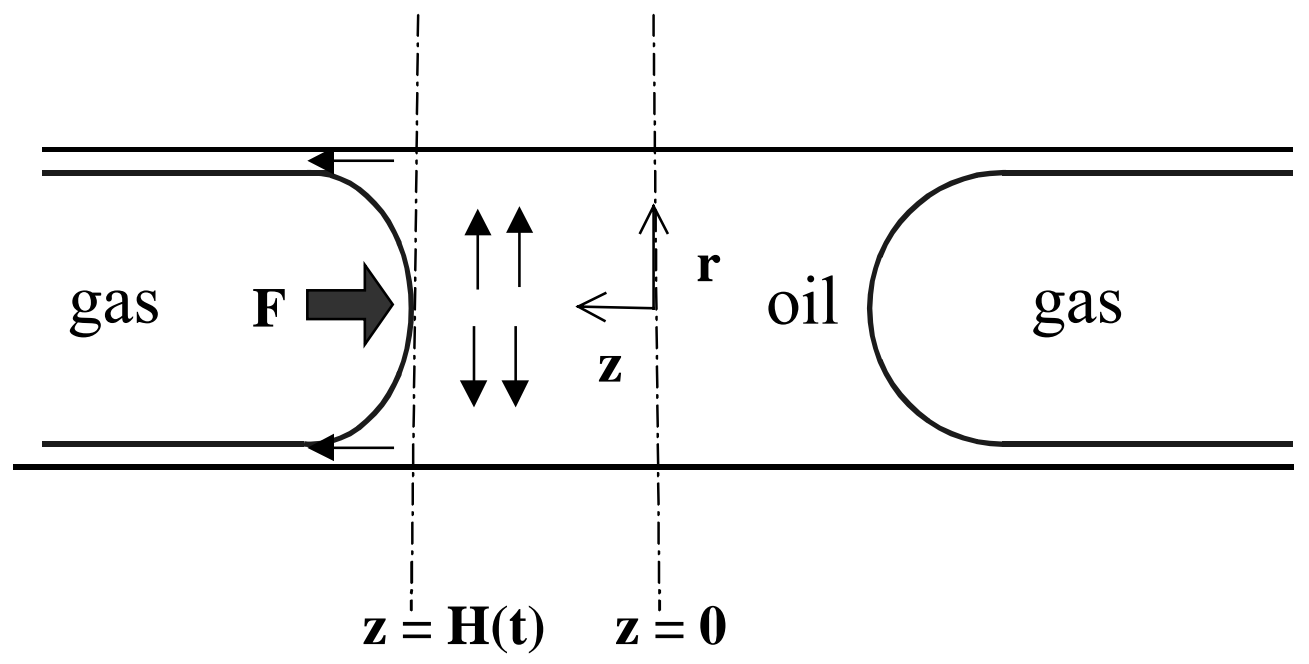

(a)

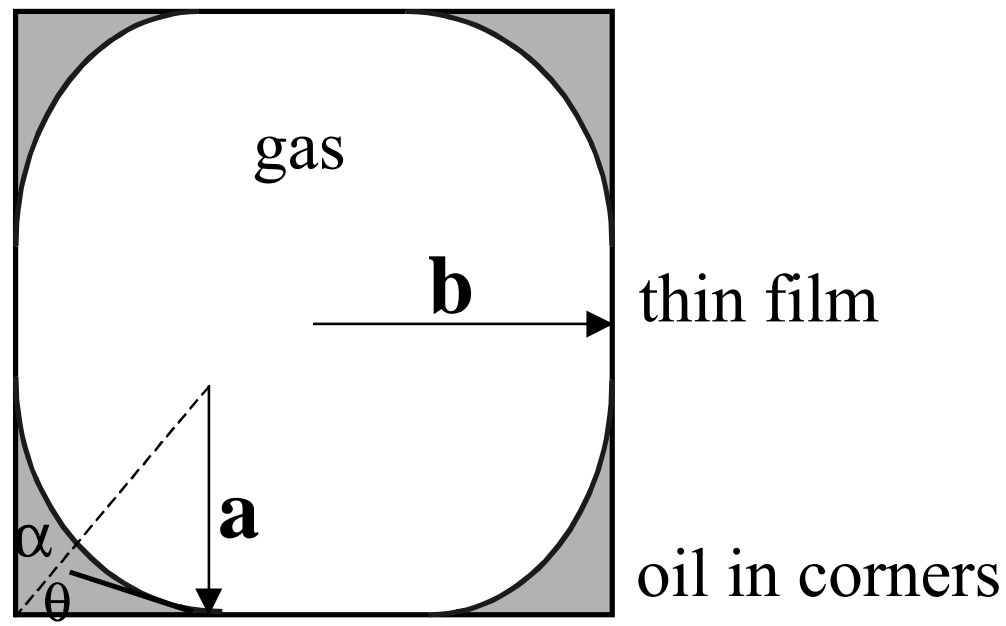

(b)

Figure 3(a) Side view of two gas bubbles moving towards each other in a narrow pore;

(b) cross-sectional view showing wetting-liquid distribution in a square pore and illustrating the geometry of the corner flow problem.

The domain is divided in half by virtue of symmetry, as illustrated in Fig. 3. This describes coalescence scenario (i) or (ii) above. The plane of symmetry is a no-flow boundary. At this point, the problem becomes analogous to a "squeeze film" (Denn, 1980). Gas bubbles move 
towards the symmetry plane and the oil flows out through the pore corners. The boundary conditions are

$$
\mathrm{v}_{\mathrm{z}}=\mathrm{v}_{\mathrm{r}}=0
$$

at the symmetry plane, where $\mathrm{v}_{\mathrm{z}}$ is the liquid velocity in the $\mathrm{z}$ direction and $\mathrm{v}_{\mathrm{r}}$ represents velocity in the $r$ direction. The oil-gas bubble interface moves towards the symmetry plane, with velocity $\mathrm{V}(\mathrm{t})$. Therefore, at the moving surface $(\mathrm{z}=\mathrm{H}(\mathrm{t}))$ :

$$
\begin{gathered}
\mathrm{v}_{\mathrm{z}}=\mathrm{V}(\mathrm{t}) \\
\mathrm{v}_{\mathrm{r}}=0
\end{gathered}
$$

The creeping flow approximation to the Navier Stokes equations is appropriate to the geometry and boundary conditions. The relevant equations are (Denn, 1980)

$$
\begin{array}{r}
v_{r}=\frac{3 r z V(t)}{H^{2}(t)}\left\lfloor 1-\frac{z}{H(t)}\right\rfloor \\
V(t)=-\frac{d H}{d t}=\left\lfloor\frac{2 F}{3 \pi \mu R^{4}}\right\rfloor H^{3}
\end{array}
$$

where $H(t)$ is the distance between the symmetry plane and the bubble front at time $t, \mu$ is the viscosity of the wetting fluid, $\mathrm{F}$, is the net imposed force at the surface represented by $\mathrm{H}(\mathrm{t})$, and $\mathrm{R}$ is the effective radius of the bubble in the pore cross section. The effective radius is related to the projected cross-sectional area, $A_{p}$, of the gas-liquid interfaces as $R=\left(A_{p} / \pi\right)^{0.5}$.

The task now is to calculate the time needed for the two bubbles separated by distance $\mathrm{H}_{0}$, to drain the oil lens so that coalescence may occur. Solution of Eq. 9 and some rearrangement, exposes the time dependence explicitly:

$$
\left.\mathrm{t}=\left\lfloor\frac{3 \pi \mu \mathrm{R}^{4}}{4 \mathrm{~F}}\right\rfloor \mid \frac{1}{\mathrm{H}^{2}(\mathrm{t})}-\frac{1}{\mathrm{H}_{\mathrm{o}}^{2}}\right\rfloor
$$

In the above equation $\mathrm{R}, \mathrm{H}$, and $\mathrm{H}_{\mathrm{o}}$ can be measured and quantified from visual micromodel experiments, to follow. The viscosity of the oil, $\mu$, is readily measured. The only unknown that needs additional mathematical treatment is the imposed force $\mathrm{F}$.

The net force, $\mathrm{F}$, is the net pressure drop pressure times the projected cross-sectional area of the gas-liquid interface of the bubble:

$$
F=\left(\Delta P-\Delta P_{c}\right) A_{p}
$$


where $\Delta \mathrm{P}$ is the pressure drop across the bubble and $\Delta \mathrm{P}_{\mathrm{c}}$ is the pressure drop for oil flow in the four pore corners. The resistance to flow in the pore corners opposes thinning of the lens. The expression for the term $\Delta \mathrm{P}_{\mathrm{c}}$ is (Ransohoff and Radke,1988)

$$
\Delta P_{c}=\frac{q_{w} \mu \beta L}{a^{2} A_{w}}
$$

where $q_{w}$ is the volumetric oil flow rate through the corners, $\beta$ is the dimensionless flow resistance coefficient, $\mathrm{L}$ is the length of the bubble, $\mathrm{A}_{\mathrm{w}}$ is the area of the corners occupied by the oil, and $\mathrm{a}$ is the interfacial radius of curvature, as shown in Fig $3 \mathrm{~b}$. The volumetric flow rate $\mathrm{q}_{\mathrm{w}}$ out of the oil lens is obtained by integrating Eq. 8 over the wetted perimeter times the thickness of the lens and back substituting V(t) from Eq. 9 to yield

$$
q_{w}=\left\lfloor\frac{6 \pi R^{2} V(t)}{H^{2}(t)}\right\rfloor\left\lfloor\frac{z^{2}}{2}-\frac{z^{3}}{3 H(t)}\right\rfloor=\frac{2 F H^{3}(t)}{3 \mu R^{2}}
$$

Now substituting this result for $\mathrm{q}_{\mathrm{w}}$ into the expression for $\Delta \mathrm{P}_{\mathrm{c}}$ in Eq. 12 gives

$$
\Delta P_{c}=\frac{2 F H^{3}(t) \beta L}{3 a^{2} R^{2} A_{w}}
$$

Finally substituting Eq. 11 into Eq. 14 to eliminate F and rearranging results in

$$
\Delta P_{c}=\left\lfloor\frac{\lambda(t)}{1+\lambda(t)}\right\rfloor \Delta P
$$

where

$$
\lambda(t)=\left\lfloor\frac{2 \pi H^{3}(t) \beta L}{3 a^{2} A_{w}}\right\rfloor
$$

At this point, the relationship is established between $\Delta \mathrm{P}$ and $\Delta \mathrm{P}_{\mathrm{c}}$ and the time required to drain the oil lens is calculable. An example illustrates the effect of oil viscosity on bubble coalescence in the pressure-driven regime, Fig. 4. The pore cross section is taken as square; the radius, $b$, of the largest circle that may be inscribed within the square is $10 \mu \mathrm{m}$. Fluid viscosity is set to $1,51,95,178,490$, and $950 \mathrm{cP}$. The bubble length and lens thickness are set $100 \mu \mathrm{m}$ and $50 \mu \mathrm{m}$, respectively. For a no-slip condition, sharp corner, zero degree contact angle, and square pore, the theoretical value of $\beta$ is 93.93 (Ransohoff and Radke, 1988). The value of $\Delta \mathrm{p}$ is taken as $1.4 \mathrm{KPa}(0.2 \mathrm{psi})$ to correspond approximately with the micromodel experiments to follow. The value for interfacial radius, a, is calculated from the work of Ransohoff et al. (1987). They tabulate values for the equilibrium dimensionless interfacial entry curvature, $C_{d m}=C_{m} b$, in a variety of noncircular cross sections. For a square cross-section, $\mathrm{C}_{\mathrm{dm}}$ equals 1.89 . For a pore radius of $(b=) 10.0 \mu \mathrm{m}$, the interfacial radius of curvature in the cross section illustrated in Fig. 3(b) equals $\left(\mathrm{a}=\mathrm{b} / \mathrm{C}_{\mathrm{dm}}\right) 5.29 \mu \mathrm{m}$. The area wetted by the oil at the four corners, $\mathrm{A}_{\mathrm{w}}$, is related to 
interfacial radius a by the geometric expression $A_{w}=4 a^{2}(1-\pi / 4)$. A zero degree contact angle is assumed. For the pore dimensions above, $\mathrm{A}_{\mathrm{w}}$ equals $24.0 \mu \mathrm{m}^{2}$. This detail to obtain the interfacial radius of curvature and wetted area is necessary because cornered pores allow bubbles to adopt equilibrium entry curvatures that are slightly smaller than $2 / \mathrm{b}$ corresponding to a hemispherical bubble (cf. Ransohoff et al, 1987). For a square pore, it follows that the projected cross-sectional area of the gas-liquid interface is $\left(=4 \mathrm{~b}^{2}-\mathrm{A}_{\mathrm{w}}\right) 376 . \mu \mathrm{m}^{2}$. Consequently, the effective radius of the bubble in the square pore is $\left(=\left(\left(4 b^{2}-A_{w}\right) / \pi\right)^{0.5}\right) 11.0 \mu \mathrm{m}$.

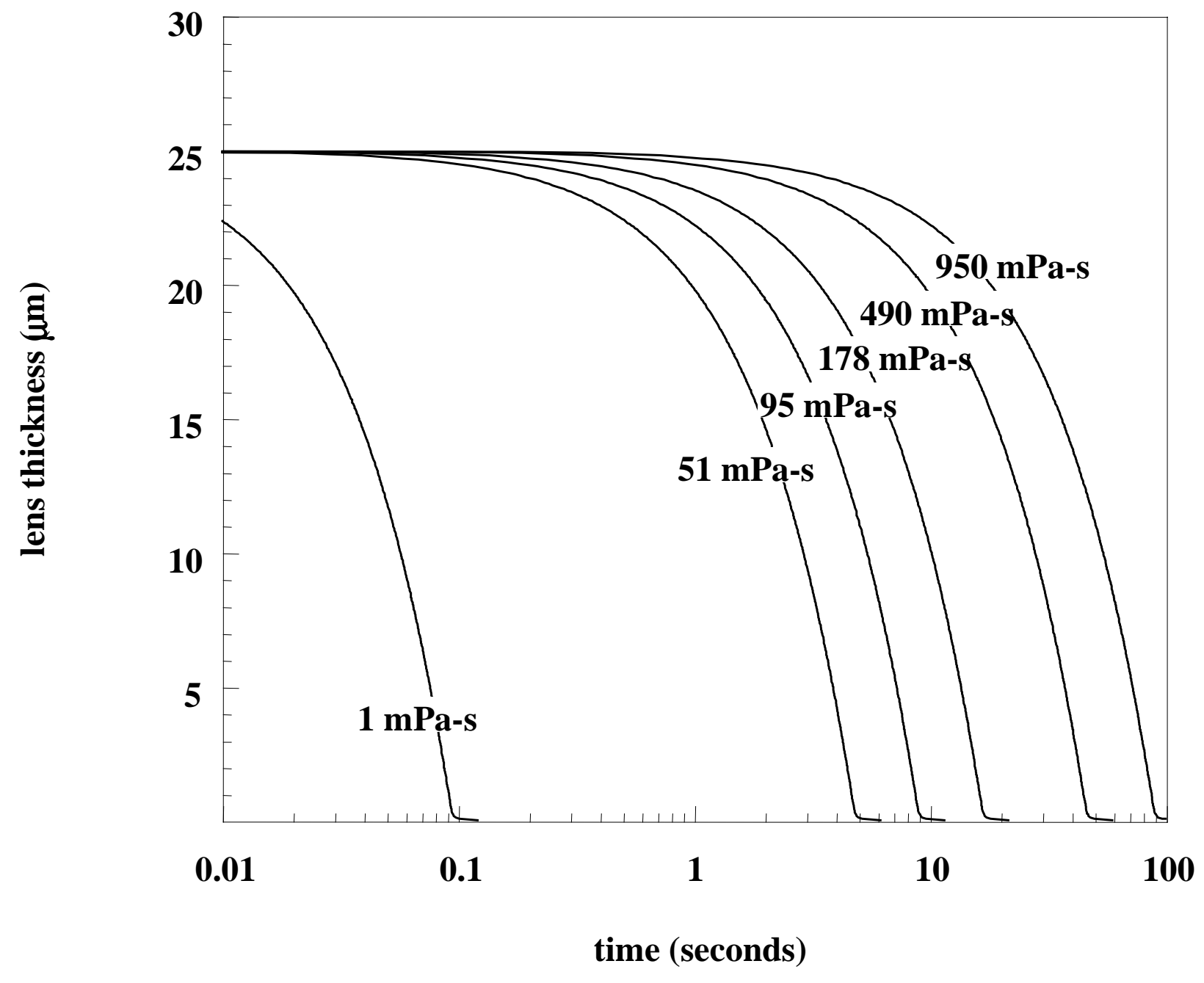

Figure 4. Example calculation illustrating the effect of oil viscosity on pressure-driven coalescence of two gas bubbles in a cornered capillary.

Figure 4 presents lens thickness versus time for this example. A logarithmic scale is used because times for thinning vary substantially over the three orders of magnitude of $\mu$. The time interval for coalescence is found by computing time versus interface position according to Eq. 10 from the initial position to $1 \mu \mathrm{m}$ of separation. The solution is then extrapolated to an interface separation of zero. At small thickness of the oil lens, the squeeze-film approximation predicts infinite coalescence times. Thin-film forces at small thickness, however, determine ultimately film instability. Figure 4 does plot the thin-film portion but this part of the solution is obscured by 
the solution for the thinning of the lens. Note that the time required for coalescence in Fig. 4 increases considerably with oil viscosity. The times are relatively short because the pressure drop across the bubble is rather large and translates to $14 \mathrm{MPa} / \mathrm{m}(600 \mathrm{psi} / \mathrm{ft})$. This pressure drop, however, is consistent with the micromodel experiments to follow. To understand the role of viscosity on bubble coalescence at field conditions, assume that the field gradient is $22.6 \mathrm{KPa} / \mathrm{m}$ $(1 \mathrm{psi} / \mathrm{ft})$. Then, the times in Fig. 4 increase by a factor of roughly 500 .

\section{Experimental}

The experimental apparatus for this study consists of the micromodel, pressure vessel, optical equipment, and a system of hardware that controls fluid flow and pressure, as shown in Fig. 5. For some experiments, a cornered glass capillary is substituted for the micromodel, as described shortly. Etched-silicon-wafer micromodels of the type developed by Hornbrook et al. (1991) were used. The micromodels contain a $50 \mathrm{~mm}$ by $50 \mathrm{~mm}$ etched pore pattern as illustrated in Fig. 6. There are two etched inlet/outlet ports at opposite corners of the pore network. Holes are drilled into the silicon wafer at these ports to provide locations that allow fluid to enter and exit the micromodel. These micromodels contain a modification introduced by Castanier and Sagar (1998): narrow etched channels adjacent to the edges of the pore network carry fluid to the inlet/outlet ports as shown in Fig. 6. The result is approximately linear flow, as opposed to radial flow, near the inlet and outlet of the micromodel. The porosity of the micromodel is roughly 0.2 . The permeability of the micromodel is approximately $0.1 \mathrm{mD}$.

The etched side of a silicon wafer is bonded anodically to a borosilicate glass plate in order to produce a micromodel. The silicon wafer is placed on an anode. The glass plate rests on top of the silicon wafer. A cathode is placed on top of the glass plate. The system is heated to 600 ${ }^{\circ} \mathrm{F}$ and the electrodes are charged to $1000 \mathrm{~V}$ after alignment of the micromodel and cover plate on the bonding apparatus. During the heating process the pore surfaces of the silicon wafer are oxidized because they are in the presence of air at high temperature. The optimum bonding time is one hour.

\section{Pressure Vessel}

The anodic bond that holds the etched-silicon-wafer to the glass cover plate can generally sustain a difference between internal and external pressure of about 30 psi. In order to achieve solution gas drive experiments at elevated pressures, a pressure vessel to hold micromodels was designed and constructed, Fig. 5(b). The pressure vessel has a maximum design operating pressure of 1000 psig and is temperature controllable. The apparatus as built was pressure tested to 940 psi without leaks or failure. 
Pressure Vessel Lid
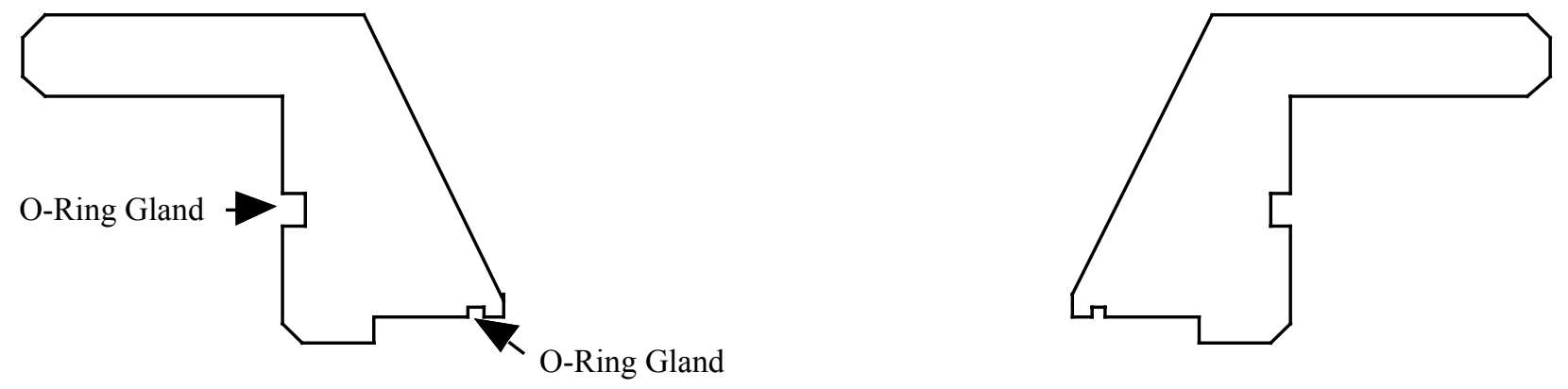

Sapphire Window

Micromodel

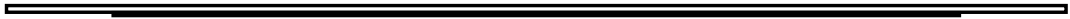

Pressure Vessel

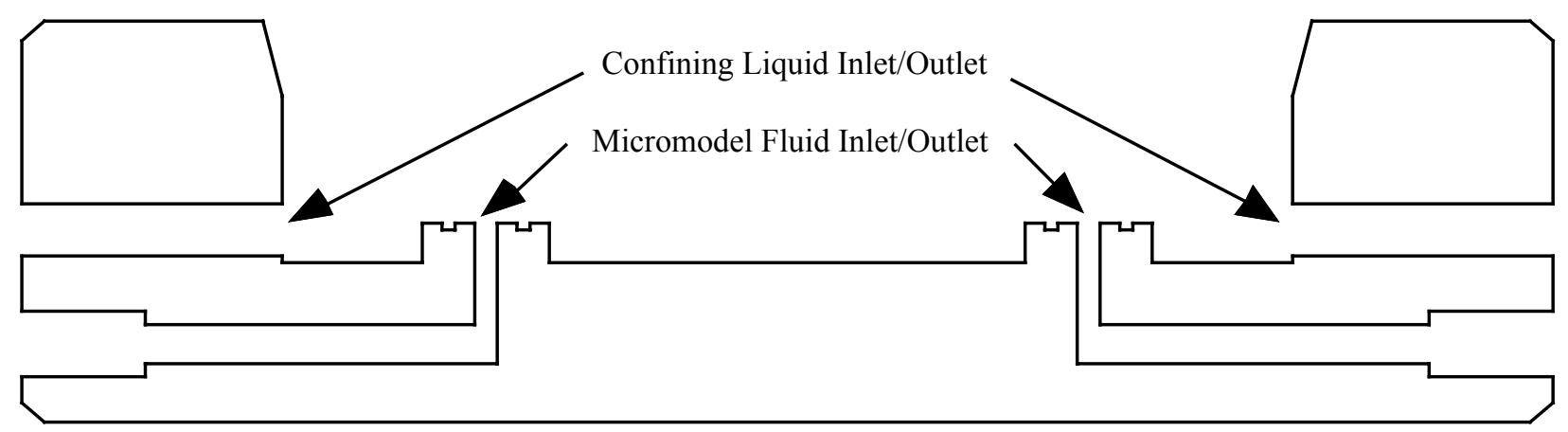

(a) 


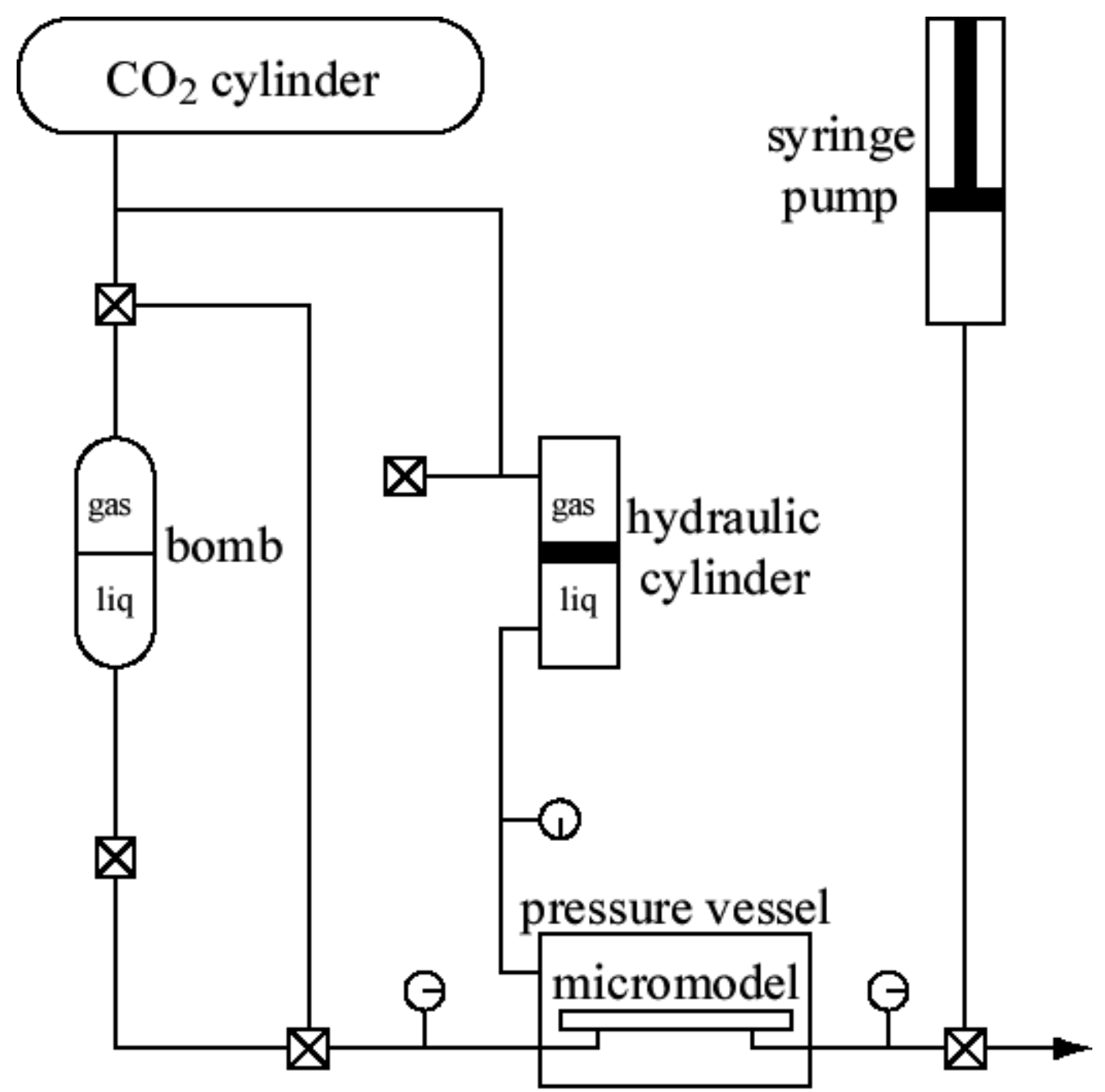

Figure 5 (a) Pressure-vessel developed for holding micromodels and (b) schematic of apparatus.

The pressure vessel was machined from 6061 T6 aluminum. Holes that serve as fluid inlet/outlet ports are drilled in the bottom of the micromodel. These holes rest directly above channels machined into the body of the pressure vessel to carry fluid to and from the micromodel. O-rings create the seal between fluid channels and the inlet/outlet ports of the micromodel. Confining liquid provides pressure support to the micromodel. If temperature control is not needed, the confining liquid remains static. The vessel is designed, however, to allow temperature-controlled confining liquid to circulate through the interior of the pressure vessel. A 0.185 in thick sapphire window is located directly above the micromodel. Sapphire was selected because of its superior mechanical and optical qualities. The window is held between the micromodel and the pressure vessel lid in a manner such that its edges are considered to be fixed. This leads to maximum mechanical strength. An O-ring between the pressure-vessel lid and the window provides a seal, and similarly, the lid seals to the body of the pressure vessel using an oring piston seal. 


\section{Optics}

A microscope (Nikon, Optiphot-M) with a photo tube that allows for the connection of a video camera was used. The silicon-wafer micromodels are opaque. Consequently, they are illuminated with an internal light source routed through the microscope objective and monitored using reflected light. A high-quality color video camera (PULNiX, TMC-7RGB, Sunnyvale, CA) is connected to the photo tube. Images are sent from the video camera to a VCR for recording. An extra-long working distance objective lens (Nikon, MPLAN-20 0.4 ELWD) was used. Total magnification is $200 \mathrm{X}$. The thickness of the sapphire window $(4.7 \mathrm{~mm})$, combined with the thickness of the micromodel cover glass $(1 \mathrm{~mm})$, results in a total cover glass thickness of 5.7 $\mathrm{mm}$. Some image quality is lost, mainly due to a loss in contrast.

The superior mechanical strength and optical qualities of sapphire provide the advantages of a short working distance and high transmission of light. Sapphire, however, has anisotropic optical properties. This sometimes causes double refraction images depending on the orientation of the window. In some cases, the image of the micromodel contains "phantom images" due to this double refraction. Although the phantom images reduce image quality, the image remains easily interpretable.

\section{Pressure and Fluid Delivery Systems}

Carbon dioxide from a high-pressure cylinder is used to pressurize confining water. To avoid carbonating the confining water, it is pressurized in a modified hydraulic cylinder (Miller Fluid Power, HV50R2N, Bensenville, IL) as shown in Fig. 5(a). A piston in the hydraulic cylinder separates the pressurized gas from the confining water. A gauge indicates the pressure of the confining liquid.

On the upstream side of the micromodel, a set of three-way valves allows $\mathrm{CO}_{2}$, dead oil, or oil saturated with $\mathrm{CO}_{2}$ to be pushed through the micromodel. Gas from a pressurized cylinder provides drive energy. On the downstream side, a three-way valve selects whether the outlet is connected to a syringe pump (ISCO, 100DM, Lincoln, NE), or to backpressure. The small micromodel volume of $0.015 \mathrm{~mL}$ necessitates the capability to withdraw fluid at very low rates. The syringe pump provides this capability, as it has a minimum refill rate of $0.0001 \mathrm{~mL} / \mathrm{min}$. Pressure gauges indicate the pressures upstream and downstream of the micromodel.

The apparatus is prepared for an experiment by assembling it and fastening the lid to the body of the pressure vessel. Copious quantities of bone-dry $\mathrm{CO}_{2}$ are flushed through the micromodel to dry any residual moisture. Back pressure is applied and the gas pressure is increased to the value desired for an experiment. Gas-saturated liquid prepared in a bomb, at the pressure of interest, is then introduced to the micromodel. This fluid is driven by increasing the pressure of the gas in the bomb. Any residual gas $\left(\mathrm{CO}_{2}\right)$ phase dissolves readily in the fluid (oil or water) and is thereby displaced. The valve is then closed that connects the micromodel to fluid supply so that experiments can be run in depletion mode. 


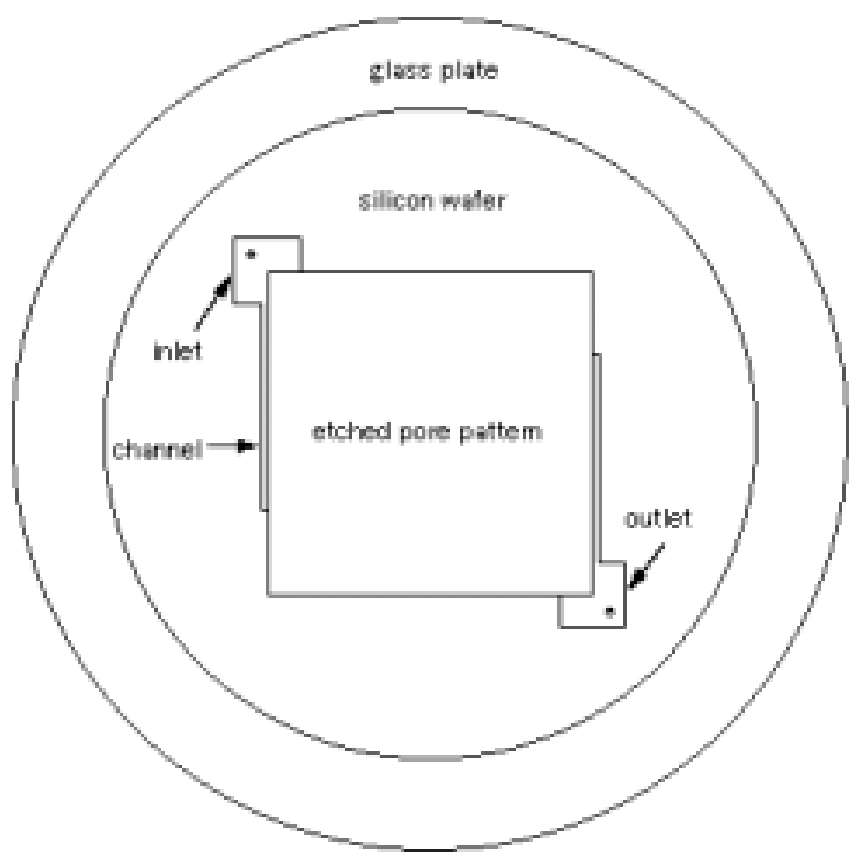

(a)

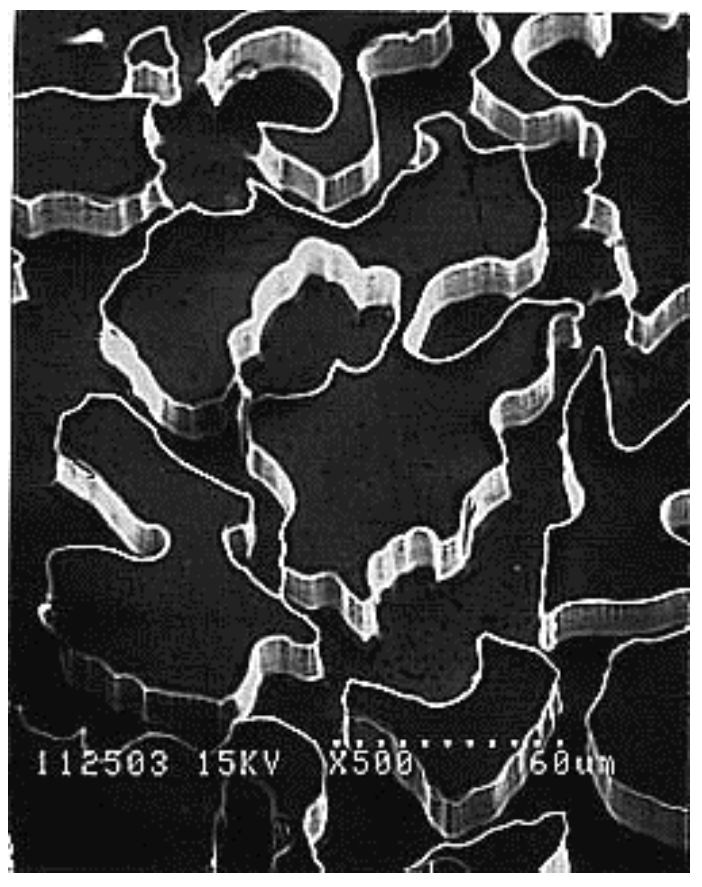

(b)

Figure 6(a) Schematic of top view of micromodel illustrating injection and production ports and channels and (b) scanning electron microphotograph of micromodel etched pore pattern at 500X magnification.

\section{Capillary Tube Experiment}

Micromodel experiments as employed here, have two significant flaws when analyzing coalescence. The first problem is with the control of events within a micromodel. It is hard to capture frequently a situation where one can observe bubble coalescence under pressure gradient across the bubble. Second, and most importantly, it is impossible to characterize exactly the forces acting on bubbles. Capillary tube experiments provide a suitable alternative for exploring some aspects of the physics of bubble coalescence. 


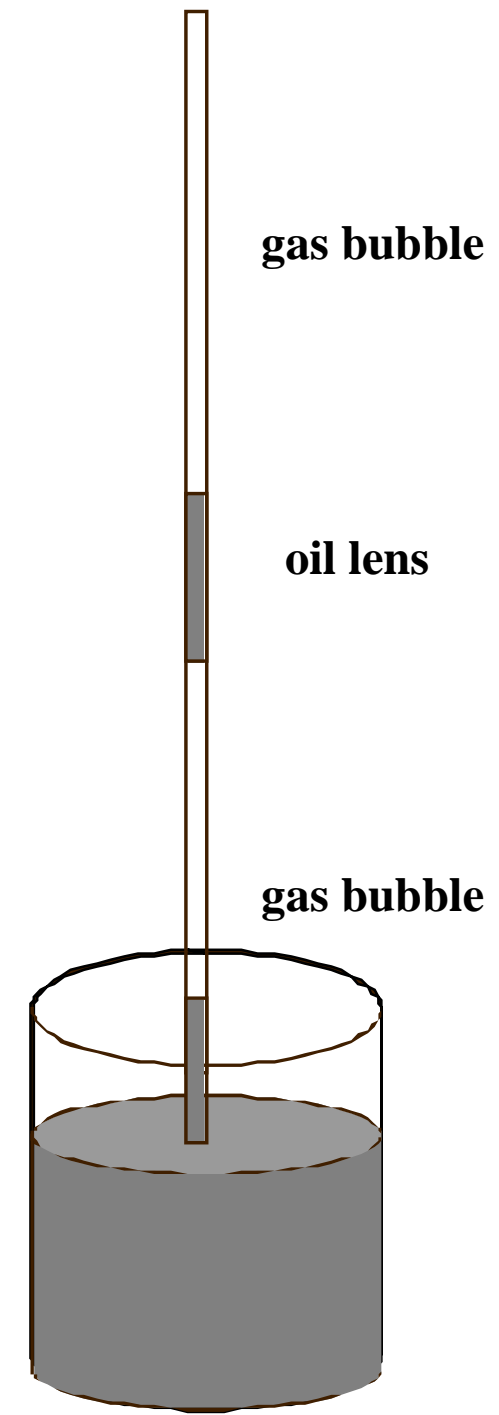

(a)

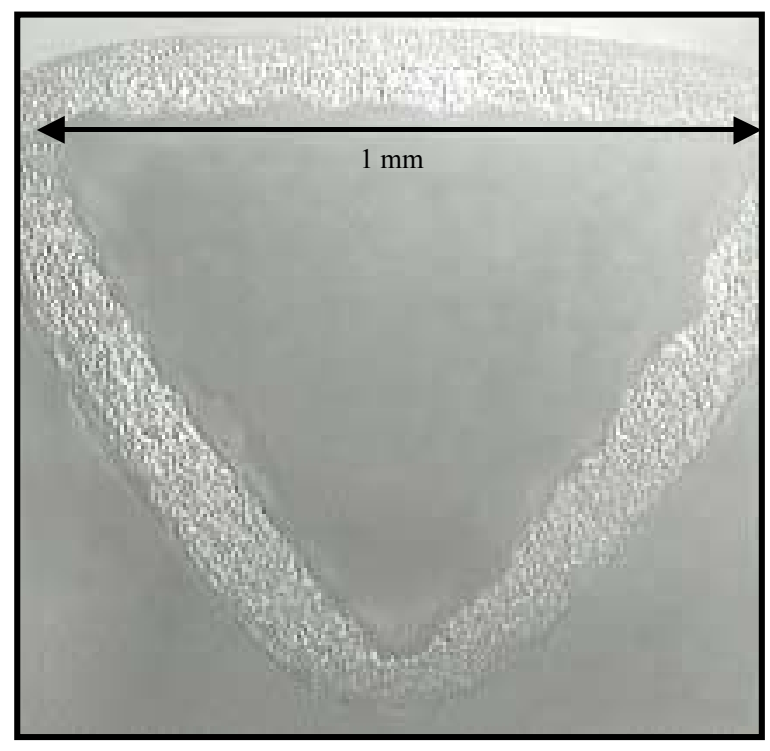

(b)

Figure 7(a) Schematic of capillary tube apparatus for gravity-driven drainage of oil lens and (b) optical microphotograph of triangular capillary cross section.

Triangular capillaries with sharp corners were used to conduct the experiments. The experimental setup is shown in Fig. 7. Oil is pulled into the capillary and allowed to drain. This establishes wetting fluid in the pore corners and the height of the oil column. The capillary tube is then held vertically with its lower end immersed in a beaker filled with the oil. An oil lens is introduced from the top of the capillary using a syringe. The air trapped between the oil bank at the bottom of the capillary and the oil lens near the top simulates a gas bubble trapped in a narrow pore. Lenses are allowed to drain to a length of $12.5 \mathrm{~mm}$ before thinning data are collected.

The bubble rises under the action of gravity, whereas, the oil in the lens drains as oil flows down the capillary corners, across the bubble, into the beaker. As shown in the Fig 7, the apparatus setup is similar to that for the micromodel experiments. These capillaries have corners as shown in Fig 7(b) consistent with the assumption of corner flow. The time it takes for the oil 
lens of given length to drain completely is measured. Experiments were repeated for a set of white oils with viscosities of 51, 95, 178, 490, and $950 \mathrm{mPa}$-s. Table 1 lists the properties of these oils. They are primarily viscosity standards for calibrating viscometers.

Gravity is the force driving drainage. For a vertically oriented tube, the pressure difference driving liquid drainage around the bubble is composed of the buoyancy of the bubble, $\left(\rho_{\mathrm{o}}-\rho_{\mathrm{g}}\right) \mathrm{gL}$ plus the gravity head, $\Delta \mathrm{p}=\rho_{\mathrm{o}} \mathrm{gL}$, imposed by the fluid in the lens above the bubble and the oil in the corners of the capillary. The gravity head aids the drainage of liquid from pore corners. As gas density is negligible compared to oil density the net force acting on the upper interface of the moving bubble is written as

$$
F=\left(2 \rho_{o} g L-\Delta P_{c}\right) A_{p}
$$

where $A_{p}$ is again the projected area of the gas-liquid bubble interface and $\Delta P_{c}$ is the pressure drop for flow through the pore corners.

\section{Results and Discussion}

The first task undertaken with the apparatus was to characterize the sequence of bubble nucleation, growth, and coalescence as they occurred in the micromodel. Special attention was paid to locating physical sites where bubbles came out of solution. For this task, $\mathrm{CO}_{2}$ and water were used in the micromodel. The relatively low viscosity of water made it easy to saturate the micromodel and conduct repeat experiments. Water in a bomb was allowed to saturate with $\mathrm{CO}_{2}$ at 110 psig for roughly a day. This $\mathrm{CO}_{2}$-saturated water was injected into the micromodel and the system pressure raised to $170 \mathrm{psig}$. Initially, the depletion rate was set at $0.004 \mathrm{ml} / \mathrm{min}$. At 12 $\mathrm{min}$, the rate was decreased to $0.003 \mathrm{ml} / \mathrm{min}$. Late into the experiment, at $400 \mathrm{~min}$, the flow rate was increased to $0.01 \mathrm{ml} / \mathrm{min}$ and then at $455 \mathrm{~min}$ the rate was set to $1 \mathrm{ml} / \mathrm{min}$. Figure 8 reports the pressure measured at the upstream and downstream portions of the micromodel. As gas builds in the micromodel, the difference between inlet and outlet pressure increases. The difference in slope between the early time and late time periods is also diagnostic of the evolution of the gas phase.

During the early stages of depletion, repeated bubble nucleation and mobilization at given sites on the solid portions of the micromodel was observed. No pre-existing bubble of gas were visible at these sites. Recall magnification is $200 \mathrm{X}$ and that the lower limit of resolution is roughly $1 \mu \mathrm{m}$. Nucleation was heterogeneous and typically found to occur at sites containing dirt or solid material that did not originate from the silicon wafer. Two pathways for dirt to enter the micromodel were found. First, the micromodel is exposed to air during preparation and bonding. Although the micromodel is washed, it may capture dust or dander from the air. Second, the gaswater interface may accumulate solid, nonsiliceous material. This material is deposited as gas bubbles go into solution while the micromodel is being pressurized for use. Formation of dirt spots was observed several times by the second mechanism (George, 1999). 


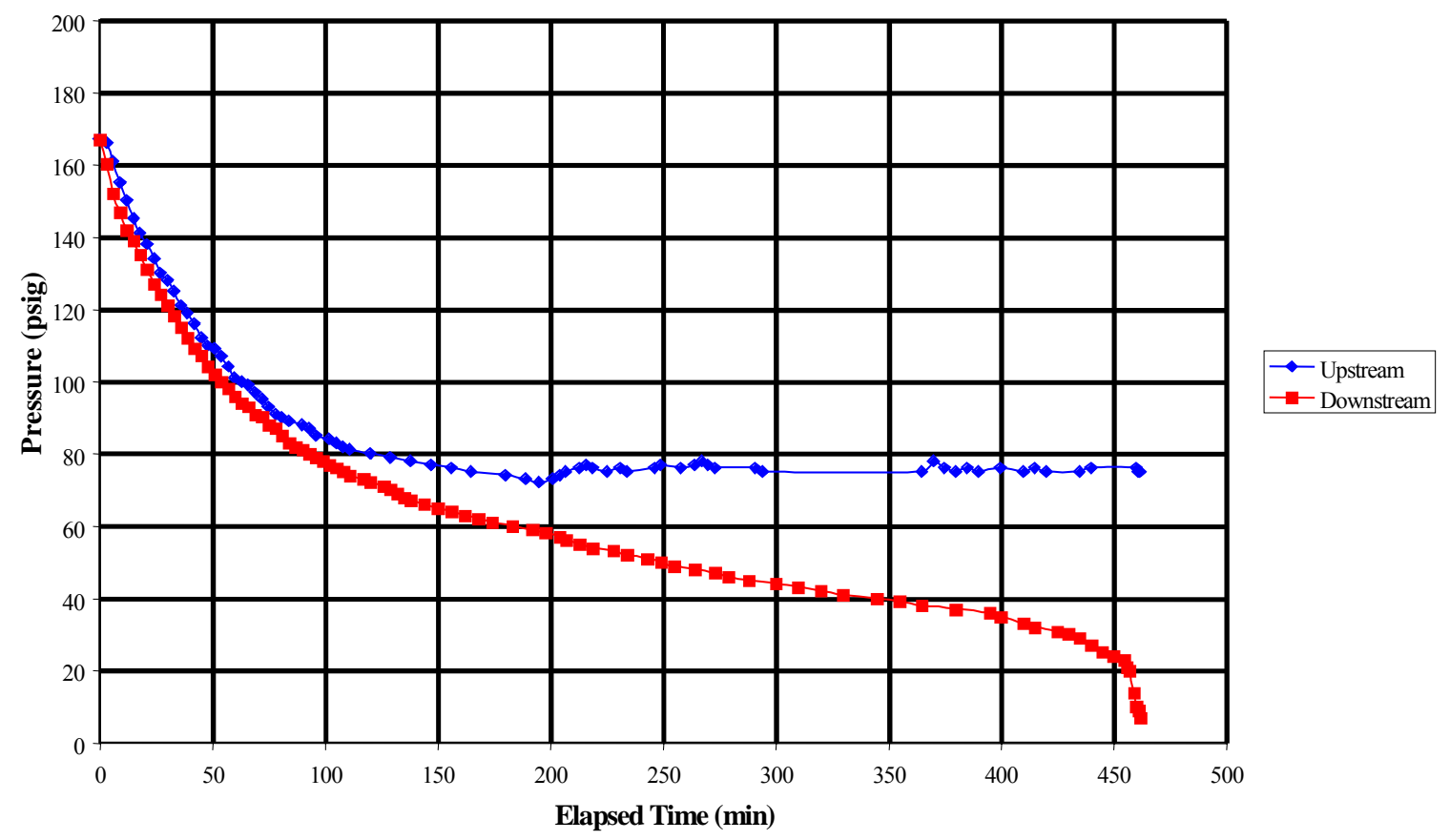

Figure 8. Pressure versus time for pressure depletion of $\mathrm{CO}_{2}$ saturated water in a micromodel.

Figures 9 and 10 document repeated heterogeneous bubble nucleation and expansion at the same site during the pressure depletion of a micromodel saturated with $\mathrm{CO}_{2}$. At the beginning of the sequence in Fig. 9a (0 s elapsed time) a recently nucleated gas bubble is just barely visible. Various features in this frame are labeled to orient the reader. The bubble is located on the boundary of the dirt spot, and near a pore wall. By $0.17 \mathrm{~s}$ in Fig. 9b, expansion of the gas bubble is clearly observed. The bubble at the nucleation site and another bubble to the right expand, as seen at 0.9 s, Fig. 9c, and are only separated by a small solid grain. The bubbles expand significantly so that they come into contact and immediately coalesce by $1.2 \mathrm{~s}$, resulting in a single large bubble visible in Fig. 9d. The gas expands to fill the pores surrounding the small grain, as observed in Fig. 9e, at $2.4 \mathrm{~s}$. Gas flows subsequently through a pore throat at $2.8 \mathrm{~s}$, leaving the nucleation site exposed, as observed in Fig. 9f.

Nucleation was often observed almost immediately after a gas bubble vacated the pore. Figure 10 presents nucleation at the same site as Fig. 9. After the bubble exits the nucleation site, water fills in the pore space vacated by the bubble, Fig. 10a. A new $\mathrm{CO}_{2}$ bubble is observed at the nucleation site that grows steadily as displayed in Fig. 10b, at $0.5 \mathrm{~s}$. Carbon dioxide diffuses steadily to the nucleation site. By $0.8 \mathrm{~s}$, the bubble has grown to fill the pore space in Fig. 10c. Continued expansion of the gas bubbles into adjacent pore space is observed in Fig. 10d at $1.0 \mathrm{~s}$. Once this bubble grows to sufficient size, it is mobilized leaving the nucleation site unoccupied so that nucleation may repeat. Eventually, this site ceases to produce bubbles as the system is depleted of supersaturation. 


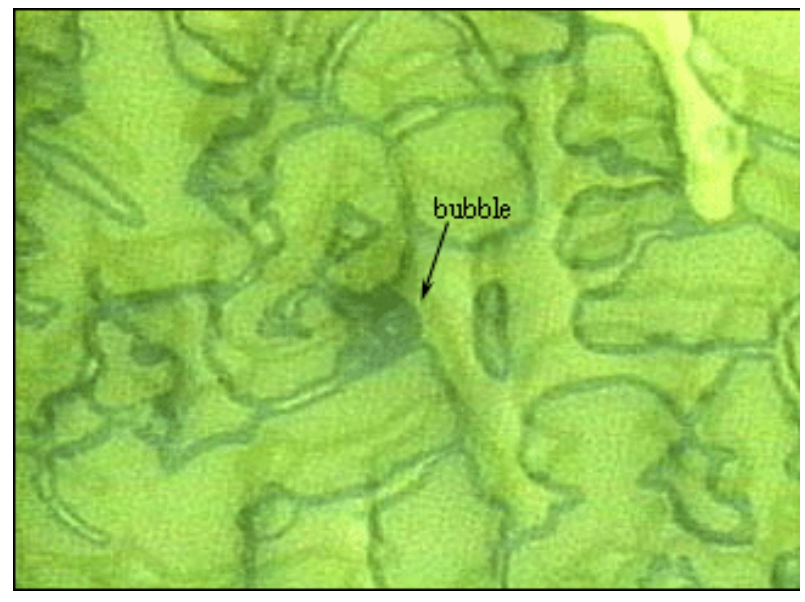

(a)

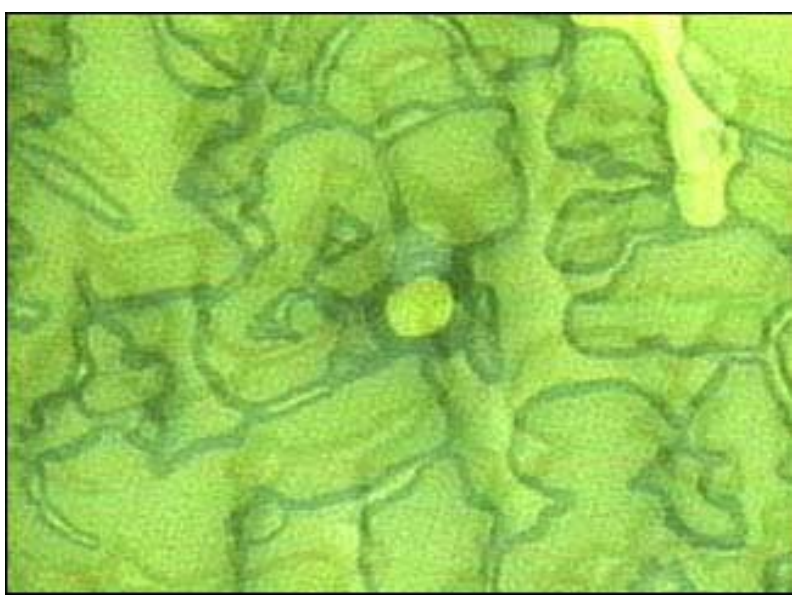

(c)

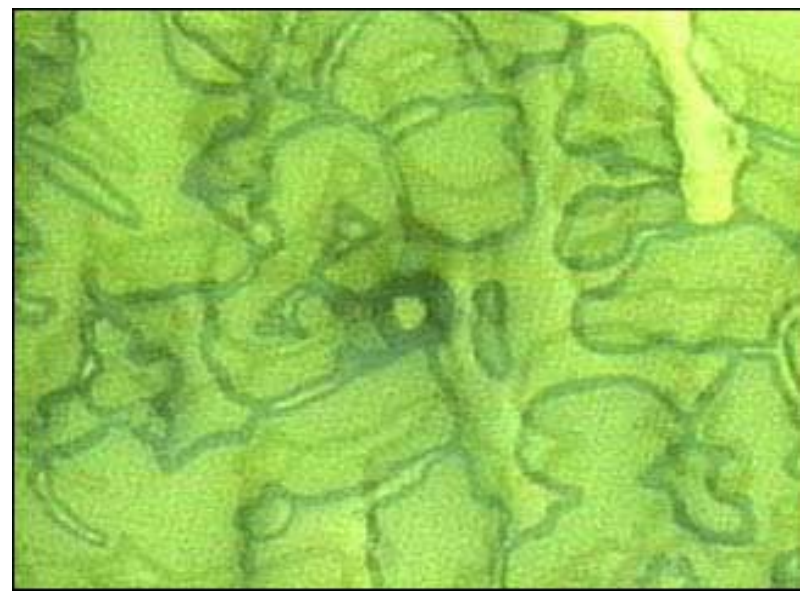

(b)

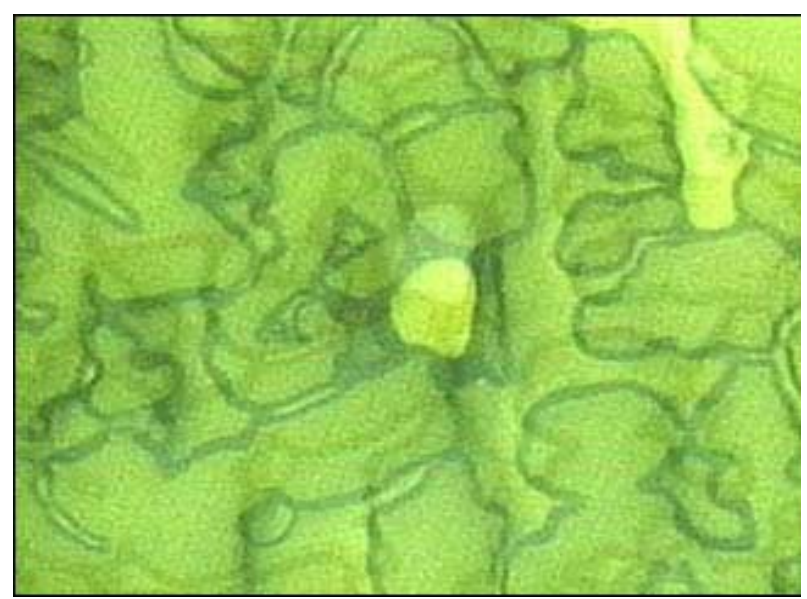

(d)

Figure 9. Bubble nucleation and growth in $\mathrm{CO}_{2}$ saturated water: (a) recently nucleated bubble at $0 \mathrm{~s}$, (b)bubble growth at $0.2 \mathrm{~s}$, (c) gas bubble at $0.9 \mathrm{~s}$ after growing to fill pore body, (d) bubbles expand and coalesce at $1.2 \mathrm{~s}$, (e)at $2.4 \mathrm{~s}$ bubble expands, (f) bubble is mobilized and leaves the pore space where it was nucleated at $2.8 \mathrm{~s}$. 


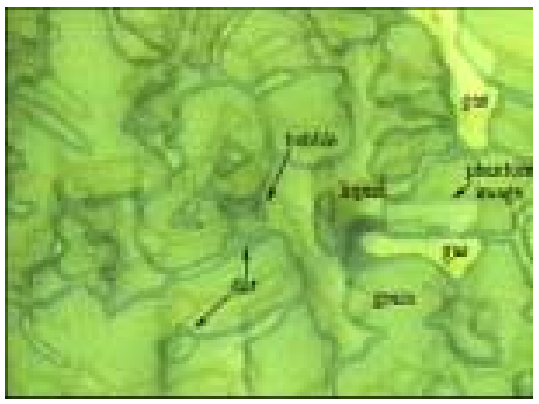

(a)

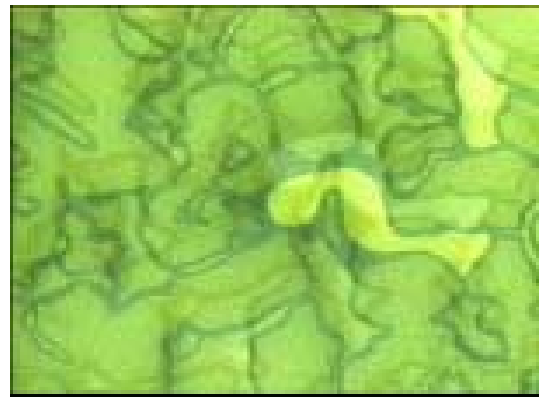

(d)

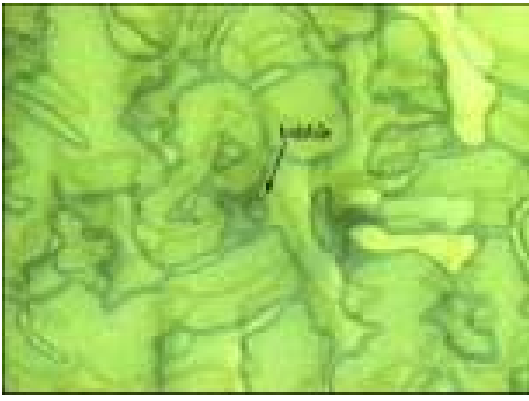

(b)

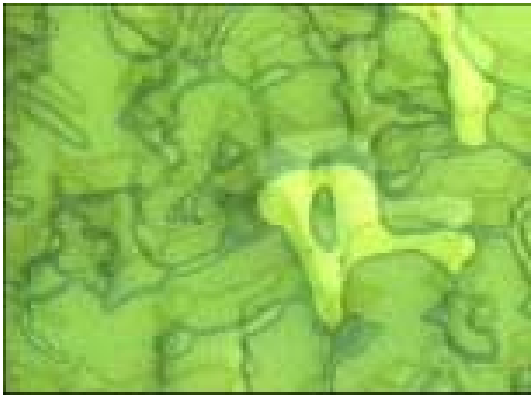

(e)

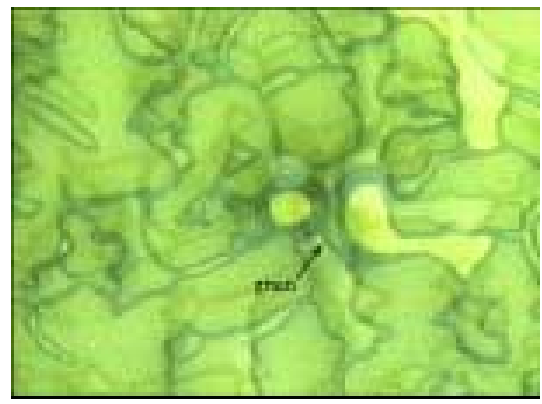

(c)

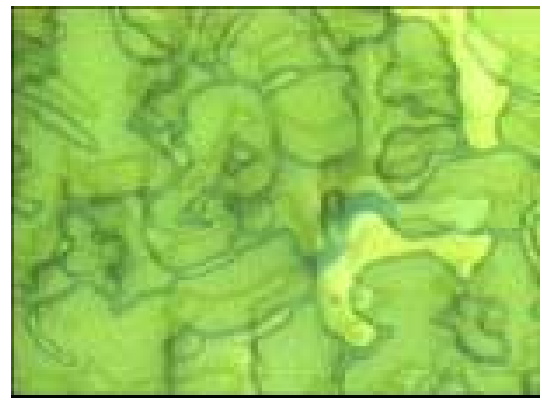

(f)

Figure 10. Repeated bubble nucleation and growth at the same site as shown in Fig. 9(a) recently nucleated bubble at $0 \mathrm{~s}$, (b) bubble growth at $0.5 \mathrm{~s}$, (c) gas bubble at $0.8 \mathrm{~s}$ after growing to fill pore body, (d) bubbles expands into adjacent pore space at $1.0 \mathrm{~s}$.

\section{Viscous Oil}

Experiments continued with the white mineral oil Kaydol saturated with $\mathrm{CO}_{2}$. Oil viscosity is $220 \mathrm{cP}$ at ambient conditions. Figure 11 displays the measured solution gas-oil ratio versus pressure function for this system. At 100 to $150 \mathrm{psi}$, the volume of gas in solution varies from about 90 to $200 \mathrm{SCF} / \mathrm{STB}$. These values are roughly comparable to many heavy-oil systems. Consequently, Kaydol was saturated with $\mathrm{CO}_{2}$ at about 100 to 150 psi for these experiments.

Figure 12 reports the pressure versus time curve at a constant depletion rate of 0.0042 $\mathrm{ml} / \mathrm{min}$ for an experiment where the initial pressure is $150 \mathrm{psig}$. Depletion begins at the bubble point. The downstream pressure declines rapidly due to the relatively large volumetric expansion rate. The upstream pressure declines smoothly, but begins to level off around $800 \mathrm{~min}$. This is similar to pressure history results for the $\mathrm{CO}_{2}$ and water experiments above. 


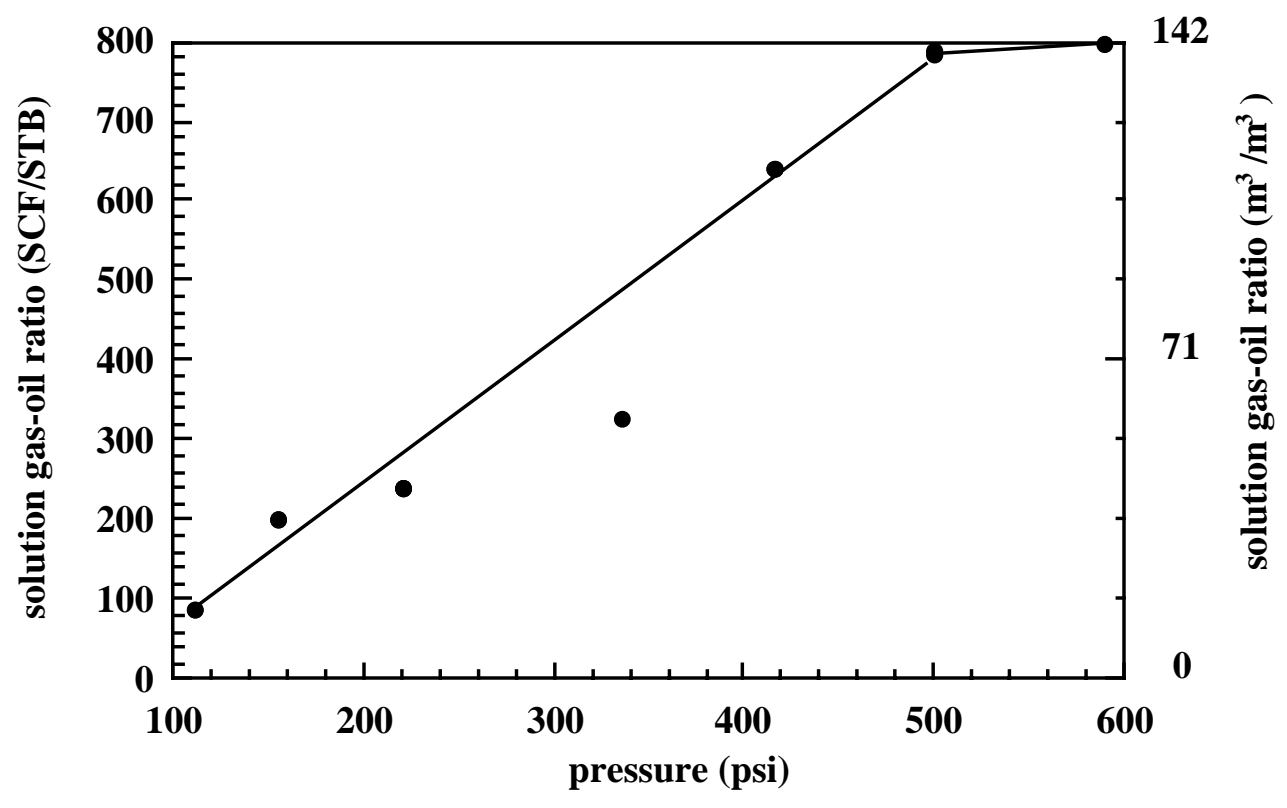

Figure 11. Solubility of $\mathrm{CO} 2$ in the viscous mineral oil Kaydol as a function of pressure.

Visual observations revealed that bubbles again nucleated heterogeneously and originated from crevices and other imperfections along micromodel surfaces. The sequence of nucleation and growth is substantially slower as compared to results for water- $\mathrm{CO}_{2}$. Figure 13 illustrates a sequence of nucleation through mobilization of the resulting gas bubble. To improve clarity, the bubble has been outlined in black. At the beginning of the gas-phase evolution sequence $(0 \mathrm{~s})$ displayed in Figure 13(a), a recently nucleated gas bubble is located at a pore wall. The pressure is $150 \mathrm{psig}$; the same as the saturation pressure. There is a crevice in the section of the pore wall occupied by a small bubble. At $1 \mathrm{~min} 31 \mathrm{~s}$, gas expansion is observed in Fig. 13b. Fig. 13c displays continuing expansion at $2 \mathrm{~min} 50 \mathrm{~s}$. The bubble almost fills a large pore by $3 \min 6 \mathrm{~s}$, but, as observed in Fig. 13d, begins to flow through a pore throat. Figure 13e indicates that the gas has almost left the pore space by $3 \min 8 \mathrm{~s}$. Note that the time scale for bubble growth is on the order of minutes whereas under similar pressure conditions for the water- $\mathrm{CO}_{2}$ system, bubble nucleation, growth, and mobilization was accomplished in seconds. The oil viscosity appears to be slowing bubble dynamics significantly. 


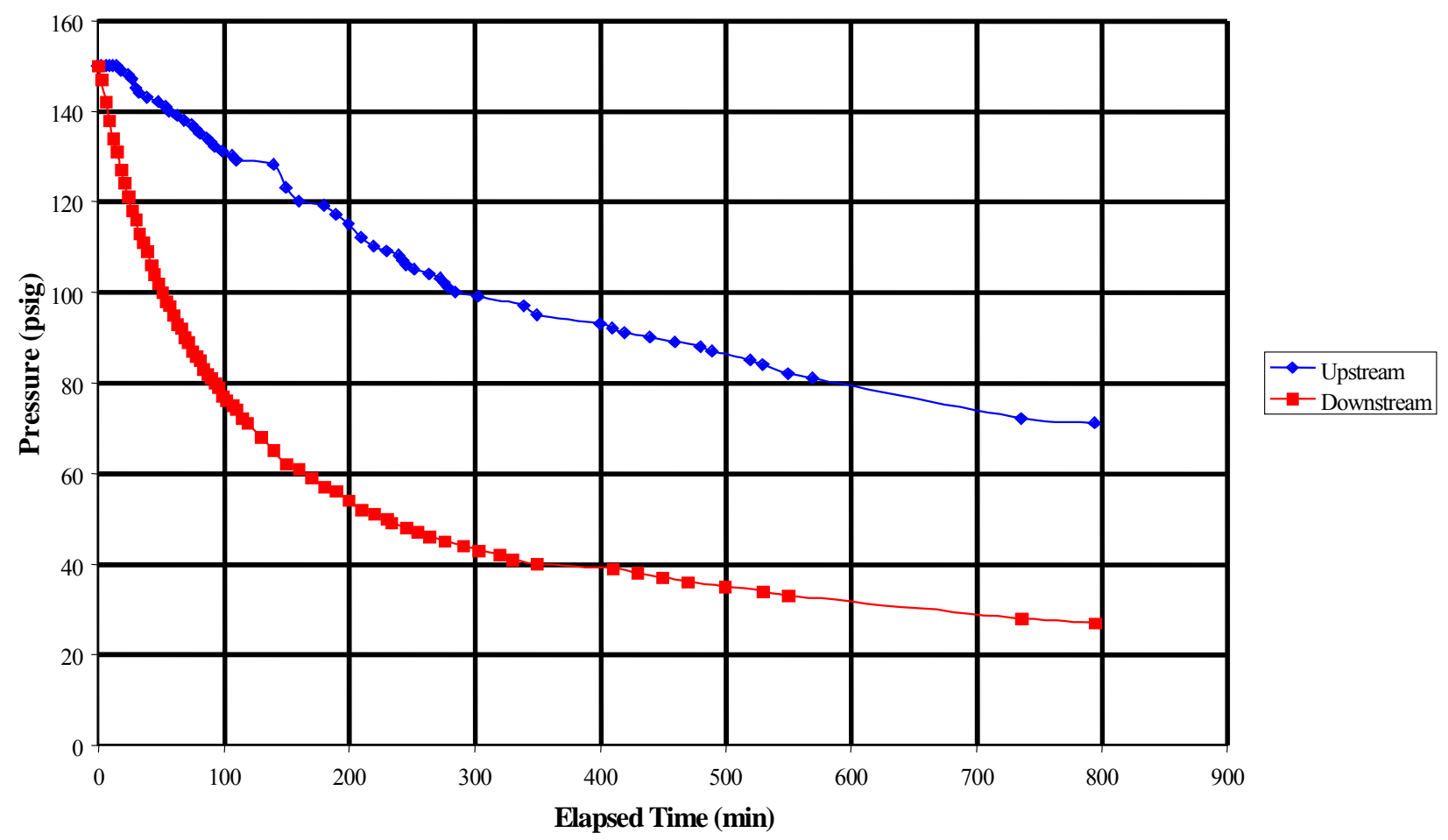

Figure 12. Pressure versus time for pressure depletion of $\mathrm{CO}_{2}$ saturated viscous oil in a micromodel.

Bubble coalescence occurs mainly by the collision of expanding gas bubbles. Figure 14 displays a typical sequence of coalescence. In Fig. 14a, three discrete gas bubbles are visible. Between the frames in Figs 14a and 14b, additional gas flows into the pores in the upper portion of the view. The two small bubbles in Figs. 14a and 14b coalesce with this incoming gas and form a larger bubble. This bubble surrounds a small grain. Figures $14 b$ and $14 c$ illustrate the relatively slow drainage of liquid from the liquid lens separating the two large gas bubbles. Liquid drains around the bubbles and perhaps through the small pore throat above and to the right of the lower bubble. The time required for this sequence of coalescence is roughly $30 \mathrm{~s}$. At this stage, the pressure drop across the micromodel is about $60 \mathrm{psi}$ and the gradient is substantial. A lesser gradient results in longer times for coalescence.

The effect of oil viscosity on bubble coalescence was intriguing, and these observations, in fact, motivated the simpler capillary tube experiments. Figure 15 shows the effect of oil viscosity on coalescence where gravity is the driving force for bubble movement. Dashed lines indicate the observed bubble movement. The upper interface of the oil lens remains stationary during the drainage and it acts as a plane of symmetry. Table 1 reports the bubble length for each of the different viscosity oils. Experimental procedure did not allow exactly identical bubble lengths to be placed in the capillary tube. Increased oil-phase viscosity significantly increases the time required for coalescence. The $950 \mathrm{mPa}$-s oil requires about 4 hours to coalesce two bubbles separated by an oil lens that is initially $12.5 \mathrm{~mm}$ thick. 


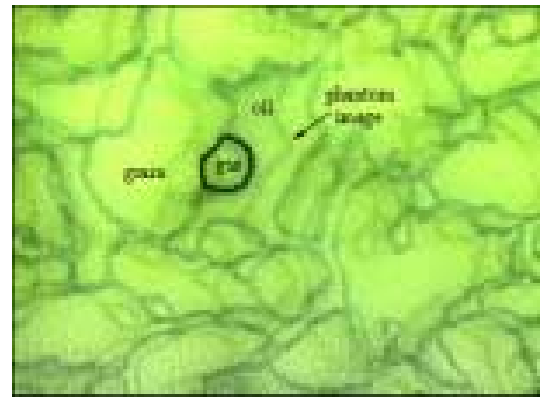

(a)

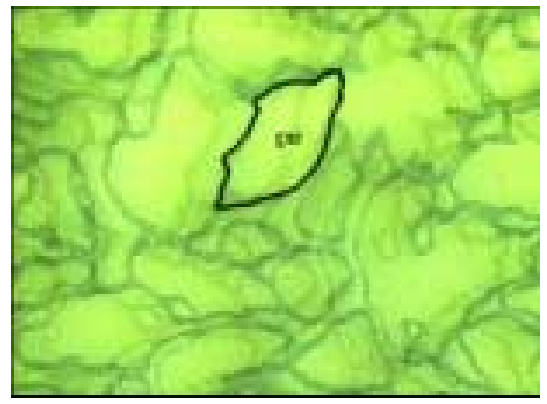

(d)

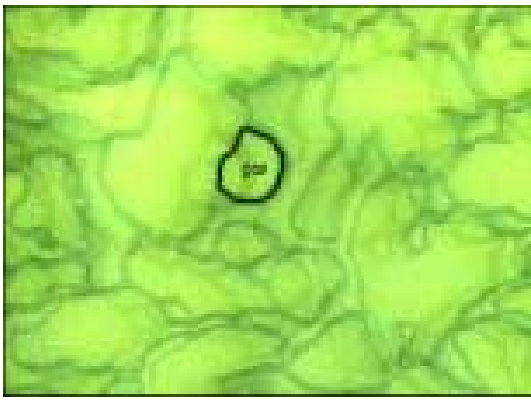

(b)

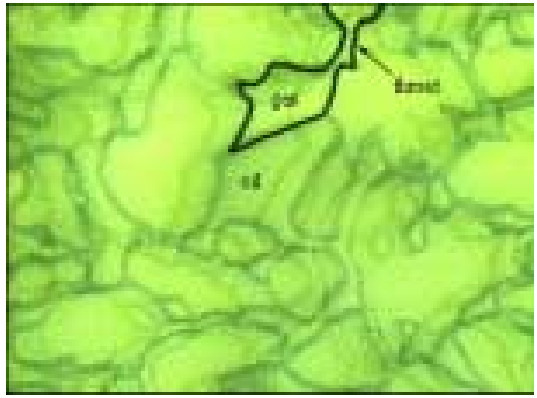

(e)

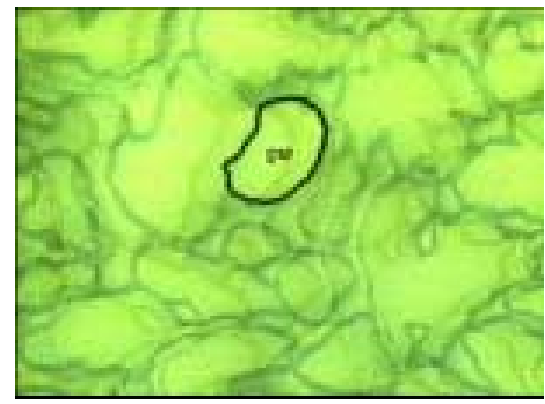

(c)

Figure 13. Bubble nucleation and growth in $\mathrm{CO}_{2}$ saturated viscous oil: (a) recently nucleated bubble at $0 \mathrm{~s}$, (b)bubble growth at $1 \mathrm{~min} 31 \mathrm{~s}$, (c) gas bubble grows to fill pore body at $2 \min 50$ $\mathrm{s}$, (d) bubbles expands into adjacent pore space at $3 \min 6 \mathrm{~s}$, (f) bubble is mobilized and leaves the pore space where it was nucleated at $3 \mathrm{~min} 8 \mathrm{~s}$.

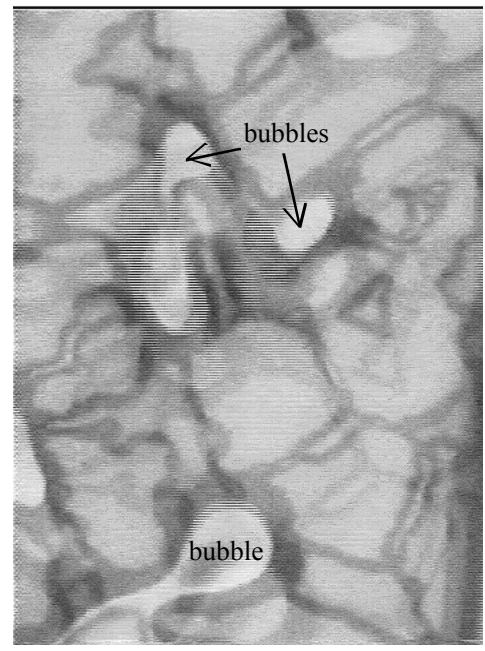

(a)

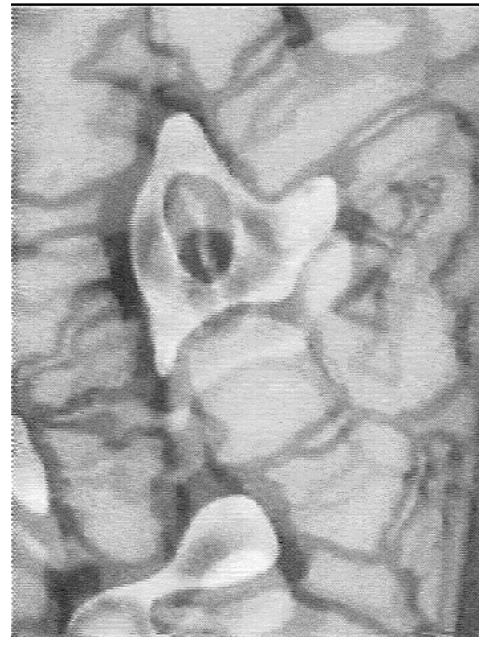

(b)

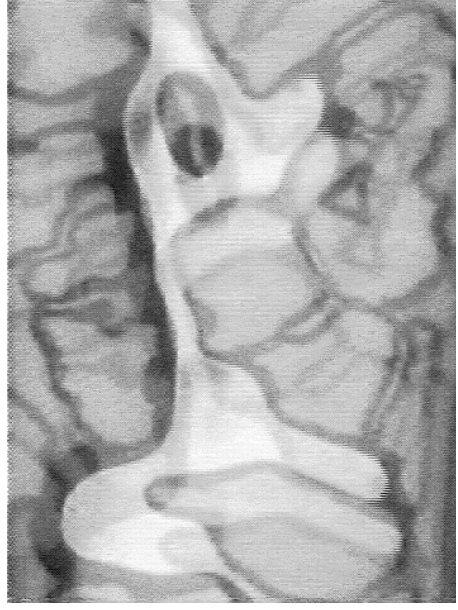

(c)

Figure 14. Bubblecoalescence in viscous oil: (a) three bubbles arrayed in pores at $0 \mathrm{~s}$, (b) coalescence of upper two bubbles at $10 \mathrm{~s}$ as gas flows into pore space, (c) drainage of thick liquid lens and coalescence of gas bubble at $42 \mathrm{~s}$. 


\section{Comparison Among Observations and Calculations}

The objective of this section is to conduct a quantitative comparison among the visual observations and the relatively simple theories for coalescence. Begin with the micromodel experiments reported in Figs. 13 and 9. The difference in the gas-free wetting phase viscosity is about 200 to 1 and the volumetric expansion rates are roughly equal. The time required for the gas bubble to grow to fill a pore body is about 3 minutes when viscous oil fills the micromodel in Fig. 13. On the other hand, the time for bubble growth to a pore filling size is about 1 second in Fig. 9. This difference in timing is roughly 180 to 1. Figure 2, while computed for a significantly lower volumetric expansion rate, does indicate that the growth rate of gas bubbles decreases substantially as the solution viscosity increases.

Next, consider pressure-driven coalescence and the experiments reported in Fig. 14. Values of parameters are taken from the example reported in Fig. 4. The length of the oil lens in Fig $14 \mathrm{~b}$ is about $50 \mu \mathrm{m}$. The SEM microphotograph of micromodel pore geometry in Fig. $6 \mathrm{~b}$ indicates that the $\beta$ value of 93.93 for sharp square corners is appropriate. The pressure drop across the bubble, $\Delta P$ is obtained by measuring the gradient across the entire micromodel and equating the $\Delta P / L_{\text {model }}$ with $\Delta P / L_{\text {pore }}$. For example the micromodel used here is a $50 \mathrm{~mm}$ square with pressure drop of about $41.4 \mathrm{KPa}(60 \mathrm{psi})$ across its length. The ratio $\Delta P / L_{\text {model }}$ for this model is about $8.3 \mathrm{MPa} / \mathrm{m}(30.5 \mathrm{psi} / \mathrm{in})$. For a typical pore of length $125 \mu \mathrm{m}$ the value of $\Delta \mathrm{P}$ is about $1040 \mathrm{~Pa}(0.15 \mathrm{psi})$. The time required for the bubble to travel from its initial position to the midpoint of the lens is calculated. There are no chemical agents present in the viscous mineral oil to stabilize the interface and prevent coalescence. Thus, coalescence is assumed to occur when the bubble interfaces (gas-oil) touch. The time interval for coalescence is found by computing time versus interface position according to Eq. 9. With this procedure the calculated time for coalescence is 28 seconds, whereas the experimentally measured time is $32 \mathrm{~s}$. The two agree well.

The capillary tube experiments provide much more data for comparison. A set of five different viscosities was tested and the results compared with the physical model in Fig 15. Equation 9 is used to calculate the time for coalescence with the gas-oil interface of the upper bubble taken as the no-flow boundary. The dimensionless flow resistance, $\beta$, is needed for the triangular capillary. A cursory examination of Fig. $7 \mathrm{~b}$ teaches that the capillary corners are rounded and filled in with glass. A small filling in of the corners can have a substantial effect on the resistance to liquid flow. From geometric characterization of Fig. 7b, it is measured that 5.3\% of the area of the cross section is filled in. Following Ransohoff and Radke (1986), we estimate that the degree of roundedness is 0.48 . When the degree of roundedness is 0 , the corner is perfectly sharp, whereas when it is 1 the liquid in the corner exists only as a thin film. From the tabulations of $\beta$ given by Ransohoff and Radke (1986), $\beta$ is set to 46.01 in accordance with a roundedness of 0.48 . The remaining geometrical parameters $a, A_{p}$, and $A_{w}$ are $0.163 \mathrm{~mm}, 0.378$

$\mathrm{mm}^{2}$ and $0.0547 \mathrm{~mm}^{2}$, respectively, as appropriate for the cross-sectional geometry in Fig. $7 \mathrm{~b}$. 


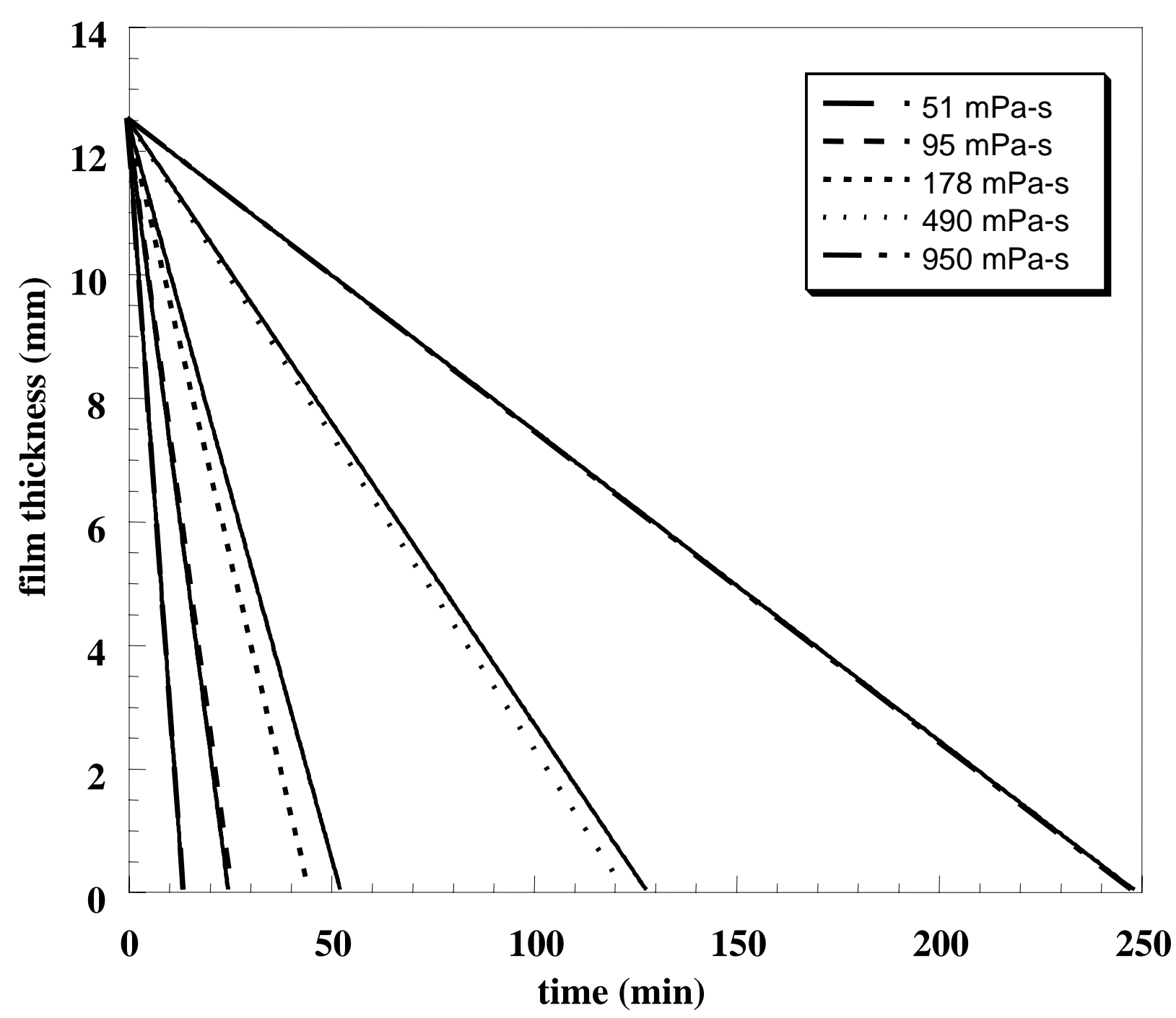

Figure 15. Distance-time plot of coalescence in capillary tubes.

Solid line shows calculations using physical model; dashed lines are experiments.

The effect of viscosity on time for coalescence is apparent in Fig. 15. It takes longer for bubbles trapped in high viscosity oils to come close and coalesce. An increase in the solution viscosity by an order of magnitude increases the time required for coalescence by roughly an order of magnitude. As Fig 15 illustrates, the model (solid lines) well represents the coalescence time from experiments (dashed lines). All parameters are obtained a priori. The match among experiment and calculation with no parameter adjustment provides pleasing affirmation of the underlying physical picture.

A final note with respect to lens drainage calculations is in order. The initial lens thickness in the experiments and calculations is a relatively small $12.5 \mathrm{~mm}$ to allow experiments to complete in a reasonably short period of time. In reservoir media, bubbles may be separated by greater distances if the number of bubbles nucleated per volume of rock is small. Additional distance between bubbles adds substantially to the time for coalescence. 


\section{Summary and Conclusions}

The effect of viscosity on bubble coalescence in viscous oils is studied. It is shown that viscosity of oil has a profound effect on coalescence dynamics during both earlier and later time regimes. In the earlier time stage when coalescence occurs primarily due to bubble growth by diffusion, high viscosity of the oil-phase slows the diffusion of dissolved solution gas in the oil thus reducing the rate of bubble growth. This results in longer time for coalescence of bubbles trapped in more viscous oils. Calculations for the rate of growth of bubbles trapped in oils with different viscosity confirm these statements quantitatively.

At a later stage, coalescence is governed by the pressure drop across the bubbles, pushing them together, draining the oil lens between the bubbles and finally resulting in coalescence. Intuitively, it should be more difficult to drain the oil between the bubbles as oil-phase viscosity increases. This observation is also verified quantitatively. A physical model based on a rather simple set of equations is developed.

The dependence of time for coalescence on viscosity of oil is in agreement with observed high recoveries for heavy-oil reservoirs under solution gas drive. These heavy-oil reservoirs are able to maintain substantial drive energy because gas bubble coalescence is delayed by viscous oil. Low bubble coalescence rates lead to substantial disconnected gas that is difficult to mobilize. Correspondingly, oil-phase relative permeability remains high contributing to relatively efficient oil recovery despite high oil-phase viscosity. This helps to explain why some heavy-oil reservoirs show higher than expected production rates and producing GOR that remains close to solution GOR. Any surface agents present in the crude oil enhance the effect of viscosity by further preventing coalescence.

\section{Nomenclature}

a shape factor of a bubble

a interfacial radius

A kinetic constant of the classical nucleation rate expression

$A_{w}$ wetting area in the corners

$A_{w}$ projected cross-sectional area of the bubble interface

$\mathrm{B}$ thermodynamic constant of the classical bubble nucleation rate expression

$\mathrm{C}$ pressure decline rate

$\mathrm{C}_{\mathrm{dm}}$ dimensionless interfacial entry curvature

$\mathrm{C}_{\mathrm{m}}$ interfacial entry curvature

D diffusion coefficient

F force across the gas bubble

g gravity

$\mathrm{G}$ growth rate of bubble

$\mathrm{J}$ nucleation rate

$\mathrm{K}$ kinetic constant of bubble growth

$\mathrm{K}_{\mathrm{s}}$ solubility constant

$\mathrm{L}$ length of the bubble 
$\mathrm{N}^{\mathrm{o}}$ density of bubbles nucleated instantaneously at $\mathrm{t}=0$

$\mathrm{P}$ pressure in the liquid phase

$\mathrm{P}$ bubblepoint pressure

$\Delta \mathrm{p}$ pressure gradient across the bubble

$\Delta \mathrm{p}_{\mathrm{c}}$ pressure gradient of oil in corners

$\mathrm{p}_{\mathrm{e}}$ equilibrium pressure

$\mathrm{q}_{\mathrm{w}}$ volumetric flow rate of oil

Q $a(\omega+v)^{3} V^{0} N^{0} K^{3}$

$\mathrm{R}$ effective radius of a bubble

$\mathrm{R}$ universal gas constant

$\mathrm{S}$ supersaturation

t time

$\mathrm{T}$ temperature

$\mathrm{V}_{\mathrm{b}}$ volume of a single bubble

$\mathrm{v}_{\mathrm{z}}$ velocity of oil in $\mathrm{z}$-direction

$\mathrm{V}_{\mathrm{r}}$ velocity of oil in radial direction

$\mathrm{V}$ velocity of bubble

$\mathrm{V}^{\mathrm{o}}$ initial total volume

$\mathrm{V}_{\mathrm{g}}$ volume of gas phase

$\mathrm{V}_{\mathrm{A}}$ molar volume of gas $\mathrm{A}$

$\mathrm{V}_{\mathrm{C}}$ critical volume of gas

\section{Greek Letters}

$\beta$ slope of the two phase equilibrium line

$\beta$ dimensionless flow resistance coefficient

$\rho_{\mathrm{o}}$ oil density

$\rho_{\mathrm{g}}$ gas density

$\mu$ oil viscosity

$\checkmark$ growth-parameter power

$\omega$ growth-parameter power 


\section{References}

Arora P. and Kovscek, A. R. 2001. Mechanistic modeling of solution gas drive in viscous oils, SPE 69717, Presented at the SPE International Thermal Operations and Heavy Oil Symposium, Porlamar, Venezuela March 12-14.

Arora P. and Kovscek, A. R. 2003. A mechanistic modeling and experimental study of solution gas drive. Transport in Porous Media. 51, 237-265.

Bora, R., Maini, B.B., and Chakma, 2000. Flow visualization studies of solution gas drive process in heavy oil reservoirs with a glass micromodel. Soc. Pet. Eng. Res. Eval. \& Eng. 3(3), 224-229.

Castanier, L.M. and Sagar, N.S., 1998. Pore level visualization of oil-foam interactions in a silicon micromodel, SPE 39512. Presented at the SPE India and Gas Conference and Exhibition, New Delhi, India April 7-9.

Dake, L. P., 1978. Fundamentals of reservoir engineering; Developments in Petroleum Science, 8; Elsevier: Amsterdam 1978.

De Mirabal M., Gordillo R., Rojas G., Rodriguez H., and Huerta M., 1996. Impact of foamy oil mechanism on the Hamaca oil reserves, Orinoco belt-Venezuela, SPE 36140. Presented at the Fourth Latin American and Caribbean Petroleum Engineering Conference and Exhibition held in Port of Spain, Trinidad and Tobago, Apr. 23-26.

De Mirabal, Rodriguez, H. et al. 1997. Production improvement strategy for foamy Hamaca crude oil: a field case, SPE 37544. Presented at the SPE International Thermal Operations and Heavy Oil Symposium, Bakersfield, CA, Feb. 10-12.

Denn, M M., 1980. Process Fluid Mechanics, Prentice Hall: Englewood Cliffs NJ USA, 255 261.

Dusseault, M., 1993. Cold production and enhanced oil recovery, J Can Pet. Tech, Nov 16.

El Yousfi, A., C. Zarcone, and S. Bories, 1997. Physical mechanisms for bubble growth during solution gas drive, SPE 38921. Proceedings of the Annual Technical Conference and Exhibition of the SPE, San Antonio, TX Oct 5-8.

Firoozabadi, A. and D. Kashchiev, 1996. Pressure and volume evolution during gas phase formation in solution gas drive processes," Soc. Pet. Eng. J., 1(3), 219-227.

Firoozabadi, A., 1997. Author's reply to pressure and volume evolution during gas phase formation in solution gas drive processes," Soc. Pet. Eng. J. 2(2), 228-231.

Firoozabadi, A., 2001. Mechanisms of solution gas drive in heavy oil reservoirs. J. Can. Pet. Tech. 40(3) 15-20. 
George, D.S., 1999. Visualization of solution gas drive in viscous oils, MS Thesis, Stanford University, Stanford CA.

Hayduk, W., and Minhas B.S., 1982. Correlation for prediction of molecular diffusion in liquids, Can. J. Chem. Eng. 60, 294-299.

Hornbrook, J.W., Castanier, L.M. and Petit, P.A., 1991. Observation of foam/oil interactions in a new, high-resolution micromodel, SPE 22631. Presented at the $66^{\text {th }}$ Annual Technical Conference and Exhibition of the SPE, Dallas, Oct. 6-9, 1991.

Huang, W.S., Marcum, B.E. Chase, M.R. and Yu, C.L. 1998. Cold production of heavy oil from horizontal wells in the frog lake field," Soc. Pet. Eng. Res. Eval. \& Eng. 1(6), 551-555.

Kamath, K. and Boyer, R.E.1995. Critical gas saturation and supersaturation in low-permeability rocks. Soc. Pet. Eng. Form. Eval. 10(4), 247-253.

Li, X. and Y.C. Yortsos, 1995. Theory of multiple bubble growth in porous media by solute diffusion. Chem. Eng. Sci., 50(8), 1247-1271.

Press, W. H., Teukolsky, S. A., Vettering, W. T., and Flannery, B. P., 1992. Numerical Recipes in $C$ The Art of Scientific Computing, $2^{\text {nd }}$ Ed. Cambridge University Press: Cambridge, 708-714.

Pooladi-Darvish, M., and Firoozabadi, A., 1999. Solution-gas drive in heavy oil reservoirs. $J$. Can. Pet. Tech, 38( 4) 54-61.

Ransohoff, T.C. and Radke, C.J., 1988. Laminar flow of a wetting liquid along the corners of a predominantly gas-occupied noncircular pore. J. Coll. Int. Sci. 121(2) 392-400.

Ransohoff, T.C. Radke, C.J. and Gauglitz, P.A., 1987. Snap-off of gas bubbles in smoothly constricted noncircular capillaries. Am. Inst. Chem Eng J. 33(5) 753-765.

Reid, R.C., Prausnitz, J.M., and Poling, B.E., 1987. The properties of gases \& liquids, $4^{\text {th }}$ Ed., McGraw-Hill Inc.: New York, 598.

Satik,C., Li,X. and Yortos,Y.C., 1995, Scaling of single-bubble growth in a porous medium. Phys. Rev. E. 51(4) 3286-3295.

Scriven, L.E., 1959. On the Dynamics of Phase Growth, Chem. Eng. Sci., 10(1/2), 1-13.

Tremblay, B., Sedgwick, G. and Vu, D, 1999. CT imaging of wormhole growth under solution gas drive. Soc. Pet. Eng. Res. Eval. \& Eng. 2(1), 37-45.

Tyn, M. T and Calus, W. F., 1975. Processing. 21(4). 116.

Wilt, P.M., 1986. Nucleation rates and bubble stability in water carbon dioxide solutions, J. Coll. Int. Sci., 112(2), 530-538. 


\section{Heavy Oil Solution Gas Drive: Effect of Oil Composition and Rock Consolidation}

\section{(Task 2a)}

An experimental study of heavy oil production resulting from solution gas drive was conducted to probe production mechanisms. This work is documented in two papers prepared for presentation at meetings sponsored by the Society of Petroleum Engineers (Tang et al., 2003, 2004). A brief summary is given here as the full text is available elsewhere.

A series of viscous mineral oil and heavy-oil primary depletion experiments were conducted to differentiate the mechanisms of heavy-oil solution gas drive. Our study was conducted under a variety of test conditions (temperature: $25-80^{\circ} \mathrm{C}$, solution GOR: $14-44 \mathrm{vol} / \mathrm{vol}$, oil viscosity: 258-2800 cp, oil type: crude oil and mineral oil, depletion rate: 0.0035-0.035 $\mathrm{PV} / \mathrm{hr}$ ). Our study shows that solution-gas drive is an effective process to recover heavy crude oil. Recoveries for crude oil range from 28 to $45 \%$ OOIP (original oil in place) in the laboratory. Recovery of mineral oil, under similar conditions, was substantially less and ranged from 12 to $18 \%$ of the OOIP. Generally, the greater the depletion rate, the greater the recovery of oil.

For heavy crude oil, gas bubbles analyzed at the sandpack outlet displayed a variety of morphologies ranging from dispersed pore-sized bubbles (foam-like) to free gas. High depletion rate tends to generate dispersed, pore-sized gas bubble flow in the crude oils. Dispersed bubbles are much less mobile than continuous gas as witnessed here by large pressure drops when the system was expanded at a fixed rate. The low gas mobility associated with small pore-sized bubbles correlates with greater oil recovery. Oil-phase composition appeared to play a significant role in the coalescence of the gas phase into free gas. Although oil-phase viscosities were roughly the same among pairs of mineral and crude oil, the crude oil tests exhibited significantly greater recovery. Coalescence of bubbles into a continuous gas phase occurred over a significantly greater period of time in crude oil.

The effect of gravity was apparent in X-ray CT scan images. Gas saturation within the sandpack is not uniform. High gas saturation exists at the outlet region. For mineral oil, no foamlike flow behavior is witnessed.

Sandpacks have been widely implemented to study heavy oil solution-gas drive. Sandpacks provide a high permeability porous medium, that is usually similar to those, encountered in a heavy oil reservoir. Difficulties with weakly consolidated sands with high permeability and porosity is another reason that drove researchers to use sandpacks for laboratory tests. However, most investigations with sandpack have neglected the overburden pressure. In this work, we compared solution gas drive in heavy oil between consolidated and unconsolidated rock. It is found that overburden pressure affects heavy oil solution gas drive as follows: (1) over burden pressure supports pore pressure delaying its decline that in turn affects the features of flowing gas bubbles, (2) fluid flow resistance is greater in consolidated sand, (3) critical gas saturation and recovery performance are sensitive to rate and overburden pressure, and (4) gas and oil relative permeability is greater for the consolidated rock.

For the test system, the heavy oil recovery by solution gas drive is about $22-32 \%$ OOIP. Additionally, the gas recovery is about $60-90 \%$ OGIP. The critical gas saturation is about 7-19 \% 
and it increases with depletion rate significantly. With overburden pressure presence, the critical gas saturation is about 3\% higher. A modified relative permeability model established for solution gas drive in heavy oil was proposed. For non uniform gas saturation distribution within the system, change in gas saturation and pressure at each section is required. The measured gas relative permeability for the test system is about $10^{-5}$ to $10^{-6}$ and is not a strong function of gas saturation.

\section{References}

Tang, G.-Q., Y. Leung, L. M. Castanier, A. Sahni, F. Gadelle, M. Kumar, and A. R. Kovscek, 2003. "An Investigation of the Effect of Oil Composition on Heavy Oil Solution Gas Drive," SPE 84197, Proceedings of the SPE Annual Technical Conference and Exhibition, Denver, CO, Oct. 5-8.

Tang, G.-Q., A. Sahni, F. Gadelle, M. Kumar, and A. R. Kovscek, 2004. "Heavy-Oil Solution Gas Drive in Consolidated and Unconsolidated Rock," SPE 87226, Proceedings of the SPE International Thermal Operations and Heavy Oil Symposium and Western Regional Meeting, Bakersfield, CA, Mar 16-18. 


\section{Model and Simulation of Cold Production Using a Population Balance Approach}

\section{(Task 3b)}

\section{Introduction}

The previous sections illustrate that cold production of heavy oil involves significant flow of dispersed gas. Initially, it was believed that production of reservoir sand along with oil might be necessary for foamy-oil behavior. More recently, several reservoirs have been identified that exhibit high recovery of heavy oil as foamy oil, but produce little or no sand (Claridge and Prats, 1995). Thus, flow mechanisms can be considered independently from sand production. The rate of oil production appears to contribute to foamy-oil formation. Rapid depletion of reservoir pressure during primary production leads to larger than usual recoveries. Nevertheless, there are currently no estimates for the rates necessary to improve production response.

Although we are certain nucleation generates bubbles, the microstructure of the dispersed gas within the porous medium is unknown. In the case of aqueous foams, a detailed accounting of the pore-level morphology of foam and the mechanisms for changing bubble size were keys to unlocking, understanding, and predicting aqueous foam flow behavior (Kovscek et al., 1995). A similar quantitative tool to interpret gas-bubble microstructure would likely benefit our understanding of heavy-oil solution gas drive. The foamy-oil case is more complicated in some regards. Gas bubbles are generated by nucleation, and thus, are smaller than pore throat and body dimensions initially. Once formed, these bubbles may grow larger than pore size by gas diffusion and the coalescence of multiple bubbles into a single bubble.

The focus of this work was to prove the feasibility and develop a bubble population balance modeling framework to describe solution gas drive behavior in viscous oils and address the question of gas-bubble microstructure. We incorporate pore-level bubble nucleation and growth mechanisms, including the idea of a crevice-size distribution from which nucleated bubbles emerge, into a continuum displacement model consistent with standard simulation of multi-phase flow in porous media. Theoretical predictions are verified by comparison to prior and new experimental results. The model is based upon a bubble population balance framework (Patzek, 1988; Randolph and Larson, 1971). In such methods, the bubble conservation equation resembles the usual continuum mass balance equations for chemical species flowing in porous media (Patzek 1988; Falls et al., 1988). Importantly, a population balance approach allows us to blend previous, and new, experimental knowledge on heavy-oil solution gas drive with models for nucleation and growth physics of bubbles in porous media.

The theoretical framework, experimental design, and comparison of experiment and theory are reported in the literature (Arora and Kovscek, 2003). Accordingly, the following is an abbreviated summary. 


\section{Population Balance Model of Solution Gas Drive}

A population balance formulation quantifies directly the evolution of bubble size as pressure declines. Gas mobility is assessed from the concentration of bubbles. Further, the method is mechanistic in that documented and/or proposed pore-level events are portrayed in bubble nucleation, growth, breakup, and coalescence equations. The method provides a general framework where the relevant physics of heavy-oil solution gas drive are tested and expressed. Deriving continuum-averaged equations that accurately represent pore-level physics was a difficult task that formed the majority of this work.

Instantaneous nucleation (IN) (Firoozabadi and Kashchiev, 1996) and progressive nucleation (PN) (Li and Yortsos, 1995) models of bubble nucleation were both incorporated into the population balance model.

\section{Results and Discussion}

Next, we discuss the results of simulations for only the IN model. Full results are given in the paper (Arora and Kovscek, 2003). Porous medium properties are listed in Table 1 and the fluid properties in Table 2. Viscosity data for gas saturated n-decane versus pressure is given by Lee et al. (1966). The parameters $\omega$ and $v$ are both set to 0.5 , whereas the grid size is 40 blocks horizontally by 3 blocks vertically. Initially the core is totally filled with gas-saturated oil. At time $t=0$, withdrawal of oil from the core is started at a constant volumetric flow rate of 1.44 $\mathrm{cm}^{3} /$ day $\left(5.1 \times 10^{-5} \mathrm{ft}^{3} /\right.$ day $)$.

Table 1. Porous Medium Characteristics

\begin{tabular}{|c|c|c|}
\hline Parameter & $\begin{array}{c}\text { Firoozabadi et al. } \\
(1992)\end{array}$ & This Work \\
\hline \hline $\begin{array}{c}\text { Length of core } \\
(\mathrm{cm})\end{array}$ & 49.40 & $40.6 \pm 0.1$ \\
\hline $\begin{array}{c}\text { Cross Sectional } \\
\text { Area }\left(\mathrm{cm}^{2}\right)\end{array}$ & 12.01 & $20.3 \pm 0.2$ \\
\hline Porosity & 0.223 & $0.320 \pm 0.001$ \\
\hline Pore Volume $\left(\mathrm{cm}^{3}\right)$ & 132 & $264 \pm 1$ \\
\hline Permeability (d) & 0.605 & $25.7 \pm 0.1$ \\
\hline
\end{tabular}

The model is compared to available experimental data. Firoozabadi et al. (1992) conducted pressure depletion experiments on a Berea sandstone core saturated with a $C_{1} / C_{2} / n-C_{10}$ mixture. In these experiments, the oil withdrawal rate was fixed and the pressure of the core was recorded as a function of time prior to the start of bubble flow. The bubble point is $1071 \mathrm{psi}$ ( 7.4 $\mathrm{MPa}$ ). The core and hydrocarbon mixture properties are given in Tables 1 and 2. Grid size is 40 blocks in the horizontal direction by 3 blocks in the vertical direction. 
Table 2. Fluid Properties

\begin{tabular}{|c|c|c|c|}
\hline Parameter & $\begin{array}{c}\text { High Tension } \\
\text { Firoozabadi et al. } \\
(1992)\end{array}$ & $\begin{array}{c}\text { Low Tension } \\
\text { Firoozabadi et al. } \\
(1992)\end{array}$ & This Work \\
\hline $\begin{array}{c}\text { Initial oil } \\
\text { composition } \\
(\text { mole } \%)\end{array}$ & $\begin{array}{c}\mathrm{C}_{1} 28.52 \% \\
\mathrm{C}_{2} 0.02 \% \\
\mathrm{n}-\mathrm{C}_{10} 71.46 \% \\
\end{array}$ & $\begin{array}{c}\mathrm{C}_{1} 65.14 \% \\
\mathrm{C}_{2} 0.22 \% \\
\mathrm{n}-\mathrm{C}_{10} 34.29 \% \\
\end{array}$ & $\begin{array}{c}\mathrm{CO}_{2} 0.59 \% \\
\text { Kaydol } 0.41 \%\end{array}$ \\
\hline $\begin{array}{c}\text { Surface tension } \\
(\text { dyne } / \mathrm{cm})\end{array}$ & 13 & 2.1 & 5 \\
\hline $\begin{array}{l}\text { Bubble point } \\
\text { pressure (psi) }\end{array}$ & 1071 & 3655 & $525 \pm 5$ \\
\hline $\mathrm{D}\left(\mathrm{ft}^{2} / \mathrm{sec}\right)$ & $6.5 \times 10^{-8}$ & $6.5 \times 10^{-8}$ & $3.4 \times 10^{-9}$ \\
\hline$\beta\left(\mathrm{psi} / \mathrm{cm}^{3}\right)$ & 14 & 45.9 & 3.53 \\
\hline $\mathrm{K}_{\mathrm{s}}\left(\mathrm{lb}-\mathrm{mol} / \mathrm{psi}-\mathrm{ft}^{3}\right)$ & $1.1 \times 10^{-4}$ & $3.3 \times 10^{-4}$ & $\begin{array}{c}\text { refer to solid line in } \\
\text { Fig. } 2\end{array}$ \\
\hline $\begin{array}{l}\text { dead-oil viscosity } \\
\text { (cP or mPa-s) }\end{array}$ & 0.84 & 0.84 & 220 \\
\hline
\end{tabular}

Figure 1 shows a comparison of the IN model simulation with the experimental data for a withdrawal rate of $1.44 \mathrm{~cm}^{3} /$ day $\left(5.1 \times 10^{-5} \mathrm{ft}^{3} /\right.$ day $)$. Note that in the experiments, the fluid pressure was adjusted to roughly $1200 \mathrm{psi}(8.2 \mathrm{MPa})$ by expanding the system prior to the constant rate depletion (Firoozabadi et al., 1992). Simulation begins at $1175 \mathrm{psi}(8.099 \mathrm{MPa})$ and a volume expansion of roughly $0.5 \mathrm{~cm}^{3}$ in concert with the experimental starting point. The number density of nucleated bubbles per volume of rock is fixed to $350 \mathrm{bubbles} / \mathrm{ft}^{3}(0.0124$ bubbles $/ \mathrm{cm}^{3}$ ) rock for this run. The simulation result matches the data quite well until a volume expansion of about $0.75 \mathrm{~cm}^{3}\left(2.6 \times 10^{-5} \mathrm{ft}^{3}\right)$ and tends to deviate somewhat thereafter. The pressure from the simulation is larger than the experimental data at late time, but the error is only about 2 $\%$.

An important point to note is that no critical supersaturation is imposed on the system for the simulation runs. Firoozabadi et al. (1992) asserted that bubbles nucleated at the pressure corresponding to the minima on the $\mathrm{P}$ vs. $\mathrm{t}$ curve $(6.9 \mathrm{MPa}, 1010 \mathrm{psi})$. Thus, according to them, the value of supersaturation was around $60 \mathrm{psi}(0.41 \mathrm{MPa})$. In these simulations, bubble growth begins at the bubble point pressure. Although bubbles nucleate at the thermodynamic bubble point of the mixture, gas and liquid are not in equilibrium. Bubbles grow according to the kinetic equations above, and the volume of gas as a function of time lags behind what is expected for equilibrium conditions. There is competition between pressure increase due to mass transfer of gas to the bubbles and pressure decrease due to the expanding system volume. These competing effects result in the characteristic pressure minimum and rebound witnessed in solution gas drive. One advantage of our approach is that it eliminates the need for determining experimentally, the value of critical supersaturation as a function of rate for every system. 


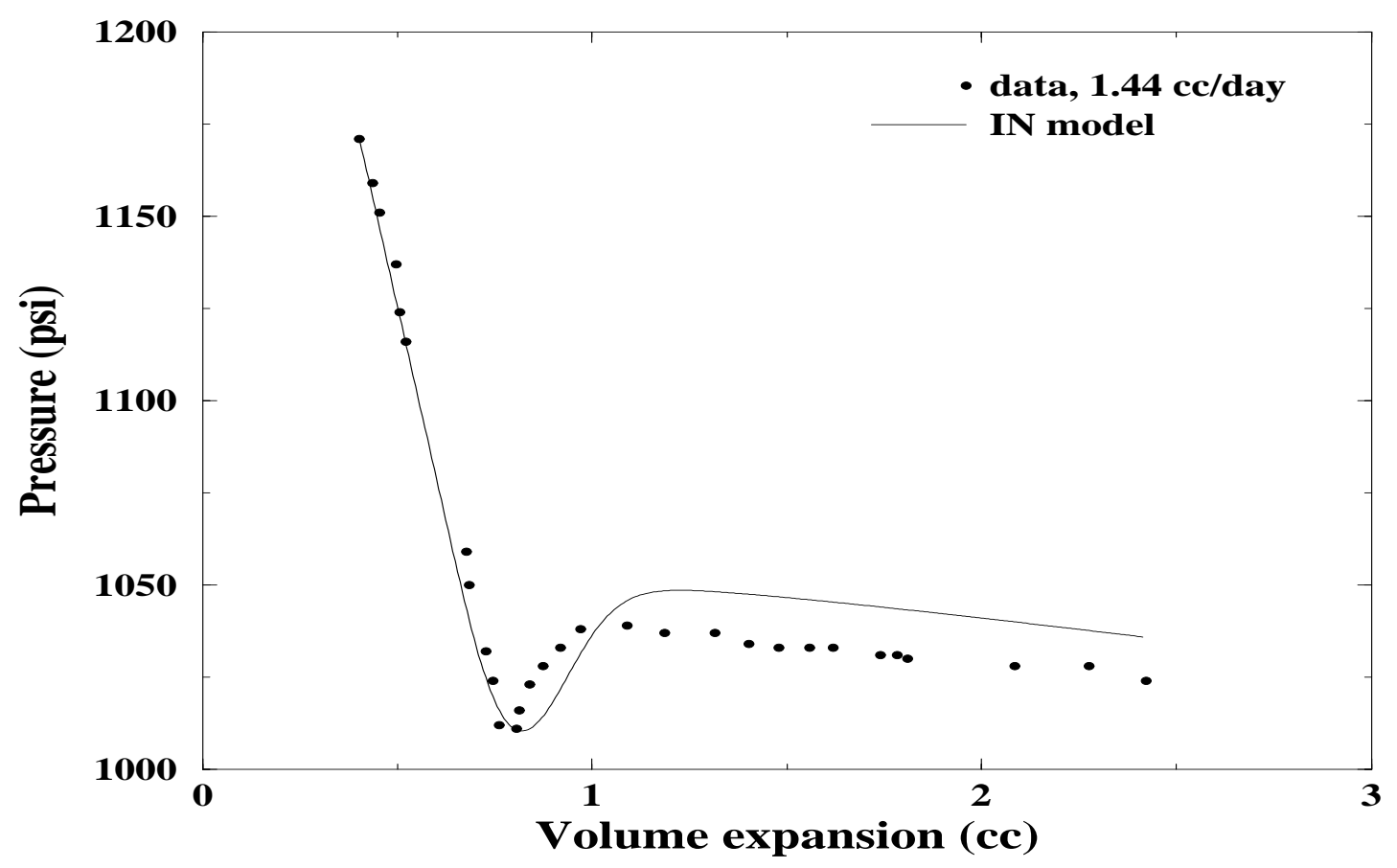

Figure 1. Match of the IN bubble population balance model to the experimental pressure data of Firoozabadi et al. (1992). Withdrawal rate is $1.44 \mathrm{~cm}^{3} /$ day $\left(5.10 \times 10-5 \mathrm{ft}^{3} /\right.$ day) and the number of bubbles nucleated is 0.0124 bubbles $/ \mathrm{cm}^{3}$ (350 bubbles $\left./ \mathrm{ft}^{3}\right)$.

Figure 2 shows the results of the simulation and experimental pressure data for a liquid withdrawal rate of $7.2 \mathrm{~cm}^{3} /$ day $\left(2.54 \times 10^{-4} \mathrm{ft}^{3} /\right.$ day $)$. The bubble number density of nucleated bubbles was adjusted to 1000 bubbles $/ \mathrm{ft}^{3}\left(0.0353 \mathrm{bubbles} / \mathrm{cm}^{3}\right)$ to obtain a match with data. All the other parameters are governed by the properties of either the liquid or the porous medium and are not affected by a change in the withdrawal rate. Again, gas bubbles nucleate and begin to grow at the thermodynamic bubble point. The pressure match for this case is again quite good. The final gas saturation corresponding to a volume expansion of $3.25 \mathrm{~cm}^{3}\left(1.1 \times 10^{-4} \mathrm{ft}^{3}\right)$ is around $1.8 \%$ which is close to the value of $2 \%$ reported by Firoozabadi et al. (1992).

Note, the bubble density increases as the liquid withdrawal rate increases for the IN model. The explicit implication is that more nucleation sites are activated as the withdrawal rate is increased. Additionally, we observe that the bubble population balance approach has directly modeled the increase in apparent critical supersaturation evident in the data (compare Figs. 1 and 2). 


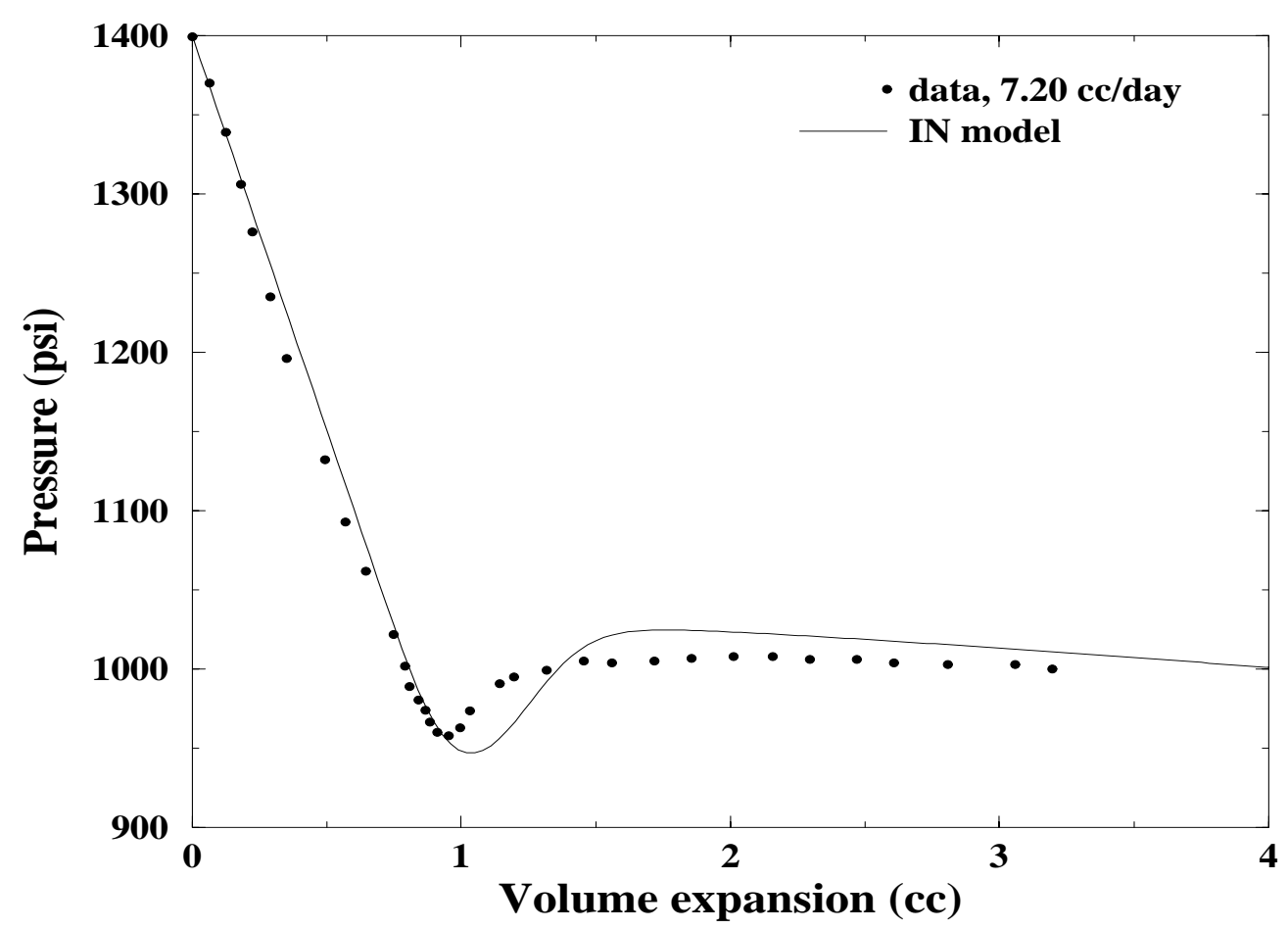

Figure 2. Match of the IN bubble population balance model to the experimental pressure data of Firoozabadi et al. (1992). Withdrawal rate is $7.20 \mathrm{~cm}^{3} /$ day $\left(2.50 \times 10^{-4} \mathrm{ft}^{3} /\right.$ day $)$ and the number of bubbles nucleated is 0.0353 bubbles $/ \mathrm{cm}^{3}\left(1000\right.$ bubbles $\left./ \mathrm{ft}^{3}\right)$.

The bubble population balance model was also applied to the viscous-oil solution gas drive experiments. The properties of the mineral oil (Kaydol) are summarized in Table 2, and the properties of the sand pack are given in Table 1. No data is available regarding the viscosity of this mineral oil as a function of pressure and gas content. For calculations, the generalized correlations presented by McCain (1990) are used. The number of bubbles nucleated is 14,000 per $\mathrm{ft}^{3}$ of rock $\left(0.49\right.$ bubbles $\left./ \mathrm{cm}^{3}\right)$ consistent with the large volumetric expansion rate. Figure 3 is a plot of average core pressure versus time for a run with an oil withdrawal rate of $6 \mathrm{~cm}^{3} / \mathrm{hr}$ $\left(2.1 \times 10^{-4} \mathrm{ft}^{3} / \mathrm{hr}\right)$. The match between the experiment and the simulation is quite good. The simulation overestimates the pressure after $t=80 \mathrm{mins}$. Gas begins to flow at about $5.25 \mathrm{hrs} \mathrm{(314}$ min) corresponding to a gas saturation of $6 \%$. Our model does not match the data well after 5.25 hrs because it does not yet take into account mobile gas. 


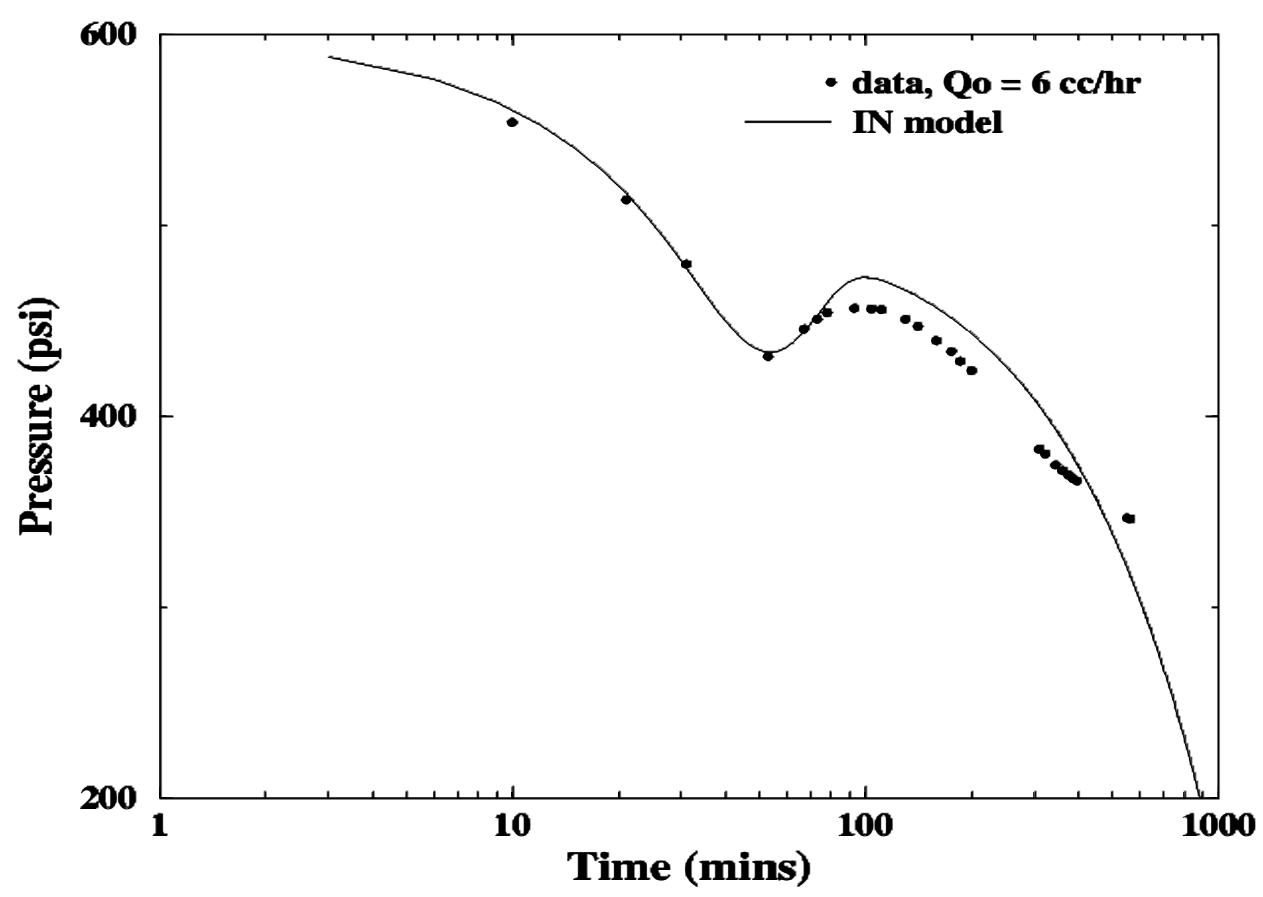

Figure 3. Match of the IN bubble population balance model to viscous-oil solution gas drive pressure data. Withdrawal rate is $6.00 \mathrm{~cm}^{3} /$ hour $\left(2.10 \times 10^{-4} \mathrm{ft}^{3} / \mathrm{hr}\right)$ and the number of bubbles nucleated is $0.490 \mathrm{bubbles} / \mathrm{cm}^{3}(14,000$ bubbles $\left./ \mathrm{ft}^{3}\right)$.

\section{Conclusion}

Solution gas drive is a production mechanism commonly encountered in oil reservoirs that is not very well understood when the oil is viscous. A mechanistic population balance model has been developed that incorporates instantaneous and progressive mechanisms for solution gas drive. The population balance approach is physical in that the nucleation and growth of the gas phase are modeled explicitly. Of course, expressions are required that model bubble mechanics accurately. The population balance equations resemble the mass balance equations for reservoir simulation and so can be incorporated into existing reservoir simulation framework.

Both the IN and PN bubble-population balance models were applied successfully to experimental data for the initial period of solution gas drive (before the onset of gas flow) in light and viscous oils. All bubbles nucleate at the thermodynamic bubble point of the gas-liquid system. Computational results taught that bubbles grew to dimensions that exceeded those of individual pores, in all cases. The IN model showed good matches to pressure data for both light and viscous oils. The average gas saturation at the onset of gas flow for the two cases was also close to the experimentally observed values. The number density $\left(N_{o}\right)$ of bubbles nucleated per volume of rock is an adjustable parameter. It increased when the oil withdrawal rate increased; likewise, $N_{o}$ increased when the surface tension of oil decreased. An important feature of our IN model is that no critical supersaturation threshold is required to match experimental data. 
The match of the PN model results to experimental data for light and heavy oils was very good and judged superior than those for the IN model. Extra computational work was associated with this improved agreement. Bubbles were released from cavities when the supersaturation overcame the capillary forces associated with a particular crevice size. The period of active bubble nucleation for the PN model, however, was relatively small compared to the total time of the pressure depletion runs. This is in agreement with experimental observations in the literature (El Yousfi et al., 1997).

\section{References}

Arora, P. and A. R. Kovscek, "A Mechanistic Modeling and Experimental Study of Solution Gas Drive," Transport in Porous Media, 51, 237-265 (2003).

Claridge, E.L. and M. Prats, "A Proposed Model and Mechanism For Anomalous Foamy Heavy Oil Behavior," SPE 29243, in Proceedings of the International Heavy Oil Symposium, Calgary, Alberta (Jun., 1995).

El Yousfi, A., C. Zarcone, and S. Bories, "Physical Mechanisms for Bubble Growth During Solution Gas Drive," SPE 38921, in Proceedings of the Soc. Pet. Eng. Ann. Tech. Conf. and Exhibition, San Antonio, TX (Oct 5-8, 1997).

Firoozabadi, A., B. Ottesen, and M. Mikkelsen, "Measurement of Supersaturation and Critical Gas Saturation," Soc. Pet. Eng. Formation Evaluation, Dec, 337-344 (1992).

Firoozabadi, A. and D. Kashchiev, "Pressure and Volume Evolution During Gas Phase Formation in Solution Gas Drive Processes," Soc. Pet. Eng. J., 1(3), 219-227 (1996).

Li, X. and Y.C. Yortsos, "Theory of Multiple Bubble Growth in Porous Media by Solute Diffusion," Chem. Eng. Sci., 50(8), 1247-1271 (1995).

McCain, W. D., The Properties of Petroleum Fluids, $2^{\text {nd }}$ Ed., PennWell Publishing Co.: Tulsa, OK. p.328-333 (1990).

Patzek, T.W., Description of Foam Flow in Porous Media by the Population Balance Approach, in Surfactant-Based Mobility Control: Progress in Miscible-Flood Enhanced Oil Recovery, D.H. Smith, Editor., American Chemical Society: Washington, D. C. p. 326341 (1988).

Randolph, A. D. and Larson, M. A., Theory of Particulate Processes, Academic Press: New York p. 41-64 (1971). 


\section{Area 4. In-Situ Combustion}

Steam injection is the most common form of enhanced oil recovery, but steam is not applicable to all reservoirs. In situ combustion is useful in reservoirs where steam injection is difficult or impossible. Heat is produced in situ by burning part of the oil; hence, heat losses at the surface and in the injection wells are non existent. This allows implementation of the combustion process in deep reservoirs or in reservoirs where heat losses may be a problem (permafrost and offshore to name two examples).

This is a brief summary of the work performed in Area 4 in-situ combustion. Complete details of our efforts are given by Castanier and Brigham (2003A, 2003B). Specifically, we have focused on understanding some of the main aspects of in-situ combustion. The scope of work involved three main research directions: (i) understand-ing water soluble metallic additives effect on combustion reactions, (ii) feasibility study of a combination of solvent injection and combustion processes, and (iii) improving the performance prediction of field projects. 


\section{Solvent and Air Injection Processes}

\section{(Task 4a)}

\section{Introduction}

This work is an attempt to estimate the potential of using solvents in a cyclic manner to recover and upgrade heavy oil. Preliminary analytical calculations based on an adaptation of the Boberg and Lanz model as described by Prats (1982) to solvent injection showed that such a combination could be technically and economically viable

\section{Experimental}

Cold Lake Heavy crude was mixed with decane and the viscosity of the mixtures was measured using a rotational viscometer. Filtration of the mixtures determined that solid precipitation occurred at about $60 \%$ by volume of decane. The results of the first series of experiments are shown in Table 1.

Table 1. Dilution of Cold Lake Crude Oil

\begin{tabular}{|l|l|}
\hline Percentage n-decane (volume) & Viscosity $(\mathrm{cP})$ \\
\hline 0 & 37,000 \\
\hline 10 & 4,000 \\
\hline 20 & 310 \\
\hline 30 & 22 \\
\hline 50 & 11 \\
\hline 55 & 7 \\
\hline 60 and over & no measured, asphalt precipitates \\
\hline
\end{tabular}

Figure 1 displays graphically the log of mixture viscosity versus the solvent concentration. One interesting point to note is that by graphing those data on ASTM paper, a relatively good fit with a straight line is obtained. It should be possible as proposed by Burger et al. (1984) to obtain an analytical expression for viscosity of the mixture. This expression is of the form of $\log$ (log of solvent concentration). Of course more measurements using various pairs of solvents and oils are needed to assess better if this relationship is generally valid. 


\section{Results and Discussion}

In the process envisioned, a slug of solvent is injected into the reservoir where it mixes with the oil. The amount to be injected is determined by reservoir simulation, calculations, or experience. We expect in most cases, that the amount to be injected is a small fraction of the pore volume of the reservoir considered. The mixture viscosity is substantially lower as compared to the viscosity of the original oil. This viscosity reduction is evaluated if the solvent is a liquid by using the relationship described above. Note that at $20 \%$ solvent concentration, the mixture viscosity is decreased by more than 2 orders of magnitude. If primary production exists, the improvement in oil production is estimated using an approach similar to that used by Boberg for cyclic steam stimulation and taking into account only the change of viscosity caused by the solvent (cf. Eq. 9.6 and/or Table 9.2 of Prats, 1982). Production after the injection of the first slug of solvent is thus easily calculated. At this stage, simple calculations give estimates of the production in the field.

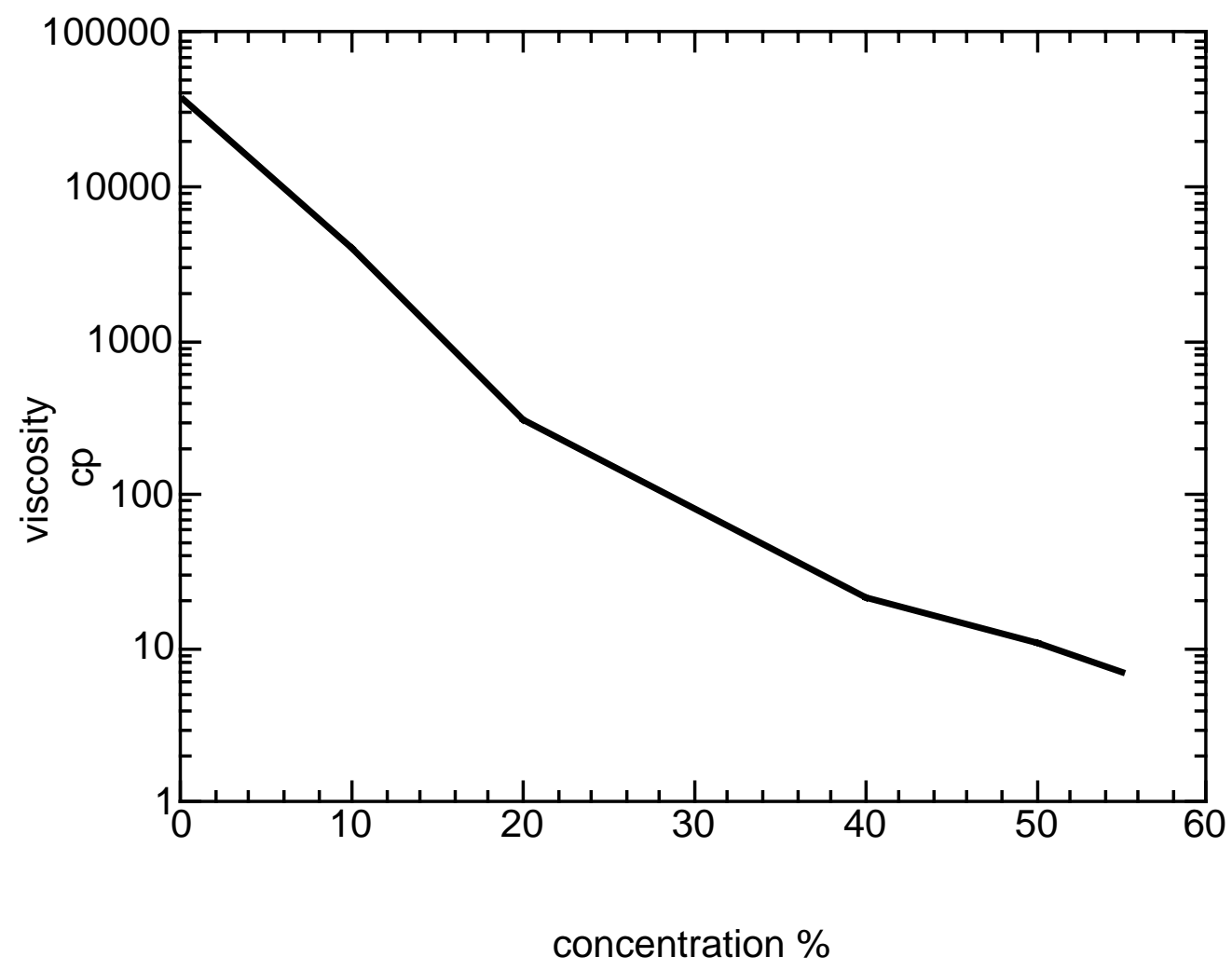

Figure 1. Viscosity of mixtures of Cold Lake oil and n-decane. Concentration reported in volume per cent.

For the sake of illustration, we perform calculations for a well in a reservoir containing oil similar to the Cold Lake but producing before solvent injection at about 20 $\mathrm{bbl} /$ day. The producing interval thickness is 10 meters and a slug of $500 \mathrm{~m}^{3}$ of solvent 
similar to decane is injected. Assume that the mixing is such that the concentration of solvent is linear with distance to the well and that no gravity effects exist. We also assume a drainage radius of 100 meters and no skin effect. The well radius is assumed to be 0.2 meters.

The concentration profile as a function of distance to the well is calculated by material balance and results are summarized in Table 2. For convenience, the viscosity of the mix is also given in this table.

Table 2. Example Calculation for Solvent Injection

\begin{tabular}{|l|l|l|}
\hline Distance $(\mathrm{m})$ & Solvent volume $(\%)$ & Viscosity $(\mathrm{cP})$ \\
\hline 0 & 100 & 5 \\
\hline 1.5 & 75 & 5 \\
\hline 3 & 50 & 11 \\
\hline 4.5 & 25 & 130 \\
\hline 6 & 0 & 37,000 \\
\hline
\end{tabular}

We assume that near the well viscosity is $5 \mathrm{cp}$ when solvent concentration is over $60 \%$. With these very conservative assumptions, the initial flow rate is 3.5 times the rate before stimulation. Working on a step by step basis, we calculate a production schedule and a solvent recovery schedule. The production data are summarized in Table 3.

Table 3: Example Production Data

\begin{tabular}{|l|l|l|l|}
\hline time (days) & Oil $(\mathrm{bbl} / \mathrm{d})$ & Solvent $(\mathrm{bbl} / \mathrm{d})$ & Solvent recovery\% \\
\hline 5 & 3.5 & 66.5 & 10.6 \\
\hline 15 & 10.2 & 57.8 & 29.1 \\
\hline 25 & 16.2 & 48.75 & 44.7 \\
\hline 35 & 21.7 & 40.3 & 57.6 \\
\hline 55 & 25.2 & 30.8 & 77.3 \\
\hline 75 & 26.4 & 21.6 & 91.2 \\
\hline 95 & 28 & 12 & 98.8 \\
\hline 105 & 20 & & \\
\hline
\end{tabular}

The total oil recovered is about $2300 \mathrm{bbl}$ after 100 days meaning an increase of $300 \mathrm{bbl}$ over primary. This is a small number but one notices that most of the solvent is recovered and most of the oil production occurs at the end of the cycle. Mixtures of recovered solvent and oil could be used in subsequent cycles without refining, thus improving the economics. 
The mixing characteristics of the oil and the solvent are important. If instead of assuming a linear concentration profile with distance to the well, we assume that the two liquids mix $50 \%$ to $50 \%$, the recovery of oil will be around 3,000 bbls after 100 days and solvent recovery at that time will be over $98 \%$. Although this is not a very realistic case, it should be considered as the upper limit possible. It is obvious that the economics of such processes depend strongly on solvent costs and availability. 


\section{Metallic Additives}

(Task 4b)

\section{Introduction}

Some water soluble metallic salts change the kinetics of the reactions leading to fuel formation and combustion (Castanier et al., 1992; Castanier and Brigham, 1996). During the period of performance we have confirmed the fact that tin, zinc and iron can be used as water soluble metallic catalysts for combustion. In all the oils and rock matrix systems tested we have noticed an increase in fuel burnt and lower energy of activation for the reactions in presence of the metals. This allows combustion of light oils where without the additive not enough fuel would be present to allow combustion. Tube runs have been run using a $34^{\circ}$ API California oil with ferric nitrate as the additive. Combustion was not effective without the additive and the process died within an hour of ignition whilst the run performed with iron nitrate yielded sustained combustion for eight hours with the front propagating through the length of our combustion tube. Obviously these results need to be expanded to more oils and reservoir rocks. Documentation of the above can be found in our annual reports for 2001 and 2002 (Kovscek and Castanier 2001, 2002).

One very interesting feature of in-situ combustion is that combustion may promote upgrading of the produced oil. In fact numerous field observations have shown upgrading by combustion ranging from 2 to $6^{\circ}$ API. This has obvious beneficial consequences on the economic viability of the process.

A paper was published on the upgrading of crude oil by in-situ combustion (Castanier and Brigham, 2003). This work first reviews the various reactions occurring during the in-situ combustion process with focus on the oil composition, fuel composition and upgrading. We show that the vast majority of chemical reactions and physical processes such as distillation do indeed promote oil improvement by removing the heavy part of the oil as fuel and creating lighter ends via cracking and pyrolisis

\section{Results and Discussion}

Combustion can also be used for removal of unwanted compounds prior to refining. Sulfur has always be a problem for refiners. In the second part of the above paper we present as an example results of three combustion experiments aiming at sulfur removal from an oil from Kuwait initially containing around 6\% sulfur by weight. By proper implementation of in-situ combustion, the laboratory results showed a reduction in sulfur content of the oil from $6 \%$ to less than $1 \%$. Figures 1 and 2 show the produced gas composition for a run without additive where sulfur removal was about $3 \%$ by weight and a run with tin chloride where only $1 \%$ by weight of sulfur remained in the oil. 


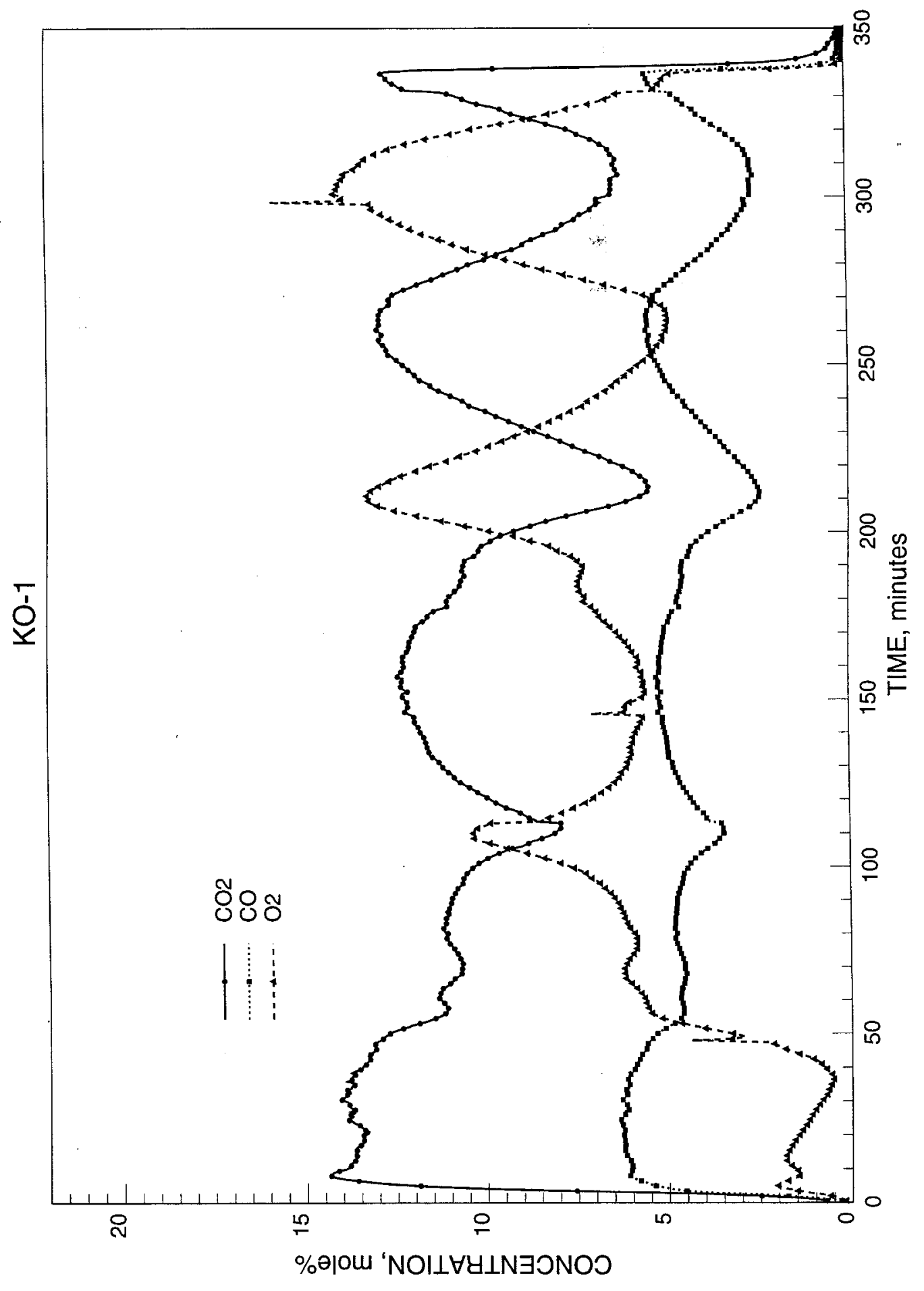

Figure 1. Effluent gas composition, no additives. 


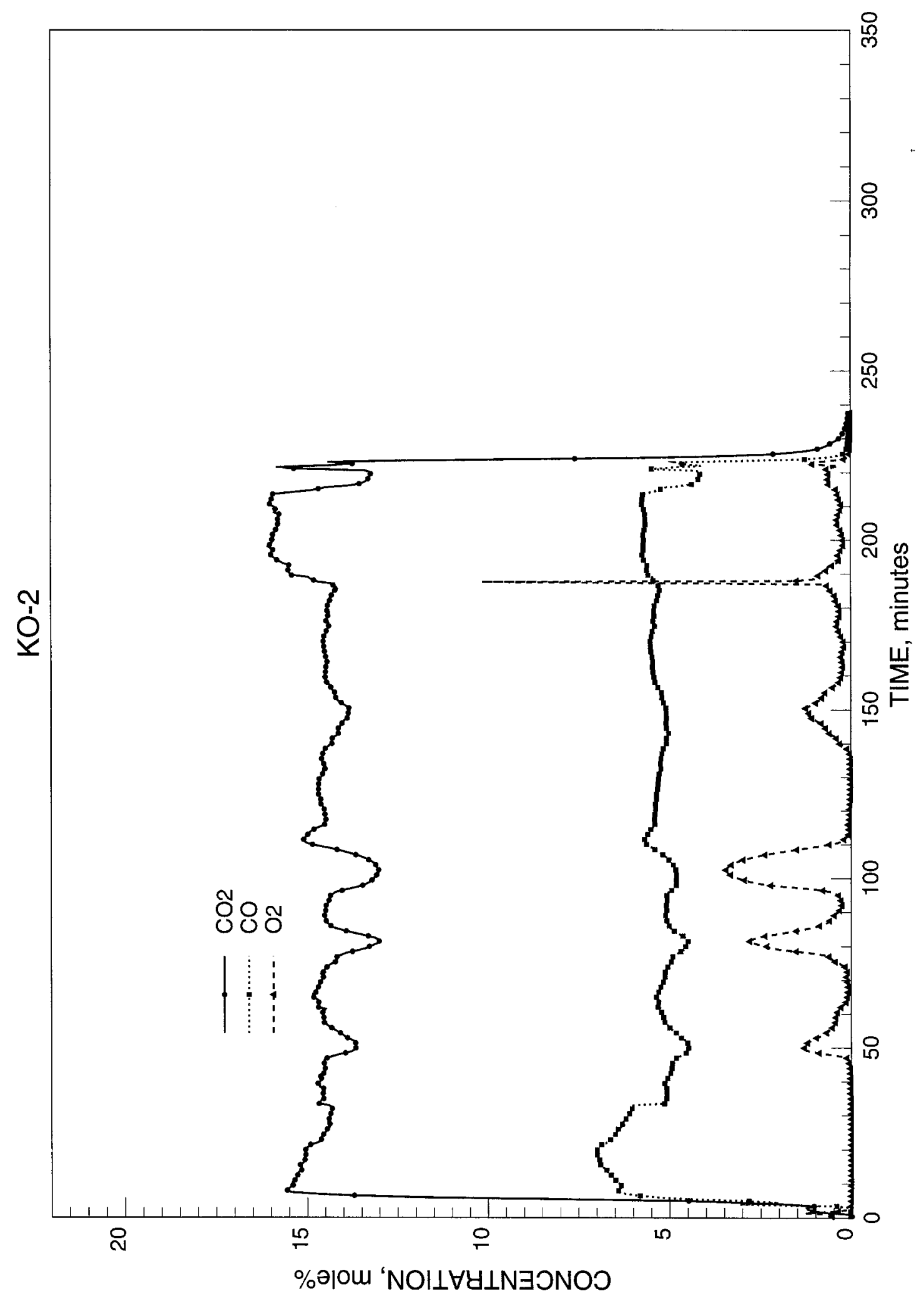

Figure 2. Effluent gas composition, tin with $5 \% \mathrm{Sn} \mathrm{cl}_{2}$ solution. 


\section{Reservoir Engineering Aspects of In-Situ Combustion}

In-situ combustion is, in fact, the oldest thermal recovery technique. Nevertheless, it is regarded as high-risk process by many and as a niche-filling technique by others. The former perception is a result primarily of spectacular failures during early field tests. Most of these failures resulted from the application of a good process (ISC) to the wrong reservoirs and/or to the poorest prospects. The latter perception developed because of the inability to combust all oil types. During the performance period it became obvious that the potential of ISC as an economically viable oil recovery technique required clarification. A manuscript was drafted listing a summary description of ISC, laboratory screening techniques, application of laboratory results to field design, a review of performance prediction methods, operational problems, and analysis of field results. This material subsequently became a portion of the Society of Petroleum Engineer's Handbook (Castanier and Brigham, 2003B).

\section{Summary}

Insitu combustion can be applied to a wide array of reservoirs. In fact it is the only thermal method that can presently be applied to deep reservoirs, though deep downhole steam generation is being tested. It can be used at any stage of reservoir depletion. It can be used in special situations such as offshore or in arctic regions. Because of the lack of heat losses at the surface and in the injection wells, it is the most thermally efficient thermal recovery method. The injectant (air) is readily available. Combustion allows wider well spacing than steam. Economic results are comparable to those of steam injection.

Several aspects of operating ISC projects are important. First is the large compression ratios and associated costs required to inject air into the formation. Second is planning and design requirements for a combustion project that are more difficult than steam. Third is extensive laboratory work to assess fuel availability, air requirements and burning characteristics of the crude that are required before designing insitu combustion projects. Fourth is the high degree of technical sophistication, and careful monitoring needed to ensure proper operation of a project. Fifth is the limitation of numerical simulation and other techniques that make predictions of recovery more difficult than most other enhanced oil recovery methods.

Considerable improvements in the application of in-situ combustion have been made since the early projects. New developments, such as application to light oil reservoirs, injection of gases at high oxygen concentrations, and the use of horizontal wells are reviving interest in ISC. This process deserves consideration for many reservoirs including those in hostile environments or not amenable to other recovery methods. 


\section{References}

Burger J., Sourrieau P. and Combarnous M. 1984. " Thermal Recovery Methods" Editions TECHNIP Paris France.

Castanier, L.M. and Brigham W.E 1996. "Modifying in-situ combustion with metallic additives" In Situ.

Castanier, L.M., C.J. Baena, R.J. Holt, C. Tavares, and W.E. Brigham 1992. "Insitu Combustion with Metallic Additives," SPE 23708, Proceedings 2nd Latin American Petroleum Conference, Caracas, Venezuela, March 8-12, 1992; also SPE 67th Annual Technical Conference and Exhibition, Washington, D.C.

Castanier L.M. and W.E. Brigham 2003A. "Upgrading of crude oil via in-situ combustion by in situ combustion" Journal of Petroleum Science and Technology 39 125-136.

Castanier L.M. and W.E. Brigham 2003B. "In Situ Combustion" Chapter 17, Reservoir Engineering Section. Society of Petroleum Engineers Handbook. Society of Petroleum Engineers, Richardson TX, to appear.

Kovscek, A. R. and L. M. Castanier 2001. SUPRI TR 127: "Heavy and Thermal Oil Recovery Mechanisms Annual Report for 2000-2001.

Kovscek, A. R. and L. M. Castanier 2002. SUPRI TR 130: "Heavy and Thermal Oil Recovery Mechanisms Annual Report for 2001-2002.

Prats M. 1982, "Thermal Recovery" Monograph 7. Society of Petroleum Engineers, Richardson, Texas. 


\section{Area 5. Reservoir Definition}

The central idea of reservoir definition is to apply reservoir engineering techniques to improve our ability to understand reservoir rock and fluid properties and reservoir architecture, and the flow paths and flow barriers that arise from this architecture. Our interest in this topic is to learn the distribution of permeability and how geology and flow patterns determine the success of a recovery process.

History matching using reservoir simulators plays an important role in reservoir engineering. It is important for prediction and data interpretation. In many cases we have tracer or water breakthrough information at producers and pressure information. These data contain much information about the permeability distribution of the reservoir, but it is difficult to infer this distribution. Most approaches to this inverse problem begin with a conventional reservoir simulator and manipulate parameters at the grid-block level corresponding to conventional simulation grids. There are many grid blocks, thus the optimization is slow. Also, conventional finite difference reservoir simulators for flow simulation are relatively slow. Streamline based reservoir simulators have been developed recently that execute much more rapidly, relative to conventional finitedifference based simulation, and are quite accurate in that numerical dispersion is minimized. In streamline simulation, the three-dimensional flow simulation is broken down into a number of one-dimensional flow paths. We have applied the concepts of streamlines to infer permeability fields based on tracer breakthrough, water cut, and injection/production pressure information.

The basic idea is to switch the optimization to the streamline level rather than optimizing parameters associated with individual grid blocks. For instance, the effective permeability along a streamline can be adjusted to match reference data rather than the permeability of each grid block. In this way, the production characteristics of wells are decoupled and broken into a series of streamlines. With streamlines, we compute the time of flight of a fluid volume along a streamline, and so we also know the breakthrough time for each individual streamline. The streamline or series of streamlines that under or over predict reservoir performance are thus easily identified. The streamline properties, for instance permeability, are adjusted to match the reference production curve and pressure histories. Once the modifications of the streamline properties are computed, these modifications can be converted to changes in the properties of grid blocks through which the streamline passes. In this way, we dramatically reduce the size of the optimization problem. 


\section{A Streamline Based Technique for History Matching Production Data}

\section{(Task 5)}

\section{Introduction}

History-matching plays an important role in monitoring the progress of displacement processes and predicting future reservoir performance. Historical production data is routinely collected and it carries much information, although convoluted, that is useful for reservoir characterization and description of reservoir heterogeneity (Vasco and Datta-Gupta, 1997; Grinestaff, 1999). In this section, the concept of streamlines is applied to develop an automatic method for inferring the permeability distribution of a reservoir based on the history of pressure, flow rate, and water cut at producers.

The properties of streamlines are used in deriving the inverse method, and so a brief review of streamline methodology follows. Streamline and streamtube techniques are approximate reservoir simulation methods proposed some years ago (Higgins and Leighton, 1962, Higgins et al., 1964, Martin and Wegner, 1979, Fay and Prats, 1951) that have undergone recent intense study (Hewett and Behrens, 1991, Thiele et al., 1996; Peddibhotla 1997). They are most accurate when heterogeneity determines flow paths and the recovery process is dominated by displacement (viscous forces) as opposed to gravity or capillarity (Blunt et al., 1996). A streamline is tangent everywhere to the instantaneous fluid velocity field and, for a symmetric permeability tensor, streamlines are perpendicular to isobars or iso-potential lines. Streamlines bound streamtubes that carry fixed volumetric flux, and in some cases, flow rate is assigned to streamlines (Thiele, 1996; Blunt et al., 1996). For this reason, we use the terms streamline and streamtube interchangeably.

The streamline method assumes that displacement along any streamline follows a onedimensional solution, and there is no communication among streamlines. Thus, the flow problem is decomposed into a set of one-dimensional flow simulations linked by common boundary conditions. A streamline must start and end at a source to maintain continuity. In a streamlinebased approach, pressure equations are solved independent of saturation equations. The decoupling of pressure equations from saturation equations speeds up significantly the simulation by reducing the number of times that the pressure field must be updated and greatly reduces the number of equations to solve.

For unit mobility ratio and constant boundary conditions, the streamline distribution remains unchanged throughout the displacement process. Therefore, the pressure field or streamline distribution only needs to be solved once and saturation solutions can be mapped along streamlines. For non-unit mobility ratio, there are two common approaches to treat streamlines. One is to fix the streamline geometry and allow the flow rate to change during the displacement process (Hewett and Yamada, 1997, Portella and Hewett, 1997). The other is to update the streamline distribution and distribute the flow rate equally among the streamtubes (Thiele et al., 1995A, 1995B). In the second case, both the pressure field and streamline geometry must be updated periodically. 
For complete descriptions of the various streamline formulations and inclusive reviews of the history of streamlines for predicting reservoir flow, please refer to references (Hewett and Behrens, 1991, Blunt et al., 1996, Batycky et al., 1997; King and Datta-Gupta, 1998).

In this study, the streamline simulator 3DSL (Batycky et al., 1997) is employed for forward simulation; however, the method is not limited to this simulator nor is it restricted to a streamline simulator. In short, after solving the pressure field and the streamline distribution, 3DSL assigns equal flow rate to each streamline. Then a one-dimensional saturation solution, either analytical or numerical, is solved along the streamlines. Periodically, the streamline saturation distribution is mapped onto the multidimensional grid, the pressure equation is resolved, and streamline geometry redetermined. There is no detectable reduction in accuracy with this technique for incompressible and viscous determined flows (Wang et al., 1999).

\section{Previous Work}

Most approaches to history-matching field data manipulate permeability at the grid-block level, and hence, demand a great amount of computational work because there are many gridblocks in a typical simulation. Integration of production data with reservoir description remains an important issue because of the prevalence of production data and the information that it carries about the reservoir. In this brief review, we focus on work that is most similar to our method to follow. Other approaches to history matching are based upon simulated annealing (Gupta et al., 1999), sensitivity coefficients (Wen et al., 1998; Vasco et al., 1998; Chu et al., 1995), and parameter estimation approaches (Landa and Horne, 1997).

Sensitivity coefficient techniques compute the sensitivity of the objective function to the change of permeability of a cell or a set of cells and solve an inverse system that can be very large and somewhat difficult to construct (Chu et al., 1995; Landa and Horne, 1997). Sensitivity coefficient methods might also be computationally expensive if the sensitivity coefficients are evaluated numerically by running multiple simulations. Chu et al. (1995) developed a generalized pulse spectrum technique to estimate efficiently the sensitivity of wellbore pressure to gridblock permeability and porosity. Other work employed sensitivity coefficients in the integration of well test information, production history, and time-lapse seismic data (Landa and Horne, 1997).

Vasco et al. (1998) combined streamlines and a sensitivity coefficient approach while integrating dynamic production data. They employ streamlines to estimate sensitivity coefficients analytically thereby greatly speeding up the procedure. The streamline analysis allows them to "line up" the first arrival of injected fluid at production wells and then match the production history. This technique remains a grid-block-level optimization approach as all of the cells from the flow simulation are used to describe reservoir heterogeneity.

Simulated annealing, as applied by Gupta et al. (1994), perturbs permeability in a set of grid-blocks and evaluates energy objective functions or the degree of misfit between simulated and desired results. This process is stochastic and it is not guaranteed that a perturbation will decrease the energy level. The decision of whether or not to accept the perturbation is based on the change of energy caused by this perturbation. Perturbations that increase the degree of 
mismatch are accepted with a frequency that decreases with increase in the error. Many iterations are usually required to obtain an acceptable solution. In general, the computational costs of incorporating production data using simulated annealing become very large if a reservoir simulation must be conducted for each iteration.

Reservoir characterization approaches such as geostatistics do not explicitly account for field production data. It is a major task to determine the geostatistical realizations that are consistent with injection and production data. Generally, this involves conducting flow simulations for many different geostatistical simulation realizations. Efficient conditioning of permeability fields to both a geostatistical model and production data is discussed by Wu et al. (1998). In other work, Wen et al. (1998A, 1998B) also present a geostatistical approach to the inverse problem of integrating well production data. They adapt the sequential self-calibration (SSC) inverse technique to single-phase, multi-well, transient pressure and production rate data. The SSC method is an iterative, geostatistically-based inverse method coupled with an optimization procedure that generates a series of coarse grid two-dimensional permeability realizations. In later work, they combine SSC with analytical computation of sensitivity coefficients using streamline distributions (Wen et al., 1998). In both instances, this approach is applied for single-phase flow.

The remainder of this section presents the streamline-based history-matching approach where streamline effective permeability is manipulated rather than grid-block level data. An algorithm for mapping inverse streamline information onto the grid is also discussed. A synthetic case is used to explore the proposed approach. Then, two substantially different techniques are outlined that allowed the history-matching method to be constrained to prior geological information. These two constraining techniques also employ multiple production wells in the verification and demonstration cases. Discussion and conclusions complete the section.

\section{Method}

We propose a two-step method to match dynamic production data and infer reservoir heterogeneity. The first step is to modify the permeability distribution at the streamline level based on the difference between simulation results and field data for water cut, pressure drop, and flow rate. By matching the fractional-flow curve through manipulation of the permeability field, we try to capture reservoir heterogeneity. The second step is to map the streamline permeability modification onto the grid-blocks. Then flow simulation is performed to check the match. The above process is iterated until convergence.

A number of assumptions or simplifications were made in order to begin. Most originated from the streamline method used for forward flow displacement calculations. Permeability is assumed to be isotropic and the fluids are incompressible. Gravity and capillary forces are taken as negligible. Throughout we consider relatively simple well development. That is, no changes in well configuration, no infill wells, no conversion of producers to injectors, or vice versa. Also, we assume that other parameters such as porosity, relative permeability, and boundary conditions are known accurately. 
Throughout, we use the terms water-cut and fractional flow interchangeably. Because of the equal flow rate property of streamlines in forward flow simulation with 3DSL, every streamline breakthrough contributes the same amount to the fractional flow. Therefore, by ordering the streamlines with respect to their breakthrough time, we discretize the fractional-flow curve and relate various segments to the breakthrough of individual streamlines. In this sense, our proposed method is similar to the work of Vasco et al. (1998); however, we do not compute sensitivity coefficients nor is our formulation of the inverse problem similar. When the fractionalflow curve of the forward simulation result for a given permeability does not match the field water-cut curve, we infer the streamlines responsible for the difference between the fractional flow curves. Then based on the relation between streamline breakthrough time and effective permeability, a modification of effective permeability along streamlines is computed to match the production data. The objective function, as defined below, indicates the error of the simulation result compared to the field data:

$$
E=w_{t} E_{t, n}+w_{p} E_{p, n}+w_{q} E_{q, n}
$$

In Eq. $1, E_{t, n}, E_{p, n}$, and $E_{q, n}$ are errors in the dimensionless breakthrough time of individual streamlines, pressure, and flow rate at the producer, respectively. The terms $w_{t}, w_{p}, w_{q}$ are weights for dimensionless breakthrough time of individual streamlines, pressure, and flow rate at producer $i$, respectively. For simplicity, we weight each term identically.

We decouple the computation of streamline permeability modification to match flow rate and/or pressure from that to match fractional flow. To capture heterogeneity, we need to solve an inverse system to match the fractional flow curve as discussed next.

\section{Inverse System}

The degree of mismatch between reference and history-matched results is computed as

$$
E_{t}=\frac{1}{N_{s I}} \sum_{i=1}^{N_{s}}\left|E_{t_{B T}, i}\right|
$$

where $N_{s l}$ is the number of streamlines connected to the producer. The error $E_{t_{B T}, i}$ refers to breakthrough time of streamline $i$ as defined below

$$
E_{t_{B T, i}}=t_{D, B T, i}^{C}-t_{D, B T, i}^{R}
$$

where $t_{D, B T, i}^{C}$ and $t_{D, B T, i}^{R}$ are the computed, C, and reference, $\mathrm{R}$, dimensionless breakthrough times (pore volume injected) for the $i^{\text {th }}$ streamline, respectively.

Permeability modification of the $i^{\text {th }}$ streamline alters the breakthrough time of not only the $i^{\text {th }}$ streamline, but possibly the other streamlines. Therefore, all the streamlines must be considered, at least initially, and a system of equations has to be solved. That system of equations after assumption of unit mobility ratio and substantial mathematical manipulation is (Wang and Kovscek, 2000). 


$$
\left.\left[\begin{array}{ccccc}
b_{11} & b_{12} & b_{13} & \cdots & b_{1 N} \\
b_{21} & b_{22} & b_{23} & \cdots & b_{2 N} \\
b_{31} & b_{32} & b_{33} & \cdots & b_{3 N} \\
\cdots & & & & \\
b_{N 1} & b_{N 2} & b_{N 3} & \cdots & b_{N N}
\end{array}\right\rfloor \mid \begin{array}{c}
\delta k_{1} \\
\delta k_{2} \\
\delta k_{3} \\
\cdots \\
\delta k_{N}
\end{array}\right\rfloor=-\left[\begin{array}{c}
e_{t, B T, 1} \\
e_{t, B T, 2} \\
e_{t, B T, 3} \\
\cdots \\
e_{t, B T, N}
\end{array}\right\rfloor
$$

where $e_{t, B T, i}$ is normalized error in breakthrough time of streamline i to be defined in Eq. $5, \delta k_{j}$ is the normalized modification of effective permeability along streamline $\mathrm{j}$, and $b_{i j}$ is the derivative of $e_{t_{B T}, i}$ with respect to $\delta k_{j}$. For the purpose of discussing updates from one iteration to the next, let $k_{j}^{\lambda+1}$ be the new value of $\mathrm{k}_{\mathrm{j}}$, and likewise, $k_{j}^{\lambda}$ be the previous value. In equation form, the normalized variables are

$$
\begin{gathered}
e_{t_{B T}, i}=E_{t_{B T}, i} / t_{D, B T, i}^{R}=\left(t_{D, B T, i}^{C}-t_{D, B T, i}^{R}\right) / t_{D, B T, i}^{R} \\
\delta k_{j}=\frac{k_{j}^{\lambda+1}-k_{j}^{\lambda}}{k_{j}^{\lambda}}=\frac{\Delta k_{j}}{k_{j}^{\lambda}} \\
b_{i j}=\frac{\partial e_{D, B T, i}}{\partial\left(\delta k_{j}\right)}=\frac{k_{j}^{\lambda}}{t_{D, B T, i}^{R}} \frac{\partial t_{D, B T, i}}{\partial k_{j}^{\lambda+1}}
\end{gathered}
$$

And after further manipulation, Eq. 7 is written

$$
b_{i j}=\left\{\begin{array}{l}
\frac{k_{i}}{\sum_{k=1}^{N_{S l}} c_{i k} V_{D, k} k_{k} / k_{i}}\left(-\frac{1}{k_{i}^{2}} \sum_{k=1, k \neq i}^{N_{S}} q_{k} V_{D, k} k_{k}\right) \approx-1, \quad \text { if } i=j \\
\frac{k_{i}}{\sum_{k=1}^{N_{S}} q_{k} V_{D, k} k_{k} / k_{i}}\left(\frac{q_{i j} V_{D, j}}{k_{i}}\right)=\frac{c_{i j} V_{D, j} k_{i}}{\sum_{k=1}^{N_{S l}} q_{k} V_{D, k} k_{k}} \approx \frac{1}{N_{s l}}, \text { if } i \neq j
\end{array}\right.
$$

With the normalization above, the elements of the matrix are now functions of only matrix size $\mathrm{N}_{\mathrm{sl}}$ for a given set of streamlines. Therefore, the inverse of the matrix is also solely a function of $\mathrm{N}_{\mathrm{sl}}$. As is shown in Eq. 8, when the number of streamlines is large, the off-diagonal terms of the matrix are close to 0 . Therefore, a unit matrix is a good approximation of this nondimensionalized matrix [B], and therefore inversion of this matrix is also unit. This demonstrates that decoupling of the streamlines is possible. Decoupling of streamlines means that the contribution of a streamline to the error of the production data is mainly related to the average permeability of the streamline. Thus, all of the elements in the inverse of the matrix are calculated directly. This observation is verified by practice as discussed later. 
The above simplification works well for unit mobility ratio. In non-unit mobility ratio cases, we do not get a matrix as simple as $[\mathrm{B}]$ in Eq. 4. An alternative for non-unit mobility ratio cases is to solve the full system of equations leading to [B] in Eq. 4. However, the streamlines evolve during the displacement process. To obtain a good history match with such a procedure, we may need to select several different streamline distributions over the time period of interest. Each of the distributions could be used to match a segment of the fractional flow curve. This is computationally intensive and so we have not implemented this alternative yet. Our prior case studies (Wang and Kovscek, 2000) indicate that it is appropriate to apply the simplification made for unit mobility ratio to non-unit mobility ratio cases and treat streamlines as non-evolving during inverse calculation.

\section{Permeability Updates}

For the forward simulation, we solve the pressure field and streamline distribution as many times as required to obtain convergence, but in the inverse process, we choose one of the streamline distributions to compute the streamline properties and update permeability along this streamline distribution. In the inverse process, parameters are non-dimensionalized, therefore, the modification of permeability is performed in a relative manner. That is, we compute a factor that is used to multiply the original permeability value.

Mapping the permeability modifications onto grid blocks is performed in the following way. When the average permeability of a streamline must be multiplied by a factor $f$ to match its breakthrough time, then the permeability of all the grid-blocks along this streamline is multiplied by $f$. This gives us the proper average permeability of the streamline. When more than one streamline passes through the same grid-block, then for this block, the factor for multiplication is the geometric average of all of the factors for the streamlines passing through this block. This leads to inexact modification of the permeability field and the need for iteration. For those gridblocks where no streamlines pass through them, we do not modify their permeability. However, when the number of streamlines is large, these grid-blocks are few.

\section{Steps of the Approach}

This approach can be implemented in a relatively simple procedure that involves no modification to the forward simulator. This is especially helpful when source code is not available. The steps include:

1. Obtain an initial permeability field by guess or geostatistical realization.

2. Run a streamline simulation on the initial permeability field with the appropriate number of re-computations of the pressure field to obtain a converged forward solution. Check whether the simulation results match the field data (reference data) including fractional-flow curve at 
the producers, flow rate, and pressure. If not, modify the permeability according to the following steps;

3. Work on the streamlines. Calculate the time of flight (or the associated pore volume) for all streamlines. Sort the streamlines in ascending order of pore volume

4. Compute the difference in fractional flow, flow rate, and pressure between the simulation result and the reference. Relate differences in the fractional-flow curve to the corresponding streamline;

5. Solve the system described above (Eq. 4) to compute the modification of the effective permeability of each streamline required for a good match to available data;

6. Map the modification at the streamline level onto grid-blocks honoring the effective permeability of each streamline;

7. Iterate steps 2 to 6 until a satisfactory match is achieved.

\section{Results and Discussion}

Several cases have been tested with this approach and the results show that this method is robust with the stated assumptions and converges quickly (Wang and Kovscek, 2000). A summary case is presented below. First, a reference permeability field is generated, and then 3DSL run to obtain the reference production data. Water cut and pressure drop are the data from the reference case that we try to match. An initial permeability field is guessed to start the procedure. The permeability field is generated by a geostatistical sequential Gaussian simulation method (Deutsch and Journel, 1998) where the field is conditioned to sparsely distributed data. For simplicity, this case is a quarter of a five-spot pattern. Our formulation is not restricted to this pattern, nor is the method restricted to two-dimensional areal cases. The injector is located at the lower left corner, and the producer at the upper right corner of the pattern. The pressure at the producer is constant and the fluid is incompressible.

In this case, the injection rate undergoes several step changes during the course of the displacement process. The fluids are again incompressible. The endpoint mobility ratio is 2.5 , requiring multiple pressure solves in the forward solution. The relative permeability functions are described by

$$
\begin{aligned}
& k_{r w}=k_{r w}^{0}\left(\frac{S_{w}-S_{w c}}{1-S_{o r}-S_{w c}}\right)^{2} \\
& k_{r o}=k_{r o}^{0}\left(\frac{1-S_{o r}-S_{w}}{1-S_{o r}-S_{w c}}\right)^{2}
\end{aligned}
$$


The viscosities for water and oil are $1 c p$ and $10 c p$, respectively. Two permeability fields are generated by sequential Gaussian simulation (Deutsch and Journel, 1998). One is used as a reference and the other as an initial permeability field as shown in Fig. 1a and b. In the inversion, the streamline distribution from the first pressure solve is chosen to compute the properties of streamlines and the permeability modifications.

With the procedure given above and fixing the injection rate schedule to match the history of the reference case, a very good match to the fractional flow curve and the pressure at the injector is obtained as shown in Figs. 2 and 3, respectively. The error during iteration is given in Fig. 4. Only three iterations are needed to converge despite the relatively complicated injection conditions. The inferred permeability field is shown in Fig. 1c. Visually the final match appears to be closer to the reference permeability field of Fig. 1a than is the initial permeability in Fig. 1b 


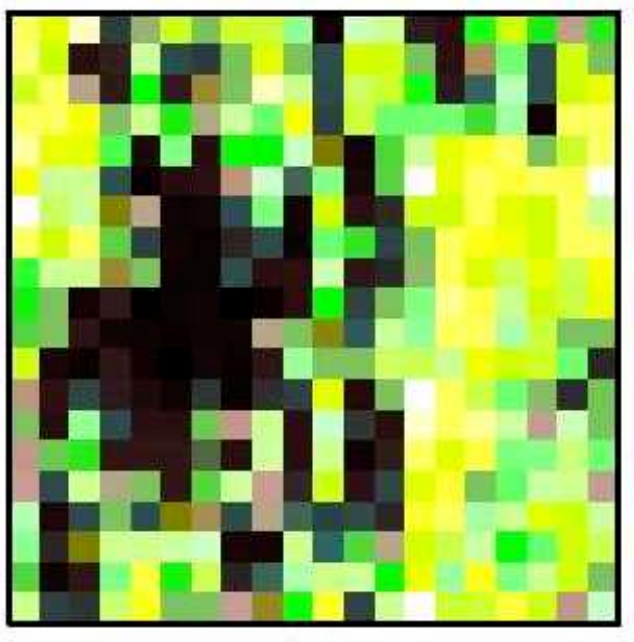

(a)
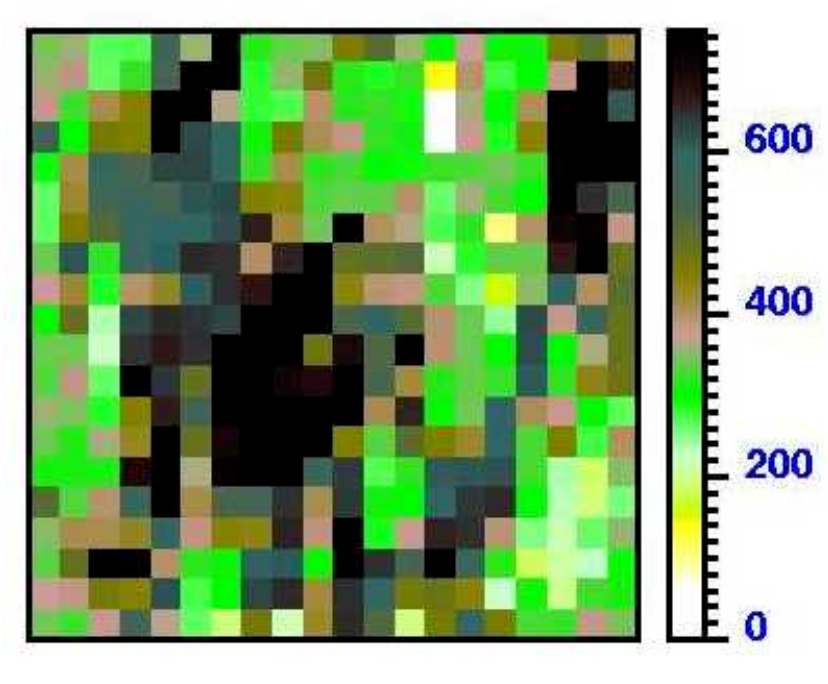

(c)
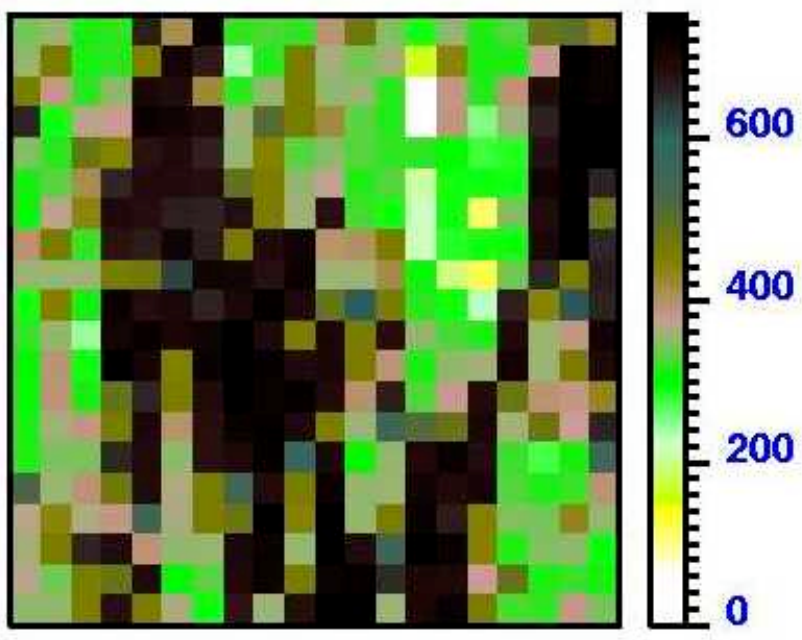

(b)

Figure 1. Comparison of permeability fields (a) reference, (b) initial, and (c) history-matched. Shading indicates permeability in md. 


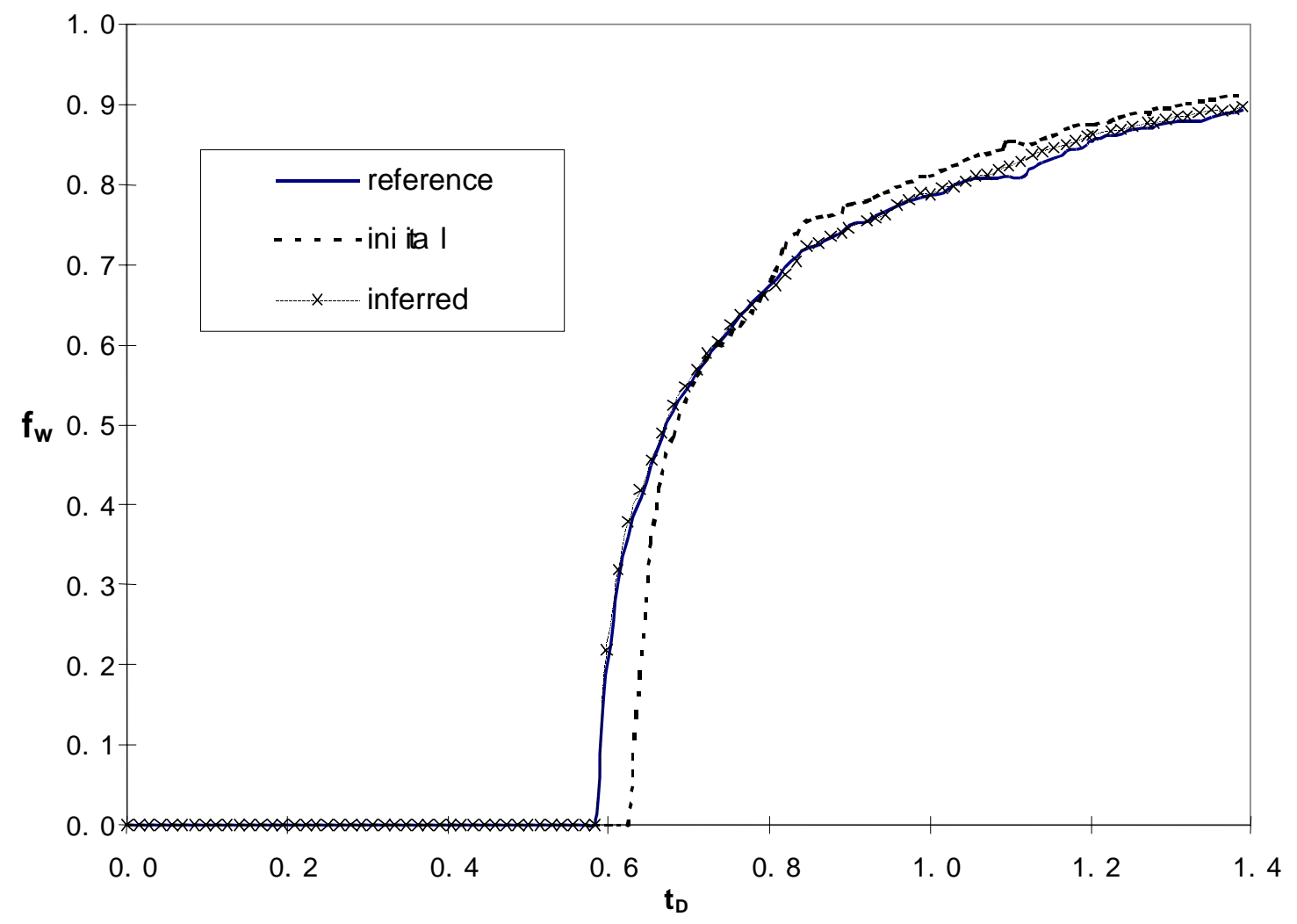

Figure 2. Fractional flow curves for test case.

The example applications show that this approach is robust within the stated limitations and converges quickly. Each iteration significantly reduces the errors in fractional flow, pressure drop, and flow rate. In all of the examples, an acceptable match to the production data is obtained within a small number of iterations. One strength of this approach is that the input of the inverse problem is simply the usual output of the streamline simulation. Namely, streamline coordinates, production data including fractional flow, flow rate, and pressure. Therefore, this approach stands alone. Importantly, it appears to be expandable to integrate geostatistical data or to honor geological and seismic data. We are actively pursuing this task and discuss this topic below.

Another strength is that repeated inversion of a matrix is not needed for the cases studied. The permeability modification for every iteration is computed directly based on differences between the streamline breakthrough time from field observation and the simulation result. Therefore, this process takes very little computational time. All of the examples illustrated here used the simplified inverse approach derived for unit mobility ratio cases. It appears that this idea works well for flow with unfavorable mobility ratios in heterogeneous reservoirs. Further study is required. 


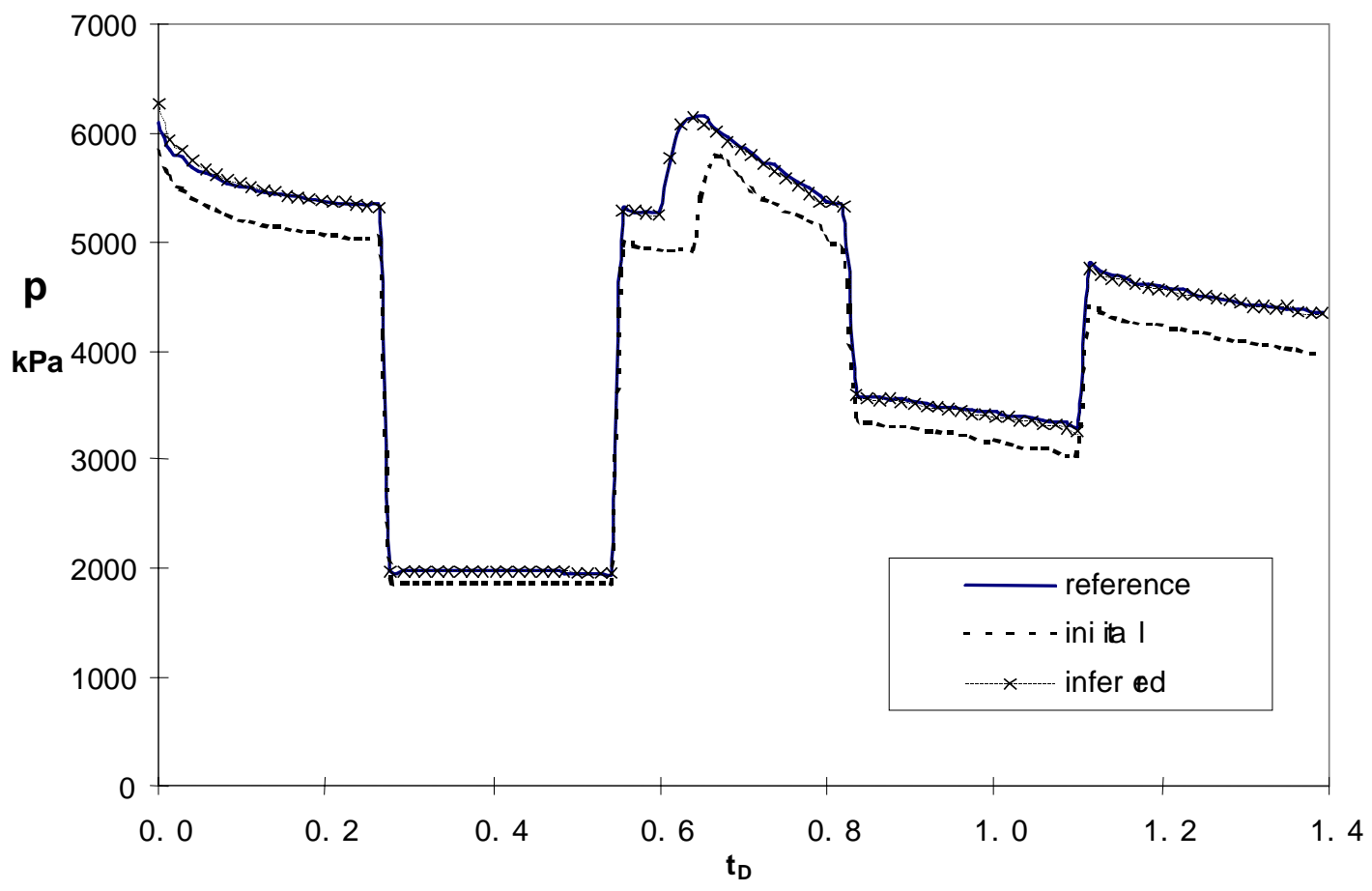

Figure 3. Pressure history for test case.

The computation of the error in water-cut for both unit and non-unit mobility ratio cases is performed using fractional-flow curves from the simulation and the reference results. The pore volume associated with each streamline is calculated for the purpose of sorting streamlines. The accuracy of computing streamline pore volume does not affect inversion results as long as the correct ordering of the breakthrough time is obtained. Therefore, for non-constant, non-unit mobility ratio cases, the method works well as long as the order of streamline breakthrough time is preserved, even though the streamlines evolve.

Streamline permeability modifications are mapped directly to grid-blocks by propagating the indicated change through all of the grid-blocks that a particular streamline passes. For example, when the effective permeability of a streamline needs to be increased by 5 percent, then the permeability of all the grid-blocks along that streamline are increased by 5\%. Mapping is not unique and different mapping schemes can produce the same effective streamline permeability. Necessarily, however, the water-cut curve is matched and the inferred field can be used to predict results of other mobility ratios. In the mapping process, it is possible to enforce constraints to honor observed geological information, if any.

Inverse solutions can be constrained in many different ways. Three approaches, as described below, appear to be consistent with this approach. 
A simple, but probably inefficient, method is to generate a number of permeability fields by sequential Gaussian simulation based on available geological information, and to check whether the effective permeability distribution from a particular realization matches that from the inversion. The check can be performed in the following way. Solve the pressure field and the streamline distribution once for permeability field from geostatistical simulation. A unit mobility ratio should suffice. Sort the streamlines in the order of breakthrough time as we did for the inverse process. Compute the effective permeability and time of flight of each streamline, compare it with the effective permeability and time of flight from the inversion. In this manner, realizations are sorted. Those realizations within an acceptable value of the error in permeability are expected to generate a good match in production response, and therefore are accepted. It is easy to generate geostatistical realizations and solve the pressure field once to obtain streamline distribution. This approach requires much less computational work than running a full flow simulation on each realization.

Another way to constrain the inversion is to obtain a map of the expected pattern of highs and lows in the permeability field based on geological or seismic information The high and low values would only be relative. When mapping the permeability modification from streamlines to grid-blocks, the decision of whether to modify the permeability of a grid-block could be based on whether the permeability is to be increased or decreased, and whether this change agrees with the geological information. Thus, permeability could be mapped to honor prior information about the field.

A third approach is a combination of this work with the master-point idea (Wen et al., 1998): We could compute the modification of the average permeability, and convert this modification to a number of pre-selected master points (i.e, grid-blocks). Kriging is performed to propagate the modification at the master points to other grid-blocks. When kriging, we incorporate known geological information, such as direction of continuity and correlation length, and all the grid-blocks are reached for modification.

Examples shown here are for two-dimensional flow problems. We continue to work on the generalization to three-dimensional problems with gravity. Additionally, this approach was originally designed for incompressible two-phase flow or tracer flow studies. That is, the fractional flow curve or tracer breakthrough curve at the producers is required to infer the heterogeneity of the reservoir. More study is needed to extend this procedure to compressible single-phase flow scenarios such as well test problems. Also,_streamline simulation encounters difficulties when infill wells are drilled, injectors are converted to producers, or vice versa, well completions are changed, or radical changes are made in pattern balancing. In forward flow simulation, such actions require that_streamlines be recomputed. In history matching, multiple realizations of streamline geometry would have to be honored. This is an important topic, but we must address it later.

Results, indicate that the inverse solutions are very sensitive to relative permeability. It is necessary to obtain accurate relative permeability curves in order to retrieve a good inverse solution. This is true of most history-matching approaches. As long as we use the same relative permeability curves in both the forward simulation and inverse process, the inferred permeability field will give us a good history match. 
Actual production data is never as smooth as that in our synthetic examples. In general, for continuous injection of one phase to displace another phase, the fractional flow of the injected phase at the producers is monotonically increasing. However, noise may cause it, apparently, not to be so. In such cases, we need to preprocess the data. The first step would be to use dimensionless time (pore volume) rather than real time. To reduce the effect of noise, a filter to remove small time scale variations (high frequency) is needed, while maintaining the variations in large time scale. Normally, noise is random and therefore does not have a large time scale trend. In general, it is the bulk reservoir properties that are important for predicting reservoir performance, and they are represented in large time scale variations. Therefore, removing small time scale variation, even if it is not caused by noise, has small effect for prediction.

\section{Conclusions}

This approach relates producer water-cut curves to the breakthrough time of individual streamlines. The effective permeability along streamlines is modified directly to history-match the fractional flow curve, pressure drop, and flow rate information. No matrix inversion is involved in the inverse process and therefore it is quite fast. The forward flow simulation with 3DSL is also fast and so, for the examples examined, the entire process appears to be computationally efficient.

The current work examined 2-D areal porous media, where the effect of gravity is not important, as well as incompressible two-phase flow. As with most history-matching approaches, high-quality noise-free data is needed for fast and accurate inversion. This approach works well for reservoirs where heterogeneity is a dominant factor. It also works well for unfavorable mobility ratios because the effects of heterogeneity are exacerbated by the unstable displacement. Although streamlines evolve for non-unit mobility ratio cases during the displacement process, we find it feasible to choose one streamline distribution and apply the simplified inverse system during the inversion.

Importantly, a permeability field inferred by matching production data for one mobility ratio can be used to predict reservoir performance for displacement processes at other mobility ratios.

\section{Nomenclature}

A cross-sectional area of a streamtube, $\mathrm{L}^{2}$

c constant

E Absolute error

$e \quad$ Relative error

$f_{w} \quad$ fractional flow of water

$k \quad$ permeability, $\mathrm{L}^{2}$

$k_{r w} \quad$ relative permeability of water

$k_{r o} \quad$ relative permeability of oil

$l \quad$ streamline length 


$$
\begin{array}{ll}
M & \text { mobility ratio } \\
p & \text { pressure, } \mathrm{M} /\left(\mathrm{Lt}^{2}\right) \\
q & \text { flow rate, } \mathrm{L}^{3} / \mathrm{t} \\
t_{D} & \text { dimensionless time } \\
V & \text { pore volume, } \mathrm{L}^{3} \\
V_{P} & \text { pore volume } \\
V_{D} & \text { ratio of pore volume } \\
x_{D} & \text { dimensionless length } \\
& \\
\phi & \text { porosity }
\end{array}
$$

\section{Subscripts:}

$\mathrm{n} \quad$ producer index

$\mathrm{i}, \mathrm{j}, \mathrm{k}$ streamline index

sl streamline

BT breakthrough

D dimensionless

\section{Superscripts:}

C Computed

$\mathrm{R} \quad$ Reference

$\lambda \quad$ iteration index

\section{References}

Batycky, R. P., Blunt, M. J., and Thiele, M. R.: “A 3D Field-Scale Streamline-Based Reservoir Simulator," SPERE (November 1997) 246-254.

Blunt, M.J., Liu, K., and Thiele, M.R.:"A Generalized Streamline Method to Predict Reservoir Flow," Petroleum Geoscience (1996) 2, 259-269.

Brigham, W.E. and Abbaszadeh-Dehghani, M., "Tracer Testing for Reservoir Description," JPT (May 1987) 519-527.

Chu, L., Reynolds, A. C. and Oliver, D. S.: "Computation of Sensitivity Coefficients for Conditioning the Permeability Field to Well-Test Pressure Data," In Situ , (1995) 19(2), 179-223.

Deutsch, C. V. and Journel, A. G., GSLIB, Geostatistical Software Library and User's Guide, Second Edition, Oxford University Press, New York (1998).

Dykstra, H. and Parsons, R.L., "The Prediction of Oil Recovery by Waterflood," Secondary Recovery of Oil in the United States, Principles and Practice, 2d ed., American Petroleum Institute (1950), 160-174. 
Fay, C. H. and Prats, M.. "The Application of Numerical Methods to Cycling and Flooding Problems" Proceedings of the 3rd World Petroleum Congress (1951).

Grinestaff, G.H.: "Waterflood Pattern Allocations: Quantifying the Injector to Producer Relationship with Streamline Simulation," paper SPE 54616 presented at the 1999 Western Regional Meeting, Anchorage, Alaska, 26-28 May.

Gupta, A. D., Vasco, D. W. and Long J.C.S.: "Detailed Characterization of Fractured Limestone Formation Using Stochastic Inverse Approaches," SPE Ninth Symposium (1994).

Hewett, T.A. and Behrens, R.A.: "Scaling Laws in reservoir Simulation and Their Use in a Hybrid Finite Difference/Streamtube Approach to Simulation the Effects of Permeability Heterogeneity," in Reservoir Characterization, II, L. Lake and H.B.J. Carroll (eds.), Academic Press Inc., London (1991) 402-441.

Hewett, T. A., and Yamada, T.: "Theory of the Semi-Analytical Calculation of Oil Recovery and Effective Permeabilities Using Streamlines," Advances in Water Resources (1997) 20(56), 279-295.

Higgins, R.V., Boley, D.W. and Leighton, A.J.: "Aids to Forecasting the Performance of Water Floods," JPT (September 1964) 1076-1082.

Higgins, R.V. and Leighton, A.J.: "A Computer Method to Calculate Two-Phase Flow in Any Irregularly Bounded Porous Medium," JPT (June 1962) 679-683.

King, M.J., and Datta-Gupta, A.: "Streamline Simulation: A Current Perspective," In Situ, 22(1), (1998) 91-140.

Lake. L. W., Enhanced Oil Recovery, Prentice Hall, Englewood Cliffs, NJ (1989).

Landa, J. L. and Horne, R. N.: "A Procedure to Integrate Well Test Data, Reservoir Performance History and 4-D Seismic Information," paper SPE 38653 presented at 1997 Annual Technical Conference and Exhibition in San Antonio, Texas, 5-8 October.

Lepine, O. J., Bissell, R. C., Aanonsen, S. I., Pallister, I. and W Barker, J.: "Uncertainty Analysis in Predictive Reservoir Simulation Using Gradient Information," SPE 48997, proceedings of the SPE Annual Meeting, New Orleans (September 1998).

Martin, J.C. and Wegner, R.E.: "Numerical Solution of Multiphase, Two-Dimensional Incompressible Flow Using Streamtube Relationships," SPEJ (October 1979) 313-323.

Peddibhotla, S., Datta-Gupta, A., and Xue, G.: "Multiphase Streamline Modeling in Three Dimensions: Further Generalizations and a Field Application," paper SPE 38003 presented at 1997 Reservoir Simulation Symposium in Dallas, Texas, 8-11 June. 
Portella, R. C. M. and Hewett, T. A.: "Fast 3-D Reservoir Simulation and Applications Using Streamlines," paper SPE 39061 presented at the Fifth Latin American and Caribbean Petroleum Engineering Conference and Exhibition in Rio de Janeiro, Brazil, 30 August-3 September 1997.

Rahon, D., Edoa, P. F. and Masmoudi, M.: "Identification of geological shapes in reservoir engineering by history matching production data," SPE 48969, proceedings of the SPE Annual Meeting, New Orleans (September 1998).

Thiele, M. R., Batycky, R. P., Blunt, M. J. and Orr Jr, F. M.Jr. "Simulating Flow in Heterogeneous Systems Using Streamtubes and Streamlines," SPERE (February 1996) 512.

Thiele, M.R., Blunt, M.J., and Orr, F.M. Jr.: "Modeling Flow in Heterogeneous Media Using Streamtubes--I. Miscible and Immiscible Displacements," In Situ (August 1995) 19(3), 299-339.

Thiele, M.R., Blunt, M.J., and Orr, F.M. Jr.: "Modeling Flow in Heterogeneous Media Using Streamtubes--II. Compositional Displacements," In Situ (1995) 19(4), 367-391.

Vasco, D.W. and Datta-Gupta, A.: "Integrating Multiphase Production History in Stochastic Reservoir Characterization," SPEFE (September 1997) 149-156.

Vasco, D.W., Yoon, S. and Datta-Gupta, A.: "Integrating Dynamic Data Into High- Resolution Reservoir Models Using Streamline-Based Analytic Sensitivity Coefficients," paper SPE 49002 presented at 1998 Annual Technical Conference and Exhibition in New Orleans, Louisiana, 27-30 September.

Wang, Y.D., Kovscek, A. R. and Brigham, W.E.: "Effect of Mobility Ratio on Pattern Behavior of a Homogeneous Porous Media", In-Situ, 23(1) (1999) 1-20.

Wen, X.H., Clayton, D.V. and Cullick A.S.: "High Resolution Reservoir Models Integrating Multiple-Well Production Data," SPEJ (December 1998) 344-355.

Wen, X.H., Clayton, D.V. and Cullick A.S.: "Integrating Pressure and Fractional Flow Data in Reservoir Modeling with Fast Streamline-Based Inverse Method," paper SPE 48971 presented at 1998 Annual Technical Conference and Exhibition in New Orlean, Louisiana, 27-30 September.

Wu, Z., Reynolds, A. C. and Oliver, D. S.: "Conditioning Geostatistical Models to Two-Phase Production Data," SPEJ (June 1998) 142-155. 


\section{History Matching Constrained to Geostatistical Data}

\section{(Task 5)}

\section{Introduction}

Geostatistical techniques are used primarily to generate realistic equally probable numerical reservoir models that honor known geological information. However, significant uncertainty exists in the detailed distribution of reservoir properties such as permeability (Deutsch and Srinivasan, 1996) because the known data is relatively sparse. If flow simulation is performed on various realizations to predict reservoir performance, geological reservoir model uncertainty is transferred into a relatively uncertain prediction of future production.

The reliability of geostatistical reservoir models as applied to flow simulation increases as dynamic data, such as production history, is incorporated (cf. Tran et al. 2001). However, integration of production history with geostatistical information is, to date, computationally time consuming. The problem is generally approached through inverse modeling where a constrained optimization problem is solved. That is, dynamic data, such as oil production rate, water cut, or pressure, are matched by adjusting parameters within a subspace of parameter values. Inversion is both CPU demanding and poorly determined because the number of parameters adjusted is large. For instance, a problem might be constructed where the permeability of every gridblock in a reservoir model is treated as an unknown.

The history-matched models (or inverse solutions) can be constrained to geology in many different ways. The following two approaches appear to be consistent with our streamline-based inverse method: (1) incorporating geostatistical constraint directly into our history-matching process; and (2) streamline time-of-flight ranking of reservoir models. First we focuse on the integration of a geostatistical technique called Gauss-Markov random functions with the streamline inverse method. The streamline approach is then summarized.

\section{Gauss-Markov Random Functions (GMrf)}

The GMrf technique is similar to sequential Gaussian Simulation (SGSIM) (Deutsch and Journel, 1998). GMrf uses a Markov chain Monte Carlo method to constrain realizations such that they honor a specific variogram and histogram (Caers, 2001). The main difference between $G M r f$ and SGSIM is that GMrf is iterative, while SGSIM is a non-iterative technique. SGSIM starts with an empty grid and then simulates that grid sequentially node by node along a random path. The procedure finishes when every node is simulated. The problems with SGSIM are that it is difficult to constrain the realizations to complex data types, and that only linear constraints are permitted. GMrf has the same goal as SGSIM, i.e., generating realizations with a specific variogram and histogram. The procedure operates node by node, changing the value at each node according to a probabilistic criterion. When the procedure iterates long enough, both the variogram and the histogram will be honored. The key is how to choose the probabilistic criterion, which is based on a new geostatistical idea that seems similar to the simulated 
annealing technique. The method is faster than simulated annealing, there is no objective function criterion, and we need not set any cooling schedule (Caers, 2001).

The fact that the grid is "full" while iterating, as opposed to SGSIM where the grid is filled gradually, allows us to condition the model to much richer data. Caers (2001) has extended this technique to include any type of nonlinear, multiple-point averaging constraints. In our applications, the constraint is the effective permeability of relevant streamlines. The inverse result honors the production history with effective permeabilities along streamlines, but not necessarily the variogram and/or histogram. So, the GMrf technique is used to post-process the inverse result at every iteration and turn it into a realization that honors the variogram/histogram, effective permeabilities, and the production data.

\section{Integration of GMrf into Streamline-Based History-Matching}

There are different ways to combine GMrf and our streamline inverse method such that the resulting models honor both the geology and production history. The following two ways are most appropriate. The first is to integrate GMrf and our inverse method in the true sense. In this approach, we solve the history-matching and GMrf problem simultaneously, i.e., performing the inverse process and GMrf as a whole in each iteration. The second approach is to perform the two processes sequentially. That is, in each iteration, we first perform the streamline-based inverse process, which may destroy the geological consistency slightly. Then, we run the GMrf process as a remedy to restore the correct geology into the history-matched models.

Both approaches have advantages and disadvantages. In the first approach, the two processes are tightly coupled. The modification of the model parameters in each iteration honors both geology and production history; therefore, its rate of convergence is expected to be higher than that of the second approach. However, due to the coupling, the implementation can be more complicated. In the second approach, the two processes run independently, with GMrf following the streamline inverse process. The two procedures are decoupled; therefore, they can be developed separately and are applied together to achieve our goal. Because we have developed the algorithm and computer programs for the two methods, we can readily combine the two existing parts to construct the new method. However, because the two procedures are not truly integrated, the negative effect of one process on the other cannot be eliminated by designing new algorithm and code. Consequently, the convergence rate may suffer.

We implemented the second approach. However, the two components still need minor modification to accommodate each other. For example, the streamline inverse method needs to be modified to output necessary information used in the GMrf process, including streamline coordinates, time-of-flight, and effective permeability. The GMrf code also needs to be modified so that the streamline effective permeability is honored.

Moreover, we try to minimize the negative effect of one component on the other. The streamline history-matching method may introduce streamline-related artifacts in the permeability field. If we modify the permeability field as required by the inverse process in each iteration, it may cause an excessive number of iterations within the GMrf process to restore the correct 
variogram and histogram. To reduce the GMrf cost, we introduce a dampening (or decelerating) factor $\eta<1$ in the calculation of the streamline effective permeability modification:

$$
k_{i}^{\lambda+1}=k_{i}^{\lambda}\left(1+\eta \delta_{i}^{\lambda+1}\right)
$$

where the superscripts and subscripts are the indices of iterations and streamlines, respectively; $k$ is the streamline effective permeability; and $\delta$ is the relative change of $k$.

On the other hand, if we choose a value of $\eta$ that is too small, we need more history matching iterations; consequently increasing the cost of the entire method. By our investigation, an optimal $\eta$ range is from 0.2 to 0.7 . We may use large values during initial iterations and small values in iterations near the end of the history-matching.

\section{Algorithm}

As stated above, we use the second approach to combine the GMrf technique with our streamline inverse process, i.e., performing history-matching and GMrf sequentially. The procedure is summarized as follows:

1. Choose an initial reservoir model. It is beneficial to have an initial model that honors the known or assumed geology so that less computation occurs in the GMrf process. We use $S G S I M$ to generate the initial reservoir model (expressed as a permeability distribution in our case study).

2. Perform the history-matching procedure as described in Chapter 3 to obtain: (1) the permeability value $k(u)$ for each grid-block/location $u$; (2) the geometry and time-of-flight of each streamline; and (3) the effective permeability of each streamline. After this inverse process, the match to the production data is improved, but the resulting model may violate the geology as expressed by the variogram and histogram in the permeability distribution.

3. Use the GMrf procedure (Caers, 2001) that takes the model obtained in Step 2 as its initial reservoir model and "superpose" the variogram on it using an iterative procedure as follows:

4. Transform the permeability values $k(u)$ to the standard normal space to obtain $y(u)$;

5. Traverse iteratively through the normal space grid. For each visited grid block $u_{i j k}$, propose a new value $y^{\text {new }}\left(u_{i j k}\right)$ drawn from the marginal distribution, transform it into the data space $k^{\text {new }}\left(u_{i j k}\right)$ and then accept it with the Metropolis sampling probability;

6. Continue iterations until convergence of GMrf.

7. Check the match to the historic production data. Iterate Steps 2 and 3 until a satisfactory match to production history is achieved. 
In the GMrf procedure (Step 3), the distribution of permeability values in the domain is made to honor the variogram and histogram. The effective permeability along each streamline is not changed. The effective permeability of a streamline is the time-of-flight weighted harmonic average of permeability values of all the grid-blocks the streamline passes. When proposing a modification, we use the geometry, time-of-flight and effective permeability of streamlines obtained in Step 2 so that the effective permeability of all streamlines are honored. By doing so, we assume the streamline geometry does not evolve radically as a result of this post processing.

To generate multiple geostatistical realizations, we perform the method with a different random seed each time. We can also perform the inverse process with a different initial geostatistical model from which our final inverse result will most likely have different streamline geometry. Using the multiple realizations that honor geology and production history, we quantify the degree of uncertainty in the prediction of future reservoir performance.

\section{Results and Discussion -- GMrf}

Four example applications are presented below. First, a simple five-spot pattern case with isotropic permeability distribution and unit mobility ratio demonstrates the working of this method. Next, a similar case shows this method works equally well for a nonunit mobility ratio case $(M=2.5)$. Then, a case with an anisotropic field was studied. In the last case, our method was used to match a short production history and then we examine the prediction of reservoir performance using the history-matched models. Throughout this section, we used the GSLIB program SGSIM (Deutsch \& Journel, 1998) to generate permeability models, and the streamline simulator 3DSL (Batycky et al., 1997) to perform forward flow simulation. In each example, our method worked effectively and generated reservoir models that honor both geology and historic production data.

\section{Cases 1: Five-spot, Isotropic Permeability Field, $M=1$}

We use this simple case to demonstrate the method. The case scenario is as follows: 100x100 grid-block isotropic permeability field, five-spot pattern with the injector in the center and four producers in the four corners. Water is injected at a constant rate and the pressure is fixed at the producers. First, we generate a reference permeability field; its distribution, histogram and variogram are shown in Fig. 1. Flow simulation is run on this permeability field to obtain the reference production history. Some parameters to run the flow simulation are given in Table 1. Unit mobility ratio is used. Another permeability field is generated and used as the initial model. In the inverse process, 178 streamlines are launched; an $\eta$ value of 0.3 is used for all iterations.

Nine iterations were performed to obtain an acceptable match to the production data. Figure 2 shows the gradual change of permeability distribution of iteration 1,2, 5 and 9 . The left column figures are prior to the GMrf constraining process, while the right are after the GMrf process, and therefore, consistent with geology. The permeability fields in the left column, though not visually obvious, do not satisfactorily describe the known geology. Figure 3 shows 
the final history-matched realization, its histogram and variogram. Figure 4 shows the match to the water-cut at all four producers. The match is acceptable.

Table 1 Summary of Flow Simulation Parameters for the Four Cases

\begin{tabular}{|r|c|r|c|r|r|r|r|r|c|c|r|}
\hline Pattern & Grid & $M$ & $\begin{array}{c}P V \\
(\mathrm{~m} 3)\end{array}$ & $\begin{array}{c}q_{i n j} \\
(\mathrm{~m} 3 / \mathrm{day})\end{array}$ & $S_{o r}$ & $S_{w c}$ & $k_{r w}^{0}$ & $k_{r o}^{0}$ & $\begin{array}{c}k_{r} \\
\text { eqns }\end{array}$ & $\begin{array}{c}\mu_{w} \\
(\mathrm{cp})\end{array}$ & $\begin{array}{c}\mu_{o} \\
(\mathrm{cp})\end{array}$ \\
\hline Case 1 & $100 \times 100$ & 1 & 100000 & 300 & 0 & 0 & 1.0 & 1.0 & & & 1 \\
\hline Case 2 & $100 \times 100$ & 2.5 & 100000 & 300 & 0.2 & 0.2 & 0.2 & 0.2 & Set 2 & 1 & 10 \\
\hline Case 3 & $200 \times 200$ & 1 & 100000 & 300 & 0 & 0 & 0 & 0 & Set 1 & 1 & 1 \\
\hline Case 4 & $100 \times 100$ & 5 & 100000 & 300 & 0.2 & 0.2 & 0.2 & 0.2 & Set 2 & 1 & 20 \\
\hline
\end{tabular}

Set 1: $k_{r w}=k_{r w}^{0}\left(s_{w}-s_{w c}\right), \quad k_{r o}=k_{r o}^{0}\left(s_{o}-s_{r o}\right)$

Set 2: $k_{r w}=k_{r w}^{0}\left(\frac{s_{w}-s_{w c}}{1-s_{o r}-s_{w c}}\right)^{2}, \quad k_{r o}=k_{r o}^{0}\left(\frac{1-s_{o r}-s_{w}}{1-s_{o r}-s_{w c}}\right)^{2}$

\section{Cases 2: Five-spot, Isotropic Permeability Field, $M=2.5$}

We also studied an unfavorable mobility ratio case in which water displaces oil. We use the same reference and initial permeability field and same well configuration as in the unit mobility ratio (tracer) case above, but change the fluid properties (see Table 1), resulting an endpoint mobility ratio of 2.5. During history-matching, the dampening factor used and the number of streamlines launched are the same as in the previous case.

The history-matching took eight iterations, which is smaller than in the unit mobility ratio case, to obtain an acceptable match to the production history. However, each iteration required more computation than that in the previous case, because we need to solve the pressure field more frequently to obtain an accurate flow simulation result.

Figure 5 shows the final history-matched permeability field, its histogram and variogram. Figure 6 shows the match to the water-cut history. The match is acceptable in terms of both geology and production history; though the final permeability field is different from that of the previous case. The result indicates our method works equally well for non-unit mobility ratio cases. 

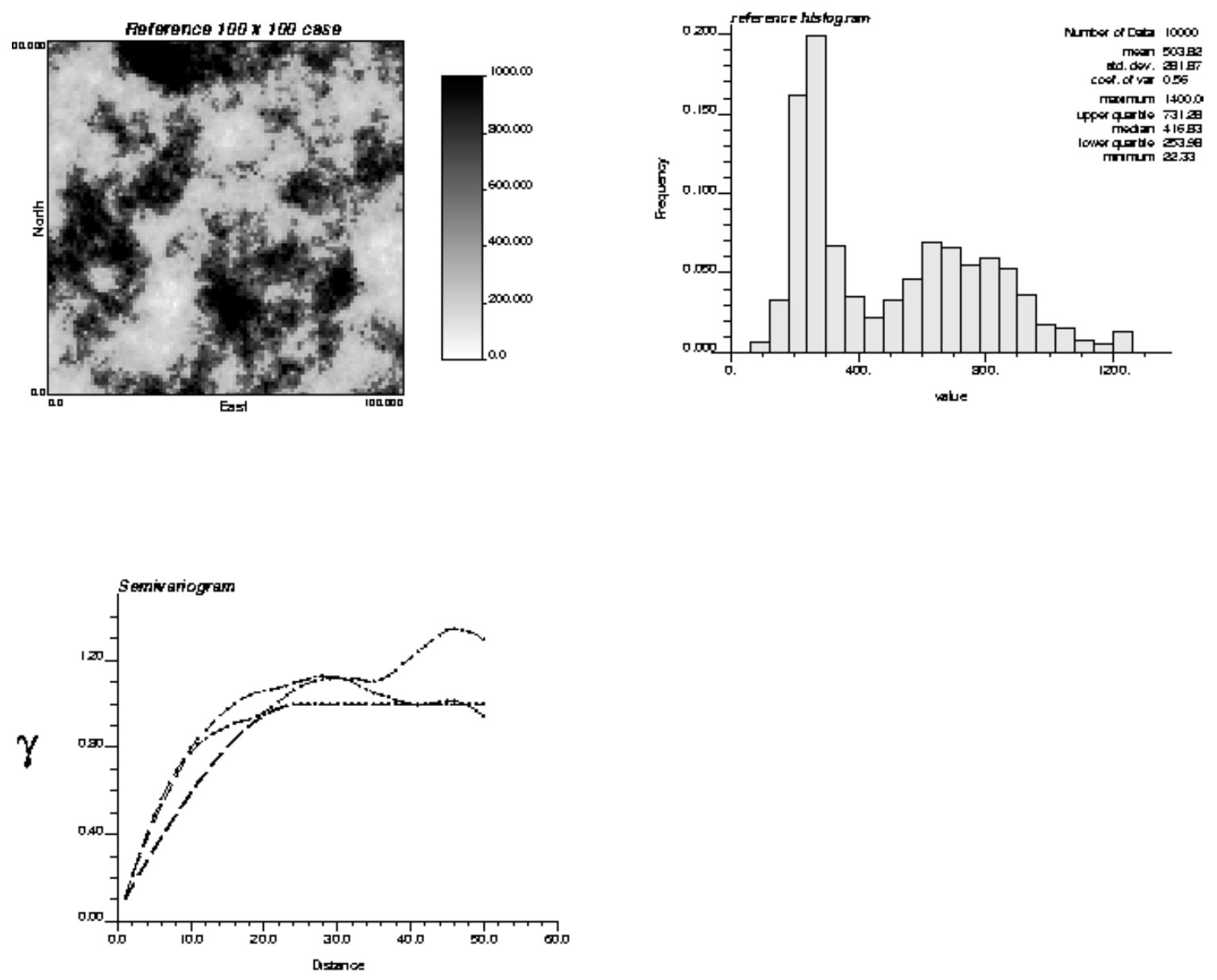

Figure 1. Reference permeability field, histogram and variogram, 100x100. 

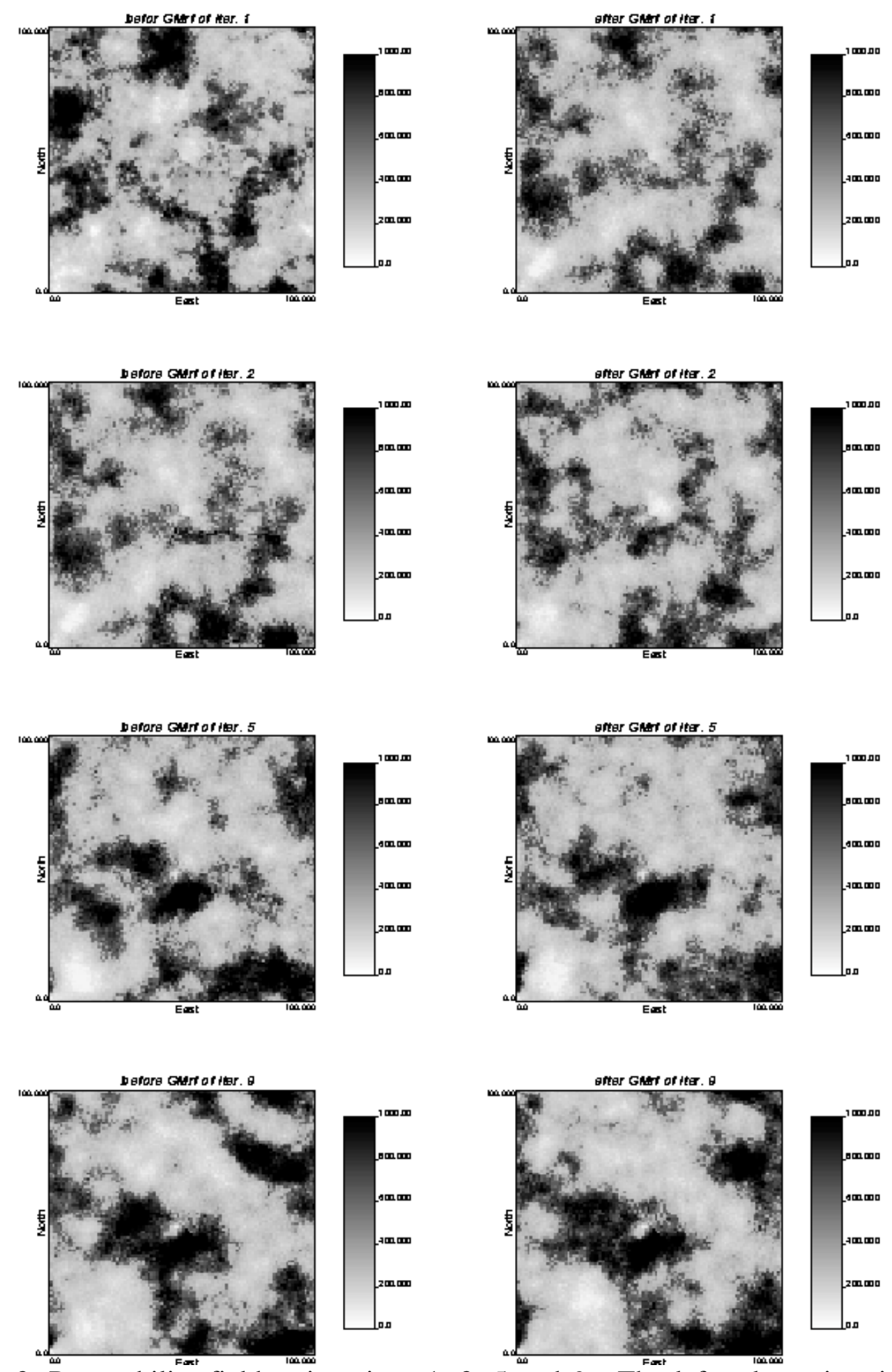

Figure 2. Permeability field at iterations $1,2,5$ and 9. The left column is prior to the GMrf process, while the right is after the GMrf and therefore consistent with geology. 

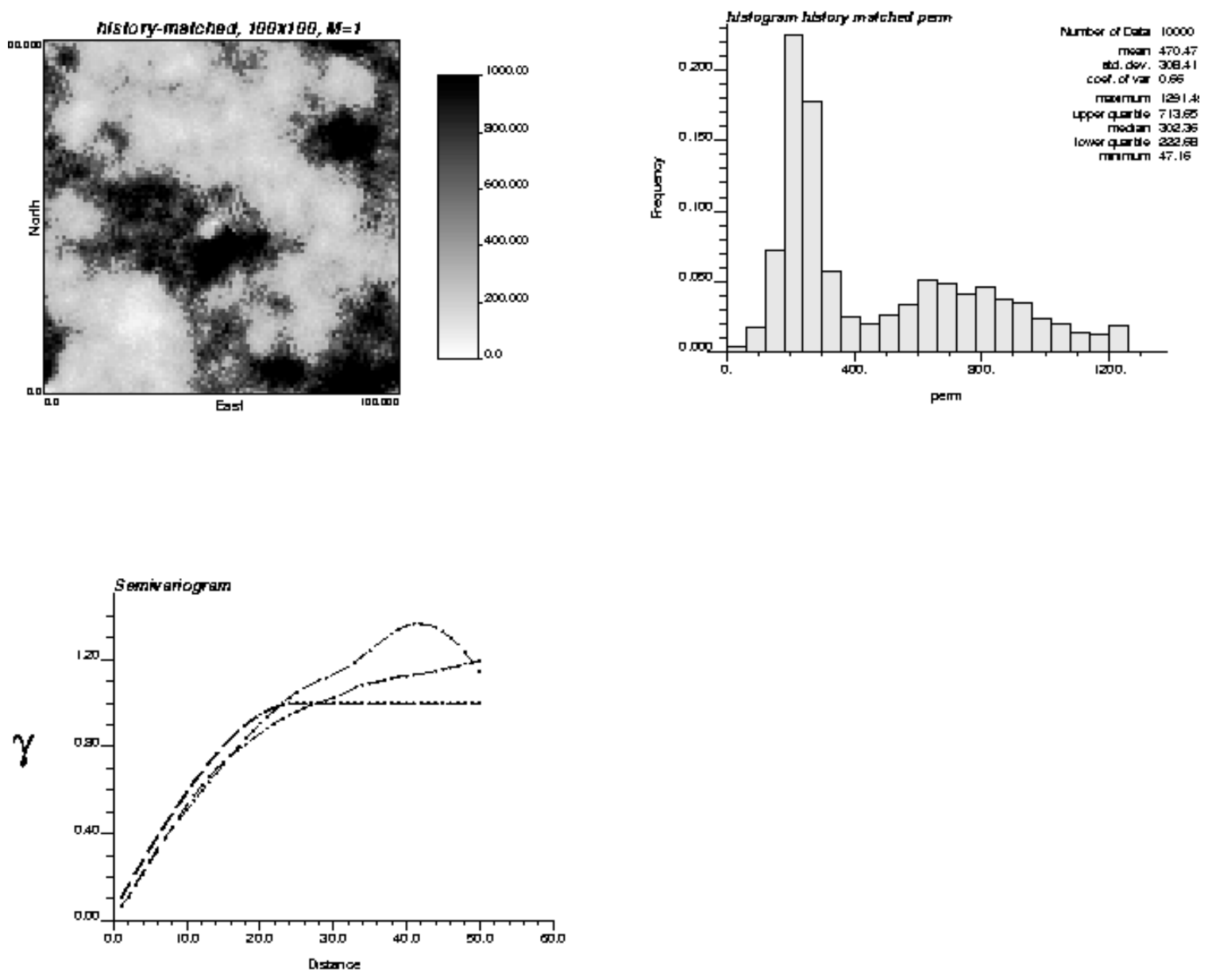

Figure 3. History-matched permeability field (100x100), five-spot, $M=1$. 
Well 1

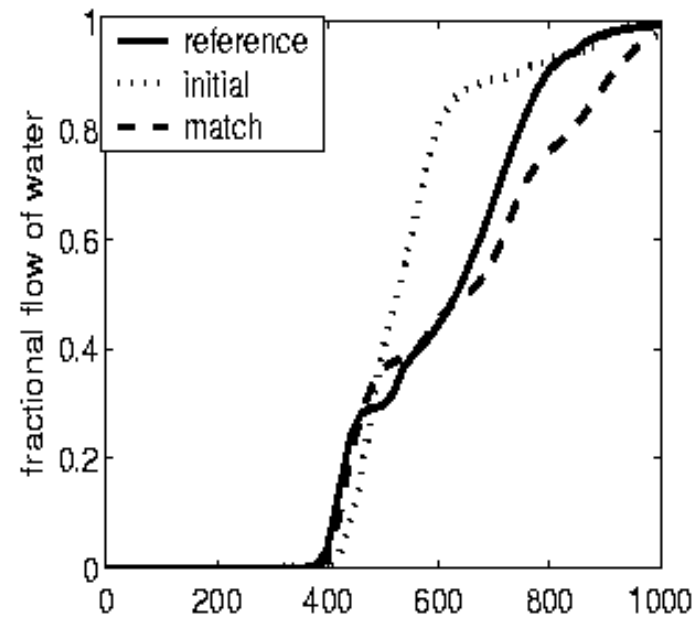

Well 3

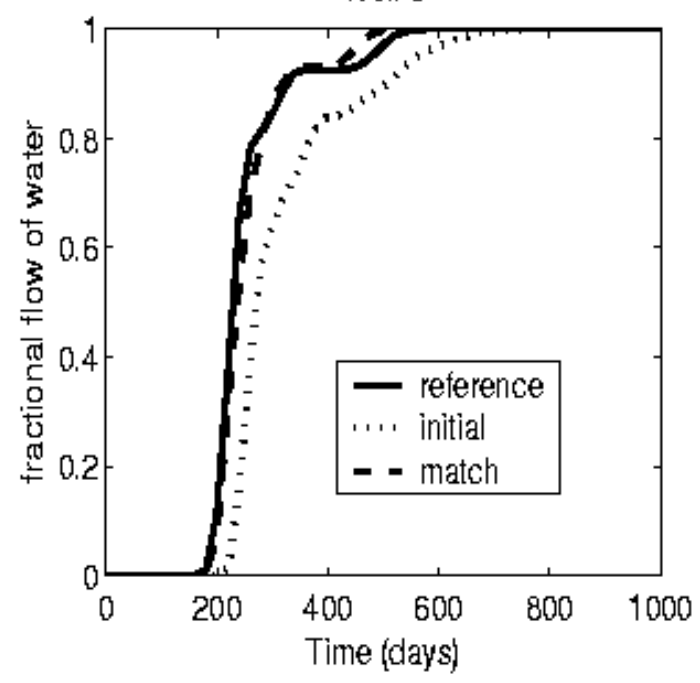

Well 2

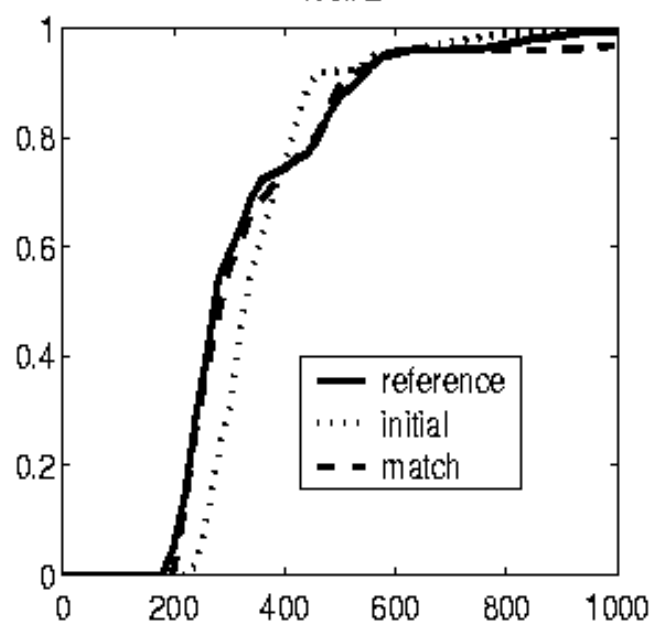

Well 4

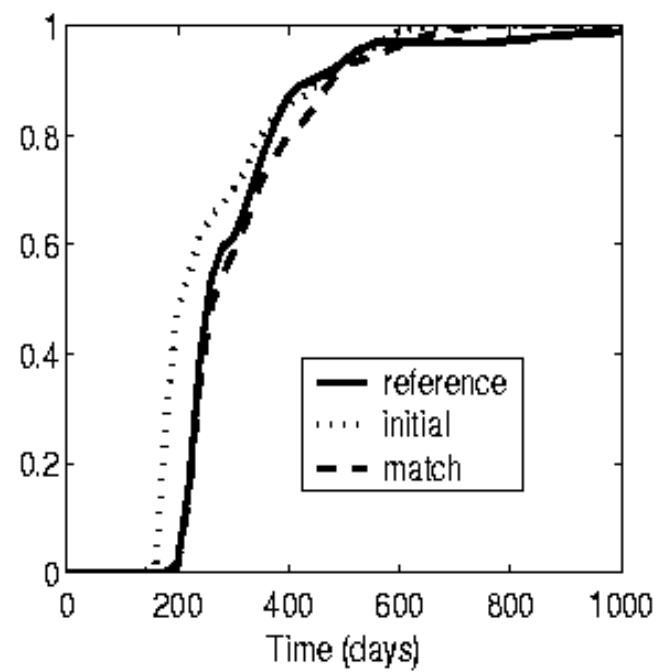

Figure 4. Match in water-cut, five-spot, 100x100 grid-blocks, $M=1$. 

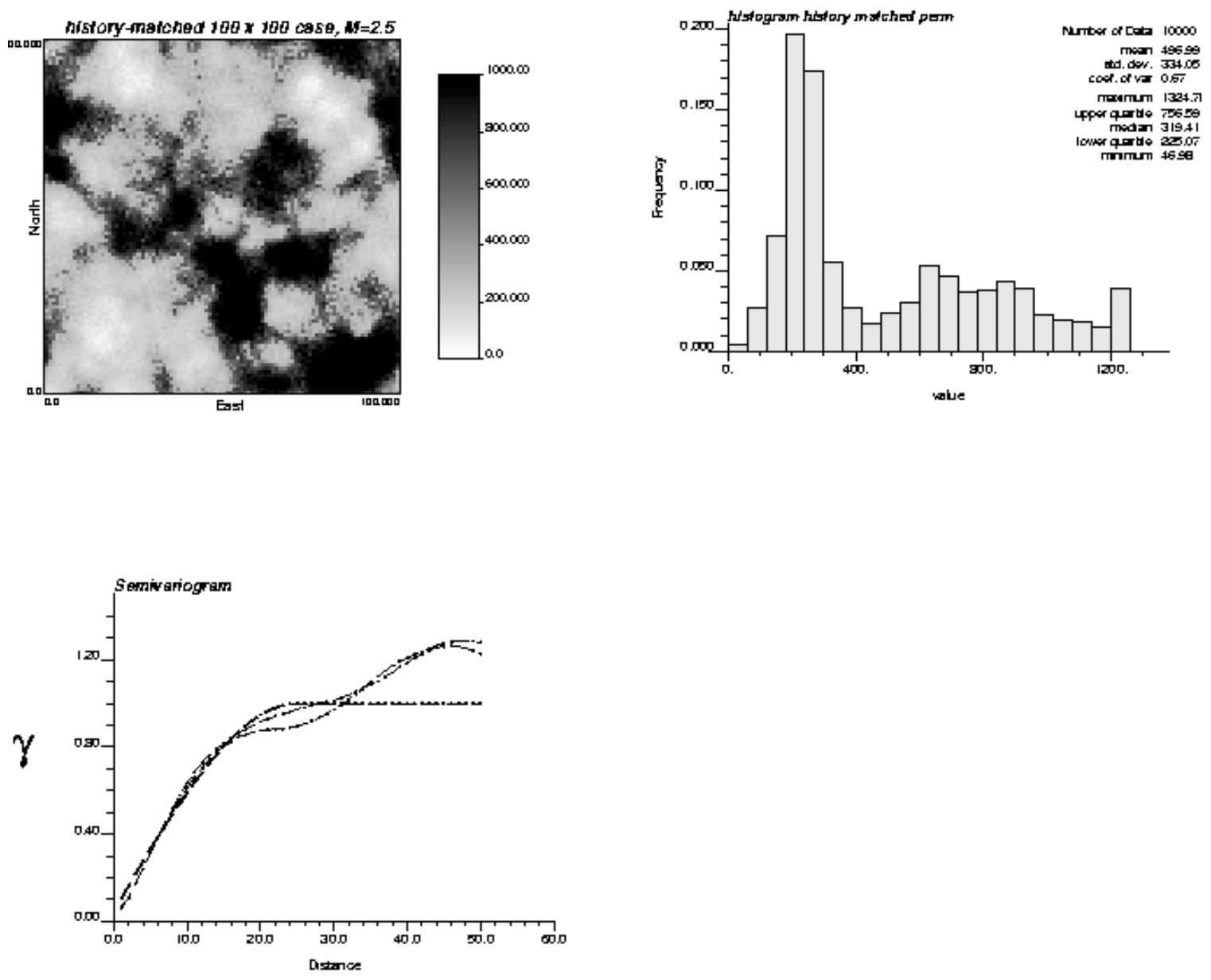

Figure 5. History-matched permeability field (100x 100), five-spot, $M=2.5$. 
Well 1

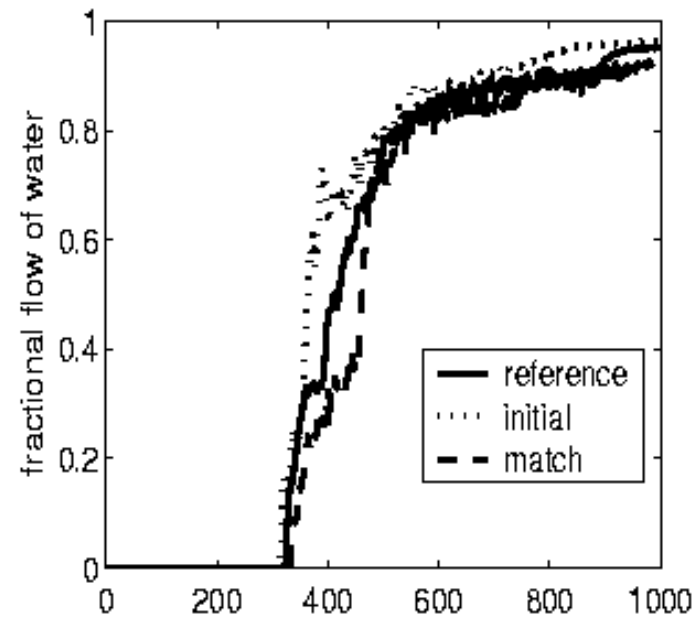

Well 3

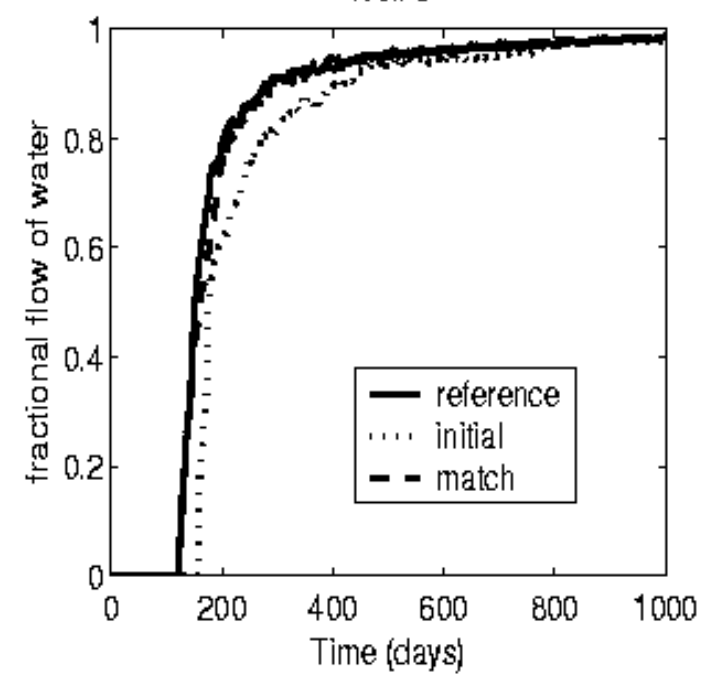

Well 2

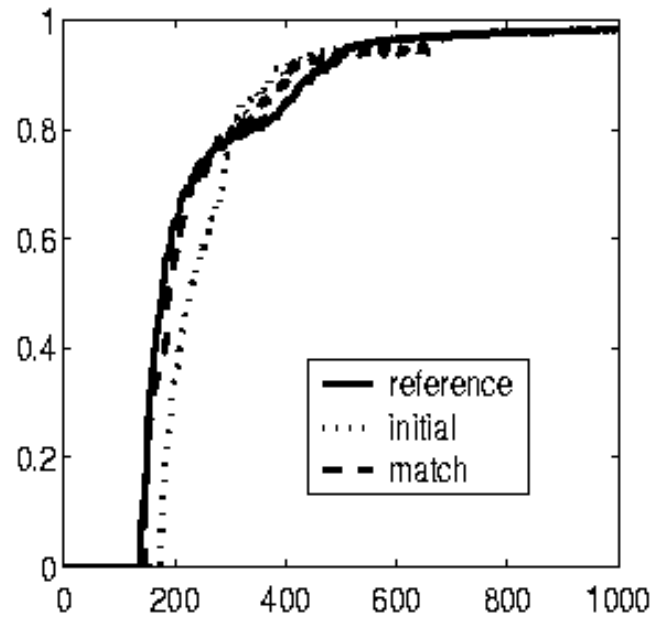

Well 4

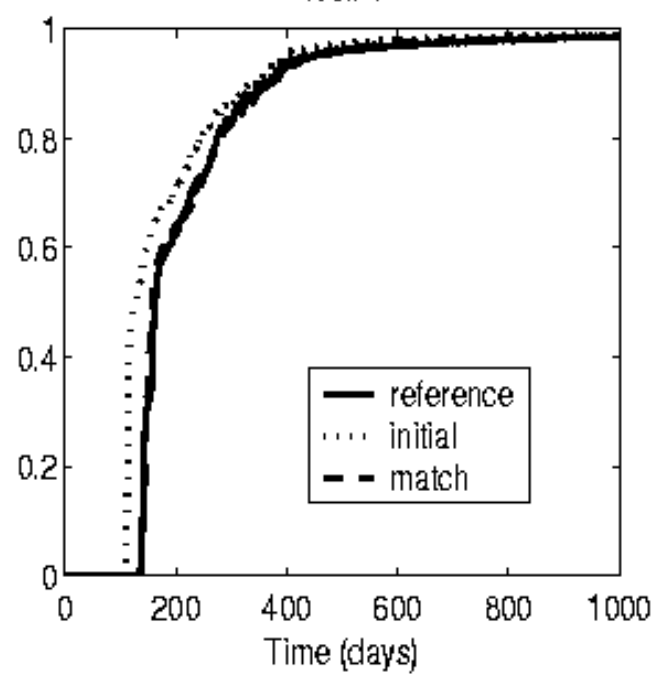

Figure 6. Match in water-cut, five-spot, 100x100 grid-blocks , $M=2.5$. 


\section{Cases 3: Five-spot, Anisotropic Permeability Fields}

We used SGSIM to generate the reference and initial permeability fields with the major direction of continuity set to N45W. There are 200x200 grid-blocks. Figure 7 shows the reference permeability field, its histogram and variogram. The same well locations and fluid properties as in the previous case are used to run the flow simulation. The key parameters used in the flow simulation are given in Table 1. A dampening factor of 0.25 was used; about 300 streamlines are launched in the inverse process.

Eight iterations were used to obtain a satisfactory history-match. Figures 8 and 9 show the history-matched realization and the match to water-cuts of all four producers. The match to the production history and the reproduction of the variogram and histogram are excellent. It took about three hours to history-match this 40,000 grid-block model and the major computational cost is for the eight runs of the streamline simulator (Table 2).

\section{Cases 4: Prediction of Reservoir Performance Using the History-Matched Model $M=5$}

In this case, we demonstrate how our method is used to obtain a reservoir model that matches a short period of production history; then, the model is used to predict the future reservoir performance, including water-cut and cumulative oil production. We use SGSIM to generate reference (Fig. 10) and initial permeability fields, and use 3DSL to create reference reservoir performance. Simulation parameters are listed in Table 1. Next, starting from an initial model, we perform the above history-matching process to match only an initial portion of the reference. Then, the history-matched model is used to run flow simulation and compare with the rest of the reference performance.

Starting from an initial permeability field, we performed the history-matching process three times with different random paths. Seven to eight iterations were used to obtain an acceptable match to the partial production history-150 days or 0.75 PVI. The three history-matched models are shown in Fig. 10. The matches and predictions of water-cut are shown in Fig. 11; the matches and predictions of cumulative oil production are given in Fig. 12. Compared with the initial model, which does not reproduce the reference results, the history-matched models produced excellent matches to the reference in terms of both the historic and the future performance. 

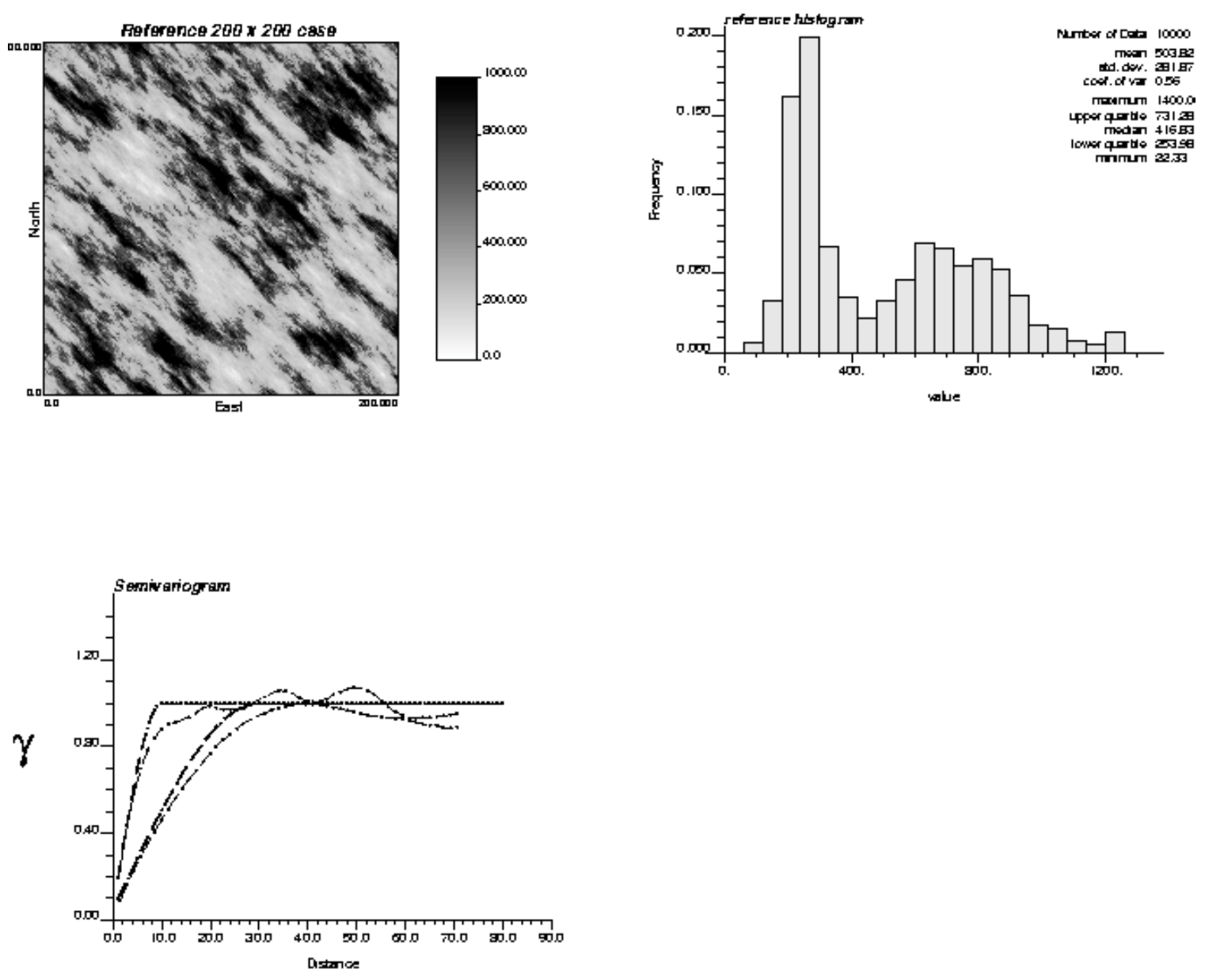

Figure 7. Reference permeability field, histogram and variogram, 200x200 grid-blocks. 

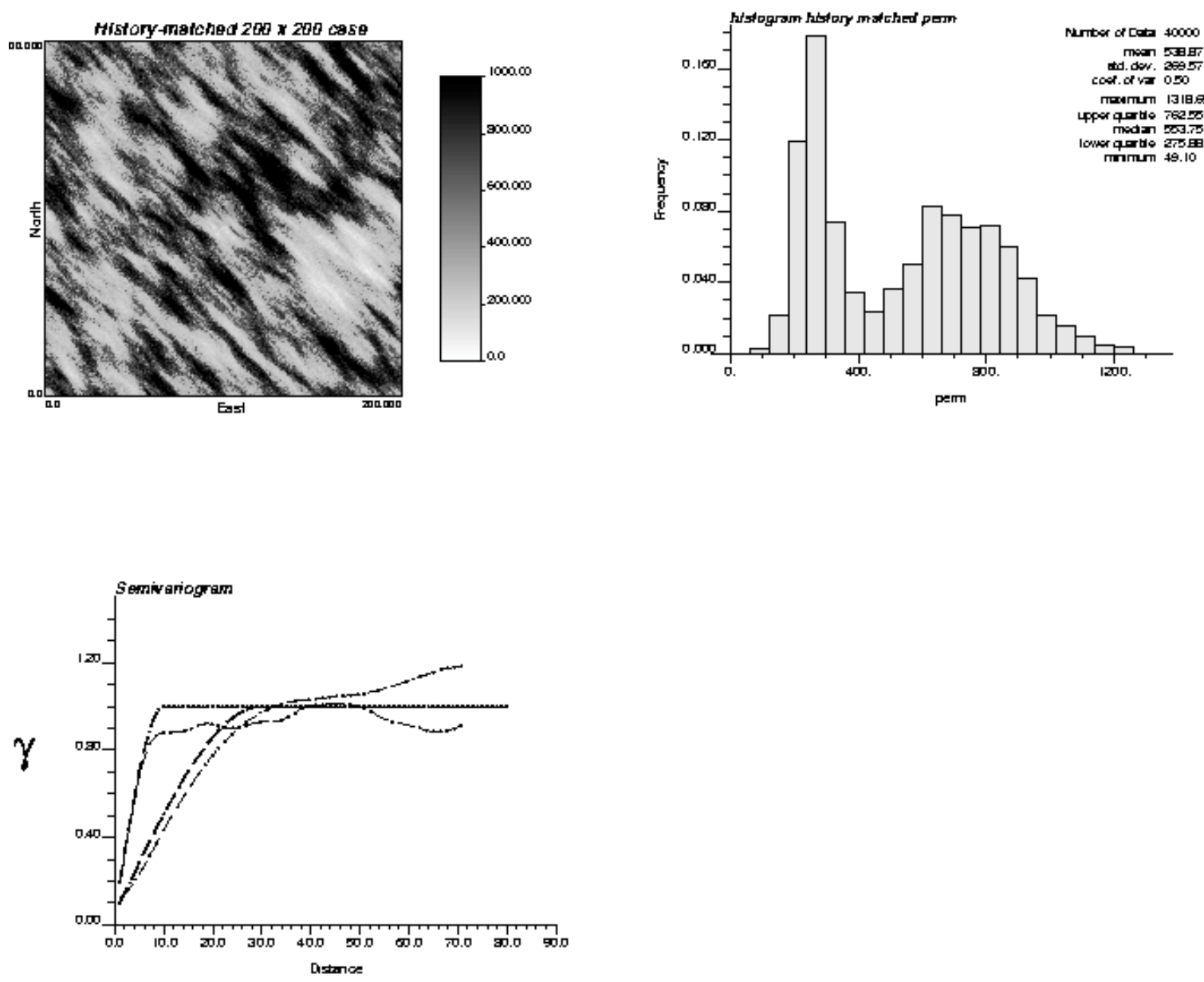

Figure 8. History-matched permeability field (200x200 grid-blocks), five-spot, $M=1$. 

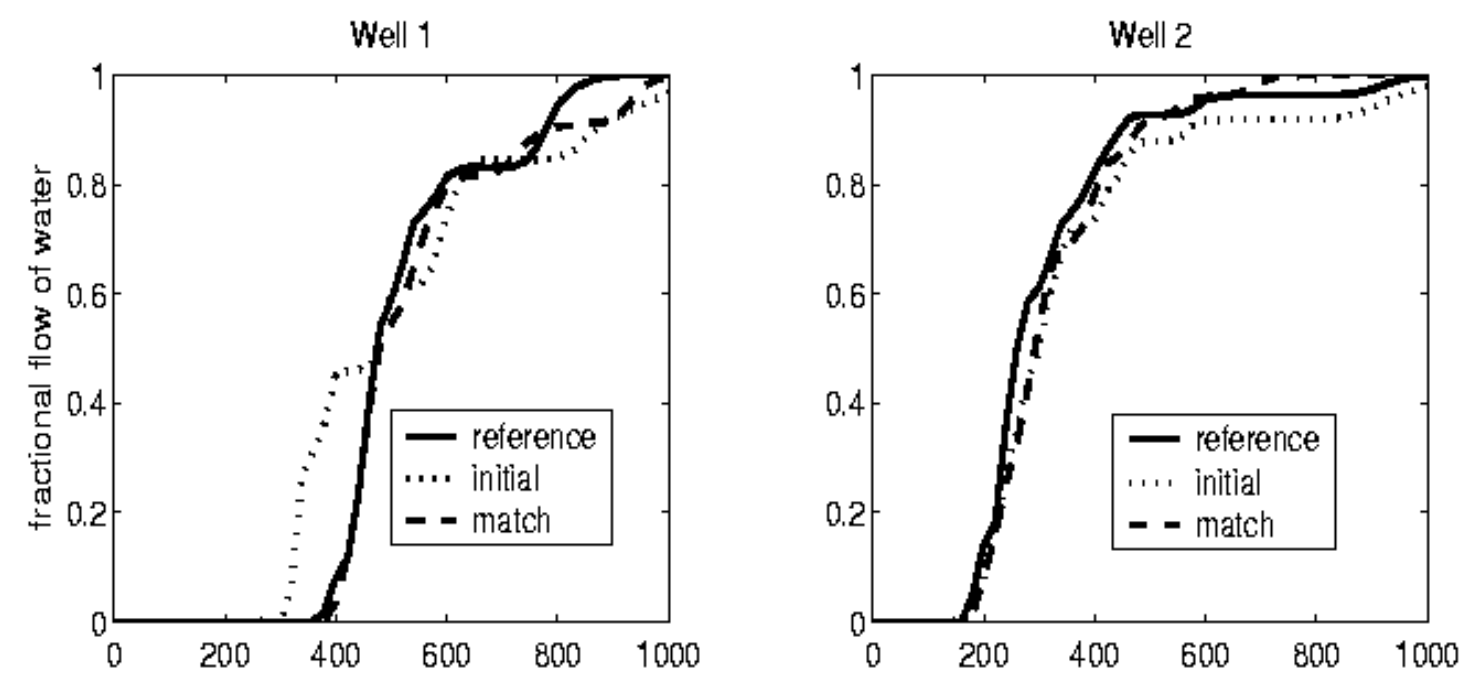

Well 3

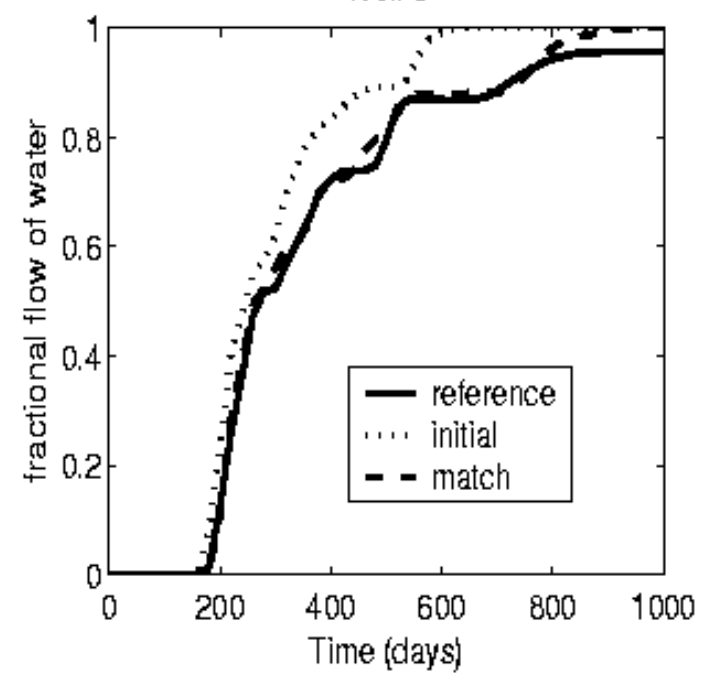

Well 4

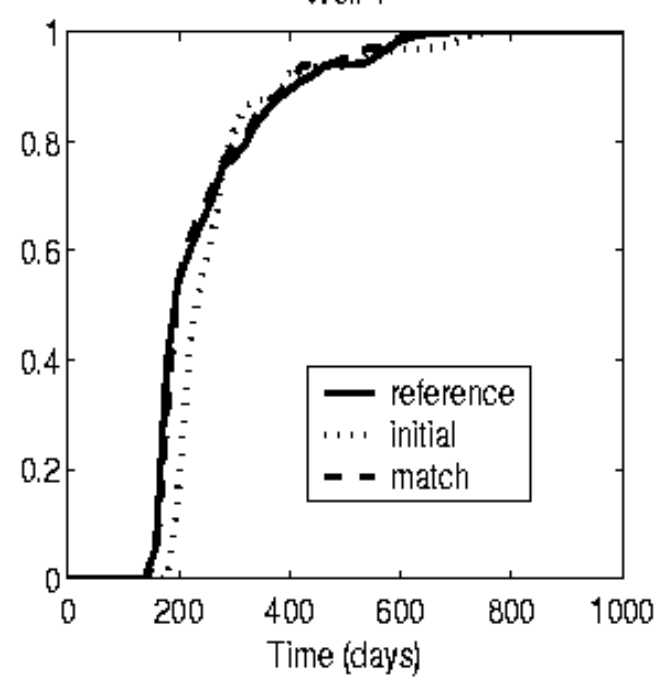

Figure 9. Match in water-cut, five-spot, 200x200 gridblocks, $M=1$. 

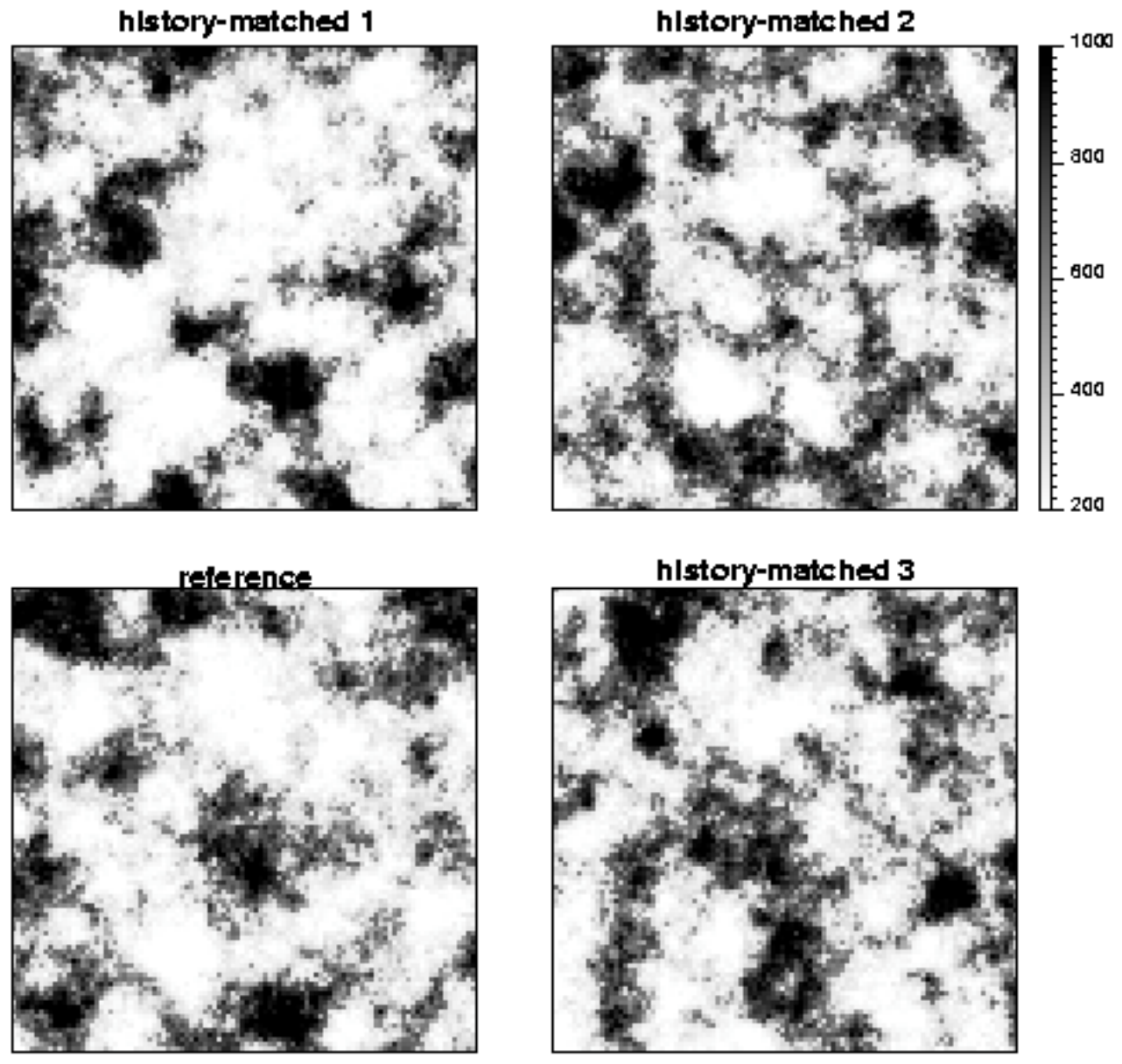

Figure 10. Reference and history-matched permeability fields. 

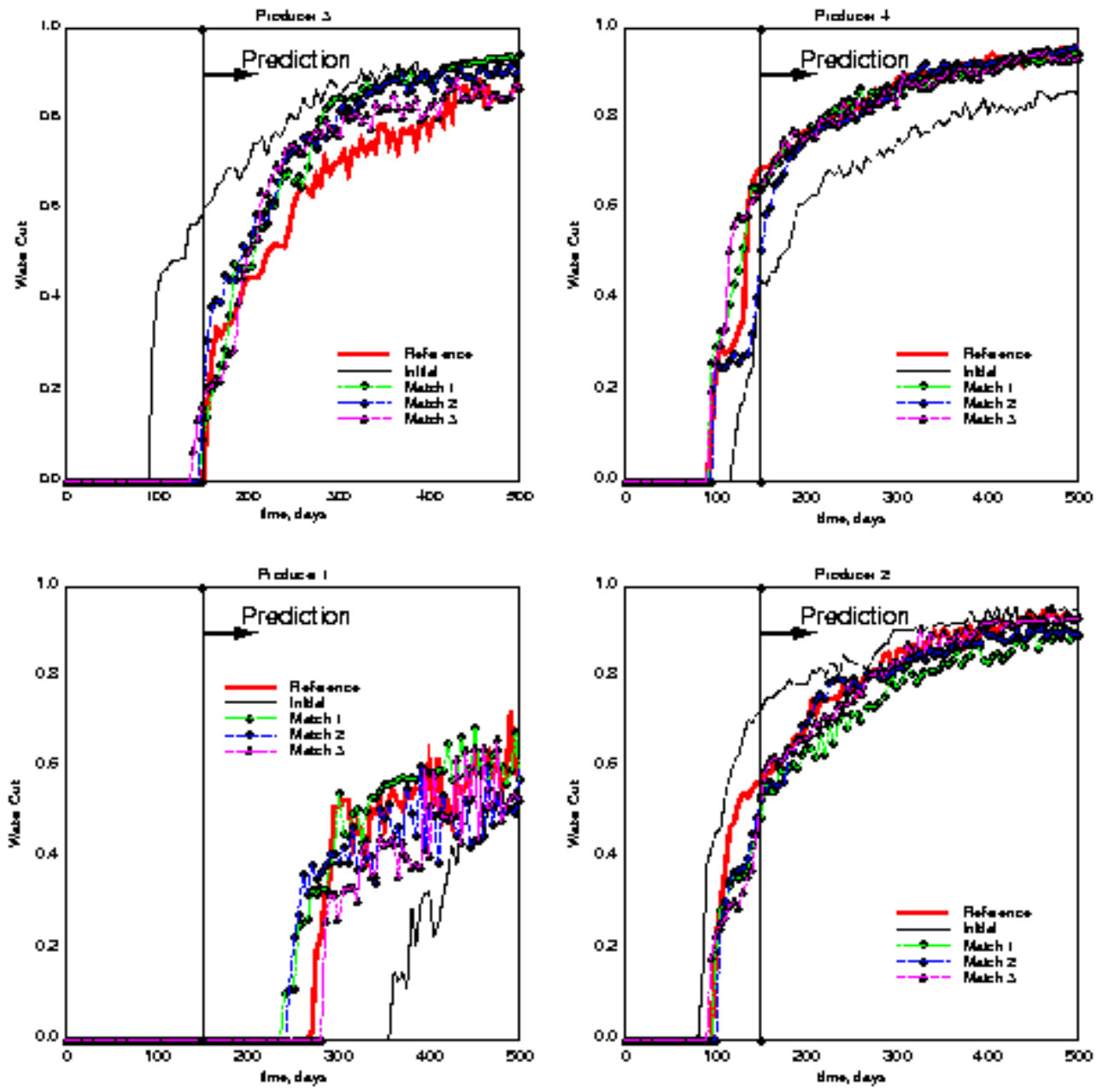

Figure 11. Match and prediction of water-cut. Matched to a production history of 150 days. The rest is prediction. 

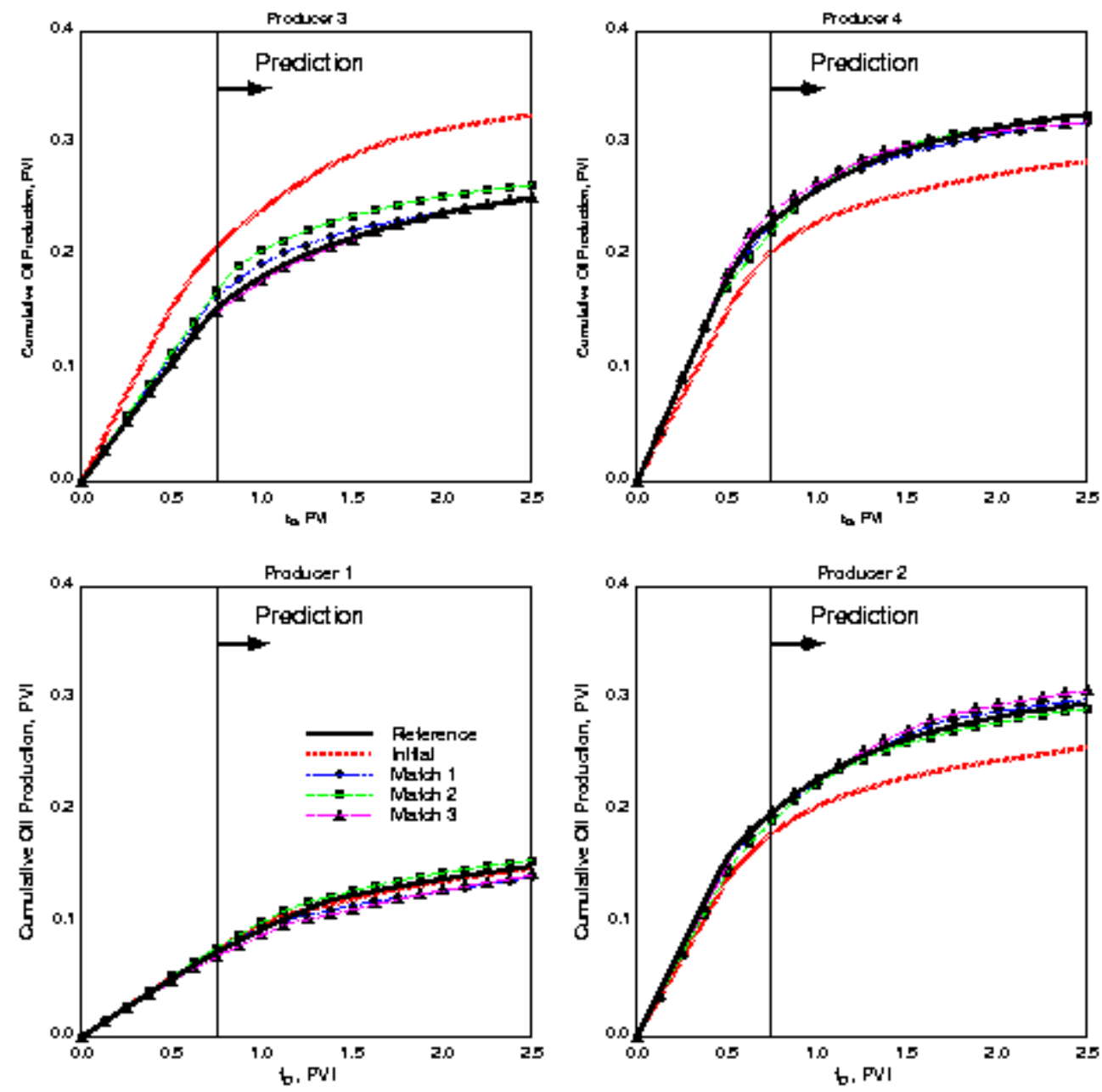

Figure 12. Match and prediction of cumulative oil production. Matched to a production history of $0.75 P V I$. The rest is prediction. 


\section{Computational Cost}

Table 2 shows the overall computational work for each case and its breakdown for each component of the inverse process. The jobs were run on a $600 \mathrm{MHz}$ DEC alpha machine.

For a small number of grid-blocks ( 50 by 50 case) and unit mobility ratio, the major cost can be attributed to GMrf. This is largely due to the computing overhead associated with the GMrf. However, as the number of grid-blocks increases, the computational work of the flow simulation increases rapidly, while that of GMrf procedure increases at slower rate. We observed that the CPU cost of the flow simulation grows quadratically with the number of grid-blocks, while that of the GMrf process grows slightly faster than linearly. Computational work by the streamline inverse process also scales linearly with the number of grid-blocks. In any case, the CPU time consumed by the streamline inverse process is negligible when compared with the other two parts (GMrf and forward simulation).

For nonunit mobility ratios, the pressure field and streamline geometry must be updated periodically during forward simulation to obtain accurate results. Hence, the CPU-time is somewhat greater for the $M=2.5$ and $M=5$ cases, as compared to the $M=1$ cases.

Table 2 Computational Work

\begin{tabular}{|c|c|c|c|c|c|c|c|c|}
\hline \multirow{2}{*}{$\begin{array}{c}\text { Case } \\
\#\end{array}$} & \multirow{2}{*}{$\begin{array}{c}\text { Case } \\
\text { Description }\end{array}$} & \multicolumn{4}{|c|}{$\mathrm{CPU}$ per iteration $(\mathrm{sec})$} & \multirow{2}{*}{$\begin{array}{c}\text { \# } \\
\text { rations }\end{array}$} & \multicolumn{2}{|c|}{ Total CPU } \\
\hline & & $3 \mathrm{dsl}$ & GMrf & SLInv & Total & & $(\mathrm{sec})$ & $(\min )$ \\
\hline 1 & 5-spot, $100 \times 100, M=1$ & 76 & 69 & 1.4 & 146 & 9 & 1318 & 22.0 \\
\hline 2 & 5-spot, $100 x 100, M=2.5^{*}$ & 1169 & 69 & 1.4 & 1239 & 8 & 9915 & 165.3 \\
\hline 3 & 5-spot, $200 \times 200, M=1$ & 1285 & 338 & 6.0 & 1629 & 8 & 13032 & 217.2 \\
\hline 4 & 5-spot, 100x100, M=5* & 1254 & 69 & 1.4 & 1324 & $7 \sim 8$ & 9933 & 165.6 \\
\hline
\end{tabular}

*--Non-unit mobility ratio, more pressure solves required than $M=1$ case.

\section{Remarks}

We propose a combined streamline-geostatistical history matching procedure. The methodology relies on decoupling of the inversion process to the greatest extent possible. Flow history and geology are matched in a two-step procedure: a streamline method calculates a desired change in streamline effective permeabilities and a geostatistical routine spreads this desired change to account for known geological variations.

No matrix inversion is necessary in the streamline inversion portion, the Gauss-Markov technique is an efficient optimization algorithm, and forward simulation is conducted with a streamline simulator. Hence, the methodology is fast, and the major computational cost is the forward flow simulation. However, the combined geostatistical and streamline-based method shares many of the advantages and limitations of its two basic components. The limitations of streamline inverse methods have been discussed in the previous section. 
Inverse solutions can be constrained in many different ways. One of the main advantages of the proposed technique lies in the decoupling of the streamline-based inversion from the geostatistical simulation algorithm. In a sense, the effective permeability along streamlines required to match production data is computed without any knowledge of geology. Geostatistics is then used to distribute grid-block permeability in a consistent manner that restores geology. However, since the harmonic averages in Step 2 of the algorithm are calculated without knowledge of the underlying histogram and variogram model, inconsistencies can occur between Step 2 and Step 3 of the algorithm. Therefore, the introduction of a dampening factor is crucial to make the combined procedure of history-matching and GMrf robust and efficient. Essentially, the dampening factor limits the degree of change and prevents overly drastic permeability modification that may introduce difficulty in convergence of the GMrf process.

All the example applications show acceptable matches to the production history and reproduction of variogram and histogram within a few iterations. Our method works equally well for cases with unit and non-unit mobility ratios. Importantly, if a short period of production history is matched using our method, the uncertainty of the model can be substantially reduced while the model is used to forecast the future reservoir performance, as opposed by using a geostatistical reservoir model that has not been history-matched.

The current methodology allows only for quantification of geology in terms of a variogram, yet the decoupled procedure could lead to an extension of the algorithm to account for other types of geological models (e.g., channels). More study is needed to pursue this concept.

\section{Streamline-Based Ranking}

To address jointly production history and constraint to a prior geological model, we also have proposed and demonstrated ranking suites of reservoir models in terms of streamline properties. A subset is then selected rationally that honor observed production data. The ranking approach is somewhat similar to importance sampling (Barker et al. 2001), but requires substantially less computation to evaluate the objective function. Additionally, results are less sensitive to the prior reservoir model. In streamline ranking, the objective function measures the mismatch of streamline properties between any given and a desired (i.e., reference) reservoir model. The mismatch is evaluated efficiently by calculating, for instance, the distribution of the TOF of streamlines reaching a producer. By sampling a sufficient number of reservoir models, a set of models that honor desired streamline properties are identified. It has been demonstrated that ranking according to $T O F$ also sorts models in regard to historic production data, such as watercut. The use of full flow simulation in minimized throughout the algorithm.

Results from this study have been published already (Wang and Kovscek 2002, 2003). By way of summary, the work proceeded by first providing the empirical observations and limited theoretical development that form the background for streamline ranking. We established the correlation among streamline properties and the corresponding comprehensive flow simulation results. The algorithm arising by the correspondence of streamline and flow simulation results was then described. Synthetic examples illustrate application of the method under various 
conditions. A brief digression showed that streamline ranking can be employed for downscaling consistently coarse-grid history-matched reservoir models.

\section{Method}

The ranking method is summarized in the following. The major assumptions include 1) the history of injected phase production is available; 2) no drastic changes of well conditions (e.g., shut in or in fill); and 3) gravity, capillary forces, and compressibility are not important.

The ranking procedure:

1. Start from an initial reservoir model and perform history matching (Wang and Kovscek, 2000) to obtain a history-matched reservoir model that is not necessarily constrained to geology. For unit mobility ratio, the water-cut curve is the same as the streamline TOF $c d f$ as shown above. In this case, the reference water-cut curve is used directly as the reference streamline $T O F c d f$.

2. For the history-matched model and each of the models to be ranked, compute the pressure distribution for unit mobility ratio, streamline geometries, and streamline TOF $c d f$ for each producer. Pollock's (1998) method is used here to compute streamline geometries.

3. For each of the reservoir models to be ranked, compute the mismatch of its streamline properties, such as TOF $c d f$, $q$, and $N_{p}^{E}$, with respect to the history-matched model. This computation is performed for each producer.

4. Rank the reservoir models based on the mismatch type of interest. For instance, if a match of the water-cut is desired, then streamline TOF $c d f$ ranking should be used.

5. Select a suitable number of models within a specified tolerance and perform comprehensive flow simulation to evaluate the match in simulation results, predict future reservoir performance, as well as quantify the uncertainty of the prediction.

\section{Conclusions}

A method is proposed to rank rapidly reservoir models. This method is based on the observation that there is approximately a linear correlation between unit mobility ratio streamline properties and nonunit mobility ratio reservoir flow simulation results, under appropriate assumptions. The streamline time-of-flight cumulative distribution function indicates the connectivity between injector-producer pairs, and consequently it is characteristic of flow simulation results regardless of mobility ratio. Exhaustive flow simulation and theoretical derivation established correlation among the following data pairs: 1) streamline TOF $c d f$ and producer water cut, 2) unit mobility flow-rate at one time value and that in comprehensive flow simulation, and 3) $N_{p}^{E}$ and $N_{p}$. With these data pairs, reservoir models ranked in terms of streamline properties are also ranked in terms of their match to the observed production history. 
Computation of streamline geometries and properties is computationally efficient. The computational effort per reservoir model is equivalent to one time-step of an IMPES flow simulation.

The ranking method requires that a history-matched model be obtained first. The history match does not necessarily need to be constrained to geology. Then, geostatistically consistent models are generated and compared to the history-matched model with regard to streamline properties. A subset of models with tolerable mismatch are selected to run flow simulation and thereby verify that they match the observed behavior. In this manner, multiple models are obtained that reproduce satisfactorily production history and are constrained to geological information. An exercise demonstrated that partial production history when used in combination with streamline-based ranking reduced significantly the uncertainty of forecasting reservoir performance.

The ranking results do not depend on the history-matching method or the initial reservoir model, but depend on the accuracy of the match used to obtain the target TOF $c d f$. Additionally, there is no restriction that the reservoir models must have the same grid-block dimensions as the history-matched model. Therefore, fine-grid models with a million cells can be ranked and selected efficiently when history matching is performed on a coarse grid. This provides an efficient way to downscale history-matched models.

\section{References}

Barker, J. W., Cuypers, M. \& Holden, L. 2001. "Quantifying Uncertainty in Production Forecasts: Another Look at the PUNQ-S3," SPE Journal, 6(4) 433-441.

Batycky, R. P., Blunt, M. J. \& Thiele, M. R. 1997. "A 3D Field-Scale Streamline-Based Reservoir Simulator," SPE Reservoir Engineering, 12, November, 246-254.

Caers, J., Krishnan, S., Wang, Y. \& Kovscek, A. R. 2002. "A Geostatistical Approach to Streamline-based History Matching” to appear SPE Journal.

Deutsch, C. V. \& Journel, A. G. 1998. GSLIB, Geostatistical Software Library and User's Guide, Second Edition, Oxford University Press, New York.

Deutsch, C. V. \& Srinivasan, S. 1996. "Improved Reservoir Management Through Ranking Stochastic Reservoir Models," SPE 35411 presented at SPE/DOE tenth Symposium on Improved Oil Recovery, Tulsa, OK, 21-24 April, 105-112.

Tran, T. T, Wen, X-H., \& Behrens, R. A. 2001. "Efficient Conditioning of 3D Fine-Scale Reservoir Model to Multiphase Production Data Using Streamline-Based Coarse-Scale Inversion and Geostatistical Downscaling", SPE Journal, 6(4), 364-374.

Wang, Y. and A. R. Kovscek, "Integrating Production History into Reservoir Models Using Streamline-Based Time-of-Flight Ranking," Petroleum Geoscience, 9(2), 163-174 (2003). 
Wang, Y. and A. R. Kovscek. "A Streamline Approach for Ranking Reservoir Models that Incorporates Production History" SPE 77377, Proceedings of the SPE Annual Technical Conference and Exhibition, San Antonio, TX, Sept. 29 - Oct. 2, 2002. 


\section{Area 6. Technology Transfer}

Technology transfer was an important part of our research efforts. It was considered so important that it was made an explicit part of the work plan and became Area 6. Technology transfer was accomplished through a variety of venues including:

- an annual industrial review meeting

- technical presentations at appropriate symposia, technical meetings, and workshops

- publication of technical papers, reviews, and chapters for the Society of Petroleum Engineers Handbook

- training of students who are subsequently hired by the domestic oil industry

- creation of a world wide web site http://ekofisk.stanford.edu/supria.html

- a short course on thermal recovery for practicing engineers

- international cooperation

Because all of our work is in the public domain, we aim for significant, rapid, and broad dissemination of our research results. Further discussion of our technology transfer efforts in each area follow.

\section{Industrial Review Meeting}

We host a review meeting annually to review with industrial representatives our current research results and research in progress. This review provides industry with an opportunity to see our work first hand and provide a critique. Meetings consist of technical presentations followed by extensive question and answer periods. Tours of our laboratory and one-on-one meetings between industry and SUPRI personnel also occur. Some time is also alotted for open discussion.

During the period of performance meetings were held April 19-20, 2001, April 18-19, 2002, and April 22-23, 2003. Attendees from D.O.E., Aera Energy LLC, ChevronTexaco, ConocoPhillips, ExxonMobil, PDVSA-Intevep, Shell,Total, Tyco Thermal Controls participated in the various meetings.

\section{Technical Presentations}

Presentation of research results at technical meetings and workshops was a principle means of technology transfer, in addition to the industrial review meetings. 
Most of the presentations occurred at Society of Petroleum Engineers (SPE) meetings as these meeting were judged to have the greatest audience with an interest in our research. The specific meetings attended to conduct technology transfer include: $6^{\text {th }}$ International Symposium on Evaluation of Reservoir Wettability and Its Affect on Oil Recovery (2000), SPE International Thermal Operations and Heavy Oil Symposium (2001), SPE Western Regional Meetings (2001, 2002, 2003), SPE Annual Technical Conference and Exhibition (2001, 2002, 2003), and the SPE/DOE Thirteenth Symposium on Enhanced Oil Recovery (2002). A listing of authors and paper titles is given at the end of this subsection under the heading "Conference Presentations".

During the period of performance, we also participated with the West Coast Petroleum Technology Transfer Council during a workshop (2001) that they hosted. The topic during the workshop was a review of diatomite physical properties and their relation to hydraulic fracturing. A variety of people from companies operating in the San Joaquin Valley attended. A variety of presentations were also made at the University of California, Berkeley (2002, 2003), ChevronTexaco, and Aera Energy LLC (2003).

\section{Conference Presentations}

Arora, P. and A. R. Kovscek, "Mechanistic Modeling of Solution Gas Drive in Viscous Oils, SPE 69717, Proceedings of the SPE International Thermal Operations and Heavy Oil Symposium, Porlamar, Venezuela March 12-14, 2001.

Hoffman, B. T. and A. R. Kovscek, "Light-Oil Steamdrive in Fractured Low Permeability Reservoirs," SPE 83491, Proceedings of the SPE Western Region/AAPG Pacific Section Joint Meeting, Long Beach, CA, May 19-24, 2003.

Kovscek, A. R. and H. J. Bertin, "Estimation of Foam Mobility in Heterogeneous Porous Media, " SPE 75181 Proceedings of the SPE/DOE Thirteenth Symposium on Improved Oil Recovery, Tulsa, OK April 13-17, 2002.

Rangel-German. E. R. and A. R. Kovscek, "Time-Dependent Matrix-Fracture Shape Factors for Partially and Completely Immersed Fractures," SPE 84411, Proceedings of the SPE Annual Technical Conference and Exhibition, Denver, CO, Oct. 5-8. 2003.

Rangel-German, E. R. and A. R. Kovscek, "Water Infiltration in Fractured Systems: Experiments and Analytical Model," SPE 71618 Proceedings of the SPE Annual Technical Conference and Exhibition, New Orleans, LA Sept. 30 - Oct. 3, 2001.

Ross, C. M. and A. R. Kovscek, "Pore Microstructure and Fluid Distribution in a Diatomaceous Reservoir, " SPE 75190 Proceedings of the SPE/DOE Thirteenth Symposium on Improved Oil Recovery, Tulsa, OK April 13-17, 2002 
Schembre, J. M. and A. R. Kovscek, " Direct Measurement of Dynamic Relative Permeability from CT-Monitored Spontaneous Imbibition Experiments," SPE 71484 Proceedings of the SPE Annual Technical Conference and Exhibition, New Orleans, LA Sept. 30 - Oct. 3, 2001.

Tang, G.Q. and A. R. Kovscek, "Experimental Study of Heavy Oil Production from Diatomite Core at Elevated Temperatures, " SPE 75132 Proceedings of the SPE/DOE Thirteenth Symposium on Improved Oil Recovery, Tulsa, OK April 13-17, 2002.

Tang, G-Q. and A. R. Kovscek, "Wettability Alteration of Diatomite Induced by Hot-Fluid Injection" SPE 77461, Proceedings of the SPE Annual Technical Conference and Exhibition, San Antonio, TX, Sept. 29 - Oct. 2, 2002.

Tang, G.-Q., Y. Leung, L. M. Castanier, A. Sahni, F. Gadelle, M. Kumar, and A. R. Kovscek, "An Investigation of the Effect of Oil Composition on Heavy Oil Solution Gas Drive," SPE 84197, Proceedings of the SPE Annual Technical Conference and Exhibition, Denver, CO, Oct. 5-8. 2003.

Wang, Y. and A. R. Kovscek. "A Streamline Approach for Ranking Reservoir Models that Incorporates Production History" SPE 77377, Proceedings of the SPE Annual Technical Conference and Exhibition, San Antonio, TX, Sept. 29 - Oct. 2, 2002.

Zhou, D., L. Jia, J. Kamath, and A. R. Kovscek, "Scaling of Counter-Current Imbibition Processes in Low-Permeability Porous Media," Proceedings of the $6^{\text {th }}$ International Symposium on Evaluation of Reservoir Wettability and Its Effect on Oil Recovery, Soccoro, NM September 27-28, 2000.

Zhou, D., L. Jia, J. Kamath, and A. R. Kovscek, "An Investigation of Counter-Current Imbibition Processes in Diatomite," SPE 68837, Proceedings of the SPE Western Regional Meeting, Bakersfield, CA March 26-30, 2001.

\section{Technical Papers}

To aid in the dissemination and documentation of technical results, a series of papers were submitted to journals. An advantage of the submission of work to journals is that papers are subjected to anonymous peer review. Thus, a form of quality control is associated with published work. A listing of authors and peer-reviewed manuscript titles is given at the end of this subsection under the heading "Reviewed Manuscripts".

A highlight of this aspect of our technology transfer efforts was the publication of a chapter on in-situ combustion for the Reservoir Engineering Section of the Society of Petroleum Engineers Handbook (Castanier and Brigham, 2003). During past Industrial Review Committee Meetings, it was suggested rather strongly by attendees that while in-situ combustion has great potential as a thermal recovery technique, it is still viewed as a high-risk process by many. They stated that we should clarify the potential of in-situ combustion as an enhanced oil recovery 
process and try to capture some of the knowledge with regard to design aspects of ISC projects. An invitation from the Society of Petroleum Engineers to prepare a chapter for the latest version of the Petroluem Engineers Handbook allowed this suggestion to take shape.

\section{Reviewed Manuscripts}

Apaydin, O. and A. R. Kovscek, "Surfactant Concentration and End Effects on Foam Flow in Homogeneous Porous Media," Transport in Porous Media, 43(3), 511-536 (2001).

Akin, S. and A. R. Kovscek, "Heavy Oil Solution Gas Drive: A Laboratory Study," Journal of Petroleum Science and Engineering, 35(1-2), 33-48 (2002).

Arora, P. and A. R. Kovscek, "A Mechanistic Modeling and Experimental Study of Solution Gas Drive," Transport in Porous Media, 51, 237-265 (2003).

Bertin, H. J. and A. R. Kovscek, " Foam Mobility in Heterogeneous Porous Media I: Scaling Concepts," Transport in Porous Media, 52, 17-35 (2003).

Bertin, H. J. and A. R. Kovscek, " Foam Mobility in Heterogeneous Porous Media II: Experimental Observations," Transport in Porous Media, 52, 37-49 (2003).

Caers, J., S. Krishnan, Y. Wang, and A. R. Kovscek, "A Geostatistical Approach to StreamlineBased History Matching," Society of Petroleum Engineers Journal, 7(3) Sept, 250-266 (2002).

Castanier, L. M. and W. E. Brigham, "In Situ Combustion”, Chapter 17, Reservoir Engineering Section, Society of Petroleum Engineers Handbook, Richardson TX: Society of Petroleum Engineers, in press 2004.

Castanier, L. M. and W. E. Brigham, "Upgrading of Crude Oil Via In Situ Combustion," Journal of Petroleum Science and Engineering, 39(1\&2), 125-136 (2003).

Diabira, I., L. M. Castanier, and A. R. Kovscek, "Porosity and Permeability Evolution Accompanying Hot Fluid Injection into Diatomite," Petroleum Science and Technology, 19(9\&10), 1167-1185 (2001).

Elliot, K. T. and A. R, Kovscek, "A Numerical Analysis of the Single-Well Steam Assisted Gravity Drainage Process," Petroleum Science and Technology, 19(7\&8), 733-760 (2001).

George, D. S., O. Hayat, and A. R. Kovscek, "A Microvisual Study of Solution Gas Drive Mechanisms in Viscous Oils," Journal of Petroleum Science and Engineering, in review, submitted March 2003. 
Hoffman, B. T. and A. R. Kovscek, "Efficiency and Oil Recovery Mechanisms of Steam Injection into Low Permeability, Hydraulically Fractured Reservoirs," Petroleum Science and Technology, to appear.

Kovscek, A. R. and C. J. Radke, "Pressure-Driven Capillary Snap off of Gas Bubbles at Low Wetting-Liquid Content", Colloids and Surfaces A: Physicochemical and Engineering Aspects, 212(2\&3), 99-108 (2003).

Tang, G.-Q. and A. R. Kovscek, "An Experimental Investigation of the Effect of Temperature on Recovery of Heavy Oil From Diatomite," Society of Petroleum Engineers Journal, in review, submitted January 2003.

Rangel-German, E. R. and A. R. Kovscek,"Experimental and Analytical Study of Multidimensional Imbibition in Fractured Porous Media," Journal of Petroleum Science and Engineering, 36(1-2), 45-60 (2002).

Schembre, J. M. and A. R. Kovscek, "A Technique for Measuring Two-Phase Relative Permeability in Porous Media via X-ray CT Measurements, Journal of Petroleum Science and Engineering, 39(1\&2), 159-174 (2003).

Wang, Y. and A. R. Kovscek, "Integrating Production History into Reservoir Models Using Streamline-Based Time-of-Flight Ranking," Petroleum Geoscience, 9(2), 163-174 (2003).

Wang, Y. and A. R. Kovscek, "Streamline Approach for History Matching Production Data, " Society of Petroleum Engineers Journal, 25, 353-362 (2000).

Zhou, D., L. Jia, J. Kamath, and A. R. Kovscek, "Scaling of Counter-Current Imbibition Processes in Low-Permeability Porous Media," Journal of Petroleum Science and Engineering, 33(1-3), 61-74 (2002).

\section{Website}

To aid in the dissemination of technical information to industry and other research institutions, we developed an easy to navigate website that describes our research activities. It also serves as a virtual library of technical results. Papers, reports, and abstracts are available in portable document format (PDF) for simple download and viewing. The website is also useful for distributing information related to our annual meeting and short course. The address is http://ekofisk.stanford.edu/supria.html

\section{Short Course}

Also in the area of industrial interaction, we have developed and taught a short course on thermal recovery methods for engineers and geologists who wish to broaden their backgrounds. 
The course is oriented toward individuals with beginning to intermediate knowledge of thermal recovery. We taught the course in July of 2003 and June of 2001.

\section{International Cooperation}

International cooperation was also a feature of the technology transfer plan. We shared research results and research approaches with PDVSA-Intevep as Venezuela also possesses significant heavy oil resources, similar to the U.S. We participated in technical exchange via the Venezuelan Memorandum of Understanding (MOU) Annex IV. These meetings occurred March 16, 2001 at Porlamar Venezuela, April 19, 2002 at Stanford CA, and September 11, 2002 at Caracas Venezuela.

Another major vehicle for international cooperation was the International Energy Agency Collaborative Project on Enhanced Oil Recovery. We attended the Annual Symposium and Workshop: September 20-22 2000 Edinburgh Scotland, September 2001 Vienna Austria, September 8-10, 2002 Caracas Venezuela, September 7-10, 2003 Regina Saskatchewan Canada. Manuscripts and talks presented are listed below.

\section{IEA Presentations}

Brigham, W.E., "Fluid Flow in Various Patterns and Implications for EOR Pilot Flooding," $24^{\text {th }}$ Annual Workshop \& Symposium Collaborative Project on Enhanced Oil Recovery International Energy Agency, Regina, Saskatchewan, September 7-10, 2003.

Kovscek, A. R. and G. Q. Tang, "An Experimental Investigation of the Effect of Temperature on Recovery of Heavy Oil from Diatomite," $24^{\text {th }}$ Annual Workshop \& Symposium Collaborative Project on Enhanced Oil Recovery International Energy Agency, Regina, Saskatchewan, September 7-10, 2003.

Castanier, L.M., "Upgrading of Crude Oil Via In-situ Combustion," $23^{\text {rd }}$ Annual Workshop \& Symposium, Collaborative Project on Enhanced Oil Recovery International Energy Agency, Caracas, Venezuela (September 8-11, 2002).

Brigham, W.E., "Doublets and Other Allied Well Patterns. Part 1. Doublet Geometry, paper presented at the Annual International Energy Agency Workshop and symposium, Vienna, Austria (September 2001).

Apaydin, O. G. and A. R. Kovscek "Transient Foam Flow in Homogeneous Porous Media: Surfactant Concentration and End Effects," paper S-10, Proceedings of the 21st Annual International Energy Agency Workshop and Symposium, Edinburgh, Scotland September 21-24, 2000. 
Brigham, W.E., "Behavior of Reservoir/Aquifer Systems. Part 2. Predicting Future Performance, paper presented at the $21^{\text {st }}$ Annual International Energy Agency Workshop and Symposium, Edinburgh, Scotland (September 20-22 2000).

Brigham, W.E., "Using Water Cut or Change in Produced Water Composition to Diagnose Reservoir Heterogeneity," paper presented at the $21^{\text {st }}$ Annual International Energy Agency Workshop and symposium, Edinburgh , Scotland (September 20-22, 2000). 